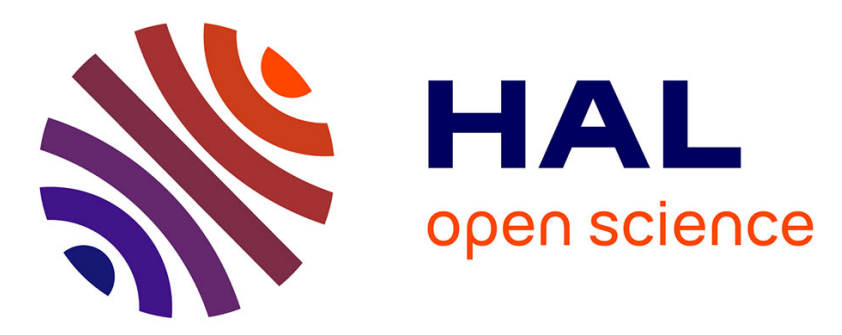

\title{
Antimicrobial resistance and virulence common mechanisms (ebook)
}

Etienne Giraud, Ivan Rychlik, Axel Cloeckaert

\section{To cite this version:}

Etienne Giraud, Ivan Rychlik, Axel Cloeckaert (Dir.). Antimicrobial resistance and virulence common mechanisms (ebook). Frontiers Media, 138 p., 2017, Frontiers in Microbiology, 9782889451814. 10.3389/978-2-88945-181-4 . hal-01605380

\section{HAL Id: hal-01605380 \\ https://hal.science/hal-01605380}

Submitted on 5 Jun 2020

HAL is a multi-disciplinary open access archive for the deposit and dissemination of scientific research documents, whether they are published or not. The documents may come from teaching and research institutions in France or abroad, or from public or private research centers.
L'archive ouverte pluridisciplinaire $\mathbf{H A L}$, est destinée au dépôt et à la diffusion de documents scientifiques de niveau recherche, publiés ou non, émanant des établissements d'enseignement et de recherche français ou étrangers, des laboratoires publics ou privés. 


\section{ANTIMICROBIAL RESISTANCE AND VIRULENCE COMMON MECHANISMS}

\section{EDITED BY: Etienne Giraud, Ivan Rychlik and Axel Cloeckaert PUBLISHED IN: Frontiers in Microbiology}

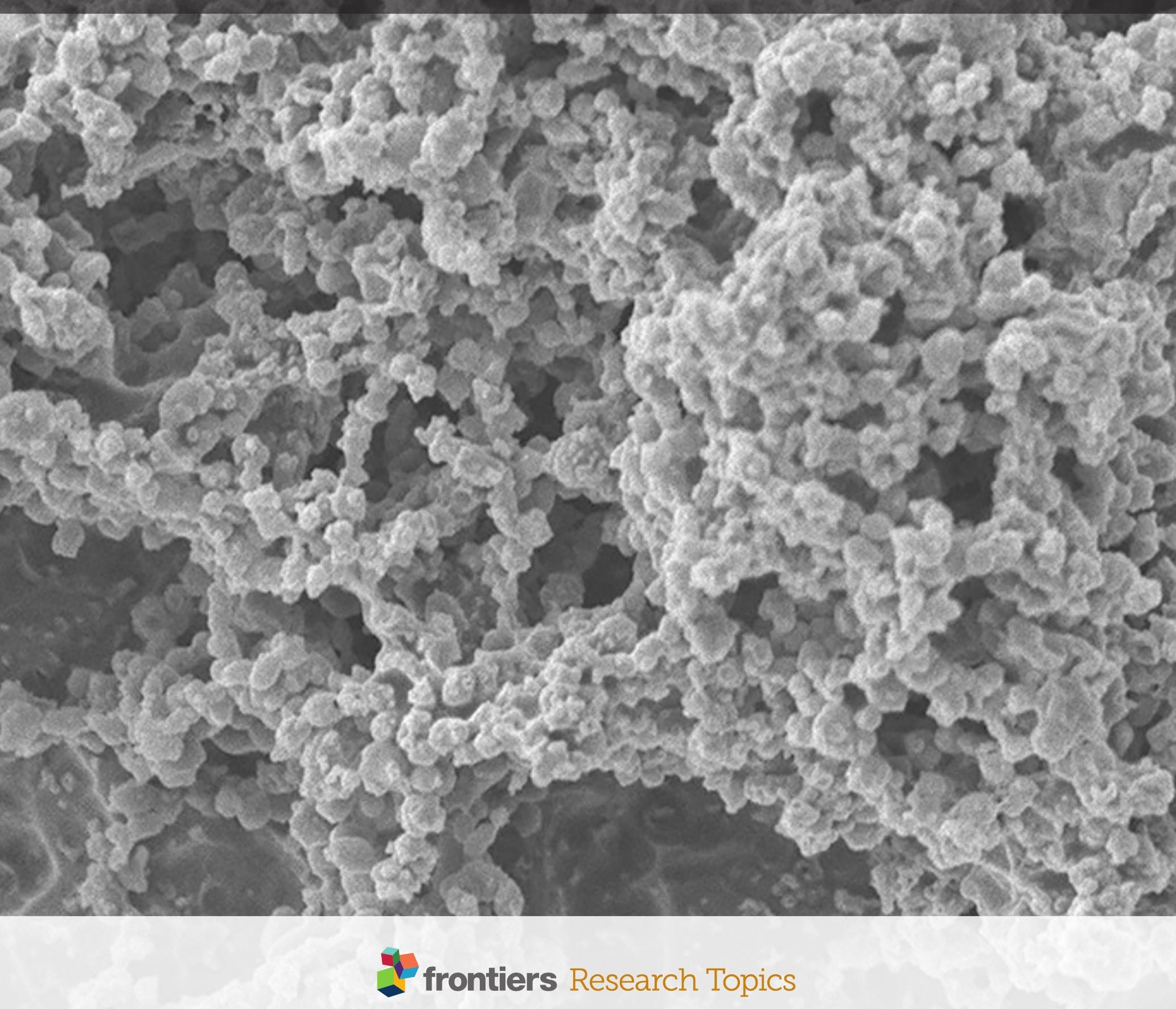




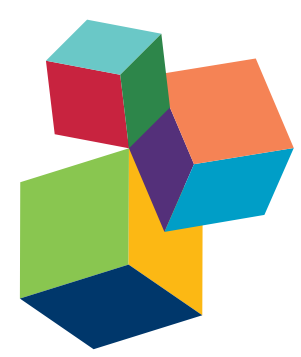

Frontiers Copyright Statement

(c) Copyright 2007-2017 Frontiers Media SA. All rights reserved.

All content included on this site, such as text, graphics, logos, button icons, images, video/audio clips, downloads, data compilations and software, is the property of or is licensed to Frontiers Media SA

("Frontiers") or its licensees and/or subcontractors. The copyright in the text of individual articles is the property of their respective authors, subject to a license granted to Frontiers.

The compilation of articles constituting this e-book, wherever published, as well as the compilation of all other content on this site, is the exclusive property of Frontiers. For the conditions for downloading and copying of e-books from Frontiers website, please see the Terms for Website Use. If purchasing Frontiers e-books from other websites or sources, the conditions of the website concerned apply.

Images and graphics not forming part of user-contributed materials may not be downloaded or copied without permission.

Individual articles may be downloaded and reproduced in accordance with the principles of the $C C-B Y$ licence subject to any copyright or other notices. They may not be re-sold as an e-book.

As author or other contributor you grant a CC-BY licence to others to reproduce your articles, including any graphics and third-party materials supplied by you, in accordance with the Conditions for Website Use and subject to any copyright notices which you include in connection with your articles and materials.

All copyright, and all rights therein, are protected by national and international copyright laws.

The above represents a summary only. For the full conditions see the Conditions for Authors and the Conditions for Website Use.

ISSN 1664-8714 ISBN 978-2-88945-181-4

DOI 10.3389/978-2-88945-181-4

\section{About Frontiers}

Frontiers is more than just an open-access publisher of scholarly articles: it is a pioneering approach to the world of academia, radically improving the way scholarly research is managed. The grand vision of Frontiers is a world where all people have an equal opportunity to seek, share and generate knowledge. Frontiers provides immediate and permanent online open access to all its publications, but this alone is not enough to realize our grand goals.

\section{Frontiers Journal Series}

The Frontiers Journal Series is a multi-tier and interdisciplinary set of open-access, online journals, promising a paradigm shift from the current review, selection and dissemination processes in academic publishing. All Frontiers journals are driven by researchers for researchers; therefore, they constitute a service to the scholarly community. At the same time, the Frontiers Journal Series operates on a revolutionary invention, the tiered publishing system, initially addressing specific communities of scholars, and gradually climbing up to broader public understanding, thus serving the interests of the lay society, too.

\section{Dedication to Quality}

Each Frontiers article is a landmark of the highest quality, thanks to genuinely collaborative interactions between authors and review editors, who include some of the world's best academicians. Research must be certified by peers before entering a stream of knowledge that may eventually reach the public - and shape society; therefore, Frontiers only applies the most rigorous and unbiased reviews.

Frontiers revolutionizes research publishing by freely delivering the most outstanding research, evaluated with no bias from both the academic and social point of view. By applying the most advanced information technologies, Frontiers is catapulting scholarly publishing into a new generation.

\section{What are Frontiers Research Topics?}

Frontiers Research Topics are very popular trademarks of the Frontiers Journals Series: they are collections of at least ten articles, all centered on a particular subject. With their unique mix of varied contributions from Original Research to Review Articles, Frontiers Research Topics unify the most influential researchers, the latest key findings and historical advances in a hot research area! Find out more on how to host your own Frontiers Research Topic or contribute to one as an author by contacting the Frontiers Editorial Office: researchtopics@frontiersin.org 


\section{ANTIMICROBIAL RESISTANCE AND VIRULENCE COMMON MECHANISMS}

Topic Editors:

Etienne Giraud, National Institute for Agricultural Research, France

Ivan Rychlik, Veterinary Research Institute, Czechia

Axel Cloeckaert, National Institute for Agricultural Research, France

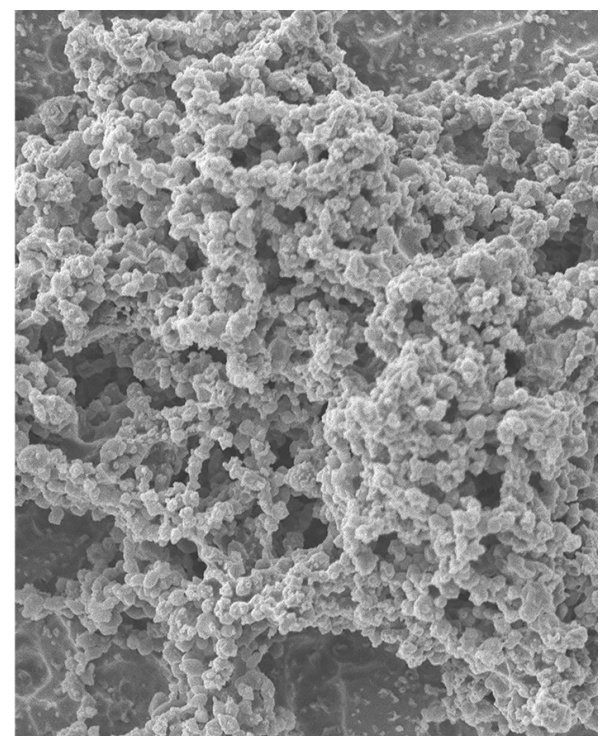

Scanning electron microscopy image of a Streptococcus suis biofilm. Certain physiological adaptations like biofilm formation may affect both virulence by increasing resistance to host defense response and resistance to antibiotics by decreasing their access to biofilm embedded bacteria.

Image taken from: Wang S, Yang Y, Zhao Y, Zhao H, Bai J, Chen J, Zhou Y, Wang C and Li Y (2016) Sub-MIC Tylosin Inhibits Streptococcus suis Biofilm Formation and Results in Differential Protein Expression. Front. Microbiol. 7:384. doi: 10.3389/fmicb.2016.00384

Multiple relationships exist between antimicrobial resistance and bacterial virulence, and the spread of clones combining multiple antibiotic resistance and a high virulence level is an increasing problem. It was previously described how mutation-driven or horizontally acquired resistance mechanisms can also have effects on virulence. It was also reported that mobile genetic elements often carry both resistance determinants and virulence-modulating genes, which favors the co-selection of both traits. In the present volume, we present a collection of articles which document additional aspects of the interactions between antimicrobial resistance and virulence in bacteria, and describe their potential therapeutic consequences.

Citation: Giraud, E., Rychlik, I., Cloeckaert, A., eds. (2017). Antimicrobial Resistance and Virulence Common Mechanisms. Lausanne: Frontiers Media. doi: 10.3389/978-2-88945-181-4 


\section{Table of Contents}

05 Editorial: Antimicrobial Resistance and Virulence Common Mechanisms

Etienne Giraud, Ivan Rychlik and Axel Cloeckaert

08 Multidrug Efflux Pumps at the Crossroad between Antibiotic Resistance and Bacterial Virulence

Manuel Alcalde-Rico, Sara Hernando-Amado, Paula Blanco and José L. Martínez

22 PSM-Mec-A Virulence Determinant that Connects Transcriptional Regulation, Virulence, and Antibiotic Resistance in Staphylococci

Li Qin, Joshua W. McCausland, Gordon Y. C. Cheung and Michael Otto

30 Sub-MIC Tylosin Inhibits Streptococcus suis Biofilm Formation and Results in Differential Protein Expression

Shuai Wang, Yanbei Yang, Yulin Zhao, Honghai Zhao, Jingwen Bai, Jianqing Chen,

Yonghui Zhou, Chang Wang and Yanhua Li

39 Absence of Protoheme IX Farnesyltransferase CtaB Causes Virulence Attenuation but Enhances Pigment Production and Persister Survival in MRSA

Tao Xu, Jian Han, Jia Zhang, Jiazhen Chen, Nan Wu, Wenhong Zhang and Ying Zhang

53 Polyphosphate Kinase Mediates Antibiotic Tolerance in Extraintestinal

Pathogenic Escherichia coli PCN033

Jing Chen, Lijie Su, Xiangru Wang, Tao Zhang, Feng Liu, Huanchun Chen and Chen Tan

63 The Small Colony Variant of Listeria monocytogenes Is More Tolerant to Antibiotics and Has Altered Survival in RAW 264.7 Murine Macrophages

Thomas D. Curtis, Lone Gram and Gitte M. Knudsen

74 Carriage of Extended-Spectrum Beta-Lactamase-Plasmids Does Not Reduce Fitness but Enhances Virulence in Some Strains of Pandemic E. coli Lineages

Katharina Schaufler, Torsten Semmler, Derek J. Pickard, María de Toro,

Fernando de la Cruz, Lothar H. Wieler, Christa Ewers and Sebastian Guenther

86 Fitness Cost of Fluoroquinolone Resistance in Clinical Isolates of Pseudomonas aeruginosa Differs by Type III Secretion Genotype

Melissa Agnello, Steven E. Finkel and Annie Wong-Beringer

98 Virulence and Genomic Feature of Multidrug Resistant Campylobacter jejuni Isolated from Broiler Chicken

Haihong Hao, Ni Ren, Jing Han, Steven L. Foley, Zahid Iqbal, Guyue Cheng,

Xiuhua Kuang, Jie Liu, Zhenli Liu, Menghong Dai, Yulian Wang and Zonghui Yuan

112 Is Quorum Sensing Interference a Viable Alternative to Treat Pseudomonas aeruginosa Infections?

Rodolfo García-Contreras 
119 PA3297 Counteracts Antimicrobial Effects of Azithromycin in Pseudomonas aeruginosa

Hao Tan, Lu Zhang, Yuding Weng, Ronghao Chen, Feng Zhu, Yongxin Jin, Zhihui Cheng, Shouguang Jin and Weihui Wu

131 Efflux Pump Blockers in Gram-Negative Bacteria: The New Generation of Hydantoin Based-Modulators to Improve Antibiotic Activity

Ewa Otrębska-Machaj, Jacqueline Chevalier, Jadwiga Handzlik, Ewa Szymańska, Jakub Schabikowski, Gérard Boyer, Jean-Michel Bolla, Katarzyna Kieć-Kononowicz, Jean-Marie Pagès and Sandrine Alibert 


\title{
Editorial: Antimicrobial Resistance and Virulence Common Mechanisms
}

\author{
Etienne Giraud ${ }^{1,2 *}$, Ivan Rychlik ${ }^{3}$ and Axel Cloeckaert ${ }^{1}$ \\ 1 ISP, Institut National de la Recherche Agronomique, Université François Rabelais de Tours, UMR 1282, Nouzilly, France, \\ 2 TOXALIM (Research Centre in Food Toxicology), ENVT, INP-Purpan, Institut National de la Recherche Agronomique, UPS, \\ Université de Toulouse, Toulouse, France, ${ }^{3}$ Department of Immunology, Veterinary Research Institute, Brno, Czechia
}

Keywords: antimicrobial resistance mechanisms, virulence, co-evolution, biofilms, fitness costs

Editorial on the Research Topic

\section{Antimicrobial Resistance and Virulence Common Mechanisms}

\section{OPEN ACCESS}

Edited by:

Rustam Aminov,

University of Aberdeen, UK

Reviewed by:

Rustam Aminov,

University of Aberdeen, UK

Jose L. Martinez,

Consejo Superior de Investigaciones

Cientificas, Spain

Kunihiko Nishino,

Osaka University, Japan

*Correspondence:

Etienne Giraud

etienne.giraud@inra.fr

Specialty section:

This article was submitted to

Antimicrobials, Resistance and

Chemotherapy,

a section of the journal

Frontiers in Microbiology

Received: 30 January 2017

Accepted: 15 February 2017

Published: 03 March 2017

Citation:

Giraud E, Rychlik I and Cloeckaert A

(2017) Editorial: Antimicrobial

Resistance and Virulence Common

Mechanisms. Front. Microbiol. 8:310.

doi: 10.3389/fmich.2017.00310
Multiple relationships exist between antimicrobial resistance and bacterial virulence, and the spread of clones combining multiple antibiotic resistance and a high virulence level is an increasing problem. These relationships have been previously reviewed, notably by Beceiro et al. (2013), who described how mutation-driven or horizontally acquired resistance mechanisms can also have effects on virulence. It was also reported that mobile genetic elements often carry both resistance determinants and virulence-modulating genes, which favors the co-selection of both traits. In the present volume, we present a collection of articles which document additional aspects of the interactions between antimicrobial resistance and virulence in bacteria, and describe their potential therapeutic consequences.

In an excellent review article, Alcade-Rico et al. established that multidrug efflux pumps are "at the cross-road between resistance and virulence of bacterial pathogens." They describe how multidrug efflux pumps are involved, besides antibiotic resistance, in many physiological and virulence-related processes controlled by complex regulatory networks. A special focus is made on the role of these efflux pumps in bacterial cell-to-cell communication and in bacterial-host interactions.

Another example of link between resistance and virulence in Staphylococci is given in a mini review by Qin et al. The authors summarized how the psm-mec locus present in some methicillin-resistant Staphylococcus aureus (MRSA) and coagulase-negative Staphylococci influences methicillin resistance and virulence-related phenotypes, including the ability to form biofilms.

In another paper also dealing with biofilms, Wang et al. used an up-to-date iTRAQ protein labeling technology to identify proteins that are differencially expressed in Streptococcus suis treated with sub-MIC concentrations of tylosin. These concentrations were indeed capable of inhibition of biofilm formation by this zoonotic pathogen, which is of interest, since biofilms are known to display an increased tolerance to antibiotics.

This antibiotic tolerance of bacteria present in biofilms is thought to be at least partially due to the presence of higher numbers of persisters than in planktonic bacterial populations. Persisters are phenotypic variants of bacteria which are able to survive antibiotic treatments in a dormancy state and to resume growth when the (antibiotic) stress is withdrawn. Like antibiotic-resistant bacteria, antibiotic-tolerant persisters are also often responsible for failures of antibiotic therapies. However, the mechanisms underlying their production are still not well-understood. An article by $\mathrm{Xu}$ et al. lifts a corner of the veil on this subject in Staphylococcus aureus by suggesting that heme biosynthesis, in addition to being necessary to a full expression of virulence, is also important in the formation of persisters in S. aureus. 
Another study by Chen et al. addressed the problem of antibiotic tolerance in extraintestinal pathogenic Escherichia coli (ExPEC). Authors studied the role of polyphosphate kinase (PPK), an enzyme already known for its implication in motility, quorum sensing or virulence, in antibiotic tolerance. Their results indicate that PPK is important for the antibiotic stress response and may therefore be a valuable antibacterial target, especially since it is absent in mammals.

Small Colony Variants (SCV) represent another state in which bacteria are more tolerant to antibiotics. The clinical importance of SCV has been well-documented in cases of chronic infections by $S$. aureus and Pseudomonas aeruginosa but their existence was only recently reported in Listeria monocytogenes, a food pathogen producing recurrent infections despite being rarely resistant to antibiotics. In an original research article, Curtis et al. described a heme mutant displaying a SCV phenotype mutant with an increased tolerance toward most of the relevant antibiotics. They suggest that the SCV phenotype of L. monocytogenes should be screened in clinical laboratories, as it might be associated to complicated antibiotic treatments.

Genetic changes leading to antimicrobial resistance are expected to result in higher fitness costs in the absence of antibiotic selection pressure. On the other hand, increased resistance is sometimes associated with increased virulence or to a better adaptation to stress conditions. This topic is developed in a study by Schaufler et al. who reported that the carriage of extended spectrum beta-lactamase (ESBL)-plasmids by strains of pandemic E. coli lineages did not lead to a fitness cost. Instead, these plasmids could enhance virulence or adaptation to specific habitats by influencing the expression of chromosomal genes.

In another article dealing with bacterial fitness, Agnello et al. presented results that possibly explain why highly virulent $P$. aeruginosa strains, which often possess the exoU toxin gene, are more frequently resistant to fluoroquinolones than the generally less virulent strains harboring the exoS toxin gene. Their results support the hypothesis that fitness cost imposed by fluoroquinolone resistance may be lower and "more easily" compensable by exoU strains than by exoS strains.

In a whole genome sequence-based study, Hao et al. analyze a multidrug resistant and virulent Campylobacter jejuni strain isolated from a broiler chicken. By comparison to reference strains, they revealed that the phenotype of this isolate was associated with large differences in the genome structure and content. This study provides an example of how a stepwise accumulation of mutations in antibiotic target genes, acquisition of exogenous resistance genes and virulence associated genes can result in the emergence of isolates that are both multidrugresistant and virulent.

Since quorum sensing is a major player in the control of virulence factors in $P$. aeruginosa, therapies based on quorum sensing interference (QSI) have been envisaged as possible alternatives to conventional antibiotic therapies against this pathogen. In a thoughtful perspective article, Garcia-Contreras, however, compiles many recent experimental evidence which actually indicate that early optimistic expectations about QSI should be put in perspective. Indeed, he estimates that a far better understanding of $P$. aeruginosa virulence and behavior during infection is needed before efficient QSI-based clinical applications can be designed.

In addition to some immunomodulating activities, azithromycin (AZM) has various inhibiting effects on $P$. aeruginosa such as bacterial killing or the repression of multiple virulence factors. This antibiotic is therefore currently used in the treatment of cystic fibrosis patients, whose lungs are commonly colonized by this pathogen. However, $P$. aeruginosa responds to AZM by yet unknown mechanisms and is able to counteract its killing and virulenceinhibitory effects. An interesting work by Tan et al. revealed that PA3297, a $P$. aeruginosa gene encoding a DEAH-box helicase, is involved in this response. Indeed, deficiency of this gene renders $P$. aeruginosa more susceptible to the killing and to virulence suppression by AZM. Authors therefore suggested that targeting the regulatory pathway of PA3297 or its function might increase the beneficial effect of AZM in chronic $P$. aeruginosa infections such as those occuring in cystic fibrosis patients.

At last, in a pharmacological perspective, Obtreska-Machaj et al. report the development of hydantoin derivatives that have increased activity as inhibitors of the AcrAB-TolC efflux pump of Enterobacter aerogenes. Close homologs of this multidrug pump are also present in other pathogenic enterobacteria and involved in their virulence. The authors defined important pharmacophoric groups whose modulation may allow to develop still more potent inhibitors capable of restoring antibiotic activity in $A c r A B$ producing bacteria.

It is likely that both resistant and virulent high risk clones will continue to emerge and spread in the next years. Several factors supports this hypothesis, among which the facts that highly pathogenic bacterial strains are more likely to be subjected to the selective pressure of antibiotics treatments, that virulence and resistance determinants are sometimes genetically associated and that fitness and virulence impairments that often accompany acquisition of resistance can be compensated in the long term by suppressor mutations (Beceiro et al., 2013; Mathers et al., 2015). In spite of these facts, virulence and antimicrobial resistance are studied usually separately rather than jointly. But now, as exemplified in this research topic, time has come for more integrative studies linking both traits and their coevolution, so that we may be better armed against future bacterial threats.

\section{AUTHOR CONTRIBUTIONS}

All authors have substantially contributed to this work and approved it for publication. 


\section{REFERENCES}

Beceiro, A., Tomás, M., and Bou, G. (2013). Antimicrobial resistance and virulence: a successful or deleterious association in the bacterial world? Clin. Microbiol. Rev. 26, 185-230. doi: 10.1128/CMR.00 059-12

Mathers, A. J., Peirano, G., and Pitout, J. D. D. (2015). The role of epidemic resistance plasmids and international high-risk clones in the spread of multidrug-resistant enterobacteriaceae. Clin. Microbiol. Rev. 28, 565-591. doi: 10.1128/CMR.00116-14
Conflict of Interest Statement: The authors declare that the research was conducted in the absence of any commercial or financial relationships that could be construed as a potential conflict of interest.

Copyright (c) 2017 Giraud, Rychlik and Cloeckaert. This is an open-access article distributed under the terms of the Creative Commons Attribution License (CC BY). The use, distribution or reproduction in other forums is permitted, provided the original author(s) or licensor are credited and that the original publication in this journal is cited, in accordance with accepted academic practice. No use, distribution or reproduction is permitted which does not comply with these terms. 


\title{
Multidrug Efflux Pumps at the Crossroad between Antibiotic Resistance and Bacterial Virulence
}

\author{
Manuel Alcalde-Rico, Sara Hernando-Amado, Paula Blanco and José L. Martínez*
}

Departamento de Biotecnología Microbiana, Centro Nacional de Biotecnología, Consejo Superior de Investigaciones Científicas, Madrid, Spain

Multidrug efflux pumps can be involved in bacterial resistance to antibiotics at different levels. Some efflux pumps are constitutively expressed at low levels and contribute to intrinsic resistance. In addition, their overexpression may allow higher levels of resistance. This overexpression can be transient, in the presence of an effector (phenotypic resistance), or constitutive when mutants in the regulatory elements of the expression of efflux pumps are selected (acquired resistance). Efflux pumps are present in all cells, from human to bacteria and are highly conserved, which indicates that they are ancient elements in the evolution of different organisms. Consequently, it has been suggested that, besides antibiotic resistance, bacterial multidrug efflux pumps would likely contribute to other relevant processes of the microbial physiology. In the current article, we discuss some specific examples of the role that efflux pumps may have in the bacterial virulence of animals' and plants' pathogens, including the processes of intercellular communication. Based in these evidences, we propose that efflux pumps are at the crossroad between resistance and virulence of bacterial pathogens. Consequently, the comprehensive study of multidrug efflux pumps requires addressing these functions, which are of relevance for the bacterial-host interactions during infection.

Keywords: multidrug efflux pumps, quorum sensing, antibiotic resistance mechanisms, virulence, global regulation

\section{INTRODUCTION}

Multidrug resistance (MDR) efflux pumps are relevant elements belonging to the microbial repertoire that bacteria harbor for resisting the action of antimicrobial drugs (Piddock, 2006a; Vila and Martínez, 2008; Li et al., 2015; Jang, 2016). Indeed, several works have shown that these elements are involved in resistance of in vitro selected mutants as well as in the reduced susceptibility to antimicrobials of clinical isolates of different bacterial pathogens. The expression of efflux pumps is usually down regulated; only some of them present a substantial level of expression under regular growing conditions in the laboratory (Grkovic et al., 2001, 2002). However, constitutive high-level expression of these elements can be achieved by means of mutations in the elements regulating their expression. Transient high-level expression of efflux pumps can also be triggered in the presence of their effectors or under some specific growing conditions. In agreement with this situation, efflux pumps contribute to antibiotic resistance at three different levels: they can be involved in intrinsic resistance when presenting a basal level of expression under any condition. 
They can contribute to acquired resistance when mutants achieving high-level of expression of the efflux pumps are selected. Finally, they can contribute to transient, noninheritable, phenotypic resistance when bacteria are growing in the presence of an effector of the efflux pump or under growing conditions that trigger their overexpression. As reviewed in Hernando-Amado et al. (2016), efflux pumps are grouped in five structural families, namely the resistancenodulation-division (RND), the small multidrug resistance (SMR), the multi antimicrobial extrusion (MATE), the major facilitator superfamily (MFS), and the ATP-binding cassette (ABC) superfamilies. Whereas some efflux pumps can work independently of any other protein, mainly in the case of Grampositive organisms, in the case of Gram-negative organisms, they form tripartite complexes capable to traverse both bacterial membranes. These complexes include the inner-membrane efflux pump, a membrane fusion protein and an outer membrane protein.

When compared with other classical resistance genes, MDR efflux pumps present some specific features that support they should have other roles in the bacterial physiology besides their well-known involvement in antibiotic resistance. First, MDR efflux pumps are ubiquitous; they are present in all living cells, from humans to bacteria (Alonso et al., 1999; Alonso and Martinez, 2001; Gould et al., 2004; Sanchez et al., 2004). Second, the genes encoding them belong to the bacterial core genome in the sense that all (or most) members of a given species harbor the same efflux pumps (Alonso et al., 1999). Third, they are redundant; a single bacterial cell usually contains more than 10 different efflux pumps (Crossman et al., 2008). Fourth, they are rather unspecific; each efflux pump is able to extrude a variety of different substrates, including synthetic antibiotics as quinolones (Hernandez et al., 2011; Redgrave et al., 2014). Fifth, as above mentioned the expression of efflux pumps is tightly regulated; this regulation includes local regulators usually encoded upstream the structural genes of the operon encoding the efflux pump, as well as global regulators (Randall and Woodward, 2002; Luong et al., 2003; Nikaido et al., 2008; De Majumdar et al., 2013), frequently controlling the expression of a set of genes involved in the adaptation to a given ecosystem, as is the infected host. Sixth, at least in occasions, antibiotics are not good effectors of the expression of efflux pumps, whereas host-produced compounds as bile salts or plant-produced signals may induce the expression of MDR pumps (Rosenberg et al., 2003; Prouty et al., 2004; García-León et al., 2014). Altogether, these characteristics support that MDR efflux pumps are ancient elements (present in all organisms), important for the bacterial physiology (all members of a given species present the same, conserved efflux pumps), likely displaying different functions besides antibiotic resistance (a single microorganism contains a large number of different efflux pumps, with overlapping substrate ranges, including synthetic antibiotics not present in nature) and frequently integrated in complex response networks (they form part of global regulons and their expression is triggered by host produced compounds). In the present article we discuss some examples of the potential functions, besides antibiotic resistance, of MDR efflux pumps with a particular focus on the role that they may have in bacterial-host interactions in animals (humans) and plants as well as in intercellular signaling (Piddock, 2006b; Martinez et al., 2009; Alvarez-Ortega et al., 2013).

\section{EFFLUX PUMPS AND CELL-TO-CELL COMMUNICATION}

The capability to sense the environment and the organisms that are living in the same niche is critical to allow the microorganisms for choosing the best strategy to survive and colonize such niche. Along evolution, a battery of different mechanisms to sense the continuously changing environment has been selected in different microbial species. One of these mechanisms consists on the cell-to-cell communication systems. These inter-cellular signaling systems are based on the production of one or more low-molecular weight compounds which are sensed by molecular receptors of other cells, promoting a specific response in the target organism. In the bacterial world, this phenomenon is known as quorum sensing (QS) because it was initially described as a mechanism to sense the density of the bacterial population belonging to the same species present in a given habitat. The QS system allows the establishment of a cooperative genetic program of the whole population that increases the microbial efficacy for colonizing a given environment, including the infected host (Williams et al., 2007). The QS signal molecules (QSSMs) are constitutively produced at low quantities by all cells in the population. Their release outside the cells allows a progressive accumulation of QS signals in the intercellular space. The consequence is an increasingly production and accumulation of QSSMs while the population size increases, activating the QS response when the concentration of the signal reaches a threshold level. Further, these molecules (known as autoinducers) are able to induce their own production when they bind to their cognate transcriptional regulators, which produce a feedforward regulation circuit. The regulator-QSSM complex is the main responsible of triggering the QS response by increasing the expression of a large number of genes, including those encoding the autoinducer synthase enzymes, which further increases the QSSM production. This response coordinates a number of physiological changes at a population-scale level, which allows among other issues improving bacterial competition for nutrients with other species, forming morphological-resistance structures to overcome environmental threats or triggering the expression of virulence factors (Swift and Downie, 2001; Williams, 2007). Further, it has been shown that several QSSMs can be sensed by other species promoting responses mediated by inter-species communication processes (Williams et al., 2007; Shimada et al., 2013; Lee and Zhang, 2015), including eukaryotic cells (interkingdom signaling; Martinez, 2014). As above stated these QSSMs have to cross the cells membranes for their extracellular accumulation, and it has been reported that some MDR efflux pumps might be involved in their transport outside the cell. In this section, we will discuss the role of MDR efflux pumps in the modulation of intercellular signaling and how the acquisition of antibiotic resistance in mutants overexpressing efflux pumps 

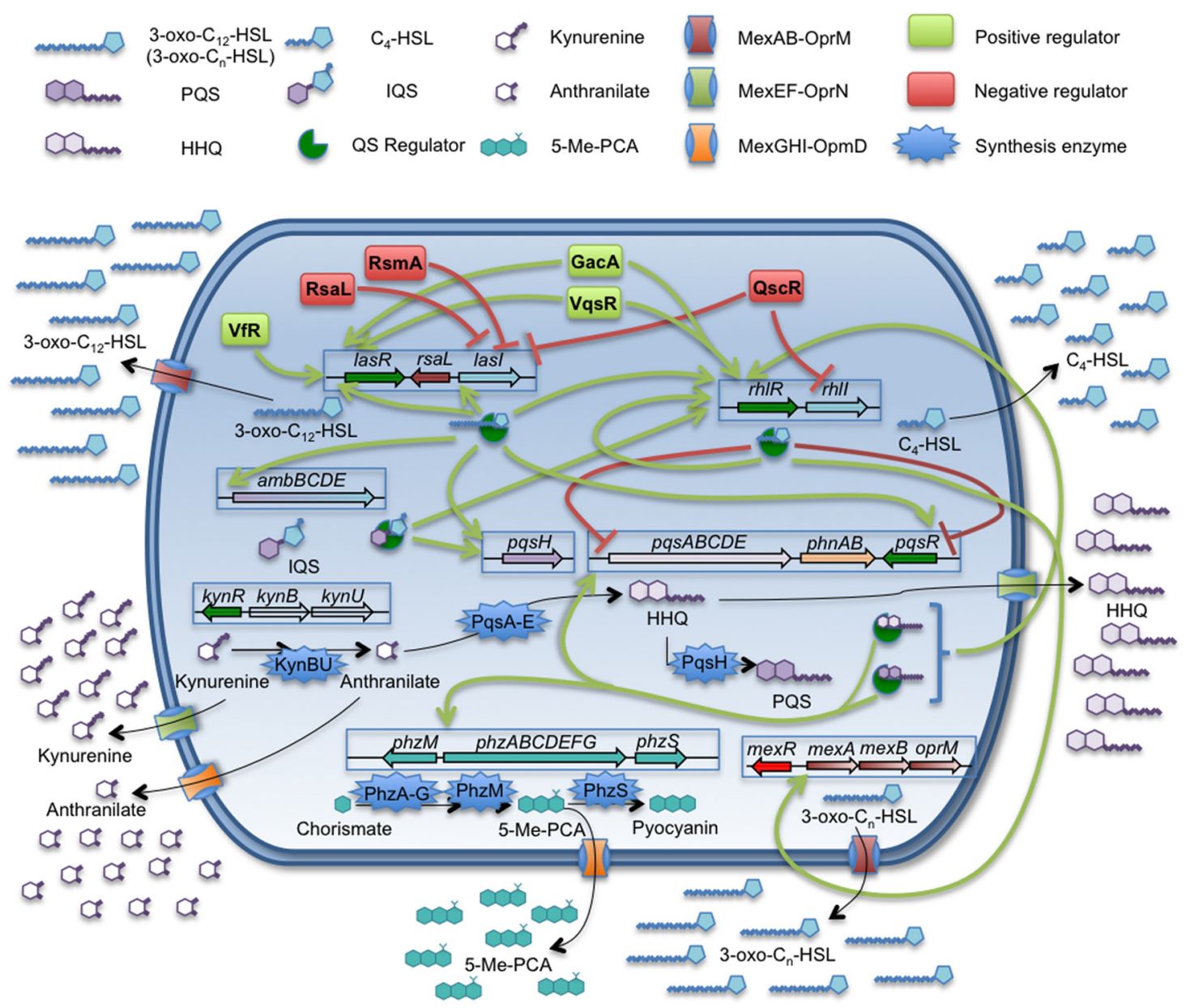

FIGURE 1 | Quorum sensing network and related efflux pumps in $\boldsymbol{P}$. aeruginosa. The QS signals produced by $P$. aeruginosa are: $3-\mathrm{O}$ - $\mathrm{xo}-\mathrm{C}_{12}-\mathrm{HSL}, \mathrm{C}_{4}-\mathrm{HSL}$, $\mathrm{PQS} / \mathrm{HHQ}$, and the recently discovered IQS. The figure sums up the complexity of the QS regulation network and the implications of MexAB-OprM, MexEF-OprN, and MexGHI-OpmD efflux pumps on the extrusion of QSSMs, their precursors or molecules which expression is QS-regulated. Despite of $\mathrm{C}_{4}-\mathrm{HSL}$ is able to cross the cell envelopes by free diffusion, the expression of mexAB-oprM is triggered in presence of this autoinducer. Further, this efflux pump is able to extrude 3-oxo- $\mathrm{C}_{12}-\mathrm{HSL}$ and others related 3-oxo- $\mathrm{C}_{n}-\mathrm{HSL}$. The MexEF-OprN system is able to extrude $\mathrm{HHQ}$ and kynurenine, a precursor of 4-alkyl-quinolones, having an impact on the QS response. In the case of MexGHI-OpmD, its role has been recently linked to the extrusion of 5-Me-PCA, a precursor of the phenazine pyocyanin, whose production is induced upon the QS response. However, it has been proposed that this system is able to efflux anthranilic acid, another AQs intermediate, which is toxic for the cell at high concentrations. P. aeruginosa is a good example for the potential role of efflux systems in modulating the cell-to-cell communication networks.

may challenge bacterial virulence through alterations in the diffusion of QS signals.

Pseudomonas aeruginosa is one of the most important opportunistic pathogens causing infections at hospitals as well as in cystic fibrosis patients (Buhl et al., 2015; Kaye and Pogue, 2015; Oliver et al., 2015; Talwalkar and Murray, 2016). This pathogen is able to produce different virulence factors, many of them being regulated by a hierarchically organized QS signaling system (Figure 1), which consists in three different and interconnected regulatory networks each one respectively governed by the transcriptional regulators LasR, RhlR, and PqsR (Lee and Zhang, 2015). These three QS regulators recognize respectively one of different QSSMs produced by $P$. aeruginosa: $N$-(3-oxododecanoyl)-L-homoserine lactone (3oxo-C12-HSL), N-butanoyl-L-homoserine lactone (C4-HSL), and Pseudomonas quinolone signal (PQS). The presence of an additional regulatory QS network has been recently described (Lee et al., 2013; Lee and Zhang, 2015). Despite being tightly regulated by the las system in standard conditions of growth, this new system is able to trigger the PQS and C4-HSL production in absence of the las system or under phosphate stress conditions. However, the mechanisms responsible of the regulation mediated by this QS system are not fully understood and consequently will not be discussed along the present review.

Some works have shown that the expression of different efflux pumps encoded in the $P$. aeruginosa chromosome may have an impact in the QS-regulation networks of this microorganism. For instance, the RND efflux pump MexAB-OprM is highly integrated within the las and $r h l$ QS regulons, since it has 
been shown that its expression can be induced by C4-HSL (Maseda et al., 2004; Sawada et al., 2004). In addition, this efflux pump is able to extrude the 3-oxo-C12-HSL QS signal (Evans et al., 1998; Pearson et al., 1999; Minagawa et al., 2012). In such a way, the constitutive high-level expression of MexABOprM entails the extrusion of 3-oxo-C12-HSL, increasing the concentration of this QS signal around the cell, but reducing its intracellular accumulation. Indeed, antibiotic resistant mutants overexpressing MexAB-OprM present defects in the production of several virulence factors and are impaired in their QS response (Evans et al., 1998).

It is important to highlight that the 3-oxo-C12-HSL autoinducer is not just an intra-specific signal compound but may also entail inter-kingdom signaling. For instance, it has been reported that this QS signal may inhibit the filamentous differentiation, which is linked to a virulent state of Candida albicans, a fungal pathogen commonly found in patients with P. aeruginosa infections (Hogan et al., 2004). Conversely, it has been also shown that the C. albicans QS compound farnesol in turn can inhibit the production of QSSMs and the production of virulence factors by $P$. aeruginosa (Méar et al., 2013), evidencing the relevance of these cell-to-cell communication "weapons" in the establishment of a competitive interaction between these two opportunistic pathogens. In addition to its role in inter-microbial interactions, a role as activator of the human immune system has been attributed to 3-oxo-C12-HSL. This autoinducer signal can act as a chemoattractant for polymorphonuclear neutrophils and is able to induce the expression of adhesion proteins and immunoglobulin receptors implicated in the recognition and localization of microbial infections (Smith et al., 2001; Zimmermann et al., 2006; Wagner et al., 2007). On the other hand, it has been shown that 3-oxo-C12-HSL can induce the apoptosis of neutrophils and macrophages (Tateda et al., 2003). The fact that 3-oxo-C12-HSL has been detected directly in the sputum of cystic fibrosis patients with $P$. aeruginosa infections indicates that this QS signal might be involved in in vivo interkingdom signaling (Erickson et al., 2002; Middleton et al., 2002).

Pseudomonas aeruginosa MDR strains overexpressing MexAB-OprM produce lower amounts of virulence factors as well as of 3-oxo-C12-HSL (Evans et al., 1998). In addition, mexAB-OprM defective mutants are avirulent in a mouse model of infection as well as in MDCK cells (Hirakata et al., 2002). These results support the notion that this efflux system is directly involved in the appropriate and coordinate las response needed for a successful host infection. Further, it has been proposed that this system is used by $P$. aeruginosa to optimize indirectly the specific binding of the LasR regulator to 3-oxo-C12-HSL by extruding other 3-oxo-Cn-HSL, which can affect the las network activation because they are able of competing with 3-oxo-C12HSL for the same binding site in LasR (Minagawa et al., 2012). All these intra- and interspecific responses mediated by 3-oxoC12-HSL suggest that MexAB-OprM is an important element modulating cell-to-cell communication and host-pathogen interactions. As a consequence, the acquisition of antimicrobial resistance through the overexpression of this system could have an impact, at several levels, on the virulence of $P$. aeruginosa.
Besides MexAB-OprM, other $P$. aeruginosa efflux pumps might be involved in the regulation of the expression of QSdependent virulence factors. One of them is MexEF-OprN, an efflux pump able to extrude both the QS signal HHQ (Lamarche and Deziel, 2011) and its precursor, kynurenine (Olivares et al., 2012), making it an important element in the QS response (Köhler et al., 2001). As we have mentioned above, the virulence and pathogenicity of $P$. aeruginosa is partially controlled by the PQS communication system. Therefore, it is not surprising that, as it happens in the case of MexAB-OprM, mutants overexpressing MexEF-OprN are affected in the production of QS-regulated virulence factors and, in consequence, they are impaired in the host infection process (Olivares et al., 2012). It is worth mentioning that the extrusion of kynurenine by this system could have an additional role in $P$. aeruginosa-host interaction. It has been shown that the expression of the MexEF-OprN system can be induced upon contact with human airway epithelial cells (Frisk et al., 2004). On the other hand, it has been suggested that $P$. aeruginosa production of kynurenine may have a role in the bacterial resistance to the toxic reactive oxygen species (ROS) produced by neutrophils, a key cell component in the innate immune system and in the inflammatory response in lung infections (Genestet et al., 2014). Based on this situation, it is possible that the increased expression of MexEF-OprN upon contact with the lung epithelial cells may allow $P$. aeruginosa to secrete high levels of kynurenine, thus promoting resistance against the neutrophil ROS production. This possibility, which has not been explored yet, would provide MexEF-OprN a new role in the infected lungs beyond the modulation of the QS response.

Another $P$. aeruginosa efflux pump with potential relevance in the virulence of this pathogen is MexGHI-OprD (Aendekerk et al., 2005; Dietrich et al., 2006). It has been shown that this efflux pump is able of extruding 5-methylphenazine-1-carboxylate (5-Me-PCA), a precursor of the phenazine pyocyanin, and anthranilate, the immediate precursor of PQS (Aendekerk et al., 2005). Further, the mexGHI-oprD expression could be induced by 5 -Me-PCA and, in consequence, is under the transcriptional control of the QS response. It is worth mentioning that the phenazine extruded by this efflux pump is required for biofilm development by P. aeruginosa (Sakhtah et al., 2016). If we take into consideration that biofilms are the regular way of growing of $P$. aeruginosa in the lungs of chronically infected patients (Martinez-Solano et al., 2008; Wagner and Iglewski, 2008), this indicates that MexGHI might have a relevant role in the adaptation of $P$. aeruginosa for colonizing that habitat.

Another relevant pathogen whose virulence has been associated to the extrusion of QS signals by efflux pumps is Burkholderia pseudomallei, the causal agent of melioidosis. Different works have suggested that the virulence of this pathogen is modulated by the stationary phase sigma factor RpoS in addition to the regulatory activity of a QS system based on both acyl-homoserine lactones (AHLs) and 2alkyl-4-quinolones (Ulrich et al., 2004; Wongtrakoongate et al., 2012; Butt et al., 2016). B. pseudomallei produces up to six different homoserine lactones: $\mathrm{N}$-octanoylhomoserine lactone (C8-HSL), $N$-decanoyl-homoserine lactone 
(C10-HSL), N-(3-hydroxy)-octanoyl-homoserine lactone (3$\mathrm{OH}$-C8-HSL), $\mathrm{N}$-(3-hydroxy)-decanoyl-homo-serine lactone (3-OH-C10-HSL), N-(3-oxo)-decanoyl-homoserine lactone (3-oxo-C10-HSL), and N-(3-oxo)-tetradecanoyl-homoserine lactone (3-oxo-C14-HSL). It has been reported that in the clinical strain KHW, the BpeAB-OprB efflux pump is strictly needed for the synthesis and full extrusion of these six AHLs and it has been suggested that another efflux pump, AmrAB-OprA could also be involved in the extrusion of 3-oxo-C10-HSL (Chan et al., 2007). In agreement with a potential role of these efflux pumps in QS communication, it has been shown that bpeAB-oprB expression is induced in presence of exogenous C8-HSL and C10-HSL (Chan and Chua, 2005). Moreover, a bpeAB-oprB defective mutant is affected in the expression of QS-dependent virulence factors as well as in biofilm formation. In addition, the mutant presents impaired invasiveness and cytotoxicity in both human macrophages and lung epithelial cells. Even though the role of BpeAB-OprB in virulence might be strain-dependent (Mima and Schweizer, 2010), all of the aforementioned results support the hypothesis that some efflux systems could be involved in the modulation of B. pseudomallei virulence and in the host-pathogen interactions through the extrusion of QS communication signals or by responding to their presence.

Other signaling networks involved in intra- and inter-specific communication are based in the use of indole and its derived compounds as signal molecules (Bommarius et al., 2013; Shimada et al., 2013; Lee J.H. et al., 2015). Indole is synthesized by plants and by many different bacteria and has different roles depending on the target species. Even though it was proposed that the efflux pump AcrEF could be implicated in indole export in Escherichia coli (Kawamura-Sato et al., 1999), other works have shown that indole is able to diffuse easily across the bacterial envelope (Gaede et al., 2005; Pinero-Fernandez et al., 2011) so that a clear role of efflux pumps in indole trafficking remains controversial. However, a crosstalk between virulence and efflux pumps-linked antibiotic resistance mediated through indole signaling is possible. Indeed, indole may affect the expression of efflux pumps, the resistance to antibiotics and the behavior of Salmonella enterica serovar Typhimurium when growing inside the host (Nikaido et al., 2012). This bacterial pathogen does not produce indole. However, it usually lives together with indole-producing species in the host and is able to respond to this signal (Nikaido et al., 2011). It is proposed that indole blocks the activity of the RamR transcriptional regulator through the interaction with its C-terminal domain, leading to the overexpression of RamA activator (Nikaido et al., 2011, 2012). This regulatory protein is able to induce the expression of $\operatorname{acr} A B$, $a c r E F$, and $t o l C$, in addition of repressing the expression of some virulence determinants (Bailey et al., 2010; Nikaido et al., 2012). This entails the development of a low-virulence phenotype, which presents, however, increased resistance to antibiotics as well as to host-produced toxic compounds as bile salts and fatty acids, a phenotype closer to a commensal behavior than to an infective one. This is another example of the relationship between the communication signals, with inter-specific function in this case and the expression of efflux pumps, a situation with clear implications in the virulence potential of bacterial pathogens.
Indeed, the role of AcrAB-TolC in the virulence of Salmonella goes beyond the acquired phenotype upon induction by indole, since, as described below, it has been shown that acr $A B$-tolC deficient mutants have reduced invasiveness in animal models (Buckley et al., 2006; Webber et al., 2009).

\section{MULTIDRUG EFFLUX PUMPS AND THEIR ROLE IN THE VIRULENCE OF HUMAN PATHOGENS}

During the course of an infection, a bacterial pathogen has to be capable of surviving from the anti-infective defense mechanisms of the host. These mechanisms include, among others, the production of a diverse set of antimicrobial compounds as fatty acids, peptides or even detergents as bile salts, which function is in food uptake, but also present antimicrobial activity (Fernando and Kumar, 2013). In addition of extruding antibiotics regularly used for treating bacterial infections, different MDR efflux pumps have the ability to extrude a wide variety of compounds, including those antimicrobials produced by the host as well as QS signals involved in the regulation of the expression of virulence determinants (see above). Consequently, these efflux pumps are relevant payers on both antibiotic resistance and virulence of bacterial populations (Piddock, 2006b; Martinez et al., 2009; Alvarez-Ortega et al., 2013).

In the case of enteric bacteria, it has been shown that the efflux of several host-derived antimicrobial compounds, such as bile salts, allows the colonization and promotes the bacterial adaptation to the animal intestinal tract. The best-studied system able to confer resistance to bile salts is the E. coli RND efflux pump AcrAB-TolC, which is also a major contributor to intrinsic resistance to antibiotics in this organism (Thanassi et al., 1997). Similar roles have been reported for different AcrAB homologs from other Enterobacteriaceae species, such as S. enterica serovar Typhimurium. In this case, it has been described that mutants lacking $a c r B$ and tolC are less proficient for adhering, invading and surviving in mouse monocyte macrophages. The same study reported that an $a c r B$ mutant was able to colonize chicks. However, it was unable to survive gastrointestinally, which suggests that AcrB is not particularly relevant for the early steps of the gut colonization but it is needed for gastrointestinal persistence. On the other hand, the tolC mutant was able to colonize and persist in the chicken intestinal tract, but with a much lower efficiency than the wild-type strain, which could be due to the bile hypersensitivity displayed by this mutant (Buckley et al., 2006). A more recent study, using total genome transcriptional analysis, showed that the inactivation of $\operatorname{acr} A$, $a c r B$, and tolC rendered changes in the level of expression of several genes involved in bacterial pathogenicity, further supporting a crosstalk between resistance and virulence (Webber et al., 2009). For instance, the disruption of $a c r B$ or tolC led to a general repression of the SPI-1 pathogenicity island, which promotes the invasion of non-phagocytic intestinal epithelial cells, as well as bacterial survival and persistence within the host, while inactivation of $\operatorname{acr} A$ is associated with a repression of SPI-2, which promotes the survival and multiplication in 
phagocytic cells (Dieye et al., 2009). Therefore, inactivation of acr $B$ led to the inability to grow anaerobically, which would negatively impact in the capacity to survive in the host gut; as well as a reduced bacterial motility (Webber et al., 2009), which is also an important factor in the S. enterica serovar Typhimurium pathogenicity (Khoramian-Falsafi et al., 1990). As above stated, RND efflux systems are tripartite protein complexes formed by an inner membrane protein (the pump itself), an outer membrane protein and a linker, membrane fusion protein (Yamaguchi et al., 2015; Daury et al., 2016). The inner membrane and the membrane fusion proteins are always encoded in the same operon and are supposed to be specifically associated in each efflux pump, while the outer membrane protein can frequently form part of different efflux pumps. Consequently, while a differential response to the inactivation of the outer membrane protein of the complex (TolC in this case) as compared with the other members of the complex is conceivable, the observed differences between the $a c r A$ and $a c r B$ are more difficult to explain and suggests that a certain degree of trans-complementation between the components of different efflux pumps may happen. Although some information on this possibility has been published (Smith and Blair, 2014), this is a topic that remains to be studied in detail, although it merits to be addresses if we wish to understand in full the role that efflux pumps may play in the bacterial physiology besides antibiotic resistance.

An homologous of $A c r A B$ is also present in the respiratory tract pathogen Moraxella catarrhalis. In addition of contributing to antibiotic resistance, this efflux pump is also involved in the efficient invasion of nasopharyngeal epithelial cells, since mutants lacking $\operatorname{acr} A, \operatorname{acr} B$, or tolC present decreased invasion levels as compared to the wild-type strain (Spaniol et al., 2015). This study also showed that exposure to cold shock $\left(26^{\circ} \mathrm{C}\right)$ led to an increase in the expression of the efflux pump genes (Spaniol et al., 2015). Since temperature is an important factor for the adaptation and survival in the respiratory tract, as well as for the colonization properties and the virulence of $M$. catarrhalis (Heiniger et al., 2005; Spaniol et al., 2013), these results indicate that the levels of expression of this efflux pump are controlled by cues with relevance for the infectious success of $M$. catarrhalis. This further supports that efflux pumps may form part of global regulatory networks that include resistance and virulence determinants among other elements (Randall and Woodward, 2002; Luong et al., 2003; Nikaido et al., 2008; De Majumdar et al., 2013).

The ability to persist and to replicate in bile-rich environments is also critical for the pathogenesis of the food-borne pathogen Listeria monocytogenes. Among the elements that contribute to its survival in bile salts are the MDR efflux pumps MdrM and MdrT, belonging to the MFS family (Quillin et al., 2011). Expression of both MDR efflux pumps is strongly induced by cholic acid, a bile component, but only the MdrT efflux pump is able to extrude this compound, which is toxic for mutants lacking this efflux pump. Besides, MdrT is an important virulence factor involved in the colonization of the gallbladder in vivo, since mutants lacking $m d r T$ are 100 -fold attenuated. This study also suggests that MdrM has a synergistic role with MdrT in L. monocytogenes liver colonization, although MdrM substrates have not been identified yet (Quillin et al., 2011).

RND efflux systems also play a role in the pathogenesis of Vibrio cholerae. It has been reported that VexAB, VexCD, VexIJK, and VexGH contribute not only to antimicrobial resistance, but also to the colonization of the infant mouse small intestine; deletion of these systems impair the colonization of the mice intestine by $V$. cholerae (Bina et al., 2008; Taylor et al., 2012). Besides being relevant factors for colonization, these efflux pumps are required for the expression of the genes that encode two of the most important virulence factors in $V$. cholerae: the cholera toxin (CT) and the toxin-coregulated pilus (TCP). In a mutant lacking $v e x B, v e x D$, vexH, and $v e x K$, the production of $\mathrm{CT}$ and TcpA (the pilin subunit of the TCP) is reduced by $45 \%$, while a six RND-null strain showed a 70\% reduction in the expression of these virulence determinants, suggesting that the remaining efflux pumps, VexF and VexM, also contribute to virulence in V. cholerae (Taylor et al., 2012).

Multidrug efflux systems are also relevant elements in the defense against oxidative stress produced in the host during phagocytosis. For example, the $\mathrm{ABC}$ family efflux pump $\mathrm{MacAB}$ is required for the survival of $S$. enterica serovar Typhimurium inside macrophages, where they are exposed to ROS. It has been observed that mutants lacking macAB showed an impaired intracellular replication in macrophages as compared with the wild-type parental strain and also failed in growing in the inflamed intestine, where neutrophils release ROS. Further, the same mutants were able to grow inside macrophages that do not produce ROS, which implies that MacAB is needed for S. enterica serovar Typhimurium replication inside macrophages and for the survival under oxidative stress conditions. Besides, the mac $A B$ deletion mutant had a defect in liver colonization in BALB/c mice after intraperitoneal infection, which indicates another function of this system in the infective program of $S$. enterica serovar Typhimurium (Bogomolnaya et al., 2013).

In addition to the role of efflux pumps in the interaction of bacterial pathogens with the compounds present in the host, they also may play a direct role in virulence. This is the case for the Mycobacterium tuberculosis RND proteins designated as MmpL (Mycobacterial membrane protein Large; Cole et al., 1998). The genome of M. tuberculosis encodes 13 of these proteins, which role seems to be transporting lipids for their incorporation on the cell envelope, providing protection against host-derived compounds and contributing to the bacterial virulence (Neyrolles and Guilhot, 2011). Domenech et al. (2005) examined the contribution of these proteins to the bacterium virulence by using a murine model of infection and mutants for each MmpL protein. Among these proteins, they found that MmpL4, MmpL7, MmpL8, and MmpL11 were required for the virulence maintenance, since there is an increase in the survival time when the host is infected with these mutants as compared with the wild-type strain. The attenuation of the mmpL7 mutant might be due to the lack of phthiocerol dimycocerosate (PDIM), which is an abundant wax of the outer cell matrix involved in the cell permeability (Camacho et al., 2001); and the mmpL8 mutant is deficient in SL-1 because MmpL8 is involved in the transport of SL-N, a precursor of 
SL-1 (Domenech et al., 2004). It has been shown that SL$\mathrm{N}$ stimulates human CD1b-restricted $\mathrm{T}$ cells (Gilleron et al., 2004), a feature that might explain the attenuation of the mmpL8 mutant if this molecule has a similar effect on murine CD1d-restricted T cells. The mechanisms by which mmpL4 and mmpL11 mutants were more attenuated have not been fully elucidated; however, it has been recently described a mutation in mmpL4a (Tyr842His) in Mycobacterium bolletii, which is responsible for the smooth-to-rough morphotype change, since MmpL4 is involved in the transport of glycopeptidolipid. This variant also contributes to the bacterial virulence in a zebrafish model (Bernut et al., 2016). The Tyr842 residue is conserved in all other mycobacterial MmpL4 orthologs and in all $13 \mathrm{MmpL}$ RND proteins in $M$. tuberculosis, which indicates the functional relevance of this residue (Szekely and Cole, 2016).

Biofilms are complex microbial associations attached to a variety of surfaces. Bacteria that grow forming biofilms are more resistant to antibiotics than planktonic cells, being also important elements in the bacterial virulence and pathogenesis. The link between antimicrobial tolerance on biofilms and efflux pumps has been reported in several microorganisms (Soto, 2013). For instance, in the opportunistic pathogen P. aeruginosa, the MerRlike regulator BrlR plays a role in the high-level tolerance to antimicrobials in biofilms because it is able to activate under these growing conditions the expression of the multidrug efflux pumps MexAB-OprM and MexEF-OprN (Liao et al., 2013). A novel efflux pump in $P$. aeruginosa involved in biofilm tolerance, named as PA1874-1877, has been identified. The expression of this efflux pump was 10 -fold increased in biofilms when compared with planktonic cells. Besides, deletion of the genes encoding this efflux pump resulted in an increased susceptibility to ciprofloxacin, gentamicin, and tobramycin (Zhang and Mah, 2008). Efflux pump expression can also impact the flagellar motility, which plays a relevant role in biofilm formation (Houry et al., 2012) and enhances pathogenicity by improving bacterial motility (Duan et al., 2013). In Stenotrophomonas maltophilia, another opportunistic pathogen, it has been observed that deletion of the RND efflux pump SmeYZ resulted in a reduced ability to form biofilm and the abolition of flagella formation. Besides, this deletion mutant was more susceptible to redox compounds, human serum and neutrophils, which indicates that this efflux pump is also involved in the protection against ROS (Lin et al., 2015).

Expression of efflux pumps not always enhance virulence; constitutive overexpression of these systems in antibiotic resistant mutants, can compromise the bacterial fitness and the virulence as well (Sanchez et al., 2002), indicating that the expression of these elements is finely regulated and deviations on this regulation, altering their expression below or above the physiological levels, may impair bacterial physiology and virulence. This is the case of the overexpression of the MDR efflux pumps MexCD-OprJ and MexEF-OprN in P. aeruginosa, which negatively affect the expression of the type III secretion system (T3SS; Linares et al., 2005). The T3SS is an important virulence mechanism, since bacteria are able to inject effector proteins manipulating the host cell function (Coburn et al., 2007). The effect of over-expression of efflux pumps on T3S was due to the lack of expression of exs $A$ gene, which is the transcriptional activator of the T3SS in P. aeruginosa (Linares et al., 2005). More recently, it was found that MexT, the positive regulator of MexEF, was able to repress the expression of the T3SS through the regulators MexS and PtrC (Jin et al., 2011), in such a way linking resistance and virulence within a single regulon.

Altogether, these works show that, in addition of being involved in antibiotic resistance, efflux pumps can participate in bacterial virulence as well. In some cases, a direct effect can be foreseen; this is the situation of efflux pumps able to extrude host-produced antimicrobial compounds. However, in other occasions the reasons behind the effect of efflux pumps on virulence are not so straightforward. In some cases, the effect on virulence is derived from the integration of the efflux pumps in a regulon that also includes virulence determinants. This could be the case of MexEF, a part of the MexT regulon that also includes the $P$. aeruginosa T3SS. Mutations in this global regulator will simultaneously alter antibiotic resistance and virulence, although the efflux pump itself is not directly involved in T3S. Other situation, also described for MexEF, is the capability of some efflux pumps for extruding intercellular signal molecules (see above) or their precursors (Olivares et al., 2012). If this efflux pump is abnormally expressed, the levels of expression of the genes belonging to the regulatory network (frequently including virulence genes) triggered by such signals will be also altered.

\section{THE FUNCTIONAL ROLE OF MULTIDRUG EFFLUX PUMPS IN PLANT-BACTERIA INTERACTIONS}

Multidrug efflux pumps, in addition of being relevant antibiotic resistance determinants, are relevant key players for the behavior of microorganisms in their natural (non-clinical) habitats. Indeed, whereas most studies in human pathogens have concentrated in the role on antibiotic resistance of these elements, the analyses of efflux pumps from plants pathogens or epiphytes, has mainly focused on their role in plant-bacteria or bacteriabacteria interactions. The rhizosphere is a natural ecosystem that includes a complex microbiome formed by microorganisms that live in contact with plants' roots. Roots' and other plants' exudates contain a large array of natural products, such as flavonoids, which confer them protection against microbial attack. However, different microorganisms have developed mechanisms to deal with the activity of those compounds. One of them is the flavonoid-responsive RND family of efflux pumps, which includes several members as MexAB-OprM from Pseudomonas syringae, AcrAB from Erwinia amylovora, AcrD from Erwinia chrysanthemi, IfeAB from Agrobacterium tumefaciens, XagID2689 from Xanthomonas axonopodis, SmeDEF from S. maltophilia, EmrAB from Sinorhizobium meliloti and BjG30 from Bradyrhizobium japonicum (Palumbo et al., 1998; Burse et al., 2004b; Vargas et al., 2011; Takeshima et al., 2013; García-León et al., 2014; Pletzer and Weingart, 2014; Rossbach et al., 2014; Chatnaparat et al., 2016). Some of these efflux pumps are implicated in plant colonization, whereas 
some others are involved in bacteria/plant symbiosis processes. As stated before MexAB-OprM is described as a significant determinant of multidrug resistance in $P$. aeruginosa (Poole, 2001) having a basal expression level enough to contribute to the intrinsic antimicrobial resistance of these bacteria ( $\mathrm{Li}$ et al., 1995) and it also has a role in virulence. Indeed, as above described, mutant strains overexpressing this efflux pump are less virulent because of the extrusion of the QS homoserine lactone 3-oxo-C 12 -HSL (Minagawa et al., 2012). In addition of extruding antibiotics or QS signaling molecules, MexAB-OprM is able to pump out plant antimicrobial compounds from leaves of Melaleuca alternifolia, supporting that, in addition of its role in clinical settings, this efflux pump (and several others, see below) may allow bacterial survival in a vegetal environment (Papadopoulos et al., 2008). Indeed, this efflux pump is required for the efficient colonization of tomato plants by $P$. syringae, since the inactivation MexAB-OprM lead to a defective colonization capacity of the plant by this bacterial species (Vargas et al., 2011). In line with the role of efflux pumps in a general bacterial response to the plant antimicrobial defense, it has been shown that flavonoids are inducers and substrates of this transporter (Vargas et al., 2011). In addition, the same flavonoids are able to inhibit the GacS/GacA two component system (TCS) of $P$. syringae, which is implicated, among other regulatory elements, in the activation of the motility and the T3SS in this species (Chatterjee et al., 2003). In fact, the absence of MexAB-OprM and the accumulation of flavonoids inside bacteria lead to a reduction of swarming and swimming motility and a significant impairment in the production of flagella and T3S (Vargas et al., 2013). Therefore, when the amount of plant flavonoids is enough to reduce bacterial virulence by inhibiting $\mathrm{GacS} / \mathrm{GacA}, \quad$. syringae may extrude such flavonoids using the MexAB efflux pump (which expression is now induced), shrewdly regulating the intracellular level of flavonoids and consequently, ensuring the viability of the bacteria by the de-repression of this TCS. This is an elegant example of the adaptive co-evolution of plant resistance and pathogen virulence in which the role of this efflux pump goes beyond being a mere detoxification system (Figure 2).

Another plant-pathogenic bacteria is E. amylovora, an Enterobacteria that causes fire blight on apple and pear trees. The flavonoid inducible AcrAB-TolC efflux pump from E. amylovora confers resistance, in addition to antibiotics, to plant compounds as phytoalexins and flavonoids (phloretin, naringenin, and quercetin) leading to the successful colonization of plants (Burse et al., 2004b; Maggiorani Valecillos et al., 2006; Al-Karablieh et al., 2009). Moreover, an AcrAB deletion mutant has a strong reduction of E. amylovora virulence in apple plants (Burse et al., 2004b). A close homolog of AcrB is AcrD, an efflux pump that is also induced by plant flavonoids, such as luteolin, but whose deletion mutant exhibits full virulence on apple and pear fruits (Pletzer and Weingart, 2014). E. chrysanthemi constitutes another example in which efflux pumps are required to infect plants. A tolC mutant of E. chrysanthemi is unable to extrude the plant antimicrobial compound berberine, being then unable to cause plant tissue maceration. Moreover, TolC plays a role also modulating the fitness of the bacteria growing in the microbial community (Barabote et al., 2003), indicating that efflux pumps might also be involved in inter-microbial interactions (see below). In addition of plant-derived antimicrobials, the AcrAB efflux pump of E. chrysanthemi is inducible by salicylic acid (SA), an important plant hormone implicated in local and systemic plant resistance (Ravirala et al., 2007). SA and others plant phenolic acids are able of reducing the expression of the T3SS, by inhibiting the GacS/A pathway, in P. syringae (Lee J.S. et al., 2015), suggesting that a role of efflux pumps in non-clinical ecosystems might be to adjust the intracellular level of plant signals, as a mechanism to deal with plant defense systems. Other efflux pump that has a role in plant colonization is IfeAB from A. tumefaciens, which extrudes coumestrol bestowing measurable ecological benefits to this bacterium in flavonoids rich environments. X. axonopodis pv. glycines is another plant pathogen, causative of bacterial pustule of Glycine max, one of the most important diseases in soybean. It contains another flavonoid inducible RND efflux pump, called XagID2689 (Chatnaparat et al., 2016), which in addition of being induced by flavonoids contributes as well to flavonoids' resistance; its deletion strongly reduces bacterial virulence in soybean. Moreover, this deletion mutant shows a higher susceptibility than the wild-type parental counterpart to the isoflavonoids phloretin, naringenin and berberine, as well as to the antibiotics acriflavine and tetracycline, suggesting a role in the intracellular reduction of the levels of several compounds produced by soybean and involved in its antimicrobial response program. In S. maltophilia, the SmeDEF efflux pump, which is the most important quinolone resistance determinant of this microorganism (Alonso and Martinez, 2000, 2001; Zhang et al., 2001; Garcia-Leon et al., 2014), is induced by flavonoids, that are able to bind to its SmeT repressor (García-León et al., 2014); in addition, a mutant lacking smeE is unable to colonize the roots of Arabidopsis plants (GarcíaLeón et al., 2014), further supporting that MDR efflux pumps, with a relevant role for antibiotic resistance at clinical settings, might have been selected in nature for different ecological purposes.

In addition of easening the bacterial plants' colonization and infection, efflux pumps have also a role in the interactions between plants and their symbiotic bacteria. For example, EmrAB from S. meliloti is an inducible flavonoid efflux pump with a role in symbiosis with Medicago sativa; the symbiotic process is impaired when the regulator of this efflux system, the TetR repressor EmrR, is deleted (Rossbach et al., 2014; Santos et al., 2014). Other examples of efflux pumps with a role in symbiotic nitrogen-fixation activity processes in G. max are $B d e A B$ and BjG30 from B. japonicum (Lindemann et al., 2010; Takeshima et al., 2013). BdeAB deficient mutants, in addition of presenting symbiotic defects, are more susceptible to aminoglycosides, highlighting the multifunctional role of efflux pumps (Lindemann et al., 2010).

Efflux pumps have also an important role in the intermicrobial interactions in the host plant and in its rhizosphere, where each bacteria have to compete for space and nutrients to survive. As with antibiotic resistance mechanisms, that can be considered as a colonization factor in the treated 


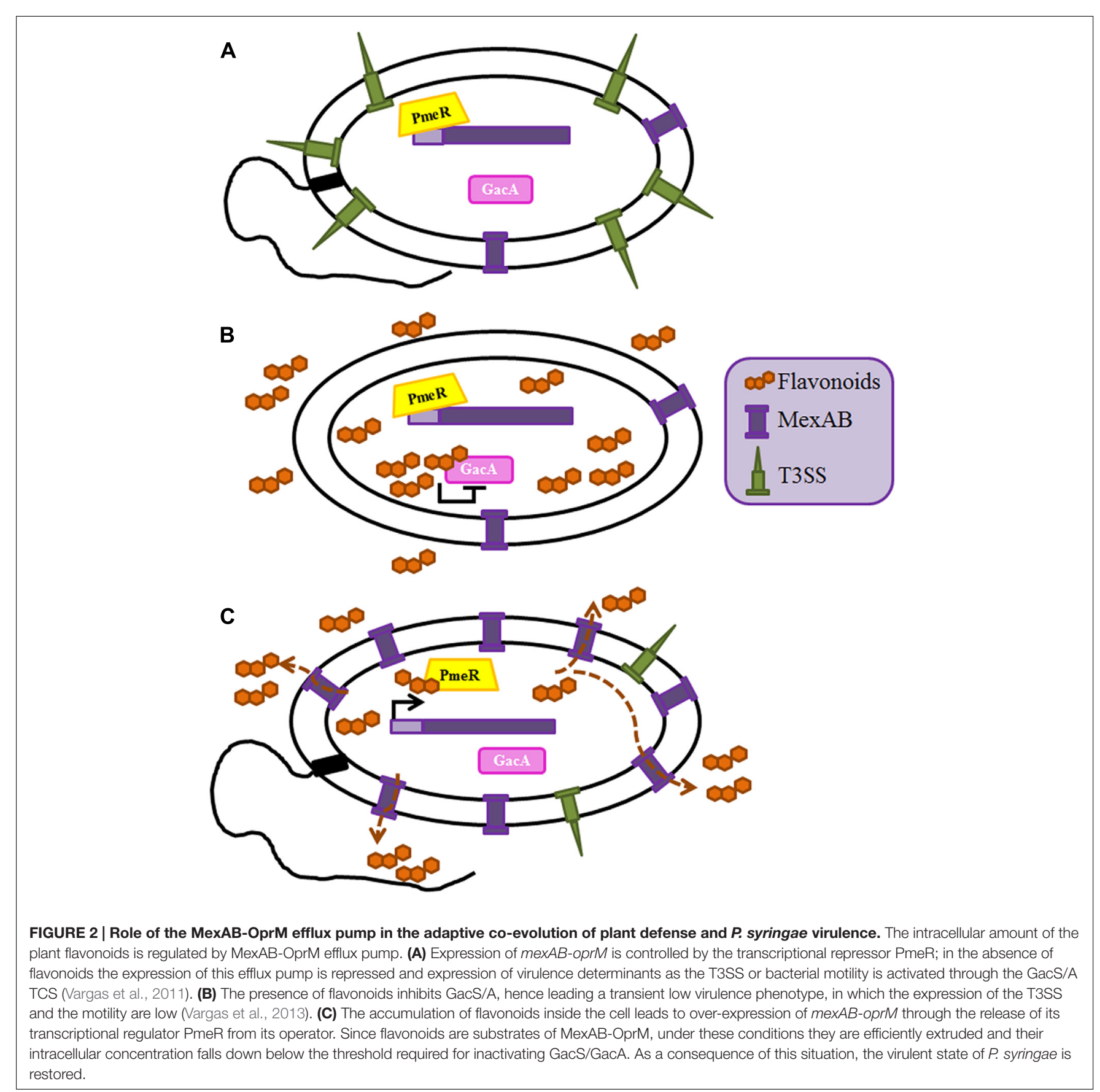

patient, where antibiotics are present (Martinez and Baquero, 2002), resistance to antimicrobials produced by epiphytes may be considered as a colonization tool of phytopathogenic bacteria and vice versa. If the epiphytes can inhibit the phytopathogens, the plant will be protected from infection, whereas if pathogens can inhibit epiphytes, this situation will provide a colonization advantage and hence better possibilities for infecting the plant. Resistance to compounds produced by bacterial competitors will then increase the chances for colonization of the epiphyte or the pathogen, with important consequence in terms of crops protection. This is the case of the efflux pump NorM from E. amylovora that, in contrast to the previously mentioned efflux pump AcrAB from E. amylovora (Burse et al., 2004b), is not able to extrude the high amount of isoflavonoids that produce the members of the Rosaceae family (Burse et al., 2004a). Moreover, a norM-deficient mutant causes comparable symptoms as the wild-type parental counterpart in plant tissues, indicating that this efflux pump does not contribute to virulence of E. amylovora against apple plants. Nevertheless, while the NorM efflux pump does not directly contribute to virulence E. amylovora, it has an important role extruding toxic molecules produced by Pantoea 
agglomerans (Pusey et al., 2011), an epiphytic bacteria that is an excellent colonizer of stigmas of apple and pear and it may effectively inhibit the multiplication of E. amylovora when both microorganisms co-colonize rosaceous (Burse et al., 2004a; Piddock, 2006b), hence constituting a potential biocontrol agent for fire blight (Kim et al., 2012). It is worth mentioning that NorM is involved in the capability E. amylovora to reach highdensity populations at low temperatures. Indeed, at $18^{\circ} \mathrm{C}$ the growth of an E. amylovora norM mutant is significantly impaired as compared with the wild-type strain. In addition, the level of expression of nor $M$ is twofold greater at $18^{\circ} \mathrm{C}$ gene than at $28^{\circ} \mathrm{C}$ (Burse et al., 2004a). Altogether these results suggest that the intrinsic resistance of E. amylovora to $P$. agglomerans competition at $18^{\circ} \mathrm{C}$ may be due to the increased expression level of norM, which might allow the colonization by E. amylovora of the stigma surface of the blossom when it coexists with $P$. agglomerans. These evidences lead to hypothesize that the utilization of $P$. agglomerans as a biocontrol mechanism for fire blight would be compromised at low temperatures or by mutations causing of overexpression of norM in E. amylovora.

\section{REFERENCES}

Aendekerk, S., Diggle, S. P., Song, Z., Hoiby, N., Cornelis, P., Williams, P., et al. (2005). The MexGHI-OpmD multidrug efflux pump controls growth, antibiotic susceptibility and virulence in Pseudomonas aeruginosa via 4-quinolonedependent cell-to-cell communication. Microbiology 151(Pt. 4), 1113-1125. doi: 10.1099/mic.0.27631-0

Al-Karablieh, N., Weingart, H., and Ullrich, M. S. (2009). The outer membrane protein TolC is required for phytoalexin resistance and virulence of the fire blight pathogen Erwinia amylovora. Microb. Biotechnol. 2, 465-475. doi: 10.1111/j.1751-7915.2009.00095.x

Alonso, A., and Martinez, J. L. (2000). Cloning and characterization of SmeDEF, a novel multidrug efflux pump from Stenotrophomonas maltophilia. Antimicrob. Agents Chemother. 44, 3079-3086. doi: 10.1128/AAC.44.11.30793086.2000

Alonso, A., and Martinez, J. L. (2001). Expression of multidrug efflux pump SmeDEF by clinical isolates of Stenotrophomonas maltophilia. Antimicrob. Agents Chemother. 45, 1879-1881. doi: 10.1128/AAC.45.6. 1879-1881.2001

Alonso, A., Rojo, F., and Martinez, J. L. (1999). Environmental and clinical isolates of Pseudomonas aeruginosa show pathogenic and biodegradative properties irrespective of their origin. Environ. Microbiol. 1, 421-430. doi: 10.1046/j.14622920.1999.00052.x

Alvarez-Ortega, C., Olivares, J., and Martínez, J. L. (2013). RND multidrug efflux pumps: what are they good for? Front. Microbiol. 4:7. doi: 10.3389/fmicb.2013.00007

Bailey, A. M., Ivens, A., Kingsley, R., Cottell, J. L., Wain, J., and Piddock, L. J. (2010). RamA, a member of the AraC/XylS family, influences both virulence and efflux in Salmonella enterica serovar typhimurium. J. Bacteriol. 192, 1607-1616. doi: 10.1128/JB.01517-09

Barabote, R. D., Johnson, O. L., Zetina, E., San Francisco, S. K., Fralick, J. A., and San Francisco, M. J. (2003). Erwinia chrysanthemi tolC is involved in resistance to antimicrobial plant chemicals and is essential for phytopathogenesis. J. Bacteriol. 185, 5772-5778. doi: 10.1128/JB.185.19.57725778.2003

Bernut, A., Viljoen, A., Dupont, C., Sapriel, G., Blaise, M., Bouchier, C., et al. (2016). Insights into the smooth-to-rough transitioning in Mycobacterium bolletii unravels a functional Tyr residue conserved in all mycobacterial MmpL family members. Mol. Microbiol. 99, 866-883. doi: 10.1111/mmi. 13283

Bina, X. R., Provenzano, D., Nguyen, N., and Bina, J. E. (2008). Vibrio cholerae RND family efflux systems are required for antimicrobial resistance, optimal

\section{AUTHOR CONTRIBUTIONS}

All authors listed, have made substantial, direct and intellectual contribution to the work, and approved it for publication.

\section{FUNDING}

Work in our laboratory is supported by grants from the Spanish Ministry of Economy and Competitiveness (BIO2014-54507$\mathrm{R}$ and JPI Water StARE JPIW2013-089-C02-01); from Madrid Autonomous Community [S2010/BMD2414 (PROMPT)]; and from the Instituto de Salud Carlos III [Spanish Network for Research on Infectious Diseases (REIPI RD12/0015)]. MA-R and $\mathrm{PB}$ are recipients of FPI fellowships from MINECO.

\section{ACKNOWLEDGMENT}

Thanks are given to Mary Higgins for grammar corrections.

virulence factor production, and colonization of the infant mouse small intestine. Infect. Immun. 76, 3595-3605. doi: 10.1128/IAI.01620-07

Bogomolnaya, L. M., Andrews, K. D., Talamantes, M., Maple, A., Ragoza, Y., Vazquez-Torres, A., et al. (2013). The ABC-type efflux pump MacAB protects Salmonella enterica serovar typhimurium from oxidative stress. MBio 4, e63013. doi: $10.1128 / \mathrm{mBio} .00630-13$

Bommarius, B., Anyanful, A., Izrayelit, Y., Bhatt, S., Cartwright, E., Wang, W., et al. (2013). A family of indoles regulate virulence and Shiga toxin production in pathogenic E. coli. PLOS ONE 8:e54456. doi: 10.1371/journal.pone. 0054456

Buckley, A. M., Webber, M. A., Cooles, S., Randall, L. P., La Ragione, R. M., Woodward, M. J., et al. (2006). The AcrAB-TolC efflux system of Salmonella enterica serovar typhimurium plays a role in pathogenesis. Cell Microbiol. 8, 847-856. doi: 10.1111/j.1462-5822.2005.00671.x

Buhl, M., Peter, S., and Willmann, M. (2015). Prevalence and risk factors associated with colonization and infection of extensively drug-resistant Pseudomonas aeruginosa: a systematic review. Expert Rev. Anti Infect. Ther. 13, 1159-1170. doi: 10.1586/14787210.2015.1064310

Burse, A., Weingart, H., and Ullrich, M. S. (2004a). NorM, an Erwinia amylovora multidrug efflux pump involved in in vitro competition with other epiphytic bacteria. Appl. Environ. Microbiol. 70, 693-703. doi: 10.1128/AEM.70.2.693703.2004

Burse, A., Weingart, H., and Ullrich, M. S. (2004b). The phytoalexin-inducible multidrug efflux pump AcrAB contributes to virulence in the fire blight pathogen, Erwinia amylovora. Mol. Plant Microbe Interact. 17, 43-54. doi: 10.1094/MPMI.2004.17.1.43

Butt, A., Halliday, N., Williams, P., Atkins, H. S., Bancroft, G. J., and Titball, R. W. (2016). Burkholderia pseudomallei kynB plays a role in AQ production, biofilm formation, bacterial swarming and persistence. Res. Microbiol. 167, 159-167. doi: 10.1016/j.resmic.2015.11.002

Camacho, L. R., Constant, P., Raynaud, C., Laneelle, M. A., Triccas, J. A., Gicquel, B., et al. (2001). Analysis of the phthiocerol dimycocerosate locus of Mycobacterium tuberculosis. Evidence that this lipid is involved in the cell wall permeability barrier. J. Biol. Chem. 276, 19845-19854. doi: 10.1074/jbc.M100662200

Chan, Y. Y., Bian, H. S., Tan, T. M., Mattmann, M. E., Geske, G. D., Igarashi, J., et al. (2007). Control of quorum sensing by a Burkholderia pseudomallei multidrug efflux pump. J. Bacteriol. 189, 4320-4324. doi: 10.1128/JB. 00003-07

Chan, Y. Y., and Chua, K. L. (2005). The Burkholderia pseudomallei BpeABOprB efflux pump: expression and impact on quorum sensing and virulence. J. Bacteriol. 187, 4707-4719. doi: 10.1128/JB.187.14.4707-4719.2005 
Chatnaparat, T., Prathuangwong, S., and Lindow, S. E. (2016). Global pattern of gene expression of Xanthomonas axonopodis pv. glycines within soybean leaves. Mol. Plant Microbe Interact. 29, 508-522. doi: 10.1094/mpmi-01-160007-r

Chatterjee, A., Cui, Y., Yang, H., Collmer, A., Alfano, J. R., and Chatterjee, A. K. (2003). GacA, the response regulator of a two-component system, acts as a master regulator in Pseudomonas syringae pv. tomato DC3000 by controlling regulatory RNA, transcriptional activators, and alternate sigma factors. Mol. Plant Microbe Interact. 16, 1106-1117. doi: 10.1094/mpmi.2003.16. 12.1106

Coburn, B., Sekirov, I., and Finlay, B. B. (2007). Type III secretion systems and disease. Clin. Microbiol. Rev. 20, 535-549. doi: 10.1128/CMR.00013-07

Cole, S. T., Brosch, R., Parkhill, J., Garnier, T., Churcher, C., Harris, D., et al. (1998). Deciphering the biology of Mycobacterium tuberculosis from the complete genome sequence. Nature 393, 537-544. doi: 10.1038/31159

Crossman, L. C., Gould, V. C., Dow, J. M., Vernikos, G. S., Okazaki, A., Sebaihia, M., et al. (2008). The complete genome, comparative and functional analysis of Stenotrophomonas maltophilia reveals an organism heavily shielded by drug resistance determinants. Genome Biol. 9:R74. doi: 10.1186/gb-2008-94-r74

Daury, L., Orange, F., Taveau, J. C., Verchere, A., Monlezun, L., Gounou, C., et al. (2016). Tripartite assembly of RND multidrug efflux pumps. Nat. Commun. 7:10731. doi: $10.1038 /$ ncomms 10731

De Majumdar, S., Veleba, M., Finn, S., Fanning, S., and Schneiders, T. (2013). Elucidating the regulon of multidrug resistance regulator RarA in Klebsiella pneumoniae. Antimicrob. Agents Chemother. 57, 1603-1609. doi: 10.1128/AAC.01998-12

Dietrich, L. E., Price-Whelan, A., Petersen, A., Whiteley, M., and Newman, D. K. (2006). The phenazine pyocyanin is a terminal signalling factor in the quorum sensing network of Pseudomonas aeruginosa. Mol. Microbiol. 61, 1308-1321. doi: 10.1111/j.1365-2958.2006.05306.x

Dieye, Y., Ameiss, K., Mellata, M., and Curtiss, R. III (2009). The Salmonella Pathogenicity Island (SPI) 1 contributes more than SPI2 to the colonization of the chicken by Salmonella enterica serovar typhimurium. BMC Microbiol. 9:3. doi: 10.1186/1471-2180-9-3

Domenech, P., Reed, M. B., and Barry, C. E. III (2005). Contribution of the Mycobacterium tuberculosis $\mathrm{MmpL}$ protein family to virulence and drug resistance. Infect. Immun. 73, 3492-3501. doi: 10.1128/IAI.73.6.34923501.2005

Domenech, P., Reed, M. B., Dowd, C. S., Manca, C., Kaplan, G., and Barry, C. E. III (2004). The role of MmpL8 in sulfatide biogenesis and virulence of Mycobacterium tuberculosis. J. Biol. Chem. 279, 21257-21265. doi: 10.1074/jbc.M400324200

Duan, Q., Zhou, M., Zhu, L., and Zhu, G. (2013). Flagella and bacterial pathogenicity. J. Basic Microbiol. 53, 1-8. doi: 10.1002/jobm. 201100335

Erickson, D. L., Endersby, R., Kirkham, A., Stuber, K., Vollman, D. D., Rabin, H. R., et al. (2002). Pseudomonas aeruginosa quorum-sensing systems may control virulence factor expression in the lungs of patients with cystic fibrosis. Infect. Immun. 70, 1783-1790. doi: 10.1128/IAI.70.4.1783-1790.2002

Evans, K., Passador, L., and Srikumar, R. (1998). Influence of the MexABOprM multidrug efflux system on quorum sensing in Pseudomonas aeruginosa. J. Bacteriol. 180, 5443-5447.

Fernando, D. M., and Kumar, A. (2013). Resistance-nodulation-division multidrug efflux pumps in gram-negative bacteria: role in virulence. Antibiotics (Basel) 2, 163-181. doi: 10.3390/antibiotics2010163

Frisk, A., Schurr, J. R., Wang, G., Bertucci, D. C., Marrero, L., Hwang, S. H., et al. (2004). Transcriptome analysis of Pseudomonas aeruginosa after interaction with human airway epithelial cells. Infect. Immun. 72, 5433-5438. doi: 10.1128/IAI.72.9.5433-5438.2004

Gaede, H. C., Yau, W. M., and Gawrisch, K. (2005). Electrostatic contributions to indole-lipid interactions. J. Phys. Chem. B 109, 13014-13023. doi: 10.1021/jp0511000

García-León, G., Hernández, A., Hernando-Amado, S., Alavi, P., Berg, G., and Martínez, J. L. (2014). A function of SmeDEF, the major quinolone resistance determinant of Stenotrophomonas maltophilia, is the colonization of plant roots. Appl. Environ. Microbiol. 80, 4559-4565. doi: 10.1128/AEM. 01058-14
Garcia-Leon, G., Salgado, F., Oliveros, J. C., Sanchez, M. B., and Martinez, J. L. (2014). Interplay between intrinsic and acquired resistance to quinolones in Stenotrophomonas maltophilia. Environ. Microbiol. 16, 1282-1296. doi: $10.1111 / 1462-2920.12408$

Genestet, C., Le Gouellec, A., Chaker, H., Polack, B., Guery, B., Toussaint, B., et al. (2014). Scavenging of reactive oxygen species by tryptophan metabolites helps Pseudomonas aeruginosa escape neutrophil killing. Free Radic. Biol. Med. 73, 400-410. doi: 10.1016/j.freeradbiomed.2014.06.003

Gilleron, M., Stenger, S., Mazorra, Z., Wittke, F., Mariotti, S., Bohmer, G., et al. (2004). Diacylated sulfoglycolipids are novel mycobacterial antigens stimulating CD1-restricted T cells during infection with Mycobacterium tuberculosis. J. Exp. Med. 199, 649-659. doi: 10.1084/jem.20031097

Gould, V. C., Okazaki, A., Howe, R. A., and Avison, M. B. (2004). Analysis of sequence variation among smeDEF multi drug efflux pump genes and flanking DNA from defined 16S rRNA subgroups of clinical Stenotrophomonas maltophilia isolates. J. Antimicrob. Chemother. 54, 348-353. doi: 10.1093/jac/dkh367

Grkovic, S., Brown, M. H., and Skurray, R. A. (2001). Transcriptional regulation of multidrug efflux pumps in bacteria. Semin. Cell Dev. Biol. 12, 225-237. doi: $10.1006 / \mathrm{scdb} .2000 .0248$

Grkovic, S., Brown, M. H., and Skurray, R. A. (2002). Regulation of bacterial drug export systems. Microbiol. Mol. Biol. Rev. 66, 671-701. doi: 10.1128/MMBR.66.4.671-701.2002

Heiniger, N., Troller, R., Meier, P. S., and Aebi, C. (2005). Cold shock response of the UspA1 outer membrane adhesin of Moraxella catarrhalis. Infect. Immun. 73, 8247-8255. doi: 10.1128/IAI.73.12.8247-8255.2005

Hernandez, A., Sanchez, M. B., and Martinez, J. L. (2011). Quinolone resistance: much more than predicted. Front. Microbiol. 2:22. doi: 10.3389/fmicb.2011.00022

Hernando-Amado, S., Blanco, P., Alcalde-Rico, M., Corona, F., Reales-Calderón, J. A., Sánchez, M. B., et al. (2016). Multidrug efflux pumps as main players in intrinsic and acquired resistance to antimicrobials. Drug Resist. Updat. 28, 13-27. doi: 10.1016/j.drup.2016.06.007

Hirakata, Y., Srikumar, R., Poole, K., Gotoh, N., Suematsu, T., Kohno, S., et al. (2002). Multidrug efflux systems play an important role in the invasiveness of Pseudomonas aeruginosa. J. Exp. Med. 196, 109-118. doi: 10.1084/jem. 20020005

Hogan, D. A., Vik, A., and Kolter, R. (2004). A Pseudomonas aeruginosa quorumsensing molecule influences Candida albicans morphology. Mol. Microbiol. 54, 1212-1223. doi: 10.1111/j.1365-2958.2004.04349.x

Houry, A., Gohar, M., Deschamps, J., Tischenko, E., Aymerich, S., Gruss, A., et al. (2012). Bacterial swimmers that infiltrate and take over the biofilm matrix. Proc. Natl. Acad. Sci. U.S.A. 109, 13088-13093. doi: 10.1073/pnas. 1200 791109

Jang, S. (2016). Multidrug efflux pumps in Staphylococcus aureus and their clinical implications. J. Microbiol. 54, 1-8. doi: 10.1007/s12275-016-5159-Z

Jin, Y., Yang, H., Qiao, M., and Jin, S. (2011). MexT regulates the type III secretion system through MexS and PtrC in Pseudomonas aeruginosa. J. Bacteriol. 193, 399-410. doi: 10.1128/JB.01079-10

Kawamura-Sato, K., Shibayama, K., Horii, T., Iimuma, Y., Arakawa, Y., and Ohta, M. (1999). Role of multiple efflux pumps in Escherichia coli in indole expulsion. FEMS Microbiol. Lett. 179, 345-352. doi: 10.1111/j.15746968.1999.tb08748.x

Kaye, K. S., and Pogue, J. M. (2015). Infections caused by resistant gram-negative bacteria: epidemiology and management. Pharmacotherapy 35, 949-962. doi: 10.1002/phar.1636

Khoramian-Falsafi, T., Harayama, S., Kutsukake, K., and Pechere, J. C. (1990). Effect of motility and chemotaxis on the invasion of Salmonella typhimurium into HeLa cells. Microb. Pathog. 9, 47-53. doi: 10.1016/0882-4010(90) 90039-S

Kim, I. Y., Pusey, P. L., Zhao, Y., Korban, S. S., Choi, H., and Kim, K. K. (2012). Controlled release of Pantoea agglomerans E325 for biocontrol of fire blight disease of apple. J. Control. Release 161, 109-115. doi: 10.1016/j.jconrel.2012.03.028

Köhler, T., Delden, C. V., Van Delden, C., Curty, L. K., Hamzehpour, M. M., and Pechere, J. C. (2001). Overexpression of the MexEF-OprN multidrug efflux system affects cell-to-cell signaling in Pseudomonas aeruginosa. J. Bacteriol. 183, 5213-5222. doi: 10.1128/JB.183.18.5213 
Lamarche, M. G., and Deziel, E. (2011). MexEF-OprN Efflux Pump Exports the Pseudomonas Quinolone Signal (PQS) Precursor HHQ (4-hydroxy2-heptylquinoline). PLoS ONE 6:e24310. doi: 10.1371/journal.pone. 0024310

Lee, J., Wu, J., Deng, Y., Wang, J., Wang, C., Wang, J., et al. (2013). A cellcell communication signal integrates quorum sensing and stress response. Nat. Chem. Biol. 9, 339-343. doi: 10.1038/nchembio.1225

Lee, J., and Zhang, L. (2015). The hierarchy quorum sensing network in Pseudomonas aeruginosa. Protein Cell 6, 26-41. doi: 10.1007/s13238-0140100-x

Lee, J. H., Wood, T. K., and Lee, J. (2015). Roles of indole as an interspecies and interkingdom signaling molecule. Trends Microbiol. 23, 707-718. doi: 10.1016/j.tim.2015.08.001

Lee, J. S., Ryu, H. R., Cha, J. Y., and Baik, H. S. (2015). The hrp pathogenicity island of Pseudomonas syringae pv. tomato DC3000 is induced by plant phenolic acids. J. Microbiol. 53, 725-731. doi: 10.1007/s12275-015-5256-4

Li, X. Z., Nikaido, H., and Poole, K. (1995). Role of mexA-mexB-oprM in antibiotic efflux in Pseudomonas aeruginosa. Antimicrob. Agents Chemother. 39, 19481953. doi: 10.1128/AAC.39.9.1948

Li, X.-Z., Plésiat, P., and Nikaido, H. (2015). The challenge of efflux-mediated antibiotic resistance in Gram-negative bacteria. Clin. Microbiol. Rev. 28, 337418.

Liao, J., Schurr, M. J., and Sauer, K. (2013). The MerR-like regulator BrlR confers biofilm tolerance by activating multidrug efflux pumps in Pseudomonas aeruginosa biofilms. J. Bacteriol. 195, 3352-3363. doi: 10.1128/JB. 00318-13

Lin, Y. T., Huang, Y. W., Chen, S. J., Chang, C. W., and Yang, T. C. (2015). The SmeYZ efflux pump of Stenotrophomonas maltophilia contributes to drug resistance, virulence-related characteristics, and virulence in mice. Antimicrob. Agents Chemother. 59, 4067-4073. doi: 10.1128/aac.00372-15

Linares, J. F., Lopez, J. A., Camafeita, E., Albar, J. P., Rojo, F., and Martinez, J. L. (2005). Overexpression of the multidrug efflux pumps MexCD-OprJ and MexEF-OprN is associated with a reduction of type III secretion in Pseudomonas aeruginosa. J. Bacteriol. 187, 1384-1391. doi: 10.1128/JB.187.4.1384-1391.2005

Lindemann, A., Koch, M., Pessi, G., Muller, A. J., Balsiger, S., Hennecke, H., et al. (2010). Host-specific symbiotic requirement of BdeAB, a RegR-controlled RND-type efflux system in Bradyrhizobium japonicum. FEMS Microbiol. Lett. 312, 184-191. doi: 10.1111/j.1574-6968.2010.02115.x

Luong, T. T., Newell, S. W., and Lee, C. Y. (2003). Mgr, a novel global regulator in Staphylococcus aureus. J. Bacteriol. 185, 3703-3710. doi: 10.1128/JB.185.13.3703-3710.2003

Maggiorani Valecillos, A., Rodriguez Palenzuela, P., and Lopez-Solanilla, E. (2006). The role of several multidrug resistance systems in Erwinia chrysanthemi pathogenesis. Mol. Plant Microbe Interact. 19, 607-613. doi: 10.1094/MPMI19-0607

Martinez, J. L. (2014). Interkingdom signaling and its consequences for human health. Virulence 5, 243-244. doi: 10.4161/viru.28073

Martinez, J. L., and Baquero, F. (2002). Interactions among strategies associated with bacterial infection: pathogenicity, epidemicity, and antibiotic resistance. Clin. Microbiol. Rev. 15, 647-679. doi: 10.1128/CMR.15.4.647679.2002

Martinez, J. L., Sanchez, M. B., Martinez-Solano, L., Hernandez, A., Garmendia, L., Fajardo, A., et al. (2009). Functional role of bacterial multidrug efflux pumps in microbial natural ecosystems. FEMS Microbiol. Rev. 33, 430-449. doi: 10.1111/j.1574-6976.2008.00157.x

Martinez-Solano, L., Macia, M. D., Fajardo, A., Oliver, A., and Martinez, J. L. (2008). Chronic Pseudomonas aeruginosa infection in chronic obstructive pulmonary disease. Clin. Infect. Dis. 47, 1526-1533. doi: 10.1086/ 593186

Maseda, H., Sawada, I., Saito, K., Uchiyama, H., Nakae, T., and Nomura, N. (2004). Enhancement of the mexAB-oprM efflux pump expression by a quorumsensing autoinducer and its cancellation by a regulator, MexT, of the mexEFoprN efflux pump operon in Pseudomonas aeruginosa. Antimicrob. Agents Chemother. 48, 1320-1328. doi: 10.1128/AAC.48.4.1320-1328.2004

Méar, J.-B., Kipnis, E., Faure, E., Dessein, R., Schurtz, G., Faure, K., et al. (2013). Candida albicans and Pseudomonas aeruginosa interactions: more than an opportunistic criminal association? Méd. Mal. Infect. 43, 146-151. doi: 10.1016/j.medmal.2013.02.005

Middleton, B., Rodgers, H. H. C., Cámara, M., Knox, A. J., Williams, P., and Hardman, A. (2002). Direct detection of $\mathrm{N}$-acylhomoserine lactones in cystic fibrosis sputum. FEMS Microbiol. Lett. 207, 1-7. doi: 10.1016/S03781097(01)00556-0

Mima, T., and Schweizer, H. P. (2010). The BpeAB-OprB efflux pump of Burkholderia pseudomallei $1026 \mathrm{~b}$ does not play a role in quorum sensing, virulence factor production, or extrusion of aminoglycosides but is a broadspectrum drug efflux system. Antimicrob. Agents Chemother. 54, 3113-3120. doi: 10.1128/AAC.01803-09

Minagawa, S., Inami, H., Kato, T., Sawada, S., Yasuki, T., Miyairi, S., et al. (2012). RND type efflux pump system MexAB-OprM of Pseudomonas aeruginosa selects bacterial languages, 3-oxo-acyl-homoserine lactones, for cell-to-cell communication. BMC Microbiol. 12:70. doi: 10.1186/14712180-12-70

Neyrolles, O., and Guilhot, C. (2011). Recent advances in deciphering the contribution of Mycobacterium tuberculosis lipids to pathogenesis. Tuberculosis 91, 187-195. doi: 10.1016/j.tube.2011.01.002

Nikaido, E., Giraud, E., Baucheron, S., Yamasaki, S., Wiedemann, A., Okamoto, K., et al. (2012). Effects of indole on drug resistance and virulence of Salmonella enterica serovar typhimurium revealed by genome-wide analyses. Gut Pathog. 4:5. doi: 10.1186/1757-4749-4-5

Nikaido, E., Shirosaka, I., Yamaguchi, A., and Nishino, K. (2011). Regulation of the AcrAB multidrug efflux pump in Salmonella enterica serovar typhimurium in response to indole and paraquat. Microbiology 157(Pt. 3), 648-655. doi: 10.1099/mic.0.045757-0

Nikaido, E., Yamaguchi, A., and Nishino, K. (2008). AcrAB multidrug efflux pump regulation in Salmonella enterica serovar typhimurium by RamA in response to environmental signals. J. Biol. Chem. 283, 24245-24253. doi: 10.1074/jbc.M804544200

Olivares, J., Alvarez-Ortega, C., Linares, J. F., Rojo, F., Kohler, T., and Martinez, J. L. (2012). Overproduction of the multidrug efflux pump MexEF-OprN does not impair Pseudomonas aeruginosa fitness in competition tests, but produces specific changes in bacterial regulatory networks. Environ. Microbiol. 14, 19681981. doi: 10.1111/j.1462-2920.2012.02727.x

Oliver, A., Mulet, X., Lopez-Causape, C., and Juan, C. (2015). The increasing threat of Pseudomonas aeruginosa high-risk clones. Drug Resist. Updat. 2, 41-59. doi: 10.1016/j.drup.2015.08.002

Palumbo, J. D., Kado, C. I., and Phillips, D. A. (1998). An isoflavonoidinducible efflux pump in Agrobacterium tumefaciens is involved in competitive colonization of roots. J. Bacteriol. 180, 3107-3113.

Papadopoulos, C. J., Carson, C. F., Chang, B. J., and Riley, T. V. (2008). Role of the MexAB-OprM efflux pump of Pseudomonas aeruginosa in tolerance to tea tree (Melaleuca alternifolia) oil and its monoterpene components terpinen-4-ol, 1,8-cineole, and alpha-terpineol. Appl. Environ. Microbiol. 74, 1932-1935. doi: 10.1128/AEM.02334-07

Pearson, J. P., Van Delden, C., and Iglewski, B. H. (1999). Active efflux and diffusion are involved in transport of Pseudomonas aeruginosa cell-to-cell signals. J. Bacteriol. 181, 1203-1210.

Piddock, L. J. (2006a). Clinically relevant chromosomally encoded multidrug resistance efflux pumps in bacteria. Clin. Microbiol. Rev. 19, 382-402. doi: 10.1128/CMR.19.2.382-402.2006

Piddock, L. J. (2006b). Multidrug-resistance efflux pumps - not just for resistance. Nat. Rev. Microbiol. 4, 629-636. doi: 10.1038/nrmicro1464

Pinero-Fernandez, S., Chimerel, C., Keyser, U. F., and Summers, D. K. (2011). Indole transport across Escherichia coli membranes. J. Bacteriol. 193, 17931798. doi: 10.1128/JB.01477-10

Pletzer, D., and Weingart, H. (2014). Characterization and regulation of the resistance-nodulation-cell division-type multidrug efflux pumps MdtABC and MdtUVW from the fire blight pathogen Erwinia amylovora. BMC Microbiol. 14:185. doi: 10.1186/1471-2180-14-185

Poole, K. (2001). Multidrug resistance in Gram-negative bacteria. Curr. Opin. Microbiol. 4, 500-508. doi: 10.1016/S1369-5274(00)00242-3

Prouty, A. M., Brodsky, I. E., Falkow, S., and Gunn, J. S. (2004). Bile-salt-mediated induction of antimicrobial and bile resistance in Salmonella typhimurium. Microbiology 150(Pt. 4), 775-783. doi: 10.1099/mic.0.26769-0 
Pusey, P. L., Stockwell, V. O., Reardon, C. L., Smits, T. H., and Duffy, B. (2011). Antibiosis activity of Pantoea agglomerans biocontrol strain E325 against Erwinia amylovora on apple flower stigmas. Phytopathology 101, 1234-1241. doi: 10.1094/phyto-09-10-0253

Quillin, S. J., Schwartz, K. T., and Leber, J. H. (2011). The novel Listeria monocytogenes bile sensor BrtA controls expression of the cholic acid efflux pump MdrT. Mol. Microbiol. 81, 129-142. doi: 10.1111/j.13652958.2011.07683.x

Randall, L. P., and Woodward, M. J. (2002). The multiple antibiotic resistance (mar) locus and its significance. Res. Vet. Sci. 72, 87-93. doi: 10.1053/rvsc.2001.0537

Ravirala, R. S., Barabote, R. D., Wheeler, D. M., Reverchon, S., Tatum, O., Malouf, J., et al. (2007). Efflux pump gene expression in Erwinia chrysanthemi is induced by exposure to phenolic acids. Mol. Plant Microbe Interact. 20, 313-320. doi: 10.1094/MPMI-20-3-0313

Redgrave, L. S., Sutton, S. B., Webber, M. A., and Piddock, L. J. (2014). Fluoroquinolone resistance: mechanisms, impact on bacteria, and role in evolutionary success. Trends Microbiol. 22, 438-445. doi: 10.1016/j.tim.2014.04.007

Rosenberg, E. Y., Bertenthal, D., Nilles, M. L., Bertrand, K. P., and Nikaido, H. (2003). Bile salts and fatty acids induce the expression of Escherichia coli AcrAB multidrug efflux pump through their interaction with Rob regulatory protein. Mol. Microbiol. 48, 1609-1619. doi: 10.1046/j.1365-2958.2003. 03531.x

Rossbach, S., Kunze, K., Albert, S., Zehner, S., and Gottfert, M. (2014). The Sinorhizobium meliloti EmrAB efflux system is regulated by flavonoids through a TetR-like regulator (EmrR). Mol. Plant Microbe Interact. 27, 379-387. doi: 10.1094/MPMI-09-13-0282-R

Sakhtah, H., Koyama, L., Zhang, Y., Morales, D. K., Fields, B. L., PriceWhelan, A., et al. (2016). The Pseudomonas aeruginosa efflux pump MexGHI-OpmD transports a natural phenazine that controls gene expression and biofilm development. Proc. Natl. Acad. Sci. U.S.A. 113:E3538-47. doi: 10.1073/pnas.1600424113

Sanchez, P., Alonso, A., and Martinez, J. L. (2004). Regulatory regions of smeDEF in Stenotrophomonas maltophilia strains expressing different amounts of the multidrug efflux pump SmeDEF. Antimicrob. Agents Chemother. 48, 2274 2276. doi: 10.1128/AAC.48.6.2274-2276.2004

Sanchez, P., Linares, J. F., Ruiz-Diez, B., Campanario, E., Navas, A., Baquero, F., et al. (2002). Fitness of in vitro selected Pseudomonas aeruginosa nalB and nfxB multidrug resistant mutants. J. Antimicrob. Chemother. 50, 657-664. doi: 10.1093/jac/dkf185

Santos, M. R., Marques, A. T., Becker, J. D., and Moreira, L. M. (2014). The Sinorhizobium meliloti EmrR regulator is required for efficient colonization of Medicago sativa root nodules. Mol. Plant Microbe Interact. 27, 388-399. doi: 10.1094/MPMI-09-13-0284-R

Sawada, I., Maseda, H., Nakae, T., Uchiyama, H., and Nomura, N. (2004). A quorum-sensing autoinducer enhances the mexAB-oprM effluxpump expression without the MexR-mediated regulation in Pseudomonas aeruginosa. Microbiol. Immunol. 48, 435-439. doi: 10.1111/j.1348-0421.2004. tb03533. X

Shimada, Y., Kinoshita, M., Harada, K., Mizutani, M., Masahata, K., Kayama, H., et al. (2013). Commensal bacteria-dependent indole production enhances epithelial barrier function in the colon. PLOS ONE 8:e80604. doi: 10.1371/journal.pone.0080604

Smith, H. E., and Blair, J. M. (2014). Redundancy in the periplasmic adaptor proteins AcrA and AcrE provides resilience and an ability to export substrates of multidrug efflux. J. Antimicrob. Chemother. 69, 982-987. doi: 10.1093/jac/dkt481

Smith, R. S., Fedyk, E. R., Springer, T. A., Mukaida, N., Iglewski, B. H., and Phipps, R. P. (2001). IL-8 production in human lung fibroblasts and epithelial cells activated by the Pseudomonas autoinducer N-3-oxododecanoyl homoserine lactone is transcriptionally regulated by NF-kappa B and activator protein-2. J. Immunol. 167, 366-374. doi: 10.4049/jimmunol.167.1.366

Soto, S. M. (2013). Role of efflux pumps in the antibiotic resistance of bacteria embedded in a biofilm. Virulence 4, 223-229. doi: 10.4161/viru.23724

Spaniol, V., Bernhard, S., and Aebi, C. (2015). Moraxella catarrhalis AcrABOprM efflux pump contributes to antimicrobial resistance and is enhanced during cold shock response. Antimicrob. Agents Chemother. 59, 1886-1894. doi: 10.1128/AAC.03727-14

Spaniol, V., Wyder, S., and Aebi, C. (2013). RNA-Seq-based analysis of the physiologic cold shock-induced changes in Moraxella catarrhalis gene expression. PLoS ONE 8:e68298. doi: 10.1371/journal.pone.0068298

Swift, S., and Downie, J. A. (2001). Quorum sensing as a population-densitydependent determinant of bacterial physiology. Adv. Microbial Physiol. 45, 199-270.

Szekely, R., and Cole, S. T. (2016). Mechanistic insight into mycobacterial MmpL protein function. Mol. Microbiol. 99, 831-834. doi: 10.1111/mmi.13306

Takeshima, K., Hidaka, T., Wei, M., Yokoyama, T., Minamisawa, K., Mitsui, H., et al. (2013). Involvement of a novel genistein-inducible multidrug efflux pump of Bradyrhizobium japonicum early in the interaction with Glycine max (L.) Merr. Microbes Environ. 28, 414-421. doi: 10.1264/jsme2.ME13057

Talwalkar, J. S., and Murray, T. S. (2016). The approach to Pseudomonas aeruginosa in cystic fibrosis. Clin. Chest Med. 37, 69-81. doi: 10.1016/j.ccm.2015.10.004

Tateda, K., Ishii, Y., Horikawa, M., Matsumoto, T., Miyairi, S., Pechere, J. C., et al. (2003). The Pseudomonas aeruginosa autoinducer N-3-oxododecanoyl homoserine lactone accelerates apoptosis in macrophages and neutrophils. Infect. Immun. 71, 5785-5793. doi: 10.1128/IAI.71.10.5785-5793.2003

Taylor, D. L., Bina, X. R., and Bina, J. E. (2012). Vibrio cholerae VexH encodes a multiple drug efflux pump that contributes to the production of cholera toxin and the toxin co-regulated pilus. PLOS ONE 7:e38208. doi: 10.1371/journal.pone.0038208

Thanassi, D. G., Cheng, L. W., and Nikaido, H. (1997). Active efflux of bile salts by Escherichia coli. J. Bacteriol. 179, 2512-2518.

Ulrich, R. L., Deshazer, D., Brueggemann, E. E., Hines, H. B., Oyston, P. C., and Jeddeloh, J. A. (2004). Role of quorum sensing in the pathogenicity of Burkholderia pseudomallei. J. Med. Microbiol. 53(Pt. 11), 1053-1064. doi: 10.1099/jmm.0.45661-0

Vargas, P., Farias, G. A., Nogales, J., Prada, H., Carvajal, V., Baron, M., et al. (2013). Plant flavonoids target Pseudomonas syringae pv. tomato DC3000 flagella and type III secretion system. Environ. Microbiol. Rep. 5, 841-850. doi: 10.1111/1758-2229.12086

Vargas, P., Felipe, A., Michan, C., and Gallegos, M. T. (2011). Induction of Pseudomonas syringae pv. tomato DC3000 MexAB-OprM multidrug efflux pump by flavonoids is mediated by the repressor PmeR. Mol. Plant Microbe Interact. 24, 1207-1219. doi: 10.1094/MPMI-03-11-0077

Vila, J., and Martínez, J. L. (2008). Clinical impact of the over-expression of efflux pump in nonfermentative Gram-negative bacilli, development of efflux pump inhibitors. Curr. Drug Targets 9, 797-807. doi: 10.2174/138945008785747806

Wagner, C., Zimmermann, S., Brenner-Weiss, G., Hug, F., Prior, B., Obst, U., et al. (2007). The quorum-sensing molecule N-3-oxododecanoyl homoserine lactone (3OC12-HSL) enhances the host defence by activating human polymorphonuclear neutrophils (PMN). Anal. Bioanal. Chem. 387, 481-487. doi: 10.1007/s00216-006-0698-5

Wagner, V. E., and Iglewski, B. H. (2008). Pseudomonas aeruginosa biofilms in CF infection. Clin. Rev. Allergy Immunol. 35, 124-134. doi: 10.1007/s12016-0088079-9

Webber, M. A., Bailey, A. M., Blair, J. M., Morgan, E., Stevens, M. P., Hinton, J. C., et al. (2009). The global consequence of disruption of the AcrAB-TolC efflux pump in Salmonella enterica includes reduced expression of SPI-1 and other attributes required to infect the host. J. Bacteriol. 191, 4276-4285. doi: 10.1128/JB.00363-09

Williams, P. (2007). Quorum sensing, communication and cross-kingdom signalling in the bacterial world. Microbiology 153(Pt. 12), 3923-3938. doi: 10.1099/mic.0.2007/012856-0

Williams, P., Winzer, K., Chan, W. C., and Camara, M. (2007). Look who's talking: communication and quorum sensing in the bacterial world. Philos. Trans. R. Soc. Lond. B Biol. Sci. 362, 1119-1134. doi: 10.1098/rstb.2007.2039

Wongtrakoongate, P., Tumapa, S., and Tungpradabkul, S. (2012). Regulation of a quorum sensing system by stationary phase sigma factor RpoS and their coregulation of target genes in Burkholderia pseudomallei. Microbiol. Immunol. 56, 281-294. doi: 10.1111/j.1348-0421.2012.00447.x

Yamaguchi, A., Nakashima, R., and Sakurai, K. (2015). Structural basis of RND-type multidrug exporters. Front. Microbiol. 6:327. doi: $10.3389 /$ fmicb. 2015.00327 
Zhang, L., Li, X. Z., and Poole, K. (2001). SmeDEF multidrug efflux pump contributes to intrinsic multidrug resistance in Stenotrophomonas maltophilia. Antimicrob. Agents Chemother. 45, 3497-3503. doi: 10.1128/AAC.45.12.34973503.2001

Zhang, L., and Mah, T. F. (2008). Involvement of a novel efflux system in biofilm-specific resistance to antibiotics. J. Bacteriol. 190, 4447-4452. doi: 10.1128/JB.01655-07

Zimmermann, S., Wagner, C., Müller, W., Brenner-Weiss, G., Hug, F., Prior, B., et al. (2006). Induction of neutrophil chemotaxis by the quorum-sensing molecule N-(3-oxododecanoyl)-L-homoserine lactone. Infect. Immun. 74, 5687-5692. doi: 10.1128/IAI.01940-05
Conflict of Interest Statement: The authors declare that the research was conducted in the absence of any commercial or financial relationships that could be construed as a potential conflict of interest.

Copyright (c) 2016 Alcalde-Rico, Hernando-Amado, Blanco and Martínez. This is an open-access article distributed under the terms of the Creative Commons Attribution License (CC BY). The use, distribution or reproduction in other forums is permitted, provided the original author(s) or licensor are credited and that the original publication in this journal is cited, in accordance with accepted academic practice. No use, distribution or reproduction is permitted which does not comply with these terms. 


\title{
PSM-Mec-A Virulence Determinant that Connects Transcriptional Regulation, Virulence, and Antibiotic Resistance in Staphylococci
}

\author{
Li Qin ${ }^{1,2}$, Joshua W. McCausland ${ }^{1}$, Gordon Y. C. Cheung ${ }^{1}$ and Michael Otto ${ }^{1 *}$ \\ ${ }^{1}$ Pathogen Molecular Genetics Section, Laboratory of Bacteriology, National Institute of Allergy and Infectious Diseases, \\ National Institutes of Health, Bethesda, MD, USA, ${ }^{2}$ Department of Dermatology, Wuhan No. 1 Hospital, Tongji Medical \\ College, Huazhong University of Science and Technology, Wuhan, China
}

\section{OPEN ACCESS}

Edited by:

Axel Cloeckaert,

French National Institute for Agricultural Research (INRA), France

Reviewed by:

Jan Maarten Van Dijl, University Medical Center Groningen, Netherlands

Dinesh Sriramulu, Shres Consultancy (Life Sciences),

India

${ }^{*}$ Correspondence: Michael Otto

motto@niaid.nih.gov

Specialty section:

This article was submitted to

Antimicrobials, Resistance and

Chemotherapy

a section of the journal

Frontiers in Microbiology

Received: 07 June 2016 Accepted: 05 August 2016

Published: 22 August 2016

Citation:

Qin L, McCausland JW, Cheung GYC and Otto M (2016) PSM-Mec-A Virulence Determinant that Connects Transcriptional Regulation, Virulence,

and Antibiotic Resistance in Staphylococci.

Front. Microbiol. 7:1293.

doi: 10.3389/fmicb.2016.01293
PSM-mec is a secreted virulence factor that belongs to the phenol-soluble modulin (PSM) family of amphipathic, alpha-helical peptide toxins produced by Staphylococcus species. All known PSMs are core genome-encoded with the exception of PSM-mec, whose gene is found in specific sub-types of SCCmec methicillin resistance mobile genetic elements present in methicillin-resistant Staphylococcus aureus and coagulase-negative staphylococci. In addition to the cytolytic translational product, PSM-mec, the psm-mec locus encodes a regulatory RNA. In S. aureus, the psm-mec locus influences cytolytic capacity, methicillin resistance, biofilm formation, cell spreading, and the expression of other virulence factors, such as other PSMs, which results in a significant impact on immune evasion and disease. However, these effects are highly strain-dependent, which is possibly due to differences in PSM-mec peptide vs. psm-mec RNA-controlled effects. Here, we summarize the functional properties of PSM-mec and the psm-mec RNA molecule and their roles in staphylococcal pathogenesis and physiology.

Keywords: Staphylococcus aureus, Staphylococcus epidermidis, phenol-soluble modulin, PSM-mec, regulatory RNA, SCCmec, virulence

\section{INTRODUCTION}

Staphylococcus aureus is a serious human pathogen responsible for a multitude of human diseases, which range from acute skin and soft tissue infections to more severe illnesses such as catheter-associated bacteremia, necrotizing pneumonia, and osteomyelitis. Since the emergence of methicillin-resistant S. aureus (MRSA) in the early 1960s, S. aureus has continued to cause significant morbidity, mortality, and a considerable financial burden for public health systems. For S. aureus and its closely related cousin, Staphylococcus epidermidis, the acquisition of antibiotic resistance, coupled with the ability to attach to surfaces and form sticky, multicellular agglomerations (biofilms; Costerton, 1999) on indwelling catheters, are two defining hallmarks that account for the ongoing difficulty in treating immuno-compromised individuals (Otto, 2008) with infections caused by Hospital-Associated MRSA [HA-MRSA] (Otto, 2012) and S. epidermidis (Otto, 2009).

Genetic mutations and/or the acquisition of mobile genetic elements (MGEs) that harbor drug resistance genes are two common mechanisms that are responsible for antibiotic resistance in microorganisms. Arguably the most important staphylococcal MGE is Staphylococcus cassette 
chromosome mec (SCCmec), which codes for methicillin resistance. Recently, a unique locus was discovered in specific types of SSCmec elements in S. aureus and S. epidermidis, which comprises a small regulatory (sr)RNA and an embedded gene encoding a cytolytic peptide called PSM-mec (Queck et al., 2009). PSM-mec belongs to the phenol-soluble modulin (PSM) family of amphipathic, alpha-helical peptide toxins produced by Staphylococcus species (Wang et al., 2007, 2011; Diep and Otto, 2008; Otto, 2014; Cheung et al., 2014a), which collectively play an important role as virulence determinants in many facets of S. aureus and S. epidermidis pathogenesis (Wang et al., 2011; Periasamy et al., 2012; Cassat et al., 2013; Cheung et al., 2014a). Here, we will discuss the current knowledge on the PSM-mec and the psm-mec srRNA molecule, with a focus on their roles in $S$. aureus pathogenesis.

\section{THE DISTRIBUTION OF THE psm-mec LOCUS IN SCCmec}

SCCmec elements, which range in size from 21 to $67 \mathrm{~kb}$, comprise as characteristic components, the mecA gene that codes for a penicillin-binding protein, a $c c r$ gene complex for site-specific recombination, and flanking repeat sequences for integration into the genome (International Working Group on the Classification of Staphylococcal Cassette Chromosome Elements, 2009). SCCmec elements are classified into 11 different "types," as defined by the presence of different $c c r$ and mec resistance genes (International Working Group on the Classification of Staphylococcal Cassette Chromosome Elements, 2009; Li et al., 2011; Shore et al., 2011) and further divided into "subtypes" based on the presence of other resistance genes and transposons in the non-essential $\mathrm{J}$ regions (Ito et al., 2003). The mec gene complex has four core elements; IS431, mecA, mecR1, and mecI, which represent the genes coding for insertion sequence IS431, a modified penicillin binding protein $2 \mathrm{a}(\mathrm{PBP} 2 \mathrm{a})$, the signal transducer protein, and the methicillin resistance regulatory protein (International Working Group on the Classification of Staphylococcal Cassette Chromosome Elements, 2009; Shore and Coleman, 2013; Figure 1A).

The psm-mec locus is found in the $\mathrm{J} 2$ region adjacent to mecI of the class A mec gene complex in several widespread HA-MRSA lineages, but most prominently in Sequence Type (ST) 5, ST36, ST45, ST225, and ST239 strains (Deurenberg and Stobberingh, 2009; Nübel et al., 2010; Castillo-Ramirez et al., 2012; Schulte et al., 2013), which all have either SCCmec types II or III (Chatterjee et al., 2011; Monecke et al., 2012; Josten et al., 2014). However, more detailed analysis revealed that the psm-mec locus is found in SCCmec types II, IIA, IIB, IID, III, and VIII of many Staphylococcus species (Monecke et al., 2012; Shore and Coleman, 2013).

Antibiotic resistance in bacteria is often associated with a reduced fitness cost to the bacterial host (Otto, 2013a). We recently reported that the expression of the PSM-mec peptide impacted oxacillin resistance in S. aureus (Cheung et al., 2014b), suggesting that a delicate balance may exist between virulence gene expression and methicillin resistance in MRSA strains carrying the psm-mec locus.

\section{EXPRESSION AND REGULATION OF THE PSM-mec PEPTIDE}

The production of the PSM-mec peptide, which can be identified in bacterial culture filtrates by high-pressure liquid chromatography/mass spectrometry (HPLC/MS) (Queck et al., 2009) or Matrix-assisted laser desorption/ionization Time of Flight (MALDI-TOF) analysis (Josten et al., 2014), is a reliable indicator of methicillin resistance. However, the restricted distribution of the psm-mec locus severely limits the potential use of HPLC-based detection of PSM-mec for routine clinical screening of methicillin resistance.

The expression of all PSMs including the MGE-encoded PSMmec is positively regulated by the accessory gene regulatory (agr) quorum sensing system (Novick, 2003; Queck et al., 2008; Chatterjee et al., 2011). This up-regulation is dependent on the binding of activated AgrA, the response regulator of $a g r$, to promoter sequences upstream of the psm genes (Queck et al., 2008), but this has not directly been demonstrated for the psm-mec promoter. Interestingly, PSM expression is also enhanced in the presence of calf serum (Oogai et al., 2011) and up-regulated within intracellular environments after the activation of the stringent response (Geiger et al., 2012). Whether this is also the case for PSM-mec is not known.

PSM-mec production was shown to be especially frequent among clinical isolates of $S$. epidermidis compared to isolates from the skin of healthy individuals (Queck et al., 2009), which is a direct consequence of the genetic connection of psm-mec with methicillin resistance, which occurs much more frequently in clinical isolates. However, expression levels of PSM-mec can vary strongly among isolates (Queck et al., 2009). The mechanistic reasons for varying levels of PSM-mec expression between isolates cannot be fully explained but a documented $-7 \mathrm{~T}>\mathrm{C}$ mutation in the psm-mec promoter is known to diminish PSMmec expression in a subset of HA-MRSA strains (Kaito et al., 2011, 2013; Aoyagi et al., 2014).

\section{CHARACTERIZATION OF PSM-mec PEPTIDE}

Like all members of the PSM family, PSM-mec is expressed with an N-terminal formyl-methionine and secreted without a signal peptide (Wang et al., 2007; Queck et al., 2009). Although this has not yet been shown directly for PSM-mec, it is likely that it is also exported by one or both of the two dedicated ATP-Binding Cassette (ABC) transporters that secrete PSMs (Chatterjee et al., 2013; Yoshikai et al., 2016). The 22 amino acid PSM-mec fits into the class of shorter $\alpha$-type PSMs, which are anywhere between 20-25 amino acids in length, as opposed to the $\beta$-type, which are approximately twice as long (43-45 amino acids) (Cheung et al., 2014a).

PSM-mec has a unique cysteine residue at position 17, while cysteine residues are not present in the sequences of any other 


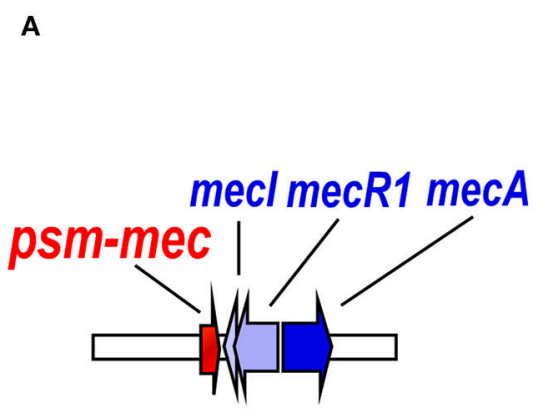

Class A mecA complex
C

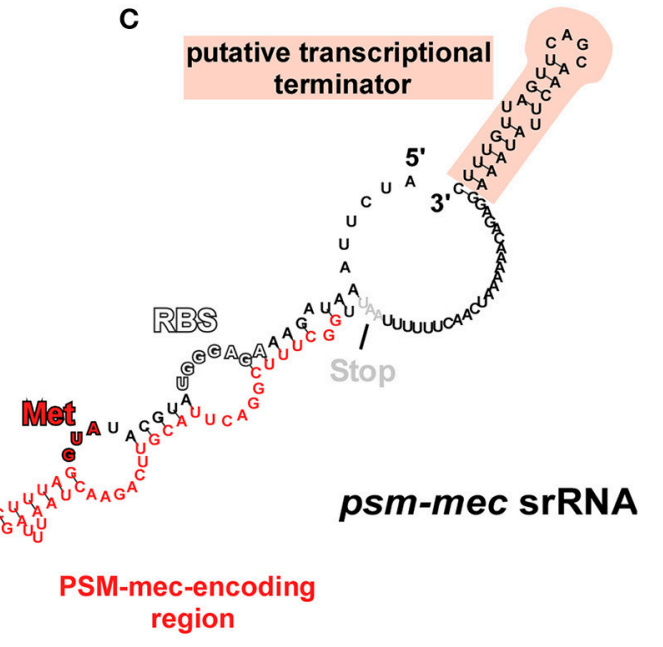

B

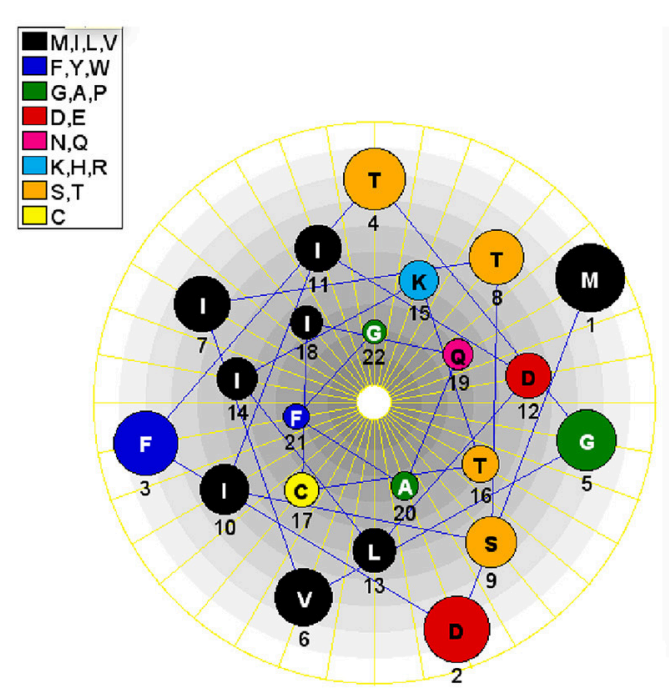

\section{fM D F T G VITSIIDLIKTCIQAF G}

FIGURE 1 | The psm-mec locus and its products. (A) The psm-mec gene is located on the class A mecA complex in SCCmec elements of types II, III, and VIII, next to the $m e c / / m e c R 1 / m e c A$ genes that confer methicillin resistance and its regulation. (B) The PSM-mec peptide product of the psm-mec locus forms an $\alpha$-helix with pronounced amphiphathy; helical wheel presentation. (C) The psm-mec gene is part of a regulatory RNA with pronounced predicted secondary structure. The psm-mec gene is entirely embedded in a highly folded, large stem loop. The psm-mec srRNA only slightly extends toward the $3^{\prime}$ end and ends with a characteristic predicted terminator stem loop structure (Note, however, that Kaito et al. reported the srRNA to be slightly longer at the $5^{\prime}$ end). RBS, ribosomal binding site; Start codon coding for ( $\mathrm{N}$-formyl) methionine.

known PSM (Figure 1B). The notion of a hypothetical dimeric form of PSM-mec, due to the formation of disufide bridges created from oxidized cysteine residues, was formally discounted after size exclusion chromatography experiments showed no difference in extracted ion chromatograms of PSM-mec mutants compared to that of the unaltered PSM-mec peptide (Queck et al., 2009). However, the cysteine residue was crucial for pro-inflammatory and cytolytic activity, probably because it is essential for the PSM-mec secondary structure (Queck et al., 2009).

\section{THE PRO-INFLAMMATORY AND CYTOLYTIC PROPERTIES OF PSM-mEC}

At nanomolar concentrations, the PSMs are powerful pro-inflammatory agents and have an extraordinary capacity for activating and stimulating neutrophils (Wang et al., 2007), which is exerted through their interactions with human formyl peptide receptor 2 (FPR2; Kretschmer et al., 2010), a G proteincoupled receptor expressed on multiple immune cell types (Migeotte et al., 2006). Although differences in activity are 
observed between PSM members, PSM-mec readily up-regulates both CD11b and gp91phox expression, induces calcium flux, chemotaxis, and IL-8 release in neutrophils (Queck et al., 2009).

On the other hand, several PSM members of the $\alpha$-type possess potent cytolytic activities, and an ever-increasing number of immune and non-immune cells are being discovered that are subject to PSM-mediated cytolysis (Cheung et al., 2014a). The lysis of eukaryotic cells by PSMs, which occurs at micromolar concentrations, is likely mediated in a receptor-independent manner (Kretschmer et al., 2010), While the involvement of receptors cannot be formally excluded, the facts that PSMs are surfactant molecules, lyse artificial phospholipid vesicles (Duong et al., 2012; Laabei et al., 2014), and FPR2 is not involved in lysis (Kretschmer et al., 2010), speak for such a receptor-independent mechanism. Lytic activity is, however, heavily influenced by membrane composition (Laabei et al., 2014). It is believed to be crucial for PSM-mediated immune evasion and progression of staphylococcal pathogenesis. PSM-mec follows the characteristic cytolytic profile of some $\alpha$-type PSMs and can lyse human erythrocytes and neutrophils at a comparatively moderate level that is similar to that exerted by $\delta$-toxin (Queck et al., 2009).

\section{THE psm-mec srRNA}

The first clue for the presence of an srRNA within the psm-mec locus stemmed from experiments with MRSA strains transformed with a plasmid carrying 575 nucleotides amplified from SCCmec type II, termed the "F region" (Kaito et al., 2008). The authors reported that an open reading frame (ORF) transcribed in the opposite direction of the psm-mec gene, coined "fudoh," encoded a protein that controlled colony spreading (Kaito et al., 2008). It was later discovered from experiments using $S$. aureus isogenic fudoh point-deletion mutants that the phenotypic differences were in fact influenced by an srRNA (Kaito et al., 2011), which contains the psm-mec gene (Kaito et al., $2011,2013)$, in a way similar to the genetic layout of $\delta$-toxin, whose gene is found within the regulatory RNA of the agr system, RNAIII (Novick, 2003). Interestingly, our group reported that the psm-mec srRNA transcript length was $143 \mathrm{bp}, 14$ nucleotides shorter than that described by Kaito et al. (2013) (Figure 1C). Notably, in any case, the psm-mec srRNA only barely exceeds the ORF coding for PSM-mec. This contrasts with RNAIII, which is considerably longer than the $\delta$-toxin gene, $h l d$. Additionally, the psm-mec srRNA is estimated to have a half-life of approximately $20 \mathrm{~min}$ (Kaito et al., 2013), which is significantly shorter than the $\sim 45$ min half-life described for RNAIII (Huntzinger et al., 2005). The only reported mechanism by which the psm-mec srRNA exerts it gene regulatory activity is through its interaction with the agrA transcript resulting in the overall decrease of AgrA activity (Kaito et al., 2013), which is entirely dependent on the first 60 nucleotides of the transcript (Kaito et al., 2013), where key anti-sense base-pair interactions occur (Cheung et al., 2014b). Because AgrA strictly regulates activity of the $p s m \alpha$ and $p s m \beta$ promoters (Queck et al., 2008), many of the psm-mec srRNAmediated phenotypes likely are mostly due to a repression of the production of the strongly cytolytic and pro-inflammatory PSM $\alpha$ peptides (Table 1). Kaito et al. initially observed up to eight-fold reduction in PSM expression when the psm-mec locus was over-expressed on a plasmid in methicillin-sensitive S. aureus strains or strains with SCCmec type IV (Kaito et al., 2011). However, the differences in PSM reduction were less dramatic, and often inconsistent, in isogenic psm-mec mutants naturally harboring SCCmec types II and III (Chatterjee et al., 2011), indicating a high degree of strain dependence regarding the role of the psm-mec srRNA in $S$. aureus.

\section{THE IMPACT OF THE psm-mec srRNA ON BIOFILM FORMATION, COLONY SPREADING, AND EXPRESSION OF S. aureus VIRULENCE FACTORS}

PSM peptides have a considerable impact on biofilm formation, which is based on their detergent-like properties (Vuong et al., 2000; Kong et al., 2006). PSMs cause the structuring and maturation of biofilms, which includes the formation of nutrienttransporting channels (Wang et al., 2011; Periasamy et al., 2012). PSMs also facilitate the detachment of biofilm clusters in vitro and in vivo (Wang et al., 2011; Periasamy et al., 2012; Otto, 2013b). It was shown that isogenic agr mutants (Vuong et al., 2000, 2004), total psm deletion mutants in all psm genes, and even single mutants in either the $p s m \alpha, p s m \beta$, or hld loci (Wang et al., 2011; Periasamy et al., 2012) formed thicker biofilms compared to their wild type counterparts. Therefore, it was surprising to find that several isogenic psm-mec $S$. aureus mutants showed a reverse phenotype, albeit the change in biofilm formation was overall minor (Queck et al., 2009; Kaito et al., 2013). A phenotype of increased aggregation similar to that conferred by other PSMs (Dastgheyb et al., 2015) was only found in a strain in which the PSM-mec peptide was expressed in high relative amounts as compared to other PSMs (Queck et al., 2009). In most psm-mec-positive $S$. aureus strains, the regulatory effect of the psm-mec srRNA on the expression of other biofilm-dispersing

TABLE 1 | Activities of the PSM-mec peptide and the psm-mec srRNA.

\begin{tabular}{|c|c|}
\hline $\begin{array}{l}\text { Virulence determinant } \\
\text { within the psm-mec locus }\end{array}$ & Known in vitro activities in S. aureus \\
\hline PSM-mec peptide & $\begin{array}{l}\text { FPR2-mediated pro-inflammatory activity at nM } \\
\text { concentrations: } \\
\text { - Increased CD11b expression } \\
\text { - Increased gp91phox expression } \\
\text { - Increased IL-8 expression } \\
\text { - Increased calcium flux } \\
\text { - Increased chemotaxis } \\
\text { Receptor-independent cytolytic activity at } \mu \mathrm{M} \\
\text { concentrations toward: } \\
\text { - Erythrocytes } \\
\text { - Neutrophils } \\
\text { Peptide expression decreases oxacillin resistance }\end{array}$ \\
\hline psm-mec srRNA & $\begin{array}{l}\text { Represses AgrA activity through direct interaction } \\
\text { - Decreased PSM production }{ }^{\mathrm{b}} \\
\text { - Enhanced biofilm formation and aggregation }{ }^{\mathrm{b}} \\
\text { - Suppresses colony spreading } \\
\text { - Up-regulates SpA expression }{ }^{\mathrm{b}}\end{array}$ \\
\hline
\end{tabular}

a Only tested in one background strain.

${ }^{b}$ Strain-specific phenotype. 
PSMs (Periasamy et al., 2012) appears to over-shadow the lack of the direct dispersion effect of the PSM-mec peptide in psmmec mutants (Kaito et al., 2013). Thus, in most strains, the impact of the psm-mec locus on biofilm formation is opposite to that of other psm loci, although that effect is generally not very pronounced.

For many pathogenic bacteria, motility plays an important role in colonization and virulence (Cossart, 2002; Josenhans and Suerbaum, 2002; Krukonis and DiRita, 2003). Motility is commonly dependent on the expression of flagella and type IV pili (Kearns, 2010). Even though S. aureus has always been historically regarded as non-motile, as it does not have the genes for flagella or pili, S. aureus has repeatedly been shown to spread efficiently across the surface of soft agar (Kaito et al., 2011; Tsompanidou et al., 2012) and more recently, on fresh pork meat (Tsompanidou et al., 2013), through a PSMdependent mechanism (Lin et al., 2016), an effect now known as "colony-spreading" (Kaito and Sekimizu, 2007).

Originally, the observations of colony spreading and several other phenotypes were made using plasmid-based expression of the psm-mec locus in MRSA strains that did not carry the psmmec locus, such as $S$. aureus isolates that were either methicillinsensitive or MRSA strains that have recently emerged in the community (Community-Associated MRSA, CA-MRSA (Lowy, 1998; DeLeo et al., 2010). CA-MRSA strains are genetically distinct and notably more aggressive than HA-MRSA strains, show enhanced production of virulence factors, and are capable of causing disease in otherwise healthy individuals outside public healthcare settings (Tacconelli et al., 2004; Diep and Otto, 2008; Li et al., 2009; DeLeo et al., 2010). Furthermore, CA-MRSA strains characteristically possess SCCmec types IV or V (Diep and Otto, 2008; DeLeo et al., 2010) and therefore lack the psm-mec locus. Interestingly, CA-MRSA strains were reported to have a greater capacity to spread on soft agar compared to HA-MRSA strains (Kaito et al., 2008) and the absence of the psm-mec locus from CA-MRSA strains was speculated to cause that phenotype. Indeed, CA-MRSA strains transformed with plasmids containing the psm-mec locus were impaired in colony spreading. Conversely, isogenic HA-MRSA psm-mec mutants showed enhanced colony spreading (Kaito et al., 2013). Moreover, the inability of synthetic PSM-mec to rescue the colony spreading phenotype in an agr deletion strain of $S$. aureus (Tsompanidou et al., 2013) indicated that this regulation is predominantly mediated by the psm-mec srRNA. However, other studies showed that colony spreading is positively regulated by agr (Tsompanidou et al., 2011) and mainly controlled by mechanisms independent of the psm-mec srRNA, which include the production of several staphylococcal virulence factors, such as PSMs (Omae et al., 2012; Tsompanidou et al., 2013) and teichoic acids (Kaito and Sekimizu, 2007). In contrast, colony spreading is suppressed in the presence of multiple staphylococcal cell surface proteins (Tsompanidou et al., 2012). Taken together, the molecular mechanisms behind colony spreading in $S$. aureus reveal a somewhat complex network involving different, and often opposing, signals that affect the final phenotypic outcome. Lastly, it remains unknown whether colony spreading on agar surface is a mechanism universally adopted by all staphylococci and to what extent it matters for the colonization of epithelial surfaces in vivo.

In an effort to investigate $p s m$-mec-dependent regulation in the natural strain background without artificial plasmid expression-induced effects, our group used microarray profiling to compare the transcriptomes of wild-type strains compared with those of isogenic psm-mec mutants (Cheung et al., 2014b). Most strikingly, the primary conserved target of psm-mecdependent gene regulation was $s p a$, the gene coding for the immune evasion protein, surface protein A (SpA), which was independently confirmed (Kaito et al., 2011). We also found that the strength of psm-mec srRNA transcription affected SpA expression (Cheung et al., 2014b). The up-regulation of SpA by the psm-mec srRNA is especially interesting because it adds to a number of existing transcriptional regulators known to control spa (Gao and Stewart, 2004). The up-regulated expression of SpA may play an important but yet unidentified role in the manifestation of staphylococcal disease (Gomez et al., 2004; Cheng et al., 2009; Merino et al., 2009; Kim et al., 2011) in some psm-mec-harboring $S$. aureus strains.

\section{THE IMPACT OF THE psm-mec LOCUS ON S. aureus VIRULENCE}

Our group demonstrated that the ability of an isogenic psmmec $S$. aureus mutant to form skin abscesses and cause sepsis in mouse models of infection was severely attenuated compared to the parent strain (Queck et al., 2009), indicating that the PSMmec peptide contributed significantly to $S$. aureus pathogenesis. However, this phenotype could only be produced using an isogenic psm-mec S. aureus mutant whose parent strain produced more PSM-mec relative to other PSMs as compared to most psm-mec-harboring strains. Kaito et al. reported that the psmmec srRNA contributes to virulence, but in a fashion opposite to that mediated by the PSM-mec peptide (Kaito et al., 2011, 2013). Furthermore, those authors proposed that the psm-mec srRNA was generally responsible for the differences in virulence between HA- and CA-MRSA strains (Kaito et al., 2013). They also speculated that point mutations in the psm-mec locus underlie increased virulence of a certain lineage, ST764, but this was not directly shown using isogenic mutants (Suzuki et al., 2016). However, a phenotype of increased virulence is not always observed in isogenic psm-mec HA-MRSA strains (Chatterjee et al., 2011), suggesting that the contribution of psm-mec srRNA toward S. aureus pathogenesis is strongly strain-dependent and cannot serve as a generally applicable explanation for differences in virulence between CA- and HA-MRSA strains.

\section{THE ROLES OF THE PSM-mec PEPTIDE AND THE psm-mec REGULATORY RNA IN OTHER Staphylococcus SPECIES}

In addition to $S$. aureus, the psm-mec locus is detected in many different methicillin-resistant Staphylococcus species (Monecke et al., 2012). However, PSM-mec expression levels have only been rigorously studied in S. epidermidis (Queck et al., 2009). Our 
group showed that PSM-mec expression was only detected in methicillin-resistant $S$. epidermidis infection isolates, but never in methicillin-sensitive S. epidermidis strains (Queck et al., 2009). There is no amino acid or nucleotide sequence difference (100\% identity) between the $S$. aureus and S. epidermidis PSM-mec peptide or srRNA, respectively, based on a comparison of strains S. aureus MRSA252 and S. epidermidis RP62A, and differences are also absent or very minor in other strains. This is in accordance with the notion of a relatively recent move of SCCmec from coagulase-negative species to $S$. aureus.

To date, only one study has studied the impact of the psmmec locus on the phenotypes in S. epidermidis and S. haemolyticus (Ikuo et al., 2014). The results, which were based on S. epidermidis and S. haemolyticus strains without SCCmec transformed with plasmids harboring the psm-mec locus, showed a decrease in PSM production, as demonstrated in similar experiments with S. aureus (Kaito et al., 2011). Intriguingly, an increase of biofilm formation was only observed in experiments with S. epidermidis, as reported previously (Kaito et al., 2011), but not S. haemolyticus, suggesting that the impact of the psm-mec locus on virulence phenotypes among staphylococci is also highly strain-dependent. The role of PSM-mec in coagulase-negative staphylococci has not yet been directly investigated using isogenic deletion mutants.

\section{CONCLUSION}

It has become clear that the effects exerted by the psm-mec locus in $S$. aureus on virulence and virulence phenotypes

\section{REFERENCES}

Aoyagi, T., Kaito, C., Sekimizu, K., Omae, Y., Saito, Y., Mao, H., et al. (2014). Impact of psm-mec in the mobile genetic element on the clinical characteristics and outcome of SCCmec-II methicillin-resistant Staphylococcus aureus bacteraemia in Japan. Clin. Microbiol. Infect. 20, 912-919. doi: 10.1111/1469-0691.12575

Cassat, J. E., Hammer, N. D., Campbell, J. P., Benson, M. A., Perrien, D. S., Mrak, L. N., et al. (2013). A secreted bacterial protease tailors the Staphylococcus aureus virulence repertoire to modulate bone remodeling during osteomyelitis. Cell Host Microbe 13, 759-772. doi: 10.1016/j.chom.2013.05.003

Castillo-Ramirez, S., Corander, J., Marttinen, P., Aldeljawi, M., Hanage, W. P., Westh, H., et al. (2012). Phylogeographic variation in recombination rates within a global clone of methicillin-resistant Staphylococcus aureus. Genome Biol. 13:R126. doi: 10.1186/gb-2012-13-12-r126

Chatterjee, S. S., Chen, L., Joo, H. S., Cheung, G. Y., Kreiswirth, B. N., and Otto, M. (2011). Distribution and regulation of the mobile genetic element-encoded phenol-soluble modulin PSM-mec in methicillin-resistant Staphylococcus aureus. PLoS ONE 6:e28781. doi: 10.1371/journal.pone.0028781

Chatterjee, S. S., Joo, H. S., Duong, A. C., Dieringer, T. D., Tan, V. Y., Song, Y., et al. (2013). Essential Staphylococcus aureus toxin export system. Nat. Med. 19, 364-367. doi: 10.1038/nm.3047

Cheng, A. G., Kim, H. K., Burts, M. L., Krausz, T., Schneewind, O. and Missiakas, D. M. (2009). Genetic requirements for Staphylococcus aureus abscess formation and persistence in host tissues. FASEB J. 23, 3393-3404. doi: 10.1096/fj.09-135467

Cheung, G. Y., Joo, H. S., Chatterjee, S. S., and Otto, M. (2014a). Phenol-soluble modulins-critical determinants of staphylococcal virulence. FEMS Microbiol. Rev. 38, 698-719. doi: 10.1111/1574-6976.12057

Cheung, G. Y., Villaruz, A. E., Joo, H. S., Duong, A. C., Yeh, A. J. Nguyen, T. H., et al. (2014b). Genome-wide analysis of the regulatory function mediated by the such as biofilm formation are highly strain-dependent. They are also commonly rather moderate in extent compared to those conferred by other members of the PSM family. While great strides have been made to better understand the molecular underpinnings of gene regulatory psm-mec-dependent mechanisms in S. aureus pathogenesis, in the future the focus should be shifted toward the investigation of how the psm-mec locus affects the pathogenesis in other Staphylococcus species, especially S. epidermidis and S. haemolyticus, which, alongside $S$. aureus, are the most frequent and pathogenic agents of hospitalassociated infections around the world. As it is a commonly accepted notion that SCCmec elements originate from coagulasenegative staphylococci (Otto, 2013c), such investigation is bound to shed light on the "original" role of the psm-mec locus in staphylococcal physiology, with the potential to also explain the contrasting roles of psm-mec in the $S$. aureus background.

\section{AUTHOR CONTRIBUTIONS}

LQ, JM, GC, and MO contributed to the drafting of the manuscript and approved the final version.

\section{FUNDING}

This work was supported by the Intramural Research Program of the National Institute of Allergy and Infectious Diseases, U.S. National Institutes of Health (grant ZIA AI000904-16). small regulatory psm-mec RNA of methicillin-resistant Staphylococcus aureus. Int. J. Med. Microbiol. 304, 637-644. doi: 10.1016/j.ijmm.2014.04.008

Cossart, P. (2002). Molecular and cellular basis of the infection by Listeria monocytogenes: an overview. Int. J. Med. Microbiol. 291, 401-409. doi: 10.1078/1438-4221-00146

Costerton, J. W. (1999). Introduction to biofilm. Int. J. Antimicrob. Agents 11, 217-221. discussion: 237-239.

Dastgheyb, S. S., Villaruz, A. E., Le, K. Y., Tan, V. Y., Duong, A. C., Chatterjee, S. S., et al. (2015). Role of phenol-soluble modulins in formation of Staphylococcus aureus biofilms in synovial fluid. Infect. Immun. 83, 2966-2975. doi: 10.1128/IAI.00394-15

DeLeo, F. R., Otto, M., Kreiswirth, B. N., and Chambers, H. F. (2010). Communityassociated meticillin-resistant Staphylococcus aureus. Lancet 375, 1557-1568. doi: 10.1016/S0140-6736(09)61999-1

Deurenberg, R. H., and Stobberingh, E. E. (2009). The molecular evolution of hospital- and community-associated methicillin-resistant Staphylococcus aureus. Curr. Mol. Med. 9, 100-115. doi: 10.1111/j.1469-0691.2006.01573.x

Diep, B. A., and Otto, M. (2008). The role of virulence determinants in community-associated MRSA pathogenesis. Trends Microbiol. 16, 361-369. doi: 10.1016/j.tim.2008.05.002

Duong, A. C., Cheung, G. Y., and Otto, M. (2012). Interaction of phenolsoluble modulins with phosphatidylcholine vesicles. Pathogens 1, 3-11. doi: $10.3390 /$ pathogens 1010003

Gao, J., and Stewart, G. C. (2004). Regulatory elements of the Staphylococcus aureus protein A (Spa) promoter. J. Bacteriol. 186, 3738-3748. doi: 10.1128/JB.186.12.3738-3748.2004

Geiger, T., Francois, P., Liebeke, M., Fraunholz, M., Goerke, C., Krismer, B., et al. (2012). The stringent response of Staphylococcus aureus and its impact on survival after phagocytosis through the induction of intracellular PSMs expression. PLoS Pathog. 8:e1003016. doi: 10.1371/journal.ppat.1 003016 
Gomez, M. I., Lee, A., Reddy, B., Muir, A., Soong, G. Pitt, A., et al. (2004). Staphylococcus aureus protein A induces airway epithelial inflammatory responses by activating TNFR1. Nat. Med. 10, 842-848. doi: 10.1038/nm1079

Huntzinger, E., Boisset, S., Saveanu, C., Benito, Y., Geissmann, T., Namane, A., et al. (2005). Staphylococcus aureus RNAIII and the endoribonuclease III coordinately regulate spa gene expression. EMBO J. 24, 824-835. doi: 10.1038/sj.emboj.7600572

Ikuo, M., Nagano, G., Saito, Y., Mao, H., Sekimizu, K., and Kaito, C. (2014). Inhibition of exotoxin production by mobile genetic element SCCmec-encoded psm-mec RNA is conserved in staphylococcal species. PLoS ONE 9:e100260. doi: 10.1371/journal.pone.0100260

Ito, T., Okuma, K., Ma, X. X., Yuzawa, H., and Hiramatsu, K. (2003). Insights on antibiotic resistance of Staphylococcus aureus from its whole genome: genomic island SCC. Drug Resist. Updat. 6, 41-52. doi: 10.1016/S1368-7646(03)00003-7

International Working Group on the Classification of Staphylococcal Cassette Chromosome Elements. (2009). Classification of staphylococcal cassette chromosome mec (SCCmec): guidelines for reporting novel SCCmec elements. Antimicrob. Agents Chemother. 53, 4961-4967. doi: 10.1128/AAC.00579-09

Josenhans, C., and Suerbaum, S. (2002). The role of motility as a virulence factor in bacteria. Int. J. Med. Microbiol. 291, 605-614. doi: 10.1078/1438-4221-00173

Josten, M., Dischinger, J., Szekat, C., Reif, M., Al-Sabti, N., Sahl, H., et al. (2014). Identification of agr-positive methicillin-resistant Staphylococcus aureus harbouring the class A mec complex by MALDI-TOF mass spectrometry. Int. J. Med. Microbiol. 304, 1018-1023. doi: 10.1016/j.ijmm.2014.07.005

Kaito, C., Omae, Y., Matsumoto, Y., Nagata, M., Yamaguchi, H., Aoto, T., et al. (2008). A novel gene, fudoh, in the SCCmec region suppresses the colony spreading ability and virulence of Staphylococcus aureus. PLoS ONE 3:e3921. doi: 10.1371/journal.pone.0003921

Kaito, C., Saito, Y., Ikuo, M., Omae, Y., Mao, H., Nagano, G., et al. (2013). Mobile genetic element SCCmec-encoded psm-mec RNA suppresses translation of agrA and attenuates MRSA virulence. PLoS Pathog. 9:e1003269.doi: 10.1371/journal.ppat.1003269

Kaito, C., Saito, Y., Nagano, G., Ikuo, M., Omae, Y., Hanada, Y., et al. (2011). Transcription and translation products of the cytolysin gene psm-mec on the mobile genetic element SCCmec regulate Staphylococcus aureus virulence. PLoS Pathog. 7:e1001267. doi: 10.1371/journal.ppat.1001267

Kaito, C., and Sekimizu, K. (2007). Colony spreading in Staphylococcus aureus. J. Bacteriol. 189, 2553-2557. doi: 10.1128/JB.01635-06

Kearns, D. B. (2010). A field guide to bacterial swarming motility. Nat. Rev. Microbiol. 8, 634-644. doi: 10.1038/nrmicro2405

Kim, H. K., Kim, H. Y., Schneewind, O., and Missiakas, D. (2011). Identifying protective antigens of Staphylococcus aureus, a pathogen that suppresses host immune responses. FASEB J. 25, 3605-3612. doi: 10.1096/fj.11-187963

Kong, K. F., Vuong, C., and Otto, M. (2006). Staphylococcus quorum sensing in biofilm formation and infection. Int. J. Med. Microbiol. 296, 133-139. doi: 10.1016/j.ijmm.2006.01.042

Kretschmer, D., Gleske, A. K., Rautenberg, M., Wang, R., Köberle, M., Bohn, E., et al. (2010). Human formyl peptide receptor 2 senses highly pathogenic Staphylococcus aureus. Cell Host Microbe 7, 463-473. doi: 10.1016/j.chom.2010.05.012

Krukonis, E. S., and DiRita, V. J. (2003). From motility to virulence: sensing and responding to environmental signals in Vibrio cholerae. Curr. Opin. Microbiol. 6, 186-190. doi: 10.1016/S1369-5274(03)00032-8

Laabei, M., Jamieson, W. D., Yang, Y., van den Elsen, J., and Jenkins, A. T. (2014). Investigating the lytic activity and structural properties of Staphylococcus aureus phenol soluble modulin (PSM) peptide toxins. Biochim. Biophys. Acta 1838, 3153-3161. doi: 10.1016/j.bbamem.2014.08.026

Li, M., Diep, B. A., Villaruz, A., E., Braughton, K., R., Jiang, X., DeLeo, F. R., et al. (2009). Evolution of virulence in epidemic community-associated methicillinresistant Staphylococcus aureus. Proc. Natl. Acad. Sci. U.S.A. 106, 5883-5888. doi: 10.1073/pnas.0900743106

Li, S., Skov, R. L., Han, X., Larsen, A. R., Larsen, J., Sørum, M., et al. (2011). Novel types of staphylococcal cassette chromosome mec elements identified in clonal complex 398 methicillin-resistant Staphylococcus aureus strains. Antimicrob. Agents Chemother. 55, 3046-3050. doi: 10.1128/AAC.01475-10

Lin, M. H., Ke, W. J., Liu, C. C., and Yang, M. W. (2016). Modulation of Staphylococcus aureus spreading by water. Sci. Rep. 6:25233. doi: $10.1038 /$ srep 25233
Lowy, F. D. (1998). Staphylococcus aureus infections. N. Engl. J. Med. 339, 520-532. doi: 10.1056/NEJM199808203390806

Merino, N., Toledo-Arana, A., Vergara-Irigaray, M., Valle, J., Solano, C., Calvo, E., et al. (2009). Protein A-mediated multicellular behavior in Staphylococcus aureus. J. Bacteriol. 191, 832-843. doi: 10.1128/JB.01222-08

Migeotte, I., Communi, D., and Parmentier, M. (2006). Formyl peptide receptors: a promiscuous subfamily of $G$ protein-coupled receptors controlling immune responses. Cytokine Growth Factor Rev. 17, 501-519. doi: 10.1016/j.cytogfr.2006.09.009

Monecke, S., Engelmann, I., Archambault, M., Coleman, D. C., Coombs, G. W., Cortez de Jäckel, S., et al. (2012). Distribution of SCCmec-associated phenol-soluble modulin in staphylococci. Mol. Cell. Probes 26, 99-103. doi: 10.1016/j.mcp.2012.01.001

Novick, R. P. (2003). Autoinduction and signal transduction in the regulation of staphylococcal virulence. Mol. Microbiol. 48, 1429-1449. doi: 10.1046/j.13652958.2003.03526.x

Nübel, U., Dordel, J., Kurt, K., Strommenger, B., Westh, H., Shukla, S., et al. (2010). A timescale for evolution, population expansion, and spatial spread of an emerging clone of methicillin-resistant Staphylococcus aureus. PLoS Pathog. 6:e1000855. doi: 10.1371/journal.ppat.1000855

Omae, Y., Sekimizu, K., and Kaito, C. (2012). Inhibition of colony-spreading activity of Staphylococcus aureus by secretion of delta-hemolysin. J. Biol. Chem. 287, 15570-15579. doi: 10.1074/jbc.M112.357848

Oogai, Y., Matsuo, M., Hashimoto, M., Kato, F., Sugai, M., and Komatsuzawa, H. (2011). Expression of virulence factors by Staphylococcus aureus grown in serum. Appl. Environ. Microbiol. 77, 8097-8105. doi: 10.1128/AEM. 05316-11

Otto, M. (2008). Staphylococcal biofilms. Curr. Top. Microbiol. Immunol. 322, 207-228. doi: 10.1007/978-3-540-75418-3_10

Otto, M. (2009). Staphylococcus epidermidis-the 'accidental' pathogen. Nat. Rev. Microbiol. 7, 555-567. doi: 10.1038/nrmicro2182

Otto, M. (2012). MRSA virulence and spread. Cell. Microbiol. 14, 1513-1521. doi: 10.1111/j.1462-5822.2012.01832.x

Otto, M. (2013a). Community-associated MRSA: what makes them special? Int. J. Med. Microbiol. 303, 324-330. doi: 10.1016/j.ijmm.2013.02.007

Otto, M. (2013b). Staphylococcal infections: mechanisms of biofilm maturation and detachment as critical determinants of pathogenicity. Annu. Rev. Med. 64, 175-188. doi: 10.1146/annurev-med-042711-140023

Otto, M. (2013c). Coagulase-negative staphylococci as reservoirs of genes facilitating MRSA infection: staphylococcal commensal species such as Staphylococcus epidermidis are being recognized as important sources of genes promoting MRSA colonization and virulence. Bioessays 35, 4-11. doi: 10.1002/bies.201200112

Otto, M. (2014). Staphylococcus aureus toxins. Curr. Opin. Microbiol. 17, 32-37. doi: 10.1016/j.mib.2013.11.004

Periasamy, S., Joo, H. S., Duong, A. C., Bach, T. H., Tan, V. Y., Chatterjee S. S., et al. (2012). How Staphylococcus aureus biofilms develop their characteristic structure. Proc. Natl. Acad. Sci. U.S.A. 109, 1281-1286. doi: 10.1073/pnas.1115006109

Queck, S. Y., Jameson-Lee, M., Villaruz, A. E., Bach, T. H., Khan, B. A., Sturdevant, D. E., et al. (2008). RNAIII-independent target gene control by the agr quorum-sensing system: insight into the evolution of virulence regulation in Staphylococcus aureus. Mol. Cell 32, 150-158. doi: 10.1016/j.molcel.2008.08.005

Queck, S. Y., Khan, B. A., Wang, R., Bach, T. H., Kretschmer, D., Chen, L., et al. (2009). Mobile genetic element-encoded cytolysin connects virulence to methicillin resistance in MRSA. PLoS Pathog. 5:e1000533. doi: 10.1371/journal.ppat.1000533

Schulte, B., Bierbaum, G., Pohl, K., Goerke, C., and Wolz, C. (2013). Diversification of clonal complex 5 methicillin-resistant Staphylococcus aureus strains (Rhine-Hesse clone) within Germany. J. Clin. Microbiol. 51, 212-216. doi: 10.1128/JCM.01967-12

Shore, A. C., and Coleman, D. C. (2013). Staphylococcal cassette chromosome mec: recent advances and new insights. Int. J. Med. Microbiol. 303, 350-359. doi: 10.1016/j.ijmm.2013.02.002

Shore, A. C., Deasy, E. C., Slickers, P., Brennan, G., O'Connell, B., Monecke, S., et al. (2011). Detection of staphylococcal cassette chromosome mec type XI carrying highly divergent mecA, mecI, mecR1, blaZ, and ccr genes in human clinical isolates of clonal complex 130 methicillin-resistant 
Staphylococcus aureus. Antimicrob. Agents Chemother. 55, 3765-3773. doi: 10.1128/AAC.00187-11

Suzuki, T., Yamamoto, T., Kaito, C., Miyamoto, H., and Ohashi, Y. (2016). Impact of psm-mec in methicillin-resistant Staphylococcus aureus (ST764) strains isolated from keratitis patients. Microb. Drug Resist. 22, 137-140. doi: 10.1089/mdr.2015.0315

Tacconelli, E., Venkataraman, L., De Girolami, P. C, and DAgata, E. M. (2004). Methicillin-resistant Staphylococcus aureus bacteraemia diagnosed at hospital admission: distinguishing between community-acquired versus healthcare-associated strains. J. Antimicrob. Chemother. 53, 474-479. doi: 10.1093/jac/dkh107

Tsompanidou, E., Denham, E. L., Becher, D., de Jong, A., Buist, G., van Oosten, M., et al. (2013). Distinct roles of phenol-soluble modulins in spreading of Staphylococcus aureus on wet surfaces. Appl. Environ. Microbiol. 79, 886-895. doi: 10.1128/AEM.03157-12

Tsompanidou, E., Denham, E. L., Sibbald, M. J., Yang, X. M., Seinen, J., Friedrich, A. W., et al. (2012). The sortase A substrates FnbpA, FnbpB, ClfA and ClfB antagonize colony spreading of Staphylococcus aureus. PLoS ONE 7:e44646. doi: 10.1371/journal.pone.0044646

Tsompanidou, E., Sibbald, M. J., Chlebowicz, M. A., Dreisbach, A., Back, J. W., van Dijl, J. M., et al. (2011). Requirement of the agr locus for colony spreading of Staphylococcus aureus. J. Bacteriol. 193, 1267-1272. doi: 10.1128/JB.01276-10

Vuong, C., Kocianova, S., Yao, Y., Carmody, A. B., and Otto, M. (2004). Increased colonization of indwelling medical devices by quorum-sensing mutants of Staphylococcus epidermidis in vivo. J. Infect. Dis. 190, 1498-1505. doi: $10.1086 / 424487$
Vuong, C., Saenz, H. L., Götz, F., and Otto, M. (2000). Impact of the agr quorumsensing system on adherence to polystyrene in Staphylococcus aureus. J. Infect. Dis. 182, 1688-1693. doi: 10.1086/317606

Wang, R., Braughton, K. R., Kretschmer, D., Bach, T. H., Queck, S. Y., Li, M., et al. (2007). Identification of novel cytolytic peptides as key virulence determinants for community-associated MRSA. Nat. Med. 13, 1510-1514. doi: 10.1038/nm1656

Wang, R., Khan, B. A., Cheung, G. Y., Bach, T. H., Jameson-Lee, M., Kong, K. F., et al. (2011). Staphylococcus epidermidis surfactant peptides promote biofilm maturation and dissemination of biofilm-associated infection in mice. J. Clin. Invest. 121, 238-248. doi: 10.1172/JCI42520

Yoshikai, H., Kizaki, H., Saito, Y., Omae, Y., Sekimizu, K., and Kaito, C. (2016). Multidrug-resistance transporter AbcA secretes Staphylococcus aureus cytolytic toxins. J. Infect. Dis. 213, 295-304. doi: 10.1093/infdis/ jiv376

Conflict of Interest Statement: The authors declare that the research was conducted in the absence of any commercial or financial relationships that could be construed as a potential conflict of interest.

Copyright (C) 2016 Qin, McCausland, Cheung and Otto. This is an open-access article distributed under the terms of the Creative Commons Attribution License (CC BY). The use, distribution or reproduction in other forums is permitted, provided the original author(s) or licensor are credited and that the original publication in this journal is cited, in accordance with accepted academic practice. No use, distribution or reproduction is permitted which does not comply with these terms. 


\title{
Sub-MIC Tylosin Inhibits Streptococcus suis Biofilm Formation and Results in Differential Protein Expression
}

\author{
Shuai Wang ${ }^{1}$, Yanbei Yang ${ }^{1}$, Yulin Zhao ${ }^{1}$, Honghai Zhao ${ }^{2}$, Jingwen Bai ${ }^{1}$, Jianqing Chen ${ }^{1}$, \\ Yonghui Zhou ${ }^{1}$, Chang Wang ${ }^{1}$ and Yanhua $L^{1{ }^{1 *}}$ \\ ${ }^{1}$ Department of Veterinary Pharmacy, College of Veterinary Medicine, Northeast Agricultural University, Harbin, China, \\ ${ }^{2}$ Department of Biotechnology, Heilongjiang Vocational College for Nationalities, Harbin, China
}

OPEN ACCESS

Edited by:

Etienne Giraud,

Institut National de la Recherche

Agronomique, France

Reviewed by:

Rui Vitorino,

University of Aveiro, Portugal

Raymond Allan,

University of Southampton, UK

*Correspondence:

Yanhua $L i$

liyanhua1970@163.com

Specialty section:

This article was submitted to Antimicrobials, Resistance and

Chemotherapy,

a section of the journal

Frontiers in Microbiology

Received: 19 November 2015

Accepted: 11 March 2016

Published: 30 March 2016

Citation:

Wang S, Yang Y, Zhao Y, Zhao H, Bai J, Chen J, Zhou Y, Wang $C$ and Li Y (2016) Sub-MIC Tylosin Inhibits

Streptococcus suis Biofilm Formation and Results in Differential Protein

Expression. Front. Microbiol. 7:384 doi: 10.3389/fmicb.2016.00384
Streptococcus suis (S.suis) is an important zoonotic pathogen that causes severe diseases in humans and pigs. Biofilms of $S$. suis can induce persistent infections that are difficult to treat. In this study, the effect of tylosin on biofilm formation of $S$. suis was investigated. 1/2 minimal inhibitory concentration (MIC) and 1/4 MIC of tylosin were shown to inhibit $S$. suis biofilm formation in vitro. By using the ITRAQ strategy, we compared the protein expression profiles of $S$. suis grown with sub-MIC tylosin treatment and with no treatment. A total of 1501 proteins were identified by iTRAQ. Ninety-six differentially expressed proteins were identified (Ratio $> \pm 1.5, p<0.05$ ). Several metabolism proteins (such as phosphoglycerate kinase) and surface proteins (such as ABC transporter proteins) were found to be involved in biofilm formation. Our results indicated that $S$. suis metabolic regulation, cell surface proteins, and virulence proteins appear to be of importance in biofilm growth with sub-MIC tylosin treatment. Thus, our data revealed the rough regulation of biofilm formation that may provide a foundation for future research into mechanisms and targets.

Keywords: proteomics, S. suis, biofilms, tylosin, iTRAQ

\section{INTRODUCTION}

Streptococcus suis (S. suis) is an important zoonotic pathogen that causes a wide range of diseases in pigs, including meningitis, septicaemia, pneumonia, endocarditis, and arthritis (Gottschalk et al., 2010). In addition, this pathogen is an emerging zoonotic agent and an important public health issue in East and Southeast Asia (Sriskandan and Slater, 2006; Gottschalk et al., 2007). During a single outbreak in China in 2005, more than 200 human cases of S. suis were reported, with a death total of 39 (Yang et al., 2006). Current studies show that $S$. suis can cause persistent infections by forming biofilms in vivo (Wang et al., 2011).

Biofilms are assemblages of microorganisms characterized by cells that are irreversibly attached to a substratum and embedded in a matrix of self-produced extracellular polymeric substances such as exopolysaccharides (EPS), proteins, nucleic acids and other substances; this type of sessile community-based existence is a critical characteristic for bacterial persistence (Davey and O'Toole, 2000). The physical and biological properties of the biofilm, such as slow growth and mechanical barrier, have a substantial role in the development of increased antimicrobial tolerance. Because the bacteria in chronic infections are aggregated and are in close proximity, genes coding for resistance 
to antimicrobials can be passed horizontally from one bacterium to the another (Bjarnsholt et al., 2013). The bacteria in biofilms could be 1000-times more difficult to kill by antibiotics than the same organism growing planktonically (Gilbert et al., 1997). Therefore, the control of biofilms is understood to be crucial.

Apart from surgical intervention (when applicable), antibiotics are the main option for the treatment of biofilm infections (Bjarnsholt et al., 2013). Previous studies showed that macrolides successfully inhibited Staphylococcus aureus biofilm formation and reduced its virulence when used at sub-inhibitory concentrations (Fujimura et al., 2008). In addition, a subminimal inhibitory concentration of erythromycin can inhibit $S$. suis biofilm formation (Zhao et al., 2015). Tylosin, a macrolide antibiotic produced by Streptomyces fradiae, is widely used as a veterinary medicine. However, there is still not much effective research of sub-MIC tylosin inhibiting biofilm formation of $S$. suis in vitro.

At present, most of the available information regarding biofilm formation by drug intervention is based on transcriptomic analyses. However, a limitation of transcriptomic analysis for identifying biofilm-regulated gene network raised concerns among investigators. The method of proteomics is thought to be an essential complement to transcriptomic analysis for discovering key regulators of biofilm (Sauer, 2003). Different immunogenic components of planktonically grown S. suis proteins such as secreted or cell wall-associated proteins had been studied by using immunoproteomic assays (Zhang and $\mathrm{Lu}$, 2007a,b; Geng et al., 2008; Zhang et al., 2008). Additionally, our lab found that quorum-sensing played a crucial role leading to biofilm formation through quantitative proteomic analysis of $S$. suis biofilm inhibited by sub-MIC erythromycin treatment in vitro (Zhao et al., 2015). However, there are no reports regarding the proteomic analysis of sub-MIC tylosin inhibiting biofilm formation of S. suis in vitro.

We identified several proteins in sub-MIC tylosin inhibiting biofilm formation of $S$. suis by using iTRAQ technology in this study. The findings from the present study may provide a theoretical foundation for therapy of S. suis biofilm infection and provide references for finding new potential therapeutic targets.

\section{MATERIALS AND METHODS}

\section{Growth of S. suis Planktonic Cells}

S. suis (ATCC 700794) was grown in Todd-Hewitt yeast Broth (THB; Summus Ltd., Harbin, Heilongjiang, China) for $16-18 \mathrm{~h}$ at $37^{\circ} \mathrm{C}$ with constant shaking for biofilm assays (Wang et al., 2011).

\section{Observation by Scanning Electron Microscopy (SEM)}

Mid-exponential growth phase cultures of S. suis ATCC 700794 were adjusted to an optical density of 0.1 at $600 \mathrm{~nm}$ (OD600). Then, $2 \mathrm{~mL}$ cultures were transferred to the wells of a 6-well microplate containing an $11 \times 11 \mathrm{~mm}$ sterilized rough glass slide (Mosutech Co., Ltd., Shanghai, China) on the bottom. After culturing for $72 \mathrm{~h}$ at $37^{\circ} \mathrm{C}$ without shaking, the glass slide was removed with tweezers, and the biofilms on the rough glass slide were washed with sterile PBS. The remaining biofilms were fixed with fixative solution $[4 \%(\mathrm{w} / \mathrm{v})$ paraformaldehyde, $2.5 \%(\mathrm{w} / \mathrm{v})$ glutaraldehyde, $2 \mathrm{mM} \mathrm{CaCl}$ in $0.2 \mathrm{M}$ cacodylate buffer, $\mathrm{pH} 7.2$ ] for $6 \mathrm{~h}$ and washed three times with $0.1 \mathrm{M}$ PBS $10 \mathrm{~min}$ each, then fixed in $2 \%$ osmium tetroxide containing $2 \mathrm{mM}$ potassium ferrocyanide and $6 \%(\mathrm{w} / \mathrm{v})$ sucrose in cacodylate buffer. The samples were dried, gold sputtered with an ion sputtering instrument (current $15 \mathrm{~mA}, 2 \mathrm{~min}$ ) and observed using SEM (FEI Quanta, Netherland).

\section{Effect of Tylosin on Biofilm Formation Determined by the TCP Assay}

Mid-exponential growth phase cultures of $S$. suis were adjusted to 0.2 of OD600. Then, $100 \mu \mathrm{L}$ of cultures were added to each wells of a 96-well microplate with equal volume of tylosin solution with the final concentrations of $1 / 2 \mathrm{MIC}(0.25 \mu \mathrm{g} / \mathrm{mL}), 1 / 4$ MIC $(0.125 \mu \mathrm{g} / \mathrm{mL}), 1 / 8 \mathrm{MIC}(0.0625 \mu \mathrm{g} / \mathrm{mL})$, and 1/16 MIC $(0.03125 \mu \mathrm{g} / \mathrm{mL})$, respectively. In addition, a negative control (with THB alone) and a positive control (with bacteria alone) were also included. After incubation at $37^{\circ} \mathrm{C}$ for $72 \mathrm{~h}$ without shaking, the medium was removed by aspiration and the wells were washed three times with sterile physiological saline. The remaining attached bacteria were fixed with $200 \mu \mathrm{L}$ of $99 \%$ methanol (Guoyao Ltd., China) per well, and the plates were emptied after $15 \mathrm{~min}$ and left to dry. Then, the plates were stained for $5 \mathrm{~min}$ with $200 \mu \mathrm{L}$ of $2 \%$ crystal violet (Guoyao Ltd., China) per well. The excess stain was rinsed off by placing the plate under running tap water. After the plates were air dried, the dye bound to the adherent cells was resolubilized with $200 \mu \mathrm{L}$ of $33 \%(\mathrm{v} / \mathrm{v})$ glacial acetic acid (Guoyao Ltd., China) per well. The amount of released stain was quantified by measuring the absorbance at $570 \mathrm{~nm}$ with a microplate reader (DG5033A, Huadong Ltd., Nanjing, Jiangsu, China). The reported values are the means of three measurements. The experiments were performed in triplicate.

\section{Colony Forming Unit (CFU) Enumeration}

Overnight cultures of $S$. suis were adjusted to an OD 600 of 0.2 . Then, the bacteria were inoculated into 96-well microtiter plate wells containing $200 \mu \mathrm{L}$ of THB alone (untreated wells) or adding the tylosin solution with the final concentrations of $1 / 4 \mathrm{MIC}$ $(0.125 \mu \mathrm{g} / \mathrm{mL})$. In addition, a negative control (with THB alone) and a positive control (with bacteria alone) were also included. After incubation at $37^{\circ} \mathrm{C}$ for $72 \mathrm{~h}$ without shaking, the medium was removed by aspiration, and the wells were washed three times with sterile physiological saline. Biofilm cells were removed from wells by sonication for $5 \mathrm{~min}$ in $200 \mu \mathrm{L}$ of THB. The cell suspensions $(n=3)$ underwent 10 -fold dilutions in THB, and 100 $\mu \mathrm{L}$ of each dilution was spot plated onto THB soft-agar plates and incubated at $37^{\circ} \mathrm{C}$ for $24 \mathrm{~h}$. All the experiments were performed in triplicate.

\section{Preparation of Protein Extracts}

For biofilm cultures, S. suis was grown in THB in $100 \mathrm{~mm}$ polystyrene petri dishes at $37^{\circ} \mathrm{C}$ for $24 \mathrm{~h}$. Then, the supernatant was removed and the dishes were washed twice with Tris$\mathrm{HCl}$ buffer $(50 \mathrm{mM}, \mathrm{pH}$ 7.5). The biofilms were detached by scraping. After being sonicated for $5 \mathrm{~min}$ (Bransonic 220; Branson Consolidated Ultrasonic Pvt. Ltd., Australia), the cells were centrifuged at $12,000 \times \mathrm{g}$ for $10 \mathrm{~min}$ at $4^{\circ} \mathrm{C}$. Then, the cell 
pellets were washed twice with Tris- $\mathrm{HCl}$ buffer (Wang et al., 2012).

\section{Protein Digestion and iTRAQ Labeling}

Protein digestion was performed according to the reported FASP procedure (Wisniewski et al., 2009). In brief, $200 \mu \mathrm{g}$ of proteins at two different conditions (1/4 MIC of tylosin treated cells and nontreated cells) were added into $30 \mu \mathrm{L}$ STD buffer ( $4 \%$ SDS, $100 \mathrm{mM}$ DTT, $150 \mathrm{mM}$ Tris- $\mathrm{HCl} \mathrm{pH} 8.0$ ) and ultrafiltered (Microcon units, $30 \mathrm{kD}$ ) with UA buffer $(8 \mathrm{M}$ Urea, $150 \mathrm{mM}$ Tris- $\mathrm{HCl} \mathrm{pH}$ 8.0). To block reduced cysteine residues, $100 \mu \mathrm{L}$ $0.05 \mathrm{M}$ iodoacetamide was added into UA buffer and incubated for $20 \mathrm{~min}$ in the dark. The filters were washed three times with $100 \mu \mathrm{L}$ UA buffer and twice with $100 \mu \mathrm{L}$ DS buffer $(50 \mathrm{mM}$ triethylammoniumbicarbonate at $\mathrm{pH}$ 8.5). Finally, the proteins were digested with $2 \mu \mathrm{g}$ trypsin (Promega) in $40 \mu \mathrm{L}$ DS buffer at $37^{\circ} \mathrm{C}$ for $16-18 \mathrm{~h}$. Then, the resulting peptides were collected as a filtrate. The peptide content was estimated by UV light spectral density at $280 \mathrm{~nm}$ using an extinctions coefficient of 1.1 of $0.1 \%(\mathrm{~g} / \mathrm{l})$ solution calculated on the basis of the frequency of tryptophan and tyrosine in vertebrate proteins.

For the iTRAQ labeling, the peptides were labeled with the 8plex iTRAQ reagent by following the manufacturer's instructions (Applied Biosystems). Each iTRAQ reagent was dissolved in $70 \mu \mathrm{L}$ of ethanol and added to the respective peptide mixture. The peptides from the S. suis biofilms treated by tylosin were labeled with 115 isobaric reagent, and the peptides from the nontreated S. suis biofilms were labeled with 116 isobaric reagent. Then, the samples were multiplexed and vacuum dried. Three independent biological experiments were performed.

\section{Peptide Fractionation with Strong Cation Exchange (SCX) Chromatography}

SCX chromatography using the AKTA Purifier system (GE Healthcare) was used to fractionate the iTRAQ labeled peptides. After being reconstituted and acidified with $2 \mathrm{~mL}$ buffer A $\left(10 \mathrm{mM} \mathrm{KH}_{2} \mathrm{PO}_{4}\right.$ in $25 \%$ of $\left.\mathrm{ACN}, \mathrm{pH} 2.7\right)$, the peptides were loaded onto a PolySULFOETHYL $4.6 \times 100 \mathrm{~mm}$ column $(5 \mu \mathrm{m}$, $200 \AA$ A, PolyLC Inc., Maryland, U.S.A.). Then, the peptides were eluted at $1 \mathrm{ml} / \mathrm{min}$ with a gradient of $0-10 \%$ buffer B $(500 \mathrm{mM}$ $\mathrm{KCl}, 10 \mathrm{mM} \mathrm{KH} \mathrm{PO}_{4}$ in $25 \%$ of $\mathrm{ACN}, \mathrm{pH} 2.7$ ) for $2 \mathrm{~min}, 10$ $20 \%$ buffer B for $25 \mathrm{~min}, 20-45 \%$ buffer B for $5 \mathrm{~min}$, and $50-100 \%$ buffer $\mathrm{B}$ for $5 \mathrm{~min}$. The elution was monitored by absorbance at $214 \mathrm{~nm}$, and the fractions were collected after every $1 \mathrm{~min}$. The collected fractions ( $\sim 30$ fractions) were combined into 10 pools and desalted on C18 Cartridges [Empore ${ }^{\mathrm{TM}}$ SPE Cartridges C18 (standard density), bed I.D. $7 \mathrm{~mm}$, volume $3 \mathrm{ml}$, Sigma]. Each pooled fraction was concentrated by vacuum centrifugation and reconstituted in $40 \mu \mathrm{l}$ of $0.1 \%(\mathrm{v} / \mathrm{v})$ trifluoroacetic acid and stored at $-80^{\circ} \mathrm{C}$ for LC-MS/MS analysis.

\section{Liquid Chromatography (LC) Electrospray lonization (ESI) Tandem Ms (MS/MS) Analysis by $Q$ Exactive}

Experiments were performed on a $\mathrm{Q}$ Exactive mass spectrometer that was coupled to Easy nLC (Thermo Fisher Scientific). A sample $(10 \mu \mathrm{L})$ of each fraction was injected for the nano LCMS/MS analysis. The peptide mixture $(5 \mu \mathrm{g})$ was loaded onto a C18-reversed phase column (Thermo Scientific Easy Column, $10 \mathrm{~cm}$ long, $75 \mu \mathrm{m}$ inner diameter, $3 \mu \mathrm{m}$ resin) in buffer $\mathrm{A}$ $(0.1 \%$ formic acid $)$ and separated with a linear gradient of buffer B ( $80 \%$ acetonitrile and $0.1 \%$ formic acid) at $250 \mathrm{nl} / \mathrm{min}$ controlled by IntelliFlow technology for $140 \mathrm{~min}$. MS data were acquired using a data-dependent top 10 method dynamically choosing the most abundant precursor ions from the survey scan $(300-1800 \mathrm{~m} / \mathrm{z})$ for HCD fragmentation. Determination of the target value was based on predictive Automatic Gain Control (pAGC). The dynamic exclusion duration was $60 \mathrm{~s}$. Survey scans were acquired at a resolution of 70,000 at $\mathrm{m} / \mathrm{z} 200$, and the resolution for HCD spectra was set to 17,500 at $\mathrm{m} / \mathrm{z} 200$. The normalized collision energy was $30 \mathrm{eV}$, and the underfill ratio, which specifies the minimum percentage of the target value likely to be reached at maximum fill time, was defined as $0.1 \%$. The instrument was run with the peptide recognition mode enabled.

\section{Sequence Database Searching and Data Analysis}

The MS/MS spectra were searched using the MASCOT engine (Matrix Science, London, UK; version 2.2) in the Proteome Discoverer 1.3 (Thermo Electron, San Jose, USA.) against the Uniprot S. suis fasta database $(38,369$ sequences, downloaded March 4th, 2013) and the decoy database. False discovery rates (FDR) were calculated by running all spectra against a decoy database using the MASCOT software. To identify proteins, the following options were used: Peptide mass tolerance $=20 \mathrm{ppm}$, MS/MS tolerance $=0.1 \mathrm{Da}$, Enzyme $=$ Trypsin, Missed cleavage $=2$, Fixed modification: Carbamidomethyl $(C)$, iTRAQ 8plex (K), iTRAQ 8plex (N-term), Variable modification: Oxidation (M). The quantification was performed based on the peak intensities of the reporter ions in the MS/MS spectra. The ratio of label 115 and 116 represents the expression of proteins with the protein identification confidence of a 1\% FDR (Unwin et al., 2010). The proteins were considered over expressed when the ITRAQ ratio was above 1.5 and under expressed when the ITRAQ ratio was lower than 0.67. The Proteome Discoverer tool was used to categorize the proteins detected by Gene Ontology (GO) annotation according to the cellular component, biological process and molecular function.

\section{RESULTS}

\section{Effect of Tylosin against Biofilm Formation In vitro by the TCP Assay}

We evaluated the action of tylosin on biofilm growth in vitro. The MIC against $S$. suis was $0.5 \mu \mathrm{g} \cdot \mathrm{mL}^{-1}$. Tylosin at $1 / 2 \mathrm{MIC}$ and $1 / 4$ MIC caused a significantly higher reduction in the biofilmforming ability of $S$. suis compared with positive control $(p<$ $0.05)$. However, there was no pronounced effect for $1 / 16$ MIC and 1/8 MIC of tylosin on biofilm formation of S. suis $(p>0.05$; Figure 1). 


\section{Direct Observation of Biofilm Formation In vitro by Sem}

SEM analysis was performed to observe the $1 / 4$ MIC of tylosin treated cells and nontreated cells biofilm formation by $\mathrm{S}$. suis under same growth conditions. As shown in Figure 2A, the surface of the glass slide is almost entirely covered by the aggregates and microcolonies of $S$. suis when growth was carried out in the culture medium without tylosin. However, when the culture medium was added to $1 / 4$ MIC of tylosin, the biofilms were characterized by the presence of small clusters of cells interspersed amongst individual cells (Figure 2B). This result showed that the biofilm formation of $S$. suis was inhibited by the tylosin of $1 / 4$ MIC in vitro.

\section{Colony Forming Unit (CFU) Enumeration}

To better assess action of tylosin on biofilm quantitatively, the CFUs of S. suis were counted. The viability of S. suis treated with 1/4 MIC of tylosin was different from the viability of untreated S. suis. The number of CFUs/mL in treated biofilms $\left(5.3 \log _{10}\right.$ CFUs $/ \mathrm{mL}$ ) was significantly fewer than in nontreated biofilms (6.5 $\log _{10}$ CFUs $/ \mathrm{mL} ; p<0.05$ ). The number of CFUs $/ \mathrm{mL}$ in treated biofilms $\left(5.3 \log _{10} \mathrm{CFUs} / \mathrm{mL}\right.$ ) was significantly fewer than in nontreated biofilms $\left(6.5 \log _{10}\right.$ CFUs/mL; $\left.p<0.05\right)$ (Figure 3). The findings demonstrated that $1 / 4$ MIC of tylosin remained effective in decreasing the viability of $S$. suis.
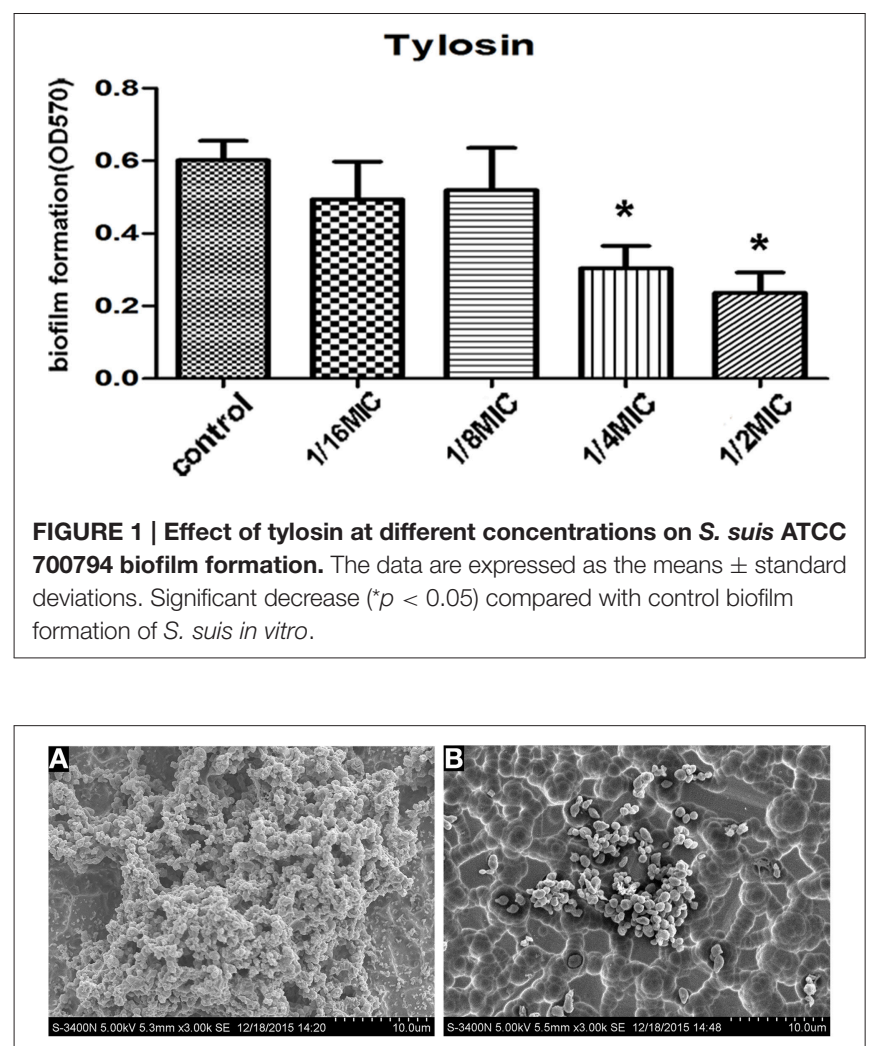

FIGURE 2 | (A) Biofilm formation of S. suis without tylosin. (B) Biofilm formation of $S$. suis with $1 / 4 \mathrm{MIC}$ tylosin treatment.

\section{Sub-MIC Tylosin Inhibits Biofilm Formation and Differentially Expressed Proteins by ITRAQ}

A total of 1501 proteins were identified by iTRAQ. Detailed information is shown in the Supplementary File. A ratio of proteins with $>1.5$ or $<0.67(p<0.05)$ was considered to be differentially expressed. Based on this criterion, 96 differentially expressed proteins were identified in 1/4 MIC tylosin treated cells and nontreated cells. Detailed information could is shown Table 1. These proteins were detected by GO annotation. Thirty-five proteins were deleted from the database. The remaining 61 proteins were classified into biological process, molecular function and cellular component (Figure 4). The results regarding the biological process were as follows: single-organism process $(8,13 \%)$, response to stimulus $(4$, $7 \%)$, localization $(4,7 \%)$, biological adhesion $(1,2 \%)$, cellular component organization or biogenesis $(6,10 \%)$, cellular process $(25,41 \%)$, biological regulation $(3,5 \%)$, metabolic process $(30$, $49 \%)$. The results regarding molecular function were as follows: binding $(29,48 \%)$, nucleic acid binding transcription factor activity $(1,2 \%)$, transporter activity $(3,5 \%)$, structural molecule activity $(5,8 \%)$, catalytic activity $(25,41 \%)$. The results regarding cellular component were as follows: organelle $(6,10 \%)$, virion (1, $2 \%)$, cell $(13,21 \%)$, extracellular region $(1,2 \%)$, membrane $(6$, $10 \%)$, macromolecular complex $(8,13 \%)$.

\section{DISCUSSION}

A TCP assay is based on the ability of bacteria to form biofilms on the bottom of tissue culture plates and is mainly used to identify the formation of bacterial biofilms (Mathur et al., 2006; Okajima et al., 2006; Presterl et al., 2007). Bacteria are grown in cell culture plates, and the quantity of a biofilm is detected by staining the wells with crystal violet according to the correlation between OD values and biofilm formation (Mathur et al., 2006).

To further confirm S. suis biofilm formation, the structure of the $S$. suis biofilm was identified by scanning electron microscopy

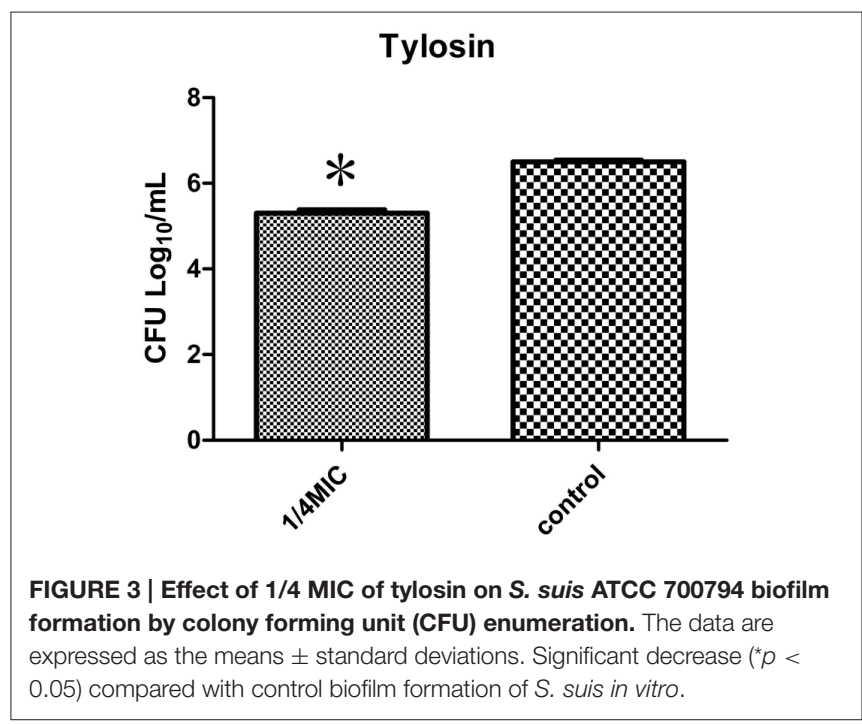


TABLE 1 | List of differentially expressed proteins in tylosin treated cells.

\begin{tabular}{|c|c|c|}
\hline Accession & Description & Fold change $^{a}$ \\
\hline G7SDH9 & Putative uncharacterized protein & 0.555777 \\
\hline A4W1G9 & $\begin{array}{l}\text { Phosphotransferase system cellobiose-specific } \\
\text { component IIC }\end{array}$ & 0.521547 \\
\hline G7SA24 & Cold-shock DNA-binding domain protein & 0.394546 \\
\hline F4EDP5 & Putative uncharacterized protein & 0.568016 \\
\hline R4NL31 & MF3-like protein & 0.63094 \\
\hline E9NQ29 & CPS16V & 0.452748 \\
\hline G5KZ73 & $\begin{array}{l}\text { NADH:flavin oxidoreductase/NADH oxidase family } \\
\text { protein }\end{array}$ & 0.459622 \\
\hline G7S4C2 & $\begin{array}{l}\text { Major membrane immunogen, } \\
\text { membrane-anchored lipoprotein }\end{array}$ & 0.643938 \\
\hline A8CUL3 & Integrase & 0.46773 \\
\hline G7SM99 & $\begin{array}{l}\text { Type I site-specific restriction-modification system, } \\
\text { R (Restriction) subunit and related helicase }\end{array}$ & 0.506277 \\
\hline G7SLJ0 & Putative competence-damage inducible protein & 0.564703 \\
\hline A4W1W3 & FOF1-type ATP synthase, subunit a & 0.586873 \\
\hline G7RZWO & Sugar $A B C$ transporter permease & 1.508325 \\
\hline G7S2T8 & Alcohol dehydrogenase & 0.610195 \\
\hline G7SDG9 & Laminin binding protein & 1.524593 \\
\hline R4NLJ6 & $\begin{array}{l}\text { Oligopeptide ABC transporter, periplasmic } \\
\text { oligopeptide-binding protein OppA }\end{array}$ & 0.644177 \\
\hline G7S371 & Fructose-bisphosphate aldolase & 0.623705 \\
\hline M1UGE2 & Uncharacterized protein & 0.647168 \\
\hline A4VZM2 & Cell cycle protein GpsB & 0.631018 \\
\hline A4W2D2 & Cation transport ATPase & 1.676356 \\
\hline G5L1V6 & $50 S$ ribosomal protein L9 & 0.6638821 \\
\hline G7SEP2 & Putative uncharacterized protein & 1.502566 \\
\hline A4VZ3 & tRNA dimethylallyltransferase & 1.510883 \\
\hline G7S5B8 & 3-isopropylmalate dehydrogenase & 1.513117 \\
\hline G7SA68 & UDP-N-acetylmuramate-alanine ligase & 0.656503 \\
\hline G5L098 & Transcriptional regulator Spx & 1.514636 \\
\hline E8UKC3 & Uncharacterized protein & 1.507837 \\
\hline G7SFU4 & Putative uncharacterized protein & 1.543591 \\
\hline A4V999 & DNA repair protein & 1.545594 \\
\hline A4W2Z7 & FMN-dependent NADH-azoreductase & 1.527496 \\
\hline F4EC05 & Putative uncharacterized protein & 1.57743 \\
\hline C6GNL8 & 30 S ribosomal protein S21 & 1.545471 \\
\hline K7ZNG8 & DNA recombination/repair protein (Fragment) & 1.558023 \\
\hline G7S3K5 & $\begin{array}{l}\text { ABC-type cobalt transport system, ATPase } \\
\text { component }\end{array}$ & 1.583854 \\
\hline G7SM56 & Ribosomal RNA small subunit methyltransferase $\mathrm{H}$ & 1.594936 \\
\hline A2VC24 & Muramidase-released protein & 1.579304 \\
\hline A4W3C1 & Putative phosphotyrosine protein phosphatase & 1.60975 \\
\hline G7S4U0 & Putative uncharacterized protein & 1.596783 \\
\hline G7SP92 & Putative uncharacterized protein & 1.658448 \\
\hline G7S7E3 & Helicase & 1.516925 \\
\hline M1VK55 & Glycosyltransferase & 1.662795 \\
\hline G7S3P9 & Putative uncharacterized protein & 1.673475 \\
\hline A4W106 & Signal transduction histidine kinase & 1.62492 \\
\hline BOMOG7 & Phosphomethylpyrimidine kinase (Fragment) & 1.633436 \\
\hline A5JSJ7 & Putative uncharacterized protein & 1.681661 \\
\hline G5L226 & $\begin{array}{l}\text { Type III restriction-modification system, restriction } \\
\text { endonuclease subunit }\end{array}$ & 1.697991 \\
\hline
\end{tabular}

TABLE 1 | Continued.

\begin{tabular}{|c|c|c|}
\hline Accession & Description & Fold change $\mathrm{a}^{\mathrm{a}}$ \\
\hline G7S7K4 & Putative uncharacterized protein & 1.645719 \\
\hline B9WY95 & Peptidase M20 & 1.647536 \\
\hline G7S4T2 & $\begin{array}{l}\text { Branched-chain alpha-keto acid dehydrogenase } \\
\text { subunit E2 }\end{array}$ & 1.673032 \\
\hline G5KX48 & $\begin{array}{l}\text { ABC-type uncharacterized transport system, } \\
\text { permease component }\end{array}$ & 1.705109 \\
\hline G7S178 & Putative uncharacterized protein & 1.774886 \\
\hline M1VDL7 & $A B C$ transporter permease protein & 1.942183 \\
\hline G7S6Z3 & Putative uncharacterized protein & 1.956584 \\
\hline J7KIA5 & $\begin{array}{l}\text { Abortive infection bacteriophage resistance } \\
\text { related protein }\end{array}$ & 1.999106 \\
\hline G7SJZ7 & Primosomal protein N' & 1.720079 \\
\hline G7SHW7 & Putative uncharacterized protein & 2.001559 \\
\hline Q9EZW2 & Elongation factor Tu (Fragment) & 1.739971 \\
\hline A4VJ5 & Uncharacterized protein & 2.141695 \\
\hline M1VJZ8 & Zeta toxin & 1.74309 \\
\hline G5L2VO & $\begin{array}{l}\text { NADPH:quinone reductase and related } \\
\text { Zn-dependent oxidoreductase }\end{array}$ & 1.79706 \\
\hline R4NU43 & $\begin{array}{l}\text { Aromatic amino acid aminotransferase gamma } \\
@ \text { N-acetyl-L,L-diaminopimelate } \\
\text { aminotransferase }\end{array}$ & 2.144605 \\
\hline A4W296 & Uncharacterized protein & 1.837367 \\
\hline G7SKQ0 & CHAP domain containing protein & 2.179882 \\
\hline B9WVD6 & Putative uncharacterized protein (Fragment) & 2.226792 \\
\hline G5L2N5 & L-fucose isomerase & 1.859887 \\
\hline B9WTA1 & Putative uncharacterized protein & 2.290848 \\
\hline B9WXU6 & $\mathrm{ABC}$ transporter related protein & 1.873566 \\
\hline G8DU82 & Transposase & 2.376768 \\
\hline G7S242 & Putative $\mathrm{ABC}$ transporter & 1.883471 \\
\hline A4VZ91 & 50 S ribosomal protein L32 & 1.915877 \\
\hline G7SDX6 & $50 S$ ribosomal protein $\mathrm{L} 7 / \mathrm{L} 12$ & 1.918196 \\
\hline G5KZ86 & $\begin{array}{l}\text { Phosphatidylserine/phosphatidylglycerophosphate/ } \\
\text { cardiolipin synthase-like protein }\end{array}$ & 1.965488 \\
\hline G7SIQ5 & Putative uncharacterized protein & 2.451703 \\
\hline G7S7J2 & Putative uncharacterized protein & 2.481527 \\
\hline G7SPA1 & Putative scaffolding protein & 2.584249 \\
\hline G7S7A9 & $\begin{array}{l}\text { FAD-dependent pyridine nucleotide-disulfide } \\
\text { oxidoreductase }\end{array}$ & 1.970356 \\
\hline G7S8Q2 & Peptidase M22 glycoprotease & 1.978772 \\
\hline M1TIM7 & $\begin{array}{l}\text { Methylated DNA-protein cysteine } \\
\text { methyltransferase }\end{array}$ & 2.161583 \\
\hline G7SBK2 & Elongation factor Ts & 2.266517 \\
\hline M1VE47 & Fic/DOC family protein & 2.606274 \\
\hline M1UFP9 & Thiamine-phosphate synthase & 2.298028 \\
\hline G7S535 & Fructose-6-phosphate aldolase & 2.553516 \\
\hline G5L351 & NsuB & 2.694988 \\
\hline B9WUV5 & Transcriptional regulator, DeoR family & 2.65073 \\
\hline R4NW55 & $\begin{array}{l}\text { Plasmid replication protein Rep and AAA-class } \\
\text { ATPase domain protein }\end{array}$ & 2.779765 \\
\hline G7RZ18 & Sortase-like protein & 2.791504 \\
\hline S6B433 & RecN protein (Fragment) & 3.007243 \\
\hline G5KZR3 & Glutathione S-transferase & 3.262393 \\
\hline R4NLK5 & SSU ribosomal protein S1p & 3.46851 \\
\hline R4NWB6 & Uncharacterized protein & 8.306942 \\
\hline
\end{tabular}

(Continued) 
TABLE 1 | Continued.

\begin{tabular}{llc}
\hline Accession & Description & Fold change $^{\text {a }}$ \\
\hline G5L259 & $\begin{array}{l}\text { NADP-dependent glyceraldehyde-3-phosphate } \\
\text { dehydrogenase, putative }\end{array}$ & 2.781098 \\
D5AFN4 & Xaa-Pro dipeptidyl-peptidase & 3.095053 \\
G7SMG3 & Elongation factor G & 3.667679 \\
G7SD52 & ABC superfamily ATP binding cassette & 3.201581 \\
& transporter, membrane protein & 3.796473 \\
G5L1N9 & Nucleoid DNA-binding protein & 11.34908 \\
G7S8P5 & Phosphoglycerate kinase &
\end{tabular}

Fold change ${ }^{a}$, the ratio of different expression level between Sub-MIC tylosin treated cells and nontreated cells.

(SEM). The biofilm examination relied heavily on SEM. Because of its high magnification, the biofilm microstructure is observed clearly. These findings further confirm that S. suis can form biofilms in vitro. Although these procedures have their advantages, the TCP procedure lacks specificity and sensitivity because crystal violet stains all of the components of the biofilm and could potentially stain non-biofilm material. More specific and sensitive techniques can be performed to assess each of the individual components. This method is good for screening purposes.

Tylosin is a frequently used drug for the treatment of $S$. suis infection. Upon treatment with this antimicrobial agent, $S$. suis is inevitably exposed to a sub-inhibitory level of the agent. Therefore, we studied its effect in sub-inhibitory concentrations on S. suis biofilm formation. Tylosin can inhibit S. suis biofilm formation at sub-inhibitory concentrations in a dose-dependent manner. In addition, the inhibition of biofilm formation varied among the antimicrobial agents. Numerous reports have showed biofilm formation in the presence of sub-inhibitory concentrations of antimicrobial agents (Dunne, 1990; CarsentiEtesse et al., 1993; Rupp and Hamer, 1998; Rachid et al., 2000; Yang et al., 2015). Our results are in agreement with these previous findings. Our results showed a better activity of tylosin against $S$. suis biofilm formation. However, further studies should be conducted to confirm and clarify the relationship between the relative adherence-inhibiting properties of tylosin and their mechanisms. These results may provide information regarding the clinical use of antimicrobial agents against biofilm-forming bacteria.

Our data identified proteins related to biofilm growth that have previously been uncharacterized. iTRAQ analyses showed that the regulation of metabolism plays a key role during $S$. suis biofilm growth. First, the iTRAQ quantitative data revealed that carbohydrate metabolism may be particularly important during S. suis biofilm growth. It was reported that fructose bisphosphate aldolase and glyceraldehyde-3-phosphate dehydrogenase were significantly decreased in Streptococcus pneumoniae biofilms (Allan et al., 2014). In our study, there was a >two-fold increase of glyceraldehyde-3-phosphate dehydrogenase in the tylosin inhibiting biofilm formation. In addition, the levels of fructose bisphosphate aldolase (fold change: 0.62) were down-regulated. It was reported that phosphoglycerate kinase was up-regulated in S. suis biofilms compared with planktonic cells by comparative proteomic analysis (Wang et al., 2012).
Similarly, biofilms of Pseudomonas aeruginosa (Sauer et al., 2002) and Staphylococcus xylosus (Planchon et al., 2009) exhibit up-regulated phosphoglycerate kinase. There was a $>11$-fold increase in the level of phosphoglycerate kinase in the tylosin inhibiting biofilm formation. Phosphoglycerate kinase is a glycolytic enzyme that functions in the conversion of glyceraldehyde 3-phosphate into 1, 3-diphosphoglycerate. Glycolysis may play a pivotal role during $S$. suis biofilm growth. Furthermore, histidine metabolism may play an important role in biofilm formation. Histidine kinase (fold change: 1.62) was up-regulated in the tylosin-inhibited biofilm formation. Compared with other amino acids, L-His had the strongest effect on biofilm induction (Cabral et al., 2011). Histidine metabolism is also found to be involved in biofilm formation and is confirmed by gene disruption (Cabral et al., 2011). Histidine kinase is an important signaling molecule in biofilm formation in gram-positive and negative bacteria (McLoon et al., 2011; Shemesh and Chai, 2013; Yang et al., 2014; Grau et al., 2015). In addition, several ABC transporter system proteins were significantly up-regulated in the tylosin inhibiting biofilm formation. This finding is consistent with the previous results of our laboratory showing that sub-MIC erythromycin inhibits S. suis biofilm formation (Zhao et al., 2015). The carbohydrate substrate selection and fermentation determine $\mathrm{ABC}$ transporter proteins were significantly up-regulated in tylosin-treated cells, suggesting that $S$. suis had the capability of metabolizing a wide range of carbohydrates during biofilm development (Hardy et al., 2001; Marion et al., 2011; Bidossi et al., 2012; Haertel et al., 2012). Moreover, ABC transporters are important because they regulate respiration and biofilm formation, which in turn affect the rate of electricity production and bioremediation (Selvaraj et al., 2014). Furthermore, in the mutants of a pneumococcal biofilm screen, $\mathrm{ABC}$ transporters were shown to be defective in colonization (Munoz-Elias et al., 2008).

Cell surface proteins play a crucial role in biofilms. Within the biofilm, bacterial cells are embedded in a self-produced extracellular matrix. This matrix protects bacteria against a number of environmental insults. However, the matrix also confines bacterial access to fresh nutrients. Thus, the verified increase in the expression of transmembrane channels appears to be an essential requirement for the entrance of important nutrient-containing fluid (Costerton et al., 1994). In addition to acting, as channels, porins may act as potential targets for adhesion to other cells and may mediate cell attachment through binding to the proteins released for biofilm formation (Dallo et al., 2010). The outer membrane proteins may mediate cell attachment through binding to the released proteins for biofilm formation. Membrane proteins such as OmpA mediate cell adhesion in Acinetobacter baumannii (Dallo et al., 2010). The key developmental stages of biofilm development have been reported to be adhesion to surfaces, aggregation of micro colonies, and further expansion of the microbial community. The putative involvement of many genes encoding large cell surface proteins is adhesion to the epithelium and biofilm formation (Pridmore et al., 2004; Walter et al., 2005; Frese et al., 2011). Pham (Pham et al., 2010) found increased expression of outer membrane proteins in the Tannerella forsythia biofilm cells by using quantitative non-gel-based proteomic techniques. In 


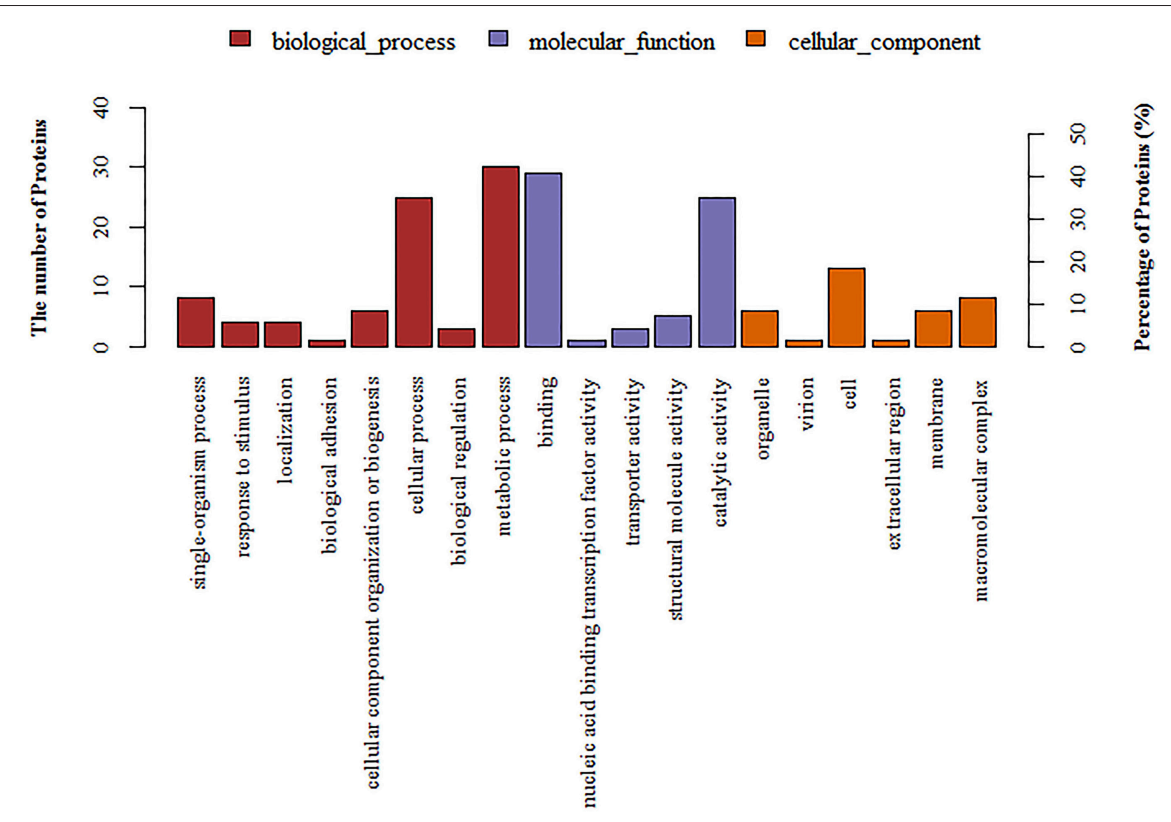

FIGURE 4 | Annotation of differentially regulated protein (ratio $>1.5$ or $<0.67$ ) functions by Gene Ontology (GO).

our study, the expression of membrane-anchored lipoprotein, phosphoglycerate kinase, sugar $\mathrm{ABC}$ transporter permease, ABC superfamily ATP binding cassette transporter membrane protein and L-fucose isomerase changed; these proteins belong to membrane proteins and cell-surface proteins and might be involved in some molecular functions including catalytic activity, motor activity, nucleotide binding, protein binding and transporter activity. For example, phosphoglycerate kinase is a S. suis surface protein that promotes cell adhesion and plays a key role in bacterial infection and invasion (Brassard et al., 2004; Wang and Lu, 2007). We predicted that these membrane proteins might affect the bacterial cell-cell interaction. Thus, membrane proteins might play a significant role in biofilm formation.

S. suis virulence proteins related to infection, persistence and competitive fitness were mostly down regulated when sub-MIC tylosin was used to inhibit biofilm formation. There was a $>1.5-$ fold decrease in the level of $\mathrm{NADH}$ oxidase. $\mathrm{NADH}$ oxidase regulates competence, virulence, and pneumococcal persistence by its actions as an oxygen sensor, in detoxifying oxygen, and in increasing the efficiency of glucose breakdown (Auzat et al., 1999) and plays an important role in pneumococcal infection in animal models of pneumonia (Yu et al., 2001). NADH oxidase is encoded by nox. The virulence and persistence in mice of a blood isolate was attenuated by a nox insertion mutation (Auzat et al., 1999). Thus, this protein appears to be of major importance in the growth of biofilms.

\section{CONCLUSIONS}

Our study suggested that sub-MICs of tylosin could inhibit $S$. suis biofilm formation in vitro. We used a robust and reliable comparative proteomic technique (iTRAQ) to compare the abundances of proteins from 1/4 MIC of tylosin treated cells and nontreated cells. Finally, in sub-MIC tylosin inhibiting cells, we identified 96 differentially expressed proteins when the protein had a fold-change of more than a ratio $>1.5$ or $<0.67$ ( $p<0.05)$. Our proteomic data suggested general changes in metabolism (such as phosphoglycerate kinase) and surface proteins (such as $\mathrm{ABC}$ transporter proteins) involved in biofilm formation. Overall, our results indicated that $S$. suis metabolic regulation, cell-surface proteins, and virulence proteins appear to be of importance in biofilm growth by sub-MIC tylosin treatment. Thus, our data revealed the rough regulation of biofilm formation that might potentially be utilized to manage biofilm infections of $S$. suis.

\section{AUTHOR CONTRIBUTIONS}

SW the design whole experiment. YL directed the completion of the experiment. YY, YZhao, HZ, JB, JC, YZhou, CW provided help during the experiment.

\section{ACKNOWLEDGMENTS}

This work was supported by the National Natural Science Foundation of China (No. 31472231) and Guangdong Technology Research Center for Traditional Chinese Veterinary Medicine and Natural Medicine (No.20141020002). We thank Shanghai Applied Protein Technology Co. Ltd. for the help with iTRAQ.

\section{SUPPLEMENTARY MATERIAL}

The Supplementary Material for this article can be found online at: http://journal.frontiersin.org/article/10.3389/fmicb. 2016.00384 


\section{REFERENCES}

Allan, R. N., Skipp, P., Jefferies, J., Clarke, S. C., Faust, S. N., HallStoodley, L., et al. (2014). Pronounced metabolic changes in adaptation to biofilm growth by Streptococcus pneumoniae. PLoS ONE 9:e107015. doi: 10.1371/journal.pone.0107015

Auzat, I., Chapuy-Regaud, S., Le Bras, G., Dos Santos, D., Ogunniyi, A. D., Le Thomas, I., et al. (1999). The NADH oxidase of Streptococcus pneumoniae: its involvement in competence and virulence. Mol. Microbiol. 34, 1018-1028. doi: 10.1046/j.1365-2958.1999.01663.x

Bidossi, A., Mulas, L., Decorosi, F., Colomba, L., Ricci, S., Pozzi, G., et al. (2012). A functional genomics approach to establish the complement of carbohydrate transporters in Streptococcus pneumoniae. PLoS ONE 7:e33320. doi: 10.1371/journal.pone.0033320

Bjarnsholt, T., Ciofu, O., Molin, S., Givskov, M., and Hoiby, N. (2013). Applying insights from biofilm biology to drug development - can a new approach be developed? Nat. Rev. Drug Discov. 12, 791-808. doi: 10.1038/nrd4000

Brassard, J., Gottschalk, M., and Quessy, S. (2004). Cloning and purification of the Streptococcus suis serotype 2 glyceraldehyde-3-phosphate dehydrogenase and its involvement as an adhesin. Vet. Microbiol. 102, 87-94. doi: 10.1016/j.vetmic.2004.05.008

Cabral, M. P., Soares, N. C., Aranda, J., Parreira, J. R., Rumbo, C., Poza, M., et al. (2011). Proteomic and functional analyses reveal a unique lifestyle for Acinetobacter baumannii biofilms and a key role for histidine metabolism. J. Proteome Res. 10, 3399-3417. doi: 10.1021/pr101299j

Carsenti-Etesse, H., Durant, J., Entenza, J., Mondain, V., Pradier, C., Bernard, E., et al. (1993). Effects of subinhibitory concentrations of vancomycin and teicoplanin on adherence of staphylococci to tissue culture plates. Antimicrob. Agents Chemother. 37, 921-923. doi: 10.1128/AAC.37.4.921

Costerton, J. W., Lewandowski, Z., DeBeer, D., Caldwell, D., Korber, D., and James, G. (1994). Biofilms, the customized microniche. J. Bacteriol. 176, 2137-2142.

Dallo, S. F., Denno, J., Hong, S., and Weitao, T. (2010). Adhesion of Acinetobacter baumannii to extracellular proteins detected by a live cell-protein binding assay. Ethn. Dis. 20(1 Suppl. 1):S1-7-11.

Davey, M. E., and O’Toole, G. A. (2000). Microbial biofilms: from ecology to molecular genetics. Microbiol. Mol. Biol. Rev. 64, 847-867. doi: 10.1128/MMBR.64.4.847-867.2000

Dunne, W. M. Jr. (1990). Effects of subinhibitory concentrations of vancomycin or cefamandole on biofilm production by coagulase-negative staphylococci. Antimicrob. Agents Chemother. 34, 390-393. doi: 10.1128/AAC.34.3.390

Frese, S. A., Benson, A. K., Tannock, G. W., Loach, D. M., Kim, J., Zhang, M., et al. (2011). The evolution of host specialization in the vertebrate gut symbiont Lactobacillus reuteri. PLoS Genet. 7:e1001314. doi: 10.1371/journal.pgen.1001314

Fujimura, S., Sato, T., Mikami, T., Kikuchi, T., Gomi, K., and Watanabe, A. (2008). Combined efficacy of clarithromycin plus cefazolin or vancomycin against Staphylococcus aureus biofilms formed on titanium medical devices. Int. J. Antimicrob. Agents 32, 481-484. doi: 10.1016/j.ijantimicag.2008. 06.030

Geng, H., Zhu, L., Yuan, Y., Zhang, W., Li, W., Wang, J., et al. (2008). Identification and characterization of novel immunogenic proteins of Streptococcus suis serotype 2. J. Proteome Res. 7, 4132-4142. doi: 10.1021/pr800196v

Gilbert, P., Das, J., and Foley, I. (1997). Biofilm susceptibility to antimicrobials. Adv. Dent. Res. 11, 160-167. doi: 10.1177/08959374970110010701

Gottschalk, M., Segura, M., and Xu, J. (2007). Streptococcus suis infections in humans: the Chinese experience and the situation in North America. Anim. Health Res. Rev. 8, 29-45. doi: 10.1017/S1466252307001247

Gottschalk, M., Xu, J. G., Calzas, C., and Segura, M. (2010). Streptococcus suis: a new emerging or an old neglected zoonotic pathogen? Future Microbiol. 5, 371-391. doi: 10.2217/fmb.10.2

Grau, R. R., de Ona, P., Kunert, M., Lenini, C., Gallegos-Monterrosa, R., Mhatre, E., et al. (2015). A duo of potassium-responsive histidine kinases govern the multicellular destiny of Bacillus subtilis. mBio 6:e00581-15. doi: 10.1128/mBio.00581-15

Haertel, T., Eylert, E., Schulz, C., Petruschka, L., Gierok, P., Grubmueller, S., et al. (2012). Characterization of central carbon metabolism of Streptococcus pneumoniae by isotopologue profiling. J. Biol. Chem. 287, 4260-4274. doi: 10.1074/jbc.M111.304311
Hardy, G. G., Magee, A. D., Ventura, C. L., Caimano, M. J., and Yother, J. (2001). Essential role for cellular phosphoglucomutase in virulence of type 3 Streptococcus pneumoniae. Infect. Immun. 69, 2309-2317. doi: 10.1128/IAI.69.4.2309-2317.2001

Marion, C., Aten, A. E., Woodiga, S. A., and King, S. J. (2011). Identification of an ATPase, MsmK, which energizes multiple carbohydrate ABC transporters in Streptococcus pneumoniae. Infect. Immun. 79, 4193-4200. doi: 10.1128/IAI.05290-11

Mathur, T., Singhal, S., Khan, S., Upadhyay, D. J., Fatma, T., and Rattan, A. (2006). Detection of biofilm formation among the clinical isolates of Staphylococci: an evaluation of three different screening methods. Indian J. Med. Microbiol. 24, 25-29. doi: 10.4103/0255-0857.19890

McLoon, A. L., Kolodkin-Gal, I., Rubinstein, S. M., Kolter, R., and Losick, R. (2011). Spatial regulation of histidine kinases governing biofilm formation in Bacillus subtilis. J. Bacteriol. 193, 679-685. doi: 10.1128/JB.01186-10

Munoz-Elias, E. J., Marcano, J., and Camilli, A. (2008). Isolation of Streptococcus pneumoniae biofilm mutants and their characterization during nasopharyngeal colonization. Infect. Immun. 76, 5049-5061. doi: 10.1128/IAI.00425-08

Okajima, Y., Kobayakawa, S., Tsuji, A., and Tochikubo, T. (2006). Biofilm formation by Staphylococcus epidermidis on intraocular lens material. Invest. Ophthalmol. Vis. Sci. 47, 2971-2975. doi: 10.1167/iovs.05-1172

Pham, T. K., Roy, S., Noirel, J., Douglas, I., Wright, P. C., and Stafford, G. P. (2010). A quantitative proteomic analysis of biofilm adaptation by the periodontal pathogen Tannerella forsythia. Proteomics 10, 3130-3141. doi: 10.1002/pmic.200900448

Planchon, S., Desvaux, M., Chafsey, I., Chambon, C., Leroy, S., Hebraud, M., et al. (2009). Comparative subproteome analyses of planktonic and sessile Staphylococcus xylosus C2a: new insight in cell physiology of a coagulasenegative staphylococcus in biofilm. J. Proteome Res. 8, 1797-1809. doi: $10.1021 / \mathrm{pr} 8004056$

Presterl, E., Suchomel, M., Eder, M., Reichmann, S., Lassnigg, A., Graninger, W., et al. (2007). Effects of alcohols, povidone-iodine and hydrogen peroxide on biofilms of Staphylococcus epidermidis. J. Antimicrob. Chemother. 60, 417-420. doi: $10.1093 / \mathrm{jac} / \mathrm{dkm} 221$

Pridmore, R. D., Berger, B., Desiere, F., Vilanova, D., Barretto, C., Pittet, A. C., et al. (2004). The genome sequence of the probiotic intestinal bacterium Lactobacillus johnsonii NCC 533. Proc. Natl. Acad. Sci. U.S.A. 101, 2512-2517. doi: 10.1073/pnas.0307327101

Rachid, S., Ohlsen, K., Witte, W., Hacker, J., and Ziebuhr, W. (2000). Effect of subinhibitory antibiotic concentrations on polysaccharide intercellular adhesin expression in biofilm-forming Staphylococcus epidermidis. Antimicrob. Agents Chemother. 44, 3357-3363. doi: 10.1128/AAC.44.12.3357-3363.2000

Rupp, M. E., and Hamer, K. E. (1998). Effect of subinhibitory concentrations of vancomycin, cefazolin, ofloxacin, L-ofloxacin and D-ofloxacin on adherence to intravascular catheters and biofilm formation by Staphylococcus epidermidis. J. Antimicrob. Chemother. 41, 155-161. doi: 10.1093/jac/41.2.155

Sauer, K. (2003). The genomics and proteomics of biofilm formation. Genome Biol. 4:219. doi: 10.1186/gb-2003-4-6-219

Sauer, K., Camper, A. K., Ehrlich, G. D., Costerton, J. W., and Davies, D. G. (2002). Pseudomonas aeruginosa displays multiple phenotypes during development as a biofilm. J. Bacteriol. 184, 1140-1154. doi: 10.1128/jb.184.4.1140-1154.2002

Selvaraj, A., Sumantran, V., Chowdhary, N., and Kumar, G. R. (2014). Prediction and classification of $\mathrm{ABC}$ transporters in Geobacter sulfurreducens PCA using computational approaches. Curr. Bioinform. 9, 166-172. doi: $10.2174 / 1574893608999140109113236$

Shemesh, M., and Chai, Y. (2013). A combination of glycerol and manganese promotes biofilm formation in Bacillus subtilis via histidine kinase KinD signaling. J. Bacteriol. 195, 2747-2754. doi: 10.1128/JB.00028-13

Sriskandan, S., and Slater, J. D. (2006). Invasive disease and toxic shock due to zoonotic Streptococcus suis: an emerging infection in the East? PLoS Med. 3:e187. doi: 10.1371/journal.pmed.0030187

Unwin, R. D., Griffiths, J. R., and Whetton, A. D. (2010). Simultaneous analysis of relative protein expression levels across multiple samples using iTRAQ isobaric tags with 2D nano LC-MS/MS. Nat. Protoc. 5, 1574-1582. doi: $10.1038 /$ nprot.2010.123

Walter, J., Chagnaud, P., Tannock, G. W., Loach, D. M., Dal Bello, F., Jenkinson, H. F., et al. (2005). A high-molecular-mass surface protein (Lsp) and methionine sulfoxide reductase B (MsrB) contribute to the ecological performance of 
Lactobacillus reuteri in the murine gut. Appl. Environ. Microbiol. 71, 979-986. doi: 10.1128/AEM.71.2.979-986.2005

Wang, K., and Lu, C. (2007). Adhesion activity of glyceraldehyde-3phosphate dehydrogenase in a Chinese Streptococcus suis type 2 strain. Berl. Munch. Tierarztl. Wochenschr. 120, 207-209. doi: 10.2376/0005-9366120-207

Wang, Y., Yi, L., Wu, Z., Shao, J., Liu, G., Fan, H., et al. (2012). Comparative proteomic analysis of Streptococcus suis biofilms and planktonic cells that identified biofilm infection-related immunogenic proteins. PLoS ONE 7:e33371. doi: 10.1371/journal.pone.0033371

Wang, Y., Zhang, W., Wu, Z. F., and Lu, C. P. (2011). Reduced virulence is an important characteristic of biofilm infection of Streptococcus suis. FEMS Microbiol. Lett. 316, 36-43. doi: 10.1111/j.1574-6968.2010. 02189.x

Wisniewski, J. R., Zougman, A., Nagaraj, N., and Mann, M. (2009). Universal sample preparation method for proteome analysis. Nat. Methods 6, 359-362. doi: $10.1038 /$ nmeth. 1322

Yang, K., Meng, J., Huang, Y.-C., Ye, L.-H., Li, G.-J., Huang, J., et al. (2014). The role of the QseC quorum-sensing sensor kinase in epinephrine-enhanced motility and biofilm formation by Escherichia coli. Cell Biochem. Biophys. 70, 391-398. doi: 10.1007/s12013-014-9924-5

Yang, W.-Z., Yu, H.-J., Jing, H.-Q., Xu, J.-G., Chen, Z.-H., Zhu, X.-P., et al. (2006). An outbreak of human Streptococcus suis serotype 2 infections presenting with toxic shock syndrome in Sichuan, China. Zhonghua Liu Xing Bing Xue za Zhi 27, 185-191.

Yang, Y. B., Wang, S., Wang, C., Huang, Q. Y., Bai, J. W., Chen, J. Q., et al. (2015). Emodin affects biofilm formation and expression of virulence factors in Streptococcus suis ATCC700794. Arch. Microbiol. 197, 1173-1180. doi: 10.1007/s00203-015-1158-4
Yu, J., Bryant, A. P., Marra, A., Lonetto, M. A., Ingraham, K. A., Chalker, A. F., et al. (2001). Characterization of the Streptococcus pneumoniae NADH oxidase that is required for infection. Microbiology $147(P t 2), 431-438$. doi: 10.1099/00221287-147-2-431

Zhang, A., Xie, C., Chen, H., and Jin, M. (2008). Identification of immunogenic cell wall-associated proteins of Streptococcus suis serotype 2. Proteomics 8, 3506-3515. doi: 10.1002/pmic.200800007

Zhang, W., and Lu, C. P. (2007a). Immunoproteomic assay of membraneassociated proteins of Streptococcus suis type 2 china vaccine strain HA9801. Zoonoses Public Health 54, 253-259. doi: 10.1111/j.1863-2378.2007.01056.x

Zhang, W., and Lu, C. P. (2007b). Immunoproteomics of extracellular proteins of Chinese virulent strains of Streptococcus suis type 2. Proteomics 7, 4468-4476. doi: 10.1002/pmic.200700294

Zhao, Y. L., Zhou, Y. H., Chen, J. Q., Huang, Q. Y., Han, Q., Liu, B. et al. (2015). Quantitative proteomic analysis of sub-MIC erythromycin inhibiting biofilm formation of S-suis in vitro. J. Proteomics 116, 1-14. doi: 10.1016/j.jprot.2014.12.019

Conflict of Interest Statement: The authors declare that the research was conducted in the absence of any commercial or financial relationships that could be construed as a potential conflict of interest.

Copyright (c) 2016 Wang, Yang, Zhao, Zhao, Bai, Chen, Zhou, Wang and Li. This is an open-access article distributed under the terms of the Creative Commons Attribution License (CC BY). The use, distribution or reproduction in other forums is permitted, provided the original author(s) or licensor are credited and that the original publication in this journal is cited, in accordance with accepted academic practice. No use, distribution or reproduction is permitted which does not comply with these terms. 
OPEN ACCESS

Edited by:

Ivan Rychlik,

Veterinary Research Institute,

Czechia

Reviewed by:

Atte Von Wright

University of Eastern Finland, Finland

Dinesh Sriramulu,

Shres Consultancy (Life Sciences),

India

*Correspondence:

Wenhong Zhang

zhangwenhong@fudan.edu.cn

Ying Zhang

yzhang@jhsph.edu

Specialty section:

This article was submitted to Antimicrobials, Resistance and

Chemotherapy,

a section of the journal

Frontiers in Microbiology

Received: 14 July 2016 Accepted: 29 September 2016

Published: 24 October 2016

Citation:

Xu T, Han J, Zhang J, Chen J, Wu N,

Zhang $W$ and Zhang Y (2016)

Absence of Protoheme IX

Farnesyltransferase CtaB Causes Virulence Attenuation but Enhances

Pigment Production and Persister

Survival in MRSA.

Front. Microbiol. 7:1625.

doi: 10.3389/fmich.2016.01625

\section{Absence of Protoheme IX Farnesyltransferase CtaB Causes Virulence Attenuation but Enhances Pigment Production and Persister Survival in MRSA}

\author{
Tao Xu ${ }^{1}$, Jian Han ${ }^{2}$, Jia Zhang ${ }^{1}$, Jiazhen Chen ${ }^{1}$, Nan Wu ${ }^{1}$, Wenhong Zhang ${ }^{1 *}$ and \\ Ying Zhang ${ }^{1,3 *}$ \\ ${ }^{1}$ Key Laboratory of Medical Molecular Virology, Huashan Hospital, Shanghai Medical College of Fudan University, Shanghai, \\ China, ${ }^{2}$ Department of Pathogenic Biology, School of Basic Medical Sciences, Lanzhou University, Lanzhou, China, \\ ${ }^{3}$ Department of Molecular Microbiology and Immunology, Bloomberg School of Public Health, Johns Hopkins University, \\ Baltimore, MD, USA
}

The membrane protein CtaB in S. aureus is a protoheme IX farnesyltransferase involved in the synthesis of the heme containing terminal oxidases of bacterial respiratory chain. In this study, to assess the role of CtaB in S. aureus virulence, pigment production, and persister formation, we constructed a $\operatorname{ctaB}$ mutant in the methicillin-resistant Staphylococcus aureus (MRSA) strain USA500. We found that deletion of ctaB attenuated growth and virulence in mice but enhanced pigment production and formation of quinolone tolerant persister cells in stationary phase. RNA-seq analysis showed that deletion of ctaB caused decreased transcription of several virulence genes including RNAlll which is consistent with its virulence attenuation. In addition, transcription of 20 ribosomal genes and 24 genes involved in amino acid biosynthesis was significantly down-regulated in the ctaB knockout mutant compared with the parent strain. These findings suggest the importance of heme biosynthesis in virulence and persister formation of $S$. aureus.

Keywords: Staphylococcus aureus, heme, antibiotics, pigment, virulence, persister formation

\section{INTRODUCTION}

Staphylococcus aureus, named according to production of golden pigment, is an important human pathogen causing a variety of infection types including rampant skin and soft tissue infections, pneumonia, septicaemia, endocarditis, and central nervous system (CNS) infections. Methicillinresistant S. aureus (MRSA) is notorious for its development of antibiotic resistance and expression of multiple virulence factors. (Li et al., 2012; Carrel et al., 2015).

$S$. aureus virulence factors are multifactorial and previous studies have been mainly focused on toxins ( $\alpha$-toxin, $\gamma$-toxin, Panton-Valentine leucocidin, exfoliative toxin and phenol-soluble modulins, etc.), surface proteins (FnbP, Bap, SasX, etc.) that help bind to host cells, facilitate

Abbreviations: MRSA, methicillin resistant Staphylococcus aureus; FPP, farnesyl diphosphate; SCV, small colony variants; MIC, minimum inhibitory concentration. 
internalization and immune evasion. Staphyloxanthin, synthesized from farnesyl diphosphate (FPP) by CrtM and $\mathrm{CrtN}$, is the main component of $S$. aureus golden pigment (Liu et al., 2005). Staphyloxanthin not only plays a protecting role in bacterial fitness, but enhances virulence and survive attack by neutrophils (Clauditz et al., 2006). In addition, global regulatory systems (Agr, SaeRS, SarA, etc.) govern different aspects of physiology and expression of virulence traits, maintaining a balance between fitness and virulence.

It was in staphylococcus that persisters were first described in Bigger (1944). Persisters represent a certain portion of a bacterial culture that is genetically identical but phenotypically resistant or tolerant to antibiotics and stresses. In the model organism Escherichia coli, much research reveals the mechanisms of persister formation, including involvement of toxin-antitoxin, protein degradation, energy production and DNA repair (Zhang, 2014). However, less understood are the mechanisms of S. aureus persister formation. The portion of persisters in $S$. aureus is so high that a hypothesis was proposed that unlike E. coli, all $S$. aureus cells in stationary phase are persisters (Keren et al., 2004). Subsequently, however, Lechner et al. proved that stationary phase cultures of $S$. aureus are also a mixture of regular and persister cells (Lechner et al., 2012).

Though the key mechanisms of $S$. aureus persister formation are poorly understood, progress has been made recently. It has been reported that biofilm formation (Lewis, 2001; Resch et al., 2006) and small colony variants (SCV; Lechner et al., 2012) are two key features involving $S$. aureus persister formation, probably because the cells in biofilms and SCV cells have a different profile of gene expression, which makes them more readily to form persisters. Glycerol uptake has been reported to play a role in persister formation. Mutation in the glycerol transporter encoding gene $g l p F$ caused defective survival of $S$. aureus to ampicillin and norfloxacin (Han et al., 2014). A point mutation of the inorganic phosphate transporter gene pitA enhanced tolerance to daptomycin (Mechler et al., 2015). Mutations in purine biosynthesis genes ( $p u r B$, purF, purH, purM,) amino acid, lipid, carbohydrate metabolism, and energy production genes efflux etc. were found to cause decreased persister formation in recent transposon mutant library screens (Yee et al., 2015; Wang et al., 2015).

Heme synthesis is an important pathway in Gram positive bacteria and provide substrate to production of terminal oxidases (Mogi et al., 1994). Within vertebrates S. aureus fulfills its requirement of iron by uptaking heme-iron from transferrin or heme or hemoglobin with its several transporters including StrA, StrB, IsdA, and IsdE, etc. (Drabkin, 1951; Mazmanian et al., 2003; Liu et al., 2008; Mason and Skaar, 2009). However, in an environment without heme-iron, $S$. aureus has to synthesize heme A with a complex pathway starting from glutamate (Hammer et al., 2016). CtaB and CtaA catalyzes the last two steps of the process. $\mathrm{CtaB}$ is a heme $\mathrm{O}$ synthase (protoheme IX farnesyltransferase) and while CtaA is an integral membrane protein that converts heme O to heme A (Svensson et al., 1993; Svensson and Hederstedt, 1994; Clements et al., 1999). Heme A is essential for functional expression of the terminal oxidases. Among terminal oxidases synthesized with heme A, cytochrome aa 3 are quinol oxidases (QoxA, QoxB, etc.) and cytochrome caa 3 is a cytochrome c oxidase.

Though heme synthesis mainly contributes to the pathway of synthesis of terminal oxidases that mediate bacterial respiration, it has also been reported to participate in fitness and virulence of $S$. aureus. For example, CtaA was found to be required for starvation survival and recovery from glucose starvation (Clements et al., 1999). A correlation between heme production and pigment production was reported by Lan et al., as depletion of CtaA and QoxB both enhanced pigment production, while attenuating hemolytic activity and virulence (Lan et al., 2010). However, no study has been done to address the specific effects of $c t a B$ mutation on the heme-to-respiratory chain pathway and associated phenotypic changes. In this study, we created a CtaB deletion mutant of $S$. aureus and found associations of CtaB with heme synthesis, pigment production as well as persister cell formation. In addition, we performed a transcriptome analysis to provide new insights into the basis of the above associations.

\section{MATERIAL AND METHODS}

\section{Bacterial Strains, Growth, and Chemical Reagents}

S. aureus USA500 (Diep et al., 2006) was used for construction of gene knockout and complementation strains. E. coli DC10B (Monk et al., 2012) was used for shuttle plasmid construction. Luria Broth medium was composed of 1\% tryptone (Oxoid), $0.5 \%$ yeast extract (Oxoid) and $0.5 \% \mathrm{NaCl}$; BM (B-Medium) was composed of $1 \%$ tryptone, $0.5 \%$ yeast extract, $0.5 \%$ glucose, $0.1 \% \mathrm{~K}_{2} \mathrm{HPO}_{4}$ and $0.5 \% \mathrm{NaCl} ; \mathrm{BM}$ and TSB (Tryptic soy broth, Oxoid) were used for $S$. aureus cultivation. Bacterial strains were inoculated in $\mathrm{BM}$, and their growth rate at $37^{\circ} \mathrm{C}$ was monitored by measuring the $\mathrm{OD}$ values at $600 \mathrm{~nm}$. Anhydrotetracycline (ATc) was used for induction of $\sec Y$ antisense RNA during gene knockout. Antibiotics were added to medium at the following concentrations: chloramphenicol, $10 \mu \mathrm{g} / \mathrm{ml}$; ampicillin, $100 \mu \mathrm{g} / \mathrm{ml}$, levofloxacin, $50 \mu \mathrm{g} / \mathrm{ml}$.

\section{Construction of Plasmids for Homologous Recombination and Complementary Strains}

We constructed plasmid pMX10 by replacing $c c d B$ element with multiple cloning sites in pKOR1 (Bae and Schneewind, 2006) and used it for construction of gene knock out strains. Primers pMX10-f and pMX10-r were mixed equally to a final concentration of $100 \mathrm{uM}$, incubated at $72^{\circ} \mathrm{C}$ for $20 \mathrm{~min}$ and slowly cooled to $4^{\circ} \mathrm{C}$. The resulting dimers were digested with BamHI and KpnI and ligated to pKOR1 backbone digested with the same restriction enzymes. To construct $\Delta c t a B$ in USA500, the upstream (us) fragment (about $1000 \mathrm{bp}$ ) at the upstream of $c t a B$ gene of USA500 strain was amplified with primer ctaB-uf and ctaB-ur, while the downstream (ds) fragment with primers ctaB$\mathrm{df}$ and ctaB-dr. The two fragments were then used as templates for fusion PCR with primer ctaB-uf and ctaB-dr. The final PCR product was digested with KpnI and MluI and then ligated into pMX10. The recombinant plasmids was transformed into 
USA500 by electroporation and mutants were selected according to the method reported by Bae et al. (Bae and Schneewind, 2006). To construct the complementation strain $\triangle c t a B:: p R B c t a B$, a fragment containing the promoter region and coding sequence of $c t a B$ gene was amplified with primers cp-ctaB-f and cp-ctaB-r. The PCR product was digested with EcoRI and BamHI and then ligated into plasmid PRB473. The resulting plasmid was transformed into the $\triangle c t a B$ mutant via electroporation. The sequences of primers are listed in Table $\mathbf{1 .}$

\section{Detection of Pigment Production and Hemolytic Activity}

To compare pigment production, USA500 and USA500 $\Delta$ ctaB were dropped onto TSA plates and USA500 $\Delta$ ctaB with pRB473 or $\mathrm{pRBctaB}$ on TSA plates with $10 \mu \mathrm{g} / \mathrm{ml}$ chloramphenicol. The plates were incubated at $37^{\circ} \mathrm{C}$ for $24 \mathrm{~h}$ and pictured. For quantitate assay of pigment production, the same strains were cultured in TSB at $37^{\circ} \mathrm{C}$ for $24 \mathrm{~h}$. For each sample, pigment was extracted with methanol and detected with a parameter (GeneSpec III, Hitachi, Japan), following a previously reported protocol (Morikawa et al., 2001). For hemolytic activity determination, the strains were analyzed by growing the strains on $5 \%$ sheep blood agar at $37^{\circ} \mathrm{C}$ for $48 \mathrm{~h}$. The result represents three independent experiments.

\section{Mouse Infection}

The mouse virulence test was performed on Balb/C mice. USA500 and the $\Delta c t a B$ mutant strains were cultured for $18 \mathrm{~h}$ and $1 \mathrm{ml}$ of the each culture was mixed with $2 \%$ Cytodex- 1 beads by 1:1. The mice were randomized into two groups ( 5 mice/group). Each mouse was challenged with $200 \mu \mathrm{l}$ bacterial mixture (each containing approximately $2 \times 10^{5}$ bacterial cells) via injection under skins on the back. After $48 \mathrm{~h}$, the mice were sacrificed and the abscess under skin was homogenized in $2 \mathrm{ml}$ PBS. The samples were diluted and plated on TSA plates at $37^{\circ} \mathrm{C}$ for $18 \mathrm{~h}$. CFU counting was performed and a Student's $t$-test was used for statistical analysis using Microsoft Excel.

Animal studies on mice were performed according to relevant national and international guidelines (the Regulations for the Administration of Affairs Concerning Experimental Animals, China) and were approved by the Institutional Animal Care and Use Committee (IACUC) of Shanghai Medical College, Fudan University (IACUC Animal Project Number: 20110630). Standard operation procedures were followed to carry out animal experiments in bio-safety level 2 labs.

\section{Susceptibility Testing}

The MIC of each antimicrobial compound was determined in triplicate by a conventional broth microdilution technique in TSB medium, following the protocol previously published (Andrews, 2001) and the CLSI guidelines. The MIC was defined as the lowest antibiotic concentration that inhibited visible bacterial growth (also according to OD600 measurements) after $24 \mathrm{~h}$ of incubation at $37^{\circ} \mathrm{C}$.
TABLE 1 | Primers used in this study.

\begin{tabular}{|c|c|c|}
\hline Primers & Sequence $5^{\prime}-3^{\prime}$ & Purpose \\
\hline pMX10-f & $\begin{array}{l}\text { GGGGTACCGCTAGCCGGCCGG } \\
\text { GGCCCACGCGTGAATTCCG }\end{array}$ & Construction of pMX10 \\
\hline pMX10-r & $\begin{array}{l}\text { CGGAATTCACGCGTGGGCCCC } \\
\text { GGCCGGCTAGCGGTACCCC }\end{array}$ & \\
\hline ctaB-uf & $\begin{array}{l}\text { GGGGTACCGCTGTATAACCAT } \\
\text { AATGAACAGTACG }\end{array}$ & Construction of $\Delta c t a B$ \\
\hline ctaB-ur & $\begin{array}{l}\text { САТССТААСТТААТТААТАТС } \\
\text { ССССТССТТАААТТТТТС }\end{array}$ & \\
\hline ctaB-df & $\begin{array}{l}\text { AATIAAGGAGGGGGATTATTAAT } \\
\text { TAAGTTAGGATGAAAAATATGGG }\end{array}$ & \\
\hline ctaB-dr & $\begin{array}{l}\text { CGACGCGTAGAAGTAAGCACTT } \\
\text { TAATATCTITACC }\end{array}$ & \\
\hline$c p-c t a B-f$ & $\begin{array}{l}\text { CGGAATTCAAAAAGAACTTA } \\
\text { ATCGTAATGATIIITATTG }\end{array}$ & Construction of $\Delta c t a B:: p R B c t a B$ \\
\hline cp-ctaB-r & $\begin{array}{l}\text { CGGGATCCCTTAATTAATCT } \\
\text { AGATCAAAGTAAGTAATGAAAC }\end{array}$ & \\
\hline RThld-f & CACTGTGTCGATAATCCATT & Real-time PCR \\
\hline RThld-r & ATTAAGGAAGGAGTGATाTCAAT & \\
\hline RTesaB-f & ACTTAGCAGTACCAGCATAT & \\
\hline RTesaB-r & AATATCTCCATCAGCGATITG & \\
\hline RTset18-f & CAGAGCGATTAGCAATGATAA & \\
\hline RTset18-r & GCGTTCTTGTCTTGTTTA & \\
\hline RThtrA-f & TGTGCTATTGAACGATAACG & \\
\hline RThtrA-r & CTTGCTCTGCTTGATAACTC & \\
\hline RTarcB2-f & TGAACCTGATGAAGTATGGA & \\
\hline RTarcB2-r & TGGAAAGATGGTAAGCAATG & \\
\hline RTsdhA2-f & CAGCAGATITAGCATTAGCA & \\
\hline RTsdhA2-r & TACGACCAACCTTATCCATT & \\
\hline RTnrdE-f & CGATGGTATGGCTATTCCTA & \\
\hline RTnrdE-r & CGATTGGCATTACAGAACTT & \\
\hline RTpyrF-f & TAGATGGCGTTGTTTGTC & \\
\hline RTpyrF-r & GTAATACGGTGTTGGTCATT & \\
\hline$R \operatorname{TrpmC}-f$ & TTAGAGACTTAACCACTTCAGA & \\
\hline RTrpmC-r & CTICACGAGCAACAGTाT & \\
\hline RTagrD-f & AACATTGGTAACATCGCAG & \\
\hline RTagrD-r & GTGTTAATTCTITGGTACTTCA & \\
\hline RTdltA-f & TGGTTCATTCAAGGTCGTA & \\
\hline RTdltA-r & GCATTGTCCGTAACTTCAG & \\
\hline RTrrs1-f & GTGCTACAATGGACAATACAA & \\
\hline RTrrs1-r & ACTACAATCCGAACTGAGAA & \\
\hline
\end{tabular}

\section{Persister Assay}

To determine the number of persister cells in exponential phase, cells were grown overnight in $4 \mathrm{ml}$ and were inoculated to $10 \mathrm{ml}$ of fresh medium to an initial OD600 of 0.05. Cultures were shaken for 1.5-2 h (for normally growing cells), until an OD600 of approximately 0.5 was reached. To determine the number of persister cells in stationary phase, overnight cultures (16 or $24 \mathrm{~h}$ ) were used without dilution.

For heat stress assay, stationary phase cultures were incubated at $57^{\circ} \mathrm{C}$ for up to $3 \mathrm{~h}$. For oxidative stress assay, stationary phase cultures were diluted by 1:100 in TSB that contained $50 \mathrm{mM}$ hydrogen peroxide $\left(\mathrm{H}_{2} \mathrm{O}_{2}\right)$ for $4 \mathrm{~h}$. 
For starvation stress assay, stationary phase cultures were centrifuged, washed and resuspended in $3 \% \mathrm{NaCl}$. The survival of bacteria was determined by CFU counting at each hour. All stress assays were conducted using at least three biological replicates.

For antibiotic exposure, $2 \mathrm{ml}$ of the overnight or the exponential phase cultures was transferred to $14 \mathrm{ml}$ culture tubes (Greiner), antimicrobials were added at 100-fold MIC as indicated and the cultures were shaken for $12 \mathrm{~h}$, or for 7 days during long-term experiments. For CFU determination, $100 \mu \mathrm{l}$ was taken before and during antimicrobial challenge on an hourly basis during the first $8 \mathrm{~h}$ and after $24 \mathrm{~h}$, or after $1,2,3$, 5, 6 days during long-term experiments. Cells were washed in PBS and spotted as $10 \mu \mathrm{l}$ aliquots of serial dilutions onto TSA plates. Colonies were counted after incubation for $24 \mathrm{~h}$ at $37^{\circ} \mathrm{C}$. The lower limit of quantification was $100 \mathrm{CFU} / \mathrm{ml}$. All timekill experiments were conducted using at least three biological replicates.

\section{RNA Isolation, mRNA Enrichment and Sequencing}

S. aureus USA500 parent strain and $\triangle c t a B$ mutant were cultured for 6 or $24 \mathrm{~h}$ as log phase and stationary phase cultures. The cultures were divided into 3 aliquots and treated with RNAprotect (Qiagen) and frozen at $-80^{\circ} \mathrm{C}$. Total RNA was extracted from bacterial cells using the RNeasy Mini kit (Qiagen) as described (Atshan et al., 2012). The quality of RNA samples was examined with Bioanalyzer 2100 RNA-6000 Nano Kit. To remove $16 \mathrm{~S}$ and $23 \mathrm{~S}$ rRNAs, $10 \mu \mathrm{g}$ of high-quality total RNA was processed using the Ribo-Zero ${ }^{\mathrm{TM}}$ Gold Kit before precipitating with ethanol and resuspending into $25 \mu \mathrm{L}$ of nuclease-free water. The cDNA libraries with 150- to 250-bp multiplexed cDNA were generated from the enriched mRNA samples using the TruSeq Illumina kit (Illumina, San Diego, CA), following instructions from the manufacturer.

Sequencing was performed with HiSeq2500 (Illumina). The Cufflinks suite of tools were used to assess and quantify the total number of reads. With the program Cuffdiff as part of the suite, transcripts were quantified by assessing the total number of reads for the entire transcript. Briefly, reads were mapped to annotated coding sequences (CDSs) from genome of S. aureus USA300 TCH1516 strain since USA500 is the progenitor of USA300. The samples to be compared were evaluated for variance and tested for differential expression. Reads' $P$-values were determined, and significance was assessed by conducting Benjamini-Hochberg correction for multiple testing. The transcript sequencing data were submitted to the NCBI Sequence Read Archive, available for access under a RUN number RSS3919726.

\section{Quantitative Real-Time PCR}

For quantitative Real-time PCR, the same RNA samples were taken from that used for RNA-seq. After reverse transcription with cDNA Synthesis Kit (Bio-Rad Laboratories, Hercules, CA), qRT-PCR was performed using SYBR Green PCR reagents (Takara Biotechnology) to determine the relative expression levels of the target genes with gene-specific primers listed in Table 1. The housekeeping gene rrs1 (16s RNA) was used as an endogenous control. All qRT-PCR experiments were carried out in triplicate with independent RNA samples and the $2^{-\Delta \Delta C T}$ method was performed for analysis of relative gene expression data (Livak and Schmittgen, 2001).

\section{Statistics}

The significance of experimental differences in pigment production, hemolytic activity, survival in vivo and persister assay was evaluated by unpaired Student's $t$-test.

\section{RESULTS}

\section{Construction and Properties of the S. aureus ctaB Deletion mutant $\Delta$ ctaB}

To investigate the functions of $\mathrm{CtaB}$, we constructed a $\operatorname{ctaB}$ deletion mutant, $\triangle c t a B$, via homologous recombination, as well as made a complemented strain $\triangle c t a B:: p R B c t a B$ by inserting $c t a B$ with its own promoter into plasmid pRB473. When grown in TSB, the $\triangle c t a B$ mutant showed a slight growth defect, compared with the parent strain USA500 (Figure 1A). The $\triangle c t a B$ mutant displayed enhanced golden pigment production when grown on TSA for $24 \mathrm{~h}$ compared with the control strain (Figure 1B), and complementation of the $\triangle c t a B$ mutant reduced pigment production to normal levels (Figure 1B). Quantification of pigment production by extracting carotenoid products confirmed that $\mathrm{CtaB}$ depletion afforded enhanced pigmentation than USA500 strain (Figure 1C).

\section{CtaB Affects Hemolytic Activity and Survival In vivo}

Hemolytic ability is an important aspect of $S$. aureus virulence (Wang and Muir, 2016). We analyzed the level of bacterial growth and hemolysis of the $\triangle c t a B$ mutant on sheep blood agar plates. While all strains showed similar sized colonies, deletion of $c t a B$ generated a strain with reduced hemolytic activity, which could be restored by complementation of the $\triangle c t a B$ mutant with the wild type $c t a B$ gene (Figure 2A).

Having observed that the $\triangle c t a B$ mutant enhanced pigment production but reduced hemolytic activity, we wondered whether $\triangle c t a B$ mutant would affect virulence in vivo. Skin is one of the most frequently targeted sites for S. aureus infection (Liu, 2009). To determine whether $\mathrm{CtaB}$ is associated with virulence during $S$. aureus infection, we compared the $\triangle c t a B$ mutant and the parent strain in a mouse model of skin abscess. Colony counting of bacteria from mouse skin lesions showed that inactivation of $c t a B$ attenuated bacterial survival in vivo. After $24 \mathrm{~h}$ of infection, the CFU counting of USA500 increased from $6.18 \pm 0.46 \mathrm{E}+6$ to $6.79 \pm 1.02 \mathrm{E}+6$. Within contrast, the $\triangle c t a B$ mutant survived less well with a decrease of CFU, from $4.74 \pm 0.57 \mathrm{E}+6$ to $2.96 \pm$ $1.3 \mathrm{E}+6$. (Figure 2B).

\section{CtaB is Involved in Persister Cell Formation under Stress and Antibiotic Treatment}

To determine if $\mathrm{CtaB}$ is involved in persister formation or survival, we subjected stationary cultures of USA500, $\Delta c t a B$ and $\triangle c t a B:: p R B c t a B$ under stress conditions including heat, oxidative stress, and starvation. The $\mathrm{CtaB}$ mutation attenuated the ability of 


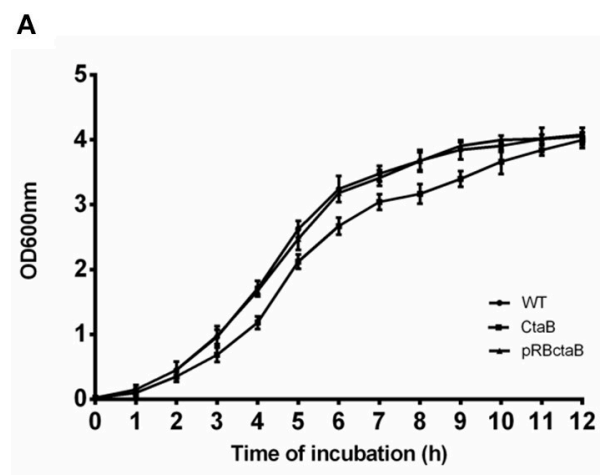

B

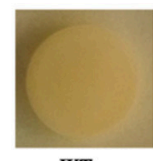

$W T$

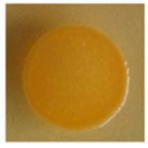

$c t a B$

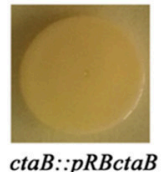

C

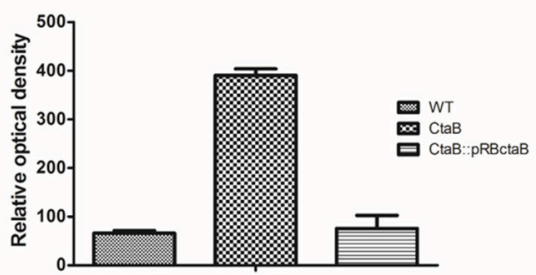

FIGURE 1 | (A) Comparison of the growth rate of USA500 and the $\triangle$ ctaB mutant. A saturated overnight culture of each strain was inoculated in a 12 ml tube and cultured at $37^{\circ} \mathrm{C}$. Cultures were monitored by measuring absorption at OD600 each hour. (B) Pigmentation display of $\mathrm{S}$. aureus strains grown on TSA plates at $37^{\circ} \mathrm{C}$ for $24 \mathrm{~h}$. (C) Measurement of the golden pigment of different strains by methanol extraction. The relative optical density units were detected at 465 nm and normalized to the USA500 strain, which was set at 100. Results are means with standard error (error bars) of three independent experiments.
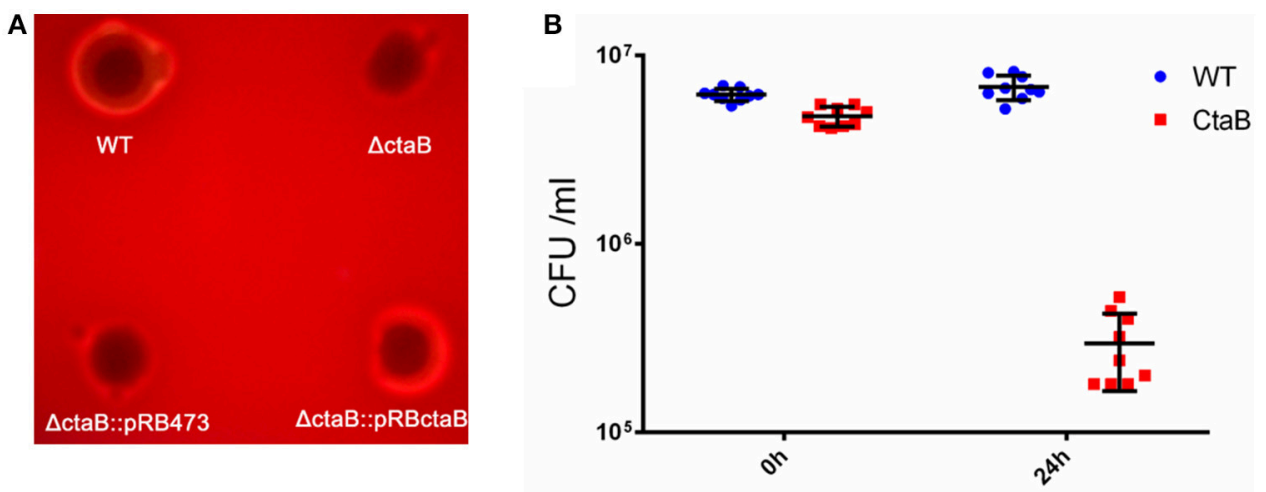

Time of infection(h)

FIGURE 2 | (A) Hemolytic activity assay. Overnight cultures of USA500, $\Delta$ ctaB mutant and complemented strains were spotted (20 $\mu$ I) on sheep blood agar plates and grown at $37^{\circ} \mathrm{C}$ for $24 \mathrm{~h}$. The result is representative of triplicate experiments. (B) Survival of $S$. aureus strains in a Balb/C challenged by subcutaneous injection. Comparison of CFU counts was performed using the Student's t-test. Results are means with standard error (errors bars).

S. aureus to survive starvation in $3 \% \mathrm{NaCl}$, which provides similar osmotic pressure as TSB, and the impact was reversed by gene complementation (Figure 3A). However, CtaB knockout did not affect survival of $S$. aureus under treatment with heat or $\mathrm{H}_{2} \mathrm{O}_{2}$ (data not shown).

Before persister assay, we measured the antibiotic sensitivity of $c t a B$ mutant and found no difference in MIC tests for multiple antibiotics (data not shown). Challenging the stationary phase cultures of USA500, $\triangle c t a B$ and $\Delta c t a B:: p R B c t a B$ with 100 $\mathrm{X}$ MIC ciprofloxacin or levofloxacin yielded disparate killing curves. As shown in Figures $(3 \mathrm{~B}, \mathrm{C})$, the surviving ratios of $\triangle c t a B$ were similar with that of USA500 in the first 3 days, but became higher than the control strain in day four and day five. Meanwhile, complementation with plasmid pRBctaB but not pRB473 partially reversed the augmentation of persister formation caused by deletion of $c t a B$ in the last 2 days of treatment. We also tested other antibiotics such as vancomycin, rifampicin, streptomycin, tobramycin, and gentamycin at $100 \mathrm{X}$ MIC concentration but found no significant difference in persister formation between the $\triangle c t a B$ mutant and the parent strain from either exponential phase or stationary phase (data not shown).

\section{RNA-seq Analysis of the $\triangle c t a B$ Mutant Compared with its Parent Strain USA500}

The above results indicate an intriguing and paradoxical role of $\mathrm{CtaB}$ in persistence and virulence of $S$. aureus, as its deletion attenuated virulence and survival in $3 \% \mathrm{NaCl}$ while increasing persister numbers for quinolone antibiotics. To gain insights into the role of $\mathrm{CtaB}$ in altered $S$. aureus virulence and persistence, we performed RNA-seq analysis of USA500 and $\triangle c t a B$ mutant grown for $6 \mathrm{~h}$ (log phase) or $24 \mathrm{~h}$ (stationary phase) in TSB medium. Based on the results of read counts of all annotated 


\section{A}
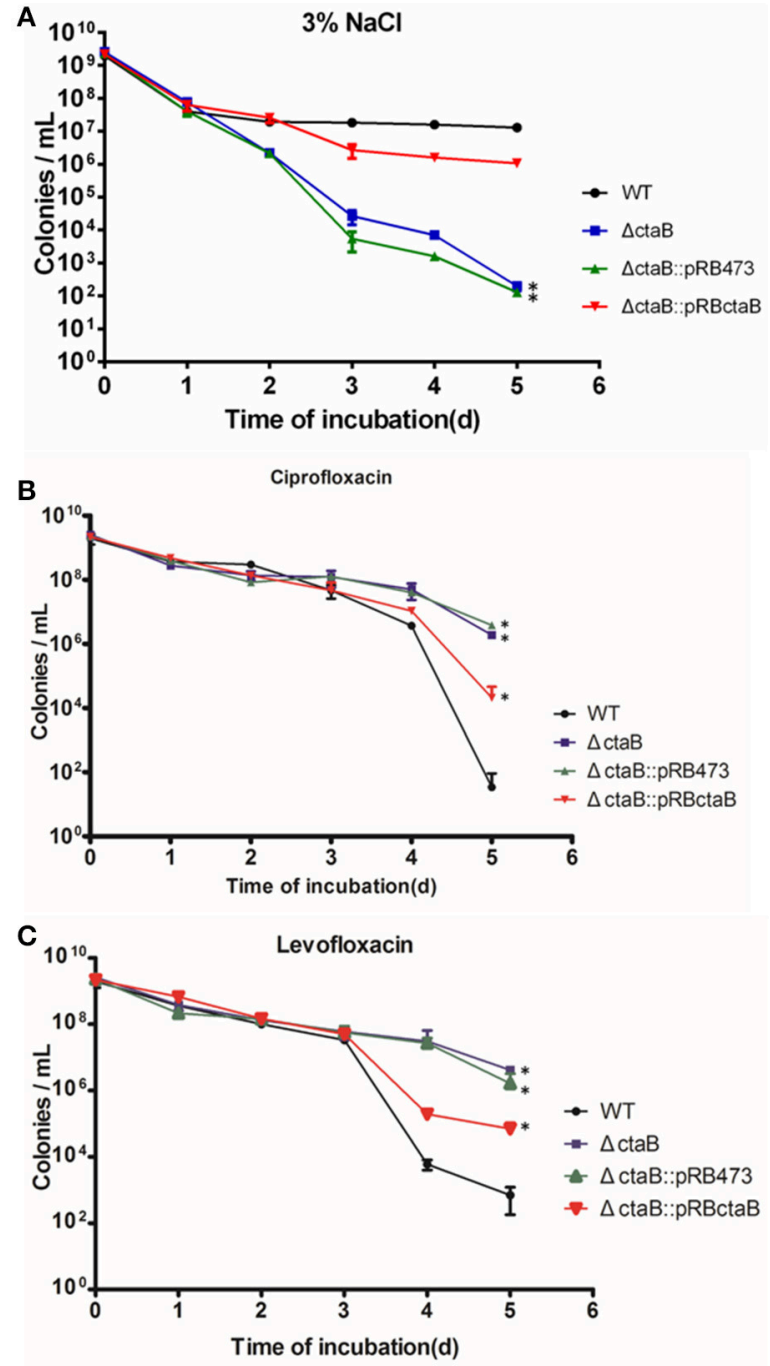

FIGURE 3 | Time dependent killing of S. aureus stationary phase bacteria. (A) Effects of starvation ( $3 \% \mathrm{NaCl}$ ) on survival kinetics of $\mathrm{S}$. aureus USA500, $\triangle$ ctaB mutant and complemented strain. Results are representative of three independent experiments. (B,C) Persister assay with antibiotics. Strains were treated with (B) $20 \mu \mathrm{g} / \mathrm{ml}$ ciprofloxacin or (C) $50 \mu \mathrm{g} / \mathrm{ml}$ levofloxacin for 6 days. The limit of detection was $100 \mathrm{CFU} / \mathrm{ml}$ throughout all killing experiments. Results are representative of three independent experiments.

genes, a total of 4 RNA-seq samples were clustered without supervision (Figure 4). The results indicated that the effect of $\mathrm{CtaB}$ knockout on the bacterial transcriptome at $24 \mathrm{~h}$ were more apparent than that at $6 \mathrm{~h}$ (Figures 5A,B).

In log phase cultures, we found 18 genes with significant changes in transcription (cutoff $>2$-fold) and $p$-values less than 0.05 between $\triangle c t a B$ mutant and the parent strain (Table 2). Most strikingly, the virulence gene hld was significantly down regulated $(0.35, p=8.47 \mathrm{E}-05)$ in the $\Delta c t a B$ mutant. In $S$. aureus, gene hld is located inside the coding sequence for small regulatory RNA RNAIII which regulates the expression of many $S$. aureus genes encoding exoproteins and cell-wall-associated

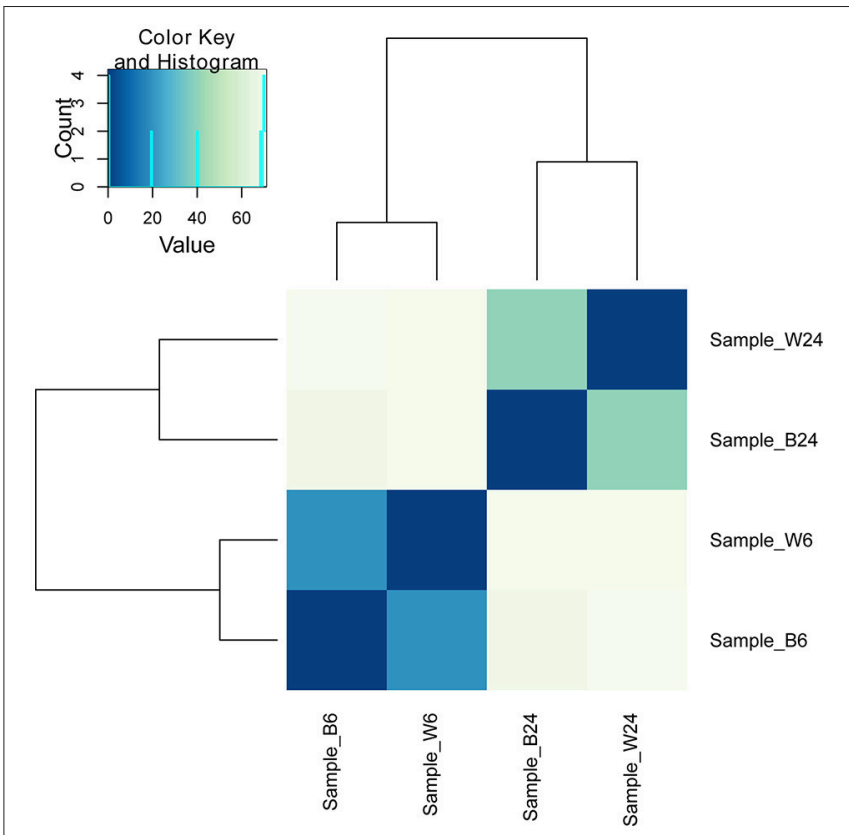

FIGURE 4 | Clustering of 4 RNA-seq samples. Sample_W6, USA500 grown to $6 \mathrm{~h}$; Sample_B6, USA500 $\Delta$ ctaB grown to $6 \mathrm{~h}$; Sample_W24, USA500 grown to $24 \mathrm{~h}$; Sample_B24, USA500 $\Delta$ ctaB grown to $24 \mathrm{~h}$. Heatmap shows the Euclidean distances between the samples as calculated from the variance-stabilizing transformation of the count data.

proteins. The data indicated that deletion of $\mathrm{CtaB}$ could attenuate expression of various virulence genes regulated by RNAIII in log phase. Interestingly, $\mathrm{CtaB}$ deletion did not affect expression of the Agr system (encoded by $\operatorname{agr} B, \operatorname{agr} D, \operatorname{agr} C$, and $\operatorname{agr} A$ ), which is the well-known upstream regulator of RNAIII and a virulence factor. The virulence gene esaB (Burts et al., 2008; Anderson et al., 2011)was also down regulated. Meanwhile, the virulence genes set 18 and set 19 were up regulated in the $\triangle \operatorname{ctaB}$ mutant. Two hemin ABC transporter super family genes $h t r B$ and $h t r A$ were significantly up regulated, as a consequence of lack of heme caused by deletion of CtaB (Table 2).

For the transcripts at $24 \mathrm{~h}, 119$ genes showed significant changes between $\triangle c t a B$ mutant and the parent strain (Table 3), indicating that $\mathrm{CtaB}$ has a major impact on stationary phase $S$. aureus. The proposed pathways that these genes participate in were analyzed (Figure 6). Nineteen genes encoding ribosome proteins were strongly down regulated, as well as nine genes involved in biosynthesis of Aminoacyl-tRNA. Genes involved in arginine, proline, cysteine, methionine, glycine, serine, threonine, lysine, phenylalanine, tyrosine, tryptophan, valine, leucine, and isoleucine were down regulated in the $\mathrm{CtaB}$ mutant. Expression of several ABC transporters was also up regulated, but other transporters such as OppA1, OppB3, OppC1, OppD1, and OppF1, were down regulated. Twenty two genes that encode factors for amino acid metabolism showed difference in expression. Expression of two genes ( $\operatorname{arcB} 2$ and $\operatorname{arcC} 1$ ) involved in arginine metabolism was up regulated while the others were down regulated. The deletion of $\mathrm{CtaB}$ also down regulated genes from pathways involved in purine 

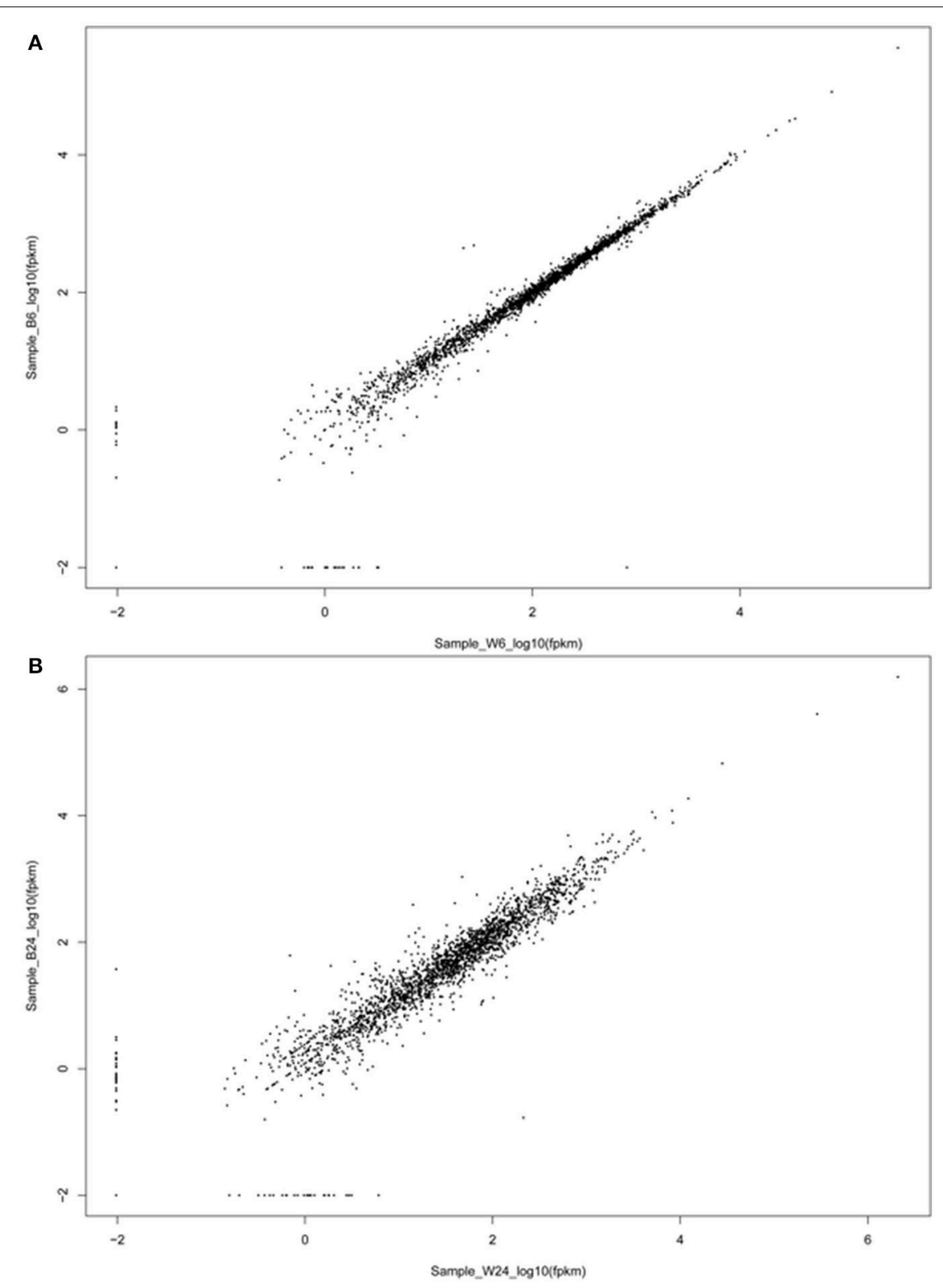

FIGURE 5 | Scatter plot of the expression levels of pairs of adjacent genes. The expression levels of two genes located within the same transcript of log phase bacteria (A) and stationary phase bacteria (B) are plotted in $\log _{10}$ scale.

metabolism, pyrimidine metabolism, and fatty acid biosynthesis. Though out of 17 genes associated with $S$. aureus infection 12 were up regulated, genes in the $d l t$ operon $(\operatorname{dlt} A, \operatorname{dlt} B, \operatorname{dlt} C$, and $d l t D$ ) were significantly down regulated (Collins et al., 2002). The CtaB deletion also induced expression of genes of five two-component systems, including PhoPR, LgrAB, SaeRS, and LytSR, indicating that these systems might play a role when $S$. aureus is confronted with lack of heme biosynthesis (Table 3).

Quantitative Real-time PCR was performed to validate the RNA-seq results. Genes were chosen from the list of genes with significant changes of transcription, favoring those associated with virulence and protein production but had a $p$-value $<0.05$. All showed similar fold change with those from the RNA-seq results (Figure 7).

\section{DISCUSSION}

The aim of this study is to address the role of $\mathrm{CtaB}$ in pigment production, virulence and persister formation in $S$. aureus. We found that deletion of $c t a B$ attenuated survival under starvation and virulence in mice but had enhanced pigment production and formation of quinolone tolerant persister cells. Our study is the first to report the complex relationship 
TABLE 2 | List of genes differentially expressed in USA500 and $\Delta \mathrm{ctaB}$ grown to $6 \mathrm{~h}$.

\begin{tabular}{|c|c|c|c|c|}
\hline Gene & Gene symbol & $\log _{2}$ fold change & $p$-value & Description \\
\hline USA300HOU_RS14135 & & -2.04 & 4.4E-05 & Hypothetical protein \\
\hline USA300HOU_RS09600 & & -1.96 & 1.1E-03 & Hypothetical protein \\
\hline USA300HOU_RS10850 & & -1.83 & $1.2 \mathrm{E}-02$ & Hypothetical bacteriophage protein \\
\hline USA300HOU_RS 10955 & hld & -1.53 & 8.5E-05 & Delta-hemolysin \\
\hline USA300HOU_RS 03725 & & -1.42 & 9.0E-04 & Hypothetical membrane protein \\
\hline USA300HOU_RS 05170 & & -1.24 & 1.9E-03 & Hypothetical protein \\
\hline USA300HOU_RS 04240 & & -1.07 & 3.7E-02 & Hypothetical protein \\
\hline USA300HOU_RS 10770 & & 1.02 & $1.8 \mathrm{E}-02$ & Hypothetical protein \\
\hline USA300HOU_RS 09400 & ribD & 1.06 & 9.6E-07 & Diaminohydroxyphosphoribosylaminopyrimidine deaminase \\
\hline USA300HOU_RS 12385 & ureB & 1.09 & 1.3E-02 & Urease beta subunit \\
\hline USA300HOU_RS 09390 & ribA & 1.13 & 2.0E-07 & Bifunctional 3,4-dihydroxy-2-butanone-4-phosphate synthase/GTP cyclohydrolase II \\
\hline USA300HOU_RS 10800 & & 1.17 & 2.8E-03 & Hypothetical bacteriophage protein \\
\hline USA300HOU_RS 09395 & ribB & 1.32 & 3.5E-08 & Riboflavin synthase alpha subunit \\
\hline USA300HOU_RS 13155 & & 1.32 & 1.6E-02 & ABC superfamily ATP binding cassette transporter, ABC/membrane protein \\
\hline USA300HOU_RS 06710 & & 1.32 & 2.3E-02 & ABC superfamily ATP binding cassette transporter, membrane protein \\
\hline USA300HOU_RS 03970 & & 1.39 & 4.5E-07 & Iron (Fe+3) ABC superfamily ATP binding cassette transporter, membrane protein \\
\hline USA300HOU_RS 12770 & htrB & 4.14 & $1.2 \mathrm{E}-51$ & Hemin ABC superfamily ATP binding cassette transporter, ABC protein \\
\hline USA300HOU_RS 12775 & htrA & 4.34 & 1.6E-55 & Hemin ABC superfamily ATP binding cassette transporter, membrane protein \\
\hline
\end{tabular}

between heme production, persister formation, and virulence in S. aureus.

We have shown that $\mathrm{CtaB}$ depletion barely affected growth in rich medium (TSB), but caused faster death under starvation stress (Figure 3A). The result echoes the finding by Clements et al., as CtaA mutation caused growth defect in glucoselimiting chemically defined medium (Clements et al., 1999). Heme production is a key step for cellular aerobic respiration and energy conversion, providing resources for synthesis of heme A-containing terminal oxidases (Svensson and Hederstedt, 1994; Hederstedt et al., 2005). The changes in the respiratory chain by mutation of $\mathrm{CtaB}$ could account for the defects. Meanwhile, it is more difficult to explain the enhanced pigment production caused by $\mathrm{CtaB}$ depletion. The production of staphyloxanthin, the main pigment of Staphylococci, is mediated by factors encoded by crtOPQMN, using FPP as the substrate (Wieland et al., 1994; Pelz et al., 2005). Regulators such as $r s b U V W$-sigB are known to regulate expression of pigment genes in S. aureus (Kullik et al., 1998; Giachino et al., 2001). In previous reports, suppression of genes from metabolic pathways (purine biosynthesis, the TCA cycle and oxidative phosphorylation) has also been found to affect pigment production (Lan et al., 2010). We detected the expression of pigment associated genes and found that $\mathrm{CtaB}$ deletion did not affect expression of $r s b U V W$-sigB, fliA (sigB) or crtOPQMN, while expression of cit $Z$ in $\triangle c t a B$ mutant was down regulated (0.45) while qoxB was induced (2.09), and the other metabolic genes were not affected (Table 4). FPP is a key intermediate in mevalonate pathway that serves as a substrate of several pathways including synthesis of heme A and staphyloxanthin (Szkopinska and Plochocka, 2005). Since $\mathrm{CtaB}$ deletion did not affect pigment production by altering expression of the currently known genes of pigment production pathway, the possibility is worth considering that the absence of competition by heme A production pathway leaves more FPP to staphyloxanthin synthesis pathway, thus enhancing pigment production provided.

From the RNA-seq data, we show the down regulation of multiple virulence genes was caused by $\mathrm{CtaB}$ depletion. Despite the depression of global regulatory RNA RNAIII and several classic virulence factors (EsaB, EsaC, EsXB, etc.), DltA-D and most of the amino acid $\mathrm{ABC}$ transporters were down regulated. The four proteins (DltA-D) incorporate D-alanine into cell wall polymers during teichoic acid synthesis (Reichmann et al., 2013) and their inactivation has been shown to impact the defense of $S$. aureus against antimicrobial agents (Peschel et al., 1999). Expression of many amino acid transporter genes (oppA1, $o p p C 1, o p p D 1$, and $o p p F 1$ ) were found down regulated in the $\mathrm{CtaB}$ knockout strain. These ABC transporters not only function by obtaining nutrients, but play important roles in adherence and processing of secreting toxins (Podbielski et al., 1996). They also showed up frequently in screening of virulence genes of $S$. aureus with transposon libraries in different animal models (Mei et al., 1997; Coulter et al., 1998; Bae et al., 2004).

Pigment production has been found to enhance fitness and virulence and help the bacteria cope with oxidative stress (Clauditz et al., 2006). However, our results seem to contradict this finding of association of pigment production and virulence as we see enhanced pigment production of $\mathrm{CtaB}$ mutant but less virulence. Nevertheless, $\mathrm{CtaB}$ deletion had multiple effects on $S$. aureus, despite enhanced pigment production, it caused attenuated hemolytic activity and survival in animal model. It is likely the virulence attenuation of $\mathrm{CtaB}$ mutant is combined effect of more important attenuated hemolytic activity over the increased pigment production such that the net outcome is 
TABLE 3 | List of genes differentially expressed in USA500 and $\Delta$ ctaB grown to $24 \mathrm{~h}$.

\begin{tabular}{|c|c|c|c|c|}
\hline Gene & Gene symbol & $\log _{2}$ fold change & $p$-value & Description \\
\hline USA300HOU_RS01190 & & -3.37 & 3.36E-06 & Acetyl-CoA C-acetyltransferase \\
\hline USA30OHOU_RS01195 & & -3.30 & 7.44E-06 & 3-hydroxyacyl-CoA dehydrogenase \\
\hline USA300HOU_RS01205 & & -3.16 & $1.08 \mathrm{E}-05$ & Long-chain-fatty-acid-CoA ligase \\
\hline USA30OHOU_RS02265 & cobW1 & -2.78 & $6.77 \mathrm{E}-04$ & Cobalamin (vitamin B12) biosynthesis protein \\
\hline USA300HOU_RS02055 & $x p r T$ & -2.66 & 1.47E-03 & Xanthine phosphoribosyltransferase \\
\hline USA300HOU_RS12265 & & -2.57 & 2.28E-03 & Hypothetical protein \\
\hline USA30OHOU_RS14500 & lip & -2.16 & $2.42 \mathrm{E}-03$ & Triacylglycerol lipase \\
\hline USA300HOU_RS02060 & pbuX & -2.16 & $3.25 \mathrm{E}-03$ & NCS2 family nucleobase:cation symporter-2 \\
\hline USA30OHOU_RS05895 & & -2.14 & 1.26E-02 & Antibacterial protein \\
\hline USA30OHOU_RS11075 & ilvC & -2.03 & 1.12E-02 & Ketol-acid reductoisomerase \\
\hline USA30OHOU_RS00655 & & -2.03 & 3.65E-02 & Hypothetical membrane protein \\
\hline USA300HOU_RS01875 & & -2.01 & $4.74 \mathrm{E}-02$ & Hypothetical protein \\
\hline USA30OHOU_RS04875 & $\mathrm{fabH1}$ & -1.95 & 4.11E-03 & 3-oxoacyl-[acyl-carrier-protein] synthase \\
\hline USA30OHOU_RS02840 & rplL1 & -1.94 & 5.40E-03 & Ribosomal protein L7/L12 \\
\hline USA300HOU_RS12130 & $r p s Q$ & -1.93 & $5.02 \mathrm{E}-03$ & Ribosomal protein S17 \\
\hline USA30OHOU_RS04900 & oppD1 & -1.91 & 4.92E-03 & Oligopeptide ABC superfamily ATP binding cassette transporter, ABC protein \\
\hline USA30OHOU_RS11060 & $i l v D$ & -1.89 & 1.79E-02 & Dihydroxy-acid dehydratase \\
\hline USA30OHOU_RS07110 & $\operatorname{dap} B$ & -1.84 & 1.91E-02 & Dihydrodipicolinate reductase \\
\hline USA300HOU_RS13735 & & -1.82 & 1.46E-02 & Transcriptional regulator \\
\hline USA30OHOU_RS08760 & $r p / U$ & -1.79 & 1.10E-02 & Ribosomal protein L21 \\
\hline USA30OHOU_RS02835 & rp/J & -1.65 & 1.75E-02 & Ribosomal protein L10 \\
\hline USA30OHOU_RS02375 & gltB1 & -1.63 & 2.23E-02 & Glutamate synthase (NADPH), large subunit \\
\hline USA30OHOU_RS06050 & pyrF & -1.62 & 3.08E-02 & Orotidine-5'-phosphate decarboxylase \\
\hline USA30OHOU_RS04905 & oppF1 & -1.58 & $1.92 \mathrm{E}-02$ & Oligopeptide ABC superfamily ATP binding cassette transporter, ABC protein \\
\hline USA30OHOU_RS01935 & rpsF & -1.56 & 2.29E-02 & Ribosomal protein S6 \\
\hline USA30OHOU_RS04620 & $d l t A$ & -1.55 & $2.45 \mathrm{E}-02$ & Long-chain-fatty-acid-CoA ligase \\
\hline USA300HOU_RS07105 & dapA & -1.52 & 4.99E-02 & dihydrodipicolinate synthase \\
\hline USA30OHOU_RS04635 & $d l t D$ & -1.51 & 3.00E-02 & D-alanine transfer protein DltD \\
\hline USA30OHOU_RS07190 & & -1.51 & 4.86E-02 & Nitric-oxide reductase \\
\hline USA30OHOU_RS12610 & hutl & -1.50 & 2.99E-02 & Imidazolonepropionase \\
\hline USA30OHOU_RS02380 & gltD & -1.50 & 4.97E-02 & Glutamate synthase (NADPH) small subunit \\
\hline USA30OHOU_RS05945 & murD & -1.50 & $2.66 \mathrm{E}-02$ & UDP-N-acetylmuramoylalanine-D-glutamate ligase \\
\hline USA30OHOU_RS08555 & & -1.49 & 2.77E-02 & Biotin carboxylase \\
\hline USA300HOU_RS12615 & hutU & -1.49 & 3.30E-02 & Urocanate hydratase \\
\hline USA30OHOU_RS13410 & & -1.47 & 3.09E-02 & Possible decarboxylase \\
\hline USA30OHOU_RS12085 & rpmD & -1.46 & 2.95E-02 & Ribosomal protein L30 \\
\hline USA30OHOU_RS11770 & & -1.46 & 3.46E-02 & Hypothetical membrane protein \\
\hline USA30OHOU_RS06545 & glpK & -1.45 & 3.79E-02 & Glycerol kinase \\
\hline
\end{tabular}


TABLE 3 | Continued

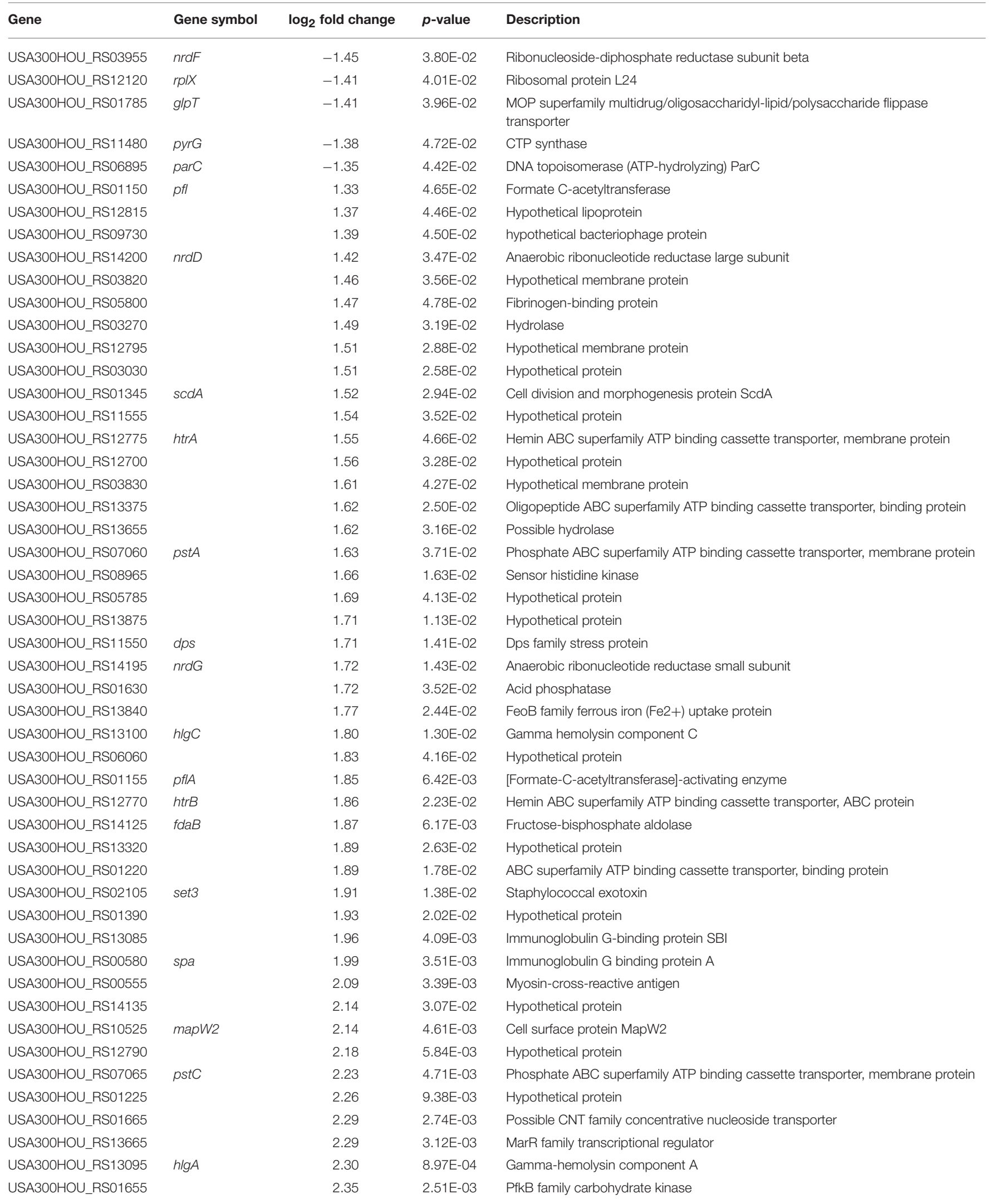


TABLE 3 | Continued

\begin{tabular}{|c|c|c|c|c|}
\hline Gene & Gene symbol & $\log _{2}$ fold change & $p$-value & Description \\
\hline USA300HOU_RS03725 & & 2.41 & 1.29E-02 & Hypothetical membrane protein \\
\hline USA300HOU_RS04290 & nuc & 2.48 & 3.60E-03 & Micrococcal nuclease \\
\hline USA300HOU_RS13635 & & 2.50 & 2.35E-03 & ABC superfamily ATP binding cassette transporter, membrane protein \\
\hline USA300HOU_RS13830 & $c / p$ & 2.51 & 2.93E-04 & S14 family endopeptidase Clp \\
\hline USA300HOU_RS13660 & & 2.53 & 1.74E-03 & Possible lactoylglutatione lyase \\
\hline USA300HOU_RS01660 & & 2.62 & 9.10E-04 & Hypothetical protein \\
\hline USA300HOU_RS05775 & & 2.62 & 2.08E-04 & Hypothetical protein \\
\hline USA30OHOU_RS05855 & & 2.80 & 1.39E-02 & Exotoxin \\
\hline USA300HOU_RS10450 & & 2.80 & 8.14E-04 & Hypothetical protein \\
\hline USA300HOU_RS07070 & pstS & 2.85 & $5.80 \mathrm{E}-05$ & Phosphate ABC superfamily ATP binding cassette transporter, binding protein \\
\hline USA300HOU_RS05795 & & 2.90 & 8.96E-05 & Fibrinogen-binding protein \\
\hline USA300HOU_RS01235 & hmp & 2.96 & 5.40E-05 & Possible nitric oxide dioxygenase \\
\hline USA300HOU_RS10965 & $\operatorname{agr} D$ & 3.45 & $6.02 \mathrm{E}-04$ & Accessory gene regulator protein $D$ \\
\hline USA300HOU_RS04615 & & 4.03 & 5.89E-03 & Hypothetical protein \\
\hline USA300HOU_RS13630 & & 4.07 & $6.62 \mathrm{E}-06$ & ABC superfamily ATP binding cassette transporter, ABC protein \\
\hline USA30OHOU_RS01365 & $\operatorname{lrg} B$ & 4.08 & 9.13E-08 & Murein hydrolase regulator LrgB \\
\hline USA300HOU_RS01360 & $\operatorname{lrg} A$ & 4.36 & $1.25 \mathrm{E}-08$ & Murein hydrolase regulator LrgA \\
\hline USA300HOU_RS01230 & & 6.07 & 1.16E-06 & Hypothetical protein \\
\hline
\end{tabular}

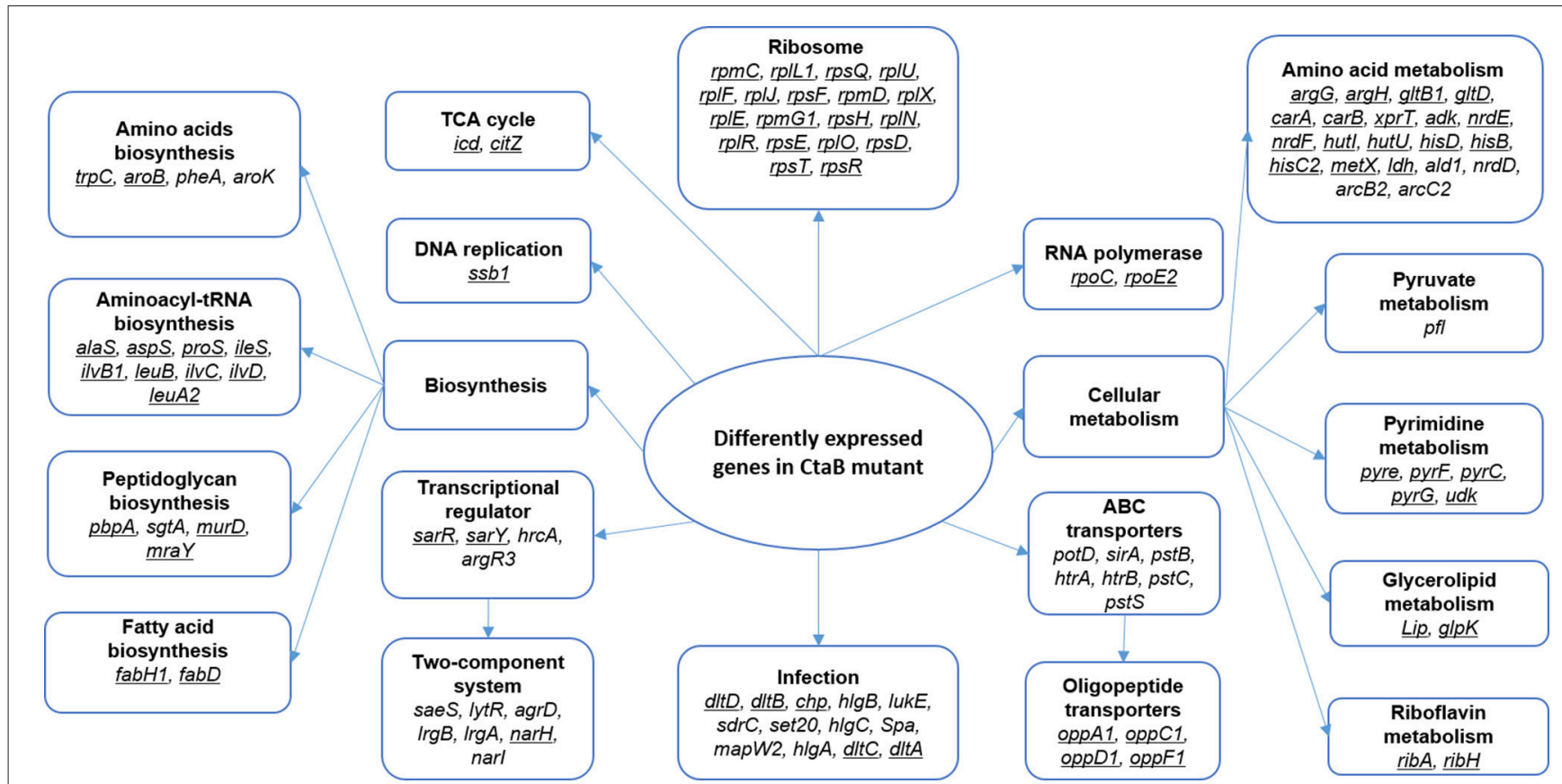

FIGURE 6 | Proposed pathways affected by depletion of CtaB in stationary phase S. aureus. Pathways enriched in genes with higher than 2-fold change in CtaB mutant at $24 \mathrm{~h}$ are framed and bold. Genes that were down regulated in the CtaB mutant are underlined, while those up regulated not underlined.

still attenuated virulence despite increased pigment production which is often associated with virulence.

Persister formation is a phenomenon with highly complex mechanisms. Energy production and protein translation are two vital pathways for bacterial survival and reproduction, and it is generally believed that an overall suppression of metabolism and replication is a universal cause for bacterial persister formation (Lewis, 2012; Kwan et al., 2013). In E. coli, deficiency of energy production genes such as $s u c B$ and $u b i F$ has been found to decrease persister survival (Ma et al., 2010). It has also been shown in E. coli that bacteriostatic antibiotic treatment enhances persister formation via suppression of 


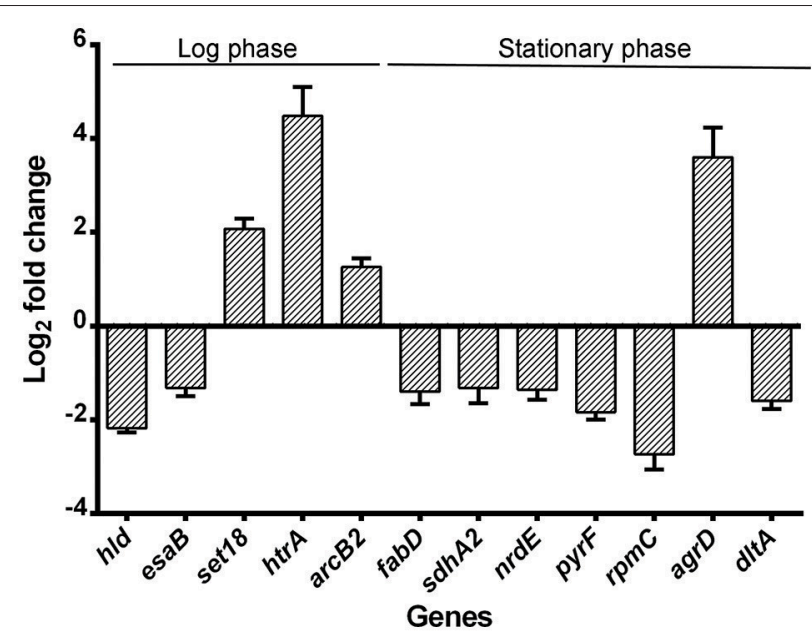

FIGURE 7 | Validation of RNA-seq by quantitative real-time PCR. Relative mRNA levels of transcripts corresponding to USA500 and $\triangle \mathrm{ctaB}$ mutant grown to 6 or $24 \mathrm{~h}$ were determined. RNA was obtained from the same samples for RNA-seq and experiments were performed in triplicate. Bars show the fold change of $\triangle$ ctaB mutant vs. USA500 and error bars indicate standard deviations calculated with the $2^{-\Delta \Delta C T}$ method based on three independent experiments.

TABLE 4 | Transcription change of pigment production associated genes.

\begin{tabular}{lcc}
\hline Genes & Fold change (ActaB vs. USA500) & SD \\
\hline rsbU & 0.90 & 0.122 \\
crtM & 1.18 & 0.143 \\
qoxB & 2.09 & 0.276 \\
citZ & 0.45 & 0.089 \\
fliA (sigB) & 0.91 & 0.129 \\
purA & 0.56 & 0.113 \\
USA300HOU_0726 (NWMN_0672) & 1.38 & 0.127 \\
USA30OHOU_(NWMN_1144) & 3.54 & 0.323 \\
\hline
\end{tabular}

cellular respiration (Lobritz et al., 2015). In S. aureus, a recent study correlated the drop of ATP level to enhanced persister formation in stationary phase (Pontes et al., 2015). $\mathrm{CtaB}$ is a key factor in $S$. aureus respiratory chain and energy production. In stationary phase when most glucose is consumed, $S$. aureus turns to utilize amino acids such as arginine and histidine for energy production (Makhlin et al., 2007). We also found that in stationary phase, multiple genes involved in amino acid metabolism ( $\arg G$, hutI, hisD, etc.) were inhibited in $\triangle c t a B$ strain (Table 3). Based on these findings, $\mathrm{CtaB}$ depletion might account for augumented persister formation. However, the correlation between respiration and persister formation is far from unveiled. A counter-example has been provided by Mehmet et al. who reported that inhibition of respiration during stationary phase with $\mathrm{KCN}$ reduced persister levels in E. coli (Orman and Brynildsen, 2015).

While more work needs to be done to investigate the role of respiratory chain in persister formation, it is also important to further investigate how repression of protein production affects persister formation. The well-understood mechanism of HipAB Toxin-antitoxin system affecting persister formation in E. coli, relies on (p)ppGpp to trigger a regulatory cascade involving inorganic polyphosphate (polyP) and Lon, which eventually results in accumulation of (p)ppGpp and persister formation (Rodionov and Ishiguro, 1995; Korch et al., 2003; Germain et al., 2013; Maisonneuve et al., 2013). In our study, CtaB depletion caused strong inhibition of translation by repressing genes involved in multiple aspects of protein production, including amino acid transport ( $o p p A 1, o p p C 1, o p p D 1$, etc.), amino acid synthesis (trpC, aroB,), aminoacyl-tRNA biosynthesis (aspS, alaS, ileS, etc.) and ribosome proteins $(r p m C, r p l F, r p s E$, etc.) (Table 3; Figure 6).

It is generally assumed that elevated persistence is associated with better survival and therefore higher virulence in animal models. However, our observation that mutation of CtaB caused attenuated virulence but elevated persister formation, seems paradoxical. Indeed, many infectious diseases are difficult to be treated with antibiotics due to persisters but not resistance (Mulcahy et al., 2010; Welsh et al., 2011). However, we propose that in most cases with MRSA infection the role of virulence is greater than persister formation because: the proportion of persisters is generally small; after antibiotics kill the majority of infecting population of bacteria, the host immune system generally eliminates persisters non-selectively. Nevertheless, the importance of persister formation by MRSA should not be neglected. Future studies are needed to better understand the roles virulence and persistence play in $S$. aureus infection.

In our study, the $\mathrm{CtaB}$ mutant only showed elevated persister formation with levofloxacin and ciprofloxacin but no other antibiotics. This may be attributed to the multi-drug resistance of the MRSA strain, which may have masked the defect in persistence to other antibiotics. The difference in persister formation between MRSA and methicillin-sensitive $S$. aureus (MSSA) is worth further investigation.

Our study suggests the importance of heme synthesis in virulence and persister formation of $S$. aureus and provide new insights into the role of $\mathrm{CtaB}$ in bacterial respiration in S. aureus virulence and persistence. However, one limitation of the study is that we have not dealt specifically with the metabolic aspects of $\mathrm{CtaB}$ mutation, such as the efficiency of the respiratory chain in the mutant and possible changes in components of the TCA cycle as well as comparing the phenotypes of $S$. aureus and the $c t a B$ mutant in anaerobic conditions. Future studies are needed to address these issues and better understand the relationship between $S$. aureus respiration and virulence and persistence.

\section{AUTHOR CONTRIBUTIONS}

$\mathrm{YZ}$, TX, and WZ designed the work and revised the manuscript; TX, JH, JZ, JC, and NW completed all the experiments; TX and $\mathrm{JH}$ performed the statistically analysis and made the figures; TX and $\mathrm{YZ}$ wrote the manuscript. 


\section{ACKNOWLEDGMENTS}

We thank Timothy J. Foster, Moyne Institute of Preventive Medicine, Department of Microbiology, Trinity College, Dublin, Ireland, for providing the E. coli strain DC10B. We also thank Ralph Bertram, Klinikum Nürnberg Medical School GmbH, Research Department, Paracelsus Medical

\section{REFERENCES}

Anderson, M., Chen, Y. H., Butler, E. K., and Missiakas, D. M. (2011). EsaD, a secretion factor for the Ess pathway in Staphylococcus aureus. J. Bacteriol. 193, 1583-1589. doi: 10.1128/JB.01096-10

Andrews, J. M. (2001). Determination of minimum inhibitory concentrations. J. Antimicrob. Chemother. 48(Suppl. 1), 5-16. doi: 10.1093/jac/48.suppl_1.5

Atshan, S. S., Shamsudin, M. N., Lung, L. T., Ling, K. H., Sekawi, Z., Pei, C. P., et al. (2012). Improved method for the isolation of RNA from bacteria refractory to disruption, including S. aureus producing biofilm. Gene 494, 219-224. doi: 10.1016/j.gene.2011.12.010

Bae, T., Banger, A. K., Wallace, A., Glass, E. M., Aslund, F., Schneewind, O., et al. (2004). Staphylococcus aureus virulence genes identified by bursa aurealis mutagenesis and nematode killing. Proc. Natl. Acad. Sci. U.S.A. 101, 12312-12317. doi: 10.1073/pnas.0404728101

Bae, T., and Schneewind, O. (2006). Allelic replacement in Staphylococcus aureus with inducible counter-selection. Plasmid 55, 58-63. doi: 10.1016/j.plasmid.2005.05.005

Bigger, J. (1944). Treatment of staphylococcal infections with penicillin by intermittent sterilisation. Lancet 244, 497-500. doi: 10.1016/S01406736(00)74210-3

Burts, M. L., DeDent, A. C., and Missiakas, D. M. (2008). EsaC substrate for the ESAT-6 secretion pathway and its role in persistent infections of Staphylococcus aureus. Mol. Microbiol. 69, 736-746. doi: 10.1111/j.1365-2958.2008. 06324.x

Carrel, M., Perencevich, E. N., and David, M. Z. (2015). USA300 methicillinresistant Staphylococcus aureus, United States, 2000-2013. Emer. Infect. Dis. J. 21, 1973-1980. doi: 10.3201/eid2111.150452

Clauditz, A., Resch, A., Wieland, K. P., Peschel, A., and Götz, F. (2006). Staphyloxanthin plays a role in the fitness of Staphylococcus aureus and its ability to cope with oxidative stress. Infect. Immun. 74, 4950-4953. doi: 10.1128/IAI.00204-06

Clements, M. O., Watson, S. P., Poole, R. K., and Foster, S. J. (1999). CtaA of Staphylococcus aureus is required for starvation survival, recovery, and cytochrome biosynthesis. J. Bacteriol. 181, 501-507.

Collins, L. V., Kristian, S. A., Weidenmaier, C., Faigle, M., Van Kessel, K. P., Van Strijp, J. A., et al. (2002). Staphylococcus aureus strains lacking D-alanine modifications of teichoic acids are highly susceptible to human neutrophil killing and are virulence attenuated in mice. J. Infect. Dis. 186, 214-219. doi: $10.1086 / 341454$

Coulter, S. N., Schwan, W. R., Ng, E. Y., Langhorne, M. H., Ritchie, H. D., Westbrock-Wadman, S., et al. (1998). Staphylococcus aureus genetic loci impacting growth and survival in multiple infection environments. Mol. Microbiol. 30, 393-404.

Diep, B. A., Carleton, H. A., Chang, R. F., Sensabaugh, G. F., and PerdreauRemington, F. (2006). Roles of 34 virulence genes in the evolution of hospitaland community-associated strains of methicillin-resistant Staphylococcus aureus. J. Infect. Dis. 193, 1495-1503. doi: 10.1086/503777

Drabkin, D. L. (1951). Metabolism of the hemin chromoproteins. Physiol. Rev. 31, 345-431.

Germain, E., Castro-Roa, D., Zenkin, N., and Gerdes, K. (2013). Molecular mechanism of bacterial persistence by HipA. Mol. Cell 52, 248-254. doi: 10.1016/j.molcel.2013.08.045

Giachino, P., Engelmann, S., and Bischoff, M. (2001). Sigma(B) activity depends on RsbU in Staphylococcus aureus. J. Bacteriol. 183, 1843-1852. doi: 10.1128/JB.183.6.1843-1852.2001
University, Nuremberg, Germany for helpful discussions about S. aureus gene manipulation and persister assay. This work was supported by the National Natural Science Foundation of China (81572046 and 81471987), and the Key Technologies Research and Development Program for Infectious Diseases of China (2013ZX10003008-003). YZ was supported in part by NIH grants AI99512 and AI108535.
Hammer, N. D., Schurig-Briccio, L. A., Gerdes, S. Y., Gennis, R. B., and Skaar, E. P. (2016). CtaM is required for menaquinol oxidase $\mathrm{aa}_{3}$ function in Staphylococcus aureus. mBio 7:e00823-16. doi: 10.1128/mBio.00823-16

Han, J., He, L., Shi, W., Xu, X., Wang, S., Zhang, S., et al. (2014). Glycerol uptake is important for L-form formation and persistence in Staphylococcus aureus. PLoS ONE 9:e108325. doi: 10.1371/journal.pone.0108325

Hederstedt, L., Lewin, A., and Throne-Holst, M. (2005). Heme A synthase enzyme functions dissected by mutagenesis of Bacillus subtilis CtaA. J. Bacteriol. 187, 8361-8369. doi: 10.1128/JB.187.24.8361-8369.2005

Keren, I., Kaldalu, N., Spoering, A., Wang, Y., and Lewis, K. (2004). Persister cells and tolerance to antimicrobials. FEMS Microbiol. Lett. 230, 13-18. doi: 10.1016/S0378-1097(03)00856-5

Korch, S. B., Henderson, T. A., and Hill, T. M. (2003). Characterization of the hipA7 allele of Escherichia coli and evidence that high persistence is governed by (p)ppGpp synthesis. Mol. Microbiol. 50, 1199-1213. doi: 10.1046/j.13652958.2003.03779.x

Kullik, I., Giachino, P., and Fuchs, T. (1998). Deletion of the alternative sigma factor sigmaB in Staphylococcus aureus reveals its function as a global regulator of virulence genes. J. Bacteriol. 180, 4814-4820

Kwan, B. W., Valenta, J. A., Benedik, M. J., and Wood, T. K. (2013). Arrested protein synthesis increases persister-like cell formation. Antimicrob. Agents Chemother. 57, 1468-1473. doi: 10.1128/AAC.02135-12

Lan, L., Cheng, A., Dunman, P. M., Missiakas, D., and He, C. (2010). Golden pigment production and virulence gene expression are affected by metabolisms in Staphylococcus aureus. J. Bacteriol. 192, 3068-3077. doi: 10.1128/JB.00928-09

Lechner, S., Lewis, K., and Bertram, R. (2012). Staphylococcus aureus persisters tolerant to bactericidal antibiotics. J. Mol. Microbiol. Biotechnol. 22, 235-244. doi: $10.1159 / 000342449$

Lewis, K. (2001). Riddle of biofilm resistance. Antimicrob. Agents Chemother. 45, 999-1007. doi: 10.1128/AAC.45.4.999-1007.2001

Lewis, K. (2012). "Persister cells: molecular mechanisms related to antibiotic tolerance," in Antibiotic Resistance, ed A. R. M. Coate (Heidelberg: Springer), 121-133.

Li, M., Du, X., Villaruz, A. E., Diep, B. A., Wang, D., Song, Y., et al. (2012). MRSA epidemic linked to a quickly spreading colonization and virulence determinant. Nat. Med. 18, 816-819. doi: 10.1038/nm.2692

Liu, G. Y. (2009). Molecular pathogenesis of Staphylococcus aureus infection. Pediatr Res 65(5 Pt 2), 71R-77R. doi: 10.1203/PDR.0b013e31819dc44d

Liu, G. Y., Essex, A., Buchanan, J. T., Datta, V., Hoffman, H. M., Bastian, J. F., et al. (2005). Staphylococcus aureus golden pigment impairs neutrophil killing and promotes virulence through its antioxidant activity. J. Exp. Med. 202, 209-215. doi: $10.1084 /$ jem. 20050846

Liu, M., Tanaka, W. N., Zhu, H., Xie, G., Dooley, D. M., and Lei, B. (2008). Direct hemin transfer from IsdA to IsdC in the iron-regulated surface determinant (Isd) heme acquisition system of Staphylococcus aureus. J. Biol. Chem. 283, 6668-6676. doi: 10.1074/jbc.M708372200

Livak, K. J., and Schmittgen, T. D. (2001). Analysis of relative gene expression data using real-time quantitative PCR and the $2(-$ Delta Delta $\mathrm{C}(\mathrm{T}))$ method Methods 25, 402-408. doi: 10.1006/meth.2001.1262

Lobritz, M. A., Belenky, P., Porter, C. B., Gutierrez, A., Yang, J. H., Schwarz, E. G., et al. (2015). Antibiotic efficacy is linked to bacterial cellular respiration. Proc. Natl. Acad. Sci. U.S.A. 112, 8173-8180. doi: 10.1073/pnas.1509743112

Ma, C., Sim, S., Shi, W., Du, L., Xing, D., and Zhang, Y. (2010). Energy production genes sucB and ubiF are involved in persister survival and tolerance to multiple antibiotics and stresses in Escherichia coli. FEMS Microbiol. Lett. 303, 33-40. doi: 10.1111/j.1574-6968.2009.01857.x 
Maisonneuve, E., Castro-Camargo, M., and Gerdes, K. (2013). (p)ppGpp controls bacterial persistence by stochastic induction of toxin-antitoxin activity. Cell 154, 1140-1150. doi: 10.1016/j.cell.2013.07.048

Makhlin, J., Kofman, T., Borovok, I., Kohler, C., Engelmann, S., Cohen, G., et al. (2007). Staphylococcus aureus ArcR controls expression of the arginine deiminase operon. J. Bacteriol. 189, 5976-5986. doi: 10.1128/JB. 00592-07

Mason, W. J., and Skaar, E. P. (2009). Assessing the contribution of heme-iron acquisition to Staphylococcus aureus pneumonia using computed tomography. PLoS ONE 4:e6668. doi: 10.1371/journal.pone.00 06668

Mazmanian, S. K., Skaar, E. P., Gaspar, A. H., Humayun, M., Gornicki, P., Jelenska, J., et al. (2003). Passage of heme-iron across the envelope of Staphylococcus aureus. Science 299, 906-909. doi: 10.1126/science.10 81147

Mechler, L., Herbig, A., Paprotka, K., Fraunholz, M., Nieselt, K., and Bertram, R. (2015). A novel point mutation promotes growth phase-dependent daptomycin tolerance in Staphylococcus aureus. Antimicrob. Agents Chemother. 59, 5366-5376. doi: 10.1128/AAC.00643-15

Mei, J. M., Nourbakhsh, F., Ford, C. W., and Holden, D. W. (1997). Identification of Staphylococcus aureus virulence genes in a murine model of bacteraemia using signature-tagged mutagenesis. Mol. Microbiol. 26, 399-407.

Mogi, T., Saiki, K., and Anraku, Y. (1994). Biosynthesis and functional role of haem $\mathrm{O}$ and haem A. Mol. Microbiol. 14, 391-398. doi: 10.1111/j.13652958.1994.tb02174.x

Monk, I. R., Shah, I. M., Xu, M., Tan, M. W., and Foster, T. J. (2012). Transforming the untransformable: application of direct transformation to manipulate genetically Staphylococcus aureus and Staphylococcus epidermidis. MBio 3:e00277-11. doi: 10.1128/mBio.00277-11

Morikawa, K., Maruyama, A., Inose, Y., Higashide, M., Hayashi, H., and Ohta, T. (2001). Overexpression of Sigma Factor, $\zeta$ B, urges Staphylococcus aureus to thicken the cell wall and to resist $\beta$-Lactams. Biochem. Biophys. Res. Commun. 288, 385-389. doi: 10.1006/bbrc.2001.5774

Mulcahy, L. R., Burns, J. L., Lory, S., and Lewis, K. (2010). Emergence of Pseudomonas aeruginosa strains producing high levels of persister cells in patients with cystic fibrosis. J. Bacteriol. 192, 6191-6199. doi: 10.1128/JB. 01651-09

Orman, M. A., and Brynildsen, M. P. (2015). Inhibition of stationary phase respiration impairs persister formation in E. coli. Nat. Commun. 6:7983. doi: 10.1038/ncomms8983

Pelz, A., Wieland, K. P., Putzbach, K., Hentschel, P., Albert, K., and Götz, F. (2005). Structure and biosynthesis of staphyloxanthin from Staphylococcus aureus. J. Biol. Chem. 280, 32493-32498. doi: 10.1074/jbc.M5050 70200

Peschel, A., Otto, M., Jack, R. W., Kalbacher, H., Jung, G., and Götz, F. (1999). Inactivation of the dlt operon in Staphylococcus aureus confers sensitivity to defensins, protegrins, and other antimicrobial peptides. J. Biol. Chem. 274, 8405-8410.

Podbielski, A., Pohl, B., Woischnik, M., Körner, C., Schmidt, K. H., Rozdzinski, E., et al. (1996). Molecular characterization of group A streptococcal (GAS) oligopeptide permease (opp) and its effect on cysteine protease production. Mol. Microbiol. 21, 1087-1099.
Pontes, M. H., Sevostyanova, A., and Groisman, E. A. (2015). When too much ATP is bad for protein synthesis. J. Mol. Biol. 427, 2586-2594. doi: 10.1016/j.jmb.2015.06.021

Reichmann, N. T., Cassona, C. P., and Gründling, A. (2013). Revised mechanism of D-alanine incorporation into cell wall polymers in Gram-positive bacteria. Microbiology 159(Pt 9), 1868-1877. doi: 10.1099/mic.0.069898-0

Resch, A., Leicht, S., Saric, M., Pásztor, L., Jakob, A., Götz, F., et al. (2006). Comparative proteome analysis of Staphylococcus aureus biofilm and planktonic cells and correlation with transcriptome profiling. Proteomics 6, 1867-1877. doi: 10.1002/pmic.200500531

Rodionov, D. G., and Ishiguro, E. E. (1995). Direct correlation between overproduction of guanosine $3^{\prime}, 5^{\prime}$-bispyrophosphate (ppGpp) and penicillin tolerance in Escherichia coli. J. Bacteriol. 177, 4224-4229.

Svensson, B., and Hederstedt, L. (1994). Bacillus subtilis CtaA is a hemecontaining membrane protein involved in heme A biosynthesis. J. Bacteriol. $176,6663-6671$.

Svensson, B., Lübben, M., and Hederstedt, L. (1993). Bacillus subtilis CtaA and CtaB function in haem A biosynthesis. Mol. Microbiol. 10, 193-201. doi: 10.1111/j.1365-2958.1993.tb00915.x

Szkopinska, A., and Plochocka, D. (2005). Farnesyl diphosphate synthase; regulation of product specificity. Acta Biochim. Pol. 52, 45-55. doi: 055201045

Wang, B., and Muir, T. W. (2016). Regulation of virulence in Staphylococcus aureus: molecular mechanisms and remaining puzzles. Cell Chem. Biol. 23, 214-224. doi: 10.1016/j.chembiol.2016.01.004

Wang, W., Chen, J., Chen, G., Du, X., Cui, P., Wu, J., et al. (2015). Transposon mutagenesis identifies novel genes associated with Staphylococcus aureus persister formation. Front. Microbiol. 6:1437. doi: 10.3389/fmicb.2015.01437

Welsh, K. J., Skrobarcek, K. A., Abbott, A. N., Lewis, C. T., Kruzel, M. C., Lewis, E. M., et al. (2011). Predictors of relapse of methicillin-resistant Staphylococcus aureus bacteremia after treatment with vancomycin. J. Clin. Microbiol. 49, 3669-3672. doi: 10.1128/JCM.05287-11

Wieland, B., Feil, C., Gloria-Maercker, E., Thumm, G., Lechner, M., Bravo, J. M., et al. (1994). Genetic and biochemical analyses of the biosynthesis of the yellow carotenoid 4,4'-diaponeurosporene of Staphylococcus aureus. J. Bacteriol. 176, 7719-7726

Yee, R., Cui, P., Shi, W., Feng, J., and Zhang, Y. (2015). Genetic screen reveals the role of purine metabolism in Staphylococcus aureus persistence to rifampicin. Antibiotics 4, 627-642. doi: 10.3390/antibiotics4040627

Zhang, Y. (2014). Persisters, persistent infections and the Yin-Yang model. Emerg. Microbes Infect. 3:e3. doi: 10.1038/emi.2014.3

Conflict of Interest Statement: The authors declare that the research was conducted in the absence of any commercial or financial relationships that could be construed as a potential conflict of interest.

Copyright (c) $2016 \mathrm{Xu}$, Han, Zhang, Chen, Wu, Zhang and Zhang. This is an openaccess article distributed under the terms of the Creative Commons Attribution License (CC BY). The use, distribution or reproduction in other forums is permitted, provided the original author(s) or licensor are credited and that the original publication in this journal is cited, in accordance with accepted academic practice. No use, distribution or reproduction is permitted which does not comply with these terms. 


\title{
Polyphosphate Kinase Mediates Antibiotic Tolerance in Extraintestinal Pathogenic Escherichia coli PCN033
}

\author{
Jing Chen ${ }^{1}$, Lijie Su ${ }^{2}$, Xiangru Wang ${ }^{1}$, Tao Zhang ${ }^{1}$, Feng Liu ${ }^{1}$, Huanchun Chen ${ }^{1,3}$ and \\ Chen $\operatorname{Tan}^{1,3 *}$
}

${ }^{1}$ State Key Laboratory of Agricultural Microbiology, College of Veterinary Medicine, Huazhong Agricultural University, Wuhan, China, ${ }^{2}$ School of Public Health, Guangzhou Medical University, Guangzhou, China, ${ }^{3}$ Key Laboratory for the Development of Veterinary Diagnostic Products, The Cooperative Innovation Center for Sustainable Pig Production, Ministry of Agriculture, Huazhong Agricultural University, Wuhan, China

\section{OPEN ACCESS}

Edited by:

Etienne Giraud,

Institut National de la Recherche

Agronomique, France

Reviewed by:

César De La Fuente-Núñez, Massachusetts Institute of

Technology, USA

Herman Sintim,

Purdue University, USA

*Correspondence:

Chen Tan

tanchen@mail.hzau.edu.cn

Specialty section: This article was submitted to Antimicrobials, Resistance and Chemotherapy

a section of the journal

Frontiers in Microbiology

Received: 01 December 2015

Accepted: 02 May 2016

Published: 19 May 2016

Citation:

Chen J, Su L, Wang X, Zhang T, Liu F

Chen $H$ and Tan C (2016)

Polyphosphate Kinase Mediates Antibiotic Tolerance in Extraintestinal Pathogenic Escherichia coli PCNO33.

Front. Microbiol. 7:724.

doi: 10.3389/fmich.2016.00724
Extraintestinal pathogenic Escherichia coli (ExPEC) causes a variety of acute infections in its hosts, and multidrug-resistant strains present significant challenges to public health and animal husbandry. Therefore, it is necessary to explore new drug targets to control E. coli epidemics. Previous studies have reported that ppk mutants of Burkholderia pseudomallei and Mycobacterium tuberculosis are more susceptible than the wild types (WTs) to stress. Therefore, we investigated the stress response to antibiotics mediated by polyphosphate kinase (PPK) in ExPEC strain PCN033. We observed that planktonic cells of a ppk knockout strain ( $\Delta p p k$ ) were more susceptible to antibiotics than was WT. However, biofilm-grown $\Delta p p k$ cells showed similar susceptibility to that of the WT and were more tolerant than the planktonic cells. During the planktonic lifestyle, the expression of genes involved in antibiotic tolerance (including resistance-conferring genes, and antibiotic influx, and efflux genes) did not change in the $\Delta p p k$ mutant without antibiotic treatment. However, the resistance-conferring gene bla and efflux genes were upregulated more in the WT than in the $\Delta p p k$ mutant by treatment with tazobactam. After treatment with gentamycin, the efflux genes and influx genes were upregulated and downregulated, respectively, more in the WT than in the $\Delta p p k$ mutant. The expression of genes involved in biofilm regulation also changed after treatment with tazobactam or gentamycin, and which is consistent with the results of the biofilm formation. Together, these observations indicate that PPK is important for the antibiotic stress response during the planktonic growth of EXPEC and might be a potential drug target in bacteria.

Keywords: ExPEC, antibiotic resistance, PPK, gene expression, RNA-seq, qPCR

\section{INTRODUCTION}

Extraintestinal pathogenic Escherichia coli (ExPEC) is a major cause of urinary tract infections in women, abdominal sepsis, and septicemia in elderly or immunocompromised individuals, and meningitis in newborns, with high morbidity and mortality (Gaschignard et al., 2011; Weston et al., 2011; Mellata, 2013). ExPEC strains commonly colonize domestic animals, such as pigs, chickens, and cattle, causing significant losses in animal husbandry and threating human health (Girardeau et al., 2003; Johnson et al., 2005; Bergeron et al., 2012). We previously investigated the prevalence 
of ExPEC in swine across China and detected ExPEC in 10.1\% of porcine samples. The frequency of ExPEC isolated from pigs increased between 2004 and 2007 from 3.1 to 14.6\% (Tan et al., 2012). The emergence of multidrug-resistant strains has significantly hindered the prevention and control of ExPEC epidemics (Sedláková et al., 2014; Sidrach-Cardona et al., 2014). Therefore, it is urgent that we identify new drug targets to control these increasing E. coli outbreaks.

Polyphosphate kinase (PPK) is an essential enzyme in polyphosphate (polyP) synthesis and has been implicated in many intracellular biological processes. Pseudomonas aeruginosa in which ppk was deleted showed impairments in motility, quorum sensing, and virulence (Rashid et al., 2000), compacted nucleoids, membrane distortion, extracellular polymer production, and a susceptibility to desiccation (Fraley et al., 2007). PPK also plays a prominent role in the stress response, and a Burkholderia pseudomallei ppk mutant was susceptible to hydrogen peroxide under oxidative stress conditions (Tunpiboonsak et al., 2010). A ppk1 mutant strain of Mycobacterium tuberculosis displayed a survival defect in response to nitrosative stress, and the negligible levels of polyP were associated with its increased susceptibility to certain tuberculosis drugs (Singh et al., 2013). PPK is highly conserved in bacteria, but is absent in higher mammals (Brown and Kornberg, 2004), indicating that PPK has potential utility as an antibacterial drug target.

As an opportunistic pathogen, E. coli mainly causes acute infections in immunocompromised individuals (Chaudhuri and Henderson, 2012; Mellata, 2013); further, acute infections are associated with its planktonic growth mode (Li et al., 2014). Therefore, we explored the role of PPK in antibiotic resistance in the planktonic cells of ExPEC strain PCN033. Biofilm formation contributes to chronic bacterial infections, such as the recurrent pyelonephritis caused by uropathogenic E. coli in children (Tapiainen et al., 2014). Therefore, we also studied the role of PPK in antibiotic tolerance in biofilm-grown cells.

\section{MATERIALS AND METHODS}

\section{Bacterial Strains and Culture Conditions}

The wild-type (WT) strain used in this study, PCN033, was isolated from a diseased swine in Hubei Province, Central China (Liu et al., 2015). The $\Delta p p k$ mutant was obtained by in-frame deletion with the suicide plasmid pRE112 (He et al., 2012). Details of both these strains and the primers used in this study are listed in Table S1 (available as Supplementary Data). The antibiotic susceptibility and biofilm formation assays were performed in MOPS broth (an inorganic phosphorus $\left[\mathrm{P}_{\mathrm{i}}\right]$-limited medium) at $28^{\circ} \mathrm{C}$ (Neidhardt et al., 1974). The antibiotics used in the biofilm inhibition assay were added below the minimum inhibitory concentrations (MICs), and had no bactericidal effect on the planktonic cells.

\section{Growth Characteristics}

Fresh colonies of both strains were taken from Luria-Bertani (LB) agar plates, used to inoculate LB broth, and then cultured in MOPS in a shaker incubator for $12 \mathrm{~h}$. The growth characteristics were monitored turbidimetrically at $600 \mathrm{~nm}$ on a spectrophotometer (Eppendorf, Hamburg, Germany) and the colony-forming units (cfu) were counted at $1 \mathrm{~h}$ intervals. The generation times were calculated with the formula (Penfold and Norris, 1912):

$$
G=\frac{T}{\log _{2}^{\frac{b}{a}}}
$$

where $G$ is the generation time; $T$ is the length of the logarithmic phase; $a$ is the initial number of bacteria; and $b$ is the final number of bacteria.

\section{Susceptibility Assay}

Each MIC was determined with a series of two-fold dilutions of the antibiotic in MOPS broth, according to the Clinical Laboratory and Standards Institute guidelines. A pre-grown inoculum of each strain was diluted in MOPS to a final concentration of $10^{7} \mathrm{cfu} / \mathrm{mL}$, and the concentration of antibiotic added varied from 0.25 to $512 \mathrm{mg} / \mathrm{L}$. The plates were incubated for $24 \mathrm{~h}$, and the MICs were determined as the lowest antibiotic concentrations that produced no visible growth.

The susceptibility assay of the biofilms was performed as described previously (Benthall et al., 2015), with some modifications. The MIC on the biofilm was determined by allowing a biofilm to form in a 96-well-plate for $24 \mathrm{~h}$. The unattached cells were washed off three times with $0.9 \%$ saline. The biofilm was incubated for $24 \mathrm{~h}$ with a range of antibiotic concentrations from 512 to $0.25 \mathrm{mg} / \mathrm{L}$. The MIC was defined as the lowest antibiotic concentration at which no bacterial growth was detected. To determine the effect of the biofilm on the bacterial susceptibility to antibiotics, the viability of planktonic and biofilm-grown cells was calculated after the antibiotic treatments. About $10^{7} \mathrm{cfu}$ were incubated with antibiotic concentrations of $2 \times$ MIC for $3 \mathrm{~h}$, and the cfu were then counted.

To quantify the bactericidal activity of these antibiotics on the biofilm, their activity percentage was assessed according to a previous report (Sánchez-Gómez et al., 2015), with some modifications. The ability of these antibiotics to remove the biofilm attached to the microplate was determined with crystal violet $(\mathrm{CV})$ staining. For this purpose, the treated biofilm was stained with $\mathrm{CV}$ for $30 \mathrm{~min}$ at room temperature. The excess stain was then rinsed off with saline and the CV remaining on the biofilm was dissolved in 33\% acetic acid. The absorbance was measured at $595 \mathrm{~nm}$ with a Synergy HT microplate reader (BioTek, USA). The activity percentage was calculated according to Sánchez-Gómez et al., with the formula:

$$
\text { Activity Percentage }=\frac{(C-B)-(T-B)}{C-B} \times 100
$$

where $C$ is the absorbance of the control well-containing untreated biofilm; $T$ is the absorbance of the well-containing treated biofilm; and $B$ is the absorbance of the blank well (i.e., no biofilm). 


\section{Biofilm Formation Assay}

Static cultures of biofilm grown at $28^{\circ} \mathrm{C}$ were analyzed in flatbottom 96-well-microtiter plates (Corning, USA) using CV. Briefly, approximately $10^{7} \mathrm{cfu}$ were inoculated with sub-MIC antibiotics and cultured for $24 \mathrm{~h}$. The unattached cells were then washed off as described above, fixed with absolute ethanol for $30 \mathrm{~min}$, dried, and stained with $0.1 \% \mathrm{CV}$ solution for $1 \mathrm{~h}$. The microplates were then washed three times to remove any unattached CV. The CV in the stained biofilm was then dissolved in $33 \%$ acetic acid solution and the absorbance read at 595 $\mathrm{nm}$. Each experiment was repeated twice with three technical replicates.

\section{RNA-seq Assay and Quantitative \\ Real-Time (qRT)-PCR Validation}

RNA samples from each strain were prepared for RNA sequencing. Sequencing was performed on an Illumina Hiseq 2500 sequencer (Illumine Inc.) by Shanghai Hanyu Biotechnology Co., Ltd (Shanghai, China). The RNA-seq results were confirmed with qRT-PCR. Before qRT-PCR, the RNA of both strains was extracted with RNAiso Plus reagent (Takara, China). Any genomic DNA contamination was eliminated, and the RNA was reverse transcribed to cDNA with the PrimeScript ${ }^{\mathrm{TM}} \mathrm{RT}$ reagent Kit with gDNA Eraser (Takara). Quantitative real-time PCR was performed in triplicate in optical 96-well-reaction plates (Life Technologies, China) using Power SYBR Green PCR Master Mix (Life Technologies). The primers are listed in Table S1. The mRNA levels of the target genes were normalized to the internal 16S rRNA control with the $\Delta \Delta$ Ct method (Kubista et al., 2006). The planktonic cells of the WT and $\Delta p p k$ mutant were cultured to an optical density at a wavelength of $600 \mathrm{~nm}\left(\mathrm{OD}_{600}\right)$ of about 0.5 . Tazobactam or gentamycin was added at a concentration of $2 \times$ MIC and the cells were incubated for $2 \mathrm{~h}$. The cells were then collected for RNA extraction and qRT-PCR.

\section{Statistical Analysis}

Statistical analysis was performed with the SPSS software (SPSS, Inc., Chicago, IL, USA) on a Windows XP system. Biofilm formation, differential expression, and generation times were compared with one-way analysis of variance (ANOVA). Values are expressed as means $\pm S D$, and statistically significant differences are marked with asterisks. To identify the levels of gene expression, the RNA-seq data were analyzed with an MA-plot-based method with the random sampling model (MARS) in the DEGseq software (http://www.bioconductor. org/packages/release/bioc/html/DESeq.html). Genes showing two-fold changes in expression, a false discovery rate $<0.001$, and reads per kilobase per million $>20$ in at least one sample were considered to be differentially expressed under the conditions used.

\section{RESULTS}

\section{Growth Characteristics}

In MOPS minimal medium, the density of the $\Delta p p k$ cultures was slightly higher than that of the WT at each time point tested
(Figure 1). However, the generation times, calculated from both the optical density and cfu, for $\Delta p p k\left(G_{\mathrm{OD}}=56.5 \pm 4.73, G_{\mathrm{CFU}}\right.$ $=42.0 \pm 1.88)$ were not significantly different $(P>0.05)$ from those for WT $\left(G_{\mathrm{OD}}=56.3 \pm 3.64, G_{\mathrm{cfu}}=45.9 \pm 4.35\right)$.

\section{Planktonic $\Delta p p k$ Cells are More Susceptible to Antibiotics than WT Cells}

As shown in Table 1, the 17 antibiotics screened in this study were categorized based on their targets: cell wall biosynthesis (type A), protein biosynthesis (type B), nucleotide metabolism (type C), and cell membrane (type D). There were no significant differences in the MIC values for the $\Delta p p k$ strain and WT in LB broth. However, in MOPS broth, $\triangle p p k$ was more susceptible to antibiotics than WT. Specifically, $\Delta p p k$ was much more susceptible to type B antibiotics than was WT, followed by type A antibiotics. Because the WT strain can accumulate more polyP in MOPS broth than in other medi (Ault-Riché et al., 1998), we performed all further assays in MOPS.

\section{Biofilm-Grown $\Delta p p k$ and WT Cells Are Similarly Tolerant and More Tolerant than Planktonic Cells}

To clarify the role of biofilms in antibiotic resistance, an MIC assay using biofilm-grown cells was performed as described previously (Benthall et al., 2015). As shown in Table 2, biofilmgrown $\Delta p p k$ cells showed almost no difference from WT cells in their antibiotic susceptibility, and biofilm-grown cells of both strains were more tolerant than the corresponding planktonic cells. Consistent with this, there was no significant difference in the ability of antimicrobial compounds to kill biofilm-grown cells of the WT and $\triangle p p k$ strains (Figure S1). However, more planktonic cells of the $\Delta p p k$ strain were killed than WT cells (Figure 2). In a future study, we will investigate the role of PPK in antibiotic tolerance within the planktonic growth mode.

\section{Expression of Antibiotic-Resistance Genes without Antibiotic Treatment}

RNA-seq data for the $\Delta p p k$ and WT strains regarding the expression of genes involved in antibiotic resistance, including resistance-conferring genes and antibiotic efflux and influx genes, are presented in Table S2. Of 53 genes known to be involved in antibiotic resistance or multidrug resistance, the expression of one resistance-conferring gene (tet $B$ ), two efflux genes ( $m d t E$ and $m d t G)$, and one influx gene $(\operatorname{omp} C)$ was upregulated, and the expression of three efflux genes ( $\operatorname{mar} A, \operatorname{mar} B$, and $m d t A$ ) was downregulated in the mutant compared with their expression in WT (Figure 3A). The expression of some of these genes was confirmed with qRT-PCR (Figure 3B).

\section{Expression of Resistance-Conferring Genes after Antibiotic Treatment}

Five resistance-conferring genes were selected for analysis when WT was treated with tazobactam, as shown in Figure 4. The expression of beta-lactamase (bla) was upregulated, as was that of aminoglycoside $3^{\prime} \mathrm{N}$-acetyltransferase III $(a a c)$, fused UDP-L-Ara4N formyltransferase ( $\operatorname{arnA}$ ), and nitroreductase A 

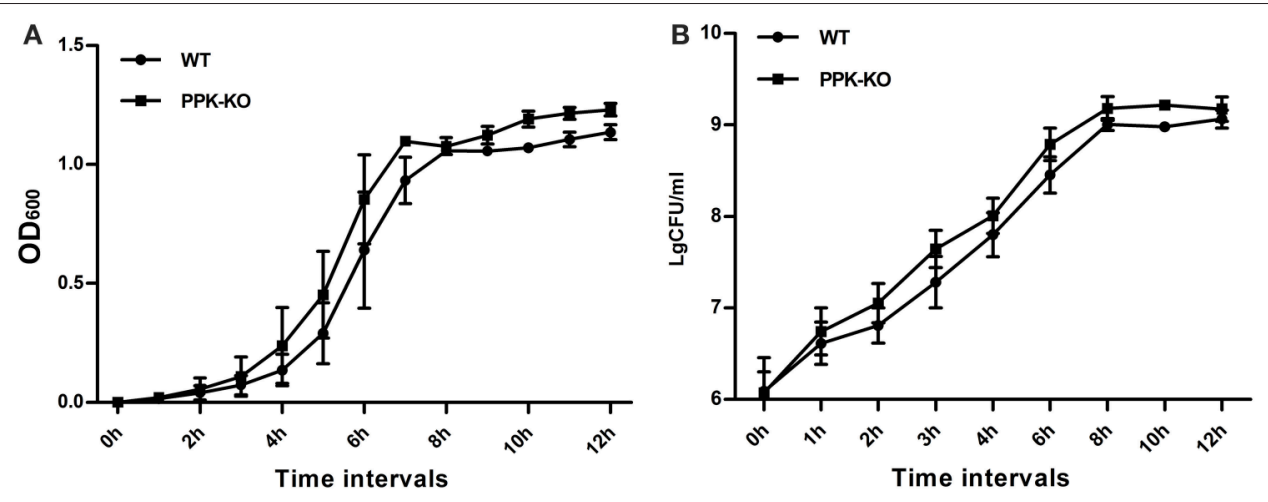

FIGURE 1 | Growth characteristics of E. coli strains PCN033 (wild type) and $\Delta p p k$ in MOPS broth: (A) optical density, and (B) colony-forming units.

TABLE 1 | Assay of the susceptibility of planktonic cells to antibiotics ( $\mathrm{mg} / \mathrm{L})$.

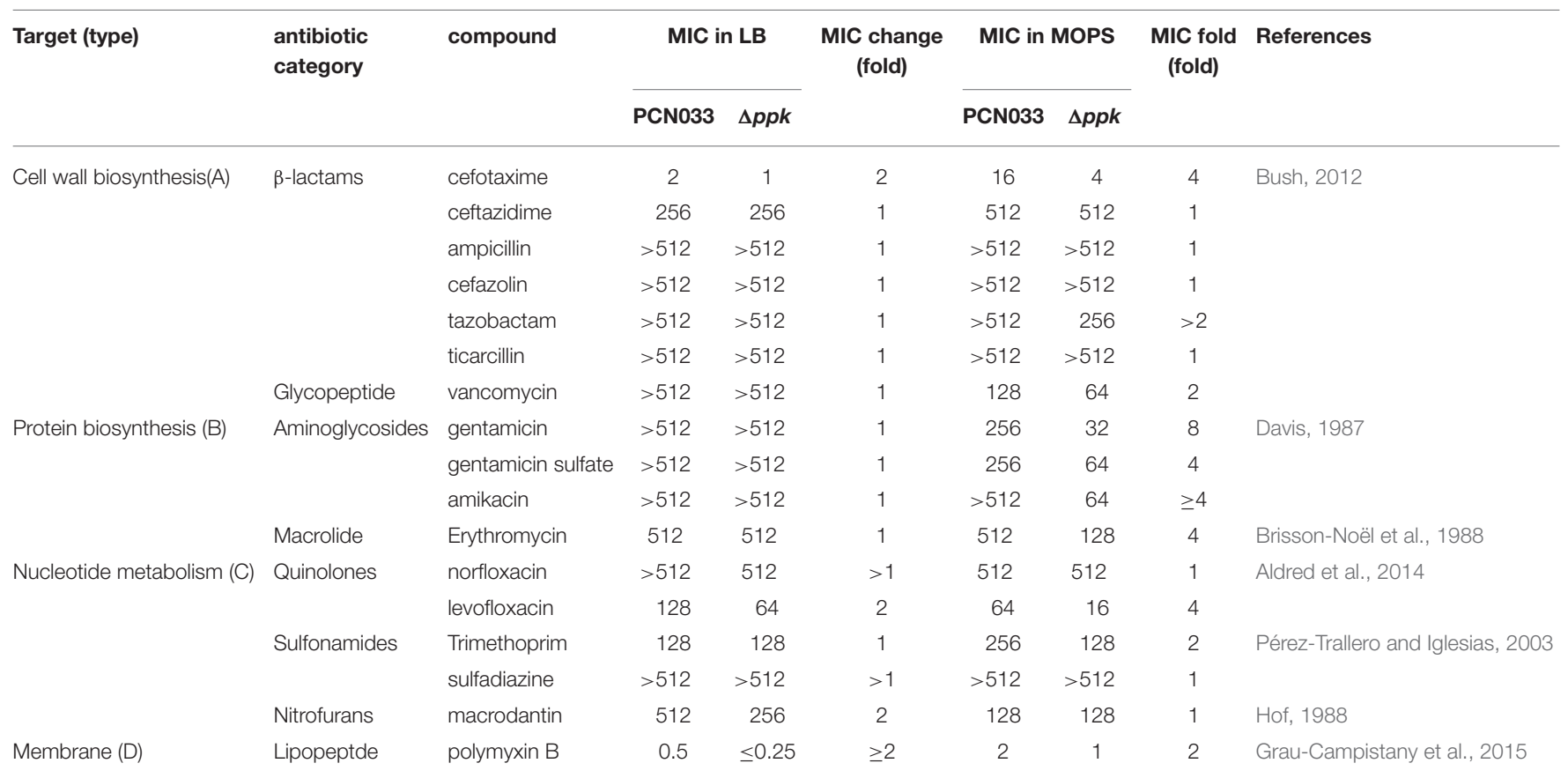

$(n f s A)$. The bla, arnA, and sulfate adenylyltransferase (cys $N)$ genes were also upregulated in the $\Delta p p k$ mutant after tazobactam treatment, but their expression was higher in WT than in the $\Delta p p k$ mutant. The expression of resistance-conferring gene was not significantly altered by gentamycin treatment, except for cys $N$.

\section{Expression of Genes Involved in Antibiotic Transport Was Altered More Strongly in WT than in the $\Delta p p k$ Mutant}

The expression of six genes involved in antibiotic efflux was determined, as shown in Figure 5A. The efflux genes were upregulated in both the WT and $\triangle p p k$ mutant strains by tazobactam and gentamycin. However, the expression of acrA, cus $C$, and marA was higher in WT than in the $\triangle p p k$ mutant after treatment with tazobactam. After treatment with gentamycin, the expression of acrA and marA was also higher in WT than in the $\triangle p p k$ mutant. The expression of three genes involved in antibiotic influx was determined, as shown in Figure 5B. All the genes tested were downregulated by antibiotic treatment. However, ompF expression decreased more strongly in WT than in the $\Delta p p k$ mutant when treated with tazobactam. The expression of $o m p F, o m p C$, and $p h o E$ also decreased more strongly in WT than in the $\Delta p p k$ mutant after treatment with gentamycin.

\section{Effects of Tazobactam and Gentamycin in Biofilm Formation}

The RNA-seq data showed that the transcription levels of some genes associated with biofilm formation were altered 
TABLE 2 | Assay of the susceptibility of biofilm-grown cells to antibiotics (mg/L).

\begin{tabular}{|c|c|c|c|c|c|}
\hline \multirow[t]{2}{*}{ Target (type) } & \multirow[t]{2}{*}{ antibiotic category } & \multirow[t]{2}{*}{ compound } & \multicolumn{2}{|c|}{ MIC for biofilm } & \multirow[t]{2}{*}{ MIC change (fold) } \\
\hline & & & PCN033 & $\Delta p p k$ & \\
\hline \multirow[t]{6}{*}{ Cell wall biosynthesis $(A)$} & $\beta$-lactams & cefotaxime & 128 & 256 & 0.5 \\
\hline & & ceftazidime & 512 & 512 & 1 \\
\hline & & ampicillin & $>512$ & $>512$ & 1 \\
\hline & & cefazolin & $>512$ & $>512$ & 1 \\
\hline & & tazobactam & 512 & $>512$ & $\leq 0.5$ \\
\hline & & ticarcillin & $>512$ & $>512$ & 1 \\
\hline \multirow[t]{6}{*}{ Protein biosynthesis (B) } & Aminoglycosides & gentamicin & 512 & 512 & 1 \\
\hline & & gentamicin sulfate & 512 & 256 & 2 \\
\hline & & amikacin & 512 & 512 & 1 \\
\hline & Macrolide & erythromycin & 512 & 512 & 1 \\
\hline & Quinolones & norfloxacin & 512 & 512 & 1 \\
\hline & & levofloxacin & 32 & 32 & 1 \\
\hline \multirow[t]{3}{*}{ Nucleotide metabolism (C) } & Sulfonamides & Trimethoprim & 512 & 512 & 1 \\
\hline & & sulfadiazine & $>512$ & $>512$ & 1 \\
\hline & Nitrofurans & macrodantin & 512 & 512 & 1 \\
\hline Membrane (D) & Lipopeptde & polymyxin B & 4 & 4 & 1 \\
\hline
\end{tabular}
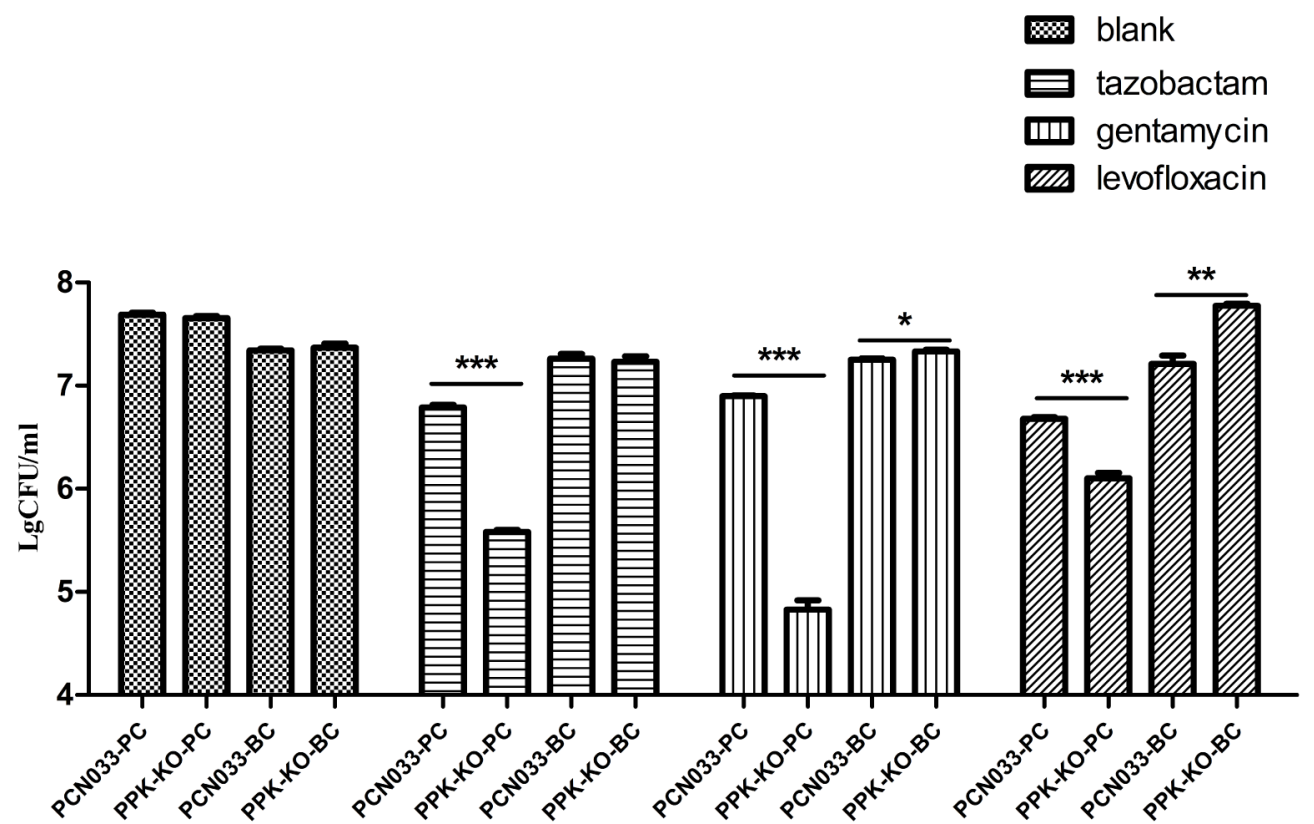

FIGURE 2 | Antibiotic killing assay of planktonic and biofilm-grown cells. PCNO33-PC indicates planktonic WT cells; PPK-KO-PC indicates planktonic cells of the $\Delta$ ppk mutant; PCN033-BC indicates biofilm-grown WT cells; PPK-KO-BC indicates biofilm-grown cells of the $\Delta p p k$ mutant; blank indicates no antibiotic treatment. ${ }^{* * *} p<0.000,{ }^{* \star} p<0.01,{ }^{*} p<0.05$.

in the $\Delta p p k$ strain (Tables S3, S4), especially those encoding the flagella cluster, which simultaneously promotes biofilm generation and impedes biofilm maturation (Laverty et al., 2014). The expression of fimbrial and curli genes was also reduced in the $\Delta p p k$ mutant. The expression levels of some genes were confirmed with qRT-PCR (Figure 6A). The expression of four genes ( $y d d V, m c b R$, bolA, and $c s g D)$ involved in biofilm regulation was determined during antibiotic treatment, as shown in Figure 6B (Laverty et al., 2014; Lord et al., 2014; Dressaire et al., 2015; Wu et al., 2015). The expression of $y d d V$, $m c b R$, and bolA was upregulated in WT but downregulated in the $\triangle p p k$ mutant when treated with tazobactam, and all four genes were upregulated in WT but downregulated in the $\Delta p p k$ mutant when treated with gentamycin. Biofilm formation was also evaluated in the presence of tazobactam or gentamycin, as shown Figure 6C. Biofilm formation increased in WT planktonic 

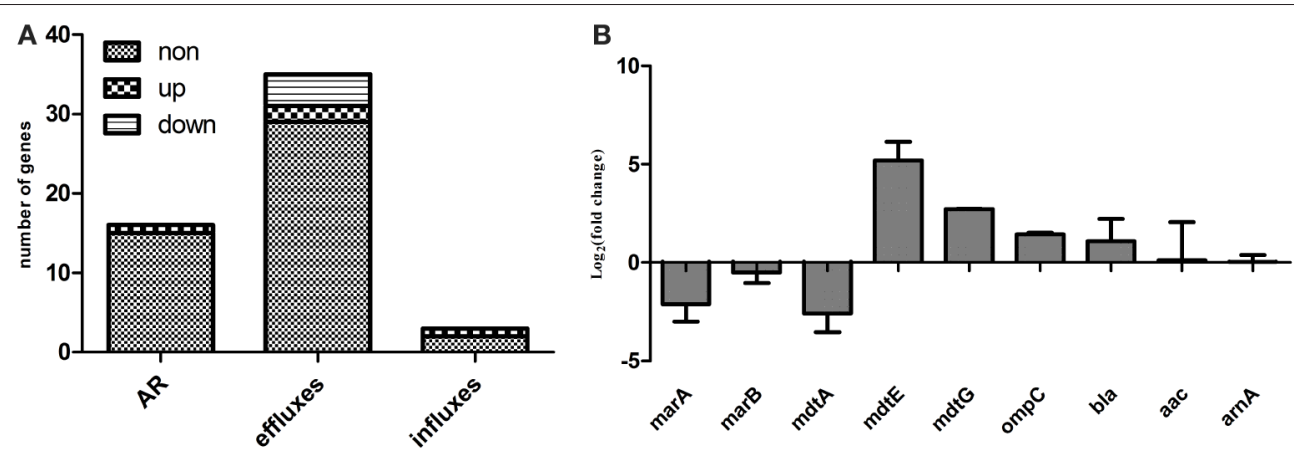

FIGURE 3 | Expression levels of genes involved in antibiotic resistance, antibiotic efflux, or antibiotic influx. (A) Expression levels determined with RNA-seq; (B) qRT-PCR confirmation. AR indicates genes that confer resistance, including beta-lactamase bla, aminoglycoside $3^{\prime} \mathrm{N}$-acetyltransferase III (aac) etc.; efflux genes include acrAB-to/C, acrDEF, cusCFBA, emrAB, emrKY, mdtABCEF, etc.; and influx genes include ompC, ompF, and phoE, details are available in Table $\mathbf{S 2}$.

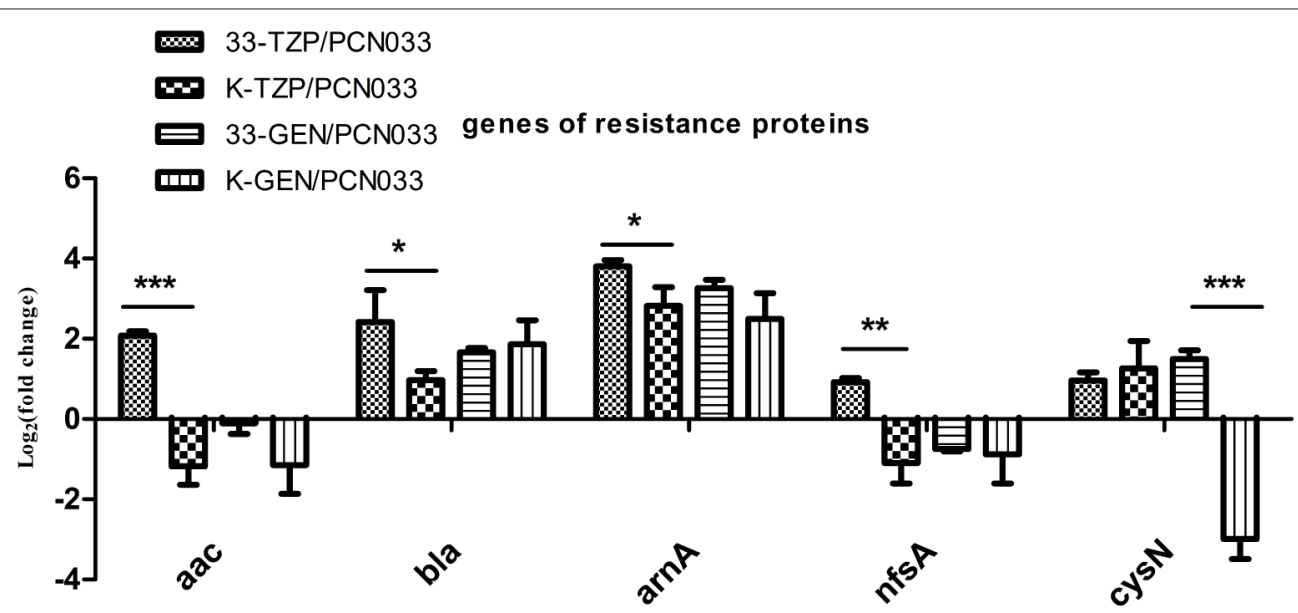

FIGURE 4 | Expression levels of genes that confer resistance, during tazobactam and gentamycin treatment. Expression levels were compared with those of WT (PCNO33). 33-TZP/PCN033 indicates the expression levels in PCNO33 during tazobactam treatment compared with those in untreated PCN033; K-TZP/PCN033 indicates expression levels in the $\triangle$ ppk mutant during tazobactam treatment compared with those in untreated PCN033; 33-GEN/PCN033 indicates expression levels in PCNO33 during gentamycin treatment compared with those in untreated PCN033; K-GEN/PCNO33 indicates expression levels in the $\triangle p p k$ mutant during gentamycin treatment compared with those in untreated PCN033. ${ }^{* * *} p<0.000,{ }^{* *} p<0.01,{ }^{*} p<0.05$.

A

A

口 33-GENPCNO33

${ }^{8} 7$ 口-GENPCNO33

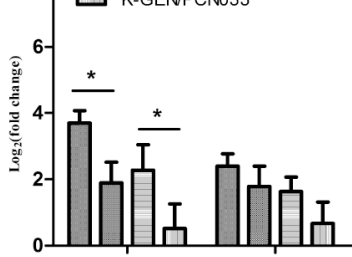

$2^{c^{5}}$ genes involved in efflux

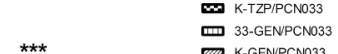

B $\square$ 33-ТZP PCNO33 K-GENPCNO33 genes involved in influx

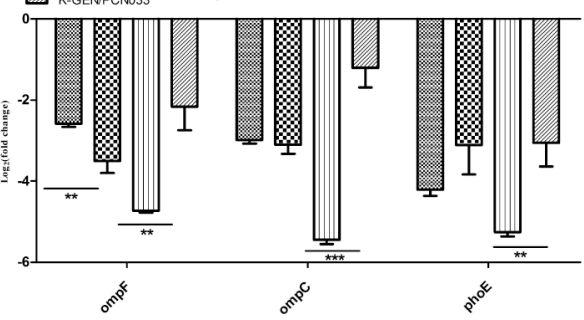

FIGURE 5 | Expression levels of genes involved in antibiotic efflux and influx during tazobactam and gentamycin treatments. (A) Expression of efflux genes, (B) expression of influx genes. Expression levels of the genes were compared with those of WT (PCNO33). 33-TZP/PCN033 indicates the expression levels in PCN033 during tazobactam treatment compared with those in untreated PCNO33; K-TZP/PCNO33 indicates the expression levels in the $\triangle p p k$ mutant during untreated PCN033.K-GEN/PCNO33 indicates the expression levels in the $\triangle p p k$ mutant during gentamycin treatment compared with those in untreated PCN033.

${ }^{* \star *} p<0.000,{ }^{* *} p<0.01,{ }^{*} p<0.05$. 
A

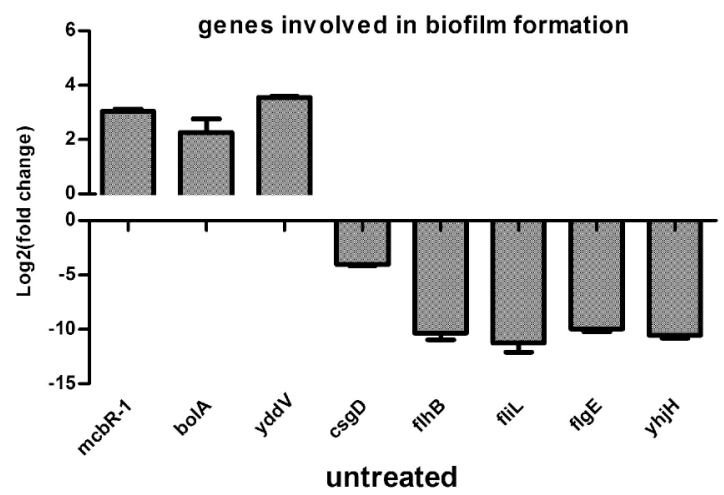

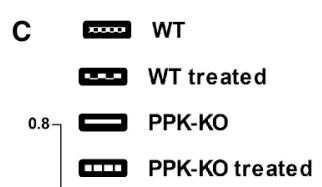

$0.6-$ PPK-KO treated

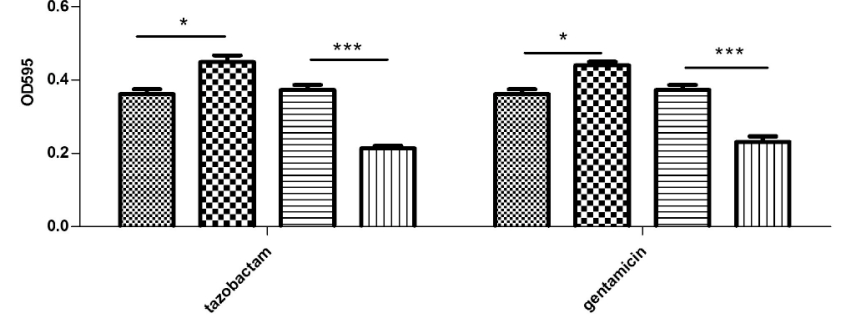

treated

B

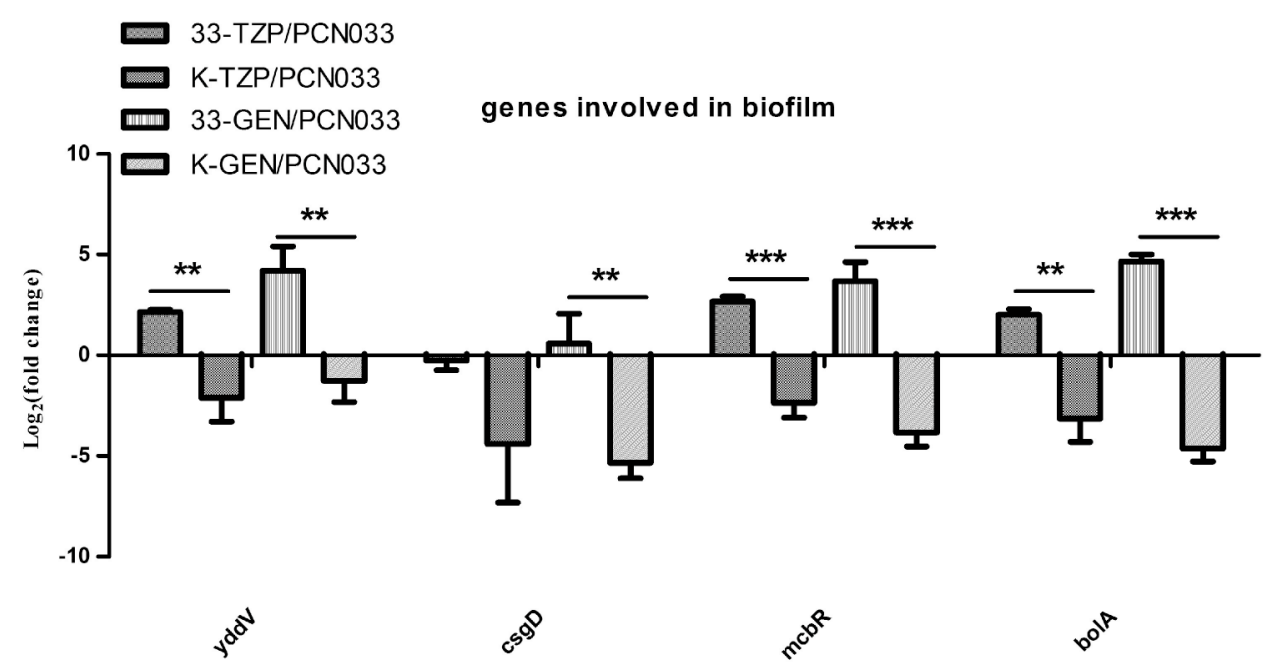

FIGURE 6 | Biofilm formation assay. (A) Validation of the expression of genes involved in biofilm formation. (B) Expression levels of genes involved in biofilm regulation during tazobactam and gentamycin treatment. (C) Biofilm formation during tazobactam or gentamycin treatment. Expression levels of genes were compared with those in WT (PCNO33). 33-TZP/PCN033 indicates the expression levels in PCN033 during tazobactam treatment compared with those in untreated PCN033; K-TZP/PCNO33 indicates the expression levels in the $\triangle p p k$ mutant during tazobactam treatment compared with those in untreated PCN033; 33-GEN/PCNO33 indicates the expression levels in PCN033 during gentamycin treatment compared with those in untreated PCN033; K-GEN/PCN033 indicates the expression levels in the $\triangle p p k$ mutant during gentamycin treatment compared with those in untreated PCNO33. WT indicates PCNO33 without antibiotic treatment; WT treated indicates PCNO33 with antibiotic treatment; PPK-KO indicates the $\triangle p p k$ mutant without antibiotic treatment; PPK-KO treated indicates the $\triangle p p k$ mutant with antibiotic treatment. ${ }^{* * *} p<0.000,{ }^{*} p<0.01,{ }^{*} p<0.05$.

cells but decreased in $\Delta p p k$ planktonic cells when treated with antibiotics.

\section{DISCUSSION}

Multidrug-resistant strains of ExPEC present significant challenges to public health and animal husbandry (Girardeau et al., 2003; Johnson et al., 2005; Bergeron et al., 2012). Because pathogenic E. coli mainly causes acute infections in its planktonic growth mode (Li et al., 2014), we initially investigated the role of PPK in the antibiotic resistance of ExPEC in the planktonic growth mode. We investigated in detail its susceptibility to different types of antibiotics, mediated by PPK, in E. coli. We found that PPK is very important in aminoglycoside tolerance, regulating the expression levels of antibiotic efflux and influx genes in the planktonic growth mode. Our findings indicate that PPK could have utility as a novel antimicrobial drug target.

As reported previously, resistance-conferring proteins and antibiotic efflux and influx porins play important roles in multidrug-resistance. Efflux proteins contribute to antibiotic tolerance by transporting compounds to the extracellular environment, whereas influx proteins have the opposite effect (Wilson, 2014). The expression levels of these genes were determined with RNA-seq, and showed that without antibiotic treatment, they did not differ significantly between WT and the $\Delta p p k$ mutant. Because PPK is reported to play prominent 
roles in the stress responses elicited by other stimuli (Alcántara et al., 2014), we investigated the role of PPK in the antibiotic stress response. Gentamycin and tazobactam were selected to treatthe planktonic cells of WT and the $\Delta p p k$ mutant. With gentamycin treatment, the expression of the efflux genes acrA and marA increased more strongly in WT than that in the $\Delta p p k$ mutant, and the influx porin genes $о m p C$ and $o m p F$ decreased more strongly in WT than in the $\Delta p p k$ mutant. Gentamycin binds the $30 \mathrm{~S}$ ribosomal subunit and interrupts protein synthesis, thus inhibiting bacterial multiplication (Wargo and Edwards, 2014). According to Gray et al., compounds that interrupt protein metabolism cause intracellular polyP accumulation (Gray et al., 2014). Because it is a high-energy phosphate compound, polyP can be used to phosphorylate the response regulators of twocomponent systems to regulate gene expression (Sureka et al., 2007). As reported previously, the two-component systems CpxR and $\mathrm{BaeR}$ are implicated in antibiotic resistance by regulating the efflux genes of the acr operon and mar operon (Hu et al., 2011; Weatherspoon-Griffin et al., 2014; Pletzer et al., 2015). We speculated that phosphorylation of BaeR or CpxR using polyP as phosphate donar to modulate expression of acrA and marA during gentamycin treatment. The expression of porin genes $o m p F$ and $o m p C$ is upregulated by cAMP (Dalhoff, 1983), and the level of cAMP is negatively regulated by polyP, which potently inhibits the activity of the class III adenylate cyclases (Guo et al., 2009). Therefore, we speculated that the expression of $o m p C$ and $o m p F$ was downregulated by polyP during gentamycin treatment. Therefore, polyP may influence gentamycin tolerance by regulating the expression of antibiotic efflux and influx genes.

With tazobactam treatment, the expression of the resistance gene bla and efflux genes acr $A, c u s C$, and marA was upregulated. Tazobactam binds to the periplasmic $\beta$-lactamase, and the efflux pump is implicated in resistance to beta-lactams and beta-lactamase inhibitors (Zhanel et al., 2014). However, there are insufficient data to clarify the role of PPK in regulating the expression of efflux pump genes induced by $\beta$-lactams. It will be interesting to explore the role of PPK in this process.

Biofilms contribute to antibiotic tolerance and chronic infection; thus, we also investigated the role of PPK in the antibiotic resistance of biofilm-grown cells. We observed that biofilm formation was impaired in the $\Delta p p k$ mutant when treated with antibiotics. The genes involved in biofilm formation (such as those encoding the fimbriae cluster, flagella cluster, and biofilm regulators BolA and $\mathrm{McbR}$ ), were downregulated in the $\Delta p p k$ mutant by both antibiotic treatments. PolyP acts as a "chemical chaperone," stabilizing cytoplasmic proteins intracellularly, similarly to heat shock proteins (Gray et al., 2014), and chaperones are known to be involved in biofilm formation. For example, the chaperone CsgE directs the intracellular localization of CsgA, the major subunit of the extracellular amyloid protein known as curli, which is essential for biofilm formation (Andersson et al., 2013). The FliS protein acts as a chaperone for FliC, a flagellar structural protein that promotes biofilm generation (Xu et al., 2014). The universal heat shock protein chaperones are also implicated in biofilm formation by fungi, such as Candida albicans (Robbins et al., 2011; Becherelli et al., 2013), and by Gram-negative bacteria, such as E. coli (Grudniak et al., 2013). Ultimately, these different effects reduce biofilm impairment during antibiotic treatment. Interestingly, PPK did not affect the antibiotic tolerance of ExPEC in the biofilm growth mode. Biofilms manifest antibiotic tolerance through many different mechanisms, including preventing the passage of antimicrobial compounds into the cytoplasm and possessing densely adherent growth (Qu et al., 2010). As reported previously, the planktonic and biofilm modes of growth are two distinct bacterial "lifestyles" (Chua et al., 2014), so it will be interesting to explore the roles of PPK in these distinct contexts.

\section{AUTHOR CONTRIBUTIONS}

JC and CT designed and supervised the research project. JC wrote the paper, and LS, XW, HC, and TZ revised the manuscript. JC, TZ, and FL performed the experiments. JC and LS processed the data and performed the statistical analysis. All the authors have read and approved the manuscript.

\section{FUNDING}

This work was supported by grants from the National Natural Science Foundation of China (grant numbers 31472201, 31030065, and 31421064) and the Programme for Introducing Talents of Discipline to Universities (grant number B12005).

\section{ACKNOWLEDGMENTS}

The authors wish to thank Dr. Janine Miller from the University of Tasmania for writing improvement.

\section{SUPPLEMENTARY MATERIAL}

The Supplementary Material for this article can be found online at: http://journal.frontiersin.org/article/10.3389/fmicb. 2016.00724

Figure S1 | Percentage activity of different antibiotics in removing biofilm. (A) Targeting the cell wall, (B) targeting protein biosynthesis, and (C) targeting nucleotide metabolism.

Table S1 | Bacteria strains and primers.

Table S2 | Transcription level of genes involved in antibiotic and multi-drug resistance.

Table S3 | Expression of genes implicated in biofilm.

Table S4 | Genes involved in biofilm formation (part results of RNA-seq). 


\section{REFERENCES}

Alcántara, C., Blasco, A., Zúñiga, M., and Monedero, V. (2014). Accumulation of polyphosphate in Lactobacillus spp. and its involvement in stress resistance. Appl. Environ. Microbiol. 80, 1650-1659. doi: 10.1128/AEM.03997-13

Aldred, K. J., Kerns, R. J., and Osheroff, N. (2014). Mechanism of quinolone action and resistance. Biochemistry 53, 1565-1574. doi: 10.1021/bi5000564

Andersson, E. K., Bengtsson, C., Evans, M. L., Chorell, E., Sellstedt, M., and Lindgren, A. E. (2013). Modulation of curli assembly and pellicle biofilm formation by chemical and protein chaperones. Chem. Biol. 20, 1245-1254. doi: 10.1016/j.chembiol.2013.07.017

Ault-Riché, D., Fraley, C. D., Tzeng, C. M., and Kornberg, A. (1998). Novel assay reveals multiple pathways regulating stress-induced accumulations of inorganic polyphosphate in Escherichia coli. J. Bacteriol. 180, 1841-1847.

Becherelli, M., Tao, J., and Ryder, N. S. (2013). Involvement of heat shock proteins in Candida albicans biofilm formation. J. Mol. Microbiol. Biotechnol. 23, 396-400. doi: 10.1159/000351619

Benthall, G., Touzel, R. E., Hind, C. K., Titball, R. W., Sutton, J. M., and Thomas, R. J. (2015). Evaluation of antibiotic efficacy against infections caused by planktonic or biofilm cultures of Pseudomonas aeruginosa and Klebsiella pneumoniae in Galleria mellonella. Int. J. Antimicrob. Agents 46, 538-545. doi: 10.1016/j.ijantimicag.2015.07.014

Bergeron, C. R., Prussing, C., Boerlin, P., Daignault, D., Dutil, L., and ReidSmith, R. J. (2012). Chicken as reservoir for extraintestinal pathogenic Escherichia coli in humans, Canada. Emerg. Infect. Dis. 18, 415-421. doi: 10.3201/eid1803.111099

Brisson-Noël, A., Trieu-Cuot, P., and Courvalin, P. (1988). Mechanism of action of spiramycin and other macrolides. J. Antimicrob. Chemother. 22 (Suppl B), 13-23.

Brown, M. R., and Kornberg, A. (2004). Inorganic polyphosphate in the origin and survival of species. Proc. Natl. Acad. Sci. U.S.A. 101, 16085-16087. doi: 10.1073/pnas.0406909101

Bush, K. (2012). Antimicrobial agents targeting bacterial cell walls and cell membranes. Rev. Sci. Tech. 31, 43-56. doi: 10.20506/rst.31.1.2096

Chaudhuri, R. R., and Henderson, I. R. (2012). The evolution of the Escherichia coli phylogeny. Infect. Genet. Evol. 12, 214-226. doi: 10.1016/j.meegid.2012.01.005

Chua, S. L., Liu, Y., Yam, J. K., Chen, Y., Vejborg, R. M., and Tan, B. G. (2014). Dispersed cells represent a distinct stage in the transition from bacterial biofilm to planktonic lifestyles. Nat. Commun. 21, 4462. doi: 10.1038/ncomms5462

Dalhoff, A. (1983). Transport of aminoglycosides in Escherichia coli. Z. Bakteriol. Mikrobiol. Hyg. A. 254, 379-387.

Davis, B. D. (1987). Mechanism of bactericidal action of aminoglycosides. Microbiol. Rev. 51, 341-350.

Dressaire, C., Moreira, R. N., Barahona, S., Alves de Matos, A. P., and Arraiano, C. M. (2015). BolA is a transcriptional switch that turns off motility and turns on biofilm development. MBio 6, e02352-e02314. doi: 10.1128/mBio.02352-14

Fraley, C. D., Rashid, M. H., Lee, S. S., Gottschalk, R., Harrison, J., and Wood, P. J. (2007). A polyphosphate kinase 1 (ppk1) mutant of Pseudomonas aeruginosa exhibits multiple ultrastructural and functional defects. Proc. Natl. Acad. Sci. U.S.A. 104, 3526-3531. doi: 10.1073/pnas.0609733104

Gaschignard, J., Levy, C., Romain, O., Cohen, R., Bingen, E., and Aujard, Y. (2011). Neonatal bacterial meningitis: 444 cases in 7 years. Pediatr. Infect. Dis. J. 30, 212-217. doi: 10.1097/INF.0b013e3181fable7

Girardeau, J. P., Lalioui, L., Said, A. M., De Champs, C., and Le Bouguénec, C. (2003). Extended virulence genotype of pathogenic Escherichia coli isolates carrying the afa- 8 operon: evidence of similarities between isolates from humans and animals with extraintestinal infections. J. Clin. Microbial. 41, 218-226. doi: 10.1128/JCM.41.1.218-226.2003

Grau-Campistany, A., Manresa, Á., Pujol, M., Rabanal, F., and Cajal, Y. (2015). Tryptophan-containing lipopeptide antibiotics derived from polymyxin B with activity against Gram positive and Gram negative bacteria. Biochim. Biophys. Acta 1858, 333-343. doi: 10.1016/j.bbamem.2015.11.011

Gray, M. J., Wholey, W. Y., Wagner, N. O., Cremers, C. M., Mueller-Schickert, A., and Hock, N. T. (2014). Polyphosphate is a primordial chaperone. Mol. Cell. 53, 689-699. doi: 10.1016/j.molcel.2014.01.012

Grudniak, A. M., Pawlak, K., Bartosik, K., and Wolska, I. K. (2013). Physiological consequences of mutations in the htpG heat shock gene of Escherichia coli. Mutat. Res. 745-746, 1-5. doi: 10.1016/j.mrfmmm.2013.04.003
Guo, Y. L., Mayer, H., Vollmer, W., Dittrich, D., Sander, P., and Schultz, A. (2009). Polyphosphates from Mycobacterium bovis-potent inhibitors of class III adenylate cyclases. FEBS J. 276, 1094-1103. doi: 10.1111/j.17424658.2008.06852.x

He, Y., Xu, T., Fossheim, L. E., and Zhang, X. H. (2012). FliC, a flagellin protein, is essential for the growth and virulence of fish pathogen Edwardsiella tarda. PLOS ONE. 7:e45070. doi: 10.1371/journal.pone.0045070

Hof, H. (1988). Antimicrobial therapy with nitroheterocyclic compounds, for example, metronidazole and nitrofurantoin. Immun. Infekt. 16, 220-225.

Hu, W. S., Chen, H. W., Zhang, R. Y., Huang, C. Y., and Shen, C. F. (2011). The expression levels of outer membrane proteins STM1530 and OmpD, which are influenced by the CpxAR and BaeSR two-component systems, play important roles in the ceftriaxone resistance of Salmonella enterica serovar Typhimurium. Antimicrob. Agents Chemother. 55, 3829-3837. doi: 10.1128/AAC. 00216-11

Johnson, J. R., Kuskowski, M. A., Smith, K., O’Bryan, T. T., and Tatini, S. (2005). Antimicrobial-resistant and extraintestinal pathogenic Escherichia coli in retail foods. J. Infect. Dis. 191, 1040-1049. doi: 10.1086/428451

Kubista, M., Andrade, J. M., Bengtsson, M., Forootan, A., Jonák, J., and Lind, K. (2006). The real-time polymerase chain reaction. Mol. Aspects Med. 27, 195-125. doi: 10.1016/j.mam.2005.12.007

Laverty, G., Gorman, S. P., and Gilmore, B. F. (2014). Biomolecular mechanisms of Pseudomonas aeruginosa and Escherichia coli biofilm formation. Pathogens 3 , 596-632. doi: 10.3390/pathogens3030596

Li, Y., Petrova, O. E., Su, S., Lau, G. W., Panmanee, W., and Na, R. (2014). BdlA, DipA and induced dispersion contribute to acute virulence and chronic persistence of Pseudomonas aeruginosa. PLoS Pathog. 10:e1004168. doi: 10.1371/journal.ppat.1004168

Liu, C., Zheng, H., Yang, M., Xu, Z., Wang, X., and Wei, L. (2015). Genome analysis and in vivo virulence of porcine extraintestinal pathogenic Escherichia coli strain PCN033. BMC Genomics. 16:717. doi: 10.1186/s12864-015-1890-9

Lord, D. M., Uzgoren Baran, A., Soo, V. W., Wood, T. K., Peti, W., and Page, R. (2014). McbR/YncC: implications for the mechanism of ligand and DNA binding by a bacterial GntR transcriptional regulator involved in biofilm formation. Biochemistry 53, 7223-7231. doi: 10.1021/bi500871a

Mellata, M. (2013). Human and avian extraintestinal pathogenic Escherichia coli: infections, zoonotic risks, and antibiotic resistance trends. Foodborne Pathog. Dis. 10, 916-932. doi: 10.1089/fpd.2013.1533

Neidhardt, F. C., Bloch, P. L., and Smith, D. F. (1974). Culture medium for enterobacteria. J. Bacteriol. 119, 736-747.

Penfold, W. J., and Norris, D. (1912). The relation of concentration of food supply to the generation-time of bacteria. J. Hyg. 12, 527-531. doi: $10.1017 /$ S0022172400005180

Pérez-Trallero, E., and Iglesias, L. (2003). Tetracyclines, sulfonamides and metronidazole. Enferm. Infecc. Microbiol. Clin. 21, 520-528. doi: 10.1016/S0213-005X(03)72999-1

Pletzer, D., Stahl, A., Oja, A. E., and Weingart, H. (2015). Role of the cell envelope stress regulators BaeR and CpxR in control of RND-type multidrug efflux pumps and transcriptional cross talk with exopolysaccharide synthesis in Erwinia amylovora. Arch. Microbiol. 197, 761-772. doi: 10.1007/s00203-0151109-0

Qu, Y., Daley, A. J., Istivan, T. S., Rouch, D. A., and Deighton, M. A. (2010). Densely adherent growth mode, rather than extracellular polymer substance matrix build-up ability, contributes to high resistance of Staphylococcus epidermidis biofilms to antibiotics. J. Antimicrob. Chemother. 65, 1405-1411. doi: $10.1093 / \mathrm{jac} / \mathrm{dkq} 119$

Rashid, M. H., Rumbaugh, K., Passador, L., Davies, D. G., Hamood, A. N., and Iglewski, B. H. (2000). Polyphosphate kinase is essential for biofilm development, quorum sensing, and virulence of Pseudomonas aeruginosa. Proc. Natl. Acad. Sci.U.S.A. 97, 9636-9641. doi: 10.1073/pnas.170283397

Robbins, N., Uppuluri, P., Nett, J., Rajendran, R., Ramage, G., and Lopez-Ribot, J. L. (2011). Hsp90 governs dispersion and drug resistance of fungal biofilms. PLoS Pathog. 7:e1002257. doi: 10.1371/journal.ppat.1002257

Sánchez-Gómez, S., Ferrer-Espada, R., Stewart, P. S., Pitts, B., Lohner, K., and Martínez de Tejada, G. (2015). Antimicrobial activity of synthetic cationic peptides and lipopeptides derived from human lactoferricin against Pseudomonas aeruginosa planktonic cultures and biofilms. BMC Microbiol. 15:137. doi: 10.1186/s12866-015-0473-x 
Sedláková, M. H., Urbánek, K., Vojtová, V., Suchánková, H., Imwensi, P., and Kolár., M. (2014). Antibiotic consumption and its influence on the resistance in Enterobacteriaceae. BMC Res. Notes. 7:454. doi: 10.1186/1756-0500-7-454

Sidrach-Cardona, R., Hijosa-Valsero, M., Marti, E., Balcázar, J. L., and Becares, E. (2014). Prevalence of antibiotic-resistant fecal bacteria in a river impacted by both an antibiotic production plant and urban treated discharges. Sci. Total Environ. 488, 220-227. doi: 10.1016/j.scitotenv.2014.04.100

Singh, R., Singh, M., Arora, G., Kumar, S., Tiwari, P., and Kidwai, S. (2013). Polyphosphate deficiency in Mycobacterium tuberculosis is associated with enhanced drug susceptibility and impaired growth in guinea pigs. J. Bacteriol. 195, 2839-2851. doi: 10.1128/JB.00038-13

Sureka, K., Dey, S., Datta, P., Singh, A. K., Dasgupta, A., and Rodrigue, S. (2007). Polyphosphate kinase is involved in stress-induced mprAB-sigE-rel signalling in mycobacteria. Mol. Microbiol. 65, 261-276. doi: 10.1111/j.13652958.2007.05814.x

Tan, C., Tang, X., Zhang, X., Ding, Y., Zhao, Z., and Wu, B. (2012). Serotypes and virulence genes of extraintestinal pathogenic Escherichia coli isolates from diseased pigs in China. Vet. J. 192, 483-488. doi: 10.1016/j.tvjl.2011.06.038

Tapiainen, T., Hanni, A. M., Salo, J., Ikäheimo, I., and Uhari, M. (2014). Escherichia coli biofilm formation and recurrences of urinary tract infections in children. Eur. J. Clin. Microbiol. Infect. Dis. 33, 111-115. doi: 10.1007/s10096-013-1935-4

Tunpiboonsak, S., Mongkolrob, R., Kitudomsub, K., Thanwatanaying, P., Kiettipirodom, W., and Tungboontina, Y. (2010). Role of a Burkholderia pseudomallei polyphosphate kinase in an oxidative stress response, motilities, and biofilm formation. J. Microbiol. 48, 63-70. doi: 10.1007/s12275-010-9138-5

Wargo, K. A., and Edwards, J. D. (2014). Aminoglycoside-induced nephrotoxicity. J. Pharm. Pract. 27, 573-577. doi: 10.1177/0897190014546836

Weatherspoon-Griffin, N., Yang, D., Kong, W., Hua, Z., and Shi, Y. (2014). The CpxR/CpxA two-component regulatory system up-regulates the multidrug resistance cascade to facilitate Escherichia coli resistance to a model antimicrobial peptide. J. Biol. Chem. 289, 32571-32582. doi: 10.1074/jbc.M114.565762
Weston, E. J., Pondo, T., Lewis, M. M., Martell-Cleary, P., Morin, C., and Jewell, B. (2011). The burden of invasive early-onset neonatal sepsis in the United States, 2005-2008. Pediatr. Infect. Dis. J. 30, 937-941. doi: 10.1097/INF.0b013e318223bad2

Wilson, D. N. (2014). Ribosome-targeting antibiotics and mechanisms of bacterial resistance. Nat. Rev. Microbiol. 12, 35-48. doi: 10.1038/nr micro3155

Wu, Y., Ding, Y., Cohen, Y., and Cao, B. (2015). Elevated level of the second messenger c-di-GMP in Comamonas testosteroni enhances biofilm formation andbiofilm-based biodegradation of 3-chloroaniline. Appl. Microbiol. Biotechnol. 99, 1967-1976. doi: 10.1007/s00253-01 4-6107-7

Xu, S., Peng, Z., Cui, B., Wang, T., Song, Y., and Zhang, L. (2014). FliS modulates FlgM activity by acting as a non-canonical chaperone to control late flagellar gene expression, motility and biofilm formation in Yersinia pseudotuberculosis. Environ. Microbiol. 16, 1090-1104. doi: 10.1111/1462-2920. 12222

Zhanel, G. G., Chung, P., Adam, H., Zelenitsky, S., Denisuik, A., and Schweizer, F. (2014). Ceftolozane/tazobactam: a novel cephalosporin/ $\beta$-lactamase inhibitor combination with activity against multidrug-resistant gram-negative bacilli. Drugs 74, 31-51. doi: 10.1007/s40265-013-0168-2

Conflict of Interest Statement: The authors declare that the research was conducted in the absence of any commercial or financial relationships that could be construed as a potential conflict of interest.

Copyright (c) 2016 Chen, Su, Wang, Zhang, Liu, Chen and Tan. This is an open-access article distributed under the terms of the Creative Commons Attribution License (CC $B Y)$. The use, distribution or reproduction in other forums is permitted, provided the original author(s) or licensor are credited and that the original publication in this journal is cited, in accordance with accepted academic practice. No use, distribution or reproduction is permitted which does not comply with these terms. 
OPEN ACCESS

Edited by:

Etienne Giraud,

Institut National de la Recherche

Agronomique, France

Reviewed by:

Stephen Peter Kidd,

University of Adelaide, Australia

Barbara C. Kahl,

University Hospital Münster, Germany

*Correspondence:

Lone Gram

gram@bio.dtu.dk

Specialty section:

This article was submitted to Antimicrobials, Resistance

and Chemotherapy,

a section of the journal

Frontiers in Microbiology

Received: 01 March 2016 Accepted: 23 June 2016

Published: 05 July 2016

Citation:

Curtis TD, Gram L and Knudsen GM (2016) The Small Colony Variant of Listeria monocytogenes Is More

Tolerant to Antibiotics and Has Altered Survival in RAW 264.7 Murine

Macrophages.

Front. Microbiol. 7:1056.

doi: 10.3389/fmicb.2016.01056

\section{The Small Colony Variant of Listeria monocytogenes Is More Tolerant to Antibiotics and Has Altered Survival in RAW 264.7 Murine Macrophages}

\author{
Thomas D. Curtis, Lone Gram* and Gitte M. Knudsen \\ Gram Lab, Department of Systems Biology, Technical University of Denmark, Kongens Lyngby, Denmark
}

Small Colony Variant (SCV) cells of bacteria are a slow-growing phenotype that result from specific defects in the electron transport chain. They form pinpoint colonies on agar plates and have a variety of phenotypic characteristics, such as altered carbon metabolism, decreased toxin and lytic enzyme production, aminoglycoside resistance, and increased intracellular persistence. They are clinically relevant in Staphylococcus aureus and Pseudomonas aeruginosa, serving as a reservoir for recurrent or prolonged infections. Here, we found that a SCV mutant in the foodborne pathogen Listeria monocytogenes (strain SCV E18), similar to the high persister mutant phenotype, survived significantly better than the wild type when exposed over a 48-h period to concentrations above Minimal Inhibitory Concentration for most tested antibiotics. SCV E18 survived more poorly than the wildtype in unactivated RAW264.7 macrophage cells, presumably because of its reduced listeriolysin O expression, however, it survived better in reactive oxygen species producing, phorbol 12-myristate 13-acetate-activated macrophages. Although SCV E18 was sensitive to oxygen as it entered the stationary phase, it was significantly more tolerant to $\mathrm{H}_{2} \mathrm{O}_{2}$ than the wild type, which may result from a shift in metabolism, however, further investigation is needed to resolve this. SCV E18 is a spontaneous mutant with a point mutation in the hemA gene. A wild type copy of hemA was complemented on plasmid pSOG30222, which restored the wild type phenotype. The results reported here suggest that the SCV of $L$. monocytogenes could be of clinical importance and highlight a need for adequate clinical screening for this phenotype, as it could affect antibiotic treatment outcomes.

Keywords: Listeria monocytogenes, small colony variants, antibiotic tolerance, persister cells, cell invasion, oxidative stress

\section{INTRODUCTION}

Listeria monocytogenes is a Gram-positive, foodborne pathogen that can cause the rare, but often lethal infection listeriosis. This typically affects pregnant women, neonates, the elderly and the immunocompromised, and can cause mortality rates of up to $34 \%$ (Farber and Peterkin, 1991). Upon entry into the gastrointestinal tract, L. monocytogenes infects host epithelial cells 
and immediately escapes the phagosome where it is free to replicate within the cytosol. Using actin-mediated mobility it spreads to neighboring cells and eventually enters the bloodstream, causing systemic listeriosis if unchecked by the innate immune system (Portnoy et al., 2002). Macrophages are a key part of the innate immune response and control the initial infection by engulfing and killing the bacteria with of a variety of antimicrobial compounds, such as reactive oxygen and nitrogen species (Shaughnessy and Swanson, 2007).

Treatment options for listeriosis are limited as many antibiotics are only bacteriostatic against L. monocytogenes. Ampicillin is the treatment of choice, and is sometimes used in combination with gentamicin, although the necessity of this supplemental treatment has been debated (Temple and Nahata, 2000; Mitjà et al., 2009). For patients who cannot tolerate ampicillin, a combination of trimethoprim and sulfamethoxazole, known as co-trimoxazole (SXT), or macrolides are substituted. (Temple and Nahata, 2000). While resistance in L. monocytogenes is uncommon, particularly for the clinically relevant antibiotics (Morvan et al., 2010), we recently demonstrated that this bacterium can form multi-antibiotic tolerant persister cells (Knudsen et al., 2013). However, it is not known if such cells are formed in vivo during infection, which could further complicate antibiotic treatment.

Persister cells are a very small subpopulation of bacteria that enter a dormant-like state making them refractory to most antibiotics. Their tolerance to antibiotics is not genetically inherited, and can be operationally defined by a biphasic killing curve when treated with bactericidal antibiotics and no observed increase in the Minimum Inhibitory Concentration (MIC) (Lewis, 2010). Persister cells are thought to exist in all bacteria and have been linked to recurrent infections with a number of diseases (Maisonneuve and Gerdes, 2014). Their formation can be either stochastic and the result of phenotypic switching from normally growing cells to those with reduced growth rates (Balaban et al., 2004), or they can be induced by certain conditions, such as biofilms (Lewis, 2010) or internalization by macrophages (Helaine et al., 2014). One of the primary genetic mechanisms of persister formation identified so far are toxin/antitoxin (TA) modules, which consist of a stable toxin that slows bacterial growth or metabolism and an unstable antitoxin that neutralizes the activity of the toxin under growth conditions. By repeatedly exposing Escherichia coli to high concentrations of ampicillin, Fridman et al. (2014) found that bacteria can also tolerate antibiotics by extending the lag-phase by mutations in a variety of pathways, essentially rendering the cells dormant until the transient antibiotic pressure is removed.

Another potential reservoir of persistent and recurrent bacterial infections is the so-called Small Colony Variant (SCV) (Kahl et al., 2016). The SCV is a slow growing phenotypic variant that forms pinpoint colonies when plated on agar, and is the result of either thymidine auxotrophy caused by mutations in the thymidylate synthase gene (Besier et al., 2007; Chatterjee et al., 2008) or an interruption in the electron transport chain (ETC), specifically resulting from an absence of menadione or hemin biosynthesis and metabolism. These variants can either be fixed, which is the result of a mutation in one of the biosynthesis or metabolism genes for the aforementioned compounds, or transient, alternating between the wild type and SCV phenotypes during replication (Edwards, 2012). Additional characteristics include altered carbon metabolism, reduced toxin and lytic enzyme production, and resistance to aminoglycosides (Proctor et al., 2014). Aminoglycosides have also been shown to select for the SCV phenotype, as the reduction in the membrane potential resulting from the disrupted ETC will lower the active transport necessary for aminoglycosides to cross the membrane (Balwit et al., 1994). The combination of trimethoprim/sulfamethoxazole (co-trimoxazole) has been found to select specifically for thymidine auxotrophic SCVs due to the anti-folate action of the drug (Garcia et al., 2013). While SCVs have been primarily studied in Staphylococcus aureus, they have been described in a number of bacteria including Pseudomonas aeruginosa, Eacherichia coli, Vibrio cholera, Salmonella, and Lactobacillus acidophilus (Proctor et al., 2006). Furthermore, they have been linked to a number of recurrent infections including those caused by S. aureus (Proctor et al., 1995), P. aeruginosa (Haussler et al., 1999) and E. coli (Roggenkamp et al., 1998; for a comprehensive review see: Kahl et al., 2016). Although typically less virulent due to their slower growth and reduced expression of virulence factors, such as toxins and lytic enzymes, their ability to adhere, invade and persist within the host cell increases, which is thought to be a crucial factor in the ability of SCVs to cause recurrent or persistent infections (Sendi and Proctor, 2009).

We have previously found that exposure to sub-lethal concentrations of triclosan with subsequent selection on gentamicin could generate stable L. monocytogenes SCV cells (Christensen et al., 2011), which all had mutations in one of the heme biosynthesis or metabolism genes and exhibited the same traits observed in SCVs of other species (e.g., colony size, decreased haemolytic activity, aminoglycoside resistance and altered carbon utilization). Our SCV E18 strain also showed reduced growth typical of SCVs from other species, taking roughly four more hours to reach the same maximal cell density as the wild type strain $\left(\sim 10^{9} \mathrm{CFU} / \mathrm{ml}\right)$, however, we also observed a sensitivity to aerated growth conditions (Kastbjerg et al., 2014), which to our knowledge has not been shown in SCVs from other species.

The phenotypic switching of the SCV and the persister cell are thought to be part of a bacterial bet-hedging strategy, allowing a small percentage of the population to survive and repopulate following stress exposure (Sousa et al., 2012). These two phenotypes share a number of other characteristics including slowed growth, intracellular persistence and a link to chronic infections. Thus, given these similarities, we speculate that a stable L. monocytogenes heme deficient SCV would show a similar tolerance toward a broad range of antibiotics that persister cells do. Furthermore, we sought to determine if L. monocytogenes SCVs are better at invading and surviving intracellularly, as they have been shown to in S. aureus (Sendi and Proctor, 2009). Taken together, these results will help to determine the clinical significance of Small Colony Variants in L. monocytogenes. 


\section{MATERIALS AND METHODS}

\section{Bacterial Strains and Growth Conditions}

Listeria monocytogenes strain N53-1 and a mutant thereof (E18) were included in the present study. N53-1 represents a foodprocessing plant persistent molecular subtype and was isolated from a fish smokehouse (Wulff et al., 2006). Strain E18 [denoted as strain (1) 1-1 in Christensen et al. (2011)] is a stable SCV of N53-1, with a Single Nucleotide Polymorphism (SNP) in the glutamyl tRNA reductase (hemA) gene (Kastbjerg et al., 2014), generated through an adaptive evolution experiment where N531 was exposed to sub-lethal levels of triclosan and subsequently selected on gentamicin (Christensen et al., 2011). Strains were stored at $-80^{\circ} \mathrm{C}$ and grown on Brain Heart Infusion (BHI; Oxoid $\mathrm{CM} 1135$ ) agar at $37^{\circ} \mathrm{C}$ for $24 \mathrm{~h}$. Overnight cultures were achieved by inoculating single colonies in $10 \mathrm{ml}$ of BHI broth (Oxoid CM 1135 ) and incubating at $37^{\circ} \mathrm{C}$ with shaking at $250 \mathrm{rpm}$.

\section{Preparation of Antibacterial Agents}

Fresh antibiotic solutions were prepared for each experiment: ampicillin (dissolved in sterile MilliQ water; Sigma-Aldrich A9518), erythromycin (dissolved in 96\% ethanol; Sigma-Aldrich E6376), gentamicin (dissolved in sterile MilliQ water; SigmaAldrich G3632), norfloxacin (dissolved in sterile MilliQ with 1\% glacial acetic acid; Fluka N9890) vancomycin (dissolved in sterile MilliQ; Sigma-Aldrich V2002) and co-trimoxazole, which is comprised of one part trimethoprim (dissolved in sterile MilliQ with $1 \%$ glacial acetic acid; Sigma-Aldrich 92131) and five parts sulphamethoxazole (dissolved in acetone; Fluka S7507). $\mathrm{H}_{2} \mathrm{O}_{2}$ (30\% in water; Sigma-Aldrich 216763) was diluted to $20 \mathrm{mM}$ in sterile MilliQ water.

\section{Minimal Inhibitory Concentration (MIC) Assay}

Antibiotic MIC values were determined in BHI broth as previously described (Cockerill et al., 2012). In brief, stationary phase cultures of N53-1 and SCV E18 were adjusted to $\mathrm{OD}_{600}=0.2$ and further diluted 1000-fold, corresponding to a $\mathrm{CFU} / \mathrm{ml}$ of $10^{5}$. The adjusted cultures were tested against norfloxacin, ampicillin, gentamicin, and co-trimoxazole (trimethoprim/sulfamethoxazole) using a twofold dilution series of concentrations ranging from 100 to $0.10 \mu \mathrm{g} / \mathrm{ml}$ in 96-well plates (Thermo Scientific 163320) and incubated at $37^{\circ} \mathrm{C}$. MIC for N53-1 and SCV E18 was determined by visual inspection after 24 and $48 \mathrm{~h}$ of incubation at $37^{\circ} \mathrm{C}$, respectively. Two biological replicates were performed.

\section{Killing Kinetic Assays}

To compare the antibiotic and $\mathrm{H}_{2} \mathrm{O}_{2}$ sensitivity of SCV E18 and N53-1, killing kinetics were performed according to Knudsen et al. (2013). Overnight cultures were diluted $10^{6}$-fold in $10 \mathrm{ml}$ BHI broth and incubated into early stationary phase for $16 \mathrm{~h}$ at $37^{\circ} \mathrm{C}$ with shaking at $250 \mathrm{rpm}$. Each culture was then adjusted to an optical density at $600 \mathrm{~nm}\left(\mathrm{OD}_{600}\right)$ of 0.2 in $2 \mathrm{ml} \mathrm{BHI}$ broth and challenged with antibiotic (30X MIC except nitrofurantoin, which was $10 \mathrm{X} \mathrm{MIC})$ at $37^{\circ} \mathrm{C}$. Bacterial counts were performed at $0,4,10,24,48$, and $72 \mathrm{~h}$. The antibiotics and concentrations used were norfloxacin $(100 \mu \mathrm{g} / \mathrm{ml})$, ampicillin (185 $\mu \mathrm{g} / \mathrm{ml})$, erythromycin $(6 \mu \mathrm{g} / \mathrm{ml})$, vancomycin $(47 \mu \mathrm{g} / \mathrm{ml})$, and co-trimoxazole $(95 \mu \mathrm{g} / \mathrm{ml})$. Cultures treated with $\mathrm{H}_{2} \mathrm{O}_{2}$ were grown and adjusted as described above, then treated with $20 \mathrm{mM}$ and incubated under stagnant conditions at $37^{\circ} \mathrm{C}$ for $2 \mathrm{~h}$. Three independent biological replicates were performed for each experiment and the limit of detection was $10^{2} \mathrm{CFU} / \mathrm{ml}$.

\section{Anaerobic Culturing Conditions}

To verify that E18 was sensitive to oxygen, it, along with N531 , were cultured in anaerobic conditions. Oxygen was removed from the media by autoclaving, followed by incubation in a HP0011 anaerobic jar (Oxoid) with an anaerobe gas generation bag (Sigma-Aldrich 68061) for $24 \mathrm{~h}$. Ten microliters of an overnight culture was added to the anaerobic BHI, along with a new anaerobe gas generation bag and incubated on the lab bench for $3 \mathrm{~h}$ to allow for an anaerobic atmosphere to be generated before incubation with shaking. The cultures were grown for $72 \mathrm{~h}$ at $37^{\circ} \mathrm{C}$ with shaking at $200 \mathrm{rpm}$. Two biological replicates were performed.

\section{hemA Complementation}

E18 carries several single nucleotide polymorphisms (SNPs) of which one is in the hemA gene (Kastbjerg et al., 2014). To verify the role of the SNP in hemA in the SCV E18 strain, a hemA complementation strain was constructed. The hemA gene with a $128 \mathrm{bp}$ upstream region including the native promoter was amplified from genomic DNA of N53-1 with the forward primer $5^{\prime}$-AAACTCGAGTCATCCGTTAACTCCTCG and the reverse primer 5'-AAAGAATTCATAGAAGGAGTTGGAATG GA, which contained terminal XhoI and EcoRI sites, respectively, using Phusion High Fidelity DNA Polymerase (NEB, M0530S). The pSOG30222 plasmid (Hain et al., 2008) and hemA amplicon were digested with XhoI and EcoRI, and ligated overnight using a 3:1 insert to vector ratio, then electroporated into $60 \mu \mathrm{l}$ of electrocompetent DH5 $\alpha$ cells using a MicroPulser (BioRad 165-2100). Transformants were selected on Luria-Bertani plates with $100 \mu \mathrm{g} / \mathrm{ml}$ erythromycin and verified by colony PCR and sequencing (GATC Biotech). Plasmids containing the correct pSOG30222::hemA construct, along with an unmodified pSOG30222 control plasmid, were transformed into the SCV E18 strain. Transformants were selected on BHI agar with $5 \mu \mathrm{g} / \mathrm{ml}$ erythromycin and confirmed with colony PCR and sequencing. With the exception of the killing kinetic assay, all complementation experiments were carried out in the presence of $5 \mu \mathrm{g} / \mathrm{ml}$ erythromycin. Colony pictures were taken of each strain with an Olympus BX51 microscope at $40 \times$ magnification.

\section{Macrophage Internalization and Survival Assay}

A murine macrophage cell line, RAW 264.7 (Sigma-Aldrich 91062702), was used to test bacterial internalization and intracellular survival using a protocol adapted from Bateman and Seed (Bateman and Seed, 2012). RAW 264.7 macrophages were grown in Dulbecco's Modified Eagle's Medium - high glucose 
(Sigma-Aldrich D6546), 10\% heat inactivated fetal bovine serum (Sigma-Aldrich F9665) and L-glutamine (Sigma-Aldrich G7513) in $150 \mathrm{cc}$ tissue culture flasks, for $10-15$ passages prior to use. A cell scraper was used to disjoin the adherent cells from the surface. RAW 264.7 cells were seeded into 24 -well plates at a density of $2 \times 10^{6}$ cells $/ \mathrm{ml}$ in RAW 264.7 culture media and then incubated for a total of $48 \mathrm{~h}$ at $37^{\circ} \mathrm{C}$ with $5 \% \mathrm{CO}_{2}$. During the $48 \mathrm{~h}$, one group of RAW 264.7 cells was stimulated for $18 \mathrm{~h}$ with $1 \mathrm{ng} / \mathrm{ml}$ IFN $\gamma$, required for macrophage activation, and a second group was stimulated with $100 \mathrm{nM}$ phorbol 12-myristate 13-acetate (PMA), a known inducer of nicotinamide adenine dinucleotide phosphate (NADPH) oxidase activity (Walloschke et al., 2010) for $2 \mathrm{~h}$, while a third control group was left unstimulated. Prior to infection, IFN $\gamma$ and PMA were removed from the RAW 264.7 containing wells. Overnight cultures of N53-1 and SCV E18 were diluted $10^{6}$-fold in $10 \mathrm{ml}$ of BHI media and grown at $37^{\circ} \mathrm{C}$ for $16 \mathrm{~h}$. L. monocytogenes cultures were adjusted to a density of $2 \times 10^{7} \mathrm{CFU} / \mathrm{ml}$ in $\mathrm{PBS}$ and added to the confluent RAW 264.7 containing wells, achieving a multiplicity of infection (MOI) of 10 . Plates were centrifuged for $5 \mathrm{~min}$ at $1,000 \mathrm{rpm}$ to synchronize the infection and incubated at $37^{\circ} \mathrm{C}$ for $1 \mathrm{~h}$. The plates were then incubated for an additional $2 \mathrm{~h}$ in media containing $100 \mu \mathrm{g} / \mathrm{ml}$ gentamicin, which was reduced to $50 \mu \mathrm{g} / \mathrm{ml}$ gentamicin for the remainder of the incubation. In order to verify that gentamicin did not favor the resistant SCV E18 during the $21 \mathrm{~h}$ incubation step, a control experiment was performed using the same methods as the unactivated macrophage experiment, with the exception that the media with $50 \mu \mathrm{g} / \mathrm{ml}$ gentamicin was substituted with PBS. To enumerate the surviving L. monocytogenes per well, RAW 264.7 cells were washed twice with PBS, followed by lysis with $0.1 \%$ Triton-X 100 (Sigma-Aldrich T8787) and then serial dilutions of the intracellular bacteria were plated on BHI agar plates. Internalization was measured following $3 \mathrm{~h}$ of incubation, while survival was measured after $24 \mathrm{~h}$.

\section{Statistical Analysis}

$\mathrm{CFU} / \mathrm{ml}$ values for each biological replicate were $\log _{10}$ transformed prior to statistical analysis. For the killing kinetic and $\mathrm{O}_{2}$ sensitivity assays, significant differences between the N53-1 and SCV E18 were determined using a paired $t$-test, where significance is equal to $P<0.05$. Significance between N53-1 and SCV E18 for the RAW 264.7 internalization and survival assays was determined using a paired $t$-test $(P<0.05)$ with the $\log _{10}$ transformed CFU/ml values. Significance for each strain between the RAW264.7 treatment groups was calculated using a one-way ANOVA test for the $\log _{10}$ transformed $\mathrm{CFU} / \mathrm{ml}$ values, where $P<0.05$ determined significance. Significance between the two strains within each RAW264.7 treatment group was calculated using a paired $t$-test $(P<0.05)$. The limit of detection for all $\mathrm{CFU} / \mathrm{ml}$ values was $10^{2}$. All data analysis was performed in Excel. Replicates falling below the detection limit were set to 99 for statistical analysis.

\section{RESULTS}

\section{SCV E18 Shows Increased Tolerance to Antibiotics}

The L. monocytogenes SCVs, selected on gentamicin, were resistant to other aminoglycosides (Kastbjerg et al., 2014). To determine if the SCV phenotype conveys resistance to other antibiotics, MIC values of selected antibiotics were tested for both N53-1 and SCV E18, which were found to be the same for the two strains (Table 1).

Because L. monocytogenes SCV grow slower than the wild type (Kastbjerg et al., 2014), and because of the lowered oxidative phosphorylation observed in other SCV organisms, such as S. aureus (Proctor et al., 1998), we hypothesized that L. monocytogenes SCVs would have an increased tolerance to other classes of antibiotics, which was evaluated using time dependent killing experiments. With the exception of erythromycin, the SCV E18 strain survived significantly better for each antibiotic challenge up to $48 \mathrm{~h}$ (Figure 1; ampicillin, $P=0.0007$; co-trimoxazole, $P=0.02$; vancomycin, $P=0.02$ and norfloxacin, $P=0.03$ ), with between one and threefold higher $\log _{10} \mathrm{CFU} / \mathrm{ml}$ as compared to the N53-1. When treated with norfloxacin, both N53-1 and SCV E18 exhibited a biphasic killing curve with a rapid decline to approximately three and five $\log _{10}$ $\mathrm{CFU} / \mathrm{ml}$, respectively, which remained stable throughout the $72 \mathrm{~h}$ experiment (Figure 1A). Ampicillin has a delayed bactericidal effect on L. monocytogenes (Winslow et al., 1983), which was also observed with SCV E18, as is evidenced by the roughly three-fold $\log _{10}$ reduction in CFU/ml over the $48-72 \mathrm{~h}$ time points (Figure 1B). However, this delayed bactericidal effect of ampicillin was not observed for N53-1 over the course of the

TABLE 1 | Minimum Inhibitory Concentration (MIC) values for Listeria monocytogenes wild type strain N53-1 strain and SCV E18 strain.

\begin{tabular}{|c|c|c|c|c|c|c|}
\hline \multirow[b]{2}{*}{ Strain } & \multicolumn{6}{|c|}{ MIC $\mu \mathrm{g} / \mathrm{ml}$} \\
\hline & Ampicillin & Co-trimoxazole & Norfloxacin & Erythromycin & Vancomycin & Gentamicin \\
\hline E18 & 3.13 & 0.78 & 3.13 & 0.20 & 1.56 & 12.50 \\
\hline $\begin{array}{l}\text { E18 } \\
\text { pSOG30222::hemA }\end{array}$ & 1.56 & 0.78 & 3.13 & - & 1.56 & 0.40 \\
\hline
\end{tabular}

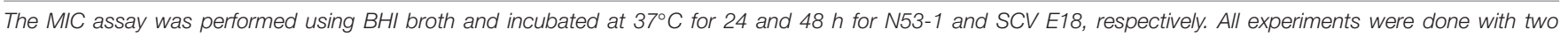
independent replicates. - , not tested. 


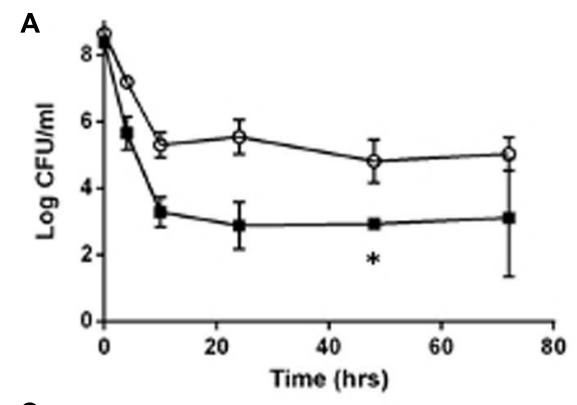

C

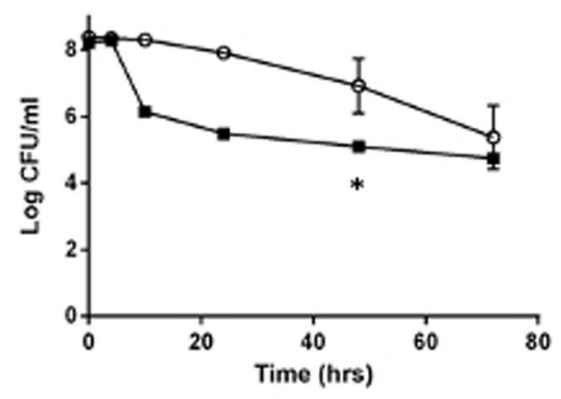

E

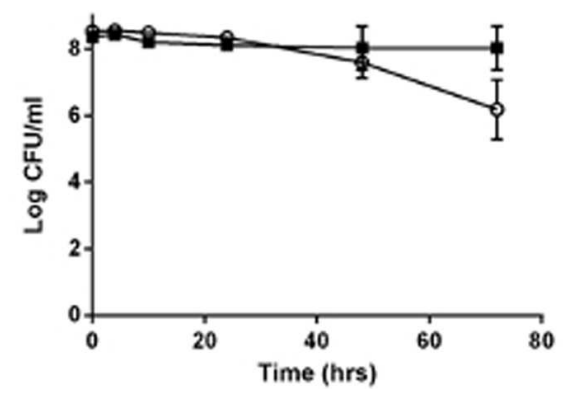

B

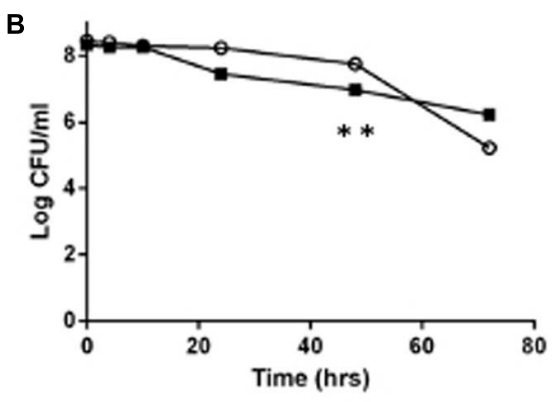

D

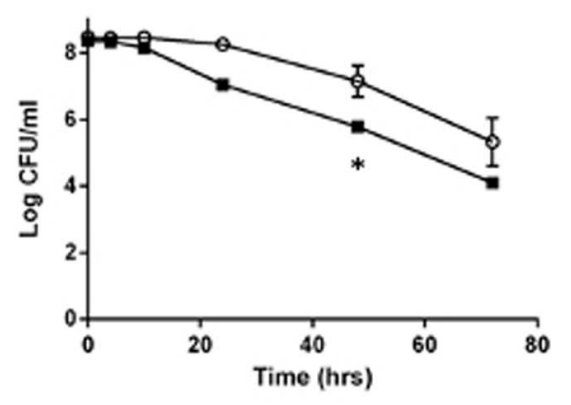

FIGURE 1 | Killing of Listeria monocytogenes N53-1 ( $\square$ ) and SCV E18 (O) over the course of $72 \mathrm{~h}$ under shaking conditions with (A) norfloxacin, (B) ampicillin, (C) co-trimoxazole, (D) vancomycin, and (E) erythromycin. Error bars represent standard deviation of the mean for three biological replicates. Significance at the 48-h time point was determined using a paired $t$-test $\left({ }^{*} p<0.05\right.$ and $\left.{ }^{* *} p<0.005\right)$.

$72 \mathrm{~h}$ killing kinetic (Figure 1B). By the end of the $72 \mathrm{~h}$ treatment with co-trimoxazole, SCV E18 decreased in a linear manner to a comparable level with N53-1 $\left(2.3 \times 10^{5}\right.$ and $5.6 \times 10^{4}$ $\mathrm{CFU} / \mathrm{ml}$, respectively; $P=0.45$; Figure $1 \mathrm{C})$. Vancomycin had an initial bacteriostatic effect on both strains, followed by linear killing, however, the bactericidal effect began earlier for N53-1 $(T=10)$, while the SCV E18 strain resisted killing until the $24 \mathrm{~h}$ time point (Figure 1D). Erythromycin was bacteriostatic on both strains up to the $48 \mathrm{~h}$ time point, however, bacterial counts for SCV E18 decreased after $48 \mathrm{~h}$, while the N53-1 remained stable (Figure 1E).

While the SCV phenotype in L. monocytogenes does not confer notable resistance to antibiotics besides the aminoglycosides, they survived significantly better under high antibiotic concentrations for up to $48 \mathrm{~h}$ under ampicillin or co-trimoxazole, and up to $72 \mathrm{~h}$ when exposed to norfloxacin or vancomycin. In contrast, by $72 \mathrm{~h}$ of exposure to erythromycin or ampicillin, the SCV has fewer $\mathrm{CFU} / \mathrm{ml}$ than the wild type.

\section{Oxygen Sensitivity, But $\mathrm{H}_{2} \mathrm{O}_{2}$ Resistance, of the SCV E18}

When grown under shaking conditions, SCV E18 grows to a maximum density of approximately $1 \times 10^{9} \mathrm{CFU} / \mathrm{ml}$ after $12 \mathrm{~h}$, where after the CFU/ml decreases rapidly, eventually dropping below the limit of detection after $24 \mathrm{~h}$ (Kastbjerg et al., 2014). Here, we grew cultures of the SCV E18, along with the N53-1, anaerobically to test if the observed decrease in CFU/ml is caused by exposure to oxygen. When grown anaerobically, cultures of the SCV E18 grow to higher densities $\left(8.1 \times 10^{8} \mathrm{CFU} / \mathrm{ml}\right)$ after $72 \mathrm{~h}$, than the N53-1 strain $\left(2.5 \times 10^{8} \mathrm{CFU} / \mathrm{ml} ; P=0.002\right)$ (Table 2), suggesting that oxygen is toxic to the SCV E18 strain. Under anaerobic growth conditions the SCV E18 strain retained the pin point colony morphology when plated, whereas the N53-1 strain exhibited variable colony size.

To test if the oxygen sensitivity was related to a general deficiency in the oxidative stress response, such as the absence of catalase in the SCV E18, we treated with $20 \mathrm{mM} \mathrm{H}_{2} \mathrm{O}_{2}$ 
TABLE 2 | Oxidative stress sensitivity of L. monocytogenes N53-1 and SCV E18 as measured by aerobic and anaerobic growth, and exposure to 20 mM $\mathrm{H}_{2} \mathrm{O}_{2}$ for $2 \mathrm{~h}$.

\begin{tabular}{|c|c|c|c|c|}
\hline & \multicolumn{4}{|c|}{ Bacterial count $\left(\log _{10} \mathrm{CFU} / \mathrm{ml}\right)$} \\
\hline & \multicolumn{2}{|c|}{ Growth in $\mathrm{BHI}$ for $72 \mathrm{~h}$} & \multicolumn{2}{|c|}{ Survival in $20 \mathrm{mM} \mathrm{H} \mathrm{H}_{2}$} \\
\hline & Aerobic & Anaerobic & $\mathbf{O h}$ & $2 \mathrm{~h}$ \\
\hline N53-1 & $9.4 \pm .01$ & $8.4 \pm 0.03$ & $7.2 \pm 0.04$ & $3.7 \pm 0.09$ \\
\hline E18 & $\mathrm{BD}$ & $8.9 \pm 0.02$ & $7.2 \pm 0.13$ & $6.3 \pm 1.02$ \\
\hline E18 pSOG30222::hemA & $8.1 \pm 0.09$ & - & $8.0 \pm 0.22$ & $5.2 \pm 0.17$ \\
\hline E18 pSOG30222 & $\mathrm{BD}$ & - & $8.0 \pm 0.06$ & $6.5 \pm 0.41$ \\
\hline
\end{tabular}

All experiments are $\log _{10} \mathrm{CFU} / \mathrm{ml}$ mean values of three biological replicates \pm standard deviation. BD, below the limit of detection; -, not tested.

for 2 h. Surprisingly, the SCV E18 survived significantly better $(P=0.008)$ than the wild type, with the number of colony forming bacteria following treatment being 33\% of the inoculum, whereas the N53-1 strain survived at a rate of $0.03 \%$ (Table 2). Thus, similar to the increased tolerance toward antibiotics, and regardless of the oxygen sensitivity and lack of catalase (Kastbjerg et al., 2014), the SCV E18 strain exhibits a greater ability to resist killing by $\mathrm{H}_{2} \mathrm{O}_{2}$ over the wild type.

\section{hemA Complementation Restores Wild Type Phenotype of SCV E18}

By genome sequencing and analyses, we previously demonstrated that all of the L. monocytogenes SCVs had several mutations, including a mutation in the hemA gene (Kastbjerg et al., 2014). To determine if the tested aspects of the SCV phenotype were due to the SNP in the hemA gene, a wild type hemA complemented strain of E18 was constructed. This complemented strain regained the large colony phenotype (Figure 2A), had restored gentamicin sensitivity identical to the N53-1 strain (Table 1), regained sensitivity to $\mathrm{H}_{2} \mathrm{O}_{2}$ and lost its sensitivity to oxygen, as demonstrated by the presence of viable colonies after culturing for $72 \mathrm{~h}$ (Table 2). Furthermore, the level of antibiotic tolerance was evaluated with a time dependent killing experiment using norfloxacin. The killing curve of the complemented hemA strain was significantly different from the empty vector control $(P=0.0000046)$, and was similar to the killing of N53-1 strain, while the empty vector control showed a very similar curve to its non-transformed counterpart SCV E18 (Figure 2B).

\section{SCV E18 Is Less Affected by Macrophage Status Than Wild Type N53-1}

We investigated the survival ability of the wild type and the SCV E18 in the IFN $\gamma$ activated and un-stimulated macrophage model. Additionally, due to the increased tolerance of SCV E18 toward $\mathrm{H}_{2} \mathrm{O}_{2}$, we used macrophages stimulated with PMA, a known activator of the NADPH oxidase pathway. Three hours following infection of the un-stimulated macrophage there was a high degree of internalization and intracellular growth of N53-1 and SCV E18, with $7.6 \log _{10} \mathrm{CFU} / \mathrm{ml}$ and $7.5 \log _{10} \mathrm{CFU} / \mathrm{ml}$ recovered, respectively, a difference that was not statistically significant $(P=0.77)$. These internalization findings were similar for the other two treatment groups (Figure 3A). By 24 h of incubation in

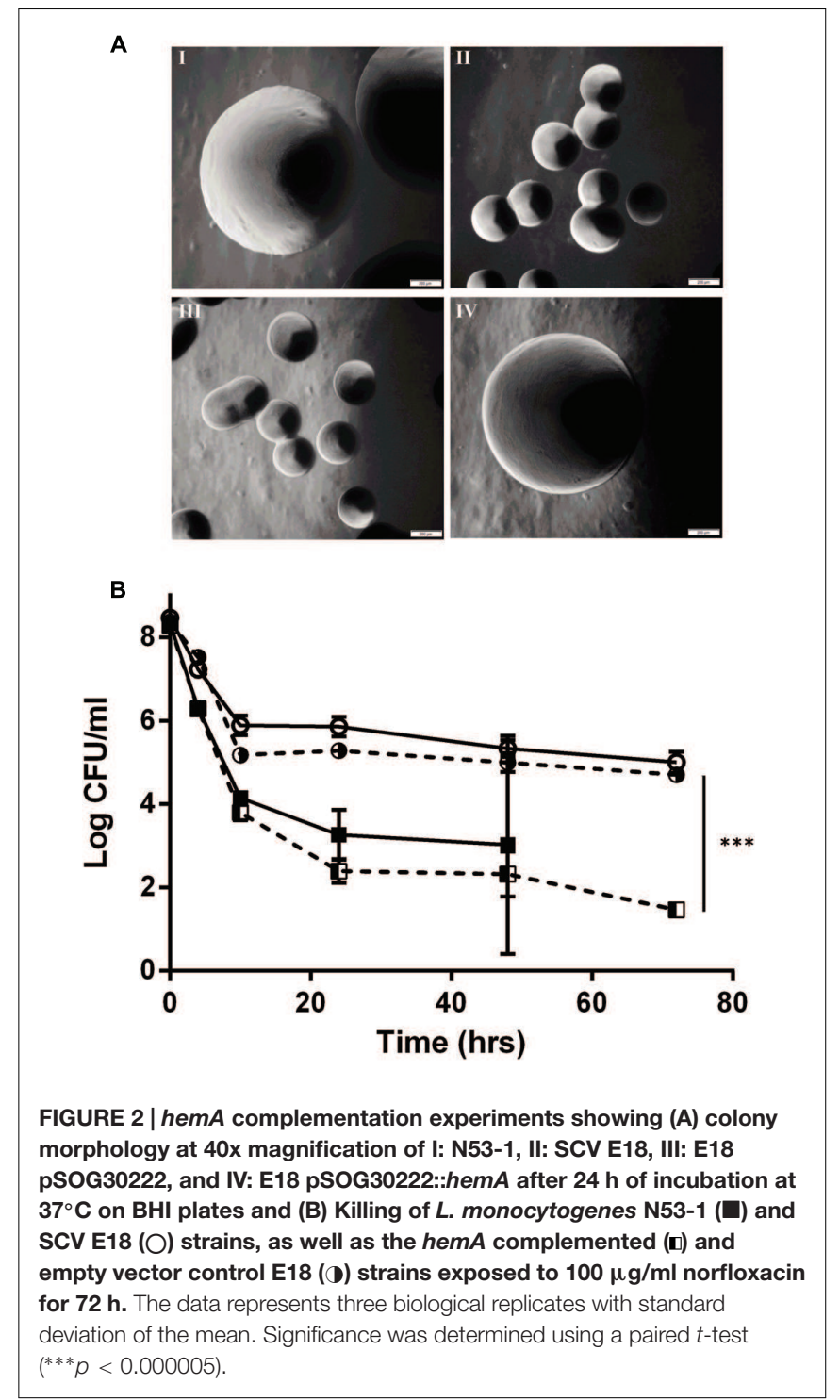

the un-stimulated macrophage, bacterial counts were drastically reduced for both strains, although N53-1 survived significantly better $(P=0.006)$ with $3.6 \log _{10} \mathrm{CFU} / \mathrm{ml}$ of the N53-1 and 2.3 $\log _{10} \mathrm{CFU} / \mathrm{ml}$ for SCV E18 (Figure 3B). When macrophages 

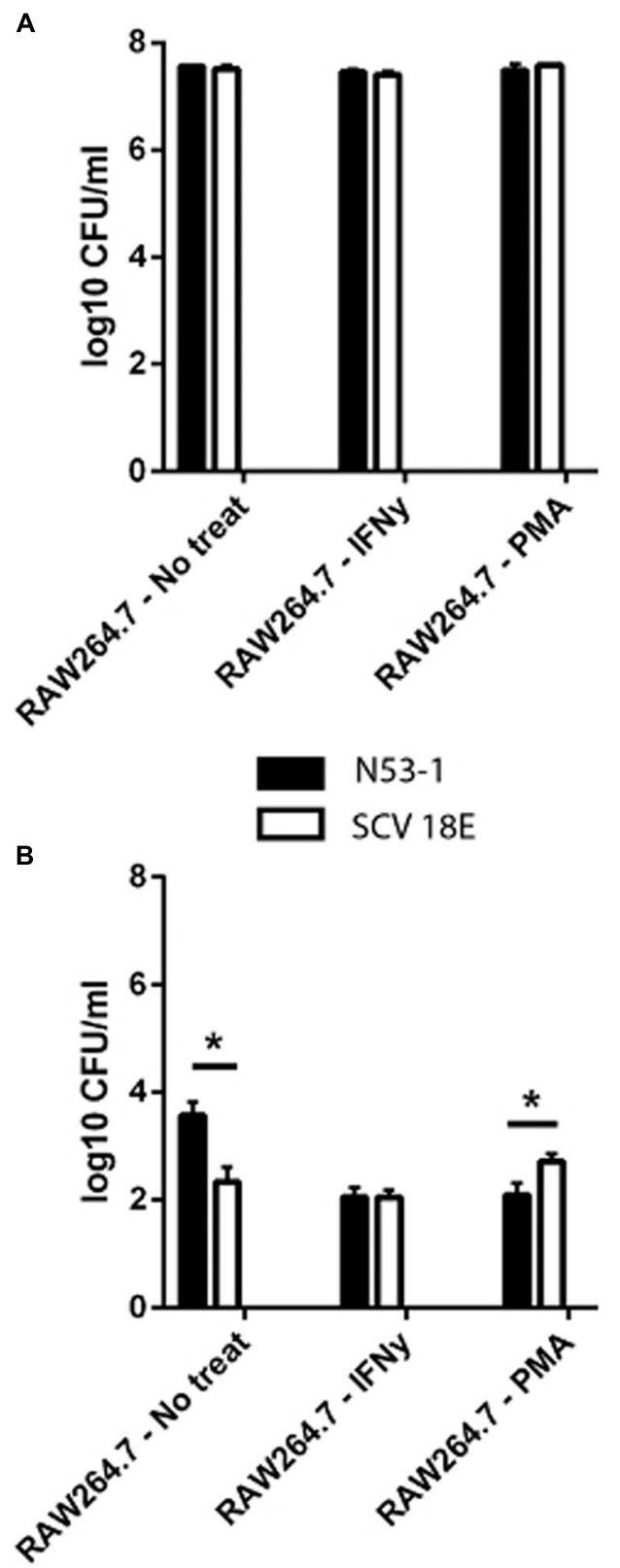

FIGURE 3 | Intracellular (A) internalization and (B) survival of L. monocytogenes N53-1 and SCV E18 in RAW264.7 cells treated with IFN $\gamma$, PMA and no treatment using a multiplicity of infection (MOI) of 10. Bacterial counts for the internalization assay were determined after $2 \mathrm{~h}$ of $100 \mu \mathrm{g} / \mathrm{ml}$ gentamicin exposure. Survival was determined after 21 additional hours of incubation with $50 \mu \mathrm{g} / \mathrm{ml}$ gentamicin. Values are displayed as the $\log _{10}$ transformed mean of three biological replicates with standard deviation. Significance was determined using a paired $t$-test $\left({ }^{*} p<0.05\right)$.

were activated with IFN $\gamma$, the survival difference between strains vanished, as the number of recovered N53-1 bacteria at $24 \mathrm{~h}$ was reduced to $2.06 \log _{10} \mathrm{CFU} / \mathrm{ml}$, while the SCV E18 burden was almost unchanged by IFN $\gamma$ activation $\left(2.15 \log _{10} \mathrm{CFU} / \mathrm{ml}\right)$ (Figure 3B). Increased reactive oxygen species (ROS) production, via PMA activation of the NADPH oxidase pathway, resulted in a slight, but significantly higher bacterial burden of macrophages infected with SCV E18 (2.7 $\left.\log _{10} \mathrm{CFU} / \mathrm{ml}\right)$, than N53-1 $\left(2.1 \log _{10}\right.$ $\mathrm{CFU} / \mathrm{ml} ; P=0.02$ ) (Figure 3B). While activation of macrophages with IFN $\gamma$ or stimulation with PMA significantly reduced the intracellular bacterial load of N53-1 $(P=0.01)$, the SCV E18 was not sensitive to either treatment and remained unchanged across groups $(P=0.17)$.

To summarize, macrophages were equally poor at controlling the bacterial burden of both N53-1 and SCV E18 in the short term $(T=3 \mathrm{~h})$, however, by $24 \mathrm{~h}$ the $\mathrm{CFU} / \mathrm{ml}$ for all intracellular bacteria had been severely reduced. While survival in the unactivated macrophage was attenuated for SCV E18, as compared to N53-1, it was unaffected by the status of the macrophage unlike N53-1, which was significantly reduced in CFU/ml upon macrophage activation with IFN $\gamma$ or stimulation with PMA.

\section{DISCUSSION}

The Small Colony Variant (SCV) has been described for decades in several bacteria such as $S$. aureus, E. coli and $P$. aeruginosa (Proctor et al., 2006). However, they were only recently identified in L. monocytogenes and, with the exception of aminoglycoside resistance (Kastbjerg et al., 2014), their importance with respect to the treatment of listeriosis has not been investigated. This study is the first to demonstrate that the SCV phenotype of L. monocytogenes caused by a single point mutation in the hem $A$ gene has the potential to complicate treatment by causing an increase in tolerance toward most of the clinically relevant antibiotics.

As shown previously (Kastbjerg et al., 2014), SCV E18 exhibited resistance to the aminoglycoside gentamicin, a defining trait of the electron-transport deficient SCV (Proctor et al., 2006). This is particularly relevant for listeriosis, as gentamicin is the most common secondary antibiotic used for treatment (Temple and Nahata, 2000). While the SCV E18 showed no increase in MIC for the other antibiotics tested, indicating a lack of acquired resistance, it was nonetheless able to survive lethal concentrations of norfloxacin, ampicillin (up to $48 \mathrm{~h}$ ), co-trimoxazole, and vancomycin significantly better than the wild type, which taken together, are the definition of antibiotic tolerance (Dimitrijovski et al., 2015). The increase in tolerance toward vancomycin, second generation fluoroquinolones (e.g., norfloxacin) and betalactams (e.g., ampicillin) has been observed with $S$. aureus SCVs and is presumably the result of their slow growing SCV phenotype (Chuard et al., 1997; Garcia et al., 2012; Lechner et al., 2012). Antibiotic tolerance poses a unique clinical dilemma, as any testing on isolates relying solely on MIC values could obscure the true antimicrobial susceptibility of the SCV and lead to sub-optimal treatment strategies.

In contrast to the other antibiotics, SCV E18 did not survive better under erythromycin pressure at any time point during the experiment and, as with ampicillin, became more sensitive after $48 \mathrm{~h}$ of killing. This could indicate that whatever protective effect the SCV phenotype contributes toward other antibiotics does not apply to the macrolide class, which target protein synthesis. Interestingly, S. aureus SCVs were not isolated from cystic fibrosis 
patients treated exclusively with the macrolide azithromycin, whereas when treated with other antibiotics, SCVs were observed in $46 \%$ of patients colonized with S. aureus (Kahl et al., 2003; Green et al., 2011). Although the SCV L. monocytogenes E18 tolerates ampicillin better than the wild type N53-1 in the short term, the sensitivity toward extended exposure (as evidenced by the pronounced decrease in bacterial count following $48 \mathrm{~h}$ of treatment) and the fact that long-term (between 2 and 8 weeks) ampicillin therapy is the primary treatment of choice for listeriosis (Temple and Nahata, 2000), could explain why the SCV phenotype has, to our knowledge, not been observed in in clinical cases. However, it is also possible that current screening procedures in the clinical laboratory are inadequate, leading the SCV sub-population to go unnoticed (Proctor et al., 2006). The slower growth of SCV E18 would likely cause it to be overshadowed in a mixed culture containing the wild type. Furthermore, the altered sugar metabolism and lack of catalase observed in SCV E18 (Kastbjerg et al., 2014), as well as the fact that some of the media used to grow L. monocytogenes contain the compounds SCVs are auxotrophic for, would complicate proper identification of any SCV L. monocytogenes clinical isolates.

We were expecting the SCV E18 to be more sensitive to $\mathrm{H}_{2} \mathrm{O}_{2}$ because it does not produce catalase, however, it was able to better withstand $\mathrm{H}_{2} \mathrm{O}_{2}$ than the wild type. As $\mathrm{H}_{2} \mathrm{O}_{2}$ reacts with the heme cofactor of proteins (Nagababu and Rifkind, 1998), our observation may simply be the result of fewer targets in the heme deficient SCV E18. Alternatively, tolerance toward $\mathrm{H}_{2} \mathrm{O}_{2}$ may be due to a change in the metabolism of the SCV, as suggested by Painter et al. (2015). In addition to showing that heme auxotrophic SCVs of $S$. aureus were more tolerant of $\mathrm{H}_{2} \mathrm{O}_{2}$, they added 4-hydroxy-2-heptylquinoline- $N$-oxide (HQNO), which can induce the SCV phenotype by blocking the electron transport chain of Gram-positive bacteria, to wild type S. aureus either hours or minutes before exposure to lethal levels of $\mathrm{H}_{2} \mathrm{O}_{2}$. Those bacteria grown for hours in the presence of HQNO survived the $\mathrm{H}_{2} \mathrm{O}_{2}$ exposure significantly better than the wild type, whereas those that received the HQNO only minutes before did not. This demonstrates that the tolerance is not simply the result of reduced electron transport, but as the authors suggest, a global physiological change caused by the loss of the electron transport chain (e.g., a switch to anaerobic metabolism), which could also explain our similar observation in L. monocytogenes.

In addition to the typical characteristics of the SCV, L. monocytogenes SCVs exhibit a unique sensitivity to growth under shaking conditions as they enter the stationary phase (Kastbjerg et al., 2014). Here, we demonstrate that this results from the presence of oxygen in the media. The addition of catalase, which converts $\mathrm{H}_{2} \mathrm{O}_{2}$ to water and oxygen, or heme (necessary for the biosynthesis of catalase) to the growth media abolished this effect, suggesting that the oxygen sensitivity is due to the inability of the heme-deficient SCV mutant to produce catalase (Kastbjerg et al., 2014). However, to our knowledge, this oxygen sensitivity has not been observed in heme-deficient SCVs of other catalase-dependent species (Roggenkamp et al., 1998; Zahra et al., 2013), and this fails to explain why the associated toxicity only manifests in the stationary phase. Few explanations seem to coincide with the observed increased tolerance toward
$\mathrm{H}_{2} \mathrm{O}_{2}$ and extended viability of SCV E18 cells when treated with antibiotics, however, we believe this is an important observation that warrants further research.

When the wild type and SCV E18 were inoculated with RAW264.7 murine macrophages over the course of $3 \mathrm{~h}$, we observed no difference between the internalization of the two strains. However, prolonged incubation of the infected macrophages revealed differences between the fates of each strain depending on the activation status of the macrophages. N53-1 survived better than SCV E18 in the naïve macrophages, however, this difference disappeared once macrophages were activated with IFN $\gamma$ where the wild type was reduced to the same CFU/ml as the mutant. The former observation is likely explained by the reduced expression of the pore-forming toxin Listeriolysin $\mathrm{O}$ (LLO) (Kastbjerg et al., 2014) in SCV E18, which has been shown to be produced in murine macrophages (Moors et al., 1999) and is necessary for L. monocytogenes to escape from the phagosome (Bielecki et al., 1990). The latter observation corresponds with studies showing that IFN $\gamma$-activated macrophages are able to block the escape of L. monocytogenes (Portnoy et al., 1989) by a process thought to be mediated by ROS inactivation of the LLO toxin (Myers et al., 2003). This suggests that macrophages need not first be activated by IFN $\gamma$ in order to control L. monocytogenes SCVs, which contrasts to SCVs from other species, and could explain the lack of observed clinical L. monocytogenes SCVs. On the other hand, LLO is the most immunogenic antigen for the $\mathrm{T}$ cell response, which mediates the ultimate removal and adaptive immunity to L. monocytogenes (Vijh and Pamer, 1997), thus a reduction in LLO expression may lead to a weaker adaptive immune response to SCV E18 and could explain the observation that mutants incapable of producing LLO persist for significantly longer in the bone marrow of infected mice (Hardy et al., 2009). Furthermore, the PMA stimulated macrophage showed that SCV E18 is significantly better at surviving the bactericidal effects of ROS within the phagosome, which is consistent with its observed increase in $\mathrm{H}_{2} \mathrm{O}_{2}$ tolerance, and could have implications on the fate of SCV L. monocytogenes in neutrophils that rely predominantly on NADPH oxidase for their bactericidal activity (Segal, 2005).

Our complementation experiment showed that this phenotype can arise through a single base-pair substitution in the hemA gene, and given the prevalence of verified SCV selection pressures L. monocytogenes faces both in the environment and during treatment (e.g., triclosan, gentamicin and co-trimoxazole), the probability of exposure to SCV L. monocytogenes seems high. Therefore, it is surprising that no clinical cases of SCV associated listeriosis have been reported. Perhaps the long treatment with ampicillin is enough to eradicate the SCV, or the immune system is better able to control SCV L. monocytogenes, as indicated by our findings with non-IFN $\gamma$ activated RAW264.7 murine macrophages. However, it seems equally likely that this elusive phenotype has gone unnoticed due to a lack of adequate screening procedures in clinical laboratories.

Much like persister cells, SCVs have also been shown to revert to the wildtype phenotype. When clinical and laboratory strains of $S$. aureus were cyclically grown with and without gentamicin, an identical proportion of SCVs upon each 
exposure to the antibiotic was observed (Massey et al., 2001). Drenkard and Ausubel (2002) found the same phenotypic switching in $P$. aeruginosa, and also identified a protein that modulates the switch between the wild type and SCV phenotypes. This phenotypic heterogeneity, often referred to as a bet-hedging strategy, allows a portion of the bacterial population to survive a stress (e.g., antibiotic exposure or the phagosome), without giving up any fitness advantages associated with mutation. It is plausible that the majority of SCVs in vivo are the result of phenotypic switching, and although, we observed no reversion with SCV E18, this mutant and others like it could represent a small subset of SCVs locked in the on position. This would add another treatment challenge, as the bacterial population as a whole would be protected from antibiotics, while simultaneously capable of full virulence (Tuchscherr et al., 2011). Reports of SCVs from a variety of bacterial species being linked to persistent and chronic infections are numerous (Proctor et al., 1995; von Eiff et al., 1997; Roggenkamp et al., 1998; Seifert et al., 1999; Häussler et al., 2003), and while uncommon, cases of recurrent listeriosis have been reported and the cause of these treatment failures were not determined (McLauchlin et al., 1991; Sauders et al., 2001; Kleemann et al., 2009).

Although L. monocytogenes SCVs may be unique in that macrophages are able to control them without first becoming activated by IFN $\gamma$, they may yet be able to better survive other aspects of the immune system, and the presence of an easily generated, multi-antibiotic tolerant sub-population

\section{REFERENCES}

Balaban, N. Q., Merrin, J., Chait, R., Kowalik, L., and Leibler, S. (2004). Bacterial persistence as a phenotypic switch. Science 305, 1622-1625. doi: 10.1126/science. 1099390

Balwit, J. M., van Langevelde, P., Vann, J. M., and Proctor, R. A. (1994). Gentamicin-resistant menadione and hemin auxotrophic Staphylococcus aureus persist within cultured endothelial cells. J. Infect. Dis. 170, 1033-1037. doi: 10.1093/infdis/170.4.1033

Bateman, S. L., and Seed, P. C. (2012). Epigenetic regulation of the nitrosative stress response and intracellular macrophage survival by extraintestinal pathogenic Escherichia coli. Mol. Microbiol. 83, 908-925. doi: 10.1111/j.13652958.2012.07977.x

Besier, S., Ludwig, A., Ohlsen, K., Brade, V., and Wichelhaus, T. A. (2007). Molecular analysis of the thymidine-auxotrophic small colony variant phenotype of Staphylococcus aureus. Int. J. Med. Microbiol. 297, 217-225. doi: 10.1016/j.ijmm.2007.02.003

Bielecki, J., Youngman, P., Connelly, P., and Portnoy, D. A. (1990). Bacillus subtilis expressing a haemolysin gene from Listeria monocytogenes can grow in mammalian cells. Nature 345, 175-176. doi: 10.1038/345175a0

Chatterjee, I., Kriegeskorte, A., Fischer, A., Deiwick, S., Theimann, N., Proctor, R. A., et al. (2008). In vivo mutations of thymidylate synthase (encoded by thyA) are responsible for thymidine dependency in clinical small-colony variants of Staphylococcus aureus. J. Bacteriol. 190, 834-842. doi: 10.1128/JB. 00912-07

Christensen, E. G., Gram, L., and Kastbjerg, V. G. (2011). Sublethal triclosan exposure decreases susceptibility to gentamicin and other aminoglycosides in Listeria monocytogenes. Antimicrob. Agents Chemother. 55, 4064-4071. doi: 10.1128/AAC.00460-11

Chuard, C., Vaudaux, P. E., Proctor, R. A., and Lew, D. P. (1997). Decreased susceptibility to antibiotic killing of a stable small colony variant of Staphylococcus aureus in fluid phase and on fibronectin-coated surfaces. J. Antimicrob. Chemother. 39, 603-608. doi: 10.1093/jac/39.5.603 is nonetheless a cause for concern. Awareness and adequate screening for SCVs in clinical isolates, such as extended incubation times and inclusion of isolates with non-standard sugar metabolism or those lacking catalase activity, would also help to determine the role (if any) this phenotype plays in human infection with $L$. monocytogenes, and we believe the evidence presented here warrants such measures being employed in clinical laboratories.

\section{AUTHOR CONTRIBUTIONS}

Conceived and designed the experiments: TC, LG, and GK. Performed the experiments: TC. Analyzed the data: TC, LG, and GK. Wrote the manuscript: TC, LG, and GK.

\section{FUNDING}

This work was supported by a Ph.D. grant from the Department of Systems Biology, Technical University of Denmark.

\section{ACKNOWLEDGMENT}

The authors would like to thank all members of the Gram Lab for their support and feedback.

Cockerill, F. R.,Wiker, M. A., Alder, J., Dudley, M. N., Eliopoulos, G. M., Ferraro, M. J., et al. (2012). Methods for Dilution Antimicrobial Susceptibility Testing for Bacteria that Grow Aerobically. Approved Standard - 9th Edn, Vol. 32. Wayne, PA: Clinical and Laboratory Standards Institute, 18-20.

Dimitrijovski, B., Jensen, S. O., Espedido, B. A., and van Hal, S. J. (2015). "Tolerance" of Misused Terminology? Enforcing Standardized Phenotypic Definitions. MBio 6:e00446-15. doi: 10.1128/mBio.00446-15

Drenkard, E., and Ausubel, F. M. (2002). Pseudomonas biofilm formation and antibiotic resistance are linked to phenotypic variation. Nature 416, 740-743. doi: $10.1038 / 416740 \mathrm{a}$

Edwards, A. M. (2012). Phenotype switching is a natural consequence of Staphylococcus aureus replication. J. Bacteriol. 194, 5404-5412. doi: 10.1128/JB.00948-12

Farber, J. M., and Peterkin, P. I. (1991). Listeria monocytogenes, a food-borne pathogen. Microbiol. Rev. 55, 476-511.

Fridman, O., Goldberg, A., Ronin, I., Shoresh, N., and Balaban, N. Q. (2014). Optimization of lag time underlies antibiotic tolerance in evolved bacterial populations. Nature 513, 418-421. doi: 10.1038/nature13469

Garcia, L. G., Lemaire, S., Kahl, B. C., Becker, K., Proctor, R. A., Denis, O., et al. (2012). Pharmacodynamic evaluation of the activity of antibiotics against hemin- and menadione-dependent small-colony variants of Staphylococcus aureus in models of extracellular (broth) and intracellular (THP-1 monocytes) infections. Antimicrob. Agents Chemother. 56, 3700-3711. doi: 10.1128/AAC.00285-12

Garcia, L. G., Lemaire, S., Kahl, B. C., Becker, K., Proctor, R. A., Denis, O., et al. (2013). Antibiotic activity against small-colony variants of Staphylococcus aureus: review of in vitro, animal and clinical data. J. Antimicrob. Chemother. 68, 1455-1464. doi: 10.1093/jac/dkt072

Green, N., Burns, J. L., Mayer-Hamblett, N., Kloster, M., Lands, L. C., Anstead, M., et al. (2011). Lack of association of small-colony-variant Staphylococcus aureus strains with long-term use of azithromycin in patients with cystic fibrosis. J. Clin. Microbiol. 49, 2772-2773. doi: 10.1128/JCM. 00835-11 
Hain, T., Hossain, H., Chatterjee, S. S., Machata, S., Volk, U., Wagner, S., et al. (2008). Temporal transcriptomic analysis of the Listeria monocytogenes EGD-e sigmaB regulon. BMC Microbiol. 8:20. doi: 10.1186/14712180-8-20

Hardy, J., Chu, P., and Contag, C. H. (2009). Foci of Listeria monocytogenes persist in the bone marrow. Dis. Mode. Mech. 2, 39-46. doi: 10.1242/dmm. 000836

Häussler, S., Lehmann, C., Breselge, C., Rohde, M., Classen, M., Tümmler, B., et al. (2003). Fatal outcome of lung transplantation in cystic fibrosis patients due to small-colony variants of the Burkholderia cepacia complex. Eur. J. Clin. Microbiol. Infect. Dis. 22, 249-253.

Haussler, S., Tummler, B., Weissbrodt, H., Rohde, M., and Steinmetz, I. (1999). Small-colony variants of Pseudomonas aeruginosa in cystic fibrosis. Clin. Infect. Dis. 29, 621-625. doi: 10.1086/598644

Helaine, S., Cheverton, A. M., Watson, K. G., Faure, L. M., Matthews, S. A., and Holden, D. W. (2014). Internalization of Salmonella by macrophages induces formation of nonreplicating persisters. Science 343, 204-208. doi: 10.1126/science. 1244705

Kahl, B. C., Becker, K., and Löffler, B. (2016). Clinical significance and pathogenesis of Staphylococcal small colony variants in persistent infections. Clin. Microbiol. Rev. 29, 401-427. doi: 10.1128/CMR.00069-15

Kahl, B. C., Duebbers, A., Lubritz, G., Haeberle, J., Koch, H. G., Ritzerfeld, B. et al. (2003). Population dynamics of persistent Staphylococcus aureus isolated from the airways of cystic fibrosis patients during a 6-year prospective study. J. Clin. Microbiol. 41, 4424-4427. doi: 10.1128/JCM.41.9.44244427.2003

Kastbjerg, V. G., Hein-Kristensen, L., and Gram, L. (2014). Triclosaninduced aminoglycoside-tolerant Listeria monocytogenes isolates can appear as small-colony variants. Antimicrob. Agents Chemother. 58, 3124-3132. doi: 10.1128/AAC.02266-13

Kleemann, P., Domann, E., Chakraborty, T., Bernstein, I., and Lohoff, M. (2009). Chronic prosthetic joint infection caused by Listeria monocytogenes. J. Med. Microbiol. 58, 138-141. doi: 10.1099/jmm.0.004234-0

Knudsen, G. M., Ng, Y., and Gram, L. (2013). Survival of bactericidal antibiotic treatment by a persister subpopulation of Listeria monocytogenes. Appl. Environ. Microbiol. 79, 7390-7397. doi: 10.1128/AEM.02184-13

Lechner, S., Lewis, K., and Bertram, R. (2012). Staphylococcus aureus persisters tolerant to bactericidal antibiotics. J. Mol. Microbiol. Biotechnol. 22, 235-244. doi: $10.1159 / 000342449$

Lewis, K. (2010). Persister cells. Annu. Rev. Microbiol. 64, 357-372. doi: 10.1146/annurev.micro.112408.134306

Maisonneuve, E., and Gerdes, K. (2014). Molecular mechanisms underlying bacterial persisters. Cell 157, 539-548. doi: 10.1016/j.cell.2014.02.050

Massey, R. C., Buckling, A., and Peacock, S. J. (2001). Phenotypic switching of antibiotic resistance circumvents permanent costs in Staphylococcus aureus. Curr. Biol. 11, 1810-1814. doi: 10.1016/S0960-9822(01)00507-3

McLauchlin, J., Audurier, A., and Taylor, A. G. (1991). Treatment failure and recurrent human listeriosis. J. Antimicrob. Chemother. 27, 851-857. doi: $10.1093 / \mathrm{jac} / 27.6 .851$

Mitjà, O., Pigrau, C., Ruiz, I., Vidal, X., Almirante, B., Planes, A.-M., et al. (2009). Predictors of mortality and impact of aminoglycosides on outcome in listeriosis in a retrospective cohort study. J. Antimicrob. Chemother. 64, 416-423. doi: $10.1093 / \mathrm{jac} / \mathrm{dkp} 180$

Moors, M. A., Levitt, B., Youngman, P., and Portnoy, D. A. (1999). Expression of listeriolysin $\mathrm{O}$ and ActA by intracellular and extracellular Listeria monocytogenes. Infect. Immun. 67, 131-139.

Morvan, A., Moubareck, C., Leclercq, A., Hervé-Bazin, M., Bremont, S., Lecuit, M., et al. (2010). Antimicrobial resistance of Listeria monocytogenes strains isolated from humans in France. Antimicrob. Agents Chemother. 54, 2728-2731. doi: 10.1128/AAC.01557-09

Myers, J. T., Tsang, A. W., and Swanson, J. A. (2003). Localized reactive oxygen and nitrogen intermediates inhibit escape of Listeria monocytogenes from vacuoles in activated macrophages. J. Immunol. 171, 5447-5453. doi: 10.4049/jimmunol.171.10.5447

Nagababu, E., and Rifkind, J. M. (1998). Formation of fluorescent heme degradation products during the oxidation of hemoglobin by hydrogen peroxide. Biochem. Biophys. Res. Commun. 247, 592-596. doi: $10.1006 /$ bbrc. 1998.8846
Painter, K. L., Strange, E., Parkhill, J., Bamford, K. B., Armstrong-James, D., and Edwards, A. M. (2015). Staphylococcus aureus adapts to oxidative stress by producing H2O2-resistant small-colony variants via the SOS response. Infect. Immun. 83, 1830-1844. doi: 10.1128/IAI.03016-14

Portnoy, D. A., Auerbuch, V., and Glomski, I. J. (2002). The cell biology of Listeria monocytogenes infection: the intersection of bacterial pathogenesis and cell-mediated immunity. J. Cell Biol. 158, 409-414. doi: 10.1083/jcb.200 205009

Portnoy, D. A., Schreiber, R. D., Connelly, P., and Tilney, L. G. (1989). Gamma interferon limits access of Listeria monocytogenes to the macrophage cytoplasm. J. Exp. Med. 170, 2141-2146. doi: 10.1084/jem.170.6.2141

Proctor, R. A., Kahl, B., Von Eiff, C., Vaudaux, P. E., Lew, D. P., and Peters, G. (1998). Staphylococcal small colony variants have novel mechanisms for antibiotic resistance. Clin. Infect. Dis. 27, 68-74. doi: 10.1086/ 514906

Proctor, R. A., Kriegeskorte, A., Kahl, B. C., Becker, K., Löffler, B., and Peters, G. (2014). Staphylococcus aureus Small Colony Variants (SCVs): a road map for the metabolic pathways involved in persistent infections. Front. Cell. Infect. Microbiol. 4:99. doi: 10.3389/fcimb.2014.00099

Proctor, R. A., van Langevelde, P., Kristjansson, M., Maslow, J. N., and Arbeit, R. D. (1995). Persistent and relapsing infections associated with smallcolony variants of Staphylococcus aureus. Clin. Infect. Dis. 20, 95-102. doi: 10.1093/clinids/20.1.95

Proctor, R. A., von Eiff, C., Kahl, B. C., Becker, K., McNamara, P., Herrmann, M., et al. (2006). Small colony variants: a pathogenic form of bacteria that facilitates persistent and recurrent infections. Nat. Rev. Microbiol. 4, 295-305. doi: 10.1038/nrmicro1384

Roggenkamp, A., Sing, A., Hornef, M., Brunner, U., Autenrieth, I. B., and Heesemann, J. (1998). Chronic prosthetic hip infection caused by a smallcolony variant of Escherichia coli. J. Clin. Microbiol. 36, 2530-2534.

Sauders, B. D., Wiedmann, M., Desjardins, M., Fenlon, C., Davenport, N., Hibbs, J. R., et al. (2001). Recurrent Listeria monocytogenes infection: relapse or reinfection with a unique strain confirmed by molecular subtyping. Clin. Infect. Dis. 33, 257-259. doi: 10.1086/321821

Segal, A. W. (2005). How neutrophils kill microbes. Annu. Rev. Immunol. 23, 197-223. doi: 10.1146/annurev.immunol.23.021704.115653

Seifert, H., Von Eiff, C., and Fätkenheuer, G. (1999). Fatal case due to methicillinresistant Staphylococcus aureus small colony variants in an AIDS patient. Emerg. Infect. Dis. 5, 450-453. doi: 10.3201/eid0503.990319

Sendi, P., and Proctor, R. A. (2009). Staphylococcus aureus as an intracellular pathogen: the role of small colony variants. Trends Microbiol. 17, 54-58. doi: 10.1016/j.tim.2008.11.004

Shaughnessy, L. M., and Swanson, J. A. (2007). The role of the activated macrophage in clearing Listeria monocytogenes infection. Front. Biosci. 12:2683-2692. doi: 10.2741/2364

Sousa, A. M., Machado, I., and Pereira, M. O. (2012). "Phenotypic switching: an opportunity to bacteria thrive," in Science against Microbial Pathogens: Communicating Current Research and Technological Advances, ed. A. MéndezVilas (Berlin: Springer), 252-262.

Temple, M. E., and Nahata, M. C. (2000). Treatment of listeriosis. Ann. Pharmacother. 34, 656-661. doi: 10.1345/aph.19315

Tuchscherr, L., Medina, E., Hussain, M., Völker, W., Heitmann, V., Niemann, S., et al. (2011). Staphylococcus aureus phenotype switching: an effective bacterial strategy to escape host immune response and establish a chronic infection. EMBO Mol. Med. 3, 129-141. doi: 10.1002/emmm.201 000115

Vijh, S., and Pamer, E. G. (1997). Immunodominant and subdominant CTL responses to Listeria monocytogenes infection. J. Immunol. 158, 3366-3371.

von Eiff, C., Bettin, D., Proctor, R. A., Rolauffs, B., Lindner, N., Winkelmann, W., et al. (1997). Recovery of small colony variants of Staphylococcus aureus following gentamicin bead placement for osteomyelitis. Clin. Infect. Dis. 25, 1250-1251. doi: 10.1086/516962

Walloschke, B., Fuhrmann, H., and Schumann, J. (2010). Macrophage cell line RAW264.7 but not P-388D1 is an appropriate in vitro-model for studying oxidative burst as well as cytokine production in context of fatty acid enrichment. Cell. Immunol. 262, 58-61. doi: 10.1016/j.cellimm.2009.12.008

Winslow, D., Damme, J., and Dieckman, E. (1983). Delayed bactericidal activity of beta-lactam antibiotics against Listeria monocytogenes: antagonism of 
chloramphenicol and rifampin. Antimicrob. Agents Chemother. 23, 555-558. doi: 10.1128/AAC.23.4.555

Wulff, G., Gram, L., Ahrens, P., and Vogel, B. F. (2006). One group of genetically similar Listeria monocytogenes strains frequently dominates and persists in several fish slaughter- and smokehouses. Appl. Environ. Microbiol. 72, 43134322. doi: 10.1128/AEM.02288-05

Zahra, M., Ferreri, M., Alkasir, R., Yin, J., Han, B., and Su, J. (2013). Isolation and characterization of small-colony variants of ornithobacterium rhinotracheale. J. Clin. Microbiol. 51, 3228-3236. doi: 10.1128/JCM. 01337-13
Conflict of Interest Statement: The authors declare that the research was conducted in the absence of any commercial or financial relationships that could be construed as a potential conflict of interest.

Copyright (c) 2016 Curtis, Gram and Knudsen. This is an open-access article distributed under the terms of the Creative Commons Attribution License (CC BY). The use, distribution or reproduction in other forums is permitted, provided the original author(s) or licensor are credited and that the original publication in this journal is cited, in accordance with accepted academic practice. No use, distribution or reproduction is permitted which does not comply with these terms. 
OPEN ACCESS

Edited by:

Ivan Rychlik,

Veterinary Research Institute,

Czech Republic

Reviewed by:

Vishvanath Tiwari,

Central University of Rajasthan, India

Dinesh Sriramulu,

Shres Consultancy (Life Sciences),

India

*Correspondence:

Katharina Schaufler

katharina.schaufler@fu-berlin.de

Specialty section:

This article was submitted to Antimicrobials, Resistance and

Chemotherapy,

a section of the journal

Frontiers in Microbiology

Received: 15 December 2015 Accepted: 03 March 2016 Published: 17 March 2016

Citation

Schaufler K, Semmler T, Pickard DJ,

de Toro $M$, de la Cruz F, Wieler LH,

Ewers $C$ and Guenther S (2016)

Carriage of Extended-Spectrum

Beta-Lactamase-Plasmids Does Not

Reduce Fitness but Enhances

Virulence in Some Strains of

Pandemic E. coli Lineages.

Front. Microbiol. 7:336

doi: 10.3389/fmicb.2016.00336

\section{Carriage of Extended-Spectrum Beta-Lactamase-Plasmids Does Not Reduce Fitness but Enhances Virulence in Some Strains of Pandemic E. coli Lineages}

\author{
Katharina Schaufler ${ }^{1 *}$, Torsten Semmler ${ }^{1,2}$, Derek J. Pickard ${ }^{3}$, María de Toro $^{4}$, \\ Fernando de la Cruz ${ }^{4}$, Lothar H. Wieler ${ }^{1,2}$, Christa Ewers ${ }^{5}$ and Sebastian Guenther ${ }^{1}$

\begin{abstract}
${ }^{1}$ Veterinary Faculty, Institute of Microbiology and Epizootics, Freie Universität Berlin, Berlin, Germany, ${ }^{2}$ Robert Koch Institute, Berlin, Germany, ${ }^{3}$ Wellcome Trust Sanger Institute, Cambridge, UK, ${ }^{4}$ Departamento de Biología Molecular, Instituto de Biomedicina y Biotecnología de Cantabria (UC-SODERCAN-CSIC), Universidad de Cantabria, Santander, Spain, ${ }^{5}$ Veterinary Faculty, Institute of Hygiene and Infectious Diseases of Animals, Justus-Liebig-Universität Giessen, Giessen, Germany
\end{abstract}

Pathogenic ESBL-producing E. coli lineages occur frequently worldwide, not only in a human health context but in animals and the environment, also in settings with low antimicrobial pressures. This study investigated the fitness costs of ESBL-plasmids and their influence on chromosomally encoded features associated with virulence, such as those involved in the planktonic and sessile behaviors of ST131 and ST648 E. coli. ESBL-plasmid-carrying wild-type E. coli strains, their corresponding ESBL-plasmid-"cured" variants (PCV), and complementary ESBL-carrying transformants were comparatively analyzed using growth curves, Omnilog ${ }^{\circledR}$ phenotype microarray (PM) assays, macrocolony and biofilm formation, swimming motility, and RNA sequence analysis. Growth curves and PM results pointed toward similar growth and metabolic behaviors among the strains. Phenotypic differences in some strains were detected, including enhanced curli fimbriae and/or cellulose production as well as a reduced swimming capacity of some ESBL-carrying strains, as compared to their respective PCVs. RNA sequencing mostly confirmed the phenotypic results, suggesting that the chromosomally encoded $\operatorname{csg} D$ pathway is a key factor involved. These results contradict the hypothesis that ESBL-plasmid-carriage leads to a fitness loss in ESBL-carrying strains. Instead, the results indicate an influence of some ESBL-plasmids on chromosomally encoded features associated with virulence in some E. coli strains. In conclusion, apart from antibiotic resistance selective advantages, ESBL-plasmid-carriage may also lead to enhanced virulence or adaption to specific habitats in some strains of pandemic ESBL-producing E. coli lineages.

Keywords: ESBL-producing E. coli, ESBL-plasmids, fitness costs, enhanced virulence, biofilm formation, plasmid and host interaction 


\section{INTRODUCTION}

The global emergence of antimicrobial resistance, including extended-spectrum beta-lactamases (ESBL), is driven not only by plasmids encoding for these factors, but is also crucially influenced by pandemic bacterial clonal lineages (Naseer and Sundsfjord, 2011). The success of the pathogenic ESBLproducing E. coli clonal lineage of sequence type ST131 and virulence-associated phylogenetic group B2 is particularly noteworthy (Nicolas-Chanoine et al., 2014). B2-ST131 is found worldwide in environments with high antimicrobial selection pressures, including human and veterinary clinics and communities (Nicolas-Chanoine et al., 2008; Ewers et al., 2010). ST131 is also found in remote areas and wildlife (Bonnedahl et al., 2014), where antimicrobial influence is thought to be of lower importance. Despite the recognition of the human clinical reservoir as most abundant, several studies have demonstrated increasing prevalence of ST131 in animals and extra-clinical settings (Ewers et al., 2012). The acquisition of ESBL-genes happened over time, and the initial spread of ST131 most likely evolved from an emergence of chromosomally encoded fluoroquinolone resistance (Nicolas-Chanoine et al., 2014). Besides ST131, several STs, including ST648, ST617, ST167, ST410, ST224, and ST117, appear to be associated with ESBLproduction, which demonstrates that ESBL-producing isolates are not equally distributed over all phylogenetic backgrounds (Ewers et al., 2012). This is expected in cases of solely plasmiddriven spread (Ewers et al., 2012). Regarding the success of pathogenic clonal lineages of B2-ST131, similar scenarios might also apply to CTX-M-producing lineages of ST648 belonging to phylogenetic group D, also known for harboring virulent isolates (Pitout, 2012; Ewers et al., 2014). Many strains of ST131 and ST648 carry plasmids encoding ESBL-enzymes, often of the CTXM-15 type, and have become problematic due to limitations in antimicrobial therapies (Johnson et al., 2010). Besides ESBLencoding genes, plasmids of these $E$. coli isolates contain antibiotic resistance determinants affecting various antimicrobial classes, which often results in multi-drug resistant phenotypes (Woodford et al., 2011). Prior studies suggest trade-offs between antibiotic-resistance and fitness in such strains (Dasilva and Bailey, 1986; Lenski, 1997; Andersson and Hughes, 2010). However, this does not necessarily apply to ST131 and ST648. In contrast, the combination of multi-resistance, virulence and phylogenetic background is hypothesized to be a recipe for their successful pandemic spread (Johnson et al., 2010; Pitout, 2012; Calhau et al., 2013). In addition to antibiotic resistance genes, ESBL-plasmids harbor non-resistance factors, which are partly unexplored. These include fertility and virulence factors, genes for plasmid maintenance including toxin-antitoxin systems, resistances against heavy metals (Seiler and Berendonk, 2012; Schaufler et al., 2013), and putative protein-coding genes (Smet et al., 2010b).

Why do ESBL-associated STs exist, and why are certain clonal lineages so successful not only in environments with high and moderate antibiotic pressures, but also in antimicrobially isolated areas? It might be due to their ubiquity and frequent detection, whereas rare lineages are found less often, or for reasons beyond antibiotic resistance such as virulence-associated factors. One possibility has rarely been studied: the interaction between ESBL-plasmids and the chromosomal content of particular clonal lineages. The influence of non-resistance genes on the chromosome may be of particular importance. For the closely related species Klebsiella pneumoniae, it was shown that acquisition of ESBL-plasmids lead to expression changes of chromosomally encoded fimbriae genes, subsequently affecting the overall invasion ability of tested strains (Sahly et al., 2008). Another main bacterial virulence factor is the chromosomally encoded ability to form biofilms. The subtle interactions between biofilm formation and its counterpart, motility, are mostly regulated by the transcriptional regulator $\operatorname{csg} D$ (curlin subunit gene D; Hammar et al., 1995; Dudin et al., 2014). Biofilm formation has previously been linked to antimicrobial resistance (Ito et al., 2009); however, the influence of ESBL-plasmids on $\operatorname{csg} D$-associated virulence features remains to be investigated.

This study addressed two hypotheses: (i) ESBLplasmid carriage does not negatively influence the host's growth/metabolic fitness; and (ii) ESBL-plasmid carriage supports the host through the ESBL-plasmid's influence on chromosomally encoded virulence-associated features.

\section{MATERIALS AND METHODS}

\section{Strains}

Seven wild-type (WT) ESBL-producing E. coli strains, their ESBL-“plasmid" cured variants (PCV) (Schaufler et al., 2013), and transformants $(\mathrm{T})$ with the reintroduced large ESBL-plasmid constructed from PCVs (Green and Sambrook, 2012) were analyzed in this study; in total, 21 strains were studied. The WT B2-ST131 and D-ST648 strains originated from different hosts including humans, companion animals, and wild birds (Supplementary Table S1).

\section{ESBL-Plasmid-"Curing" and Transformations}

As previously described by Schaufler et al. (2013), large ESBL-plasmids were extracted from seven WT ESBL-carrying E. coli strains using a heat technique (Dale and Park, 2004). Loss of the large ESBL-plasmid as well as the clonal character of WT and its corresponding PCV strain were tested via plasmid-profile analysis and XbaI-pulsed-field gel electrophoresis (PFGE) (Schierack et al., 2009). To assure genetic identity and to exclude chromosomal changes in the PCV, the whole genome sequences of WT and PCV strains were analyzed using bioinformatics. This included a pairwise comparison of the number of orthologous genes using the OrthoMCL pipeline (Chen et al., 2006) and computation of the phylogenetic distances of all strains based on their Maximum Common Genome (MCG) (Von Mentzer et al., 2014) as previously described (Schaufler et al., 2013). Transformants with the reintroduced large ESBL-plasmid were constructed to verify the observed phenotypic differences (Supplementary Table S1). PCVs functioned as electrocompetent acceptor strains; thus, the respective large ESBL-plasmid was transformed via electroporation (Green and Sambrook, 2012). Transformed 
strains were screened for ESBL-production on cefotaxime ( $4 \mu \mathrm{g} / \mathrm{mL}$; Sigma-Aldrich, Taufkirchen, Germany) containing CHROMagar $^{\text {TM }}$ (MAST Diagnostica, Reinfeld, Germany) plates for ESBL-enzyme-production. Plasmid profile analysis was used to evaluate the success of electroporation.

\section{ESBL-Plasmid Characteristics}

Whole genomes of all WT and their corresponding PCV strains were sequenced using an Illumina HiSeq2000 sequencer in collaboration with the Wellcome Trust Sanger Institute (Cambridge, United Kingdom). The resulting reads were used for a de novo assembly (CLC Genomics Workbench 6.5, CLC Bio, Denmark). PLACNET analysis (Lanza et al., 2014) was then performed for all genomes to extract putative plasmid contiguous sequences (contigs) and to assign them to the large ESBL-plasmid and to smaller, additional non-ESBL-plasmids. These were confirmed via BLAST analysis, in which they were compared to reference sequences of known plasmids. All previously defined plasmid contigs showed a high similarity to parts of plasmids that have already been described. Annotation of the contigs was performed using the annotation feature of the program Geneious version 7.1.2 (Kearse et al., 2012) with $100 \%$ similarity to an inhouse plasmid reference data base (data not shown). ESBL types, relaxase (REL) and plasmid replication initiator (RIP) proteins, incompatibility (Inc) groups, approximate ESBL-plasmid sizes, the number of additional plasmids, and virulence factors were detected based on PLACNET ESBL-plasmid sequences and using VirulenceFinder 1.4 (Joensen et al., 2014) and ResFinder 2.1 (Zankari et al., 2012; Table 1). BLAST ring image generator (BRIG) (Alikhan et al., 2011) was used to visualize the annotated PLACNET ESBL-plasmid sequences (pIMT17433, pIMT19205, pIMT27685, pIMT16316, pIMT17887, pIMT21183, and pIMT23463) of the seven WT strains with pEC_L8 as a reference ESBL-plasmid (Smet et al., 2010b; Figure 1).

\section{Growth Curves}

Growth curves in LB medium were performed in triplicate using standard protocols.

\section{Omnilog ${ }^{\circledR}$ Phenotype Microarray (PM)}

Using 96-well microtiter plates spotted with different substrates, growth/metabolic activity in 379 single substrates and sensitivity to 48 antimicrobial and chemical compounds was analyzed for all strains [Omnilog ${ }^{\circledR}$ (Biolog, Hayward, USA), Supplementary Table S2]. Three biological replicates were tested on six PM plates (PM1 and PM2: carbon sources; PM3: nitrogen sources; PM4: phosphorus and sulfur sources [http://www.biolog.com/pdf/ pm_lit/PM1-PM10.pdf]; PM13 and PM14: chemical sensitivity [http://www.biolog.com/pdf/pm_lit/PM11-PM20.pdf]). Plates were inoculated with the bacterial suspension according to Omnilog ${ }^{\circledR}$ PM protocols. Plates were incubated for $48 \mathrm{~h}$ at $37^{\circ} \mathrm{C}$. Respiration was measured by dye (tetrazolium violet) reduction every $15 \mathrm{~min}$. The calculated longitudinal respiration kinetics were analyzed in R (Vaas et al., 2013). In confidence interval plots computed with extract defined by enlisted metadata for the parameter "area under the curve," only wells in which the normalized mean point estimates of WT and T groups showed 

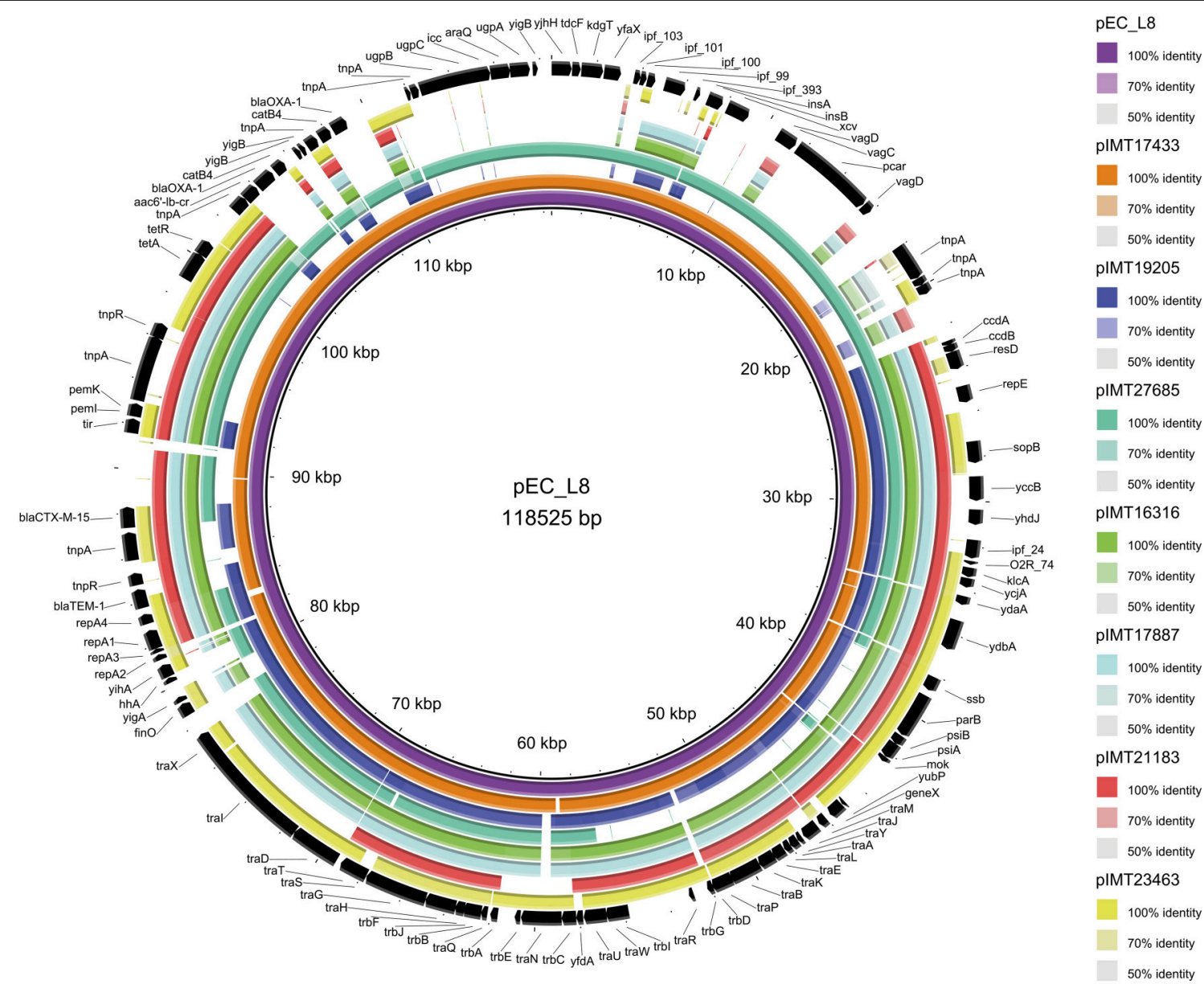

FIGURE 1 | Circular visualization of the seven wild-type ESBL-plasmid sequences as compared to E. coli ESBL-plasmid pEC_L8 using BRIG (Alikhan et al., 2011).

no overlapping $95 \%$ confidence intervals as compared to those of the PCV group, were considered to be significantly different. The group means of these wells underwent further statistical analysis using Tukey's method for multiple comparisons.

\section{Macrocolonies}

Three microliters of overnight culture (from a single colony grown in $5 \mathrm{~mL}$ BHI broth) from all strains were dropped on span agar plates (H. Carroux, Germany) with or without sodium chloride (5\%) and congo red solution $[0.5 \%$ congo red (SigmaAldrich, Taufkirchen, Germany) and $0.25 \%$ coomassie-brilliantblue (Carl Roth, Karlsruhe, Germany) diluted in ethanol]. Plates were incubated for 5 days at $28^{\circ} \mathrm{C}$ (Romling, 2005; Richter, 2011). Following an initial comprehensive screening to detect differences between WT and PCV strains regarding their cellulose and/or curli fimbriae production, follow-up runs were performed on plates containing and lacking sodium chloride for all strains (Table 2). These follow-up runs were repeated six times. Reference strains (AAEC189, Blomfield et al., 1991, IMT26949, and W3110 Hayashi et al., 2006) were included in all runs for all plates and at all temperatures.
TABLE 2 | Cellulose and curli fimbriae expression by 17433 and 16316 strains on span agar plates with congo red, with or without sodium chloride, incubated at $28^{\circ} \mathrm{C}$ for 5 days.

\begin{tabular}{lccccc}
\hline Strain & \multicolumn{2}{c}{ With sodium chloride } & & \multicolumn{2}{c}{ Without sodium chloride } \\
\cline { 2 - 3 } \cline { 5 - 6 } & Cellulose & Curli & & Cellulose & Curli \\
\hline IMT17433 & $* * * * * *$ & $* * * * * *$ & & $* * * * * *$ & $* * * * * *$ \\
PCV17433 & & & & \\
T17433 & $* * * * *$ & $* * * * * *$ & & $* * * * *$ & \\
IMT16316 & $* * * * * *$ & $* * * * * *$ & & \\
PCV16316 & & $* * * * * *$ & & \\
T16316 & $* * * * * *$ & $* * * * * *$ & & \\
& & & & &
\end{tabular}

Six repetition runs followed a comprehensive initial screening. *, positive cellulose and/or curli fimbriae phenotype per run; 16316 strains were not tested on plates lacking sodium chloride as they did not show any differences during the initial screening.

\section{Biofilm Formation Assays}

Overnight culture (from a single colony grown in $5 \mathrm{~mL} \mathrm{LB}$ and minimal medium M63 overnight, Pardee et al., 1959) was set to optical density $\mathrm{OD}_{600}=0.05$. Technical triplicates of 
the suspension were added to a 96-well plate, which was then hermetically closed and incubated for 24 or $48 \mathrm{~h}$ at 28,37 , or $42^{\circ} \mathrm{C}$. Reference strains (AAEC189 and W3110) were included in all runs in triplicate for each medium, at all temperatures and time points. After incubation, OD was measured with an ELISAreader (Synergy HT, BioTEK Instruments, Bad Friedrichshall, Germany) and bacteria were washed with aqua bidestillata, fixed in $99 \%$ methanol, stained with $0.1 \%$ crystal violet, and dissolved in 80:20 ethanol:acetone. After dissolution, OD was again measured, then biofilm formation capacity was computed (Martinez-Medina et al., 2009) and statistically analyzed using IBM SPSS Statistics for Windows, Version 20 (Dunn, 2013). Normal distributions of measuring points of all groups (strains) were tested using Kolmogorov-Smirnov (Smirnov, 1948). Based on the non-normal distributions of the measuring points of all groups, the non-parametric Wilcoxon rank-sum test (Mann-Whitney U-test; Wilcoxon, 1945) was used to estimate whether the observed biofilm formation capacity differences were statistically significant $(p=0.05)$. This assay was repeated three times in triplicate per temperature, and in two media for all strains.

\section{Motility Assays}

Overnight culture (from a single colony grown in $5 \mathrm{~mL}$ BHI broth) was set to $\mathrm{OD}_{600}=1$. One milliliter was centrifuged and washed twice with $1 \times$ phosphate buffered saline (PBS). Five microliters of the suspension were dropped onto swimagar plates (LB and $0.3 \%$ agar). Plates were incubated at 28, 37, and $42^{\circ} \mathrm{C}$. The strain MG1655 (Hayashi et al., 2006; Richter, 2011) was used as a control for a positive swimming phenotype. After 48 h, colony diameters were measured (Harshey, 2003) and statistically analyzed using IBM SPSS Statistics for Windows, Version 20 (Dunn, 2013). Normal distributions of measuring points of all groups (strains) were tested using KolmogorovSmirnov (Smirnov, 1948). Based on the non-normal distributions of the measuring points of all groups, the non-parametric Wilcoxon rank-sum test (Wilcoxon, 1945) was used to estimate whether the observed swimming differences were statistically significant. The significance level for multiple comparisons between WT, PCV and T groups was adjusted to $p=0.016$. This assay was repeated six times.

\section{RNA Sequencing}

The RNA of IMT17433, PCV17433, and T17433 was sequenced. RNA was isolated from two biological replicates from each of two macrocolony and two motility plates. RNA was isolated from cells using the RNASnap method (Stead et al., 2012) and shipped to LGC Genomics (Berlin, Germany) for RNA sequencing with an Illumina HiSeq2000 producing one channel paired end reads. The details of the company's standard protocols for quality control, RNA extraction from the RNASnap technique and rRNA depletion (using Ribo-Zero (Epicentre), Biozym, Hessisch Oldendorf, Germany) can be found on their website (http://www.lgcgroup.com/services). cDNA synthesis, library generation, indexing and cluster generation were performed using Illumina technology (TruSeq RNA Sample Preparation Kit v2). Bioinformatic mRNA differential expression analysis included the following processing steps: (a) generating FastQC reports to check the quality of sequenced reads; (b) clipping Illumina TruSeq sequencing adapters from the 3 ' ends of reads; (c) filtering rRNA reads using riboPicker (Schmieder et al., 2012); (d) aligning reads against IMT17433 as a reference using TopHat2 (Kim et al., 2013) and RSEM; (e) counting reads per gene using HTSeq-count, and per transcript and gene using RSEM (Li and Dewey, 2011); and (f) computing differential gene and transcript expression between different groups of samples (including technical and biological replicates) using R/Bioconductor packages DESeq (Anders and Huber, 2010), edgeR (Robinson et al., 2010), and Cuffdiff (part of the Cufflinks software package, Trapnell et al., 2013).

\section{RESULTS}

\section{ESBL-Plasmid-"Curing" and Transformations}

All PCVs kept their smaller plasmids and lost only the large ESBL-plasmid. Bioinformatic analysis of all WT and PCV genomes assured their genetic similarity, ruling out any changes in the PCVs' chromosomal content during the "curing" procedure (Schaufler et al., 2013; Von Mentzer et al., 2014). To verify detected phenotypic differences between WT and PCV strains, seven transformants containing the reintroduced large ESBL-plasmid were used (T17433, T19205, T27685, T16316, T17887, T21183, and T23463; Supplementary Table S1). These strains showed phenotypic cefotaxime-resistance (CLSI, 2008). Plasmid-profile analysis confirmed transformation of the large ESBL-plasmid.

\section{ESBL-Plasmid Characteristics}

Whole genomes of all WT strains were used for plasmid characterization (http://www.sanger.ac.uk/resources/downloads bacteria/escherichia-coli.html\#project_2119; IMT17433 [ERR163891], IMT19205 [ERR163889], IMT16316 [ERR163879], IMT17887 [ERR163883], IMT21183 [ERR163880], IMT23463 [ERR163881]; http://www.sanger.ac. uk/resources/downloads/bacteria/escherichia-coli.html\#project 2433; IMT27685 [ERR264283]). Table 1 summarizes the most important ESBL-plasmid characteristics. Their sizes ranged from approximately $100-166 \mathrm{~kb}$. Besides common bla $a_{\mathrm{CTX}-\mathrm{M}}$ types (mostly the CTX-M-15 enzyme), non-beta-lactam resistance genes (e.g., $\operatorname{tet}(A) /(R)$ and $\left.a a c\left(6^{\prime}\right)-I b-c r\right)$ were found. Virulenceassociated genes, mainly finO and traT, were present. All WT strains harbored at least one smaller non-ESBL-plasmid (Table 1). Analysis of PCV genomes showed complete loss of the $\mathrm{MOB}_{\mathrm{F} 12} /$ IncF ESBL-plasmids in six of the seven genomes analyzed. Strain IMT19205 contained two IncF plasmids that were unresolvable by PLACNET. This strain lost its ESBL-phenotype along with one of the IncF plasmids.

Figure 1 displays the annotated ESBL-plasmid sequences (pIMT17433, pIMT19205, pIMT27685, pIMT16316, pIMT17887, pIMT21183, and pIMT23463) as compared to ESBL-plasmid pEC_L8 (Smet et al., 2010a), showing both beta-lactam and non-beta-lactam resistance genes, as well as non-resistance genes, including icc (phosphodiesterase) and hha 
(hemolysin expression modulating protein), which were present on all plasmids. Additionally, this comparison displays typical features of conjugative plasmids (tra regions and insertions sites) and plasmid partitioning (toxin-antitoxin systems: e.g., vagC/D and $p e m I / K)$.

In summary, although they contained similar genetic backbones and resistance determinants, ESBL-plasmids were rather diverse.

\section{Growth Curves and Omnilog ${ }^{\circledR}$ Phenotype Microarray (PM)}

No differences were detected between the LB growth curves of WT ESBL-producing E. coli strains and the corresponding PCVs (data not shown).

Growth/metabolic activity and chemical sensitivity of all strains were then screened using the Omnilog ${ }^{\circledR}$ PM system. Only a minor proportion of strains showed significant differences between the 427 tested compounds (Supplementary Table S2). Exemplary for plate PM1, significant differences were observed for strain combinations 17433 and 17887. Additionally, plates PM2 and PM3 revealed few significant differences (Supplementary Table S2). In contrast, on plate PM4 (sulfur and phosphorus sources), significant differences were observed for 22 wells, of which 18 showed higher values in WT and $\mathrm{T}$ strains, while four showed higher values in $\mathrm{PCV}$ strains.

The chemical sensitivity plates PM13 and PM14 revealed expected significant differences for ESBL-carrying (WT and T) strains in antimicrobial-containing wells, which showed higher metabolic values than corresponding PCV strains (e.g., PM13, C6: Doxycycline and D4: Cefuroxime; and PM14, G8: Carbenicillin; Supplementary Table S2).

In summary, given the high numbers of substrates tested, only a small proportion of wells showed significant differences in the "area under the curve" parameter between WT, PCV, and $\mathrm{T}$ strains. The detected significant differences were bidirectional, meaning that in some cases the PCV strains showed higher growth/metabolic activity values, and in other cases the
ESBL-plasmid-carrying strains showed higher growth/metabolic activity values.

\section{Macrocolonies}

The expression of the biofilm-associated extracellular matrix components cellulose and/or curli fimbriae was tested using macrocolony assays. Table 2 shows the results of the six followup runs, which were performed for those strains with differences in cellulose and/or curli fimbriae production during the initial screening (combinations 17433 and 16316).

On plates containing sodium chloride, ESBL-carrying WT IMT17433 expressed cellulose and curli fimbriae, whereas its corresponding PCV did not (Figure 2). Another mutant (PCV16316) did not show any cellulose expression in any run as compared to its associated WT strain (IMT16316), which produced both cellulose and curli fimbriae. In both cases, in accordance with the corresponding WT strains, the transformants T17433 (Figure 2) and T16316 produced curli fimbriae and cellulose in most runs. Only combination 17433 showed the same results on plates lacking sodium chloride (Table 2).

In summary, PCV17433 and PCV16316 displayed reduced production of extracellular components as compared to their respective wild-type and transformant strains.

\section{Biofilm Formation Assays}

Virulence-associated biofilm assays revealed significant differences among three WT/PCV combinations (IMT/PCV17433, IMT/PCV27685, and IMT/PCV17887) after $24 \mathrm{~h}$ and among two combinations (IMT/PCV17433 and IMT/PCV17887) after $48 \mathrm{~h}$. IMT17433 and its corresponding PCV were particularly interesting. At all three temperatures and after both time points, the WT strain showed an enhanced biofilm formation capacity in glucose-containing M63 medium $\left(28^{\circ} \mathrm{C}, 24 \mathrm{~h}: p=0.222 ; 37^{\circ} \mathrm{C}, 24 \mathrm{~h}: p=0.008 ; 42^{\circ} \mathrm{C}, 24 \mathrm{~h}:\right.$ $p=0.094 ; 28^{\circ} \mathrm{C}, 48 \mathrm{~h}: p<0.001 ; 37^{\circ} \mathrm{C}, 48 \mathrm{~h}: p<0.001 ; 42^{\circ} \mathrm{C}$, $48 \mathrm{~h}: p=0.008$ ) as compared to the PCV strain. In contrast, biofilm formation by PCV17433 was better in LB medium

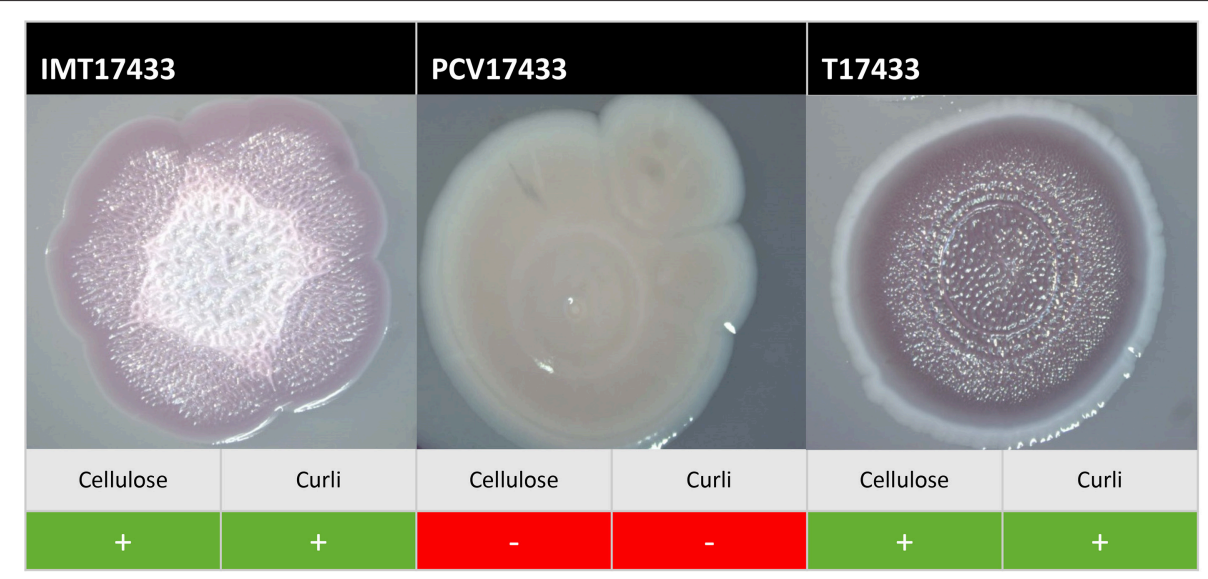

FIGURE 2 | Exemplary macrocolonies of IMT/PCV/T17433. Span agar plates with congo red and sodium chloride, incubated for 5 days at $28^{\circ} \mathrm{C}$. 
$\left(28^{\circ} \mathrm{C}, 24 \mathrm{~h}: p<0.001 ; 37^{\circ} \mathrm{C}, 24 \mathrm{~h}: p<0.001 ; 42^{\circ} \mathrm{C}, 24 \mathrm{~h}: p<\right.$ $0.001 ; 28^{\circ} \mathrm{C}, 48 \mathrm{~h}: p=0.006 ; 37^{\circ} \mathrm{C}, 48 \mathrm{~h}: p=0.004 ; 42^{\circ} \mathrm{C}, 48$ $\mathrm{h}: p=0.002$; Figures 3A,B). Transformants did not show any biofilm formation.

In summary, some WT and PCV combinations showed differences in their biofilm formation capacities in both directions; these results were medium-dependent.

\section{Motility Assays}

Motility assays were performed to test the swimming capacity of the strains at different temperatures. Two PCV strains (PCV17433 and PCV17887) showed significantly increased swimming capacity as compared to their corresponding WT strains (IMT17433 and IMT17887) at 28, 37, and $42^{\circ} \mathrm{C}$ (IMT/PCV17433: $28^{\circ} \mathrm{C}, p=0.002 ; 37^{\circ} \mathrm{C}, p=0.002 ; 42^{\circ} \mathrm{C}$, $p=0.006$; IMT/PCV17887: $28^{\circ} \mathrm{C}, p=0.002 ; 37^{\circ} \mathrm{C}, p=0.002$; $42^{\circ} \mathrm{C}, p=0.004$ ). Both transformants (T17433 and T17887) showed significantly reduced swimming capacity as compared to their corresponding PCV strains, with the exception of T17887 at $28^{\circ} \mathrm{C}$. (T/PCV17433: $28^{\circ} \mathrm{C}, p=0.002 ; 37^{\circ} \mathrm{C}, p=0.002 ; 42^{\circ} \mathrm{C}$, $p=0.002$; $\mathrm{T} / \mathrm{PCV} 17887: 28^{\circ} \mathrm{C}, p=0.032 ; 37^{\circ} \mathrm{C}, p=0.004$; $42^{\circ} \mathrm{C}, p=0.013$; Figures 4, 5A-C).

In summary, PCV17433 and PCV17887 showed higher swimming capacity than their respective WT and T strains.

\section{RNA Sequencing}

To gain insight into differential gene expression, RNA sequencing was performed for IMT/PCV/T17433. Only upor downregulated genes detected with all three software packages and a threshold of 1.5 fold bidirectional regulation were considered for subsequent analysis.

Cellulose- and curli fimbriae-related differentially regulated genes from the macrocolony assay included: $\operatorname{csg} B$ (upregulation in WT compared to PCV: 9.8), $\operatorname{csg} A$ (8.6), $\operatorname{csgE}(6.0), \operatorname{csgF}(5.5)$, $\operatorname{csg} D$ (5.5), $\operatorname{csg} G(4.0), \operatorname{csg} C$ (3.6), and $\operatorname{adrA}(2.3)$. Differentially regulated genes important for swimming in the motility assay were, among others: fliZ (downregulation in WT compared to PCV: -1.9), flgH (-2.0), flgF (-2.5), flhC (-2.7), fliD (-2.7), flgL (-3.0), flgK (-3.1), flgC (-3.4), flhD (-3.5), fliL (-4.0), $f l g D(-4.2)$, and $f l i C(-5.7)$. Most genes were found to be up- or downregulated in both assays, except for $\operatorname{csg} D$ and $\operatorname{csg} C$, which were only found to be upregulated in the macrocolony assay in the WT strain as compared to the PCV strain. Conversely, downregulation of $f h C$ and $f l h D$ was only observed in the motility assay. All cellulose- and curli-related genes that were upregulated in the macrocolony assay in IMT17433 as compared to PCV17433, were also upregulated in T17433 as compared to PCV17433. Swimming-related genes that were downregulated in the motility assay were not detected in the transformant (e.g., fliZ, $\mathrm{flgH}, \mathrm{flg} A, \mathrm{flgL}, \mathrm{flg} \mathrm{G}, \mathrm{fliC}$ ).

In summary, RNA sequencing verified the observed phenotypes for WT17433, PCV17433, and T17433 at the transcriptional level.

\section{Candidate Genes}

The observed phenotypic and transcriptomic differences were only explainable by differences at the genetic level. With only the ESBL-plasmids differing between WT/T and PCV strains and resistance determinants not explaining the results, the next step in this study was to focus on the non-resistance genes encoded by these plasmids. Table 3 shows candidate genes of the seven ESBL-plasmid sequences based on PLACNET analysis. Previous studies suggest their involvement in biofilm formation and motility. The candidate genes included hha (encoding the hemolysin expression modulating protein) and yihA (encoding a cell division protein), which were both present on pIMT17433, pIMT19205, and pIMT27685. The genes icc (encoding a phosphodiesterase) and $y f a X$ (encoding a putative transcriptional factor) were encoded on pIMT17433 and pIMT27685. All ESBLplasmids carried tra genes important for conjugation.

In summary, the detected candidate genes were mostly encoded on ESBL-plasmids of sequence type ST131 (pIMT17433, pIMT19205, and pIMT27685).

\section{DISCUSSION}

The success of ESBL-producing E. coli, particularly the pandemic pathogenic clonal lineages, cannot be explained by antimicrobial resistance alone. To assess the possibility of interactions between ESBL-plasmids and chromosomal content, we conducted a study using an unconventional approach by constructing PCVs of ESBL-plasmid-carrying WT strains of ST131 and ST648. Based on growth curves in LB medium and detailed growth/metabolic activity under different conditions, WT and PCV strains showed similar behaviors and were thus regarded suitable for analysis in subsequent phenotypic assays. Phenotypic differences between WT and PCV strains were generally verified by T strains.

In PM assays, which are a consolidation of regular LB growth curve tests (Supplementary Table S2), significant differences were detected in select wells (plates PM1-4: 3\% among all wells without combination 17433). When the results of PM4 were included for 17433, 15\% of the wells showed differences; however, these results need to be treated with caution since the negative control (A01) had high values for PCV17433 even after several repetitions of the assay. High values for negative controls in PM assays have previously been described for E. coli (Vaas et al., 2012). Differences in antimicrobial sensitivity assays on plates PM13 and PM14 between ESBL-plasmid-carrying strains and PCVs are explainable by loss of ESBL-plasmids in PCVs, which, besides ESBL-genes, carry additional antimicrobial resistance genes (Table 1; e.g., PM13, C6: Doxycycline and D4: Cefuroxime; and PM14, G8: Carbenicillin). Overall, significant differences were bidirectional, meaning that each of the ESBL-plasmidcarrying strains and the PCVs showed higher values in select wells. This indicates, as underscored by LB growth curve results, that ESBL-plasmid-carriage does not necessarily lead to fitness loss. This contradicts the hypothesis that considerable trade-offs exist between antimicrobial resistance and fitness (Dasilva and Bailey, 1986; Lenski, 1997; Andersson and Hughes, 2010). Our finding applied to all seven strain combinations irrespective of origin, ST, or ESBL-plasmid characteristics.

Biofilm-related macrocolonies result from bacterial incubation over several days, where the biosynthesis of the 

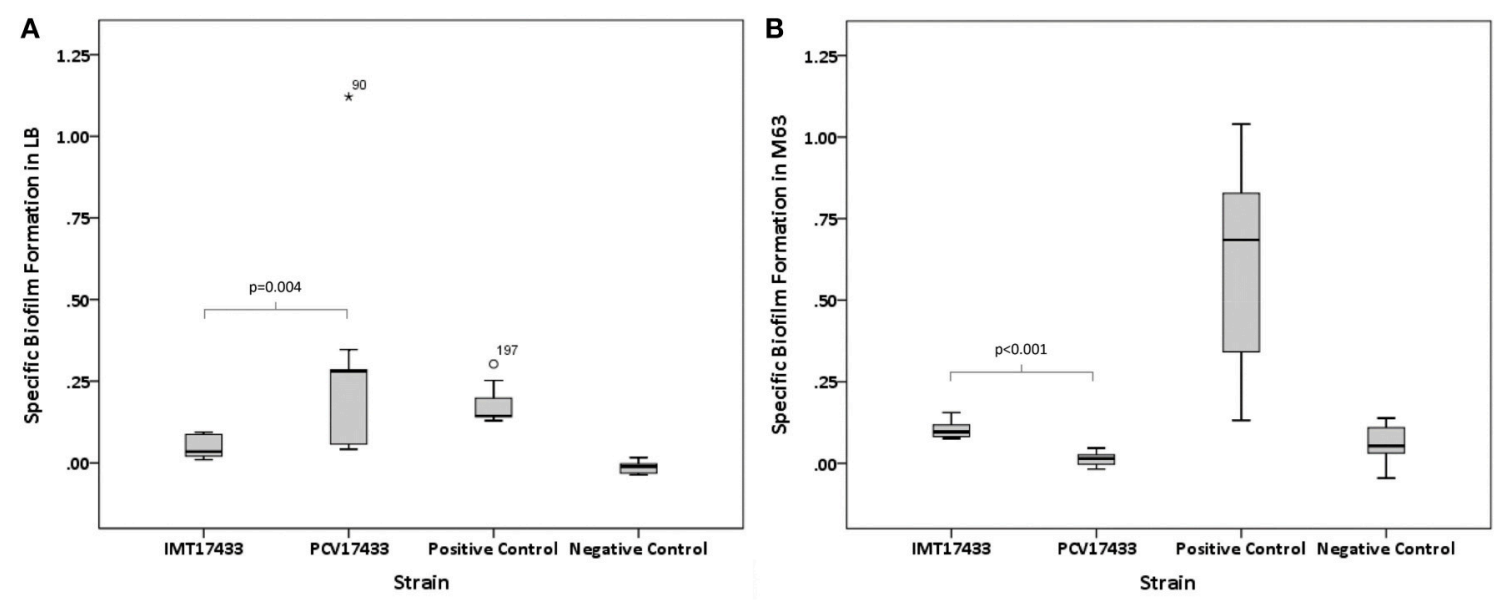

FIGURE 3 | (A) Box plots of the distributions of the specific biofilm formation capacities of IMT17433 and PCV17433 at $37^{\circ} \mathrm{C}$ after $48 \mathrm{~h}$ in LB medium. Plots were generated using IBM SPSS Statistics for Windows, Version 20. Positive control, AAEC189; Negative control, W3110. (B) Box plots of the distributions of the specific biofilm formation capacities of IMT17433 and PCV17433 at $37^{\circ} \mathrm{C}$ after $48 \mathrm{~h}$ in M63 medium. Plots were generated using IBM SPSS Statistics for Windows, Version 20. Positive control, AAEC189; Negative control, W3110.

\section{7 \\ Incubated at $28^{\circ} \mathrm{C}, 48 \mathrm{~h}$}
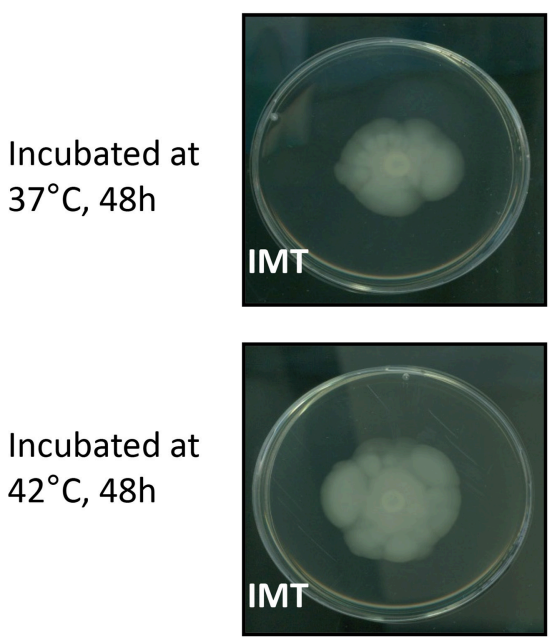
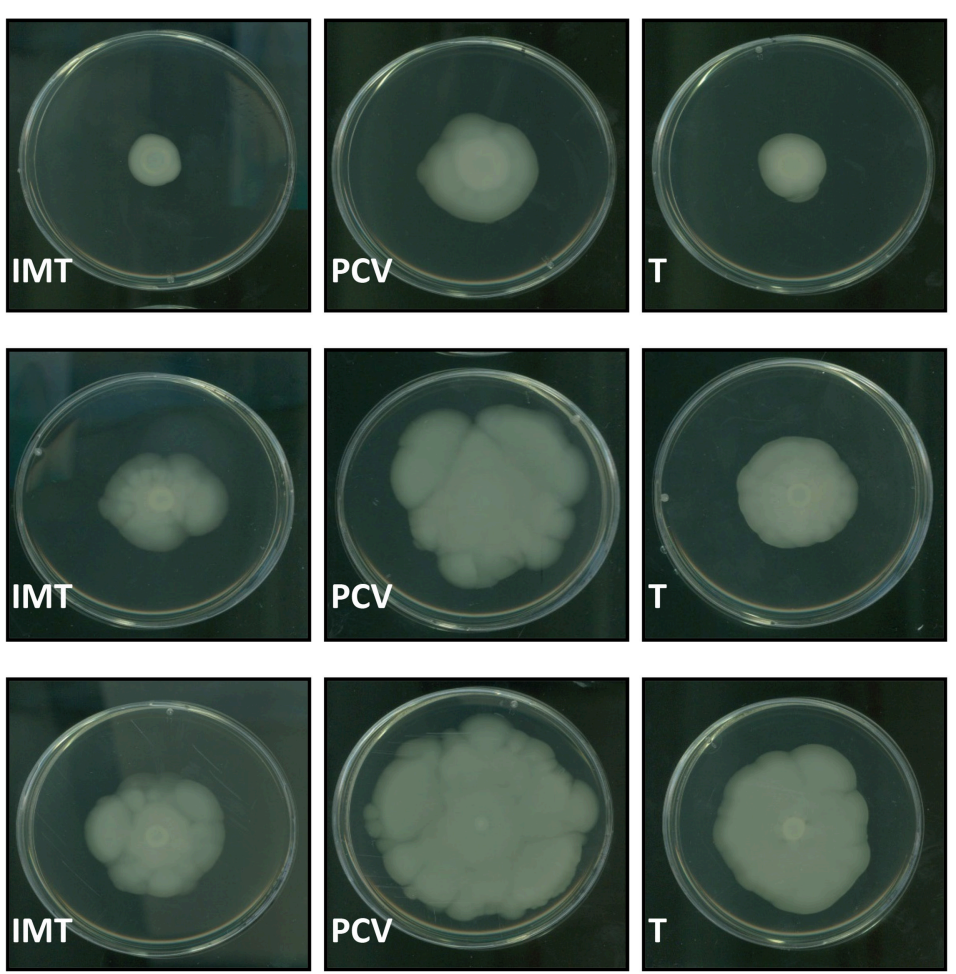

FIGURE 4 | Exemplary swimming motility of IMT/PCV/T17887. LB plates wih $0.3 \%$ agar. Incubation at 28,37 , and $42^{\circ} \mathrm{C}$ for $48 \mathrm{~h}$.

important virulence-related extracellular matrix components cellulose and curli fimbriae typically occurs below $30^{\circ} \mathrm{C}$ (Bokranz et al., 2005; Richter et al., 2014). Curli fimbriae promote adhesions to abiotic surfaces (Zogaj et al., 2001) and are associated with virulence, as they play key roles during internalization into epithelial cells (Gophna et al., 2001) and persistence in avian guts (La Ragione et al., 1999). Bacteria produce cellulose mainly as protection from both chemical and mechanical influences (Ross et al., 1991). Considering the results of the macrocolony assays for those combinations with differences, the enhanced ability of ESBL-plasmid-carrying WT and T strains to synthesize curli fimbriae and/or cellulose 

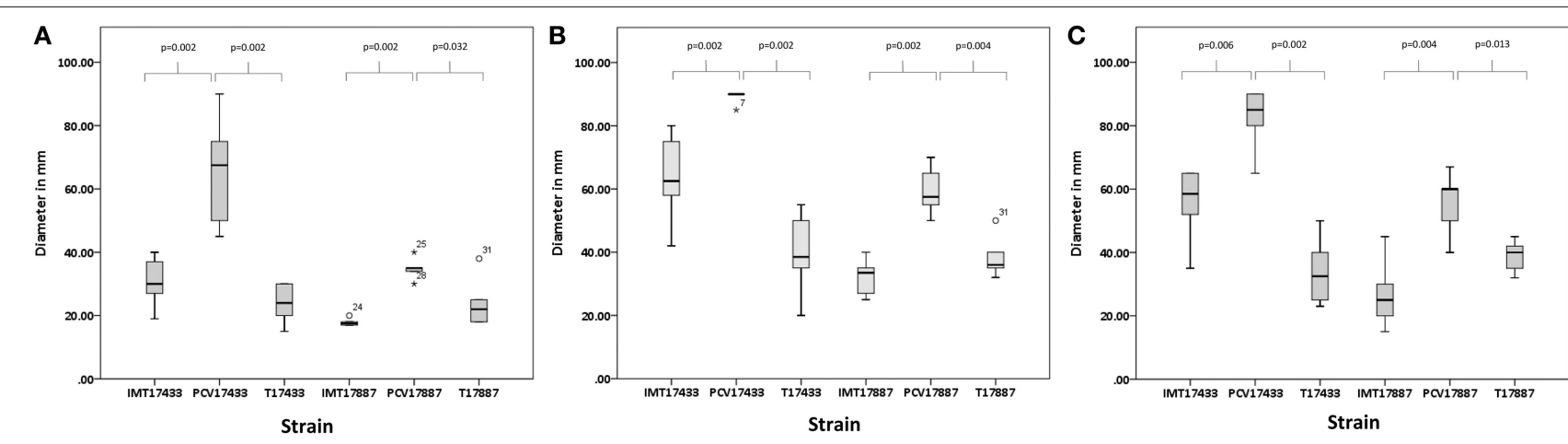

FIGURE 5 | (A) Box plots of the distributions of the diameters, in millimetres, of the swimming capacities of IMT/PCV/T17433 and IMT/PCV/T17887 at $28^{\circ} \mathrm{C}$. Plots were generated using IBM SPSS Statistics for Windows, Version 20. (B) Box plots of the distributions of the diameters, in millimetres, of the swimming capacities of IMT/PCV/T17433 and IMT/PCV/T17887 at $37^{\circ} \mathrm{C}$. Plots were generated using IBM SPSS Statistics for Windows, Version 20 . (C) Box plots of the distributions of the diameters, in millimetres, of the swimming capacities of IMT/PCV/T17433 and IMT/PCV/T17887 at $42^{\circ} \mathrm{C}$. Plots were generated using IBM SPSS Statistics for Windows, Version 20.

TABLE 3 | Candidate genes based on annotated PLACNET ESBL-plasmid sequences, literature survey, and RNA sequencing results.

\begin{tabular}{|c|c|c|c|c|}
\hline Gene & Protein & Predicted function & Presence & References \\
\hline hha & $\begin{array}{l}\text { Hemolysin expression modulating } \\
\text { protein }\end{array}$ & Involved in biofilm formation and motility & pIMT17433, pIMT19205, pIMT27685 & Barrios et al., 2006 \\
\hline$i c c$ & Phosphodiesterase & Involved in biofilm formation & pIMT17433, plMT27685 & Kalivoda et al., 2013 \\
\hline yfax & $\begin{array}{l}\text { Predicted DNA-binding } \\
\text { transcriptional regulator }\end{array}$ & Putative HTH-type transcription factor & plMT17433, pIMT27685 & $\begin{array}{l}\text { Perez-Rueda and } \\
\text { Collado-Vides, } 2000\end{array}$ \\
\hline yihA & $\begin{array}{l}\text { Cell division protein, predicted } \\
\text { checkpoint GTPase }\end{array}$ & GTP binding & pIMT17433, pIMT19205, pIMT27685 & Lehoux et al., 2003 \\
\hline tra & $\begin{array}{l}\text { Transfer regions of the } \\
\text { F-conjugative plasmid }\end{array}$ & Involved in biofilm formation & all seven pIMT ESBL-plasmids & Ghigo, 2001 \\
\hline
\end{tabular}

in contrast to their respective PCV strains was particularly notable. This indicates that ESBL-plasmid carriage confers benefits in terms of biosynthesis of virulent- and survivalassociated extracellular matrix components to some bacterial strains. Several prior studies have described an influence of conjugative plasmids on biofilm formation (Ghigo, 2001; Yang et al., 2008). May and Okabe (May and Okabe, 2008) investigated the influence of natural IncF F-plasmids on biofilm formation and maturation. In this study, both conjugative (tra regions) and non-conjugative plasmid genes seemed to play a role. In our study, all seven ESBL-plasmids belonged to incompatibility group IncF. Furthermore, all encoded different tra genes are important for conjugation; however, since not all WT strains showed differences in extracellular matrix component production as compared to PCV strains, these factors are probably not solely responsible for our observed results.

Biofilm results from IMT/PCV17433 underline the complexity of biofilm formation, which is also dependent on nutrient availability. The enhanced ability of IMT17433 to form biofilms in M63 medium as compared to PCV17433 may point toward plasmid encoded features that enable the WT strain to use limited nutrients efficiently, perhaps via phosphate-dependent pathways. PM results revealing that select phosphate-containing wells in which IMT17433 showed higher respiration values than PCV17433 reinforce the latter hypothesis. Explanations of the observed enhanced ability of the PCV strain to form biofilms in LB medium, however, remain speculative. There may have been no need for planktonic IMT17433 to transform into a biofilm due to optimal utilization of the rich LB medium. A switch from a sessile (multicellular behavior, biofilm) to a planktonic (motility) way of life, and vice versa, underlies subtle interactions at a molecular level that include numerous complex cascades. Flagellar biosynthesis, for instance, seems not only crucial for bacterial swimming but also leads to surface colonization and subsequent biofilm formation. Insufficient nutrient supply leads to detachment of the cells from the biofilm and adoption of a planktonic lifestyle (Harshey, 2003).

Explanations of the observed enhanced swimming capacity among some PCV strains as compared to their corresponding WT and $\mathrm{T}$ strains in the motility assays remain similarly speculative. Flagellar synthesis has been shown to be energetically costly, and ESBL-plasmid-carrying strains might use their energy more strategically (Zhao et al., 2007). Alternately, due to better nutrient utilization, ESBL-plasmid-carrying strains may not have to swim as well as PCV strains to reach peripheral zones with, presumably, richer nutrition supplies.

Phenotypic differences in cellulose and curli fimbriae production as well as swimming capacity, particularly those 
observed between the 17433 strains, were confirmed by RNA sequencing results showing upregulation of chromosomally encoded extracellular matrix-related genes and downregulation of chromosomally encoded flagellar-related genes in IMT17433 as compared to PCV17433. Reversible RNA sequencing results for T17433 strengthen the reliability of the differences seen at phenotypic and transcriptomic levels. The key role of the transcription factor $c s g D$ is notable in biofilm formation and motility capacity. $\operatorname{csg} D$ regulates not only the second curli fimbriae operon $\operatorname{csg} B A C$ and $a d r A$, whose product is not only accountable for several other genes involved in cellulose production, but also influences $f h D C$, the main operon for flagellar synthesis and thus motility (Ogasawara et al., 2011; Chambers and Sauer, 2013). In our study, RNA sequencing results revealed upregulation of $\operatorname{csg} D, \operatorname{csg} B A C$, and $a d r A$ and downregulation of $\mathrm{flhDC}$ in IMT17433 as compared to PCV17433. These results not only confirm the phenotypic data, but also emphasize the central importance of the CsgD protein in regulating the subtle interaction between bacterial sessile and planktonic ways of life.

Overall, the phenotypic tests performed for all seven strain combinations did not show a consistent pattern. Although, this is expected due to high diversity among the group of ESBL-plasmids as well as in the origins of host strains and their phylogenetic backgrounds, such inconsistencies weaken the generalizability of our conclusions. Rather, our results should be seen as an impetus for prospective studies. Interestingly, despite ESBL-plasmid diversity, each of pIMT17433, pIMT19205, and pIMT27685 carried different candidate genes, including hha and icc, which both have been described to be involved in biofilm formation and motility (Barrios et al., 2006; Kalivoda et al., 2013). Only further experiments that include cloning and knockout methods will provide more information on underlying molecular mechanisms.

In conclusion, this pilot study showed that ESBL-plasmid carriage does not necessarily lead to a growth/metabolic fitness loss. Some ESBL-plasmids in select strains may possess the potential to influence chromosomal gene expression, particularly of those genes that are important for the subtle interactions

\section{REFERENCES}

Alikhan, N. F., Petty, N. K., Ben Zakour, N. L., and Beatson, S. A. (2011). BLAST Ring Image Generator (BRIG): simple prokaryote genome comparisons. BMC Genomics 12:402. doi: 10.1186/1471-2164-12-402

Anders, S., and Huber, W. (2010). Differential expression analysis for sequence count data. Genome Biol. 11:R106. doi: 10.1186/gb-2010-11-10-r106

Andersson, D. I., and Hughes, D. (2010). Antibiotic resistance and its cost: is it possible to reverse resistance? Nat. Rev. Microbiol. 8, 260-271. doi: 10.1038/nrmicro2319

Barrios, A. F. G., Zuo, R. J., Ren, D. C., and Wood, T. K. (2006). Hha, YbaJ, and OmpA regulate Escherichia coli K12 biofilm formation and conjugation plasmids abolish motility. Biotechnol. Bioeng. 93, 188-200. doi: 10.1002/bit.20681

Blomfield, I. C., Mcclain, M. S., and Eisenstein, B. I. (1991). Type 1 fimbriae mutants of Escherichia coli K12: characterization of recognized afimbriate strains and construction of new fim deletion mutants. Mol. Microbiol. 5, 1439-1445. doi: 10.1111/j.1365-2958.1991.tb00790.x between the sessile and planktonic ways of life, such as $\operatorname{csgD}$. We hypothesize that this may contribute to their virulence potential and pandemic success in different habitats, although underlying mechanisms remain to be identified and characterized.

\section{AUTHOR CONTRIBUTIONS}

All authors gave their final approval of the version to be published. Conception and Design: SG, CE, KS, LW, FD. Data analysis: KS, SG, TS, DP, MD. Data acquisition: KS, SG, TS. Writing: KS, SG, LW, CE, DP, TS, MD, FD.

\section{FUNDING}

This work was supported by grants from the German Research Foundation (DFG) to SG and CE entitled, "Functional analysis of non-resistance genes of extended-spectrum beta-lactamasesassociated sequence types of Escherichia coli" (grants GU 1283/3-1 and EW116/2-1). KS was supported by a grant from SONNENFELD-Stiftung. Sequencing was partly financed by the Wellcome Trust Sanger Institute (Cambridge, United Kingdom-study number 2433ILB). The foundations that funded this work had no role in study design, data collection or interpretation, or the decision to submit the work for publication.

\section{ACKNOWLEDGMENTS}

We thank Ivonne Stamm and Peter A. Kopp (IDEXX Vet. Med. Lab., Ludwigsburg, Germany) for providing some of the wildtype strains used in this study and Gordon Dougan (Wellcome Trust Sanger Institute, Cambridge, United Kingdom) for his support during sequencing.

\section{SUPPLEMENTARY MATERIAL}

The Supplementary Material for this article can be found online at: http://journal.frontiersin.org/article/10.3389/fmicb. 2016.00336
Bokranz, W., Wang, X., Tschape, H., and Romling, U. (2005). Expression of cellulose and curli fimbriae by Escherichia coli isolated from the gastrointestinal tract. J. Med. Microbiol. 54, 1171-1182. doi: 10.1099/jmm.0.46064-0

Bonnedahl, J., Hernandez, J., Stedt, J., Waldenstrom, J., Olsen, B., and Drobni, M. (2014). Extended-spectrum beta-lactamases in Escherichia coli and Klebsiella pneumoniae in Gulls, Alaska, USA. Emerg. Infect. Dis. 20, 897-899. doi: 10.3201/eid2005.130325

Calhau, V., Ribeiro, G., Mendonca, N., and Da Silva, G. J. (2013). Prevalent combination of virulence and plasmidic-encoded resistance in ST 131 Escherichia coli strains. Virulence. 4, 726-729. doi: 10.4161/viru.26552

Chambers, J. R., and Sauer, K. (2013). Small RNAs and their role in biofilm formation. Trends Microbiol. 21, 39-49. doi: 10.1016/j.tim.2012.10.008

Chen, F., Mackey, A. J., Stoeckert, C. J. Jr., and Roos, D. S. (2006). OrthoMCL-DB: querying a comprehensive multi-species collection of ortholog groups. Nucleic Acids Res. 34, D363-D368. doi: 10.1093/nar/gkj123

CLSI (2008) Performance Standards for Antimicrobial Disk and Dilution Susceptibility Tests for Bacteria Isolated from Animals; Approved Standard, 3rd Edn. Wayne, PA: CLSI. 
Dale, J. W., and Park, S. F. (2004). Molecular Genetics of Bacteria. Chichester: John Wiley \& Sons Ltd.

Dasilva, N. A., and Bailey, J. E. (1986). Theoretical growth-yield estimates for recombinant cells. Biotechnol. Bioeng. 28, 741-746. doi: 10.1002/bit.260280514

Dudin, O., Geiselmann, J., Ogasawara, H., Ishihama, A., and Lacour, S. (2014). Repression of flagellar genes in exponential phase by CsgD and CpxR, two crucial modulators of Escherichia coli biofilm formation. J. Bacteriol. 196, 707-715. doi: 10.1128/JB.00938-13

Dunn, P. (2013). SPSS survival manual: a step by step guide to data analysis using IBM SPSS. Aust. Nz. J. Publ. Heal. 37, 597-598. doi: 10.1111/1753-6405.12166

Ewers, C., Bethe, A., Stamm, I., Grobbel, M., Kopp, P. A., Guerra, B., et al. (2014). CTX-M-15-D-ST648 Escherichia coli from companion animals and horses: another pandemic clone combining multiresistance and extraintestinal virulence? J. Antimicrob. Chemother. 69, 1224-1230. doi: 10.1093/jac/ dkt516

Ewers, C., Bethe, A., Semmler, T., Guenther, S., and Wieler, L. H. (2012). Extendedspectrum beta-lactamase-producing and AmpC-producing Escherichia coli from livestock and companion animals, and their putative impact on public health: a global perspective. Clin. Microbiol. Infect. 18, 646-655. doi: 10.1111/j.1469-0691.2012.03850.x

Ewers, C., Grobbel, M., Stamm, I., Kopp, P. A., Diehl, I., Semmler, T., et al. (2010). Emergence of human pandemic O25:H4-ST131 CTX-M-15 extendedspectrum beta-lactamase-producing Escherichia coli among companion animals. J. Antimicrob. Chemother. 65, 651-660. doi: 10.1093/jac/dkq004

Ghigo, J. M. (2001). Natural conjugative plasmids induce bacterial biofilm development. Nature 412, 442-445. doi: 10.1038/35086581

Gophna, U., Barlev, M., Seijffers, R., Oelschlager, T. A., Hacker, J., and Ron, E. Z. (2001). Curli fibers mediate internalization of Escherichia coli by eukaryotic cells. Infect. Immun. 69, 2659-2665. doi: 10.1128/IAI.69.4.2659-2665.2001

Green, A. M., and Sambrook, J. (2012). Molecular Cloning: A Labarotory Manual. Cold Springer Harbor, NY: Cold Springer Harbor Labarotory Press.

Hammar, M., Arnqvist, A., Bian, Z., Olsen, A., and Normark, S. (1995). Expression of two csg operons is required for production of fibronectin- and congo redbinding curli polymers in Escherichia coli K-12. Mol. Microbiol. 18, 661-670. doi: 10.1111/j.1365-2958.1995.mmi_18040661.x

Harshey, R. M. (2003). Bacterial motility on a surface: many ways to a common goal. Annu. Rev. Microbiol. 57, 249-273. doi: 10.1146/annurev.micro.57.030502.091014

Hayashi, K., Morooka, N., Yamamoto, Y., Fujita, K., Isono, K., Choi, S., et al. (2006). Highly accurate genome sequences of Escherichia coli K-12 strains MG1655 and W3110. Mol. Syst. Biol. 2, 2006.0007. doi: 10.1038/msb4100049

Ito, A., Taniuchi, A., May, T., Kawata, K., and Okabe, S. (2009). Increased antibiotic resistance of Escherichia coli in mature biofilms. Appl. Environ. Microbiol. 75, 4093-4100. doi: 10.1128/AEM.02949-08

Joensen, K. G., Scheutz, F., Lund, O., Hasman, H., Kaas, R. S., Nielsen, E. M., et al. (2014). Real-time whole-genome sequencing for routine typing, surveillance, and outbreak detection of verotoxigenic Escherichia coli. J. Clin. Microbiol. 52, 1501-1510. doi: 10.1128/JCM.03617-13

Johnson, J. R., Johnston, B., Clabots, C., Kuskowski, M. A., and Castanheira, M. (2010). Escherichia coli sequence type ST131 as the major cause of serious multidrug-resistant E. coli infections in the United States. Clin. Infect. Dis. 51, 286-294. doi: 10.1086/653932

Kalivoda, E. J., Brothers, K. M., Stella, N. A., Schmitt, M. J., and Shanks, R. M. Q. (2013). Bacterial cyclic AMP-phosphodiesterase activity coordinates biofilm formation. PLoS ONE 8:e71267. doi: 10.1371/journal.pone.0071267

Kearse, M., Moir, R., Wilson, A., Stones-Havas, S., Cheung, M., Sturrock, S., et al. (2012). Geneious basic: an integrated and extendable desktop software platform for the organization and analysis of sequence data. Bioinformatics 28, 1647-1649. doi: 10.1093/bioinformatics/bts199

Kim, D., Pertea, G., Trapnell, C., Pimentel, H., Kelley, R., and Salzberg, S. L. (2013). TopHat2: accurate alignment of transcriptomes in the presence of insertions, deletions and gene fusions. Genome Biol. 14:R36. doi: 10.1186/gb-2013-14-4r36

Lanza, V. F., De Toro, M., Garcillan-Barcia, M. P., Mora, A., Blanco, J., Coque, T. M., et al. (2014). Plasmid Flux in Escherichia coli ST131 sublineages, analyzed by plasmid constellation network (PLACNET), a new method for plasmid reconstruction from whole genome sequences. PLoS Genet. 10:e1004766. doi: 10.1371/journal.pgen.1004766
La Ragione, R. M., Collighan, R. J., and Woodward, M. J. (1999). Non-curliation of Escherichia coli O78:K80 isolates associated with IS1 insertion in csgB and reduced persistence in poultry infection. FEMS Microbiol. Lett. 175, 247-253. doi: 10.1111/j.1574-6968.1999.tb13627.x

Lehoux, I. E., Mazzulla, M. J., Baker, A., and Petit, C. M. (2003). Purification and characterization of YihA, an essential GTP-binding protein from Escherichia coli. Protein Expr. Purif. 30, 203-209. doi: 10.1016/S1046-5928(03)00107-4

Lenski, R. E. (1997). The cost of antibiotic resistance - from the perspective of a bacterium. antibiotic resistance: origins, evolution, selection and spread. CIBA Found. Symp. 207, 131-140.

Li, B., and Dewey, C. N. (2011). RSEM: accurate transcript quantification from RNA-Seq data with or without a reference genome. BMC Bioinformatics 12:323. doi: 10.1186/1471-2105-12-323

Martinez-Medina, M., Naves, P., Blanco, J., Aldeguer, X., Blanco, J. E., Blanco, M., et al. (2009). Biofilm formation as a novel phenotypic feature of adherentinvasive Escherichia coli (AIEC). BMC Microbiol. 9:202. doi: 10.1186/14712180-9-202

May, T., and Okabe, S. (2008). Escherichia coli harboring a natural IncF conjugative F plasmid develops complex mature biofilms by stimulating synthesis of colanic acid and Curli. J. Bacteriol. 190, 7479-7490. doi: 10.1128/JB.00823-08

Naseer, U., and Sundsfjord, A. (2011). The CTX-M Conundrum: dissemination of plasmids and Escherichia coli clones. Microb. Drug Resist. 17, 83-97. doi: $10.1089 / \mathrm{mdr} .2010 .0132$

Nicolas-Chanoine, M. H., Bertrand, X., and Madec, J. Y. (2014). Escherichia coli ST131, an intriguing clonal group. Clin. Microbiol. Rev. 27, 543-574. doi: 10.1128/CMR.00125-13

Nicolas-Chanoine, M. H., Blanco, J., Leflon-Guibout, V., Demarty, R., Alonso, M. P., Canica, M. M., et al. (2008). Intercontinental emergence of Escherichia coli clone O25:H4-ST131 producing CTX-M-15. J. Antimicrob. Chemother. 61, 273-281. doi: 10.1093/jac/dkm464

Ogasawara, H., Yamamoto, K., and Ishihama, A. (2011). Role of the biofilm master regulator CsgD in cross-regulation between biofilm formation and flagellar synthesis. J. Bacteriol. 193, 2587-2597. doi: 10.1128/JB.01468-10

Pardee, A. B., Jacob, F., and Monod, J. (1959). Genetic control and cytoplasmic expression of inducibility in the synthesis of beta-galactosidase by E. Coli. J. Mol. Biol. 1, 165-178. doi: 10.1016/S0022-2836(59)80045-0

Perez-Rueda, E., and Collado-Vides, J. (2000). The repertoire of DNA-binding transcriptional regulators in Escherichia coli K-12. Nucleic. Acids. Res. 28, 1838-1847. doi: 10.1093/nar/28.8.1838

Pitout, J. D. (2012). Extraintestinal pathogenic Escherichia coli: a combination of virulence with antibiotic resistance. Front. Microbiol. 3:9. doi: 10.3389/fmicb.2012.00009

Richter, A. (2011). Wachstumsverhalten, Motilität und Biofilm-Bildung der Escherichia coli K-12 Laborstämme W3110, MG1655 und MC4100. Ph.D. Thesis, Humboldt Universität Berlin.

Richter, A. M., Povolotsky, T. L., Wieler, L. H., and Hengge, R. (2014). Cyclic-diGMP signalling and biofilm-related properties of the Shiga toxin-producing 2011 German outbreak Escherichia coli O104:H4. EMBO Mol. Med. 6, 1622-1637. doi: 10.15252/emmm.201404309

Robinson, M. D., Mccarthy, D. J., and Smyth, G. K. (2010). edgeR: a Bioconductor package for differential expression analysis of digital gene expression data. Bioinformatics 26, 139-140. doi: 10.1093/bioinformatics/btp616

Romling, U. (2005). Characterization of the rdar morphotype, a multicellular behaviour in Enterobacteriaceae. Cell. Mol. Life Sci. 62, 1234-1246. doi: 10.1007/s00018-005-4557-x

Ross, P., Mayer, R., and Benziman, M. (1991). Cellulose biosynthesis and function in bacteria. Microbiol. Rev. 55, 35-58.

Sahly, H., Navon-Venezia, S., Roesler, L., Hay, A., Carmeli, Y., Podschun, R., et al. (2008). Extended-spectrum beta-lactamase production is associated with an increase in cell invasion and expression of fimbrial adhesins in Klebsiella pneumoniae. Antimicrob. Agents Chemother. 52, 3029-3034. doi: 10.1128/AAC.00010-08

Schaufler, K., Wieler, L. H., Semmler, T., Ewers, C., and Guenther, S. (2013). ESBL-plasmids carrying toxin-antitoxin systems can be "cured" of wild-type Escherichia coli using a heat technique. Gut. Pathog. 5:34. doi: 10.1186/17574749-5-34

Schierack, P., Romer, A., Jores, J., Kaspar, H., Guenther, S., Filter, M., et al. (2009). Isolation and characterization of intestinal Escherichia coli clones 
from wild boars in Germany. Appl. Environ. Microbiol. 75, 695-702. doi: 10.1128/AEM.01650-08

Schmieder, R., Lim, Y. W., and Edwards, R. (2012). Identification and removal of ribosomal RNA sequences from metatranscriptomes. Bioinformatics. 28, 433-435. doi: 10.1093/bioinformatics/btr669

Seiler, C., and Berendonk, T. U. (2012). Heavy metal driven co-selection of antibiotic resistance in soil and water bodies impacted by agriculture and aquaculture. Front. Microbiol. 3:399. doi: 10.3389/fmicb.2012.00399

Smet, A., Martel, A., Persoons, D., Dewulf, J., Heyndrickx, M., Claeys, G., et al. (2010a). Characterization of extended-spectrum beta-lactamases produced by Escherichia coli isolated from hospitalized and nonhospitalized patients: emergence of CTX-M-15-producing strains causing urinary tract infections. Microb. Drug Res. 16, 129-134. doi: 10.1089/mdr.2009.0132

Smet, A., Van Nieuwerburgh, F., Vandekerckhove, T. T. M., Martel, A., Deforce, D., Butaye, P., et al. (2010b). Complete nucleotide sequence of CTX-M-15-Plasmids from clinical Escherichia coli isolates: insertional events of transposons and insertion sequences. PLOS ONE 5:e11202. doi: 10.1371/journal.pone.0011202

Smirnov, N. (1948). Table for estimating the goodness of fit of empirical distributions. Ann. Math. Stat. 19, 279-279. doi: 10.1214/aoms/1177730256

Stead, M. B., Agrawal, A., Bowden, K. E., Nasir, R., Mohanty, B. K., Meagher, R. B., et al. (2012). RNAsnap (TM): a rapid, quantitative and inexpensive, method for isolating total RNA from bacteria. Nucleic Acids Res. 40, e156. doi: 10.1093/nar/gks680

Trapnell, C., Hendrickson, D. G., Sauvageau, M., Goff, L., Rinn, J. L., and Pachter, L. (2013). Differential analysis of gene regulation at transcript resolution with RNA-seq. Nat. Biotechnol. 31, 46-53. doi: 10.1038/nbt.2450

Vaas, L. A., Sikorski, J., Hofner, B., Fiebig, A., Buddruhs, N., Klenk, H. P., et al. (2013). opm: an R package for analysing $\operatorname{OmniLog}^{(R)}$ phenotype microarray data. Bioinformatics 29, 1823-1824. doi: 10.1093/bioinformatics/ btt291

Vaas, L. A., Sikorski, J., Michael, V., Goker, M., and Klenk, H. P. (2012). Visualization and curve-parameter estimation strategies for efficient exploration of phenotype microarray kinetics. PLOS ONE 7:e34846. doi: 10.1371/journal.pone.0034846
Von Mentzer, A., Connor, T. R., Wieler, L. H., Semmler, T., Iguchi, A., Thomson, N. R., et al. (2014). Identification of enterotoxigenic Escherichia coli (ETEC) clades with long-term global distribution. Nat. Genet. 46, 1321-1326. doi: 10.1038/ng.3145

Wilcoxon, F. (1945). Individual comparisons by ranking methods. Biometrics Bull. 1, 80-83. doi: 10.2307/3001968

Woodford, N., Turton, J. F., and Livermore, D. M. (2011). Multiresistant Gram-negative bacteria: the role of high-risk clones in the dissemination of antibiotic resistance. FEMS Microbiol. Rev. 35, 736-755. doi: 10.1111/j.15746976.2011.00268.x

Yang, X., Ma, Q., and Wood, T. K. (2008). The R1 conjugative plasmid increases Escherichia coli biofilm formation through an envelope stress response. Appl. Environ. Microbiol. 74, 2690-2699. doi: 10.1128/AEM.02809-07

Zankari, E., Hasman, H., Cosentino, S., Vestergaard, M., Rasmussen, S., Lund, O., et al. (2012). Identification of acquired antimicrobial resistance genes. J. Antimicrob. Chemother. 67, 2640-2644. doi: 10.1093/jac/dks261

Zhao, K., Liu, M., and Burgess, R. R. (2007). Adaptation in bacterial flagellar and motility systems: from regulon members to 'foraging'-like behavior in E. coli. Nucleic Acids Res. 35, 4441-4452. doi: 10.1093/nar/gkm456

Zogaj, X., Nimtz, M., Rohde, M., Bokranz, W., and Romling, U. (2001). The multicellular morphotypes of Salmonella typhimurium and Escherichia coli produce cellulose as the second component of the extracellular matrix. Mol. Microbiol. 39, 1452-1463. doi: 10.1046/j.1365-2958.2001.02337.x

Conflict of Interest Statement: The authors declare that the research was conducted in the absence of any commercial or financial relationships that could be construed as a potential conflict of interest.

Copyright (C) 2016 Schaufler, Semmler, Pickard, de Toro, de la Cruz, Wieler, Ewers and Guenther. This is an open-access article distributed under the terms of the Creative Commons Attribution License (CC BY). The use, distribution or reproduction in other forums is permitted, provided the original author(s) or licensor are credited and that the original publication in this journal is cited, in accordance with accepted academic practice. No use, distribution or reproduction is permitted which does not comply with these terms. 
OPEN ACCESS

Edited by:

Axel Cloeckaert,

French National Institute

for Agricultural Research, France

Reviewed by:

Henrietta Venter,

University of South Australia, Australia

César De La Fuente-Núñez,

Massachusetts Institute

of Technology, USA

Alessandra Polissi,

University of Milan, Italy

${ }^{*}$ Correspondence:

Annie Wong-Beringer anniew@usc.edu

Specialty section:

This article was submitted to Antimicrobials, Resistance

and Chemotherapy,

a section of the journal

Frontiers in Microbiology

Received: 26 April 2016 Accepted: 22 September 2016 Published: 04 October 2016

Citation:

Agnello M, Finkel SE and Wong-Beringer A (2016) Fitness Cost of Fluoroquinolone Resistance in Clinical Isolates of Pseudomonas aeruginosa Differs by Type III

Secretion Genotype.

Front. Microbiol. 7:1591. doi: 10.3389/fmicb.2016.01591

\section{Fitness Cost of Fluoroquinolone Resistance in Clinical Isolates of Pseudomonas aeruginosa Differs by Type III Secretion Genotype}

\author{
Melissa Agnello ${ }^{1}$, Steven E. Finkel ${ }^{2}$ and Annie Wong-Beringer ${ }^{1 *}$ \\ 'School of Pharmacy, University of Southern California, Los Angeles, CA, USA, ${ }^{2}$ Molecular and Computational Biology \\ Section, Department of Biological Sciences, University of Southern California, Los Angeles, CA, USA
}

Fluoroquinolone (FQ) resistance is highly prevalent among clinical strains of Pseudomonas aeruginosa, limiting treatment options. We have reported previously that highly virulent strains containing the exoU gene of the type III secretion system are more likely to be FQ-resistant than strains containing the exoS gene, as well as more likely to acquire resistance-conferring mutations in $g y r A / B$ and parC/E. We hypothesize that FQ-resistance imposes a lower fitness cost on exoU compared to exoS strains, thus allowing for better adaptation to the FQ-rich clinical environment. We created isogenic mutants containing a common FQ-resistance conferring point mutation in parC from three exoU to three exoS clinical isolates and tested fitness in vitro using head-to-head competition assays. The mutation differentially affected fitness in the exoU and exoS strains tested. While the addition of the parC mutation dramatically increased fitness in one of the exoU strains leaving the other two unaffected, all three exoS strains displayed a general decrease in fitness. In addition, we found that exoU strains may be able to compensate for the fitness costs associated with the mutation through better regulation of supercoiling compared to the exoS strains. These results may provide a biological explanation for the observed predominance of the virulent exoU genotype in FQ-resistant clinical subpopulations and represent the first investigation into potential differences in fitness costs of FQ-resistance that are linked to the virulence genotype of $P$. aeruginosa. Understanding the fitness costs of antibiotic resistance and possibilities of compensation for these costs is essential for the rational development of strategies to combat the problem of antibiotic resistance.

Keywords: Pseudomonas aeruginosa, fluoroquinolone resistance, type III secretion, fitness, virulence

\section{INTRODUCTION}

Pseudomonas aeruginosa is a Gram-negative pathogen that is the leading cause of nosocomial pneumonia (Restrepo and Anzueto, 2009; Quartin et al., 2013). Resistance to the fluoroquinolone (FQ) antibiotics has risen dramatically due to the widespread prescribing of this class of drug, limiting treatment options for P. aeruginosa infections (Linder et al., 2005). P. aeruginosa acquires resistance to the FQs through mutations in genes regulating the expression of efflux pumps and through point mutations in target site genes. The target enzymes of the FQs are the type II 
topoisomerases, GyrA/B and ParC/E (Hooper, 2001). Resistanceconferring mutations in these genes, known as target site mutations, have been well described in $P$. aeruginosa (Nakano et al., 1997; Mouneimné et al., 1999; Higgins et al., 2003).

Pseudomonas aeruginosa has the ability to cause a variety of severe infections due to its many virulence factors. Specifically, $P$. aeruginosa utilizes the type III secretion system (TTSS) during acute infections to evade phagocytosis, invade host cells, and cause cell death (Veesenmeyer et al., 2009). The TTSS consists of a molecular syringe-like apparatus that extends through the inner and outer membranes and directly contacts the host cell. This allows effector toxins (ExoU, ExoS, ExoY, and/or ExoT) to be directly injected into the cytoplasm of host cells. Although residing at entirely separate loci, the genes encoding the toxins ExoU and ExoS are mutually exclusive in most strains, with the exoS genotype accounting for about $70 \%$ of clinical and environmental strains (Feltman et al., 2001; Garey et al., 2008). While less prevalent in general, exoU strains are more virulent than exoS strains, as has been shown in animal models of acute pneumonia (Shaver and Hauser, 2004). Importantly, infection with these strains leads to poor outcomes in patients with ventilator-associated pneumonia (Roy-Burman et al., 2001; El Solh et al., 2008) as well as increased persistence and severity of disease (Schulert et al., 2003).

Alarmingly, clinical studies have shown a correlation between FQ-resistance and virulence. We have previously reported that patients infected with FQ-resistant strains of $P$. aeruginosa had threefold higher mortality or prolonged illness by an additional 5 days compared to those infected with FQ-susceptible strains (Hsu et al., 2005). In addition, clinical isolates with the more virulent exoU genotype were shown to more likely be FQresistant than exoS strains (Wong-Beringer et al., 2008; Agnello and Wong-Beringer, 2012). Others have reported similar results in isolates from various infection sites (Zhang et al., 2014; Peña et al., 2015). Furthermore, in a separate study, we found that the combined traits of FQ-resistance and exoU genotype among respiratory isolates of $P$. aeruginosa were significantly associated with the development of pneumonia rather than bronchitis or colonization (Sullivan et al., 2014), suggesting that resistance and virulence traits may be linked, negatively impacting disease severity.

In a large study of 270 clinical isolates, we found that significantly more exoU strains were FQ-resistant, compared to exoS strains ( $63 \%$ vs. $49 \%, p=0.03$ ). Sequencing of the FQ target site genes $g y r A / B$ and $p a r C / E$ revealed that exo $U$ strains were more likely than exoS strains to acquire two or more resistanceconferring target site mutations (Agnello and Wong-Beringer, 2012). Specifically, we found that while FQ-resistant exoU and exoS strains were similarly likely to possess a mutation in $g y r A$, exoU isolates were more likely to also have acquired an additional mutation in parC, resulting in greatly increased levels of resistance. While there is mounting evidence for the co-selection of resistance and virulence traits among pathogenic bacteria (Beceiro et al., 2013), the biological mechanisms underlying these observations are not well understood. Since the identification of the ExoU and ExoS toxins of the P. aeruginosa type III secretion system, many studies have investigated the roles of each toxin during infection (Allewelt et al., 2000; Schulert et al., 2003; Shaver and Hauser, 2004, 2006) while others have described the association of exoU strains with increased FQ resistance (Lakkis and Fleiszig, 2001; Maatallah et al., 2011) supporting our own observations. The aim of the current study was to gain insights into the biological basis underlying the differential development of resistance in exoU vs. exoS strains, which previous studies have not explored. We hypothesize that the greater propensity of exoU strains to acquire multiple target site mutations compared to exoS strains reflects a difference in the fitness costs associated with FQresistance mutations, favoring the strains with the exoU genetic background.

A recent meta-analysis of studies conducted on fitness costs of resistance to a variety of antibiotic classes in different organisms showed that most point mutations are generally costly (Melnyk et al., 2015). However, there is great variability depending on organism, drug, and mechanism of resistance. Furthermore, fitness costs can be mitigated through the accumulation of compensatory mutations that restore fitness to wild-type levels without loss of resistance (Andersson and Hughes, 2010). FQconferring mutations have been shown to impose variable fitness costs, depending on strain background and specific combinations of mutations (Komp Lindgren et al., 2005; Luo et al., 2005; Marcusson et al., 2009; Machuca et al., 2014; Wasels et al., 2015).

In the current study, we compared the fitness effects of a target site mutation in parC using isogenic mutants created from clinical exoU and exoS isolates. We investigated the fitness effects of this mutation using in vitro head-to-head competition experiments. Changes in supercoiling level and mutation frequency between mutants and parent strains were also assessed to explore potential mechanisms for the difference in fitness observed.

Our results suggest that the FQ resistance-conferring point mutation in parC studied here may confer less of a fitness cost to exoU strains than to exoS strains, with exoU strains showing evidence of compensation for the fitness costs. These results provide a potential biological explanation for the observed predominance of exoU strains in the clinical FQ-resistant population. The co-selection of FQ-resistance in highly virulent exoU strains will likely have a negative impact on patient outcomes, which underscores the importance to gain a better understanding of the biological basis for this observation.

\section{MATERIALS AND METHODS}

\section{Ethics Statement}

The institutional review boards at both Huntington Hospital and the University of Southern California have approved this study. Informed consent was waived from all participants since bacteria cultures were saved as part of a longitudinal epidemiological surveillance study. All data from respiratory cultures were analyzed anonymously to protect patient privacy. 
TABLE 1 | Characteristics of Strains.

\begin{tabular}{|c|c|c|c|c|c|}
\hline & Name & $\begin{array}{l}\text { LVX }^{a} \text { MIC } \\
(\mu \mathrm{g} / \mathrm{ml})\end{array}$ & $\begin{array}{l}\text { LVX + EPI } \\
M I C(\mu \mathrm{g} / \mathrm{ml})\end{array}$ & $\begin{array}{l}\text { Amino Acid } \\
\text { Change }\end{array}$ & $\begin{array}{l}\text { Doubling Time, } h \\
(-/+ \text { SEM })^{c}\end{array}$ \\
\hline \multirow[t]{6}{*}{ exou } & U-37 & 16 & 0.5 & GyrA: T83I & $2.0(1.7-2.4)$ \\
\hline & U-37PC* & 16 & 0.5 & $\begin{array}{l}\text { GyrA: T83I } \\
\text { ParC: S87L }\end{array}$ & $1.7(1.5-1.9)$ \\
\hline & U-91 & 2 & 2 & GyrA: T83। & $3.9(3.78-3.93)$ \\
\hline & U-91PC* & 16 & 2 & $\begin{array}{l}\text { GyrA: T83I } \\
\text { ParC: S87L }\end{array}$ & $3.9(3.87-3.96)$ \\
\hline & U-92 & 16 & 0.5 & GyrA: T83I & $2.5(2.3-2.7)$ \\
\hline & U-92PC* & 16 & 0.5 & $\begin{array}{l}\text { GyrA: T83I } \\
\text { ParC: S87L }\end{array}$ & $2.8(2.6-3.0)$ \\
\hline \multirow[t]{6}{*}{ exos } & S-139 & 4 & 0.5 & GyrA: T83। & $3.4(3.3-3.6)$ \\
\hline & S-139PC* & 32 & 0.25 & $\begin{array}{l}\text { GyrA: T831 } \\
\text { ParC: S87L }\end{array}$ & $3.1(2.9-3.3)$ \\
\hline & S-247 & 16 & 1 & GyrA: T83| & $2.5(2.4-2.6)$ \\
\hline & S-247PC* & 16 & 8 & $\begin{array}{l}\text { GyrA: T83I } \\
\text { ParC: S87L }\end{array}$ & $2.5(2.4-2.7)$ \\
\hline & S-215 & 16 & 1 & GyrA: T83। & $2.4(2.2-2.7)$ \\
\hline & S-215PC* & 16 & 1 & $\begin{array}{l}\text { GyrA: T831 } \\
\text { ParC: S87L }\end{array}$ & $2.3(2.2-2.5)$ \\
\hline
\end{tabular}

Mutants with Ser87Leu substitution in ParC denoted PC*. ' $L V X$, Levofloxacin; ${ }^{\mathrm{b}}$ EPI, efflux pump inhibitor; ${ }^{\mathrm{c}}$ Doubling time during exponential phase.

\section{Bacterial Strains and Culture Conditions}

Strains of $P$. aeruginosa used in this study (Table 1) were selected from a collection of isolates obtained from the respiratory tract of hospitalized patients at Huntington Hospital, Pasadena, CA, USA from 2005 to 2009 and were stored in cryovials at $-80^{\circ} \mathrm{C}$ in $30 \%$ glycerol. All strains had been previously characterized for: the presence of the exoU or exoS gene, resistance to levofloxacin, presence of mutations in the quinolone-resistance determining regions of $g y r A / B$ and $\operatorname{par} C / E$, and clonal relatedness by RAPD PCR, as described in Agnello and Wong-Beringer (2012). Strains were routinely grown as $5 \mathrm{ml}$ cultures in Luria-Bertani (LB) broth in $12 \mathrm{ml}$ polypropylene culture tubes at $37^{\circ} \mathrm{C}$ and shaking at $250 \mathrm{rpm}$ for liquid culture, or grown on Pseudomonas Isolation Agar (PIA) plates at $37^{\circ} \mathrm{C}$. Strains were routinely sub-cultured twice after inoculation from the frozen stock by 1:100 dilution (vol:vol) before use in any experiment.

\section{Susceptibility Testing and Sequencing}

Susceptibility testing to levofloxacin and rifampicin was performed by broth microdilution in twofold dilutions at concentrations ranging from 0.25 to $128 \mu \mathrm{g} / \mathrm{ml}$ according to guidelines recommended by CLSI (Clinical and Laboratory Standards Institute, 2007). In order to assess the involvement of the multidrug Mex efflux pumps to resistance, MIC of levofloxacin was also measured with the addition of an efflux pump inhibitor (EPI, MC-0228; Sigma) at $20 \mu \mathrm{g} / \mathrm{ml}$ (Kriengkauykiat et al., 2005).

For sequencing, genomic DNA was extracted and purified from isolates using the DNeasy Mini Kit (Qiagen). The quinolone-resistance determining regions of target genes gyr $A$, gyrB, parC, and parE were amplified by PCR using previously published primers and conditions and sequenced to identify mutations compared to wild-type strain PAO1 (Table 2) (Jalal and Wretlind, 1998; Lee et al., 2005; Winsor et al., 2011).

\section{Creation of Target Site Mutants}

Since most FQ-resistant isolates have gyrA mutations, three exoU and three exoS isolates with a pre-existing gyrA FQresistance mutation (Thr83 $\rightarrow$ Ile substitution) were selected for mutagenesis. A site-directed point mutation in the parC gene leading to the amino acid substitution Ser87 $\rightarrow$ Leu was introduced via electroporation of an oligonucleotide in order to create isogenic mutants, using the technique of oligonucleotide recombination as described previously (Swingle et al., 2010; Agnello and Wong-Beringer, 2014). Briefly, a single-stranded oligonucleotide 60 bases in length was designed using the parC gene sequence of reference strain PAO1 (Winsor et al., 2011) (Table 2). The oligo is identical to the PAO1 sequence from nucleotides 230 to 289 , save for the point mutation TCG $\rightarrow$ TTG at location 31 of the oligo, corresponding to nucleotide 260 in the parC gene. This point mutation, which is the most common parC mutation observed among fluoroquinolone-resistant clinical strains, gives rise to the Ser87 $\rightarrow$ Leu amino acid change in the ParC protein.

Strains were made electro-competent by several washes with $300 \mathrm{mM}$ sucrose solution as described in Choi et al. (2006) and transformed by electroporation at $2.5 \mathrm{kV}$ in a $0.2 \mathrm{~cm}$ cuvette using a Micropulser (Bio-Rad, Hercules, CA, USA). SOC medium $(1 \mathrm{ml})$ was immediately added and the cells were outgrown overnight at $37^{\circ} \mathrm{C}$ on PIA plus levofloxacin at concentrations two and fourfold above the original minimum inhibitory concentration (MIC) of the isolates for selection. Single colonies were collected and the parC gene was sequenced as described above to confirm that recombination had occurred.

\section{Growth Rate Measurements}

Independent overnight cultures were diluted to $\mathrm{OD}_{600} \sim 0.1$ and grown at $37^{\circ} \mathrm{C}$ with shaking in $50 \mathrm{ml} \mathrm{LB}$ broth in a $100 \mathrm{ml}$ flask. An aliquot of the culture $(150 \mu \mathrm{l})$ was sampled every $30 \mathrm{~min}$ for $8 \mathrm{~h}$ and turbidity was measured at $\mathrm{OD}_{600}$ using a Tecan Sunrise microplate reader (Tecan Group Ltd., Switzerland). Results were reported as an average of at least three independent experiments in terms of doubling time per hour.

\section{Insertion of Fluorescent Tag}

Mini-Tn7 vectors developed and generously shared with us by Dr. Herbert Schweizer (Choi et al., 2005) were utilized to insert cassettes containing the genes encoding YFP or CFP as well as a cassette containing the lux operon into strains used for competition experiments and supercoiling experiments, respectively. The cassette inserts into the $P$. aeruginosa genome at a single location ( $a t t \operatorname{Tn} 7$ site downstream of the $g l m S$ gene) and contains the gene for the fluorescent protein under the control of a constitutive promoter or the lux operon under the control of a supercoiling-sensitive promoter (Moir et al., 2007), as well as a gentamicin-resistance marker for selection. The delivery plasmid was co-electroporated with a helper plasmid ( $\mathrm{pTNS3}$ ) encoding the necessary transposase function for insertion. The electroporation protocol used for 
TABLE 2 | Genetic elements used.

\begin{tabular}{|c|c|c|}
\hline & Sequence & Reference \\
\hline \multicolumn{3}{|l|}{ Primer name PCR } \\
\hline glmS-up & CTGTGCGACTGCTGGAGCTGA & Choi and Schweizer, 2006 \\
\hline glmS-down & GCACATCGGCGACGTGCTCTC & Choi and Schweizer, 2006 \\
\hline Tn7-R & CACAGCATAACTGGACTGATITC & Choi and Schweizer, 2006 \\
\hline $\operatorname{Tn} 7-\mathrm{L}$ & ATTAGCTTACGACGCTACACC & Choi and Schweizer, 2006 \\
\hline \multicolumn{3}{|l|}{ Oligo for recombination } \\
\hline $\mathrm{PC}^{*}$ & $\begin{array}{l}\text { TGCTCGGCAAGTTCCACCCGCACGGCGACTTGGCCTGCTA } \\
\text { CGAGGCCATGGTGCTGATGG }\end{array}$ & Agnello and Wong-Beringer, 2012 \\
\hline Plasmid & Function & References \\
\hline pTNS3 & Helper plasmid, encodes Tn-7 transposition pathway & Choi et al., 2005 \\
\hline pUC18T-mini-Tn7T-Gm-eyfp & Delivery plasmid for $\mathrm{Gm}^{R}$ marker and YFP & Choi and Schweizer, 2006 \\
\hline pUC18T-mini-Tn7T-Gm-ecfp & Delivery plasmid for $\mathrm{Gm}^{\mathrm{R}}$ marker and CFP & Choi and Schweizer, 2006 \\
\hline TOP10/mini-Tn7-PA0614 promoter-Gm-luxCDABE & Delivery plasmid for $\mathrm{Gm}^{\mathrm{R}}$ marker and lux operon & Choi and Schweizer, 2006 \\
\hline
\end{tabular}

transformation is described in detail in Choi and Schweizer (2006); briefly, strains were made electro-competent through a series of washes with $300 \mathrm{mM}$ sucrose, subjected to electroporation as described above, and plated on LB plates containing $30 \mu \mathrm{g} / \mathrm{ml}$ gentamicin for selection. To confirm insertion had occurred, PCR was performed to amplify the insertion region using primers listed in Table 2 and protocol as previously described (Choi and Schweizer, 2006) (data not shown).

\section{In vitro Competition Assays}

To investigate the effects of the parC mutation ( $\mathrm{PC}^{*}$ ) on fitness, mutants were directly competed against isogenic parent strains in co-culture. Strains were tagged with either CFP or YFP for differentiation as described above. $\mathrm{PC}^{*}$ mutant strains were initially tagged with CFP, and competed against the respective isogenic parent strain tagged with YFP. To confirm neutrality of the tags, experiments were also performed with YFP-mutants and CFP-parents.

Strains were grown overnight in LB, and $10^{5} \mathrm{CFU}$ of each strain was co-inoculated into $10 \mathrm{ml} \mathrm{LB}$ in a $50 \mathrm{ml}$ flask, and grown with shaking at $37^{\circ} \mathrm{C}$. Every $24 \mathrm{~h}, 10 \mu \mathrm{l}$ of the culture was transferred to a new flask containing $10 \mathrm{ml}$ fresh LB, and a sample of the culture was serially diluted then plated on PIA for CFU enumeration. Colonies of each color were counted using a fluorescent wide-field microscope (Zeiss Axio Zoom.V16). Experiment duration ranged from 4 to 7 days. The ratio of the number of CFUs of the mutant-to-parent strain on each day of the experiment was calculated and used to determine the relative fitness of the mutant. Experiments were repeated a minimum of five times. Assays were also performed with the addition of levofloxacin equal to $1 / 8$ the MIC ( 2 or $0.5 \mu \mathrm{g} / \mathrm{ml})$. At day 7 of each competition experiment, colonies were selected from plates, inoculated into LB, grown overnight, and stored at $-80^{\circ} \mathrm{C}$ in $30 \%$ glycerol until ready for use in subsequent experiments. These were described as 'aged' strains. Aged strains were subjected to a secondary competition experiment vs. the original strains. Secondary competition with aged strains was performed as described above, and lasted for 4 days.

\section{Supercoiling Assay}

We adapted a reporter assay to estimate the ability to maintain supercoiling levels in mutants compared to parent strains by inserting a $\operatorname{Tn} 7$ cassette containing the $l u x$ operon under the control of a supercoiling-sensitive $P$. aeruginosa promoter from the gene PA0614 (Moir et al., 2007). The cassette was inserted as described above. Luminescent strains were grown to midexponential phase, then diluted 1:4 in LB+ levofloxacin at 1/4, $1 / 2$, and $1 x$ the measured MIC, and grown in triplicate in a deep well 96-well plate for $7 \mathrm{~h}$. Luminescence was measured using the Envision Multi-Label plate reader (Perkin-Elmer) as well as $\mathrm{OD}_{600}$ as described above. Relative Luminescence Units (RLU) was normalized to $\mathrm{OD}_{600}$ for all comparisons.

\section{Mutation Frequency Assay}

Mutation frequency was estimated during competition experiments using the spontaneous rifampicin resistance method (Oliver et al., 2000). At each 24-h interval, competition cultures were plated on PIA+ rifampicin at $5 \mathrm{x}$ the MIC (ranging from 40 to $500 \mu \mathrm{g} / \mathrm{ml}$ depending on the strain) in addition to plating on PIA. The mutation frequency reported is the number of colonies that grew on PIA + rifampicin after $48 \mathrm{~h}$ divided by the number of colonies on PIA alone. Colonies of each strain were differentiated and counted based on fluorescence as described above. For strain 139, 5x the MIC of rifampicin corresponds to $500 \mu \mathrm{g} / \mathrm{ml}$, which turned the plates a dark shade of red and prevented the differentiation of the fluorescence of the colonies. Thus, for this strain, each rifampicin-containing plate was replica-plated using sterile velvets onto a fresh PIA plate and incubated for an additional $24 \mathrm{~h}$ before colonies were counted.

\section{Statistical Analysis}

GraphPad Prism v.6 (GraphPad.com, San Diego, CA, USA) was used to perform statistical analysis. ANOVA was used to compare continuous variables where appropriate, and student's 
$t$-test was used to compare mean ratios to the null hypothesis of 1 (indicating no difference). A $p$ value $\leq 0.05$ denotes significance.

\section{RESULTS}

We previously created isogenic mutants from 6 clinical isolates of $P$. aeruginosa (three exoU and three exoS) by inserting a point mutation commonly observed in fluoroquinolone-resistant isolates into the parC gene (Agnello and Wong-Beringer, 2012). The goal of the current study was to investigate the biological effects of this target site mutation in a controlled genetic background. Mutants are denoted $\mathrm{PC}^{*}$. Interestingly, the addition of the parC mutation increased the levofloxacin MIC in only two of the six isolates. Characteristics of mutant and parent strains used in this study are summarized in Table 1.

\section{Growth Rate in Monoculture}

As an initial comparison of the relative fitness of mutant vs. parent strains, we tested the doubling time in independent cultures by measuring optical density every $30 \mathrm{~min}$ over an 8 $\mathrm{h}$ period; results are an average of at least three independent experiments (Table 1). Doubling time during exponential phase was calculated by dividing the time interval over the number of generations. Growth rates of all six mutants were comparable to parent strains.

\section{parC Mutation Differentially Affects Competitive Fitness of exoU vs. exoS Strains}

To investigate the fitness costs of the parC mutation, we performed head-to-head competition assays. Each PC* mutant was directly competed against its isogenic parent strain by growth in co-culture for 4 days. Because the strains competed are identical save for the point mutation, any fitness cost is attributable to the mutation. In each individual competition experiment, one strain is tagged with CFP (cyan fluorescent protein), and the other with YFP (yellow fluorescent protein). This allows for the direct enumeration of the number of colonies of each strain after serial dilution onto agar plates. Colonies were counted every $24 \mathrm{~h}$, and the relative fitness of the mutant is determined from the ratio of mutant-to-parent $\mathrm{CFU} / \mathrm{ml}$. Each experiment was independently repeated at least five times. We confirmed the neutrality of the YFP and CFP tags by repeating each experiment with each strain carrying the opposite tag. No difference was found, and therefore results from all experiments were combined.

Figure 1 shows the average mutant-to-parent ratios over time for each strain. Each strain has a unique pattern of fitness costs associated with this mutation; however, in general, there appears to be a higher fitness cost for the exoS strains. The exoS strains S-139PC*, S-247PC*, and S-215PC* show a consistent fitness defect over all time points, with strain S-139PC* the most affected. On the other hand, strain U-92 has the smallest fitness effect, with the mutant/parent ratios ranging from 1.2 to 2.0 over $2-4$ days of competition. Strain U-37 shows a similar pattern except the mutant starts with lower fitness (ratio 0.5 at day 2), but catches up to the parent strain by day 4 with a ratio of 0.99 . Strain U-91 is unique and interestingly shows a striking increase in fitness due to the mutation; the mutant rapidly outcompetes the parent strain and completely takes over the culture by day 3 .

\section{exoU-PC* Mutants can Better Maintain Wild-Type Supercoiling Levels than exoS-PC* Mutants}

We assessed supercoiling of parent vs. mutants in exoU strains U-92 and U-37 and exoS strains S-215 and S-139 by inserting a Tn7 genetic element in which a supercoiling sensitive promoter controls the lux operon (Moir et al., 2007). Due to challenges inserting the reporter, only two strains of each background were chosen as representative strains. Strains with the insertion were grown under levels of levofloxacin at and below the MIC in order to maximally induce expression of the reporter, and luminescence values were normalized to $\mathrm{OD}_{600}$. We measured the level of luminescence in the parent strain as an indirect reporter of the baseline level of supercoiling; any difference in luminescence expression for the $\mathrm{PC}^{*}$ strain reflects a change in supercoiling due to the parC mutation. Results show that the $\mathrm{PC}^{*}$ mutants in general have decreased luminescence compared to parent strains; however, this decrease was significantly more pronounced in exoS-PC** strains (Figure 2), suggesting that exoS strains with the $\mathrm{PC}^{*}$ mutation are somehow less able to maintain the supercoiling level of the parent strains, which may have negative effects on fitness. This is surprising given that the primary role of topoisomerase IV ( $\mathrm{ParC}$ ) is decatenation of daughter replicons and not regulation of supercoiling (Hooper, 2001); however, in this context it may suggest an overall difference in supercoiling regulation in exoU vs. exoS strains.

\section{Analysis of Aged Strains}

Because, we observed that the fitness of exoU-PC* mutants tended to increase after $24 \mathrm{~h}$ of competition, we investigated whether stable changes were occurring during competition that allowed the strains to compensate for fitness costs associated with the parC mutation. We collected colonies of parent and $\mathrm{PC}^{*}$ strains from a primary competition experiment (parent vs. mutant) after 7 days and labeled these 'aged' strains. In addition, we investigated the effect of sub-inhibitory levels of fluoroquinolone exposure on competitive fitness and ability to compensate for fitness costs by collecting aged strains from primary competition experiments in which $1 / 8$ MIC of levofloxacin was added to the media.

We were not able to collect aged colonies from the primary competition experiments of strain 91 due to the vast difference in fitness between the $\mathrm{PC}^{*}$ mutant and parent strains; the $\mathrm{PC}^{*}$ strain completely overtook the culture by day 3 . Because this left us with only two exoU strains (U-92 and U-37), we chose two exoS strains in order to have an equal number in each group for comparison. We chose strains 215 and 139 because these represent both ends of the spectrum in terms of fitness of the PC* mutants in vitro; strain S-215PC* shows the least fitness cost of 

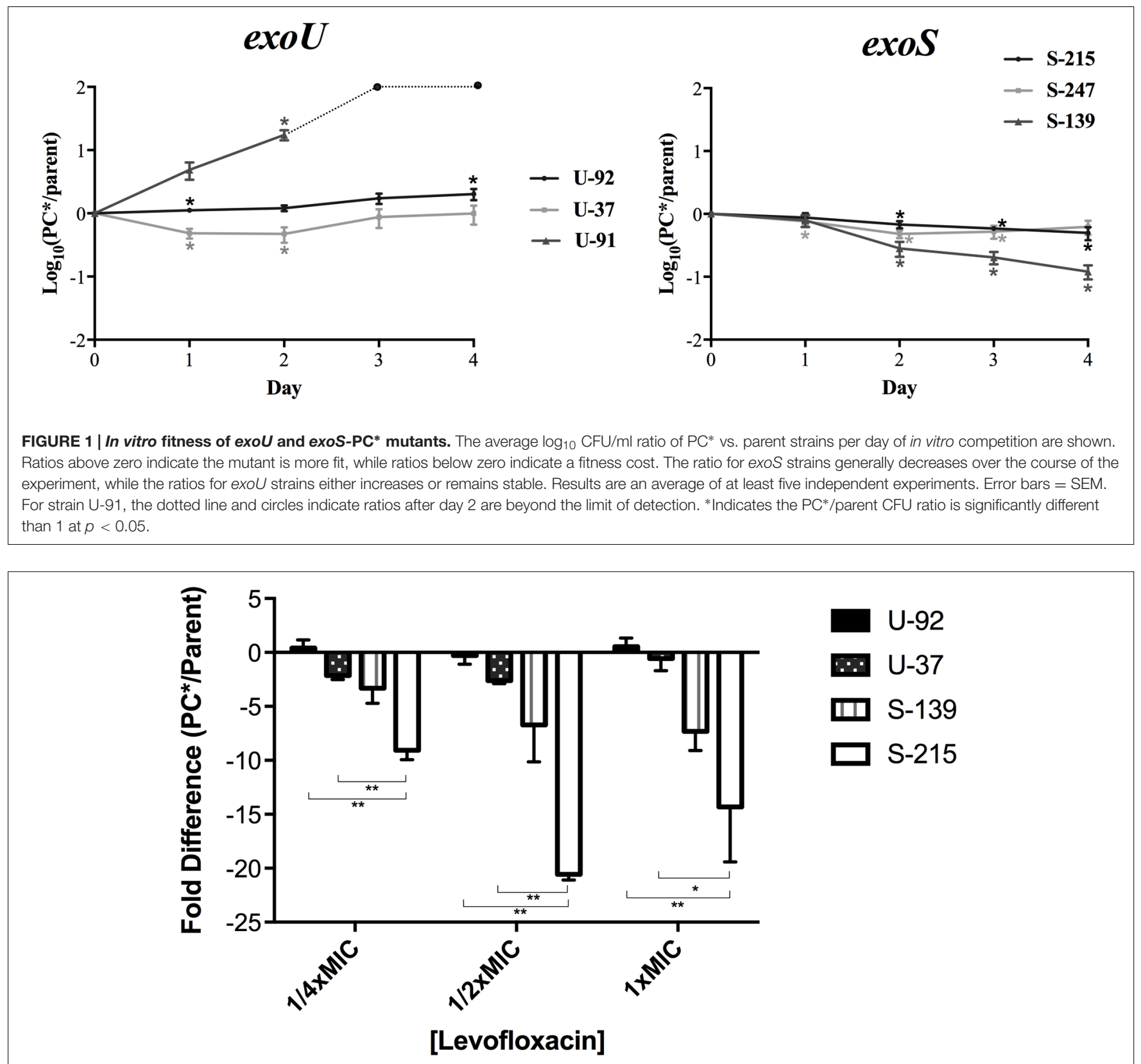

FIGURE 2 | Supercoiling changes in PC* mutants compared to parent strains. Each strain contains a chromosomal reporter construct in which the lux operon is under the control of a supercoiling-sensitive promoter. Strains were grown in concentrations of levofloxacin equal to $1 / 4,1 / 2$, and 1x the MIC. Luminescence was normalized to cell count using $\mathrm{OD}_{600}$. Results are an average of three independent experiments, and error bars represent SEM. ${ }^{*} p<0.05 ;{ }^{* *} p<0.01$.

all the exoS strains, while strain S-139PC* exhibits the greatest fitness cost.

Sequencing the FQ-target site genes of the aged strains revealed that no changes occurred in the genes associated with FQ-resistance except for exoU strain U-92, in which the parent acquired the Ser87Leu substitution in ParC during competition under levofloxacin exposure (+LVX) at 1/8 MIC. All $\mathrm{PC}^{*}$ strains maintained the parC mutation. The MIC to levofloxacin did not change in any of the aged strains (data not shown).

\section{Aged PC* Mutant exoU vs. exoS Strains Show Dramatic Difference in Fitness}

To investigate whether the aged strains had acquired adaptations during competition that rendered them more fit, we compared the fitness of each aged strain to its un-aged counterpart. Aged strains were subjected to a secondary competition experiment in which they were competed against the original, un-aged strain that contained the opposite fluorescent tag, enabling the discrimination and enumeration of each strain during competition. Results reflect the aged-to-un-aged ratio at Day 
2 (Figure 3). Because each aged strain was competed directly against an un-aged version of itself, we were able to directly observe if fitness had changed in the aged strains. All parent strains had a negligible change in fitness. Both exoU-PC* aged strains increased in fitness greater than fourfold, while the exoSPC* aged strains showed a 50\% decrease in fitness; S-139PC* was greater than 100 times less fit than before aging.

We also investigated whether growth under sub-inhibitory levels of levofloxacin affected fitness, and if mechanisms to compensate for fitness costs evolved under these conditions. The addition of levofloxacin did not affect the results of primary competition experiments (data not shown), but notable results were seen in the secondary competition experiments of the aged vs. un-aged strains, which also included 1/8 MIC of levofloxacin. Upon subsequent exposure to the drug, exoU-PC* strains were much more fit, outcompeting the un-aged strain rapidly. Specifically, the aged $\mathrm{PC}^{*}$ mutants of exoU strains U37 and U-92 outcompeted the un-aged strains significantly and rapidly, much more so than in the competition experiments without the drug. Interestingly, the measured MICs of the aged and un-aged strains remain unchanged. exoS-PC* strains showed conflicting results; S-215PC* had gained fitness, while S-139PC* had slightly decreased fitness (Figure 3).

\section{Mutation Frequency}

Compensation for potential fitness costs of a resistanceconferring mutation can evolve through the accumulation of beneficial mutations. Therefore, we investigated whether the rate of spontaneous mutation frequency could explain the increase in fitness during competition that was seen in exoU-PC* strains. Mutation frequency was estimated by calculating the frequency of spontaneous resistance to rifampicin ( $\mathrm{Rif}^{\mathrm{R}}$ ) (Oliver et al., 2000). We estimated mutation frequency during the primary competition experiments for exoU strains U-92 and U-37, and
exoS strains S-215 and S-139 by plating the mixed competition culture on agar containing $5 \mathrm{x}$ the MIC of rifampicin at each time point (Days 1-7), and counting the number of colonies based on fluorescence of each strain that were able to grow on the rifampicin-containing plates compared to the overall $\mathrm{CFU}$ counts. At Day 7, the exoU-PC* strains have both a higher mutation frequency than the parent strains as well as a higher mutation frequency compared to Day 1. exoS strain S-215PC* had a lower frequency at Day 7 compared to its parent strain as well as a lower frequency compared to Day 1. Surprisingly, exoS strain S-139PC*, which is significantly less fit than its parent strain, has a twofold higher mutation frequency than the parent strain at Day 7. However, its mutation frequency at Day 7 decreased almost threefold from Day 1 (Figure 4). Therefore, mutation frequencies seem to generally correlate with fitness results.

\section{DISCUSSION}

Pseudomonas aeruginosa is considered one of six bacteria that pose an immediate threat to public health, according to the Infectious Diseases Society of America, due to its increasing prevalence, broad arsenal of virulence factors, and emergence of resistance to all available antibiotics (Boucher et al., 2009). In its recent report entitled "Antibiotic Resistance Threats in the United States" the Centers for Disease Control named $P$. aeruginosa a serious threat that requires prompt action and continual monitoring to prevent a worsening of the problem of resistance (Centers for Disease Control [CDC], 2013). Notably, resistance rates of $P$. aeruginosa to the once-effective fluoroquinolone (FQ) antibiotics are now greater than $30 \%$ in the United States, with many of those strains also multi-drug resistant (Rosenthal et al., 2012).

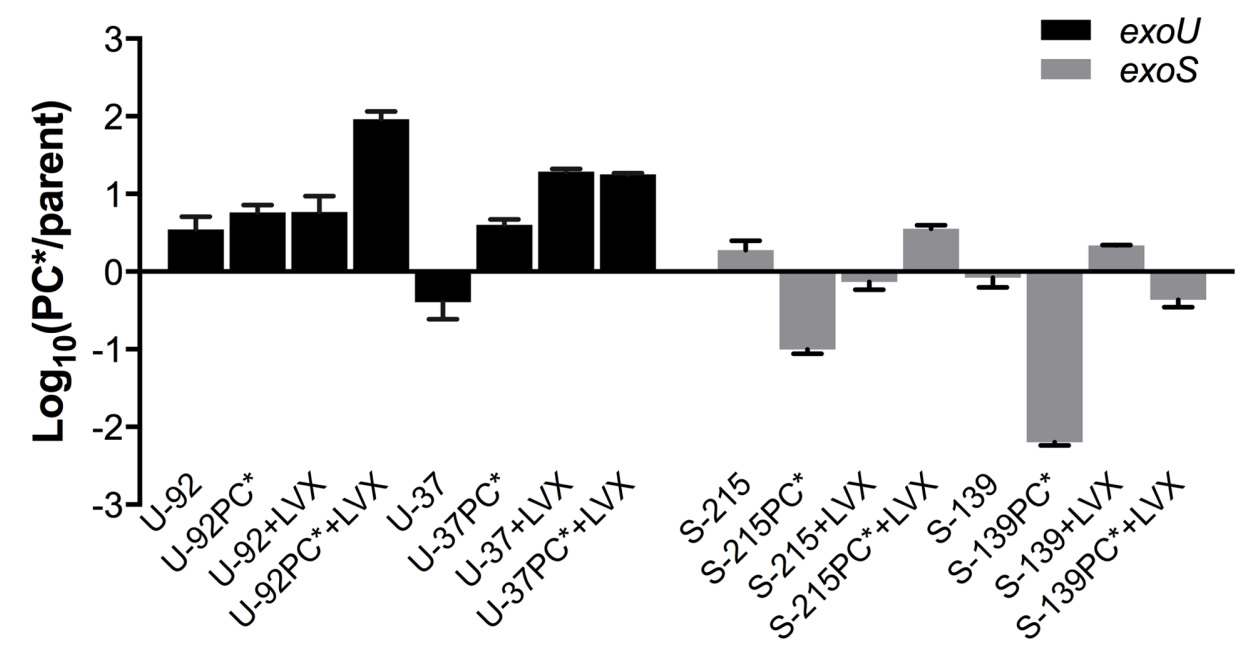

FIGURE 3 | Fitness of aged strains. Strains were collected at the end of a 7-day primary competition experiment (PC* vs. parent) with and without the addition of sub-inhibitory concentration of levofloxacin (+LVX) and subsequently re-competed in a secondary competition experiment (aged vs. un-aged) with or without levofloxacin at the same concentration. Results shown reflect the average fitness of aged strains after $48 \mathrm{~h}$ of competition. Experiments were performed in duplicate. Error bars $=$ SEM. 


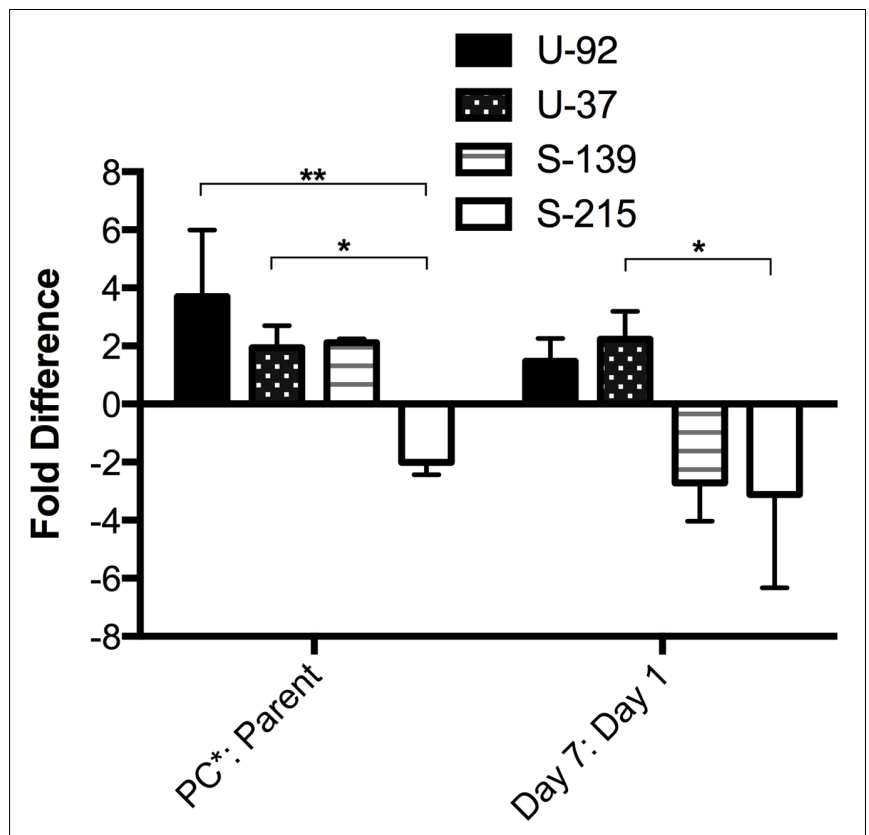

FIGURE 4 | Mutation frequency of PC* mutants vs. parent strains measured during competition experiments. The frequency of spontaneous rifampicin resistance was used to estimate mutation frequency at each time point during a 7-day competition experiment. Bars represent fold difference of the PC* vs. parent at Day 7 and the fold difference at Day 7 vs. Day 1 for each PC* strain. Error bars $=$ SEM. ${ }^{*} p<0.05 ;{ }^{* *} p<0.01$.

During acute disease, $P$. aeruginosa utilizes the toxins of the type III secretion system to circumvent the host immune system and establish infection. Of the four exotoxins, exoU is the most virulent, encoding a potent phospholipase that disrupts the plasma membrane and leads to rapid cell death (Sato et al., 2003). ExoU activity induces excessive inflammation and tissue damage in the host as well as increased bacterial dissemination that can lead to septic shock (Kurahashi et al., 1999) and higher rates of mortality compared to infections with ExoS-secreting strains (Shaver and Hauser, 2004; Zhang et al., 2014; Peña et al., 2015).

As the problem of antibiotic resistance continues to worsen, it is becoming apparent that the evolution of virulence factors and antibiotic resistance cannot be considered strictly independent processes (Beceiro et al., 2013; Hauser, 2014). Previously, we found that infection by FQ-resistant $P$. aeruginosa was an independent predictor for threefold higher mortality or prolonged illness by an additional 5 days compared to those infected with FQ-susceptible strains (Hsu et al., 2005). In a separate study, we reported that among patients from whom $P$. aeruginosa was isolated from the respiratory tract, strains with the combined traits of exoU and FQ-resistance were much more likely to cause pneumonia than exoS, FQ-resistant strains or either trait alone (Sullivan et al., 2014).

Analysis of 270 of our $P$. aeruginosa clinical isolates led to the observation that exoU strains are not only enriched in the FQ resistant sub-population, but are also more likely to have acquired two or more target site mutations than exoS strains, especially in the parC gene (Agnello and Wong-Beringer, 2012).
In order to study the biological effects of a resistance-conferring mutation in a controlled, isogenic background, we adapted a technique for inserting point mutations into bacterial genomes for use in our clinical isolates (Swingle et al., 2010). Using the technique of oligonucleotide recombination, we created 6 FQ-resistant mutants (three exoU and three exoS) (Agnello and Wong-Beringer, 2014) from clinical strains by inserting a mutation into the parC gene.

Because the target site mutation, we inserted in parC rarely occurs in clinical isolates without a pre-existing mutation in gyr $A$ (Jalal and Wretlind, 1998), we chose clinical strains that had a pre-existing gyrA mutation (Thr83Ile) in order to mimic FQresistant strains encountered in the clinical setting. These specific point mutations in gyrA and parC are commonly found in FQresistant clinical strains of $P$. aeruginosa (Nakano et al., 1997; Mouneimné et al., 1999; Higgins et al., 2003; Agnello and WongBeringer, 2012). The goal of the current study was to assess the biological effects on fitness of the parC mutation and determine if the magnitude of the effects differ in exoU vs. exoS strains.

The acquisition of resistance is generally thought to be accompanied by a fitness cost to the bacterium (Andersson and Levin, 1999); however, there are many reports of neutral fitness effects or enhanced fitness due to resistance-conferring mutations. Evidence from FQ-resistant E. coli, S. pneumoniae, and $N$. gonorrhoeae suggest that a secondary resistance mutation does not further decrease fitness and may restore the low fitness of primary mutants back to wild-type levels (Komp Lindgren et al., 2005; Rozen et al., 2007; Marcusson et al., 2009; Kunz et al., 2012; Machuca et al., 2014). Furthermore, many fitness effects seem to be strain dependent, as was shown in a study of ciprofloxacin-resistant Campylobacter jejuni, in which a gyrA mutation conferred a high cost to one strain but actually increased fitness for a different strain (Luo et al., 2005). Similar results were seen in S. pneumoniae, in which some FQ-resistant mutant strains had increased fitness while others had a significant cost (Balsalobre and de la Campa, 2008). This suggests that fitness effects are highly dependent on strain genetic background, which may explain the differences, we have seen between exoU and exoS strains.

Head-to-head competition assays are a standard method for investigating the relative fitness of a mutant strain compared to its isogenic parent strain, and it is possible to detect differences in fitness as small as 1\% (Andersson and Levin, 1999; Corzett et al., 2013). Strains are mixed together in co-culture, and must compete for the limited available resources (Finkel, 2006; Kraigsley and Finkel, 2009). In most studies, strains are differentiated based on selective growth on antibiotic plates. However, since the parC mutation, we inserted did not increase the levofloxacin MIC in all strains, we had to develop a different approach. We took advantage of a Tn7-based system developed by Schweizer (Choi et al., 2005) in order to insert a cassette containing either YFP or CFP under the control of a strong promoter.

The growth of the $\mathrm{PC}^{*}$ strains did not differ dramatically from those of the parents when grown in monoculture; however, differences were seen when strains had to compete for resources in the same culture flask during competition experiments. 
Overall, the parC mutation imposed a moderate to considerable fitness cost on exoS strains, while exoU strains were able to better tolerate the mutation. Notably, fitness of exoU-PC* strains ranged from maintaining the wild-type level of fitness to outcompeting the parent strain by more than 10 -fold, whereas exoS-PC* ${ }^{*}$ strains were consistently less fit than parent strains.

The FQ target site mutation investigated in the current study occurs in a topoisomerase enzyme and therefore may have an effect on the regulation of supercoiling. Common methods for investigating supercoiling levels rely on reporter plasmids that need to be selected for and maintained. Because, we are using clinical isolates, there is a high level of multi-drug resistance that precludes the use of standard selection antibiotics. To circumvent this, we inserted a Tn7 element (Moir et al., 2007) onto the chromosome in which a supercoiling sensitive promoter controls lux expression. exoU-PC* mutants were better able to maintain the supercoiling levels of the parent strains, while exoSPC* mutants showed a more drastic change in supercoiling, as observed by decreased lux expression. Other studies have shown that changes in supercoiling are correlated with changes in fitness (Kugelberg et al., 2005; Marcusson et al., 2009), and therefore the regulation of supercoiling may reflect the level of overall fitness and may explain the fitness differences seen in our strains. The ability of exoU-PC* strains to better regulate supercoiling levels under the stress of FQ exposure reflects increased fitness of these strains overall. The supercoiling level of the cell affects global gene expression and replication efficiency, and changes in supercoiling may alter response to environmental stressors, or even modulate pathogenesis in the host (Redgrave et al., 2014).

In the primary competition experiments, exoU-PC* mutants tended to increase in fitness over the course of the 4-day experiment. This suggests that exoU strains may be acquiring beneficial adaptations that allow for the compensation of the fitness costs associated with the $\mathrm{PC}^{*}$ mutation. Bacteria have the ability to rapidly evolve compensatory mechanisms to mitigate the fitness costs associated with antibiotic resistance, and compensatory mechanisms can reverse fitness costs without any loss of resistance (Andersson and Hughes, 2010). The exoUPC* strains that had been 'aged' through a primary competition experiment for 7 days have increased fitness compared to the unaged strains, while aged exoS-PC* strains have decreased fitness. The results suggest that the compensatory mechanisms in exoU strains most likely are acting as a repressor of the negative fitness effects of the parC mutation, as opposed to just conferring a general gain in fitness, based on the observation that the aged parent strains did not show as much of an increase in fitness as the PC* mutants. The observed ability of exoU strains to compensate for the costs of FQ-resistance has many clinically negative consequences. Compensation in clinical populations leads to the stabilization of resistant populations without the presence of drug (Andersson and Hughes, 2010). Increased fitness after acquisition of FQ-resistance adds to the already complicated problem of treating infections caused by highly virulent exoU strains.

The inability of exoS-PC* strains to regulate supercoiling suggests that the compensatory mechanisms in exoU strains are acting to maintain wild-type supercoiling levels in the PC* mutants, as has been reported in other studies of compensation of the costs of FQ-resistance (Kugelberg et al., 2005; Marcusson et al., 2009). Although, we did not identify the exact mechanisms responsible for the compensatory effects, we have shown that they are stable since strains were frozen and grown before testing for compensation in secondary competition experiments, and the results were reproducible in repeated experiments. Sequencing of the quinolone-resistance determining regions of $\operatorname{gyr} A / B$ and $p a r C / E$ revealed no additional mutations had occurred during competition; however, beneficial mutations may have occurred elsewhere. For both exoU strains tested, the mutation frequencies correlated with the increase in fitness observed during competition experiments, suggesting that higher mutation frequency may facilitate the acquisition of beneficial mutations that mitigate the fitness costs of the parC mutation.

A dramatic increase in fitness occurred in the aged exoU-PC* strains taken from a primary competition experiment in which sub-inhibitory levels (1/8 the MIC) of levofloxacin were added to culture. Although this low level of drug did not affect the growth of strains in the primary competition experiment, when the aged exoU-PC* strains were re-competed under the same concentration of levofloxacin, they were 20-100 times more fit than before aging. In contrast, exoS-PC* strains were dramatically less fit under these circumstances. However, none of the aged PC* strains had an increase in MIC, nor any additional FQ-resistance mutations in target site genes. These results suggest that although the level of levofloxacin was much below the MIC, highly fit strains were selected for rapidly, more so than in conditions without drug. Also, the highly fit strains were not more resistant, suggesting that perhaps the presence of levofloxacin accelerated the process of compensation for the already present resistance mutations. As sub-inhibitory concentrations of antibiotics are routinely present during treatment due to insufficient dosing or inadequate penetration to certain areas of the body (Baquero and Negri, 1997; Andersson and Hughes, 2014), the implications of our results are alarming and suggest that low levels of antibiotic can rapidly select for highly fit strains, preferentially the highly virulent exoU-containing strains. Furthermore, when these highly fit, highly virulent strains are re-introduced to the antibiotic, they will rapidly outcompete all other strains. The sub-inhibitory concentration was also able to select for a parC mutation in an exoU parent strain, emphasizing the known phenomenon that selection for resistance mutations can occur at sub-inhibitory levels of antibiotic (Baquero et al., 1998; Andersson and Levin, 1999; Gullberg et al., 2011; Andersson and Hughes, 2014).

Overall, these results suggest a potentially lower fitness burden associated with FQ-resistance for exoU strains than for exoS strains, which in part provides an initial biological explanation for exoU strains' greater tendency to acquire FQ-resistance in the clinical setting. Although, we have yet to identify the specific genetic components underlying the fitness differences, we suspect that genes unique to strains with the exoU genetic background may contribute to the fitness benefits seen in this study. The $P$. aeruginosa genome consists of a highly conserved core genome, but variability is introduced in the form of genomic islands, which make up the accessory genome. Genes within the islands usually encode accessory activities such as specific 
pathogenicity or symbiosis factors (Harrison et al., 2010). The exoU gene, along with its chaperone $\operatorname{spc} U$, is located on a pathogenicity island, a specialized genomic island. exoU has been identified as part of a few different pathogenicity islands. The most highly studied is from the reference strain PA14, in which the exoU gene is on an island named PAPI-2 (Kulasekara et al., 2006). Studies with PA14 have shown that virulence is dependent on the presence of the entire island and not just the exo $U$ gene alone; therefore, other as yet unknown genes contained on the pathogenicity islands contribute combinatorially to the increased virulence of exoU strains (Harrison et al., 2010). Furthermore, pathogenicity in strain PA14 requires the coordinated action of multiple virulence factors, associated with both the core and accessory genomes (Lee et al., 2006). Therefore, it is possible that other genes in the accessory genome in combination with exoU may provide fitness benefits to exoU strains that allow for increased ability to adapt to the fitness costs of FQ-resistance. Genomic islands have been shown to confer fitness benefits, and the accessory genome of $P$. aeruginosa is an important driver of the ability of strains to persist in a particular environment (Hacker and Carniel, 2001; Ozer et al., 2014).

This study has several limitations. We acknowledge that a limited number of clinical strains and their isogenic mutants were evaluated in the study. Despite having access to a large collection of clinical strains, the selection of strains to create isogenic target site mutants to study the effect of FQ-resistance on fitness proved challenging, as most of the isolates in our collection were found to already contain the resistanceconferring mutation that, we are investigating, due to the high prevalence of FQ resistance in clinical isolates of $P$. aeruginosa. Therefore, we were not able to use those to create isogenic pairs. Furthermore, genetic manipulation of these clinical isolates proved to be difficult, as these strains were not universally amenable to the creation of isogenic mutants via oligonucleotide recombination. Nonetheless, it is worth nothing that the strains evaluated in this study were carefully selected to represent a broad range of characteristics such as varying degrees of FQ resistance, virulence, and clinical outcomes. While the limited

\section{REFERENCES}

Agnello, M., and Wong-Beringer, A. (2012). Differentiation in quinolone resistance by virulence genotype in Pseudomonas aeruginosa. PLoS ONE 7:e42973. doi: 10.1371/journal.pone.0042973

Agnello, M., and Wong-Beringer, A. (2014). The use of oligonucleotide recombination to generate isogenic mutants of clinical isolates of Pseudomonas aeruginosa. J. Microbiol. Methods 98, 23-25. doi: 10.1016/j.mimet.2013. 11.014

Allewelt, M., Coleman, F. T., Grout, M., Priebe, G. P., and Pier, G. B. (2000). Acquisition of expression of the Pseudomonas aeruginosa ExoU cytotoxin leads to increased bacterial virulence in a murine model of acute pneumonia and systemic spread. Infect. Immun. 68, 3998-4004. doi: 10.1128/IAI.68.7.39984004.2000

Andersson, D. I., and Hughes, D. (2010). Antibiotic resistance and its cost: is it possible to reverse resistance? Nat. Rev. Microbiol. 8, 260-271. doi: 10.1038/nrmicro2319

Andersson, D. I., and Hughes, D. (2014). Microbiological effects of sublethal levels of antibiotics. Nat. Rev. Microbiol. 12, 465-478. doi: 10.1038/nrmic ro3270 number of strains evaluated in this study may not necessarily represent the genetic variability present among all exoU and exoS clinical strains, strain-specific differences in fitness effects were observed even among strains with the same exoU or exoS genetic background, though the trend observed appears to follow similar pattern for strains with the same exoU or exoS genetic background.

Understanding the fitness costs of antibiotic resistance and possibilities of compensation for these costs is essential for rationally combating the problem of antibiotic resistance. Importantly, this study outlines a useful model with the creation of isogenic clinical strains for investigating the biological costs of resistance in a medically important pathogen and can be applied to other drug-organism pairs.

\section{AUTHOR CONTRIBUTIONS}

MA, AW-B, and SF designed the experiments. MA and AW-B wrote the manuscript. SF edited and provided critical review of the manuscript.

\section{FUNDING}

This work was supported by award number TL1TR000132 from NIH/NCRR/NCATS to MA and award number R21AI073467 from the National Institute of Allergy and Infectious Diseases to AWB. SF was supported in part by U.S. Army Research Office grants W911NF1010444 and W911NF1210321.

\section{ACKNOWLEDGMENTS}

The authors thank Dr. Herbert Schweizer and Dr. Donald Moir for generously sharing plasmids and strains, and Juliana Brondani and Nicole Schrad for assistance with growth and competition experiments.

Andersson, D. I., and Levin, B. R. (1999). The biological cost of antibiotic resistance. Curr. Opin. Microbiol. 2, 489-493. doi: 10.1016/S1369-5274(99)00005-3

Balsalobre, L., and de la Campa, A. G. (2008). Fitness of Streptococcus pneumoniae fluoroquinolone-resistant strains with topoisomerase IV recombinant genes. Antimicrob. Agents Chemother. 52, 822-830. doi: 10.1128/AAC.00731-07

Baquero, F., and Negri, M. C. (1997). Selective compartments for resistant microorganisms in antibiotic gradients. Bioessays 19, 731-736. doi: 10.1002/bies.950190814

Baquero, F., Negri, M. C., Morosini, M. I., and Blázquez, J. (1998). Antibiotic-selective environments. Clin. Infect. Dis. 27(Suppl. 1), S5-S11. doi: 10.1086/514916

Beceiro, A., Tomás, M., and Bou, G. (2013). Antimicrobial resistance and virulence: a successful or deleterious association in the bacterial world? Clin. Microbiol. Rev. 26, 185-230. doi: 10.1128/CMR.00059-12

Boucher, H. W., Talbot, G. H., Bradley, J. S., Edwards, J. E., Gilbert, D., Rice, L. B., et al. (2009). Bad bugs, no drugs: no ESKAPE! An update from the infectious diseases society of America. Clin. Infect. Dis. 48, 1-12. doi: 10.1086/595011

Centers for Disease Control [CDC] (2013). Antibiotic Resistance Threats in the United States. Atlanta, GA: Centers for Disease Control. 
Choi, K. H., Gaynor, J. B., White, K. G., Lopez, C., Bosio, C. M., KarkhoffSchweizer, R. R., et al. (2005). A Tn7-based broad-range bacterial cloning and expression system. Nat. Methods 2, 443-448. doi: 10.1038/nmeth765

Choi, K. H., Kumar, A., and Schweizer, H. P. (2006). A 10-min method for preparation of highly electrocompetent Pseudomonas aeruginosa cells: application for DNA fragment transfer between chromosomes and plasmid transformation. J. Microbiol. Methods 64, 391-397. doi: 10.1016/j.mimet.2005.06.001

Choi, K. H., and Schweizer, H. P. (2006). mini-Tn7 insertion in bacteria with single attTn7 sites: example Pseudomonas aeruginosa. Nat. Protoc. 1, 153-161. doi: 10.1038 /nprot.2006.26

Clinical and Laboratory Standards Institute (2007). Performance Standards for Antimicrobial Susceptibility Testing: 17th Informational Supplement. Wayne, PA: Clinical and Laboratory Standards Institute, M100-M117.

Corzett, C. H., Goodman, M. F., and Finkel, S. E. (2013). Competitive fitness during feast and famine: how SOS DNA polymerases influence physiology and evolution in Escherichia coli. Genetics 194, 409-420. doi: 10.1534/genetics.113.151837

El Solh, A. A., Akinnusi, M. E., Wiener-Kronish, J. P., Lynch, S. V., Pineda, L. A., and Szarpa, K. (2008). Persistent infection with Pseudomonas aeruginosa in ventilator-associated pneumonia. Am. J. Respir. Crit. Care Med. 178, 513-519. doi: 10.1164/rccm.200802-2390C

Feltman, H., Schulert, G., Khan, S., Jain, M., Peterson, L., and Hauser, A. R. (2001). Prevalence of type III secretion genes in clinical and environmental isolates of Pseudomonas aeruginosa. Microbiology 147, 2659-2669. doi: 10.1099/00221287147-10-2659

Finkel, S. E. (2006). Long-term survival during stationary phase: evolution and the GASP phenotype. Nat. Rev. Microbiol. 4, 113-120. doi: 10.1038/nrmicro1340

Garey, K. W., Vo, Q. P., Larocco, M. T., Gentry, L. O., and Tam, V. H. (2008). Prevalence of type III secretion protein exoenzymes and antimicrobial susceptibility patterns from bloodstream isolates of patients with Pseudomonas aeruginosa bacteremia. J. Chemother. 20, 714-720. doi: 10.1179/joc.2008.20.6.714

Gullberg, E., Cao, S., Berg, O. G., Ilbäck, C., Sandegren, L., Hughes, D., et al. (2011). Selection of resistant bacteria at very low antibiotic concentrations. PLoS Pathog. 7:e1002158. doi: 10.1371/journal.ppat.1002158

Hacker, J., and Carniel, E. (2001). Ecological fitness, genomic islands and bacterial pathogenicity. a Darwinian view of the evolution of microbes. EMBO Rep. 2, 376-381.

Harrison, E. M., Carter, M. E., Luck, S., Ou, H. Y., He, X., Deng, Z., et al. (2010). Pathogenicity islands PAPI-1 and PAPI-2 contribute individually and synergistically to the virulence of Pseudomonas aeruginosa strain PA14. Infect. Immun. 78, 1437-1446. doi: 10.1128/IAI.00621-09

Hauser, A. R. (2014). Pseudomonas aeruginosa virulence and antimicrobial resistance: two sides of the same coin? Crit. Care Med. 42, 201-202. doi: 10.1097/CCM.0b013e3182a120cd

Higgins, P. G., Fluit, A. C., Milatovic, D., Verhoef, J., and Schmitz, F. J. (2003). Mutations in GyrA, ParC, MexR and NfxB in clinical isolates of Pseudomonas aeruginosa. Int. J. Antimicrob. Agents 21, 409-413. doi: 10.1016/S09248579(03)00009-8

Hooper, D. C. (2001). Emerging mechanisms of fluoroquinolone resistance. Emerg. Infect. Dis. 7, 337-341. doi: 10.3201/eid0702.010239

Hsu, D. I., Okamoto, M. P., Murthy, R., and Wong-Beringer, A. (2005). Fluoroquinolone-resistant Pseudomonas aeruginosa: risk factors for acquisition and impact on outcomes. J. Antimicrob. Chemother. 55, 535-541. doi: 10.1093/jac/dki026

Jalal, S., and Wretlind, B. (1998). Mechanisms of quinolone resistance in clinical strains of Pseudomonas aeruginosa. Microb. Drug Resist. 4, 257-261. doi: 10.1089/mdr.1998.4.257

Komp Lindgren, P., Marcusson, L. L., Sandvang, D., Frimodt-Møller, N., and Hughes, D. (2005). Biological cost of single and multiple norfloxacin resistance mutations in Escherichia coli implicated in urinary tract infections. Antimicrob. Agents Chemother. 49, 2343-2351. doi: 10.1128/AAC.49.6.2343-2351.2005

Kraigsley, A. M., and Finkel, S. E. (2009). Adaptive evolution in single species bacterial biofilms. FEMS Microbiol. Lett. 293, 135-140. doi: 10.1111/j.15746968.2009.01526.x

Kriengkauykiat, J., Porter, E., Lomovskaya, O., and Wong-Beringer, A. (2005). Use of an efflux pump inhibitor to determine the prevalence of efflux pump-mediated fluoroquinolone resistance and multidrug resistance in Pseudomonas aeruginosa. Antimicrob. Agents Chemother. 49, 565-570. doi: 10.1128/AAC.49.2.565-570.2005

Kugelberg, E., Löfmark, S., Wretlind, B., and Andersson, D. I. (2005). Reduction of the fitness burden of quinolone resistance in Pseudomonas aeruginosa. J. Antimicrob. Chemother. 55, 22-30. doi: 10.1093/jac/dkh505

Kulasekara, B. R., Kulasekara, H. D., Wolfgang, M. C., Stevens, L., Frank, D. W., and Lory, S. (2006). Acquisition and evolution of the exoU locus in Pseudomonas aeruginosa. J. Bacteriol. 188, 4037-4050. doi: 10.1128/JB.02000-05

Kunz, A. N., Begum, A. A., Wu, H., D’Ambrozio, J. A., Robinson, J. M., Shafer, W. M., et al. (2012). Impact of fluoroquinolone resistance mutations on gonococcal fitness and in vivo selection for compensatory mutations. J. Infect. Dis. 205, 1821-1829. doi: 10.1093/infdis/jis277

Kurahashi, K., Kajikawa, O., Sawa, T., Ohara, M., Gropper, M. A., Frank, D. W., et al. (1999). Pathogenesis of septic shock in Pseudomonas aeruginosa pneumonia. J. Clin. Invest. 104, 743-750. doi: 10.1172/JCI7124

Lakkis, C., and Fleiszig, S. M. (2001). Resistance of Pseudomonas aeruginosa isolates to hydrogel contact lens disinfection correlates with cytotoxic activity. J. Clin. Microbiol. 39, 1477-1486. doi: 10.1128/JCM.39.4.1477-1486.2001

Lee, D. G., Urbach, J. M., Wu, G., Liberati, N. T., Feinbaum, R. L., Miyata, S., et al. (2006). Genomic analysis reveals that Pseudomonas aeruginosa virulence is combinatorial. Genome Biol. 7:R90. doi: 10.1186/gb-2006-7-10-r90

Lee, J. K., Lee, Y. S., Park, Y. K., and Kim, B. S. (2005). Alterations in the GyrA and GyrB subunits of topoisomerase II and the ParC and ParE subunits of topoisomerase IV in ciprofloxacin-resistant clinical isolates of Pseudomonas aeruginosa. Int. J. Antimicrob. Agents 25, 290-295. doi: 10.1016/j.ijantimicag.2004.11.012

Linder, J. A., Huang, E. S., Steinman, M. A., Gonzales, R., and Stafford, R. S. (2005). Fluoroquinolone prescribing in the United States: 1995 to 2002. Am. J. Med. 118, 259-268. doi: 10.1016/j.amjmed.2004.09.015

Luo, N., Pereira, S., Sahin, O., Lin, J., Huang, S., Michel, L., et al. (2005). Enhanced in vivo fitness of fluoroquinolone-resistant Campylobacter jejuni in the absence of antibiotic selection pressure. Proc. Natl. Acad. Sci. U.S.A. 102, 541-546. doi: 10.1073/pnas.0408966102

Maatallah, M., Cheriaa, J., Backhrouf, A., Iversen, A., Grundmann, H., Do, T., et al. (2011). Population structure of Pseudomonas aeruginosa from five mediterranean countries: evidence for frequent recombination and epidemic occurrence of CC235. PLOS ONE 6:e25617. doi: 10.1371/journal.pone. 0025617

Machuca, J., Briales, A., Labrador, G., Díaz-de-Alba, P., López-Rojas, R., Docobo-Pérez, F., et al. (2014). Interplay between plasmid-mediated and chromosomal-mediated fluoroquinolone resistance and bacterial fitness in Escherichia coli. J. Antimicrob. Chemother. 69, 3203-3215. doi: 10.1093/jac/ dku308

Marcusson, L. L., Frimodt-Møller, N., and Hughes, D. (2009). Interplay in the selection of fluoroquinolone resistance and bacterial fitness. PLoS Pathog. 5:e1000541. doi: 10.1371/journal.ppat.1000541

Melnyk, A. H., Wong, A., and Kassen, R. (2015). The fitness costs of antibiotic resistance mutations. Evol. Appl. 8, 273-283. doi: 10.1111/eva.12196

Moir, D. T., Ming Di, Opperman, T., Schweizer, H. P., and Bowlin, T. L. (2007). A high-throughput, homogeneous, bioluminescent assay for Pseudomonas aeruginosa gyrase inhibitors and other DNA-damaging agents. J. Biomol. Screen. 12, 855-864. doi: 10.1177/1087057107304729

Mouneimné, H., Robert, J., Jarlier, V., and Cambau, E. (1999). Type II topoisomerase mutations in ciprofloxacin-resistant strains of Pseudomonas aeruginosa. Antimicrob. Agents Chemother. 43, 62-66.

Nakano, M., Deguchi, T., Kawamura, T., Yasuda, M., Kimura, M., Okano, Y., et al. (1997). Mutations in the gyrA and parC genes in fluoroquinolone-resistant clinical isolates of Pseudomonas aeruginosa. Antimicrob. Agents Chemother. 41, 2289-2291.

Oliver, A., Cantón, R., Campo, P., Baquero, F., and Blázquez, J. (2000). High frequency of hypermutable Pseudomonas aeruginosa in cystic fibrosis lung infection. Science 288, 1251-1254. doi: 10.1126/science.288.5469.1251

Ozer, E. A., Allen, J. P., and Hauser, A. R. (2014). Characterization of the core and accessory genomes of Pseudomonas aeruginosa using bioinformatic tools Spine and AGEnt. BMC Genomics 15:737. doi: 10.1186/1471-2164-15-737

Peña, C., Cabot, G., Gómez-Zorrilla, S., Zamorano, L., Ocampo-Sosa, A., Murillas, J., et al. (2015). Influence of virulence genotype and resistance profile 
in the mortality of Pseudomonas aeruginosa bloodstream infections. Clin. Infect. Dis. 60, 539-548. doi: 10.1093/cid/ciu866

Quartin, A. A., Scerpella, E. G., Puttagunta, S., and Kett, D. H. (2013). A comparison of microbiology and demographics among patients with healthcare-associated, hospital-acquired, and ventilator-associated pneumonia: a retrospective analysis of 1184 patients from a large, international study. $B M C$ Infect. Dis. 13:561. doi: 10.1186/1471-2334-13-561

Redgrave, L. S., Sutton, S. B., Webber, M. A., and Piddock, L. J. (2014). Fluoroquinolone resistance: mechanisms, impact on bacteria, and role in evolutionary success. Trends Microbiol. 22, 438-445. doi: 10.1016/j.tim.2014.04.007

Restrepo, M. I., and Anzueto, A. (2009). The role of gram-negative bacteria in healthcare-associated pneumonia. Semin. Respir. Crit. Care Med. 30, 61-66. doi: 10.1055/s-0028-1119810

Rosenthal, V. D., Bijie, H., Maki, D. G., Mehta, Y., Apisarnthanarak, A., Medeiros, E. A., et al. (2012). International Nosocomial Infection Control Consortium (INICC) report, data summary of 36 countries, for 2004-2009. Am. J. Infect. Control 40, 396-407. doi: 10.1016/j.ajic.2011.05.020

Roy-Burman, A., Savel, R. H., Racine, S., Swanson, B. L., Revadigar, N. S., Fujimoto, J., et al. (2001). Type III protein secretion is associated with death in lower respiratory and systemic Pseudomonas aeruginosa infections. J. Infect. Dis. 183, 1767-1774. doi: 10.1086/320737

Rozen, D. E., McGee, L., Levin, B. R., and Klugman, K. P. (2007). Fitness costs of fluoroquinolone resistance in Streptococcus pneumoniae. Antimicrob. Agents Chemother. 51, 412-416. doi: 10.1128/AAC.01161-06

Sato, H., Frank, D. W., Hillard, C. J., Feix, J. B., Pankhaniya, R. R., Moriyama, K., et al. (2003). The mechanism of action of the Pseudomonas aeruginosa-encoded type III cytotoxin ExoU. EMBO J. 22, 2959-2969.

Schulert, G. S., Feltman, H., Rabin, S. D., Martin, C. G., Battle, S. E., Rello, J., et al. (2003). Secretion of the toxin ExoU is a marker for highly virulent Pseudomonas aeruginosa isolates obtained from patients with hospital-acquired pneumonia. J. Infect. Dis. 188, 1695-1706. doi: 10.1086/379372

Shaver, C. M., and Hauser, A. R. (2004). Relative contributions of Pseudomonas aeruginosa ExoU, ExoS, and ExoT to virulence in the lung. Infect. Immun. 72, 6969-6977.

Shaver, C. M., and Hauser, A. R. (2006). Interactions between effector proteins of the Pseudomonas aeruginosa type III secretion system do not significantly affect several measures of disease severity in mammals. Microbiology 152, 143-152. doi: $10.1099 /$ mic. $0.28368-0$
Sullivan, E., Bensman, J., Lou, M., Agnello, M., Shriner, K., and WongBeringer, A. (2014). Risk of developing pneumonia is enhanced by the combined traits of fluoroquinolone resistance and type III secretion virulence in respiratory isolates of Pseudomonas aeruginosa. Crit. Care Med. 42, 48-56. doi: 10.1097/CCM.0b013e318298a86f

Swingle, B., Markel, E., Costantino, N., Bubunenko, M. G., Cartinhour, S., and Court, D. L. (2010). Oligonucleotide recombination in Gram-negative bacteria. Mol. Microbiol. 75, 138-148. doi: 10.1111/j.1365-2958.2009.06976.x

Veesenmeyer, J. L., Hauser, A. R., Lisboa, T., and Rello, J. (2009). Pseudomonas aeruginosa virulence and therapy: evolving translational strategies. Crit. Care Med. 37, 1777-1786. doi: 10.1097/CCM.0b013e31819ff137

Wasels, F., Kuehne, S. A., Cartman, S. T., Spigaglia, P., Barbanti, F., Minton, N. P., et al. (2015). Fluoroquinolone resistance does not impose a cost on the fitness of Clostridium difficile in vitro. Antimicrob. Agents Chemother. 59, 1794-1796. doi: 10.1128/AAC.04503-14

Winsor, G. L., Lam, D. K., Fleming, L., Lo, R., Whiteside, M. D., Yu, N. Y., et al. (2011). Pseudomonas Genome Database: improved comparative analysis and population genomics capability for Pseudomonas genomes. Nucleic Acids Res. 39, D596-D600. doi: 10.1093/nar/gkq869

Wong-Beringer, A., Wiener-Kronish, J., Lynch, S., and Flanagan, J. (2008). Comparison of type III secretion system virulence among fluoroquinolonesusceptible and -resistant clinical isolates of Pseudomonas aeruginosa. Clin. Microbiol. Infect. 14, 330-336. doi: 10.1111/j.1469-0691.2007. 01939.x

Zhang, J., Chu, Y., Wang, P., Ji, X., Li, X., Wang, C., et al. (2014). Clinical outcomes of multidrug resistant Pseudomonas aeruginosa infection and the relationship with type III secretion system in patients with diabetic foot. Int. J. Low. Extrem. Wounds 13, 205-210. doi: 10.1177/1534734614545878

Conflict of Interest Statement: The authors declare that the research was conducted in the absence of any commercial or financial relationships that could be construed as a potential conflict of interest.

Copyright $\odot 2016$ Agnello, Finkel and Wong-Beringer. This is an open-access article distributed under the terms of the Creative Commons Attribution License (CC BY). The use, distribution or reproduction in other forums is permitted, provided the original author(s) or licensor are credited and that the original publication in this journal is cited, in accordance with accepted academic practice. No use, distribution or reproduction is permitted which does not comply with these terms. 


\section{OPEN ACCESS}

Edited by:

Axel Cloeckaert

French National Institute for Agricultural Research (INRA), France

Reviewed by:

Elaine Allan,

University College London, UK

Xian-Zhi Li,

Health Canada, Canada

*Correspondence:

Zonghui Yuan

yuan5802@mail.hzau.edu.cn

Yulian Wang

wangyulian@mail.hzau.edu.cn

Menghong Dai

daimenghong@mail.hzau.edu.cn

${ }^{\dagger}$ These authors have contributed equally to this work

Specialty section:

This article was submitted to Antimicrobials, Resistance and

Chemotherapy,

a section of the journal

Frontiers in Microbiology

Received: 14 May 2016 Accepted: 26 September 2016

Published: 14 October 2016

Citation:

Hao H, Ren N, Han J, Foley SL, lqbal Z, Cheng G, Kuang X, Liu J,

Liu Z, Dai M, Wang Y and Yuan Z (2016) Virulence and Genomic Feature of Multidrug Resistant Campylobacter jejuni Isolated from Broiler Chicken.

Front. Microbiol. 7:1605

doi: 10.3389/fmicb.2016.01605

\section{Virulence and Genomic Feature of Multidrug Resistant Campylobacter jejuni Isolated from Broiler Chicken}

\author{
Haihong Hao 1, 2,3+, Ni Ren ${ }^{2,3+}$, Jing Han ${ }^{4}$, Steven L. Foley ${ }^{4}$, Zahid lqbal ${ }^{2}$, Guyue Cheng ${ }^{1}$, \\ Xiuhua Kuang ${ }^{2}$, Jie Liu $^{2}$, Zhenli Liu ${ }^{2}$, Menghong Dai ${ }^{2 *}$, Yulian Wang ${ }^{1 *}$ and \\ Zonghui Yuan ${ }^{1,2,3 *}$ \\ ${ }^{1}$ National Reference Laboratory of Veterinary Drug Residues (HZAU) and MOA Key Laboratory for Detection of Veterinary \\ Drug Residues, Wuhan, China, ${ }^{2}$ MOA Laboratory for Risk Assessment of Quality and Safety of Livestock and Poultry \\ Products, Wuhan, China, ${ }^{3}$ Hubei Collaborative Innovation Center for Animal Nutrition and Feed Safety, Huazhong Agricultural \\ University, Wuhan, China, ${ }^{4}$ Division of Microbiology, FDA, National Center for Toxicological Research, Jefferson, AR, USA
}

The aim of this study was to reveal the molecular mechanism involved in multidrug resistance and virulence of Campylobacter jejuni isolated from broiler chickens. The virulence of six multidrug resistant $C$. jejuni was determined by in vitro and in vivo methods. The de novo whole genome sequencing technology and molecular biology methods were used to analyze the genomic features associated with the multidrug resistance and virulence of a selected isolate (C. jejuni 1655). The comparative genomic analyses revealed a large number of single nucleotide polymorphisms, deletions, rearrangements, and inversions in C. jejuni 1655 compared to reference C. jejuni genomes. The co-emergence of Thr-86-lle mutation in gyrA gene, A2075G mutation in 23S rRNA gene, tetO, aphA and aadE genes and pTet plasmid in C. jejuni 1655 contributed its multidrug resistance to fluoroquinolones, macrolides, tetracycline, and aminoglycosides. The combination of multiple virulence genes may work together to confer the relative higher virulence in $C$. jejuni 1655. The co-existence of mobile gene elements (e.g., pTet) and CRISPR-Cas system in C. jejuni 1655 may play an important role in the gene transfer and immune defense. The present study provides basic information of phenotypic and genomic features of $C$. jejuni 1655, a strain recently isolated from a chicken displaying multidrug resistance and relatively high level of virulence.

Keywords: Campylobacter jejuni, broiler chicken, multidrug resistance, virulence, de novo genome sequencing

\section{INTRODUCTION}

Campylobacter jejuni is one of the most important foodborne pathogens worldwide (Abril et al., 2010). The macrolides (mainly erythromycin and azithromycin) and fluoroquinolones are the empirical drugs of choice for treatment of campylobacteriosis (Allos, 2001). Other antimicrobial agents, including gentamicin, meropenem, and clindamycin are alternative therapies (Iovine et al., 2008).

The National Antimicrobial Resistance Monitoring System (NARMS) in the USA and Danish Integrated Antimicrobial Resistance Monitoring and Research Program (DANMAP) data showed that multidrug resistance in $C$. jejuni has been very rare $(0.3-0.7 \%)$ from retail chicken meat (DANMAP, 2014; NARMS, 2015). Multidrug resistant $C$. jejuni have been isolated from chicken 
farms in China recently (Chen et al., 2010; Hao et al., 2015; Wang et al., 2016). The multidrug resistant $C$. jejuni in chicken may greatly threaten food safety and human public health, therefore it is important to investigate the virulence potential and mechanism involved in multidrug resistance and virulence of $C$. jejuni.

Although genomes of many human-source C. jejuni (e.g., NCTC11168, 81-176, 260.94, HB93-13, CF93-6, and 269.97) have been sequenced, only three genomes of C. jejuni (305, RM1221, and 81-176-DRH212) from poultry have been obtained (Parkhill et al., 2000; Fouts et al., 2005; Hofreuter et al., 2006). C. jejuni RM1221 (ATCC BAA-1032) is a chicken isolate with unique lipoolgosaccharide and ability to colonize chicken skin (Fouts et al., 2005). C. jejuni 305 is a turkey isolate with stress tolerance (Takamiya et al., 2011). C. jejuni 81-176-DRH212 is a C. jejuni 81-176 variant with enhanced fitness in the chicken gastrointestinal tract (Johnson et al., 2014). The genome sequences of broiler $C$. jejuni isolates with multidrug resistance and high virulence have not been published previously. In order to investigate the mechanisms involved in the multidrug resistance and increased virulence in C. jejuni isolated from broiler chickens in China, the genomic profile of a $C$. jejuni isolate with relative higher virulence and multidrug resistance was determined by the de novo sequencing technology in the present study.

\section{MATERIALS AND METHODS}

\section{Isolation and Identification of Chicken C. jejuni}

Six strains $(1442,1447,1614,1622,1655$, and 1685) were isolated from caecum of healthy broiler chicken in the chicken farms located in the center of China in 2013. These farms have a long history of usage of different antimicrobial drug for prevention and treatment chicken disease. These strains were confirmed as C. jejuni by classic biochemical test and PCR amplification of the 16S rRNA, mapA and vs1 genes (Stucki et al., 1995; Hao et al., 2013, 2015). The $C$. jejuni isolates were grown on Mueller-Hinton $(\mathrm{MH})$ agar supplemented with $5 \%$ sheep blood at $42^{\circ} \mathrm{C}$ under microaerobic conditions $\left(5 \% \mathrm{O}_{2}, 10 \% \mathrm{CO}_{2}\right.$, and $\left.85 \% \mathrm{~N}_{2}\right)$ for 24-48 h.

\section{Antimicrobial Susceptibility Test}

The minimum inhibitory concentrations (MICs) of erythromycin (ERY), tylosin (TYL), ciprofloxacin (CIP), enrofloxacin (ENR), doxycycline (DOX), tetracycline (TET), amikacin (AMK), and gentamicin (GEN) were determined using the agar dilution method as recommended by the Clinical and Laboratory Standards Institute (CLSI) M31-A3 guidelines (CLSI., 2008). The C. jejuni ATCC33560 was used as quality control for the MIC determination.

\section{Preliminary Pathogenicity Test}

Newly hatched broiler chickens were purchased from Zhengda Limited Company (Wuhan, China). Prior to the experiment, all the chickens tested negative for $C$. jejuni strains by $C$. jejuni isolation and identification methods. The chickens were randomly divided into 8 groups with 7 chickens in each group.
The chickens in groups 1-7 were administrated once with $5 \times 10^{5}$ CFU of each C. jejuni strain (1442, 1447, 1614, 1622, 1655, 1685, and RM1221) by oral injection, respectively. The eight group served as the negative control with no bacterial infection. The clinical symptoms and mortality of the chickens in each group was observed daily. The colonization rate of each $C$. jejuni strain was determined using selective medium containing $32 \alpha \mathrm{g} / \mathrm{mL}$ erythromycin.

\section{Cytotoxin Assay}

The murine macrophage RAW264.7 cell line was used to evaluate the cytotoxic effects of the six C. jejuni isolates. The tissue culture cytotoxin assay was carried out as previously described (Guerrant et al., 1987) with some modification. Briefly, C. jejuni was treated by $2000 \mu \mathrm{g} / \mathrm{ml}$ polymyxin $\mathrm{B}$ for $1 \mathrm{~h}$ at $37^{\circ} \mathrm{C}$ (Ashkenazi and Cleary, 1990) and centrifuged at $8000 \mathrm{rpm}$ for $20 \mathrm{~min}$. The supernatant were filtered through $0.22 \mu \mathrm{m}$ filter. Purified shiga toxin (Chinese Center for Disease Control and Prevention) and $0.3 \%$ Triton X-100-PBS were used as positive controls, and polymyxin B-treated broth and polymyxin B-PBS (phosphatebuffered saline) were used as negative controls. Suspensions of murine macrophage RAW264.7 cells $\left(100 \mu\right.$ l; $5 \times 10^{5}$ cells $)$ were placed in 96-well flat-bottom microtiter plates and allowed to adhere for 1 to $3 \mathrm{~h}$ to form the tissue culture monolayers. Serial twofold dilutions of the prepared C. jejuni filtrates were added to the tissue culture monolayers in $100 \mu \mathrm{l}$ volumes. The monolayers were incubated with the filtrates at $37^{\circ} \mathrm{C}$ in $5 \% \mathrm{CO}_{2}$ for $24 \mathrm{~h}$. The monolayers were then examined by phase-contrast microscopy for the percentage of cells rounded. Cell death was determined by trypan blue dye uptake (A540 value) and correlated with cell morphology after Giemsa staining. The index of cytotoxin effect was calculated by the formula.

$$
100 \times\left[1-\frac{A 540(\text { test })-A 540(\text { positive })}{A 540(\text { negative })-A 540(\text { positive })}\right] \text {. }
$$

\section{Biofilm Assays}

To measure the biofilm formation of the six C. jejuni strains, crystal violet staining was used as described previously for C. jejuni and other bacteria (Asakura et al., 2007; Fields and Thompson, 2008; McLennan et al., 2008; Reuter et al., 2010) with some modification. Briefly, the $200 \alpha \mathrm{l}$ of $C$. jejuni fresh culture $\left(\mathrm{OD}_{600}=0.05\right)$ was added to 96 -well flat-bottom microtiter plates. Plates were incubated without shaking at $42^{\circ} \mathrm{C}$ under microaerobic conditions for 24,48 , and $72 \mathrm{~h}$. For crystal violet staining, each well was washed with PBS three times to remove the planktonic cells. The $200 \alpha$ l of methanol were added and incubated for $15 \mathrm{~min}$ and then dried at room temperature. Then $200 \alpha \mathrm{l}$ of $0.1 \%$ Hucker crystal violet solution were added, and the plates were incubated at room temperature for $5 \mathrm{~min}$. Unbound crystal violet was washed off with PBS, and the plates were dried at $60^{\circ} \mathrm{C}$. Bound crystal violet was dissolved in $30 \%$ glacial acetic acid for $10 \mathrm{~min}$. The absorbance was determined using a plate reader at $570 \mathrm{~nm}$. The wells with sterile medium were used as blank control. The blank corrected absorbance values of C. jejuni strains were used for reporting biofilm production. Assays were repeated at least three times with three technical replicates. 


\section{$\mathrm{LD}_{50}$ Determination}

Based on the results from the preliminary pathogenicity experiments with chickens, cytotoxin testing and biofilm assays, three C. jejuni isolates (1442, 1622, and 1655) were selected to further determine their median lethal dose $\left(\mathrm{LD}_{50}\right)$ using 2 days old chicken orally or intraperitoneally infected with a range of concentration $\left(10^{5}-10^{7} \mathrm{CFU}\right)$ of each C. jejuni isolate. All the chickens tested negative for $C$. jejuni prior to inoculation using the $C$. jejuni isolation and identification methods described above. The chickens were randomly divided into six groups with 35 chickens in each group. The chickens in each group were orally or intraperitoneally administrated with $0,10^{5}$, $10^{6}$, or $10^{7} \mathrm{CFU}$ of the C. jejuni isolates, respectively. Seven chickens were inoculated with each of the dilution of the test strains.

\section{Adhesion and Invasion Assay}

The adhesion and invasion of these three C. jejuni strains (1442, 1622, and 1655) was determined as previously described (Almofti et al., 2011a). Briefly, $5.0 \times 10^{7} \mathrm{CFU} / \mathrm{mL}$ of the C. jejuni strain was used to inoculated the monolayers of macrophage RAW264.7 cells at multiplicity of infection (MOI) of 100 . The infected monolayers were incubated for $3 \mathrm{~h}$ to allow the occurrence of adhesion and invasion. For determination of the total number of adherent and internalized bacteria, the monolayers were washed three times with Dulbecco's Modified Eagle's Medium (DMEM) without antibiotic to remove the extracellular unbound bacteria. The monolayers were then lysed to release the intracellular bacteria. For determination of the invading bacteria, the monolayers were washed twice with aspirated DMEM medium and $100 \mathrm{mg} / \mathrm{ml}$ gentamicin was added for $1 \mathrm{~h}$ to kill the extracellular and bound bacteria. The monolayers were then washed three times and lysed to release intracellular bacteria. The number of adherent bacteria was obtained by subtraction of the internalized bacteria number from total number of adherent and internalized bacteria.

\section{Intracellular Survivability Assay}

To determine the intracellular survivability of $C$. jejuni within macrophage RAW264.7, the invasion period of each C. jejuni strain $(1442,1622$, and 1655) was extended to $3,6,10,16,24$, 48, and $72 \mathrm{~h}$ post-infection. Cells were washed and lysed as previously described in the adhesion and invasion assay. The surviving intracellular bacteria were enumerated by plating serial dilutions on blood agar and counting the resultant colonies.

\section{Motility Test}

To determine the migration of the three selected strains (1442, 1622, and 1655), a fresh culture of C. jejuni strain was inoculated into $\mathrm{MH}$ broth and cultured to their logarithmic growth phase. Then $3 \alpha$ l of bacteria with consistent concentration were stabbed into $0.4 \% \mathrm{MH}$ agar. Plates were incubated at $42^{\circ} \mathrm{C}$ under microaerophilic conditions for $24 \mathrm{~h}$ and the motility was scored by measuring the diameter of the growth in each plate. The experiment was performed in triplicate.

\section{De Novo Whole-Genome Sequencing Of C. jejuni 1655}

Total genomic DNA of C. jejuni 1655 was extracted using the TIANamp Bacteria DNA kit according to the manufacturer's protocol. The genomic DNA was sent to Shanghai Biotechnology Corporation for the de novo whole genome sequencing performed in a HiSeq 2500 platform using a multiplexed, $2 \times$ 100 nucleotide paired end approach (Illumina). The sequence analysis and assembly were carried out using CLC Genomics Workbench (ver. 6.0., Qiagen). Prediction and identification of the main components of the genome, including open reading frames (ORFs), tRNA, rRNA, ncRNA, CRISPR-Cas system, and repeat elements, were conducted using software of Glimmer 3.02 and some other online tools.

The nucleotides and predicted proteins of 1655 were compared with previously sequenced $C$. jejuni genomes in GenBank using updated nt and nr databases with BLAST software. The genomes used as reference sequences for the comparative genomic analysis were C. jejuni 81-176, C. jejuni NCTC11168, C. jejuni RM1221. The comparative genomic analysis was performed using BLASTN, BLASTX, Mummer and Quast. The sequence data from C. jejuni 1655 was compared to the results of antimicrobial resistance and virulence to identify genetic factors that correspond to the observed mechanisms.

\section{Nucleotide Sequence Accession Numbers}

The whole genome sequence of $C$. jejuni 1655 has been deposited at DDBJ/ENA/GenBank under the accession MDDM00000000.

\section{Ethics Statement}

All the experimental procedure in this study was performed according to the guidelines of the committee on the use and care of the laboratory animals in Hubei province China. The study was approved by Animal Ethics Committee of Huazhong Agricultural University (hzauch 2014-002) and the Animal Care Center, Hubei Science and Technology Agency in China (SYXK 20130044). These experiments were in line with national regulations about animal welfare ethics. All the animals were monitored throughout the study for any adverse effect signs. All efforts were made to minimize suffering of animals.

\section{RESULTS}

\section{Minimum Inhibitory Concentration (MIC)}

The minimum inhibitory concentrations (MICs) of different antimicrobial agents against the 6 C. jejuni isolates (1442, 1447, 1614, 1622, 1655, and 1685) were presented in Table 1. Each isolate exhibited resistance to all the tested drugs, while three (1622, 1655, and 1685) showed higher-level MICs to erythromycin and gentamicin than the others.

\section{Pathogenicity of C. jejuni Isolates on Chicken}

After oral infection with $10^{5} \mathrm{CFU}$ of the C. jejuni isolates, no clinical change was observed in two groups infected with 1442 or 1447 , while the other four isolates $(1614,1622,1655$, and 1685) and RM1221 caused different degrees of diarrhea and bloody 
TABLE 1 | MICs of different antimicrobial agents against six C. jejuni isolates $(\mu \mathrm{g} / \mathrm{mL})$.

\begin{tabular}{|c|c|c|c|c|c|c|c|c|}
\hline \multirow[t]{2}{*}{ Strains } & \multicolumn{8}{|c|}{ MICs of different antimicrobial agents } \\
\hline & ERY & TYL & ENR & CIP & TET & DOX & GEN & AMK \\
\hline 1442 & 128 & $>256$ & 64 & 128 & 128 & 32 & 64 & 16 \\
\hline 1447 & 64 & $>256$ & 32 & 8 & 128 & 32 & 1 & 8 \\
\hline 1614 & 256 & $>256$ & 0.25 & 16 & 64 & 64 & $>256$ & 4 \\
\hline 1622 & 512 & $>256$ & 256 & 128 & $>256$ & 32 & $>256$ & 64 \\
\hline 1655 & 512 & $>256$ & 32 & 64 & 256 & 32 & $>256$ & 8 \\
\hline 1685 & 512 & $>256$ & 16 & 32 & 64 & 32 & $>256$ & 16 \\
\hline RM1221 & 0.5 & 2.0 & 0.5 & 0.125 & 0.5 & 0.25 & 0.5 & 0.5 \\
\hline
\end{tabular}

ERY, Erythromycin; TYL, Tylosin; ENR, Enrofloxacin; CIP, Ciprofloxacin; TET, tetracycline; DOX, Doxycycline; GEN, Gentamicin; AMK, Amikacin.

\section{Colonization change of $C$. jejuni in chicken}

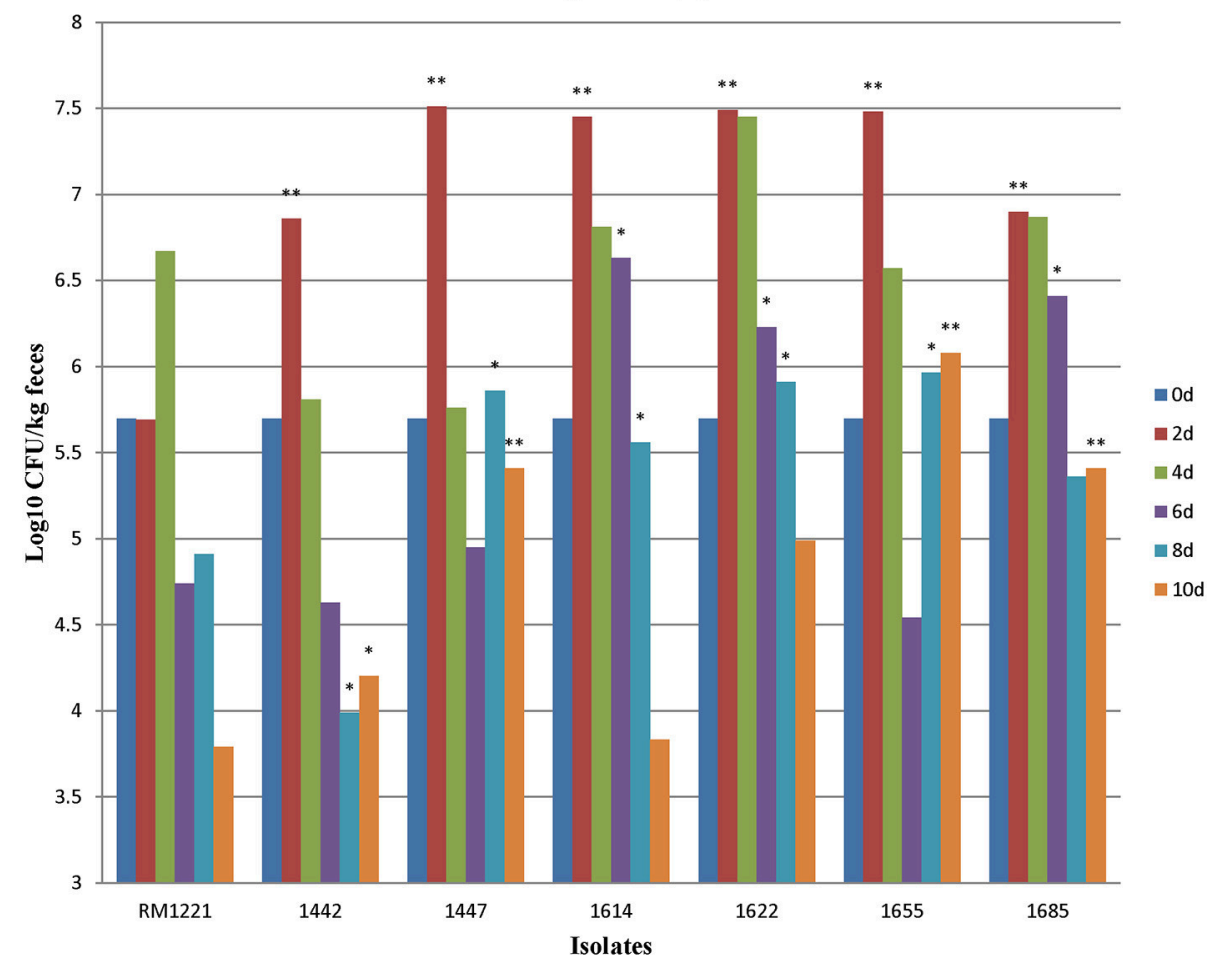

FIGURE 1 | Colonization change of $\boldsymbol{C}$. jejuni isolates in chicken after inoculation. The Y-axis is the number (Log 10 CFU/kg fece) of each strain colonizing the birds at different days. The asterisk $\left(^{\star}\right)$ and $\left(^{* \star}\right)$ represent statistical significant difference with $P \leq 0.05$ and $P \leq 0.01$ comparing with $C$. jejuni RM1221, respectively.

stools, respectively. The clinical signs of three strains $(1614,1685$, and RM 1221) were recovered at day 4, while serious diarrhea caused by 1622 and 1655 was sustained for over 8 and 10 days, respectively (Table S1). Therefore, 1655 exhibited relative higher pathogenicity on chickens than RM1221 and other five C. jejuni isolates. Within 15 days, the mortality of all the strains was $0 \%$ following oral inoculation of 2 days-old chicken.

\section{Colonization of C. jejuni Isolates in Chicken}

After oral administration with $5 \times 10^{5} \mathrm{CFU}$ of the C. jejuni isolates to 2-days old chickens, all the strains could colonize chickens' intestinal tract. Fecal samples were obtained at different time points $(2,4,6,8$, and 10 days). RM1221 colonized at concentration of $10^{5}-10^{7} \mathrm{CFU} / \mathrm{kg}$ feces at day 2 and day 4 , but the concentration was reduced to less than $10^{4} \mathrm{CFU} /$ feces at day 10 (Figure 1). The concentrations of five isolates (1442, 1447, 1622, 1655 , and 1685) were higher than $10^{4} \mathrm{CFU} / \mathrm{kg}$ feces within the ten days (Figure 1). Comparing with the colonization of RM1221, three isolates (1622, 1655, and 1685) exhibited significantly stronger colonization (Figure 1).

\section{Cytotoxin of the Six C. jejuni Isolates in Macrophage Cells}

Figure 2 showed the killing effect (index) of cytotoxin released by six strains $(1442,1447,1614,1622,1655$, and 1685) on murine 


\section{Cytotoxin production of $\mathrm{C}$. jejuni}

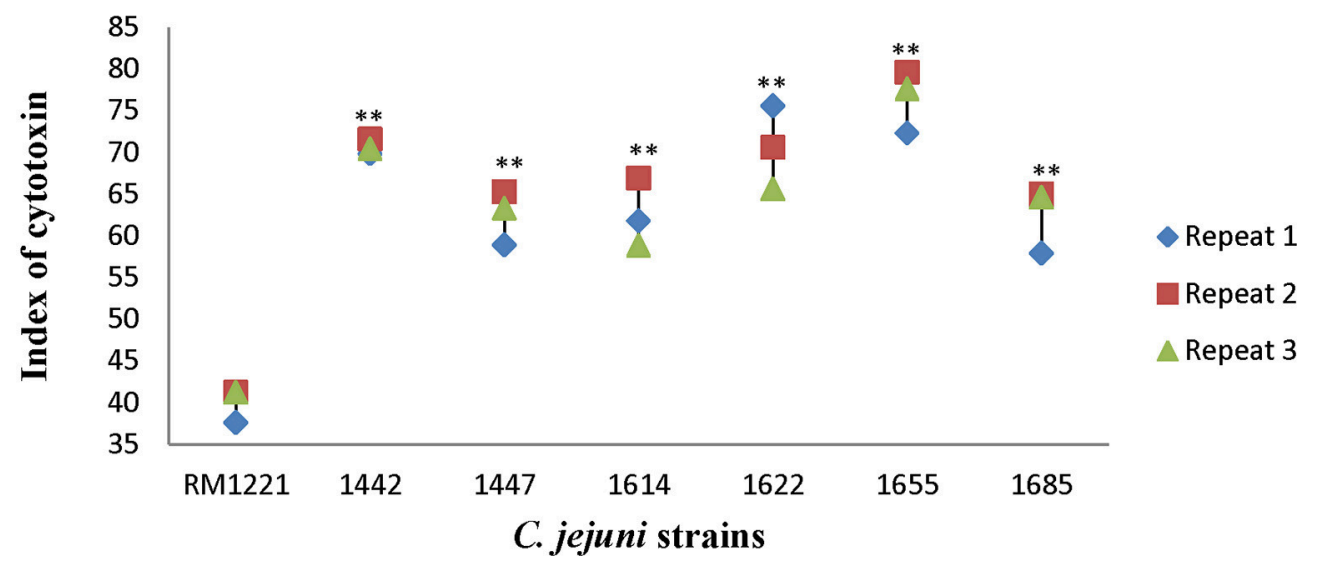

FIGURE 2 | Cytotoxic production of $\boldsymbol{C}$. jejuni isolates in murine macrophage RAW264.7 cell. The Y-axis is the index of cytotoxic production of each strain. The results were obtained from three independent repeats. The asterisk $\left(^{\star}\right)$ and $\left(^{* \star}\right)$ represent statistical significant difference with $P \leq 0.05$ and $P \leq 0.01$ comparing with C. jejuni RM1221, respectively.

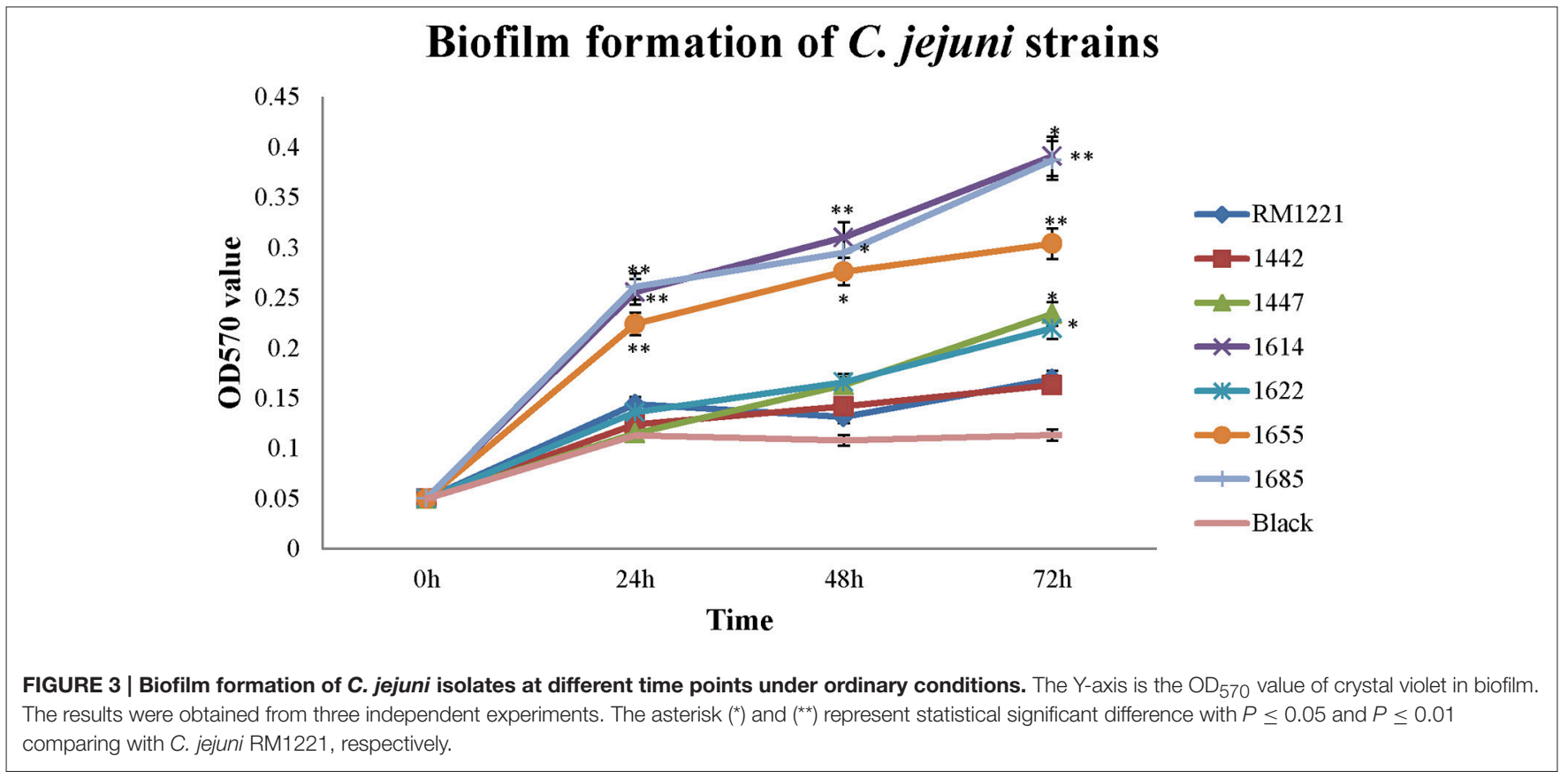

macrophage RAW264.7. The killing effect of cytotoxin from all the strains was lower than $80 \%$. However, compared to RM 1221 , significant differences of the index of cytotoxin were observed in these C. jejuni strains. Among them, 1442, 1622, and 1655 seemed to release more cytotoxin and therefore exhibited higher killing effect on murine macrophage RAW264.7.

\section{Biofilm Formation of the Six C. jejuni Isolates}

Different level of biofilm formation was observed in each C. jejuni strain (Figure 3). It seemed that the level of biofilm formation had positive correlation with the incubation time. The highest level of biofilm formation was occurred after $72 \mathrm{~h}$ incubation (Figure 3). Compared to RM1221, a significant difference of biofilm formation was observed in three isolates $(1614,1655$, and 1685) after each incubation time, indicating that these three strains may have stronger ability to form biofilm (Figure 3 ).

\section{$L_{50}$ of the Three Selected C. jejuni Isolates on Chicken}

From the preliminary pathogenicity testing on chicken, cytotoxin testing and biofilm assays, three isolates (1442, 1622, and 1655) were selected for $\mathrm{LD}_{50}$ determination. As the result shown in Table 2, different fatality rates were observed in different groups. 
After oral infection, the highest concentration $\left(3.70 \times 10^{7} \mathrm{CFU}\right)$ of 1442 and 1622 could cause mortality rate of $0 \%(0 / 7)$ and $28.6 \%$ $(2 / 7)$ respectively, while $3.7 \times 10^{7} \mathrm{CFU}$ of 1655 cause fatality rate of $55.6 \%(4 / 7)$. For intraperitoneal infection, $3.7 \times 10^{6} \mathrm{CFU}$ of 1442 and 1622 only leaded to one chicken death, while the lowest concentration $\left(3.7 \times 10^{5} \mathrm{CFU}\right)$ of 1655 leaded to $100 \%$ death of chickens. The 1655 exhibited highest pathogenicity on chicken with the calculated $\mathrm{LD}_{50}$ of $8.45 \times 10^{7} \mathrm{CFU}$ for oral infection and $\mathrm{LD}_{50}$ of less than $3.7 \times 10^{5} \mathrm{CFU}$ for Intraperitoneal infection (Table 2).

\section{Adhesion and Invasion of C. jejuni on Macrophage RAW264.7}

The three isolates $(1442,1622$, and 1655) exhibited remarkable higher adhesion and invasion to macrophage cell RAW264.7 than RM1221 (Figure 4). The significant change of adhesion to macrophage cells $(P<0.01)$ was observed in 1622 and 1655 . However, there was no significant difference in the adhesion and invasion between three selected isolates (1442, 1622, and 1655).

\section{Intracellular Survivability of $C$. jejuni in Macrophage RAW264.7}

The intracellular survivability of the three strains (1442, 1622, and 1655) was shown in Figure 5. Comparing with RM1221, three selected strains showed remarkable advantage in intracellular survivability in murine macrophage RAW 264.7. RM1221 had short survival time $(24 \mathrm{~h})$, while three strains were able to survive for more than $48 \mathrm{~h}$, although a considerable decrease in the number of all internalized was observed. Among them, 1622 and 1655 exhibited highest survivability at $72 \mathrm{~h}$. At each time point, the post-infection number of surviving 1655 was higher than 1622, suggesting that 1655 strain had strongest survivability.

\section{Motility of the Three Selected C. jejuni}

As shown in Figure 6, there was apparent difference between diameter growth rings of the three strains (1442, 1622, and 1655) on $0.4 \%$ MH agar plate. RM1221 and 1622 were not motile, while 1655 and 1442 had a strong motility.

\section{Whole-Genome Features of C. jejuni 1655}

Based on the above in vitro and in vivo studies, 1655 was selected for subsequent whole genome sequencing to analyze the potential genetic mechanisms involved in the multidrug resistance and high virulence.

The basic genome information of 1655 and other three reference strains (RM1221, 81-176, and NCTC11168) are summarized into Table 3. The 1655 genome was comprised of a chromosome and a tetracycline resistance plasmid pTet. The pTet plasmid of 1655 had $98 \%$ homology with pTet plasmid in 81176 . The genome of 1655 was 1720,061 bp long and contained 1733 predicted coding regions. The genomes of two chicken original strains (1655 and RM1221) were significantly larger than that of two human isolates (C. 81-176 and NCTC11168). The GC content of 1655 was $31.36 \%$, consistent with the reference genome sequences.
TABLE 2 | $L D_{50}$ of the three multidrug resistant strains on 2 days old chickens.

\begin{tabular}{|c|c|c|c|c|c|}
\hline $\begin{array}{l}\text { Infection } \\
\text { route }\end{array}$ & Strains & $\begin{array}{l}\text { Infection } \\
\text { dose of } \\
\text { (CFU) }\end{array}$ & $\begin{array}{c}\text { Number } \\
\text { of } \\
\text { chickens }\end{array}$ & $\begin{array}{l}\text { Number } \\
\text { of Dead } \\
\text { chicken }\end{array}$ & $\mathrm{LD}_{50}$ \\
\hline \multirow[t]{12}{*}{ Oral infection } & 1442 & $3.70 \times 10^{7}$ & 7 & 0 & NA \\
\hline & & $3.70 \times 10^{6}$ & 7 & 0 & \\
\hline & & $3.70 \times 10^{5}$ & 7 & 0 & \\
\hline & & 0 & 7 & 0 & \\
\hline & 1622 & $3.70 \times 10^{7}$ & 7 & 2 & NA \\
\hline & & $3.70 \times 10^{6}$ & 7 & 0 & \\
\hline & & $3.70 \times 10^{5}$ & 7 & 0 & \\
\hline & & 0 & 7 & 0 & \\
\hline & 1655 & $3.70 \times 10^{7}$ & 7 & 4 & $8.45 \times 10^{7}$ \\
\hline & & $3.70 \times 10^{6}$ & 7 & 1 & \\
\hline & & $3.70 \times 10^{5}$ & 7 & 1 & \\
\hline & & 0 & 7 & 0 & \\
\hline \multirow{12}{*}{$\begin{array}{l}\text { Intraperitoneal } \\
\text { infection }\end{array}$} & 1442 & $3.70 \times 10^{7}$ & 7 & 2 & NA \\
\hline & & $3.70 \times 10^{6}$ & 7 & 1 & \\
\hline & & $3.70 \times 10^{5}$ & 7 & 0 & \\
\hline & & 0 & 7 & 0 & \\
\hline & 1622 & $3.70 \times 10^{7}$ & 7 & 1 & NA \\
\hline & & $3.70 \times 10^{6}$ & 7 & 1 & \\
\hline & & $3.70 \times 10^{5}$ & 7 & 0 & \\
\hline & & 0 & 7 & 0 & \\
\hline & 1655 & $3.70 \times 10^{7}$ & 7 & 7 & $<3.70 \times 10^{5}$ \\
\hline & & $3.70 \times 10^{6}$ & 7 & 7 & \\
\hline & & $3.70 \times 10^{5}$ & 7 & 7 & \\
\hline & & 0 & 7 & 0 & \\
\hline
\end{tabular}

The genome sequence of 1655 was composed by 35 large contigs (Figure 7). The cluster of orthologous groups (COG) assignment for predicted gene products were shown in the third circle from the outside in Figure 7. A total of 1733 putative gene products were assigned to COG identifications classified into 19 COG categories. Three copies of rRNA gene cluster ( $5 \mathrm{~s}$ rRNA, $16 \mathrm{~s}$ rRNA, and 23s rRNA) and 40 tRNA genes were identified on the C. jejuni 1655 chromosome.

The global alignment of four genomes (RM1221, 81-176, NCTC11168, and 1655) is shown in Figure 8. High similarity was observed in the genome of three reference strains (RM1221, 81-176, and NCTC11168). However, disordered distribution, deletions and rearrangements were observed in the genome of 1655 when compared with genome of three reference strains.

\section{Resistance Determinants in C. jejuni 1655}

The genome sequencing data showed that 1655 contained T86I mutation in the gyrA gene. Some mutations (e.g., A2075G mutation, C2113T, position 1732-1791 mutations) were found in the 23S rRNA of C. jejuni 1655. The ribosomal L4 contained V196A mutation and ribosomal L22 had A74G mutation. The tetracycline resistant gene tet $O$ was located in pTet plasmid. The C. jejuni 1655 also contained $a p h A$ and aadE exogenous genes 


\section{Adhesion and Invasion of $C$. jejuni strains}

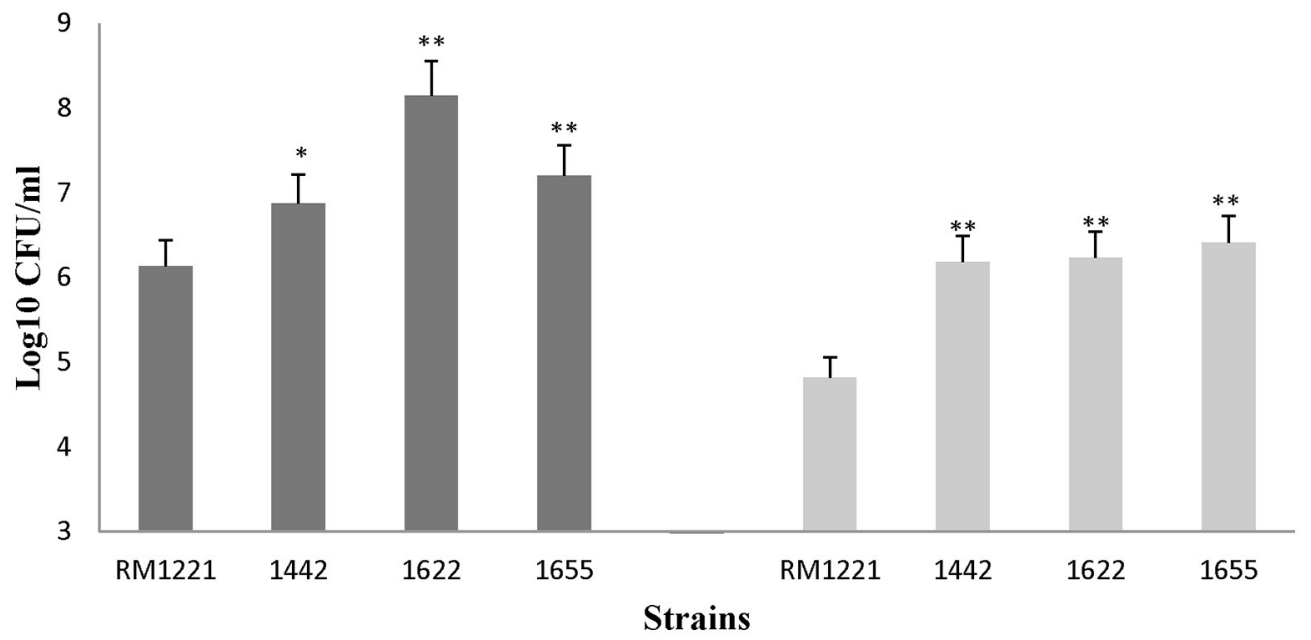

FIGURE 4 | Adhesion (left) and invasion (right) of $\mathbf{C}$. jejuni strains in macrophage RAW264.7 cell. The Y-axis is the mean of log10 CFU/mL of each strain in the cells. The results were obtained from three independent experiments. The asterisk $\left(^{*}\right)$ and $\left(^{* *}\right)$ represent statistical significant difference with $P \leq 0.05$ and $P \leq 0.01$ comparing with C. jejuni RM1221, respectively.

\section{Intracellular survivability of $C$. jejuni strains}

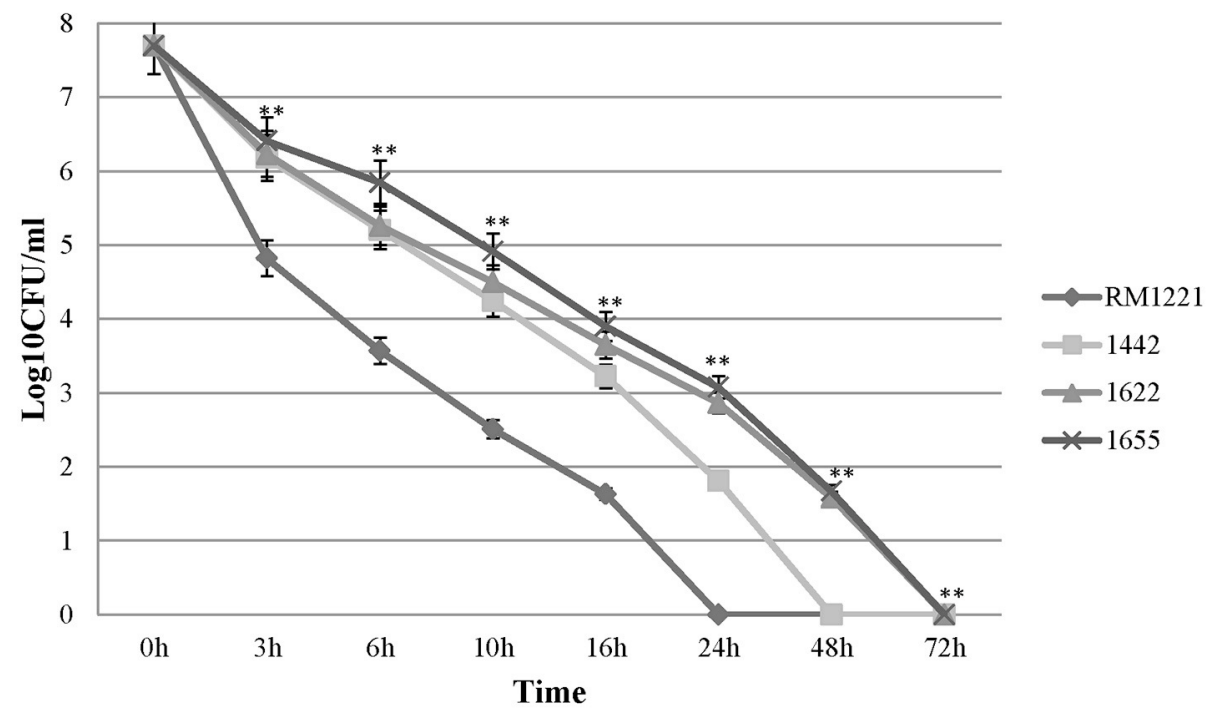

FIGURE 5 | Intra-macrophage survival assay of three selected C. jejuni strains in macrophage RAW264.7 cell. The Y-axis is the mean of log10 CFU/mL of each strain in the cell. The asterisk $\left(^{*}\right)$ and $\left(^{* *}\right)$ represent statistical significant difference with $P \leq 0.05$ and $P \leq 0.01$ comparing with $C$. jejuni RM1221, respectively.

which mediated resistance to aminoglycosides. No mutation wad found in the regulators (CmeR and CosR) of CmeABC efflux pumps.

\section{Virulence Associated Genes in C. jejuni 1655}

A large number of virulence associated genes were found in the genome of 1655 (Table 4). Among the selected genes involved in flagella synthesis and assembly (flaA, flaC, flgA, $f l g B, f l h B, f l i M$, and $f l i Y$ ), the $f l a C$ was found in the genome of 1655 and 11168. Two genes ( $c i a B$ and $n l p C$ ) associated with invasion and three genes (cadF, galE, and peb2) related to adhesion were present in 1655 but not in RM1221. Compared to the genes of CPS biosynthesis system in RM1221, the genome of 1655 contained a restriction modification enzyme, sugar nucleotidyltransferase, capsule polysaccharide protein, and alginate $\mathrm{O}$-acetyltransferase. The genome of 1655 also contained some genes ( $c f r A$ and ExbB-ExbD-TonB) involved 


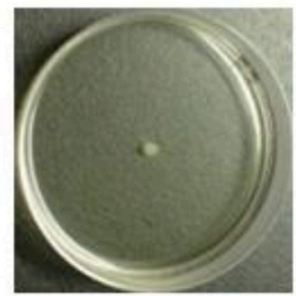

RML221

$0.5 \mathrm{~cm}$

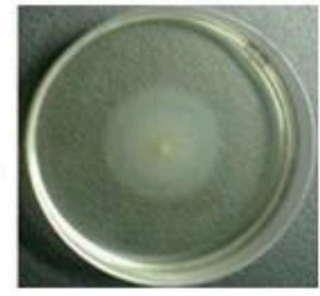

1442

$2.9 \mathrm{~cm}$

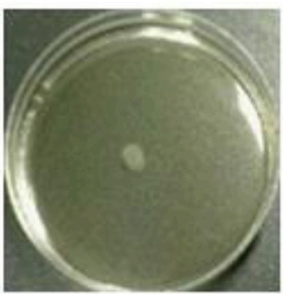

1622

$0.5 \mathrm{~cm}$

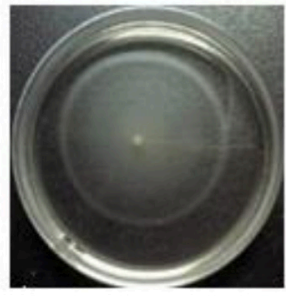

1655

$4.6 \mathrm{~cm}$
FIGURE 6 | Motility of the three selected C. jejuni strains on $0.4 \% \mathbf{M H}$ plate. The motility is positively corrleated to the diameter of growth ring of each strain. The diameter of spotted colonies was $0.5 \mathrm{~cm}$.

in iron uptake system, while RM1221 lacked TonB and 81176 lacked $c f r A$ and TonB13. The IS606 transposase, ISHa1675 transposase B and prophage Lp2 protein 6 were also found in the genome of 1655 . ISHa1675 transposase B was not found in the genome of three reference strains. The sequence of prophage Lp2 protein in C. jejuni 1655 had 99\% homology to that in 81-176. The pVir plasmid which previously found in 81-176, was not found in 1655. Three predicted genes of Clustered Regularly Interspaced Short Palindromic Repeats (CRISPR)-associated system were found in the genome of 1655. They may encode Csn1, Cas1, and Cas2 proteins respectively.

\section{DISCUSSION}

Compared to the genomes of reference strains (NCTC11168, 81-176, RM1221), the genome of 1655 contained some gene inversion, disordered distribution, deletions and rearrangements. The genome difference may be attributed to their different biological characteristics and different evolutional environment. NCTC11168 was originally isolated from a case of human enteritis (Parkhill et al., 2000). 81-176 was originally isolated from a diarrheal outbreak associated with raw milk consumption and exhibited high invasion and high pathogenicity for monkeys and humans (Korlath et al., 1985; Black et al., 1988; Tribble et al., 2010). C. jejuni RM1221 (ATCC BAA1032) was isolated from chicken and showed low virulence (Fouts et al., 2005). 1655 was a multidrug resistant strain
TABLE 3 | Genomic information of target bacteria 1655 and reference strains.

\begin{tabular}{lcccc}
\hline $\begin{array}{l}\text { Genomic } \\
\text { contents }\end{array}$ & 1655 & NCTC11168 & RM1221 & $81-176$ \\
Refseq number & & NC_002163.1 & NC_003912.7 & NC_008787 \\
Homology (\%) & - & 88.78 & 90 & 90.44 \\
Genome size & 1720061 & 1641481 & 1777831 & 1616554 \\
(bp) & & & & \\
G+C (\%) & 31.36 & 30.5 & 30.31 & 30.6 \\
Predicted coding & 1733 & - & 1898 & 1770 \\
region & & & & \\
CDS & 1732 & 1668 & 1783 & 1680 \\
Proteins & - & 1572 & 1783 & 1449 \\
Pseudo gene & 323 & 40 & 58 & 35 \\
rRNA & 9 & 9 & 9 & 9 \\
tRNA & 40 & 43 & 44 & 44 \\
Other RNA & 326 & 4 & 4 & 2
\end{tabular}

isolated from chicken fecal samples that exhibited relative higher virulence, as evidence by its higher pathogenicity to chickens, colonization of the chicken intestinal tract, cytotoxin production, biofilm formation, adhesion/invasion/intracellular survivability to macrophage cell and higher motility.

1655 had multidrug resistance to fluoroquinolones, macrolides, tetracyclines and aminoglycosides. The single T86I mutation in gyrA gene contributed to its high-level resistance to FQs (Luangtongkum et al., 2009). High level macrolide resistance in C. jejuni 1655 was likely due to A2075G mutation in 23S rRNA (Hao et al., 2009; Luangtongkum et al., 2009; Hao et al., 2010). Although there were some other mutations and deletions in $g y r B, 23 \mathrm{~s}$ rRNA genes and ribosomal L4/L22, the function of these mutations on antimicrobial resistance was not evident. The high-level resistance to tetracycline was mediated by tet $O$ gene which was located in pTet plasmid (Avrain et al., 2004). The pTet plasmid in 1655 was identical to the one found in 81-176 (Bacon et al., 2002). As a mobile gene element, pTet plasmid may act as a vehicle to pick up and spread multiple antibiotic resistance genes and virulence genes in C. jejuni (chen et al., 2013). The aadE and aphA-3 genes in 1655 could explain its resistance to amikacin and gentamicin (Gibreel et al., 2004; Iovine, 2013).

Generally it has been through that multidrug resistance is often associated with higher fitness costs or less virulence (Luangtongkum et al., 2009). However, our study suggested that the multidrug resistant 1655 had higher virulence than the reference strain RM1221 that is not multidrug resistant. Previous studies indicated that T86I mutation in gyrA gene could affect DNA supercoiling to regulate the expression of genes associated with bacterial fitness, and therefore enhance the fitness of fluoroquinolone-resistant Campylobacter in chicken host and increase the severity and duration of Campylobacterosis (Nelson et al., 2004; Luo et al., 2005; Helms et al., 2005; Evans et al., 2009; Han et al., 2012). However, mutations in $23 \mathrm{~S}$ rRNA could lead to fitness cost and reduced virulence of 


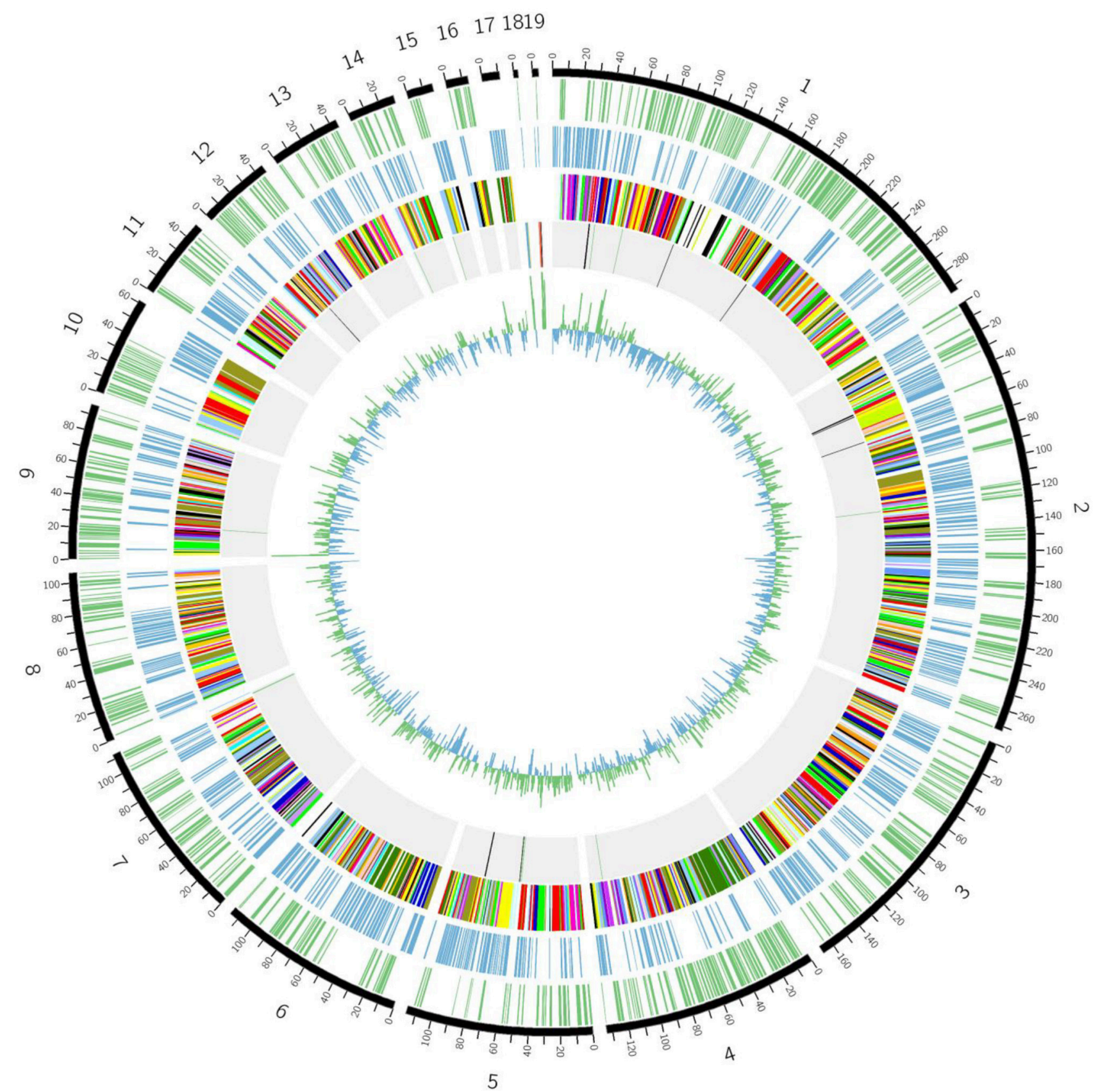

FIGURE 7 | Schematic circular genome of $\boldsymbol{C}$. jejuni 1655 strain. From outside to inside, there were five rings. The first circle shows the positive strand genes. The second circle shows the negative strand genes. The third circle were COG functional annotations (chr1-RNA processing and modification; chr2-Chromatin structure and dynamics; chr3-Energy production and conversion; chr4-Cell cycle control, cell division, chromosome partitioning; chr5-Amino acid transport and metabolism; chr6-Nucleotide transport and metabolism; chr7-Carbohydrate transport and metabolism; chr8-Coenzyme transport and metabolism; chr9-Lipid transport and metabolism; chr10-Translation, ribosomal structure and biogenesis; chr11-Transcription; chr12-Replication, recombination and repair; chr13- Cell wall/membrane/envelope biogenesis; chr14-Cell motility; chr15-Posttranslational modification, protein turnover, chaperones; chr16-Inorganic ion transport and metabolism; chr17-Secondary metabolites biosynthesis, transport and catabolism; chr18-General function prediction only; chr19-Signal transduction mechanisms). The fourth circle are rRNA and tRNA (red-16s rRNA, blue-23S rRNA, yellow-5S rRNA, black-positive chain tRNA, green-negative chain tRNA). The fitth circle is the GC contents.

macrolide resistant Campylobacter (Hao et al., 2009; Almofti et al., 2011a,b; Hao et al., 2013). Some of the clinical investigation data indicated the positive correlation between virulence and resistance, but others indicated the negative correlation (Bagger-Skjøt et al., 2007; McGowan-Spicer et al., 2008). For example, two recent studies showed that prevalence 


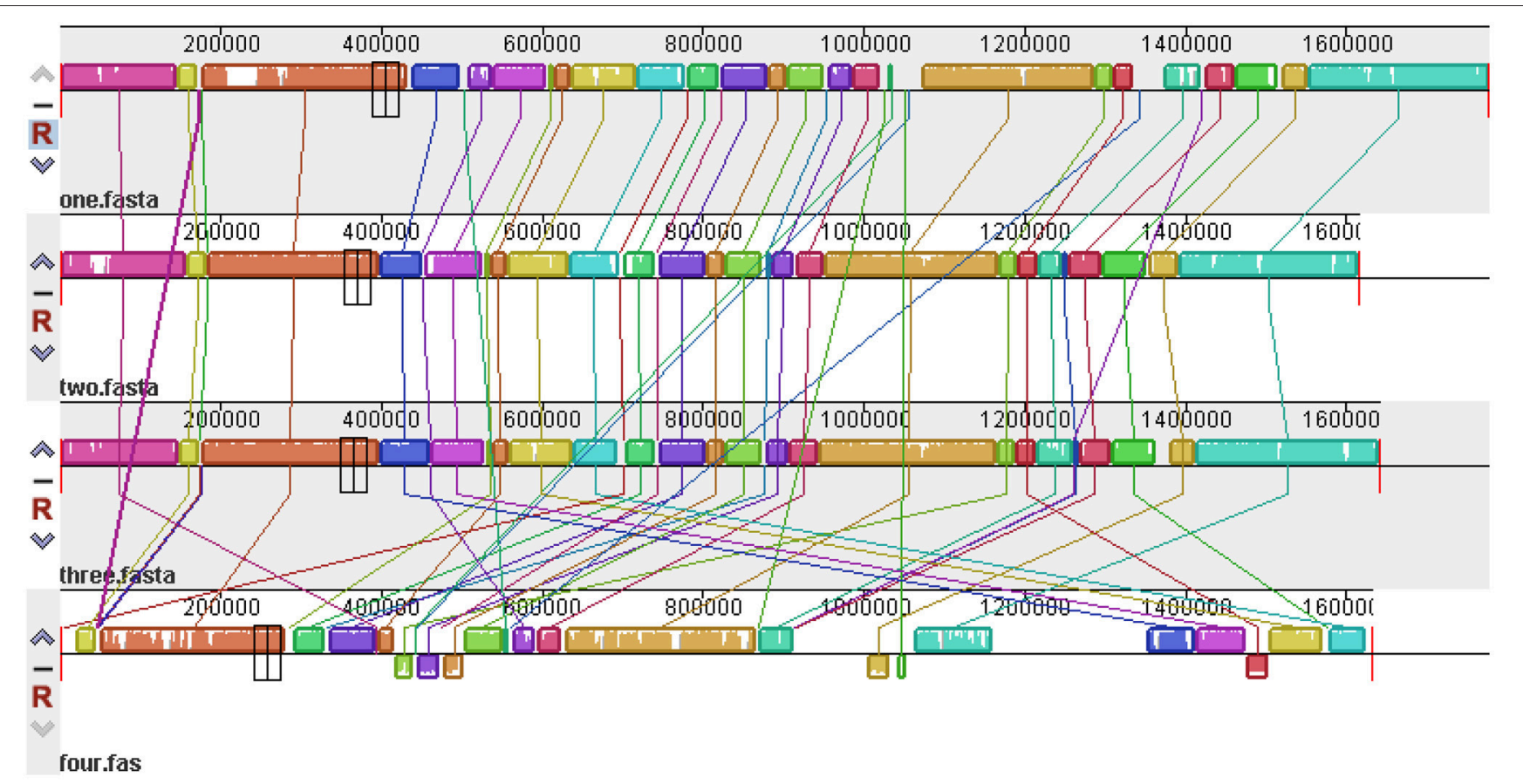

FIGURE 8 | Global multiple alignment of $\boldsymbol{C}$. jejuni genomes. From top to bottom are the genome structures of C. jejuni RM1221, 81-176, NCTC11168, and 1655 , respectively. The color region and white region represented high and low similarity respectively. Above axis was the positive strand, below was the negative strand.

of resistance to both erythromycin and ciprofloxacin was higher in isolates harboring $c i a B$ and some virulence genes (e.g., $c d t A$ and $d n a J)$ were associated with antimicrobial-resistant Campylobacter strains (Ghunaim et al., 2015; Lapierre et al., 2016). Our study revealed the relationship between multidrugresistance and virulence of some $C$. jejuni chicken isolates, indicating that complex mechanisms may work together to cause multidrug resistance and the relative higher virulence in $C$. jejuni 1655.

Comparing to the genome of RM1221, 1655 contained some special virulence-associated genes and proteins, including flaC, ciaB, nlpC, cadF, gelE, peb2, restriction modification enzyme, sugar nucleotidyltransferase, capsule polysaccharide protein, alginate $\mathrm{O}$-acetyltransferase and TonB iron uptake system. The flaC has been shown to modulate immune response in the intestinal tract and contribute to bacterial persistence of $C$. jejuni (Faber et al., 2015). The presence of ciaB, a Campylobacter invasion antigen, may contribute to the high invasion of $C$. jejuni 1655 (Rivera-Amill and Konkel, 1999). The nlpC may play a critical role in initial infection process, adherence to host cells (Padhi et al., 2016). The cadF, encoding fibronectin protein, may play an important role on the adhesion and colonization of 1655 in chicken (Konkel et al., 1997; Monteville et al., 2003). The peb2 is a membrane associated protein which may contribute to the increased gastrointestinal virulence of C. jejuni (Cordwell et al., 2008). The capsule polysaccharide (CPS), lipooligosaccharide (LOS), and restriction-modification $(\mathrm{R} / \mathrm{M})$ systems may enhance the invasion and colonization of
1655 in reservoir hosts and mediate the virulence of 1655 by evading host immune response (Bacon et al., 2001; Suerbaum et al., 2001; Ahmed et al., 2002; Guerry et al., 2002; Klena et al., 2004; Poly et al., 2004; Fouts et al., 2005; Guerry and Szymanski, 2008). The outer membrane protein CfrA and energy transporter system TonB-ExbB-ExbD are associated with iron absorption and iron uptake in gram-negative bacteria (Krewulak and Vogel, 2011). The presence of CfrA and TonBExbB-ExbD system in 1655 may contribute to its stronger ability of nutrients uptake and higher intracellular survivability (Andrews et al., 2003; Hofreuter et al., 2006; Krewulak and Vogel, 2011).

The genes encoding IS606 transposase, ISHa1675 transposase $B$ and prophage Lp2 protein 6 were identified in the genome of 1655 , suggesting that 1655 may contain transposons or phages. However, future experiments are needed to determine if these are associated with active mobile genetic elements in 1655 . The ISHa1675 transposase B and prophage Lp2 protein 6 were not found in the genome of RM1221 and 11168, indicating that these two genes may play special role in the physiological characteristic of C. jejuni 1655.

Three proteins associated with CRISPR-Cas systems (CRISPR-Cas2, CRISPR-Cas1 and a hypothetical protein associated with the CRISPR) were found in 1655. The CRISPRCas system provides bacterial defense against foreign nucleic acids derived from bacteriophages or plasmids (Barrangou et al., 2007; Marraffini, 2013). It also plays an important role in gene regulation and bacterial pathogenicity (Mojica et al., 2005; 
TABLE 4 | Virulence relative factors in C. jejuni 1655 and reference strains.

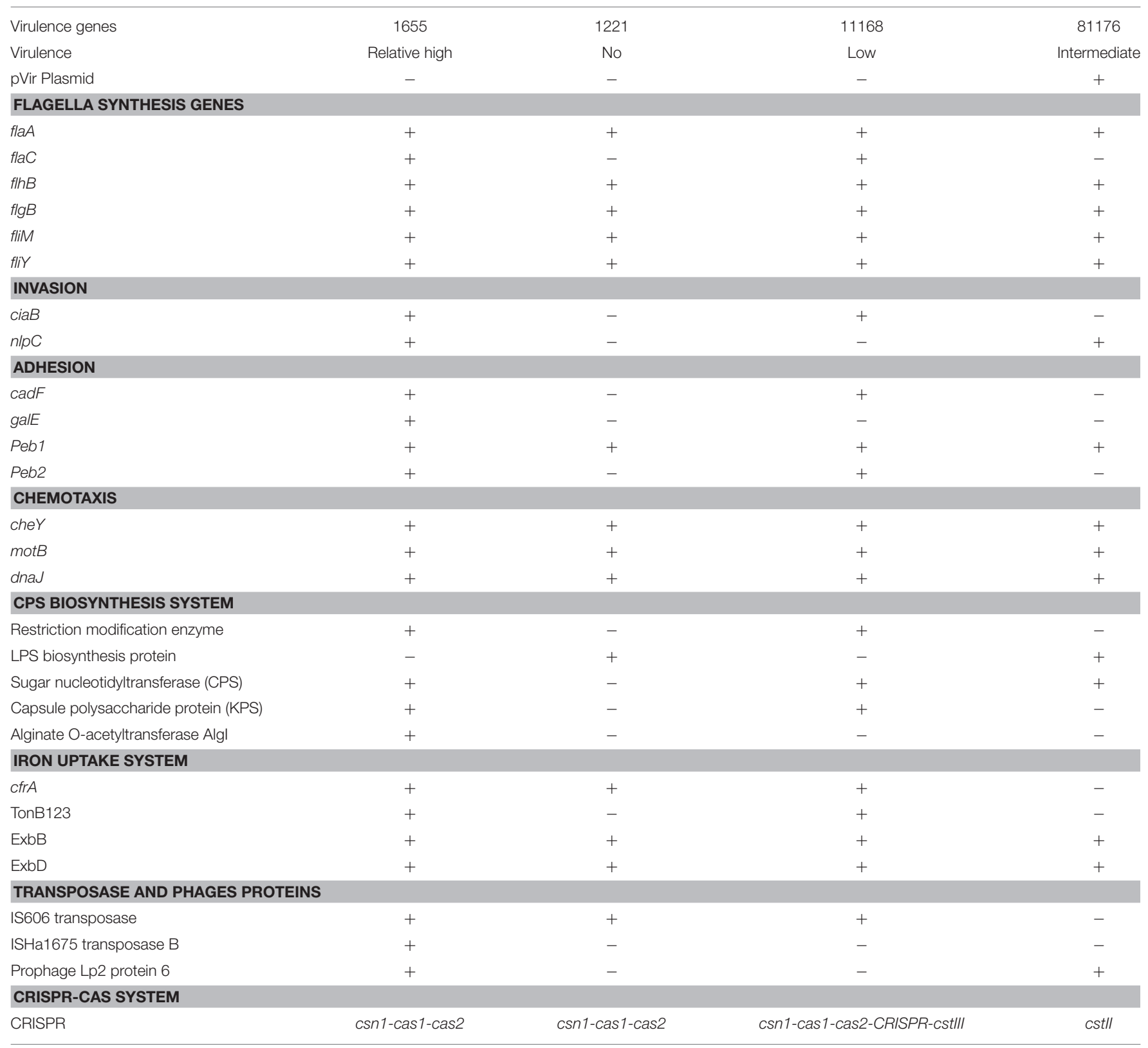

Louwen et al., 2013; Louwen and van Baarlen, 2013; Sampson and Weiss, 2014). Strains containing CRISPR-Cas system have a stronger ability of biofilm formation and colonization in mouse organs than those strains lack this system (Shimomura et al., 2011). Therefore, the presence of CRISPR-Cas system may contribute to the high virulence and biofilm formation capacity of C. jejuni 1655 strain.

\section{CONCLUSION}

1655 strain exhibited multidrug resistance to fluoroquinolone, macrolide, tetracycline, and aminoglycoside drugs and relative high in vitro and in vivo virulence. Comparing to the genome of reference strains (NCTC11168, RM1221, and 81-176), there were large difference in the genome structure and genome content in 1655 . The co-emergence of target gene mutations, resistance genes and pTet plasmid could explain its multidrug resistance. The virulence mechanism of Campylobacter may be mediated by a variety of virulence factors, including proteins involved in flagella biosynthesis, invasion, adhesion, CPS biosynthesis system, iron uptake system, transposase, phage proteins, and CRISPR-Cas system. Future experiments are needed to find the deeper molecular mechanisms and confirm the function of some important gene elements on multidrug resistance and relative higher virulence. 


\section{FINANCIAL DISCLOSURE}

This work was supported by National Basic Research Program of China (2013CB127200), National Key research and development program (2016YFD0501302), National Natural Science Foundation of China (31101856), National Key Technology R\&D Program (2012BAK01B00), Morning program of Wuhan in China (2015070404010191), Fundamental Research Funds for the Central Universities (2662015PY035), and National Program for Risk Assessment of Quality and Safety of Livestock and Poultry Products (GJFP2016008). The funders had no role in study design, data collection and analysis, decision to publish, or preparation of the manuscript. The opinions expressed in this manuscript are solely the responsibility of the authors and do not necessarily represent the official views and policy of the US Food and Drug Administration or National Institutes of Health. Reference to any commercial materials, equipment, or process does not in any way constitute approval, endorsement, or recommendation by the Food and Drug Administration.

\section{REFERENCES}

Abril, C., Brodard, I., and Perreten, V. (2010). Two novel antibiotic resistance genes, tet(44) and ant(6)-Ib, are located within a transferable pathogenicity island in Campylobacter fetus subsp. fetus. Antimicrob. Agents Chemother. 54, 3052-3055. doi: 10.1128/aac.00304-10

Ahmed, I. H., Manning, G., Wassenaar, T. M., Cawthraw, S., and Newell, D. G. (2002). Identification of genetic differences between two Campylobacter jejuni strains with different colonization potentials. Microbiology 148, 1203-1212. doi: 10.1099/00221287-148-4-1203

Allos, B. M. (2001). Campylobacter jejuni infections: update on emerging issues and trends. Clin. Infect. Dis. 32, 1201-1206. doi: 10.1086/319760

Almofti, Y. A., Dai, M., Sun, Y., Haihong, H., and Yuan, Z. (2011a). Impact of erythromycin resistance on the virulence properties and fitness of Campylobacter jejuni. Microb. Pathog. 50, 336-342. doi: 10.1016/ j.micpath.2011.02.009

Almofti, Y. A., Dai, M., Sun, Y., Hao, H., Liu, Z., Cheng, G., et al. (2011b). The physiologic and phenotypic alterations due to macrolide exposure in Campylobacter jejuni. Int. J. Food Microbiol. 151, 52-61. doi: 10.1016/j.ijfoodmicro.2011.08.002

Andrews, S. C., Robinson, A. K., and Rodríguez-Quiñones, F. (2003). Bacterial iron homeostasis. FEMS Microbiol. Rev. 27, 215-237.

Asakura, H., Yamasaki, M., Yamamoto, S., and Igimi, S. (2007). Deletion of peb4 gene impairs cell adhesion and biofilm formation in Campylobacter jejuni. FEMS Microbiol. Lett. 275, 278-285. doi: 10.1111/j.1574-6968.2007.00893.x

Ashkenazi, S., and Cleary, T. G. (1990). A method for detecting Shiga toxin and Shiga-like toxin-I in pure and mixed culture. J. Med. Microbiol. 32, 255-261. doi: 10.1099/00222615-32-4-255

Avrain, L., Vernozy-Rozand, C., and Kempf, I. (2004). Evidence for natural horizontal transfer of tetO gene between Campylobacter jejuni strains in chickens. J. Appl. Microbiol. 97, 134-140. doi: 10.1111/j.1365-2672. 2004.02306.x

Bacon, D. J., Alm, R. A., Hu, L., Hickey, T. E., Ewing, C. P., Batchelor, R. A., et al. (2002). DNA sequence and mutational analyses of the pVir plasmid of Campylobacter jejuni 81-176. Infect. Immun. 70, 6242-6250.

Bacon, D. J., Szymanski, C. M., Burr, D. H., Silver, R. P., Alm, R. A., and Guerry, P. (2001). A phase-variable capsule is involved in virulence of Campylobacter jejuni 81-176. Mol. Microbiol. 40, 769-777.

Bagger-Skjøt, L., Sandvang, D., Frimodt-Møller, N., Lester, C. H., Olsen, K. E., Porsbo, L. J., et al. (2007). Association between antimicrobial resistance and virulence genes in Escherichia coli obtained from blood and faeces. Scand. J. Infect. Dis. 39, 724-727. doi: 10.1080/00365540701242400

\section{AUTHOR CONTRIBUTIONS}

Conceived and designed the experiments: HH, NR, MD, GC, ZY. Performed the experiments: NR, XK, JL, ZI, HH. Analyzed the data: HH, NR, JH, SF, YW, ZY. Contributed reagents/materials/analysis tools: YW, ZL, MD, YW, ZY. Wrote the paper: HH, NR, JH, SF, ZI, ZY.

\section{ACKNOWLEDGMENTS}

Thanks the curious review by Dr. Huizhong Chen and Kidon Sung from FDA National Center for Toxicological Research.

\section{SUPPLEMENTARY MATERIAL}

The Supplementary Material for this article can be found online at: http://journal.frontiersin.org/article/10.3389/fmicb. 2016.01605

Barrangou, R., Fremaux, C., Deveau, H., Richards, M., Boyaval, P., Moineau, S., et al. (2007). CRISPR provides acquired resistance against viruses in prokaryotes. Science 315, 1709-1712. doi: 10.1126/science.1138140

Black, R. E., Levine, M., M., Clements, M. L., Hughes, T. P., and Blaser, M. J. (1988). Experimental Campylobacter jejuni infection in humans. J. Infect. Dis. $157,472-479$.

Chen, X., Naren, G. W., Wu, C. M., Wang, Y., Dai, L., Xia, L. N., et al. (2010). Prevalence and antimicrobial resistance of Campylobacter isolates in broilers from China. Vet. Microbiol. 144, 133-139. doi: 10.1016/j.vetmic.2009.12.035

Chen, Y., Mukherjee, S., Hoffmann, M., Kotewicz, M. L., Young, S., Abbott, J., et al. (2013). Whole-genome sequencing of gentamicin-resistant Campylobacter coli isolated from U.S. retail meats reveals novel plasmidmediated aminoglycoside resistance genes. Antimicrob. Agents Chemother. 57, 5398-5405. doi: 10.1128/AAC.00669-13

CLSI. (2008). Performance Standards for Antimicrobial Disk and Dilution Susceptibility Tests for Bacteria Isolated From Animals; Approved StandardThird Edition. Wayne, PA: Clinical and Laboratory Standards Institute (CLSI).

Cordwell, S. J., Len, A. C., Touma, R. G., Scott, N. E., Falconer, L., Jones, D., et al. (2008). Identification of membrane-associated proteins from Campylobacter jejuni strains using complementary proteomics technologies. Proteomics 8, 122-139. doi: 10.1002/pmic.200700561

DANMAP (2014). DANMAP 2014 - Use of Antimicrobial Agents and Occurrence of Antimicrobial Resistance in Bacteria from Food Animals, Food and Humans in Denmark. Copenhagen.

Evans, M. R., Northey, G., Sarvotham, T. S., Hopkins, A. L., Rigby, C. J., and Thomas, D. R. (2009). Risk factors for ciprofloxacin-resistant Campylobacter infection in Wales. J. Antimicrob. Chemother. 64, 424-427. doi: 10.1093/jac/dkp179

Faber, E., Gripp, E., Maurischat, S., Kaspers, B., Tedin, K., Menz, S., et al. (2015). Novel immunomodulatory flagellin-like protein FlaC in Campylobacter jejuni and other campylobacterales. $m$ Sphere 2, 1. doi: 10.1128/mSphere.00028-15

Fields, J. A., and Thompson, S. A. (2008). Campylobacter jejuni CsrA mediates oxidative stress responses, biofilm formation, and host cell invasion. J. Bacteriol. 190, 3411-3416. doi: 10.1128/JB.01928-07

Fouts, D. E., Mongodin, E. F., Mandrell, R. E., Miller, W. G., Rasko, D. A., Ravel, j., et al. (2005). Major structural differences and novel potential virulence mechanisms from the genomes of multiple campylobacter species. PLoS Biol. 3:e15. doi: 10.1371/journal.pbio.0030015

Ghunaim, H., Behnke, J. M., Aigha, I., Sharma, A., Doiphode, S. H., Deshmukh, A., et al. (2015). Analysis of resistance to antimicrobials and presence of virulence/stress response genes in campylobacter isolates from patients with severe diarrhoea. PLoS ONE 10:e0119268. doi: 10.1371/journal.pone.0119268 
Gibreel, A., Sköld, O., and Taylor, D. E. (2004). Characterization of plasmidmediated aphA-3 kanamycin resistance in Campylobacter jejuni. Microb. Drug Resist. 10, 98-105. doi: 10.1089/1076629041310127

Guerrant, R. L., Wanke, C. A., Pennie, R. A., Barrett, L. J., Lima, A. A., and O’Brien, A. D. (1987). Production of a unique cytotoxin by Campylobacter jejuni. Infect. Immun. 55, 2526-2530.

Guerry, P., and Szymanski, C. M. (2008). Campylobacter sugars sticking out. Trends Microbiol. 16, 428-435. doi: 10.1016/j.tim.2008.07.002

Guerry, P., Szymanski, C. M., Prendergast, M. M., Hickey, T. E., Ewing, C. P., and Pattarini D. L., et al. (2002). Phase variation of Campylobacter jejuni 81-176 lipooligosaccharide affects ganglioside mimicry and invasiveness in vitro. Infect. Immun. 70, 787-793.

Han, J., Wang, Y., Sahin, O., Shen, Z., Guo, B., Shen, J., et al. (2012). A fluoroquinolone resistance associated mutation in gyrA Affects DNA supercoiling in Campylobacter jejuni. Front. Cell. Infect. Microbiol. 2:21. doi: 10.3389/fcimb.2012.00021

Hao, H., Dai, M., Wang, Y., Chen, D., and Yuan, Z. (2010). Quantification of mutated alleles of $23 \mathrm{~S}$ rRNA in macrolide-resistant Campylobacter by TaqMan real-time polymerase chain reaction. Foodborne Pathog. Dis. 7, 43-49. doi: 10.1089/fpd.2009.0339

Hao, H., Dai, M., Wang, Y., Peng, D., Liu, Z., and Yuan, Z. (2009). 23S rRNA mutation A2074C conferring high-level macrolide resistance and fitness cost in Campylobacter jejuni. Microb. Drug Resist. 15, 239-244. doi: 10.1089/mdr.2009.0008

Hao, H., Liu, J., Kuang, X., Dai, M., Cheng, G., Wang, X., et al. (2015). Identification of Campylobacter jejuni and determination of point mutations associated with macrolide resistance using a multiplex TaqMan MGB real-time PCR. J. Appl. Microbiol. 118, 1418-1425. doi: 10.1111/jam.12793

Hao, H., Yuan, Z., Shen, Z., Han, J., Sahin, O., Liu, P., et al. (2013). Mutational and transcriptomic changes involved in the development of macrolide resistance in Campylobacter jejuni. Antimicrob. Agents Chemother. 57, 1369-1378. doi: 10.1128/AAC.01927-12

Helms, M., Simonsen, J., Olsen, K. E., and Mølbak, K. (2005). Adverse health events associated with antimicrobial drug resistance in Campylobacter species: a registry-based cohort study. J. Infect. Dis. 191, 1050-1055. doi: 10.1086/428453

Hofreuter, D., Tsai, J., Watson, R. O., Novik, V., Altman, B., Benitez, M., et al. (2006). Unique features of a highly pathogenic Campylobacter jejuni strain. Infect. Immun. 74, 4694-4707. doi: 10.1128/IAI.00210-06

Iovine, N. M. (2013). Resistance mechanisms in Campylobacter jejuni. Virulence 4, 230-240. doi: 10.4161/viru.23753

Iovine, N. M., Pursnani, S., Voldman, A., Wasserman, G., Blaser, M. J., and Weinrauch, Y. (2008). Reactive nitrogen species contribute to innate host defense against Campylobacter jejuni. Infect. Immun. 76, 986-993. doi: 10.1128/IAI.01063-07

Johnson, J. G., Carpentier, S., Spurbeck, R. R., Sandhu, S. K., and DiRita, V. J. (2014). Genome sequences of Campylobacter jejuni 81-176 variants with enhanced fitness relative to the parental strain in the chicken gastrointestinal tract. Genome Announc. 2:e00006-14. doi: 10.1128/genomeA.00006-14

Klena, J. D., Parker, C. T., Knibb, K., Ibbitt, J. C., Devane, P. M., Horn, S. T., et al. (2004). Differentiation of Campylobacter coli, Campylobacter jejuni, Campylobacter lari, and Campylobacter upsaliensis by a multiplex PCR developed from the nucleotide sequence of the lipid A gene lpxA. J. Clin. Microbiol. 42, 5549-5557. doi: 10.1128/JCM.42.12.5549-5557.2004

Konkel, M. E., Garvis, S. G., Tipton, S. L., Anderson, D. E. Jr., and Cieplak, W. Jr. (1997). Identification and molecular cloning of a gene encoding a fibronectin-binding protein (CadF) from Campylobacter jejuni. Mol. Microbiol. 24, 953-963.

Korlath, J. A., Osterholm, M. T., Judy, L. A., Forfang, J. C., and Robinson, R. A. (1985). A point-source outbreak of campylobacteriosis associated with consumption of raw milk. J. Infect. Dis. 152, 592-596.

Krewulak, K. D., and Vogel, H. J. (2011). TonB or not TonB: is that the question? Biochem. Cell Biol. 89, 87-97. doi: 10.1139/o10-141

Lapierre, L., Gatica, M. A., Riquelme, V., Vergara, C., Yañez, J. M., San Martín, B., et al., (2016). Characterization of antimicrobial susceptibility and its association with virulence genes related to adherence, invasion, and cytotoxicity in Campylobacter jejuni and Campylobacter coli isolates from animals, meat, and humans. Microb. Drug Resist. 22, 432-444. doi: 10.1089/mdr.20 15.0055
Louwen, R., Horst-Kreft, D., de Boer, A. G., van der Graaf, L., de Knegt, G., Hamersma, M., et al. (2013). A novel link between Campylobacter jejuni bacteriophage defence, virulence and Guillain-Barre syndrome. Eur. J. Clin. Microbiol. Infect. Dis. 32, 207-226. doi: 10.1007/s10096-012-1733-4

Louwen, R., and van Baarlen, P. (2013). Are bacteriophage defence and virulence two sides of the same coin in Campylobacter jejuni? Biochem. Soc. Trans. 41, 1475-1481. doi: 10.1042/BST20130127

Luangtongkum, T., Jeon, B., Han, J., Plummer, P., Logue, C. M., and Zhang, Q. (2009). Antibiotic resistance in Campylobacter: emergence, transmission and persistence. Future Microbiol. 4, 189-200. doi: 10.2217/174609 13.4.2.189

Luo, N., Pereira, S., Sahin, O., Lin, J., Huang, S., Michel, L., et al. (2005). Enhanced in vivo fitness of fluoroquinolone-resistant Campylobacter jejuni in the absence of antibiotic selection pressure. Proc. Natl. Acad. Sci. U.S.A. 102, 541-546. doi: 10.1073/pnas.0408966102

Marraffini, L. A. (2013). CRISPR-Cas immunity against phages: its effects on the evolution and survival of bacterial pathogens. PLoS Pathog. 9:e1003765. doi: 10.1371/journal.ppat.1003765

McGowan-Spicer, L. L., Fedorka-Cray, P. J., Frye, J. G., Meinersmann, R. J., Barrett, J. B., and Jackson, C. R. (2008). Antimicrobial resistance and virulence of Enterococcus faecalis isolated from retail food. J. Food Prot. 71, 760-769.

McLennan, M. K., Ringoir, D. D., Frirdich, E., Svensson, S. L., Wells, D. H., Jarrell, H., et al. (2008). Campylobacter jejuni biofilms up-regulated in the absence of the stringent response utilize a calcofluor white-reactive polysaccharide. $J$. Bacteriol. 190, 1097-1107. doi: 10.1128/JB.00516-07

Mojica, F. J. M., Díez-Villaseñor, C., García-Martinez, J., and Soria, E. (2005). Intervening sequences of regularly spaced prokaryotic repeats derive from foreign genetic elements. J. Mol. Evol. 60, 174-182. doi: 10.1007/s00239-0040046-3

Monteville, M. R., Yoon, J. E., and Konkel, M. E. (2003). Maximal adherence and invasion of INT 407 cells by Campylobacter jejuni requires the CadF outer-membrane protein and microfilament reorganization. Microbiology 149, 153-165. doi: 10.1099/mic.0.25820-0

NARMS (2015). National Antimicrobial Resistance Mornitoring System Integrated Report: 2012-2013. Available online at: http://www.fda.gov/ downloads/AnimalVeterinary/SafetyHealth/AntimicrobialResistance/ NationalAntimicrobialResistanceMonitoringSystem/UCM453398.pdf

Nelson, J. M., Smith, K. E., Vugia, D. J., Rabatsky-Ehr, T., Segler, S. D., Kassenborg, H. D., et al. (2004). Prolonged diarrhea due to ciprofloxacin-resistant campylobacter infection. J. Infect. Dis. 190, 1150-1157. doi: 10.1086/423282

Padhi, A., Naik, S. K., Sengupta, S., Ganguli, G., and Sonawane, A. (2016). Expression of Mycobacterium tuberculosis NLPC/p60 family protein Rv0024 induce biofilm formation and resistance against cell wall acting antituberculosis drugs in Mycobacterium smegmatis. Microbes Infect. 18, 224-236. doi: 10.1016/j.micinf.2015.11.007

Parkhill, J., Wren, B. W., Mungall, K., Ketley, J. M., Churcher, C., Basham, D., et al. (2000). The genome sequence of the food-borne pathogen Campylobacter jejuni reveals hypervariable sequences. Nature 403, 665-668. doi: 10.1038/35 001088

Poly, F., Threadgill, D., and Stintzi, A. (2004). Identification of Campylobacter jejuni ATCC 43431-specific genes by whole microbial genome comparisons. J. Bacteriol. 186, 4781-4795. doi: 10.1128/JB.186.14.4781-4 795.2004

Reuter, M., Mallett, A., Pearson, B. M., and van Vliet, A. H. (2010). Biofilm formation by Campylobacter jejuni is increased under aerobic conditions. Appl. Environ. Microbiol. 76, 2122-2128. doi: 10.1128/AEM. 01878-09

Rivera-Amill, V., and Konkel, M. E. (1999). Secretion of Campylobacter jejuni Cia proteins is contact dependent. Adv. Exp. Med. Biol. 473, 225-229.

Sampson, T. R., and Weiss, D. S. (2014). CRISPR-Cas systems: new players in gene regulation and bacterial physiology. Front. Cell. Infect. Microbiol. 4:37. doi: 10.3389/fcimb.2014.00037

Shimomura, Y., Okumura, K., Murayama, S. Y., Yagi, J., Ubukata, K., Kirikae, T., et al. (2011). Complete genome sequencing and analysis of a Lancefield group G Streptococcus dysgalactiae subsp. equisimilis strain causing streptococcal toxic shock syndrome (STSS). BMC Genomics 12:17. doi: 10.1186/1471-21 64-12-17 
Stucki, U., Frey, J., Nicolet, J., and Burnens, A. P. (1995). Identification of Campylobacter jejuni on the basis of a species-specific gene that encodes a membrane protein. J. Clin. Microbiol. 33, 855-859.

Suerbaum, S., Lohrengel, M., Sonnevend, A., Ruberg, F., and Kist, M. (2001). Allelic diversity and recombination in Campylobacter jejuni. J. Bacteriol. 183, 2553-2559. doi: 10.1128/JB.183.8.2553-25 59.2001

Takamiya, M., Ozen, A., Rasmussen, M., Alter, T., Gilbert, T., Ussery, D. W., et al. (2011). Genome sequences of two stress-tolerant Campylobacter jejuni poultry strains, 305 and DFVF1099. J. Bacteriol. 193, 5546-5547. doi: 10.1128/JB. 05753-11

Tribble, D. R., Baqar, S., Scott, D. A., Oplinger, M. L., Trespalacios, F., Rollins, D., et al. (2010). Assessment of the duration of protection in Campylobacter jejuni experimental infection in humans. Infect. Immun. 78, 1750-1759. doi: 10.1128/IAI.01021-09
Wang, Y., Dong, Y., Deng, F., Liu, D., Yao, H., Zhang, Q., et al. (2016). Species shift and multidrug resistance of Campylobacter from chicken and swine, China, 2008-14. J. Antimicrob. Chemother. 71, 666-669. doi: 10.1093/jac/dkv382

Conflict of Interest Statement: The authors declare that the research was conducted in the absence of any commercial or financial relationships that could be construed as a potential conflict of interest.

Copyright (C) 2016 Hao, Ren, Han, Foley, Iqbal, Cheng, Kuang, Liu, Liu, Dai, Wang and Yuan. This is an open-access article distributed under the terms of the Creative Commons Attribution License (CC BY). The use, distribution or reproduction in other forums is permitted, provided the original author(s) or licensor are credited and that the original publication in this journal is cited, in accordance with accepted academic practice. No use, distribution or reproduction is permitted which does not comply with these terms. 


\title{
Is Quorum Sensing Interference a Viable Alternative to Treat Pseudomonas aeruginosa Infections?
}

\author{
Rodolfo García-Contreras * \\ Department of Microbiology and Parasitology, Faculty of Medicine, National Autonomous University of Mexico, Mexico City, \\ Mexico
}

Quorum sensing (QS) coordinates the expression of multiple virulence factors in Pseudomonas aeruginosa; hence its inhibition has been postulated as a new alternative to treat its infections. In particular, QS interference approaches claim that they attenuate bacterial virulence without directly decreasing bacterial growth and suggest that in vivo the immune system would control the infections. Moreover, since in vitro experiments performed in rich medium demonstrate that interfering with QS decreases the production

OPEN ACCESS

Edited by:

Axel Cloeckaert,

French National Institute for Agricultural Research (INRA), France

Reviewed by:

Pierre Cornelis,

Vrije Universiteit Brussel, Belgium César De La Fuente-Núñez,

Massachusetts Institute of Technology, USA

*Correspondence: Rodolfo García-Contreras

rgarc@bq.unam.mx

Specialty section:

This article was submitted to

Antimicrobials, Resistance and Chemotherapy,

a section of the journal

Frontiers in Microbiology

Received: 01 June 2016

Accepted: 31 August 2016

Published: 14 September 2016

Citation:

García-Contreras R (2016) Is Quorum

Sensing Interference a Viable

Alternative to Treat Pseudomonas

aeruginosa Infections?

Front. Microbiol. 7:1454.

doi: 10.3389/fmicb.2016.01454 of virulence factors without affecting bacterial growth it was assumed than in vivo therapies will minimize the selection of resistant strains. Therefore, the underlying assumptions toward an effective implementation of a successful Quorum sensing interference (QSI) therapy for treating P. aeruginosa infections are that (i) QS only exerts important effects in the regulation of virulence genes but it does not affect metabolic processes linked to growth, (ii) the expression of virulence factors is only positively regulated by QS, (iii) inhibition of virulence factors in vivo do not affect bacterial growth, (iv) the immune system of the infected patients will be able to get rid of the infections, and (v) the therapy will be effective in the strains that are actively producing the infections. Nevertheless, for QSI in P. aeruginosa, substantial experimental evidence against the validity of most of these assumptions has accumulated during the past years, suggesting that a far better understanding of its virulence and its behavior during infections is needed in order to design truly solid QSI therapeutic alternatives to combat this remarkable pathogen.

Keywords: Pseudomonas infections, quorum sensing (QS), quorum quenching, resistance, clinical isolates

\section{INTRODUCTION}

Pseudomonas aeruginosa is a remarkable opportunistic pathogen that infects patients that are immunocompromised, have severe burns, cancer, or AIDS, are intubated and with prosthetic devices, and also those suffering from chronic affections like cystic fibrosis (CF). This bacterium is a major health problem worldwide being responsible of $10 \%$ of nosocomial infections (Antunes et al., 2010; Castillo-Juárez et al., 2015), since it is intrinsically resistant to several antimicrobials (Poole, 2011) and able to develop resistance against new ones, has a high biofilm production and produces an arsenal of virulence factors. One of the main mechanisms that controls the production of its virulence factors is quorum sensing (QS) which coordinates the expression of such factors 
once bacteria have reached a high population density, thus maximizing their chances to overcome the host defenses and establish the infection.

QS systems are common in bacterial pathogens; among Gram negatives, in addition to P. aeruginosa, Acinetobacter baumannii (Bhargava et al., 2010), Escherichia coli (Sperandio et al., 2002) Salmonella strains (Choi et al., 2007), and Vibrio strains (Zhu et al., 2002; Yang and Defoirdt, 2015) use them to coordinate their virulence. Hence, QS interference (QSI) or quorum quenching (QQ) is a strategy proposed to inhibit virulence as an alternative to treat the infections of several important bacterial pathogens (Castillo-Juárez et al., 2015).

In this opinion piece I focus in $P$. aeruginosa, one of the most studied organisms regarding QS and QSI, nevertheless what is exposed here may be also applicable to other bacterial pathogens.

$P$. aeruginosa is one of the more complex known bacterial pathogens; it possesses an ample genome and a high percentage of its genes are devoted to gene regulation (Stover et al., 2000). Regarding QS systems, it has a hierarchical architecture governed by the LasRI module which produces and senses $\mathrm{N}$-3-oxododecanoyl-L-Homoserine lactone and activates the expression of multiple virulence genes such as those producing elastase $\mathrm{A}$ and $\mathrm{B}$, pyocyanin, alkaline and protease, and activating a second homoserine lactone (HSL) QS module known as RhlRI, which produces and senses N-butyryl-L-Homoserine lactone. RhlR bound to its autoinducer also activates directly some virulence genes like those encoding rhamnolipids and pyocyanin (Smith and Iglewski, 2003; Jimenez et al., 2012). In addition, P. aeruginosa also has a quinolone dependent system known as PQS, which is positively regulated by LasR and negatively by RhlR, forming a complex and intricate network (Jimenez et al., 2012; Lee and Zhang, 2015). In addition to the QS intrinsic components, several regulators such as GacA/GacS, QscR, Vfr, RpoN, and RpoS, influence the expression of QS dependent factors (Lee and Zhang, 2015).

The complex relationship between QS and virulence in $P$. aeruginosa had been recently evidenced by the fact that las $R$ deficient mutants (including clinical strains) growing at slow rates or in the stationary phase, overproduce pyocyanin due a lack of repression of the phenazine genes by RsaL, a negative transcriptional regulator positively controlled by LasR (Cabeen, 2014). Moreover, in lasR mutants, the activity of the $r h l$ system is only delayed but not abolished, allowing significant production of pyocyanin, rhamnolipids and N-butyryl-L-Homoserine lactone (which are RhlR dependent) and even the production of the LasR dependent QS signals N-3-oxo-dodecanoyl-L-Homoserine lactone and PQS (Dekimpe and Deziel, 2009). These findings suggest that the inhibition of a particular component of the QS network, even the pivotal factor LasR, may be counteracted by the activation of alternative components of the network. Indeed, some recent studies have identified novel QS inhibitors for which RhlR and not LasR as the relevant in vivo target (O'Loughlin et al., 2013; Welsh et al., 2015).

In addition, recently it was discovered that an environmental strain (148 isolated from dolphin gastric juice) produces significant amounts of the QS-controlled virulence factors rhamnolipids and pyocyanin. Critically, this strain is virulent to mice, even without having a las $R$ gene and without producing N-3-oxo-dodecanoyl-L-Homoserine lactone. Hence, in this strain, the control of QS dependent virulence factors must be exerted by other regulators (Grosso-Becerra et al., 2014).

QS Interference could be achieved by the attenuation of the QS communication systems via: (i) the disruption of the QS receptors, (ii) the degradation of the autoinducers signals or (iii) the inhibition of the signal synthesis. Accordingly, several QS inhibitors or quorum quenchers (QQ) suitable for $P$. aeruginosa have been developed, under the assumptions that (i) the expression of virulence factors is only positively regulated by QS, (ii) the QQ will only exert significant effects in the regulation of virulence genes but not in metabolic processes linked to growth, hence avoiding or at least decreasing the generation of selective pressure that leads to resistance, (iii) the immune system of the infected patients will be able to get rid of the infections, and (iv) the therapy will be effective against the strains that are actively producing the infections. Nevertheless, substantial evidence against the validity of those assumptions has accumulated over the years.

\section{QUORUM SENSING INFLUENCES METABOLIC PROCESSES AND BACTERIAL GROWTH}

Although the role of QS in P. aeruginosa virulence has been extensively studied, whether it influences global metabolism and cell growth is less explored. One possible reason for this bias is that at the transcriptomic level, few significant changes in metabolicrelated genes are found when QS proficient and deficient strains are compared or when QS inhibitors are administrated to QS proficient strains (Hentzer et al., 2003; Schuster et al., 2003; Wagner et al., 2003). Nevertheless, recently Davenport and coworkers found that the metabolome of a lasI rhlI double mutant (unable to produce HSL QS autoinducers) and that of its wild-type progenitor have a notorious divergence once the wild-type strain produced the highest levels of autoinducers; remarkably, around one third of all the metabolites identified changed (Davenport et al., 2015). This phenomenon is understandable since the wild-type strain devotes many of its resources to the production of costly virulence factors such as exoproteases, phenazines, and exopolysacharides, while, in contrast, the QS mutants uses the same resources for cell division. In agreement, the QS deficient mutants achieve higher growth yields than QS wild-type strains (Diggle et al., 2007).

Although the possible implications of the metabolic divergence in QS mutants on bacterial physiology and QS interference therapies is still unexplored, Davenport and coworkers demonstrated that important metabolic changes in the membrane metabolism of the wild-type strain are driven by QS upon entering the stationary phase. These modifications include increased fatty acid saturation, chain length, and cyclopropanation, which in turn, promote the generation of robust cell membranes. In contrast, the membranes of QS mutants do not have these modifications and are therefore more susceptible to stress. 
In addition to these metabolic changes, several works have demonstrated that in $P$. aeruginosa and other bacteria, QS enhances the stress response (Bjarnsholt et al., 2005; Bhargava et al., 2014; de Oca-Mejia et al., 2015; García-Contreras et al., 2015a); for example, by upregulating antioxidant enzymes such as catalase, superoxide dismutase (Hassett et al., 1999) and NADPH-generating enzymes (García-Contreras et al., 2015a). This is important since during an infection, the immune system attacks bacteria by releasing reactive oxygen species, and at least in vitro oxidative stress is able to select functional QS systems as well as QS interference resistant mutants (García-Contreras et al., 2015a).

Moreover, growth of $P$. aeruginosa can be strongly dependent on QS either if adenosine or protein is used as sole carbon sources, since nuh, the gene encoding the nucleoside hydrolase, is under tight LasR control (Heurlier et al., 2005) and since the expression of exoproteases is under QS control (Diggle et al., 2007). During infections, adenosine utilization (Patel et al., 2007; Sheng et al., 2012) as well as the degradation of host proteins (Wretlind and Pavlovskis, 1983; Laarman et al., 2013) are important for bacterial virulence and survival.

All the above summarized facts demonstrate that QS is an important regulator of bacterial metabolism and physiology and suggest that QS interference will have the direct effect of decreasing bacterial growth and viability in vivo. In agreement, several in vivo infection studies have shown that when a QS interference therapy is able to increase animal survival (and decrease damage to the host), there is a significant decrease in bacterial counts in the infection sites (Wu et al., 2004; Christensen et al., 2007; Defoirdt et al., 2010; Jakobsen et al., 2011). The decrease in bacterial viability due QS interference may be an important potential source of in vivo selective pressure for the selection of bacterial resistance (García-Contreras et al., 2016).

\section{POTENTIAL INCREASE IN VIRULENCE UPON QUORUM SENSING INTERFERENCE}

Although the production of multiple virulence factors in $P$. aeruginosa is positively regulated by $\mathrm{QS}$, the virulence phenotype displayed by this organism is a complex combinatorial phenomenon that cannot be easily predicted with the identification of the presence or absence of a set of specific genes (Lee et al., 2006; Grosso-Becerra et al., 2014). In agreement, several studies had shown that clinical strains display a wide variety levels of virulence (Fenner et al., 2006; Lee et al., 2006; Garcia-Contreras et al., 2015b) and that environmental strains often conserve high virulence (Grosso-Becerra et al., 2014).

An overlooked fact about the role of QS in P. aeruginosa virulence was discovered in 2005 by Bleves and coworkers, who that found that in contrast to several other virulence traits, the expression of the type III secretion system (TTSS) in $P$. aeruginosa is negatively regulated by QS, specifically by RhlR and PqsR (Bleves et al., 2005; Kong et al., 2009). An important factor in such negative regulation of virulence genes by QS is that it requires low calcium levels, and hence several transcriptomics studies done in culture medium such as LB with relative high calcium levels fail to show this relationship. Since the TTSS is an important determinant of virulence in several animal infection models including pneumonia, peritonitis, bacteremia, burn infections, and keratitis (Hauser, 2009), the possibility that QS interfering therapies may be activating this system and therefore promoting virulence should not be ignored. Moreover, $P$. aeruginosa has five of the six known types of secretion systems present in Gram negative bacteria (all but type IV) and some of these systems, like type VI, are present in several copies in its genome (Bleves et al., 2010). The QS influence on the expression of these secretion systems is as of yet unknown, with the exceptions of the TTSS and the second type VI secretion system that are negatively regulated by QS (Sana et al., 2012).

More striking is the fact that QS interference using azithromycin selects the wild-type virulent phenotype against the less virulent lasR mutants in intubated patients colonized by $P$. aeruginosa (Kohler et al., 2010). This surprising fact is understandable since lasR mutants often appear and are selected in infections since they act as phenotypic cheaters that utilize the public goods such as exoproteases and siderophores produced by the cooperative wild-type individuals (Diggle et al., 2007; Sandoz et al., 2007). Hence, inhibiting QS removes the advantage of the lasR mutants and thereby selects the wild-type, thus a detrimental effect of QS interference in the long run could be to increase the prevalence of virulent genotypes in the nosocomial environment (Kohler et al., 2010).

Another key aspect of current QS inhibitors like furanones is that, depending on their concentration, they can activate rather than inhibit QS (Martinelli et al., 2004). Furthermore, related QS inhibitors such as synthetic HSLs can activate rather than inhibit some virulence factors (Welsh et al., 2015).

\section{LIMITATIONS OF THE CURRENT ANIMAL INFECTION MODELS FOR THE STUDY OF QUORUM SENSING INTERFERENCE}

To date, several animal infection models have demonstrated that QS deficient mutants are much less virulent than their QS proficient parental strains; accordingly, QS interference promotes an increase in host survival and a decrease in damage and bacterial counts (Castillo-Juárez et al., 2015). The infection models used include arthropods like Galleria mellonela, nematodes such as Caenorhabditis elegans, fruit fly, and zebrafish which are valuable and informative but that do not accurately reflect human physiology. Moreover, when mice are used as a model, immune competent individuals are evaluated; hence, the fact that $P$. aeruginosa is a strict opportunistic pathogen that does not attack individuals with competent immune systems is overlooked, and so it is not clear if the immune systems of immunosuppressed patients will be able to clear the bacteria from infections upon QS interference treatments.

In addition to attacking immunosupressed individuals, $P$. aeruginosa is the major cause of death of CF patients; accordingly, there are several mice models that incorporate 
different mutations in the CF transmembrane conductance regulator (CTFR) protein and that are able to mimic some of the characteristics of the disease in humans. However, CF is a very complex disease and there are more than one thousand reported mutations in the cftr gene associated with the disease; hence, developing a murine model that closely resembles the human disease is challenging (Guilbault et al., 2007).

Most of the current models are suitable to study acute infections and only a few like the one developed in 2005 by Hoffmann and coworkers are optimized to simulate chronic CF infections (Hoffmann et al., 2005). Importantly, this model has been used to demonstrate that QS interference with azithromycin inhibits alginate production of the mucoid NH57388A strain in vivo, attenuating the damage produced to the host, but unfortunately it was not able to significantly increase mouse survival (Hoffmann et al., 2007).

Clinical studies have demonstrated that AZM treatment improves lung function in CF patients (Saiman et al., 2003); however, besides the QSI effect, azithromycin has bactericidal and anti-inflammatory effects; hence, the improvement of both mice and patients is likely due a combination of these effects rather than an exclusive consequence of the QSI properties of azithromycin. Therefore, testing of the effect of more specific QS interference molecules in these kind of models is needed in order to elucidate the potential of these therapies for CF patients.

Another kind of mouse model that more closely resembles the situation observed in humans is the thermally-induced injury model, which consists of producing a burn of second or third degree on the dorsal side of the mouse using hot water and subsequent inoculation of the burn. The utilization of this model has confirmed that QS-deficient mutants such as lasR, lasI, rhlI, lasI rhlI, and pqsA (Rumbaugh et al., 1999a,b; Lesic et al., 2007), have less virulence than the parental strains. Moreover, it has been used to test the effect of the halogenated anthranilic acid analogs 6FABA, 6CABA, and 4CABA which are inhibitors of the synthesis of quinolone QS signals. These compounds, at the doses administrated, decrease mouse mortality significantly. Nevertheless, they were tested at a single dose (Lesic et al., 2007), so it is unknown if they exert dose response effects. This is not trivial since as mentioned before, QS inhibitors may act as QS activators depending on the used doses (Martinelli et al., 2004).

\section{INSENSIBILITY OF SOME CLINICAL STRAINS TO CURRENT QUORUM QUENCHERS}

Before 2010 it was assumed than QS interference will be impervious or at least less susceptible to promote bacterial resistance than conventional antibiotic therapies (Defoirdt et al., 2010). In 2011, it was demonstrated that $P$. aeruginosa acquires resistance easily against the canonical HSL-dependant quorum quencher furanone C-30, by activating the multidrug efflux pump MexAB-OprM (Maeda et al., 2012). The activation of this pump is mediated by mutations disrupting the transcriptional repressors MexR and NalC, and such mutations are common in clinical isolates, presumably since they are selected by intense antibiotic treatments (Tomas et al., 2010). As expected, these clinical isolates are resistant against C-30 (Maeda et al., 2012).

Recent studies have demonstrated that C-30 resistance is common in multidrug resistant strains. Importantly, resistance against other QQ compounds like 5-fluorouracil is also present in some clinical isolates and some antibiotic sensitive strains are also resistant to $\mathrm{C}-30$ by a decrease in the compound uptake. More strikingly, some of these strains produce higher amounts of the virulence factors in the presence of the furanone than in basal conditions (García-Contreras et al., 2013a,b; Garcia-Contreras et al., 2015b).

This suggests that if eventually QSI therapies are implemented in the clinic, they likely would not be effective against all the strains present in infections (García-Contreras et al., 2013a; Kalia et al., 2013), and that crucially they may fail to inhibit those strains with active antibiotic efflux pumps. Indeed, it was recently proposed that bacteria resistant to several QS inhibitors may be selected by QSI treatments (Koul et al., 2016).

\section{PERSPECTIVES}

Since recent evidence has demonstrated that QS is linked to basal metabolism and growth, it will be important to evaluate QS interference and its effect in bacteria growing in medium that more closely simulates the composition of the environment during infection (Palmer et al., 2007; GarciaContreras et al., 2015b), with attention on key metabolites that influence virulence and QS such as iron (Sokol and Woods, 1984; Mittal et al., 2008; Hazan et al., 2010), calcium (Sarkisova et al., 2005), phosphate (Zaborin et al., 2009), and adenosine (Patel et al., 2007; Sheng et al., 2012). Further, metabolomic and proteomic studies may shed light about the role of QS in bacterial physiology under in vivo-like conditions. In addition, the utilization of new technologies like microfluidics such as cell culture chips (organ-on-a-chip) would enable the interrogation of the roles of signaling, social cheating, mass transfer, and spatial organization in well-defined geometries, which is virtually impossible to asses at this moment using animal models.

Importantly, additional research about the ways $P$. aeruginosa may achieve resistance against QQ is needed in order to generate effective combination therapies less prone to the selection of resistance. One possibility is to exploit the fact that QS interference renders $P$. aeruginosa more sensitive to stress, including antibiotics and the effects of the immune system; hence, it is attractive to test if the combination of QS interference, antibiotic therapies and immunotherapy to improve the outcome of the therapies.

Another area of research that should be encouraged is the role of QS independent virulence factors and those negatively regulated by QS such as TTSS in infections and the effect of QS interference on these virulence determinants. Also, study of QS networks and the-virulence of environmental and clinical strains is needed since current evidence indicates these are highly variable (Grosso-Becerra et al., 2014; Garcia-Contreras et al., 
2015b) and that a specific QQ compound will not always be effective against all clinical strains. Also, more effort should be devoted to the study of the implementation of these therapies, since most of them are able to attenuate infections when the QQ compound is administrated shortly before the inoculation (having possible prophylactic effects), but the value of such therapies as a possible cure for an established infection should be better addressed.

Taking the available evidence together, it seems QS interference may be a valuable tool to combat $P$. aeruginosa infections; however, using QS inhibition as the sole therapy may not be an efficient strategy due several potential drawbacks. Hence, before QSI applications are used in the clinic, it is advisable to improve our current understanding of $P$. aeruginosa virulence with an emphasis on clinical strains and also on further defining the roles of QS in the physiology of this remarkable complex pathogen.

\section{REFERENCES}

Antunes, L. C., Ferreira, R. B., Buckner, M. M., and Finlay, B. B. (2010). Quorum sensing in bacterial virulence. Microbiology 156, 2271-2282. doi: 10.1099/mic.0.038794-0

Bhargava, N., Sharma, P., and Capalash, N. (2010). Quorum sensing in Acinetobacter: an emerging pathogen. Crit. Rev. Microbiol. 36, 349-360. doi: 10.3109/1040841X.2010.512269

Bhargava, N., Sharma, P., and Capalash, N. (2014). Pyocyanin stimulates quorum sensing-mediated tolerance to oxidative stress and increases persister cell populations in Acinetobacter baumannii. Infect. Immun. 82, 3417-3425. doi: 10.1128/IAI.01600-14

Bjarnsholt, T., Jensen, P. Ø., Burmølle, M., Hentzer, M., Haagensen, J. A., Hougen, H. P., et al. (2005). Pseudomonas aeruginosa tolerance to tobramycin, hydrogen peroxide and polymorphonuclear leukocytes is quorum-sensing dependent. Microbiology 151, 373-383. doi: 10.1099/mic.0.27463-0

Bleves, S., Soscia, C., Nogueira-Orlandi, P., Lazdunski, A., and Filloux, A. (2005). Quorum sensing negatively controls type III secretion regulon expression in Pseudomonas aeruginosa PAO1. J. Bacteriol. 187, 3898-3902. doi: 10.1128/JB.187.11.3898-3902.2005

Bleves, S., Viarre, V., Salacha, R., Michel, G. P., Filloux, A., and Voulhoux, R. (2010). Protein secretion systems in Pseudomonas aeruginosa: a wealth of pathogenic weapons. Int. J. Med. Microbiol. 300, 534-543. doi: 10.1016/j.ijmm. 2010.08.005

Cabeen, M. T. (2014). Stationary phase-specific virulence factor overproduction by a lasR mutant of Pseudomonas aeruginosa. PLoS ONE 9:e88743. doi: 10.1371/ journal.pone.0088743

Castillo-Juárez, I., Maeda, T., Mandujano-Tinoco, E. A., Tomas, M., Párez-Eretza, B., Garcia-Contreras, S. J., et al. (2015). Role of quorum sensing in bacterial infections. World J. Clin. Cases 3, 575-598. doi: 10.12998/wjcc.v3.i7.575

Choi, J., Shin, D., and Ryu, S. (2007). Implication of quorum sensing in Salmonella enterica serovar typhimurium virulence: the luxS gene is necessary for expression of genes in pathogenicity island 1. Infect. Immun. 75, 4885-4890. doi: 10.1128/IAI.01942-06

Christensen, L. D., Moser, C., Jensen, P. Ø., Rasmussen, T. B., Christophersen, L., Kjelleberg, S., et al. (2007). Impact of Pseudomonas aeruginosa quorum sensing on biofilm persistence in an in vivo intraperitoneal foreign-body infection model. Microbiology 153, 2312-2320. doi: 10.1099/mic.0.2007/006122-0

Davenport, P. W., Griffin, J. L., and Welch, M. (2015). Quorum sensing is accompanied by global metabolic changes in the opportunistic human pathogen Pseudomonas aeruginosa. J. Bacteriol. 197, 2072-2082. doi: 10.1128/ JB.02557-14

Defoirdt, T., Boon, N., and Bossier, P. (2010). Can bacteria evolve resistance to quorum sensing disruption? PLoS Pathog. 6:e1000989. doi: 10.1371/journal. ppat.1000989

\section{AUTHOR CONTRIBUTIONS}

The author confirms being the sole contributor of this work and approved it for publication.

\section{FUNDING}

This work was supported by grants from SEP/CONACyT-México No. 152794, CONACYT Problemas Nacionales 2015 No. 201501-402 and PAPIIT-UNAM No. IA201116.

\section{ACKNOWLEDGMENTS}

I am grateful for critical reading of the manuscript and the valuable suggestions provided by Professor Gloria SoberónChávez from the Biomedical Science Institute at UNAM, Mexico and by Professor Thomas K. Wood from Penn State University.

Dekimpe, V., and Deziel, E. (2009). Revisiting the quorum-sensing hierarchy in Pseudomonas aeruginosa: the transcriptional regulator RhlR regulates LasRspecific factors. Microbiology 155, 712-723. doi: 10.1099/mic.0.022764-0

de Oca-Mejia, M. M., Castillo-Juarez, I., Martínez-Vázquez, M., Soto-Hernandez, M., and García-Contreras, R. (2015). Influence of quorum sensing in multiple phenotypes of the bacterial pathogen Chromobacterium violaceum. Pathog. Dis. 73, 1-4. doi: 10.1093/femspd/ftu019

Diggle, S. P., Griffin, A. S., Campbell, G. S., and West, S. A. (2007). Cooperation and conflict in quorum-sensing bacterial populations. Nature 450, 411-414. doi: 10.1038/nature06279

Fenner, L., Richet, H., Raoult, D., Papazian, L., Martin, C., and La Scola, B. (2006) Are clinical isolates of Pseudomonas aeruginosa more virulent than hospital environmental isolates in amebal co-culture test? Crit. Care Med. 34, 823-828. doi: 10.1097/01.CCM.0000201878.51343.F1

García-Contreras, R., Maeda, T., and Wood, T. K. (2013a). Resistance to quorumquenching compounds. Appl. Environ. Microbiol. 79, 6840-6846. doi: 10.1128/ AEM.02378-13

García-Contreras, R., Maeda, T., and Wood, T. K. (2016). Can resistance against quorum-sensing interference be selected? ISME J. 10, 4-10. doi: 10.1038/ismej. 2015.84

García-Contreras, R., Martinez-Vazquez, M., Velazquez Guadarrama, N., Villegas Paneda, A. G., Hashimoto, T., Maeda, T., et al. (2013b). Resistance to the quorum-quenching compounds brominated furanone C-30 and 5-fluorouracil in Pseudomonas aeruginosa clinical isolates. Pathog. Dis. 68, 8-11. doi: 10.1111/ 2049-632X.12039

García-Contreras, R., Nunez-López, L., Jasso-Chávez, R., Kwan, B. W., Belmont, J. A., Rangel-Vega, A., et al. (2015a). Quorum sensing enhancement of the stress response promotes resistance to quorum quenching and prevents social cheating. ISME J. 9, 115-125. doi: 10.1038/ismej.2014.98

Garcia-Contreras, R., Perez-Eretza, B., Jasso-Chavez, R., Lira-Silva, E., RoldanSanchez, J. A., Gonzalez-Valdez, A., et al. (2015b). High variability in quorum quenching and growth inhibition by furanone C-30 in Pseudomonas aeruginosa clinical isolates from cystic fibrosis patients. Pathog. Dis. 73:ftv040. doi: 10.1093/femspd/ftv040

Grosso-Becerra, M. V., Santos-Medellin, C., Gonzalez-Valdez, A., Mendez, J. L., Delgado, G., Morales-Espinosa, R., et al. (2014). Pseudomonas aeruginosa clinical and environmental isolates constitute a single population with high phenotypic diversity. BMC Genomics 15:318. doi: 10.1186/1471-216415-318

Guilbault, C., Saeed, Z., Downey, G. P., and Radzioch, D. (2007). Cystic fibrosis mouse models. Am. J. Respir. Cell Mol. Biol. 36, 1-7. doi: 10.1165/rcmb.20060184TR

Hassett, D. J., Ma, J. F., Elkins, J. G., McDermott, T. R., Ochsner, U. A., West, S. E., et al. (1999). Quorum sensing in Pseudomonas aeruginosa controls expression of catalase and superoxide dismutase genes and mediates biofilm susceptibility 
to hydrogen peroxide. Mol. Microbiol. 34, 1082-1093. doi: 10.1046/j.13652958.1999.01672.x

Hauser, A. R. (2009). The type III secretion system of Pseudomonas aeruginosa: infection by injection. Nat. Rev. Microbiol. 7, 654-665. doi: 10.1038/nrmicro 2199

Hazan, R., He, J., Xiao, G., Dekimpe, V., Apidianakis, Y., Lesic, B., et al. (2010). Homeostatic interplay between bacterial cell-cell signaling and iron in virulence. PLoS Pathog. 6:e1000810. doi: 10.1371/journal.ppat.1000810

Hentzer, M., Wu, H., Andersen, J. B., Riedel, K., Rasmussen, T. B., Bagge, N., et al. (2003). Attenuation of Pseudomonas aeruginosa virulence by quorum sensing inhibitors. EMBO J. 22, 3803-3815. doi: 10.1093/emboj/cdg366

Heurlier, K., Dénervaud, V., Haenni, M., Guy, L., Krishnapillai, V., and Haas, D. (2005). Quorum-sensing-negative (lasR) mutants of Pseudomonas aeruginosa avoid cell lysis and death. J. Bacteriol. 187, 4875-4883. doi: 10.1128/JB.187.14.4875-4883.2005

Hoffmann, N., Lee, B., Hentzer, M., Rasmussen, T. B., Song, Z., Johansen, H. K., et al. (2007). Azithromycin blocks quorum sensing and alginate polymer formation and increases the sensitivity to serum and stationary-growth-phase killing of Pseudomonas aeruginosa and attenuates chronic P. aeruginosa lung infection in Cftr(-/-) mice. Antimicrob. Agents Chemother. 51, 3677-3687. doi: 10.1128/AAC.01011-06

Hoffmann, N., Rasmussen, T. B., Jensen, P. Ø., Stub, C., Hentzer, M., Molin, S., et al. (2005). Novel mouse model of chronic Pseudomonas aeruginosa lung infection mimicking cystic fibrosis. Infect. Immun. 73, 2504-2514. doi: 10.1128/IAI.73.4.2504-2514.2005

Jakobsen, T. H., van Gennip, M., Phipps, R. K., Shanmugham, M. S., Christensen, L. D., Alhede, M., et al. (2011). Ajoene, a sulfur-rich molecule from garlic, inhibits genes controlled by quorum sensing. Antimicrob. Agents Chemother. 56, 2314-2325. doi: 10.1128/AAC.05919-11

Jimenez, P. N., Koch, G., Thompson, J. A., Xavier, K. B., Cool, R. H., and Quax, W. J. (2012). The multiple signaling systems regulating virulence in Pseudomonas aeruginosa. Microbiol. Mol. Biol. Rev. 76, 46-65. doi: 10.1128/MMBR.05007-11

Kalia, V. C., Wood, T. K., and Kumar, P. (2013). Evolution of resistance to quorumsensing inhibitors. Microb. Ecol. 68, 13-23. doi: 10.1007/s00248-013-0316-y

Kohler, T., Perron, G. G., Buckling, A., and van Delden, C. (2010). Quorum sensing inhibition selects for virulence and cooperation in Pseudomonas aeruginosa. PLoS Pathog. 6:e1000883. doi: 10.1371/journal.ppat.1000883

Kong, W., Liang, H., Shen, L., and Duan, K. (2009). [Regulation of type III secretion system by Rhl and PQS quorum sensing systems in Pseudomonas aeruginosa]. Wei Sheng Wu Xue Bao 49, 1158-1164. Available online at: http://journals.im. ac.cn/actamicrocn/ch/reader/view_abstract.aspx?file_no=909-5

Koul, S., Prakash, J., Mishra, A., and Kalia, V. C. (2016). Potential emergence of multi-quorum sensing inhibitor resistant (MQSIR) bacteria. Indian J. Microbiol. 56, 1-18. doi: 10.1007/s12088-015-0558-0

Laarman, A. J., Bardoel, B. W., Ruyken, M., Fernie, J., Milder, F. J., van Strijp, J. A., et al. (2013). Pseudomonas aeruginosa alkaline protease blocks complement activation via the classical and lectin pathways. J. Immunol. 188, 386-393. doi: 10.4049/jimmunol.1102162

Lee, D. G., Urbach, J. M., Wu, G., Liberati, N. T., Feinbaum, R. L., Miyata, S., et al. (2006). Genomic analysis reveals that Pseudomonas aeruginosa virulence is combinatorial. Genome Biol. 7:R90. doi: 10.1186/gb-2006-7-10-r90

Lee, J., and Zhang, L. (2015). The hierarchy quorum sensing network in Pseudomonas aeruginosa. Protein Cell 6, 26-41. doi: 10.1007/s13238-0140100-x

Lesic, B., Lépine, F., Déziel, E., Zhang, J., Zhang, Q., Padfield, K., et al. (2007). Inhibitors of pathogen intercellular signals as selective anti-infective compounds. PLoS Pathog. 3, 1229-1239. doi: 10.1371/journal.ppat.0030126

Maeda, T., García-Contreras, R., Pu, M., Sheng, L., Garcia, L. R., Tomás, M., et al. (2012). Quorum quenching quandary: resistance to antivirulence compounds. ISME J. 6, 493-501. doi: 10.1038/ismej.2011.122

Martinelli, D., Grossmann, G., Sequin, U., Brandl, H., and Bachofen, R. (2004). Effects of natural and chemically synthesized furanones on quorum sensing in Chromobacterium violaceum. BMC Microbiol. 4:25. doi: 10.1186/14712180-4-25

Mittal, R., Sharma, S., Chhibber, S., and Harjai, K. (2008). Iron dictates the virulence of Pseudomonas aeruginosa in urinary tract infections. J. Biomed. Sci. 15, 731-741. doi: 10.1007/s11373-008-9274-7
O’Loughlin, C. T., Miller, L. C., Siryaporn, A., Drescher, K., Semmelhack, M. F., and Bassler, B. L. (2013). A quorum-sensing inhibitor blocks Pseudomonas aeruginosa virulence and biofilm formation. Proc. Natl. Acad. Sci. U.S.A. 110, 17981-17986. doi: 10.1073/pnas.1316981110

Palmer, K. L., Aye, L. M., and Whiteley, M. (2007). Nutritional cues control Pseudomonas aeruginosa multicellular behavior in cystic fibrosis sputum. J. Bacteriol. 189, 8079-8087. doi: 10.1128/JB.01138-07

Patel, N. J., Zaborina, O., Wu, L., Wang, Y., Wolfgeher, D. J., Valuckaite, V., et al. (2007). Recognition of intestinal epithelial HIF-1alpha activation by Pseudomonas aeruginosa. Am. J. Physiol. Gastrointest. Liver Physiol. 292, G134G142. doi: 10.1152/ajpgi.00276.2006

Poole, K. (2011). Pseudomonas aeruginosa: resistance to the max. Front. Microbiol. 2:65. doi: 10.3389/fmicb.2011.00065

Rumbaugh, K. P., Griswold, J. A., and Hamood, A. N. (1999a). Contribution of the regulatory gene las $R$ to the pathogenesis of infection of burned mice. J. Burn Care Rehabil. 20, 42-49. doi: 10.1097/00004630-199901001-00008

Rumbaugh, K. P., Griswold, J. A., Iglewski, B. H., and Hamood, A. N. (1999b). Contribution of quorum sensing to the virulence of Pseudomonas aeruginosa in burn wound infections. Infect. Immun. 67, 5854-5862.

Saiman, L., Marshall, B. C., Mayer-Hamblett, N., Burns, J. L., Quittner, A. L., Cibene, D. A., et al. (2003). Azithromycin in patients with cystic fibrosis chronically infected with Pseudomonas aeruginosa: a randomized controlled trial. JAMA 290, 1749-1756. doi: 10.1001/jama.290.13.1749

Sana, T. G., Hachani, A., Bucior, I., Soscia, C., Garvis, S., Termine, E., et al. (2012). The second type VI secretion system of Pseudomonas aeruginosa strain PAO1 is regulated by quorum sensing and Fur and modulates internalization in epithelial cells. J. Biol. Chem. 287, 27095-27105. doi: 10.1074/jbc.M112.376368

Sandoz, K. M., Mitzimberg, S. M., and Schuster, M. (2007). Social cheating in Pseudomonas aeruginosa quorum sensing. Proc. Natl. Acad. Sci. U.S.A. 104, 15876-15881. doi: 10.1073/pnas.0705653104

Sarkisova, S., Patrauchan, M. A., Berglund, D., Nivens, D. E., and Franklin, M. J. (2005). Calcium-induced virulence factors associated with the extracellular matrix of mucoid Pseudomonas aeruginosa biofilms. J. Bacteriol. 187, 4327-4337. doi: 10.1128/JB.187.13.4327-4337.2005

Schuster, M., Lostroh, C. P., Ogi, T., and Greenberg, E. P. (2003). Identification, timing, and signal specificity of Pseudomonas aeruginosa quorumcontrolled genes: a transcriptome analysis. J. Bacteriol. 185, 2066-2079. doi: 10.1128/JB.185.7.2066-2079.2003

Sheng, L., Pu, M., Hegde, M., Zhang, Y., Jayaraman, A., and Wood, T. K. (2012). Interkingdom adenosine signal reduces Pseudomonas aeruginosa pathogenicity. Microb. Biotechnol. 5, 560-572. doi: 10.1111/j.1751-7915. 2012.00338.x

Smith, R. S., and Iglewski, B. H. (2003). Pseudomonas aeruginosa quorum sensing as a potential antimicrobial target. J. Clin. Invest. 112, 1460-1465. doi: 10.1172/JCI200320364

Sokol, P. A., and Woods, D. E. (1984). Relationship of iron and extracellular virulence factors to Pseudomonas aeruginosa lung infections. J. Med. Microbiol. 18, 125-133. doi: 10.1099/00222615-18-1-125

Sperandio, V., Li, C. C., and Kaper, J. B. (2002). Quorum-sensing Escherichia coli regulator A: a regulator of the LysR family involved in the regulation of the locus of enterocyte effacement pathogenicity island in enterohemorrhagic $E$. coli. Infect. Immun. 70, 3085-3093. doi: 10.1128/IAI.70.6.3085-3093.2002

Stover, C. K., Pham, X. Q., Erwin, A. L., Mizoguchi, S. D., Warrener, P., Hickey, M. J., et al. (2000). Complete genome sequence of Pseudomonas aeruginosa PAO1, an opportunistic pathogen. Nature 406, 959-964. doi: 10.1038/35023079

Tomás, M., Doumith, M., Warner, M., Turton, J. F., Beceiro, A., Bou, G., et al. (2010). Efflux pumps, OprD porin, AmpC beta-lactamase, and multiresistance in Pseudomonas aeruginosa isolates from cystic fibrosis patients. Antimicrob. Agents Chemother. 54, 2219-2224. doi: 10.1128/AAC.00816-09

Wagner, V. E., Bushnell, D., Passador, L., Brooks, A. I., and Iglewski, B. H. (2003). Microarray analysis of Pseudomonas aeruginosa quorum-sensing regulons: effects of growth phase and environment. J. Bacteriol. 185, 2080-2095. doi: 10.1128/JB.185.7.2080-2095.2003

Welsh, M. A., Eibergen, N. R., Moore, J. D., and Blackwell, H. E. (2015). Small molecule disruption of quorum sensing cross-regulation in Pseudomonas aeruginosa causes major and unexpected alterations to virulence phenotypes. J. Am. Chem. Soc. 137, 1510-1519. doi: 10.1021/ja5110798 
Wretlind, B., and Pavlovskis, O. R. (1983). Pseudomonas aeruginosa elastase and its role in pseudomonas infections. Rev. Infect. Dis. 5(Suppl. 5), S998-S1004. doi: 10.1093/clinids/5.supplement_5.s998

Wu, H., Song, Z., Hentzer, M., Andersen, J. B., Molin, S., Givskov, M., et al. (2004). Synthetic furanones inhibit quorum-sensing and enhance bacterial clearance in Pseudomonas aeruginosa lung infection in mice. J. Antimicrob. Chemother. 53, 1054-1061. doi: 10.1093/jac/ dkh223

Yang, Q., and Defoirdt, T. (2015). Quorum sensing positively regulates flagellar motility in pathogenic Vibrio harveyi. Environ. Microbiol. 17, 960-968. doi: 10.1111/1462-2920.12420

Zaborin, A., Romanowski, K., Gerdes, S., Holbrook, C., Lepine, F., Long, J., et al. (2009). Red death in Caenorhabditis elegans caused by Pseudomonas aeruginosa PAO1. Proc. Natl. Acad. Sci. U.S.A. 106, 6327-6332. doi: 10.1073/ pnas.0813199106
Zhu, J., Miller, M. B., Vance, R. E., Dziejman, M., Bassler, B. L., and Mekalanos, J. J. (2002). Quorum-sensing regulators control virulence gene expression in Vibrio cholerae. Proc. Natl. Acad. Sci. U.S.A. 99, 3129-3134. doi: 10.1073/pnas.052694299

Conflict of Interest Statement: The author declares that the research was conducted in the absence of any commercial or financial relationships that could be construed as a potential conflict of interest.

Copyright (c) 2016 García-Contreras. This is an open-access article distributed under the terms of the Creative Commons Attribution License (CC BY). The use, distribution or reproduction in other forums is permitted, provided the original author(s) or licensor are credited and that the original publication in this journal is cited, in accordance with accepted academic practice. No use, distribution or reproduction is permitted which does not comply with these terms. 


\section{OPEN ACCESS}

Edited by: Etienne Giraud, Institut National de la Recherche Agronomique, France

Reviewed by:

Francesco Imperi, Sapienza University of Rome, Italy Yuji Morita,

Aichi Gakuin University, Japan

*Correspondence: Weihui Wu wuweihui@nankai.edu.cn: Shouguang Jin sjin@ufl.edu

Specialty section: This article was submitted to Antimicrobials, Resistance and Chemotherapy,

a section of the journal

Frontiers in Microbiology

Received: 21 December 2015 Accepted: 29 February 2016 Published: 16 March 2016

Citation:

Tan H, Zhang L, Weng Y, Chen R,

Zhu F, Jin Y, Cheng Z, Jin S and Wu W (2016) PA3297 Counteracts Antimicrobial Effects of Azithromycin in Pseudomonas aeruginosa. Front. Microbiol. 7:317. doi: 10.3389/fmicb.2016.00317

\section{PA3297 Counteracts Antimicrobial Effects of Azithromycin in Pseudomonas aeruginosa}

\author{
Hao Tan', Lu Zhang', Yuding Weng', Ronghao Chen', Feng Zhu'1, Yongxin Jin', \\ Zhihui Cheng ${ }^{1}$, Shouguang Jin ${ }^{1,2 *}$ and Weihui Wu' ${ }^{*}$
}

\footnotetext{
${ }^{1}$ State Key Laboratory of Medicinal Chemical Biology, Key Laboratory of Molecular Microbiology and Technology of the Ministry of Education, Department of Microbiology, College of Life Sciences, Nankai University, Tianjin, China, ${ }^{2}$ Department of Molecular Genetics and Microbiology, College of Medicine, University of Florida, Gainesville, FL, USA
}

Pseudomonas aeruginosa causes acute and chronic infections in human. Its increasing resistance to antibiotics requires alternative treatments that are more effective than available strategies. Among the alternatives is the unconventional usage of conventional antibiotics, of which the macrolide antibiotic azithromycin (AZM) provides a paradigmatic example. AZM therapy is associated with a small but consistent improvement in respiratory function of cystic fibrosis patients suffering from chronic $P$. aeruginosa infection. Besides immunomodulating activities, AZM represses bacterial genes involved in virulence, quorum sensing, biofilm formation, and motility, all of which are due to stalling of ribosome and depletion of cellular tRNA pool. However, how P. aeruginosa responds to and counteracts the effects of AZM remain elusive. Here, we found that deficiency of PA3297, a gene encoding a DEAH-box helicase, intensified AZM-mediated bacterial killing, suppression of pyocyanin production and swarming motility, and hypersusceptibility to hydrogen peroxide. We demonstrated that expression of PA3297 is induced by the interaction between AZM and ribosome. Importantly, mutation of PA3297 resulted in elevated levels of unprocessed 23S-5S rRNA in the presence of AZM, which might lead to increased susceptibility to AZM-mediated effects. Our results revealed one of the bacterial responses in counteracting the detrimental effects of AZM.

Keywords: RNA helicase, antibiotic resistance, azithromycin, rRNA processing, Pseudomonas aeruginosa

\section{INTRODUCTION}

Pseudomonas aeruginosa is a versatile Gram-negative pathogenic bacterium, that can cause various infections in human (de Bentzmann and Plésiat, 2011; Campa et al., 2012). During infection, P. aeruginosa produces multiple virulence factors to facilitate colonization (Sadikot et al., 2005; Kipnis et al., 2006; Hauser, 2009; Liu et al., 2015). Meanwhile, its highly intrinsic antibiotic resistance and biofilm forming ability greatly hinder the eradication of this pathogen (Høiby et al., 2005). In patients suffering from cystic fibrosis (CF) and chronic obstructive pulmonary disease (COPD), $P$. aeruginosa caused chronic respiratory infections are responsible for most of the morbidity and mortality (Rabin et al., 2004; Rada and Leto, 2013). Intensive antibiotic treatment has been used to maintain the lung function and extend lifespan of the patients (Doring et al., 2000). However, the increasing antibiotic resistance has been compromising clinical efficacy of traditional antibiotics. Thus, alternatives or unconventional usage of the antibiotics are urgently needed (Breidenstein et al., 2011; Poole, 2011; Imperi et al., 2014). 
The macrolide antibiotic azithromycin (AZM) provides a paradigmatic example of an unconventional antibacterial drug for $P$. aeruginosa treatment. Although, $P$. aeruginosa is highly resistance to macrolides owning to its low outer membrane permeability and the resistance-nodulation-cell division (RND) systems, AZM treatment benefits patients suffering from both intermittent and chronic $P$. aeruginosa infections (Saiman et al., 2003; Lister et al., 2009; Blasi et al., 2010; Steel et al., 2012; Aminov, 2013; Morita et al., 2013). AZM has been shown to have immunomodulatory activity, which attenuates the inflammatory response and promotes macrophage phagocytic activity (Legssyer et al., 2006; Steinkamp et al., 2008; Tsai et al., 2009). Furthermore, AZM exhibits bactericidal effect on stationary growth phase P. aeruginosa cells (Lovmar et al., 2004, 2009; Imamura et al., 2005; Köhler et al., 2007; Starosta et al., 2010; Gödeke et al., 2013). And sub-inhibitory concentrations of AZM suppress biofilm formation, motility, and production of multiple virulence factors, including proteases, pyocyanin, exotoxin $\mathrm{A}$, phospholipase $\mathrm{C}$ (PLC), exopolysaccharides, and other quorum-sensing (QS) regulated genes in $P$. aeruginosa (Molinari et al., 1992, 1993; Tateda et al., 2001; Favre-Bonté et al., 2003; Gillis and Iglewski, 2004). The AZM-mediated killing of stationary-phase bacterial cells and reduced expression of QS-regulated virulence factors require interaction between AZM and ribosome (Köhler et al., 2007). AZM binds in the nascent peptide exit tunnel (NPET), resulting in ribosome stalling and depletion of the intracellular pools of aminoacyl-tRNAs (Lovmar et al., 2004, 2009; Köhler et al., 2007; Starosta et al., 2010; Gödeke et al., 2013). The effects of AZM on $P$. aeruginosa can be counteracted by over expression of ErmBP or a peptidyl-tRNA hydrolase, which blocks the interaction between AZM and ribosome by modifying the $23 \mathrm{~S}$ rRNA or increases the intracellular aminoacyl-tRNA level, respectively (Köhler et al., 2007; Gödeke et al., 2013). However, how P. aeruginosa response to AZM treatment remains unclear. Understanding the mechanisms that $P$. aeruginosa uses to counteract AZM treatment may provide clues to enhance AZM-mediated virulence inhibitory and bacterial killing effects.

A large RNA helicase family named $\mathrm{DExD} / \mathrm{H}$ box helicases are characterized by a conserved $\mathrm{DExD} / \mathrm{H}$ box sequence (Cordin et al., 2006; Linder and Jankowsky, 2011), and play crucial roles in rRNA processing, translation initiation, and mRNA decay (Iost et al., 2013; Linder and Fuller-Pace, 2013). In addition, the $\mathrm{DExD} / \mathrm{H}$ box helicases have been shown to participate in bacterial responses to various stresses, such as cold shock, $\mathrm{pH}$, osmotic, and oxidative stresses (Owttrim, 2013). And several DEAD family RNA helicases, which belong to a specific subfamily of $\mathrm{DExD} / \mathrm{H}$ box helicases, have been shown to regulate virulence factors in Escherichia coli, Borrelia burgdorferi, Staphylococcus aureus, Listeria monocytogenes, and P. aeruginosa (Koo et al., 2004; Salman-Dilgimen et al., 2011, 2013; Oun et al., 2013; Bareclev et al., 2014; Intile et al., 2015). The pleiotropic functions of $\mathrm{DExD} / \mathrm{H}$ box family RNA helicases intrigued us to suspect that they might be involved in the bacterial response to AZM treatment. In this study, we found that deficiency in a DEAH box helicase, PA3297, renders $P$. aeruginosa more susceptible to the killing and virulence suppression by AZM. Our results suggest that the expression of PA3297 was up regulated in the presence of AZM, which might promote $23 \mathrm{~S}$ rRNA maturation to counteract the inhibitory effect of AZM on protein elongation.

\section{MATERIALS AND METHODS}

\section{Strains and Plasmids}

The bacterial strains and plasmids used in this study are listed in Table 1 (Simon et al., 1983; Hoang et al., 1998; Choi and Schweizer, 2006; Liberati et al., 2006). The E. coli strains $\mathrm{DH} 5 \alpha, \mathrm{S} 17-1$ and $P$. aeruginosa strains were routinely cultured in Luria-Bertani (LB) broth at $37^{\circ} \mathrm{C}$. Antibiotics were used at the following concentrations: for $E$. coli, ampicillin $100 \mu \mathrm{g} / \mathrm{ml}$, tetracycline $10 \mu \mathrm{g} / \mathrm{ml}$, and gentamicin $10 \mu \mathrm{g} / \mathrm{ml}$; for $P$. aeruginosa, carbenicillin $150 \mu \mathrm{g} / \mathrm{ml}$, tetracycline $50 \mu \mathrm{g} / \mathrm{ml}$, and gentamicin $50 \mu \mathrm{g} / \mathrm{ml}$.

\section{DNA Methods}

DNA manipulations were performed according to standard protocols or following manufacturers' instructions (Hoang et al., 1998; Zheng et al., 2004; Choi and Schweizer, 2006). The pEX18Tc:: $\triangle$ PA3297 (pTH1501) was constructed by cloning the 1002-bp upstream and 964-bp downstream fragments of PA3297 coding region into the KpnI-HindIII sites of plasmid pEX18Tc. The fragments were amplified from the PA14 chromosome with primers PA14-UPA3297-FF, PA14-UPA3297-FR, PA14DPA3297-FF, and PA14-DPA3297-FR (Table 2), respectively. Deletion of the PA3297 gene was confirmed by PCR with primers PA14-PA3297-FF and PA14-PA3297-FR (Table 2). For the complementation of PA3297, the PA3297 gene was amplified from the PA14 chromosome by PCR with the primers PA14PA3297-FF and PA14-PA3297-FR (Table 2). The PCR product was ligated into the EcoRI- SacI sites of pUC18t-mini-Tn7T$\mathrm{Gm}$, resulting in pTH1502. The plasmid was introduced into the $\triangle$ PA3297 mutant by electroporation, along with the helper plasmid pTNS3 (Choi and Schweizer, 2006). Insertion of the PA3297 gene into the chromosome was confirmed by PCR with primers $\mathrm{P}_{\mathrm{Tn} 7 \mathrm{R}}$ and $\mathrm{P}_{\text {glms-down }}$ (Table 2; Choi and Schweizer, 2006). The site-directed mutagenesis was performed as previously described (Zheng et al., 2004). The mutation sites were chosen based on the conserved critical residues of other bacterial DExD-box proteins (Koo et al., 2004; Cordin et al., 2006). Briefly, PCR amplification was performed with pTH1502 as template and with primers listed in Table 2, for K101A, D192A, and SAT224-226AAA mutations, respectively. The PCR products were treated by $\mathrm{DpnI}$ for $3 \mathrm{~h}$ at $37^{\circ} \mathrm{C}$ and purified before transformation. The correctly mutated clones were identified by DNA sequencing.

\section{Assay for Pyocyanin Production}

The pyocyanin concentration was determined as described previously (Essar et al., 1990). Briefly, $1 \mathrm{ml}$ supernatant from each 24-h-old bacteria culture grown in the absence or presence of AZM was extracted with $0.5 \mathrm{ml}$ of chloroform. Then, $0.4 \mathrm{ml}$ solution from the lower organic phase was re-extracted into 
TABLE 1 | Bacterial strains and plasmids used in this study.

\begin{tabular}{|c|c|c|}
\hline Strain or plasmid & Description $^{a}$ & Reference or origin \\
\hline \multicolumn{3}{|l|}{ E. coli strains } \\
\hline $\mathrm{DH} 5 \alpha$ & $\begin{array}{l}\mathrm{F}^{-}, \varphi 80 \mathrm{~d} / \mathrm{ac} Z \Delta M 15, \Delta(\mathrm{lac} Z Y A-\arg F) \cup 169, \text { deoR, recA1, endA1, hsdR17 }\left(r_{\mathrm{k}}^{-}, m_{\mathrm{k}}{ }^{+}\right), \text {phoA, } \\
\text { supE44, } \lambda^{-} \text {, thi1, gyrA96, relA1 }\end{array}$ & TransGen \\
\hline S17-1 & thi pro hsdR recA Tra ${ }^{+}$ & Simon et al., 1983 \\
\hline \multicolumn{3}{|l|}{ P. aeruginosa strains } \\
\hline PA14 & Wild type Pseudomonas aeruginosa strain PA14 & Liberati et al., 2006 \\
\hline PA0426::Tn & PA14 with a transposon inserted at PA0426 & Liberati et al., 2006 \\
\hline PA0455::Tn & PA14 with a transposon inserted at PA0455 & Liberati et al., 2006 \\
\hline PA2840::Tn & PA14 with a transposon inserted at PA2840 & Liberati et al., 2006 \\
\hline PA3002::Tn & PA14 with a transposon inserted at PA3002 & Liberati et al., 2006 \\
\hline PA3272::Tn & PA14 with a transposon inserted at PA3272 & Liberati et al., 2006 \\
\hline PA3297::Tn & PA14 with a transposon inserted at PA3297 & Liberati et al., 2006 \\
\hline PA3308::Tn & PA14 with a transposon inserted at PA3308 & Liberati et al., 2006 \\
\hline PA3861::Tn & PA14 with a transposon inserted at PA3861 & Liberati et al., 2006 \\
\hline PA3950::Tn & PA14 with a transposon inserted at PA3950 & Liberati et al., 2006 \\
\hline$\Delta$ PA3297 & PA14 with PA3297 in frame deletion & This study \\
\hline$\triangle$ PA3297/att7::PA3297 & $\begin{array}{l}\text { PA14 } \triangle \text { PA3297 with insertion of a single copy of PA3297 driven by its own promoter at attTn7 } \\
\text { sites }\end{array}$ & This study \\
\hline APA3297/att7::PA3297 K101A & $\begin{array}{l}\text { PA14 } \triangle \text { PA3297 complemented with a single copy of PA3297 acquired a lysine101 mutation to } \\
\text { alanine }\end{array}$ & This study \\
\hline APA3297/att7::PA3297 D192A & $\begin{array}{l}\text { PA14 } \triangle \text { PA3297 complemented with a single copy of PA3297 acquired an aspartate192 } \\
\text { mutation to alanine }\end{array}$ & This study \\
\hline APA3297/att7::PA3297SAT224AAA & $\begin{array}{l}\text { PA14 } \triangle \text { PA3297 complemented with a single copy of PA3297 acquired a serine224 and } \\
\text { threonine226 mutation to alanines }\end{array}$ & This study \\
\hline \multicolumn{3}{|l|}{ Plasmids } \\
\hline pEX18Tc & Broad-host-range gene replacement vector; sacB TET ${ }^{r}$ & Hoang et al., 1998 \\
\hline pUC18t-mini-Tn7T-Gm & For gene insertion in chromosome; GEN ${ }^{r}$ & Choi and Schweizer, 2006 \\
\hline pTNS3 & Helper plasmid & Choi and Schweizer, 2006 \\
\hline pFLP2 & Source of Flp recombinase; sacB, AMPr/CAR ${ }^{r}$ & Hoang et al., 1998 \\
\hline pTH1501 & pEX18Tc:: $\triangle$ PA3297; TET ${ }^{r}$ & This study \\
\hline pTH1502 & PA3297 gene of PA14 on pUC18T-Mini-Tn7T-Gm with its own promoter; GEN ${ }^{r}$ & This study \\
\hline
\end{tabular}

a $A M P{ }^{r}$, ampicillin resistant; TET', tetracycline resistant; GEN', gentamicin resistance; $C A R^{r}$, carbenicillin resistant.

$0.3 \mathrm{ml}$ of $0.2 \mathrm{~N} \mathrm{HCl}$ to give a pink solution, whose absorbance was measured at $520 \mathrm{~nm}$. Concentrations of pyocyanin $(\mathrm{mg} / \mathrm{ml})$ were calculated by multiplying the $\mathrm{OD}_{520}$ by 32.01 (Kurachi, 1958).

\section{Antibiotic Susceptibility Assay}

Minimum inhibitory concentrations (MICs) of P. aeruginosa to antimicrobial agents were determined by serial twofold broth dilution in LB medium, as described previously (Jo et al., 2003). MICs were recorded as the lowest concentration of antibiotic inhibiting visible growth after $24 \mathrm{~h}$ of incubation at $37^{\circ} \mathrm{C}$.

\section{Stationary-Phase Bacterial Cell Killing Assay}

The killing assay was performed as described previously (Köhler et al., 2007). Briefly, bacteria were inoculated in LB medium and grown for $16 \mathrm{~h}$ at $37^{\circ} \mathrm{C}$. The culture of each strain was diluted to an $\mathrm{OD}_{600}$ of 0.05 and cultured at $37^{\circ} \mathrm{C}$. After reaching stationary phase $\left(\mathrm{OD}_{600}, 3.0\right)$, indicated concentrations of AZM were added to the $2-\mathrm{ml}$ aliquots of the cultures. Then the bacteria were cultured for $20-22 \mathrm{~h}$ at $37^{\circ} \mathrm{C}$. The viable bacterial numbers were determined by serial dilution and plating on drug-free LB agar plates. The survival rate of each strain was calculated as live bacterial number in AZM treated sample divided by the bacterial number of the corresponding untreated sample.

\section{Biofilm Tolerance to AZM}

The biofilm resistance was measured as previously described with minor modifications (Bjerkan et al., 2009; Billings et al., 2013; Liao et al., 2013). Briefly, overnight bacterial cultures were diluted to an $\mathrm{OD}_{600}$ of $0.025 .150 \mu \mathrm{l}$ of the bacteria were incubated in each well of a 96 -well plate at $37^{\circ} \mathrm{C}$ without agitation (Brencic et al., 2009). After $24 \mathrm{~h}$, the planktonic bacteria were discarded by aspiration. Then, the biofilms were treated with $150 \mu \mathrm{l} \mathrm{LB}$ medium containing indicated concentrations of AZM for $2 \mathrm{~h}$. The medium in each well was replaced with fresh LB medium, and subjected to sonication at a frequency of $40 \mathrm{kHz}$, with a power output of $300 \mathrm{~W}$, at $37^{\circ} \mathrm{C}$ for $5 \mathrm{~min}$. The live bacteria were enumerated by serial dilution and plating.

\section{Growth Assay}

Overnight culture of each strain was diluted into fresh LB $(150 \mu \mathrm{l})$ to an $\mathrm{OD}_{600}$ of 0.05 in each well of a 96-well plate without or 
TABLE 2 | Primers used in this study.

\begin{tabular}{|c|c|}
\hline Primers $^{a}$ & Nucleotide sequence $\left(5^{\prime} \rightarrow 3^{\prime}\right)^{b}$ \\
\hline \multicolumn{2}{|c|}{ Cloning of upstream and downstream fragments for PA3297 deletion } \\
\hline PA14-UPA3297-FF & GAAAGCGGTACCGAAGTAAGTCCGCCGTTGCC (Kpn \\
\hline PA14-UPA3297-FR & CAGCTICTAGAGGTGCTGTCGTCGCTCTGGT (Xba I) \\
\hline PA14-DPA3297-FF & TTGCAGTCTAGACGCTGGATGCTGGAGGAGTA (Xba I) \\
\hline PA14-DPA3297-FR & $\begin{array}{l}\text { CGCCGGAAGCTTCACCGAGCAGTGGCTGAAGAC } \\
\text { (Hind III) }\end{array}$ \\
\hline \multicolumn{2}{|c|}{ Cloning of gene PA3297 for complementation } \\
\hline PA14-PA3297-FF & $\begin{array}{l}\text { TGAAGAGAATTCGCCAGAAGTAAGTCCGCCGTTGCC } \\
(\text { EcoR I) }\end{array}$ \\
\hline PA14-PA3297-FR & $\begin{array}{l}\text { CACCGGGAGCTCCGACCAGACCGACCTGTTCTTCA } \\
\text { CCAT (Sac I) }\end{array}$ \\
\hline$P_{\text {Tn7R }}$ & CACAGCATAACTGGACTGATTTC \\
\hline$P_{\text {glmS-down }}$ & GCACATCGGCGACGTGCTCTC \\
\hline \multicolumn{2}{|c|}{ Primers used for site-directed mutagenesis of PA3297 } \\
\hline PA3297-K101A-FF & GCGAGACCGGCTCGGGCGC̣GACCACCCAG \\
\hline PA3297-K101A-FR & TC̣GC̣CGCCCGAGCCGGTCTCGCCGGCGATC \\
\hline PA3297-D192A -FF & TACGACACGCTGATCGTCGC̣C̣AAAGCCCAC \\
\hline PA3297-D192A -FR & CGGGC̣ACGATCAGCGTGTCGTAGCGCTCCAG \\
\hline $\begin{array}{l}\text { PA3297-SAT224- } \\
\text { 226AAA-FF }\end{array}$ & GCTGATCATCACCGC̣GGCGG̣Ĉ̣ATCGACCTGGAG \\
\hline $\begin{array}{l}\text { PA3297-SAT224- } \\
\text { 226AAA-FR }\end{array}$ & TG̣GC̣CGCC̣GC̣GGTGATGATCAGCTTCAGGTCC \\
\hline \multicolumn{2}{|c|}{ RT-qPCR primers for gene expression measurements } \\
\hline 23S-R-FF & AAAGATAACCGCTGAAAG \\
\hline 23S-R-FR & CTATCAACGTCGTAGTCT \\
\hline 5S-R-FF & CGAACTCAGAAGTGAAAC \\
\hline 5S-R-FR & СTTGACGATGACCTACTC \\
\hline 23S-5S-R -FF & GTACTAATTGCCCGTGAG \\
\hline 23S-5S -R-FR & GTTCCAACGCTCTATGAT \\
\hline
\end{tabular}

a The direction of the primer is indicated at the end of the primer designation as follows: FF for forward and FR for reverse. ${ }^{b}$ The solid underlines are the sites of listed restriction enzymes; the dotted underlines are the sites for mutagenesis, with all the residues changed into alanine.

with different concentrations of AZM. The plate was incubated at $37^{\circ} \mathrm{C}$ with constant agitation (Lau et al., 2012; Guénard et al., 2014). The bacterial growth was monitored by measuring the $\mathrm{OD}_{600}$ every $30 \mathrm{~min}$ for $12 \mathrm{~h}$ by a Varioskan Flash microplate reader (Thermo Electron Corporation).

\section{$\mathrm{H}_{2} \mathrm{O}_{2}$ Susceptibility Assay}

Overnight cultures of the $P$. aeruginosa strains were diluted to an $\mathrm{OD}_{600}$ of 0.05 and cultured at $37^{\circ} \mathrm{C}$. When the $\mathrm{OD}_{600}$ reached 0.3 (about $1.5-2 \mathrm{~h}$ later), AZM $(0.5 \mu \mathrm{g} / \mathrm{ml})$ was added if needed. When the $\mathrm{OD}_{600}$ reached 2.0 (about $3 \mathrm{~h}$ later), bacteria from $500 \mu \mathrm{l}$ culture were collected and washed twice with phosphate buffered saline (PBS). Then the bacteria were resuspended in PBS with or without $10 \% \mathrm{H}_{2} \mathrm{O}_{2}$ and incubated for $15 \mathrm{~min}$. The live bacterial numbers were determined by serial dilution and plating.

\section{Motility Assay}

The swarming motility was tested on modified M9 medium plates supplemented with $0.2 \%$ glucose, $1 \mathrm{mM} \mathrm{MgSO}_{4}$, and $0.05 \%$ glutamate as the nitrogen source. $0.5 \%$ agar was used for solidification. Two microliters of exponential growth phase $P$. aeruginosa was deposited on the plates, then incubated for $18 \mathrm{~h}$ at $37^{\circ} \mathrm{C}$ (Köhler et al., 2007).

\section{Total RNA Isolation and Quantitative Real-Time PCR}

Overnight cultures of $P$. aeruginosa strain PA14 and $\triangle$ PA3297 were diluted into fresh $\mathrm{LB}$ medium to an $\mathrm{OD}_{600}$ of 0.05 . The bacteria were grown at $37^{\circ} \mathrm{C}$ with agitation $(200 \mathrm{rpm})$. When the $\mathrm{OD}_{600}$ reached 0.3 (about $1.5-2 \mathrm{~h}$ later), AZM was added to reach indicated concentrations. Samples were harvested when the $\mathrm{OD}_{600}$ reached 2.0 (about 3 h later; Skindersoe et al., 2008). Total RNA was isolated with an RNeasy Minikit (Tiangen Biotech). The cDNA from each RNA sample was synthesized with reverse transcriptase and random primers (Takara). Real-time PCR was performed with SYBR premix Ex Taq (Roche). The conserved hypothetical protein coding gene PA1769 was used as an internal control (Son et al., 2007). The primers used in quantitative real-time PCR were listed in Table 2, with a designation of "RT-qPCR."

\section{Statistical Analysis}

When indicated, Student's $t$-test (two-tailed) was used to determine whether the deletion of PA3297 resulted in any significant differences compared to the wild-type cells treated with the same concentrations of AZM.

\section{RESULTS}

\section{Deficiency of PA3297 Intensifies the Effects of AZM on Pyocyanin Production}

As pyocanin production is suppressed by AZM (Molinari et al., 1992, 1993; Tateda et al., 2001; Favre-Bonté et al., 2003; Gillis and Iglewski, 2004), which can be easily observed and quantified, we used this phenotype to test whether $\mathrm{DExD} / \mathrm{H}$ box RNA helicases are involved in bacterial response to AZM treatment. There are $17 \mathrm{DExD} / \mathrm{H}$ box RNA helicases in the genome of $P$. aeruginosa strain PA14 (www.pseudomonas.com; Winsor et al., 2011). There are nine $\mathrm{DExD} / \mathrm{H}$ box helicase mutants in the non-redundant PA14 transposon mutants library, however, the other eight $\mathrm{DExD} / \mathrm{H}$ box helicase mutants are not available (Table 1; Liberati et al., 2006; Breidenstein et al., 2011). Thus, we examined pyocyanin production of the available mutants in the absence and presence of AZM at an $\mathrm{OD}_{600}$ of 2.0. A PA3297::Tn mutant displayed a significant decrease in pyocyanin production in the presence of AZM, whereas no difference was observed in the absence of AZM (Figure 1A). To confirm the role of PA3297, we generated an in frame deletion of PA3297 in PA14. The wild type PA14 and the $\triangle$ PA3297 mutant were grown in the absence or presence of 2,5 , or $10 \mu \mathrm{g} / \mathrm{ml}$ AZM. When the bacteria reached same density $\left(\mathrm{OD}_{600}\right.$ of 2.0), we measured the pyocyanin levels. AZM inhibited the production of pyocyanin in the wild type strain in a dose dependent manner. However, the production of pyocyanin by the mutant was repressed more severely in the presence of AZM 


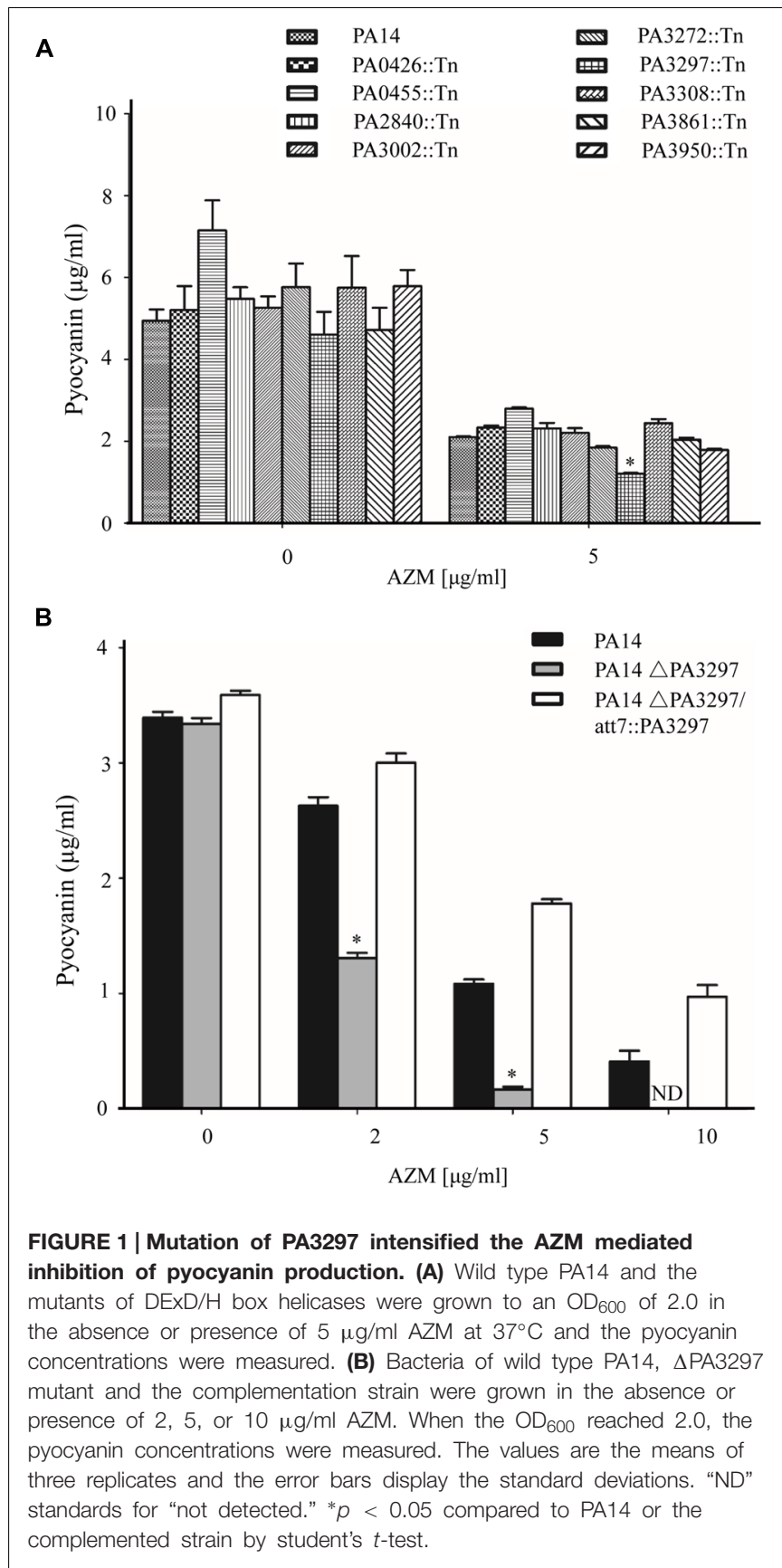

at all the tested concentrations (Figure 1B). Complementation with a PA3297 gene restored the production of pyocyanin (Figure 1B).

\section{Increased Killing of the $\triangle \mathrm{PA} 3297$ Mutant by AZM}

Stationary growth phase $P$. aeruginosa cells are susceptible to AZM (Lovmar et al., 2004, 2009; Imamura et al., 2005; Köhler et al., 2007; Starosta et al., 2010; Gödeke et al., 2013). To test whether mutation of PA3297 renders higher susceptibility, we performed the stationary-phase cells killing assay as previously

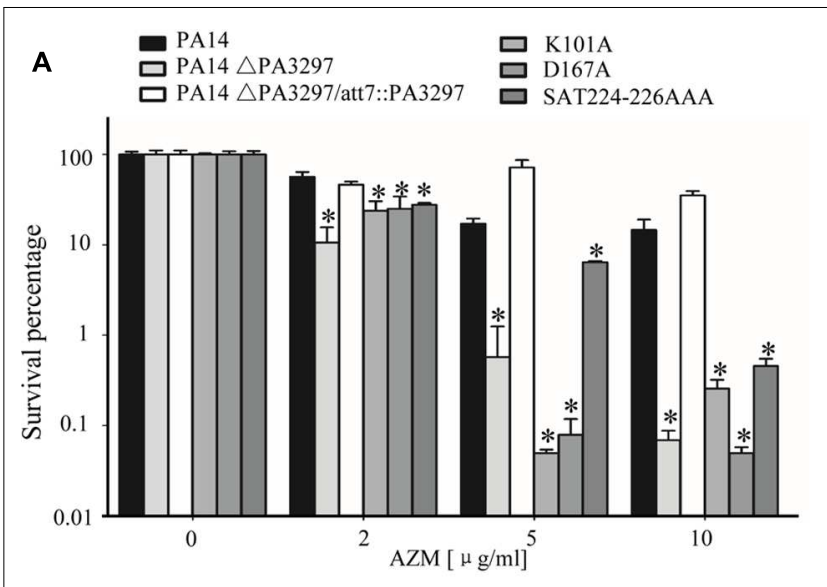

B

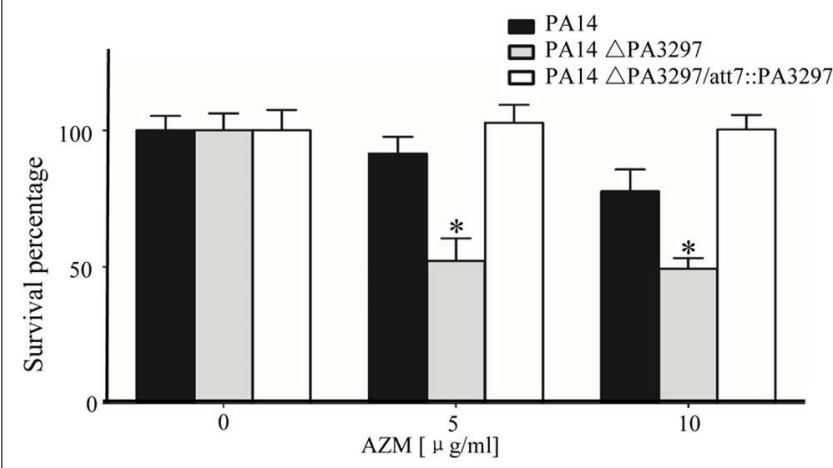

FIGURE 2 | Increased killing of the $\triangle$ PA3297 mutant by AZM. (A) AZM-mediated killing of wild type PA14, the $\triangle \mathrm{PA} 3297$ mutant and the mutant complemented with wild type or the mutated PA3297. Stationary-phase bacteria were treated with 2, 5, or $10 \mu \mathrm{g} / \mathrm{ml}$ AZM for $20 \mathrm{~h}$ at $37^{\circ} \mathrm{C}$. The numbers of live bacteria were determined by serial dilution and plating. (B) AZM-mediated killing of biofilm. 24-h-old biofilms formed by wild type PA14, the $\triangle \mathrm{PA} 3297$ mutant and the complemented strain were treated with $5 \mu \mathrm{g} / \mathrm{ml}, 10 \mu \mathrm{g} / \mathrm{ml}$ AZM or not for $2 \mathrm{~h}$. Bacteria in the biofilm were dissociated from the wells by gentle sonication. The viable bacteria were determined by serial dilution and plating. The averages and associated standard deviations from three replicates are shown. ${ }^{*} p<0.05$ compared to PA14 or the complemented strain by student's $t$-test.

described (Imamura et al., 2005; Köhler et al., 2007; Gödeke et al., 2013). Stationary-phase cells of PA14, the $\triangle$ PA3297 mutant and the complementation strain were subjected to treatment with AZM at the concentrations of 2, 5, and $10 \mu \mathrm{g} / \mathrm{ml}$. As shown in Figure 2A, the AZM-mediated killing of the $\triangle$ PA3297 mutant was significantly increased at all of the AZM concentrations tested. The most significant difference was observed at the concentration of $10 \mu \mathrm{g} / \mathrm{ml}$, where the survival rate of the mutant was approximately $1 \%$ of those of the wild type and complemented strains. In addition, the $\triangle$ PA3297 mutant was more susceptible to another macrolid antibiotic, erythromycin, with a fourfold lower MIC compared to the wild type strain PA14 (Table 3).

Next, we tested the susceptibility of the $\triangle$ PA3297 mutant to lincosamides, whose bactericidal mechanism is similar to 
macrolides (Tenson et al., 2003; Wilson, 2014). Indeed, mutation in PA3297 increased the bacterial susceptibility to lincomycin and clindamycin (Table 3 ). However, no increase of susceptibility was observed to other antibiotics, including ciprofloxacin, carbenicillin, meropenem, tetracycline, tobramycin, kanamycin, chloramphenicol, or polymyxin B (Supplementary Table S1). These results suggest that PA3297 plays an important role in the resistance against antibiotics targeting the peptide exit tunnel of ribosome.

PA3297 is a putative RNA helicase belonging to the DEAHbox family proteins, which are characterized by the presence of seven to nine conserved motifs (Tanner and Linder, 2001). In the E. coli RNA helicases DbpA and HrpA, the conserved residues GETGSGKT in motifI, DEAH in motifII, and SAT in motifIII have been shown to be required for interaction with and hydrolysis of NTP (Koo et al., 2004; Linder and Fuller-Pace, 2013). To determine whether these critical residues within motifs I, II, and III are important for PA3297 in the resistance to macrolides and lincosamides, we altered the residues by site-directed mutagenesis. Specifically, the K101 in motif I or the D167 in motif II was mutated to alanine. The S224 and T226 in motif III were both replaced with alanine. Each mutated PA3297 was transferred into the $\triangle$ PA3297 mutant and the susceptibility to antibiotics was tested. None of the mutated PA3297 was able to restore the survival rate of the mutant (Figure 2A). In addition, the mutated PA3297 was unable to restore the resistance of the $\triangle$ PA3297 mutant to macrolides, lincomycin, and clindamycin (Table 3). These results suggest that the RNA helicase function of PA3297 is required for its role in the resistance to macrolides and lincosamides.

Clinically, AZM has been used in the treatment of chronic P. aeruginosa infection (Saiman et al., 2003; Blasi et al., 2010), which is characterized by biofilm formation (Singh et al., 2000). The biofilm is notorious for high antibiotic tolerance, which severely hinders eradication of the bacteria (López et al., 2010; Breidenstein et al., 2011). We suspected that mutation of PA3297 might increase the killing efficacy of AZM on the biofilm. Indeed, the survival rates of the $\triangle$ PA3297 mutant in biofilm were lower than those of the wild type PA14 at various AZM concentrations, which were restored by complementation with a wild type PA3297 (Figure 2B).

TABLE 3 | Bacterial susceptibilities to macrolides and lincosamides.

\begin{tabular}{lccrr}
\hline \multirow{2}{*}{ Strain } & \multicolumn{4}{c}{ MIC $(\boldsymbol{\mu} \mathbf{g} / \mathbf{m l})^{\mathbf{a}}$} \\
\cline { 2 - 5 } & ERY & AZM & LIN & CLI \\
\hline PA14 & 300 & 400 & 12800 & 4800 \\
$\Delta$ PA3297 & 75 & 100 & 3200 & 2400 \\
$\Delta$ PA3297/att7::PA3297 & 300 & 400 & $>12800$ & 9600 \\
$\Delta$ PA3297/att7::PA3297 K101A & 75 & 100 & 6400 & 1600 \\
$\Delta$ PA3297/att7::PA3297 D192A & 75 & 100 & 3200 & 2400 \\
$\Delta$ PA3297/att7::PA3297 SAT224AAA & 75 & 100 & 6400 & 2400 \\
\hline aERY, erythromycin; AZM, azithromycin; & LIN, lincomycin; & CLI, clindamycin. \\
b Mutants with single or triple amino acid changes. & & &
\end{tabular}

\section{Mutation of PA3297 Increases the Bacterial Susceptibility to Hydrogen Peroxide in the Presence of AZM}

It has been shown that AZM treatment impairs the oxidative stress response in $P$. aeruginosa (Nalca et al., 2006), which intrigued us to test whether mutation of PA3297 leads to further impairment. The $\mathrm{H}_{2} \mathrm{O}_{2}$ susceptibility assay was performed in the presence of $0.5 \mu \mathrm{g} / \mathrm{ml} \mathrm{AZM}$, as the growth rates of wild type PA14 and the $\triangle$ PA3297 mutant were similar at this concentration of AZM (Supplementary Figure S1). The wild type PA14, $\triangle$ PA3297 mutant and the complemented strain were grown without or with AZM to an $\mathrm{OD}_{600}$ of 2.0, and then treated with $10 \% \mathrm{H}_{2} \mathrm{O}_{2}$ for $15 \mathrm{~min}$. In the presence of AZM, the $\triangle \mathrm{PA} 3297$ mutant was more susceptible to $\mathrm{H}_{2} \mathrm{O}_{2}$ than the wild type and complemented strains, whereas no difference was observed in the absence of AZM (Figure 3). These results suggest that PA3297 is involved in the bacterial oxidative stress response in the presence of AZM.

\section{Mutation of PA3297 Intensifies the Inhibitory Effect of AZM on Swarming Motility}

Besides oxidative stress response, AZM suppresses swarming motility (Tateda et al., 2001; Köhler et al., 2007; Gödeke et al., 2013). Same numbers of wild type PA14 and the $\triangle$ PA3297 mutant were inoculated on the plates containing various concentrations of AZM. As shown in Figure 4,10 $\mu \mathrm{g} / \mathrm{ml} \mathrm{AZM} \mathrm{suppressed}$ the swarming motility of wild type PA14 obviously, whereas the lower concentrations of AZM showed no inhibitory effect. However, starting from $2 \mu \mathrm{g} / \mathrm{ml}$, AZM suppressed the swarming motility of the $\triangle$ PA3297 mutant in a dose dependent manner. Complementation with a PA3297 gene restored the swarming motility in the presence of AZM (Figure 4).

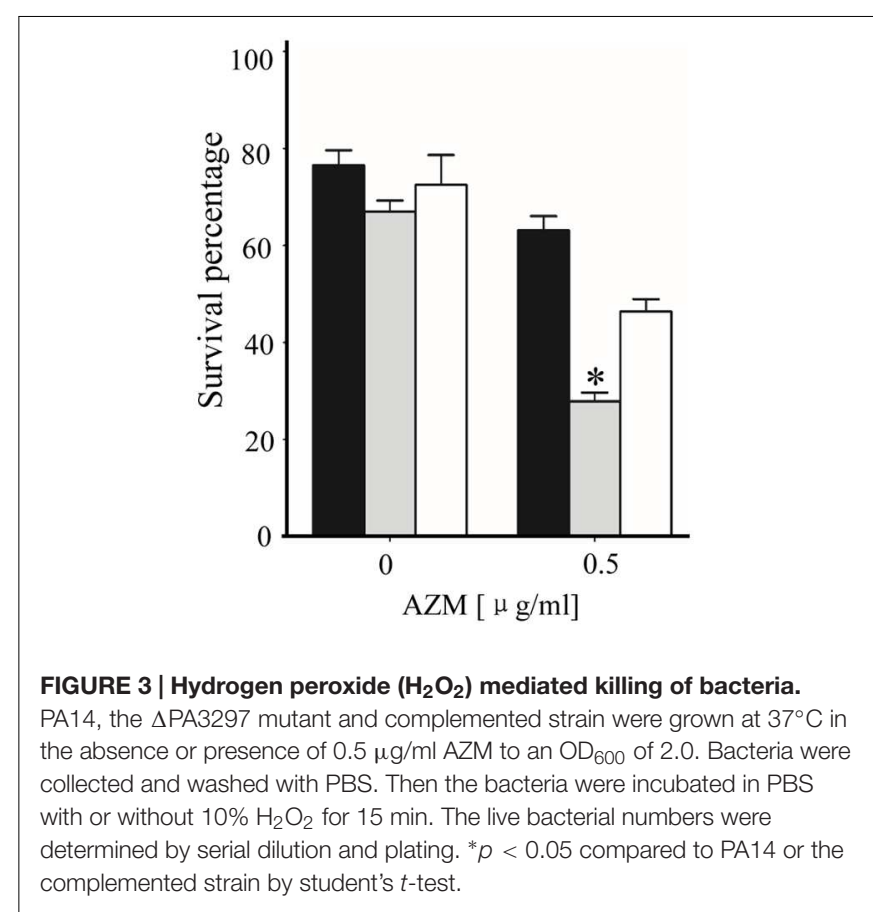




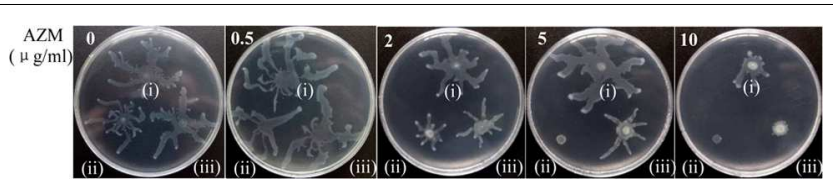

FIGURE 4 | Bacterial swarming motility. PA14 (i), the $\triangle$ PA3297 mutant (ii) and the complemented strain (iii) were inoculated on plates containing indicated concentrations of AZM.

\section{Interaction between AZM and Ribosome Induces the Expression of PA3297}

Our results so far suggested that PA3297 is involved in the bacterial response to AZM treatment. To test whether the expression of PA3297 is induced by AZM treatment, wild type PA14 was grown in the absence or presence of AZM at various concentrations and the relative RNA levels of PA3297 were determined by real time PCR. Indeed, the expression of PA3297 was induced by AZM (Figure 5A). However, overexpression of PA3297 in wild type PA14 did not further increase the bacterial

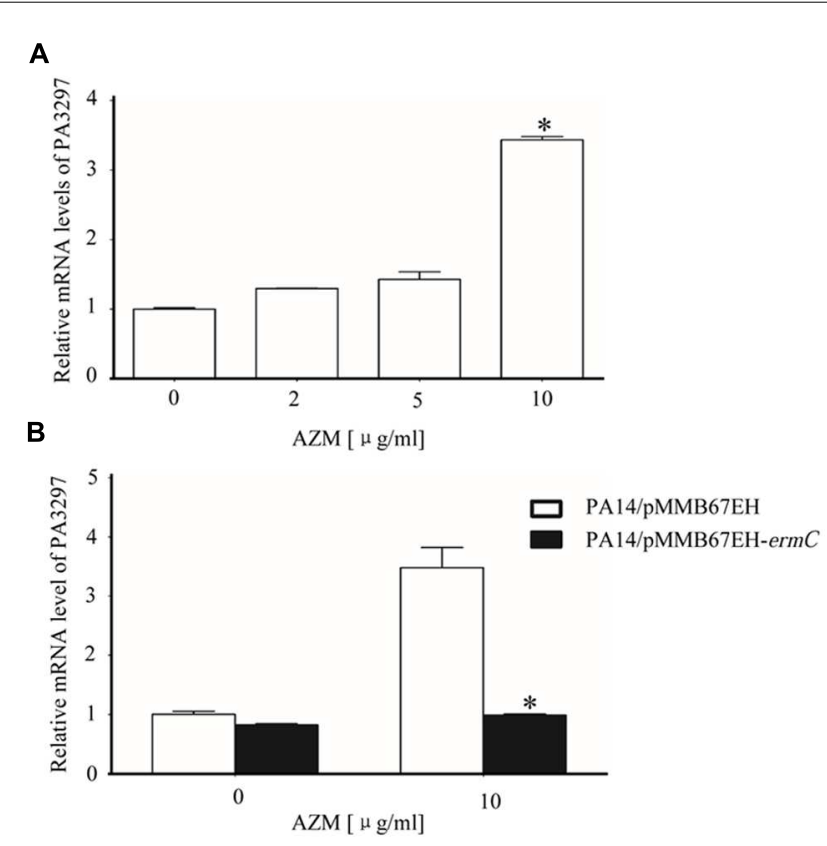

FIGURE 5 | Expression of PA3297 in the presence of AZM. (A) Wild type PA14 was grown to an $\mathrm{OD}_{600}$ of 0.3 in LB medium. The bacteria were grown further in the absence or presence of 2,5 , or $10 \mu \mathrm{g} / \mathrm{ml} A Z M$. When the $\mathrm{OD}_{600}$ reached 2.0, total bacterial RNA was isolated and the mRNA levels of PA3297 were determined with real time PCR. ${ }^{*} p<0.05$ compared to bacteria in the absence of presence of 2 or $5 \mu \mathrm{g} / \mathrm{ml}$ AZM by student's $t$-test. (B) PA14 harboring $\mathrm{pMMB67EH}$ or the ErmC over expressing plasmid (pMMB67EH-ermC) was grown at $37^{\circ} \mathrm{Cin}$ the absence of AZM. When the $\mathrm{OD}_{600}$ reached $0.3,1 \mathrm{mM}$ IPTG was added to the medium. Meanwhile, no AZM or AZM at the final concentration of $10 \mu \mathrm{g} / \mathrm{ml}$ was added to the medium. At the $\mathrm{OD}_{600}$ of 2.0, total RNA was isolated and the mRNA levels of PA3297 were determined with real time PCR. The mRNA levels of lacl from the plasmid were used as internal control. ${ }^{*} p<0.05$ compared to PA14/pMMB67EH by student's t-test.
TABLE 4 | Bacterial susceptibilities to macrolides and lincosamides.

\begin{tabular}{lcccc}
\hline Strain & \multicolumn{4}{c}{ MIC $(\boldsymbol{\mu} \mathbf{g} / \mathbf{m l})^{\mathbf{a}}$} \\
\cline { 2 - 5 } & ERY & AZM & LIN & CLI \\
\hline PA14/pMMB67EH & 600 & 200 & 12800 & 12800 \\
PA14/pMMB67-PA3297 & 600 & 200 & 12800 & 6400 \\
PA14/pMMB67-ermC & 2400 & 800 & $>12800$ & $>12800$ \\
$\Delta$ PA3297/pMMB67EH & 75 & 50 & 6400 & 3200 \\
$\Delta$ PA3297/pMMB67-PA3297 & 300 & 200 & 12800 & 3200 \\
$\Delta$ PA3297/pMMB67-ermC & 2400 & 400 & $>12800$ & $>12800$ \\
\hline
\end{tabular}

${ }^{a} E R Y$, erythromycin; $A Z M$, azithromycin; LIN, lincomycin; CLI, clindamycin.

tolerance to AZM (Table 4). We suspect that since the bacteria at exponential growth phase are highly resistant to macrolides and lincosamides, overexpression of PA3297 might not further increase the resistance significantly in the MIC test. Another possibility is that with the endogenous up regulation of PA3297, additional expression of PA3297 might be redundant.

Köhler et al. (2007) previously demonstrated that ribosome is the only target of AZM in bacteria. To test whether the induction of PA3297 is caused by the interaction between AZM and ribosome, we performed the ribosomal protection assay by overexpressing ErmC, a $23 \mathrm{~S}$ rRNA methylase that blocks the binding of macrolide antibiotics to the NPET (Köhler et al., 2007; Lawrence et al., 2008). In the presence of $10 \mu \mathrm{g} / \mathrm{ml}$ AZM, the growth speed of the ErmC overexpressing strain was similar with that of the wild type strain containing an empty vector. However, overexpression of ErmC abolished the induction of PA3297 by AZM (Figure 5B). In addition, antibiotics in the other categories, including ciprofloxacin, tobramycin, and carbenicillin did not affect the expression level of PA3297 (Supplementary Figure S2). These results suggest that the expression of PA3297 is regulated in response to AZM-mediated ribosome stalling.

\section{Ribosome Protection Rescues the $\triangle$ PA3297 Mutant from AZM-Mediated Hyperlethality}

So far, we have demonstrated that the expression of PA3297 is induced by AZM and that mutation of PA3297 renders $P$. aeruginosa hypersusceptible to AZM. These results suggest that PA3297 might play a role in counteracting the detrimental effects caused by the interaction between AZM and ribosome (Köhler et al., 2007; Gödeke et al., 2013). Thus, ribosome protection should be able to increase the tolerance of the $\triangle$ PA3297 mutant to AZM. Indeed, overexpression of ErmC increased both the growth speed and the survival rate of the $\triangle \mathrm{PA} 3297$ mutant when treated with 10 or $40 \mu \mathrm{g} / \mathrm{ml}$ AZM (Supplementary Figure S3, Figure 6). In addition, overexpression of ErmC increased the MICs of both wild type PA14 and the $\triangle$ PA3297 mutant to AZM, erythromycin, lincomycin and clindamycin (Table 4). Mutation of PA3297 did not increase the bacterial susceptibility to a variety of other antibiotics (Supplementary Table S1). These results suggest a specific role of PA3297 in responding to lincosamides and macrolides. 


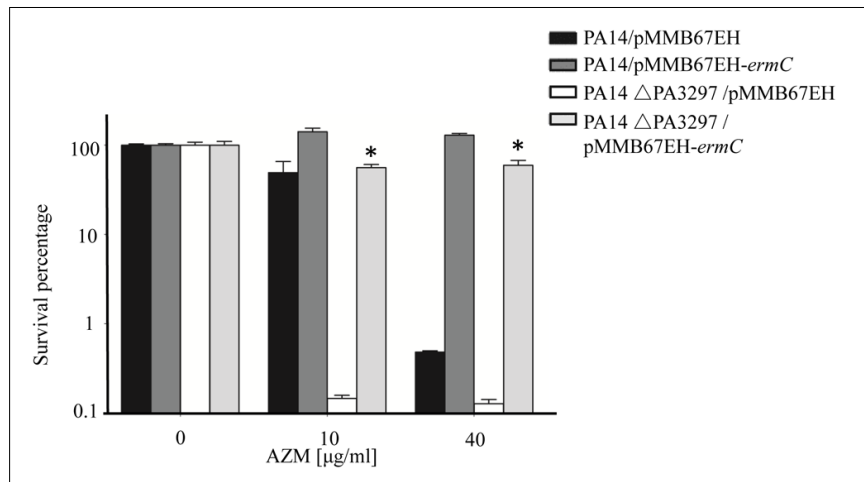

FIGURE 6 | Effects of Ribosome protection on the AZM mediated killing of the $\triangle$ PA3297 mutant. Wild type PA14 or the $\triangle$ PA3297 mutant harboring PMMB67EH or pMMB67EH-ermC was grown to an $\mathrm{OD}_{600}$ of 0.5 when $1 \mathrm{mM}$ IPTG was added to the medium. When the $\mathrm{OD}_{600}$ reached 2.0, the bacteria were treated without or with 10 or $40 \mu \mathrm{g} / \mathrm{ml} \mathrm{AZM} \mathrm{for} 20 \mathrm{~h}$ at $37^{\circ} \mathrm{C}$. The numbers of live bacteria were determined by serial dilution and plating. ${ }^{*} p<0.05$ compared to the $\triangle \mathrm{PA} 3297$ mutant harboring $\mathrm{pMMB} 67 \mathrm{EH}$ by student's t-test.

\section{Deficiency in PA3297 Compromises rRNA Processing in the Presence of AZM}

Studies in E. coli demonstrated that the $\mathrm{DExD} / \mathrm{H}$ box play crucial roles in rRNA processing (Iost et al., 2013; Linder and Fuller-Pace, 2013). Therefore, we suspected that the up regulated PA3297 might participate in rRNA maturation, which facilitates ribosome biogenesis to compensate for AZM inactivated ribosome. The rRNA coding region in the chromosome of $P$. aeruginosa PA14 is shown in Supplementary Figure S4. To examine the processing of the rRNA transcript, we designed real-time PCR primers to analyze the total $23 \mathrm{~S}$ and $5 \mathrm{~S}$ rRNA levels as well as primers across the $23 \mathrm{~S}$ and $5 \mathrm{~S}$ rRNA coding region to analyze the level of unprocessed rRNA (Supplementary Figure S4). In wild type PA14, AZM at the concentrations of 5 and $10 \mu \mathrm{g} / \mathrm{ml}$ reduced the $23 \mathrm{~S}$ and $5 \mathrm{~S}$ rRNA levels (Figures 7A,B), and $10 \mu \mathrm{g} / \mathrm{ml}$ AZM slightly increased the unprocessed 23S$5 \mathrm{~S}$ rRNA level (Figure 7C). In the absence of AZM, the total $23 \mathrm{~S}$ and 5S rRNA levels were higher in the $\triangle \mathrm{PA} 3297$ mutant than those in the wild type strain (Figures 7A,B), whereas the unprocessed 23S-5S levels were similar between the mutant and wild type strain. Interestingly, treatment with $10 \mu \mathrm{g} / \mathrm{ml} \mathrm{AZM}$ resulted in a higher level of unprocessed 23S-5S rRNA in the $\triangle$ PA3297 mutant (Figure 7C), although its total 23S and 5S rRNA levels were lower than those in the wild type strain (Figures 7A,B).

Next, we calculated the percentages of unprocessed 23S-5S rRNA in wild type PA14 and the $\triangle$ PA3297 mutant with or without AZM treatment. Standard curves were generated to determine the amplification efficiencies of the primer pairs for the detection of total 23S, $5 \mathrm{~S}$ and unprocessed 23S-5S rRNA levels in real time PCR (Supplementary Figure S5). Considering $5 \mathrm{~S}$ rRNA is more prone to be lost during RNA purification, we calculated the ratio of unprocessed 23S-5S rRNA by dividing the levels of $23 \mathrm{~S}-5 \mathrm{~S}$ rRNA by those of $23 \mathrm{~S}$ rRNA calibrated with the amplification efficiencies (Table 5). In the absence of AZM, the

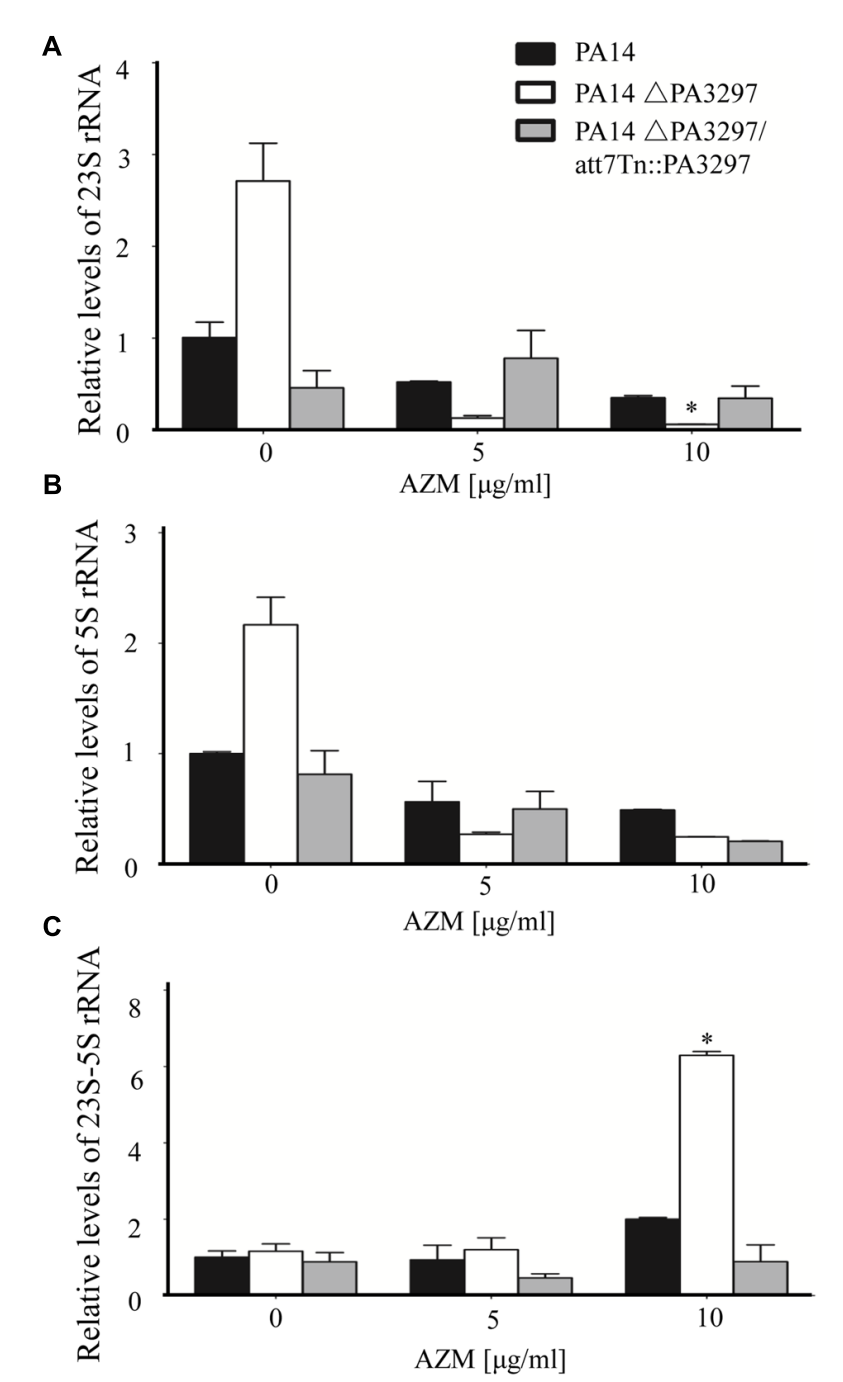

FIGURE 7 | Effects of AZM on the processing of rRNA. PA14, the $\triangle \mathrm{PA} 3297$ mutant and the complemented strain were grown at $37^{\circ} \mathrm{C}$ in the absence of $A Z M$. When the $\mathrm{OD}_{600}$ reached $0.3, \mathrm{AZM}(0,5$, and $10 \mu \mathrm{g} / \mathrm{ml})$ was added. Total RNA was harvested when the $\mathrm{OD}_{600}$ reached 2.0. The levels of total 23S (A), 5S (B), and unprocessed 23S-5S rRNA (C) were determined by real time PCR. The mRNA levels of PA1769 were used as an internal control. ${ }^{*} p<0.05$ compared to PA14 by student's $t$-test.

ratios of unprocessed 23S-5S rRNA were approximately 0.014 and $0.009 \%$ in wild type PA14 and the $\triangle$ PA3297 mutant, respectively. It seems that, without PA3297, the processing of 23S-5S rRNA is even more efficient. In the presence of 5 and $10 \mu \mathrm{g} / \mathrm{ml} \mathrm{AZM}$, the ratios of unprocessed 23S-5S rRNA rose to approximately 0.05 and $0.09 \%$ in wild type PA14, respectively. However, the ratios of unprocessed 23S-5S rRNA in the $\triangle \mathrm{PA} 3297$ mutant were 0.33 and $1.61 \%$, which were approximately 5 - and 18 -fold higher than those in PA14 under the same condition (Table 5).

Since, the growth speed of the $\triangle \mathrm{PA} 3297$ mutant in the presence of $10 \mu \mathrm{g} / \mathrm{ml}$ AZM was similar as that of the wild type PA14 in the presence of $40 \mu \mathrm{g} / \mathrm{ml} \mathrm{AZM} \mathrm{(Supplementary}$ Figure S3), we compared the rRNAs levels of the two strains 
TABLE 5 | The ratio of unprocessed 23S-5S rRNA in total 23S rRNA (\%).

\begin{tabular}{lccc}
\hline Strain & \multicolumn{3}{c}{ AZM $(\boldsymbol{\mu} \mathbf{g} / \mathbf{m l})$} \\
\cline { 2 - 4 } & $\mathbf{0}$ & $\mathbf{5}$ & $\mathbf{1 0}$ \\
\hline PA14 & 0.014 & 0.051 & 0.089 \\
$\Delta$ PA3297 & 0.009 & 0.335 & 1.608 \\
\hline
\end{tabular}

The unprocessed 23S-5S rRNA ratios were calculated according to the equation:

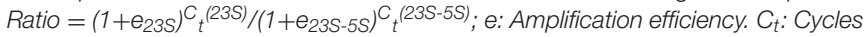
when fluorescence intensity reaches detection threshold.

grown under the two conditions. The relative levels of 23S-5S rRNA, total $5 \mathrm{~S}$ and $23 \mathrm{~S}$ rRNA in the $\triangle \mathrm{PA} 3297$ mutant were 150,55 , and $100 \%$ of those in the wild type PA14, respectively, indicating a similar rRNA processing status with the two different AZM concentrations. In combination, these results suggest that PA3297 might contribute to rRNA processing in response to AZM. And mutation of PA3297 might impair the biosynthesis of ribosome under AZM treatment, which renders the bacterium more susceptible to AZM.

\section{DISCUSSION}

By binding to $23 \mathrm{~S}$ rRNA in the $50 \mathrm{~S}$ subunit of bacterial ribosome, AZM blocks polypeptide elongation and diminishes the intracellular pools of aminoacyl-tRNAs (Tenson et al., 2003; Gödeke et al., 2013; Wilson, 2014). And it has been demonstrated that AZM reduces the expression of gacA and the small RNAs rsm $Y$ and $r s m Z$, as well as quorum sensing genes (Kai et al., 2009; Pérez-Martínez and Haas, 2011). And the stationary phase killing by sub-MIC AZM was demonstrated to be correlated with increased outer membrane permeability (Imamura et al., 2005). Here, we found that mutation of PA3297 intensified the AZM mediated inhibitory effects on pyocyanin production and swarming motility of $P$. aeruginosa. In addition, the PA3297 mutant is more susceptible to oxidative stress in the presence of AZM. During infection, host generated reactive oxygen species (ROS) is an important bacterial killing mechanism. Therefore, inhibition of PA3297 together with the treatment with AZM, might render the bacteria more susceptible to host killing.

The MIC of the $\triangle \mathrm{PA} 3297$ mutant is a quarter of that of the wild type strain. Consistently, the growth speed of the $\triangle \mathrm{PA} 3297$ mutant in the presence of $10 \mu \mathrm{g} / \mathrm{ml}$ AZM was similar as that of the wild type strain in the presence of $40 \mu \mathrm{g} / \mathrm{ml} \mathrm{AZM}$ (Supplementary Figure S3). However, in the stationary phase cell killing assay, the survival rate of the $\triangle \mathrm{PA} 3297$ mutant treated with $10 \mu \mathrm{g} / \mathrm{ml} \mathrm{AZM}$ was approximately $20 \%$ of that of the wild type strain treated with $40 \mu \mathrm{g} / \mathrm{ml} \mathrm{AZM} \mathrm{(Figure} \mathrm{6).} \mathrm{Therefore,}$ PA3297 might play a more important role in the survival of stationary phase cells under AZM treatment.

Macrolides and lincosamides bind to the 50S of ribosome and block the NPET (Tenson et al., 2003). In our experiments, mutation in PA3297 did not alter the bacterial resistance to other antibiotics, including those binding to $30 \mathrm{~S}$ or other parts of $50 \mathrm{~S}$ ribosome. In addition, the expression of PA3297 was upregulated by AZM, which was abolished by ribosome protection. Therefore, PA3297 might specifically play a role in bacterial response to macrolides and lincosamides.

PA3297 is also named HrpA, both in PAO1 and PA14 (Winsor et al., 2016). According to the Profiles from GEO Expression Database at NCBI, the expression level of PA3297 was higher in biofilm than that in planktonic cells (Anderson et al., 2008). Isolates from CF lungs displayed higher expression levels of PA3297 levels than PAO1 (Son et al., 2007; Bielecki et al., 2013). And artificial medium that mimics CF lung sputum could increase the expression level of PA3297 slightly (Fung et al., 2010). In addition, increase of PA3297 expression was also observed in antibiotic-resistant small colony variants (Wei et al., 2011). However, the expression level of PA3297 showed a significant decrease in response to airway epithelia or low oxygen conditions (Alvarez-Ortega and Harwood, 2007; Chugani and Greenberg, 2007). These results indicate that the expression of PA3297 is regulated in response to various environmental stesses.

PA3297 is predicted to locate at the cytoplasmic membrane, with a molecular weight of $149.8 \mathrm{kDa}$ (Winsor et al., 2016). It belongs to the $\mathrm{DExD} / \mathrm{H}$ box helicase family. Members of this family have been found to play crucial roles in RNA metabolism and gene regulation (Linder and Jankowsky, 2011; Iost et al., 2013; Kaberdin and Bläsi, 2013; Linder and Fuller-Pace, 2013; Putnam and Jankowsky, 2013). According to NCBI protein blast, there are 81 homologous proteins with identities of $78 \%$ or more in other microorganisms. The homolog of PA3297 in Borrelia burgdorferi was found to be required for mouse infectivity and tick transmission and involved global gene regulation (SalmanDilgimen et al., 2011; Owttrim, 2013). Another homolog in E. coli was found to be involved in fimbrial biogenesis (Koo et al., 2004). The identities they shared with PA3297 are 34 and $49 \%$, respectively. Both of them possess the conserved residues in motif I, motif II, and motif III. Meanwhile, they are also involved in RNA processing. In Listeria monocytogenes, it has been reported that defect of a DExD-box RNA helicase, Lmo1722, reduced the maturation of 23S RNA (Bareclev et al., 2014) at low temperatures.

In E. coli, the primary transcript rRNA is cleaved by RNase III, yielding precursors of the 16S rRNA (17S rRNA), $23 \mathrm{~S}$ rRNA, and 5S rRNA (9S rRNA; Shajani et al., 2011). Mutation of RNase III led to slower growth rate, reduction in cell viability and protein synthesis rates in the presence of AZM (Silvers and Champney, 2005). In E. coli, under certain stress conditions, DEAD box helicase may substitute for RhlB in the degradosome, such as CsdA under cold shock conditions (Prud'homme-Généreux et al., 2004). We found that mutation of PA3297 increased the percentage of unprocessed 23S-5S rRNA in the presence of AZM, which indicates that the cleaving function of RNase III might be impaired under the stress caused by AZM. Therefore, PA3297 might assist RNase III in rRNA processing in the presence of AZM. In addition, although the growth speed and rRNA processing in the $\triangle \mathrm{PA} 3297$ mutant treated with $10 \mu \mathrm{g} / \mathrm{ml}$ AZM were similar to those in the wild type strain treated with $40 \mu \mathrm{g} / \mathrm{ml}$ AZM (Supplementary Figure S3), the survival rate of the $\triangle$ PA3297 mutant was lower than that of the wild type strain 
under those conditions. Thus, PA3297 might play other roles in counteracting the effects of AZM.

Overall, mutation of PA3297 renders $P$. aeruginosa more susceptible to AZM mediated inhibition on virulence factors and killing effect. Therefore, targeting the regulatory pathway or the function of PA3297 might further increase the beneficial effects of AZM in the treatment of chronic $P$. aeruginosa infections.

\section{AUTHOR CONTRIBUTIONS}

Conceived and designed the experiments: WW, SJ, HT, ZC. Performed the experiments: HT, LZ, YW, RC, FZ, YJ. Analyzed the data: HT, LZ, SJ, WW. Wrote the paper: HT, SJ, WW.

\section{REFERENCES}

Alvarez-Ortega, C., and Harwood, C. S. (2007). Responses of Pseudomonas aeruginosa to low oxygen indicate that growth in the cystic fibrosis lung is by aerobic respiration. Mol. Microbiol. 65, 153-165. doi: 10.1111/j.13652958.2007.05772.x

Aminov, R. I. (2013). Biotic acts of antibiotics. Front. Microbiol. 4:241. doi: $10.3389 /$ fmicb.2013.00241

Anderson, G. G., Moreau-Marquis, S., Stanton, B. A., and O’Toole, G. A. (2008). In vitro analysis of tobramycin-treated Pseudomonas aeruginosa biofilms on cystic fibrosis-derived airway epithelial cells. Infect. Immun. 76, 1423-1433. doi: 10.1128/IAI.01373-07

Bareclev, C., Vaitkevicius, K., Netterling, S., and Johansson, J. (2014). DExD-box RNA-helicases in Listeria monocytogenes are important for growth, ribosomal maturation, rRNA processing and virulence factor expression. RNA Biol. 11, 1458-1467. doi: 10.1080/15476286.2014.996099

Bielecki, P., Komor, U., Bielecka, A., Müsken, M., Puchałka, J., Pletz, M. W., et al. (2013). Ex vivo transcriptional profiling reveals a common set of genes important for the adaptation of Pseudomonas aeruginosa to chronically infected host sites. Environ. Microbiol. 15, 570-587. doi: 10.1111/1462-2920. 12024

Billings, N., Millan, M. R., Caldara, M., Rusconi, R., Tarasova, Y., Stocker, R., et al. (2013). The extracellular matrix component psl provides fast-acting antibiotic defense in Pseudomonas aeruginosa biofilms. PLoS Pathog. 9:e1003526. doi: 10.1371/journal.ppat.1003526

Bjerkan, G., Witso, E., and Bergh, K. (2009). Sonication is superior to scraping for retrieval of bacteria in biofilm on titanium and steel surfaces in vitro. Acta Orthop. 80, 245-250. doi: 10.3109/17453670902947457

Blasi, F., Bonardi, D., Aliberti, S., Tarsia, P., Confalonieri, M., Amir, O., et al. (2010). Long-term azithromycin use in patients with chronic obstructive pulmonary disease and tracheostomy. Pulm. Pharmacol. Ther. 23, 200-207. doi: 10.1016/j.pupt.2009.12.002

Breidenstein, E. B., de la Fuente-Núñez, C., and Hancock, R. E. (2011). Pseudomonas aeruginosa: all roads lead to resistance. Trends Microbiol. 19, 419-426. doi: 10.1016/j.tim.2011.04.005

Brencic, A., McFarland, K. A., McManus, H. R., Castang, S., Mogno, I., Dove, S. L., et al. (2009). Th GacS/GacA signal transduction system of Pseudomonas aeruginosa acts exclusively through its control over the transcription of the RsmY and RsmZ regulatory small RNAs. Mol. Microbiol. 73, 434-445. doi: 10.1111/j.1365-2958.2009.06782.x

Campa, M., Bendinelli, M., and Friedman, H. (2012). Pseudomonas aeruginosa as an Opportunistic Pathogen. Berlin: Springer Science \& Business Media.

Choi, K.-H., and Schweizer, H. P. (2006). Mini-Tn7 insertion in bacteria with single attTn7 sites: example Pseudomonas aeruginosa. Nat. Protoc. 1, 153-161. doi: 10.1038/nprot.2006.24

Chugani, S., and Greenberg, E. (2007). The influence of human respiratory epithelia on Pseudomonas aeruginosa gene expression. Microb. Pathog. 42, 29-35. doi: 10.1016/j.micpath.2006.10.004

\section{FUNDING}

This work was supported by National Science Foundation of China (31370168 to WW, and 31370167 to SJ); National Basic Research Program of China (973 Program, 2012CB518700 to SJ), Program of international S\&T cooperation (2015DFG32500 to SJ) and Science and Technology Committee of Tianjin (13JCYBJC36700 to WW, 15JCZDJC33000 to SJ).

\section{SUPPLEMENTARY MATERIAL}

The Supplementary Material for this article can be found online at: http://journal.frontiersin.org/article/10.3389/fmicb. 2016.00317

Cordin, O., Banroques, J., Tanner, N. K., and Linder, P. (2006). The DEADbox protein family of RNA helicases. Gene 367, 17-37. doi: 10.1016/j. gene.2005.10.019

de Bentzmann, S., and Plésiat, P. (2011). The Pseudomonas aeruginosa opportunistic pathogen and human infections. Environ. Microbiol. 13, 16551665. doi: 10.1111/j.1462-2920.2011.02469.x

Doring, G., Conway, S., Heijerman, H., Hodson, M., Hoiby, N., Smyth, A., et al. (2000). Antibiotic therapy against Pseudomonas aeruginosa in cystic fibrosis: a European consensus. Eur. Respir. J. 16, 749-767. doi: 10.1034/j.13993003.2000.16d30.x

Essar, D., Eberly, L., Hadero, A., and Crawford, I. (1990). Identification and characterization of genes for a second anthranilate synthase in Pseudomonas aeruginosa: interchangeability of the two anthranilate synthases and evolutionary implications. J. Bacteriol. 172, 884-900.

Favre-Bonté, S., Köhler, T., and Van Delden, C. (2003). Biofilm formation by Pseudomonas aeruginosa: role of the C4-HSL cell-to-cell signal and inhibition by azithromycin. J. Antimicrob. Chemother. 52, 598-604. doi: $10.1093 / \mathrm{jac} / \mathrm{dkg} 397$

Fung, C., Naughton, S., Turnbull, L., Tingpej, P., Rose, B., Arthur, J., et al. (2010). Gene expression of Pseudomonas aeruginosa in a mucin-containing synthetic growth medium mimicking cystic fibrosis lung sputum. J. Med. Microbiol. 59, 1089-1100. doi: 10.1099/jmm.0.019984-0

Gillis, R. J., and Iglewski, B. H. (2004). Azithromycin retards Pseudomonas aeruginosa biofilm formation. J. Clin. Microbiol. 42, 5842-5845. doi: 10.1128/JCM.42.12.5842-5845.2004

Gödeke, J., Pustelny, C., and Häussler, S. (2013). Recycling of peptidyltRNAs by peptidyl-tRNA hydrolase counteracts azithromycin-mediated effects on Pseudomonas aeruginosa. Antimicrob. Agents Chemother. 57, 1617-1624. doi: 10.1128/AAC.02582-12

Guénard, S., Muller, C., Monlezun, L., Benas, P., Broutin, I., Jeannot, K., et al. (2014). Multiple mutations lead to MexXY-OprM-dependent aminoglycoside resistance in clinical strains of Pseudomonas aeruginosa. Antimicrob. Agents Chemother. 58, 221-228. doi: 10.1128/AAC. 01252-13

Hauser, A. R. (2009). The type III secretion system of Pseudomonas aeruginosa: infection by injection. Nat. Rev. Microbiol. 7, 654-665. doi: 10.1038/ nrmicro2199

Hoang, T. T., Karkhoff-Schweizer, R. R., Kutchma, A. J., and Schweizer, H. P. (1998). A broad-host-range Flp-FRT recombination system for sitespecific excision of chromosomally-located DNA sequences: application for isolation of unmarked Pseudomonas aeruginosa mutants. Gene 212, 77-86. doi: 10.1016/S0378-1119(98)00130-9

Høiby, N., Frederiksen, B., and Pressler, T. (2005). Eradication of early Pseudomonas aeruginosa infection. J. Cyst. Fibros. 4, 49-54. doi: 10.1016/j.jcf. 2005.05.018

Imamura, Y., Higashiyama, Y., Tomono, K., Izumikawa, K., Yanagihara, K., Ohno, H., et al. (2005). Azithromycin exhibits bactericidal effects on Pseudomonas aeruginosa through interaction with the outer membrane. 
Antimicrob. Agents Chemother. 49, 1377-1380. doi: 10.1128/AAC.49.4.13771380.2005

Imperi, F., Leoni, L., and Visca, P. (2014). Antivirulence activity of azithromycin in Pseudomonas aeruginosa. Front. Microbiol. 5:178. doi: $10.3389 /$ fmicb.2014.00178

Intile, P. J., Balzer, G. J., Wolfgang, M. C., and Yahr, T. L. (2015). The RNA helicase DeaD stimulates ExsA translation To promote expression of the Pseudomonas aeruginosa type III secretion system. J. Bacteriol. 197, 2664-2674. doi: $10.1128 /$ jb.00231-215

Iost, I., Bizebard, T., and Dreyfus, M. (2013). Functions of DEAD-box proteins in bacteria: current knowledge and pending questions. BBA-Gene Regul. Mech. 1829, 866-877. doi: 10.1016/j.bbagrm.2013.01.012

Jo, J. T., Brinkman, F. S., and Hancock, R. E. (2003). Aminoglycoside efflux in Pseudomonas aeruginosa: involvement of novel outer membrane proteins. Antimicrob. Agents Chemother. 47, 1101-1111. doi: 10.1128/AAC.47.3.11011111.2003

Kaberdin, V. R., and Bläsi, U. (2013). Bacterial helicases in posttranscriptional control. BBA-Gene Regul. Mech. 1829, 878-883. doi: 10.1016/j.bbagrm.2012.12.005

Kai, T., Tateda, K., Kimura, S., Ishii, Y., Ito, H., Yoshida, H., et al. (2009). A low concentration of azithromycin inhibits the mRNA expression of $\mathrm{N}$-acyl homoserine lactone synthesis enzymes, upstream of lasI or rhlI, in Pseudomonas aeruginosa. Pulm. Pharmacol. Ther. 22, 483-486. doi: 10.1016/j.pupt.2009.04.004

Kipnis, E., Sawa, T., and Wiener-Kronish, J. (2006). Targeting mechanisms of Pseudomonas aeruginosa pathogenesis. Med. Maladies Infect. 36, 78-91. doi: 10.1016/j.medmal.2005.10.007

Köhler, T., Dumas, J.-L., and Van Delden, C. (2007). Ribosome protection prevents azithromycin-mediated quorum-sensing modulation and stationaryphase killing of Pseudomonas aeruginosa. Antimicrob. Agents Chemother. 51, 4243-4248. doi: 10.1128/AAC.00613-07

Koo, J. T., Choe, J., and Moseley, S. L. (2004). HrpA, a DEAH-box RNA helicase, is involved in mRNA processing of a fimbrial operon in Escherichia coli. Mol. Microbiol. 52, 1813-1826. doi: 10.1111/j.1365-2958.2004.04099.x

Kurachi, M. (1958). Studies on the biosynthesis of pyocyanine. (II) : isolation and determination of pyocyanine. Bull. Inst. Chem. Res. Kyoto Univ. 36, 174-187.

Lau, C. H.-F., Fraud, S., Jones, M., Peterson, S. N., and Poole, K. (2012). Reduced expression of the rplU-rpmA ribosomal protein operon in mexXY-expressing pan-aminoglycoside-resistant mutants of Pseudomonas aeruginosa. Antimicrob. Agents Chemother. 56, 5171-5179. doi: 10.1128/AAC. 00846-12

Lawrence, M. G., Lindahl, L., and Zengel, J. M. (2008). Effects on translation pausing of alterations in protein and RNA components of the ribosome exit tunnel. J. Bacteriol. 190, 5862-5869. doi: 10.1128/JB.00632-638

Legssyer, R., Huaux, F., Lebacq, J., Delos, M., Marbaix, E., Lebecque, P., et al. (2006). Azithromycin reduces spontaneous and induced inflammation in DeltaF508 cystic fibrosis mice. Respir. Res. 7:134. doi: 10.1186/1465-9921-7-134

Liao, J., Schurr, M. J., and Sauer, K. (2013). The MerR-Like regulator BrlR confers biofilm tolerance by activating multidrug efflux pumps in Pseudomonas aeruginosa biofilms. J. Bacteriol. 195, 3352-3363. doi: 10.1128/jb. 00318-313

Liberati, N. T., Urbach, J. M., Miyata, S., Lee, D. G., Drenkard, E., Wu, G., et al. (2006). An ordered, nonredundant library of Pseudomonas aeruginosa strain PA14 transposon insertion mutants. Proc. Natl. Acad. Sci. U.S.A. 103, 2833-2838. doi: 10.1073/pnas.0511100103

Linder, P., and Fuller-Pace, F. V. (2013). Looking back on the birth of DEAD-box RNA helicases. BBA-Gene Regul. Mech. 1829, 750-755. doi: 10.1016/j.bbagrm.2013.03.007

Linder, P., and Jankowsky, E. (2011). From unwinding to clamping-the DEAD box RNA helicase family. Nat. Rev. Mol. Cell Biol. 12, 505-516. doi: $10.1038 / \mathrm{nrm} 3154$

Lister, P. D., Wolter, D. J., and Hanson, N. D. (2009). Antibacterialresistant Pseudomonas aeruginosa: clinical impact and complex regulation of chromosomally encoded resistance mechanisms. Clin. Microbiol. Rev. 22, 582-610. doi: 10.1128/CMR.00040-09

Liu, Y.-C., Chan, K.-G., and Chang, C.-Y. (2015). Modulation of host biology by Pseudomonas aeruginosa quorum sensing signal molecules: messengers or traitors. Front. Microbiol. 6:1226. doi: 10.3389/fmicb.2015.01226
López, D., Vlamakis, H., and Kolter, R. (2010). Biofilms. Cold Spring Harb. Perspect. Biol. 2:a000398. doi: 10.1101/cshperspect.a000398

Lovmar, M., Tenson, T., and Ehrenberg, M. (2004). Kinetics of macrolide action: the josamycin and erythromycin cases. J. Biol. Chem. 279, 53506-53515. doi: 10.1074/jbc.M401625200

Lovmar, M., Vimberg, V., Lukk, E., Nilsson, K., Tenson, T., and Ehrenberg, M. (2009). Cis-acting resistance peptides reveal dual ribosome inhibitory action of the macrolide josamycin. Biochimie 91, 989-995. doi: 10.1016/j.biochi.2009.05.002

Molinari, G., Guzman, C., Pesce, A., and Schito, G. (1993). Inhibition of Pseudomonas aeruginosa virulence factors by subinhibitory concentrations of azithromycin and other macrolide antibiotics. J. Antimicrob. Chemother. 31, 681-688. doi: $10.1093 /$ jac/31.5.681

Molinari, G., Paglia, P., and Schito, G. (1992). Inhibition of motility of Pseudomonas aeruginosa and Proteus mirabilis by subinhibitory concentrations of azithromycin. Eur. J. Clin. Microbiol. Infect. Dis. 11, 469-471. doi: 10.1007/ BF01961867

Morita, Y., Tomida, J., and Kawamura, Y. (2013). Responses of Pseudomonas aeruginosa to antimicrobials. Front. Microbiol. 4:422. doi: 10.3389/fmicb.2013.00422

Nalca, Y., Jansch, L., Bredenbruch, F., Geffers, R., Buer, J., and Hussler, S. (2006). Quorum-sensing antagonistic activities of azithromycin in Pseudomonas aeruginosa PAO1: a global approach. Antimicrob. Agents Chemother. 50, 16801688. doi: 10.1128/aac.50.5.1680-1688.2006

Oun, S., Redder, P., Didier, J. P., Francois, P., Corvaglia, A. R., Buttazzoni, E., et al. (2013). The CshA DEAD-box RNA helicase is important for quorum sensing control in Staphylococcus aureus. RNA Biol. 10, 157-165. doi: 10.4161/rna.22899

Owttrim, G. W. (2013). RNA helicases: diverse roles in prokaryotic response to abiotic stress. RNA Biol. 10, 96-110. doi: 10.4161/rna.22638

Pérez-Martínez, I., and Haas, D. (2011). Azithromycin inhibits expression of the GacA-dependent small RNAs RsmY and RsmZ in Pseudomonas aeruginosa. Antimicrob. Agents Chemother. 55, 3399-3405. doi: 10.1128/AAC.01801-10

Poole, K. (2011). Pseudomonas aeruginosa: resistance to the max. Front. Microbiol. 2:65. doi: 10.3389/fmicb.2011.00065

Prud'homme-Généreux, A., Beran, R. K., Iost, I., Ramey, C. S., Mackie, G. A., and Simons, R. W. (2004). Physical and functional interactions among RNase E, polynucleotide phosphorylase and the cold-shock protein, CsdA: evidence for a cold shock degradosome. Mol. Microbiol. 54, 1409-1421. doi: 10.1111/j.13652958.2004.04360.x

Putnam, A. A., and Jankowsky, E. (2013). DEAD-box helicases as integrators of RNA, nucleotide and protein binding. BBA-Gene Regul. Mech. 1829, 884-893. doi: 10.1016/j.bbagrm.2013.02.002

Rabin, H. R., Butler, S. M., Wohl, M. E. B., Geller, D. E., Colin, A. A., Schidlow, D. V., et al. (2004). Pulmonary exacerbations in cystic fibrosis. Pediatr. Pulmonol. 37, 400-406. doi: 10.1002/ppul.20023

Rada, B., and Leto, T. L. (2013). Pyocyanin effects on respiratory epithelium: relevance in Pseudomonas aeruginosa airway infections. Trends Microbiol. 21, 73-81. doi: 10.1016/j.tim.2012.10.004

Sadikot, R. T., Blackwell, T. S., Christman, J. W., and Prince, A. S. (2005). Pathogen-host interactions in Pseudomonas aeruginosa pneumonia. Am. J. Respir. Crit. Care Med. 171, 1209-1223. doi: 10.1164/rccm.200408-1044SO

Saiman, L., Marshall, B. C., Mayer-Hamblett, N., Burns, J. L., Quittner, A. L., Cibene, D. A., et al. (2003). Azithromycin in patients with cystic fibrosis chronically infected with Pseudomonas aeruginosa: a randomized controlled trial. JAMA 290, 1749-1756. doi: 10.1001/jama.290.13.1749

Salman-Dilgimen, A., Hardy, P. O., Dresser, A. R., and Chaconas, G. (2011). HrpA, a DEAH-Box RNA helicase, is involved in global gene regulation in the lyme disease spirochete. PLoS ONE 6: e22168. doi: 10.1371/journal.pone.0022168

Salman-Dilgimen, A., Hardy, P. O., Radolf, J. D., Caimano, M. J., and Chaconas, G. (2013). HrpA, an RNA helicase involved in RNA processing, is required for mouse infectivity and tick transmission of the lyme disease spirochete. PLoS Pathog. 9:e1003841. doi: 10.1371/journal.ppat.1003841

Shajani, Z., Sykes, M. T., and Williamson, J. R. (2011). Assembly of bacterial ribosomes. Annu. Rev. Biochem. 80, 501-526. doi: 10.1146/annurev-biochem062608-160432

Silvers, J. A., and Champney, W. S. (2005). Accumulation and turnover of 23S ribosomal RNA in azithromycin-inhibited ribonuclease mutant strains of Escherichia coli. Arch. Microbiol. 184, 66-77. doi: 10.1007/s00203-005-0017-10 
Simon, R., Priefer, U., and Puhler, A. (1983). A broad host range mobilization system for in vivo genetic engineering: transposon mutagenesis in Gramnegative bacteria. Nat. Biotechnol. 1, 784-791. doi: 10.1038/nbt1183-784

Singh, P. K., Schaefer, A. L., Parsek, M. R., Moninger, T. O., Welsh, M. J., and Greenberg, E. P. (2000). Quorum-sensing signals indicate that cystic fibrosis lungs are infected with bacterial biofilms. Nature 407, 762-764. doi: $10.1038 / 35037627$

Skindersoe, M. E., Alhede, M., Phipps, R., Yang, L., Jensen, P. O., Rasmussen, T. B., et al. (2008). Effects of antibiotics on quorum sensing in Pseudomonas aeruginosa. Antimicrob. Agents Chemother. 52, 3648-3663. doi: 10.1128/AAC.01230-07

Son, M. S., Matthews, W. J. Jr., Kang, Y., Nguyen, D. T., and Hoang, T. T. (2007). In vivo evidence of Pseudomonas aeruginosa nutrient acquisition and pathogenesis in the lungs of cystic fibrosis patients. Infect. Immun. 75, 5313-5324. doi: 10.1128/IAI.01807-06

Starosta, A. L., Karpenko, V. V., Shishkina, A. V., Mikolajka, A., Sumbatyan, N. V., Schluenzen, F., et al. (2010). Interplay between the ribosomal tunnel, nascent chain, and macrolides influences drug inhibition. Chem. Biol. 17, 504-514. doi: 10.1016/j.chembiol.2010.04.008

Steel, H. C., Theron, A. J., Cockeran, R., Anderson, R., and Feldman, C. (2012). Pathogen-and host-directed anti-inflammatory activities of macrolide antibiotics. Mediators Inflamm. 2012:584262. doi: 10.1155/2012/584262

Steinkamp, G., Schmitt-Grohe, S., Döring, G., Staab, D., Pfründer, D., Beck, G., et al. (2008). Once-weekly azithromycin in cystic fibrosis with chronic Pseudomonas aeruginosa infection. Respir. Med. 102, 1643-1653. doi: 10.1016/j.rmed.2008.03.009

Tanner, N. K., and Linder, P. (2001). DExD/H box RNA helicases: from generic motors to specific dissociation functions. Mol. Cell 8, 251-262. doi: 10.1016/S1097-2765(01)00329-X

Tateda, K., Comte, R., Pechere, J.-C., Köhler, T., Yamaguchi, K., and Van Delden, C. (2001). Azithromycin inhibits quorum sensing in Pseudomonas aeruginosa. Antimicrob. Agents Chemother. 45, 1930-1933. doi: 10.1128/AAC.45.6.19301933.2001

Tenson, T., Lovmar, M., and Ehrenberg, M. (2003). The mechanism of action of macrolides, lincosamides and streptogramin B reveals the nascent peptide exit path in the ribosome. J. Mol. Biol. 330, 1005-1014. doi: 10.1016/S00222836(03)00662-4

Tsai, W. C., Hershenson, M. B., Zhou, Y., and Sajjan, U. (2009). Azithromycin increases survival and reduces lung inflammation in cystic fibrosis mice. Inflamm. Res. 58, 491-501. doi: 10.1007/s00011-0090015-9

Wei, Q., Tarighi, S., Dötsch, A., Häussler, S., Müsken, M., Wright, V. J., et al. (2011). Phenotypic and genome-wide analysis of an antibiotic-resistant small colony variant (SCV) of Pseudomonas aeruginosa. PLoS ONE 6:e29276. doi: 10.1371/journal.pone.0029276

Wilson, D. N. (2014). Ribosome-targeting antibiotics and mechanisms of bacterial resistance. Nat. Rev. Microbiol. 12, 35-48. doi: 10.1038/nrmicro3155

Winsor, G. L., Griffiths, E. J., Lo, R., Dhillon, B. K., Shay, J. A., and Brinkman, F. S. (2016). Enhanced annotations and features for comparing thousands of Pseudomonas genomes in the Pseudomonas genome database. Nucleic Acids Res. 44, D646-D653. doi: 10.1093/nar/gkv1227

Winsor, G. L., Lam, D. K. W., Fleming, L., Lo, R., Whiteside, M. D., Yu, N. Y., et al. (2011). Pseudomonas genome database: improved comparative analysis and population genomics capability for Pseudomonas genomes. Nucleic Acids Res. 39, D596-D600. doi: 10.1093/nar/gkq869

Zheng, L., Baumann, U., and Reymond, J.-L. (2004). An efficient one-step sitedirected and site-saturation mutagenesis protocol. Nucleic Acids Res. 32:e115. doi: 10.1093/nar/gnh110

Conflict of Interest Statement: The authors declare that the research was conducted in the absence of any commercial or financial relationships that could be construed as a potential conflict of interest.

Copyright (c) 2016 Tan, Zhang, Weng, Chen, Zhu, Jin, Cheng, Jin and Wu. This is an open-access article distributed under the terms of the Creative Commons Attribution License (CC BY). The use, distribution or reproduction in other forums is permitted, provided the original author(s) or licensor are credited and that the original publication in this journal is cited, in accordance with accepted academic practice. No use, distribution or reproduction is permitted which does not comply with these terms. 
OPEN ACCESS

Edited by:

Ivan Rychlik,

Veterinary Research Institute,

Czech Republic

Reviewed by:

Helen Zgurskaya

University of Oklahoma, USA

Alessandra Polissi,

Università degli Studi di

Milano-Bicocca, Italy

Kunihiko Nishino,

The Institute of Scientific and

Industrial Research, Osaka University,

Japan

*Correspondence:

Jean-Marie Pagès

jean-marie.pages@univ-amu.fr

Specialty section:

This article was submitted to Antimicrobials, Resistance

and Chemotherapy,

a section of the journal

Frontiers in Microbiology

Received: 07 January 2016 Accepted: 15 April 2016

Published: 03 May 2016

Citation:

Otrębska-MachajE, ChevalierJ, HandzlikJ, SzymańskaE SchabikowskiJ, Boyer G, Bolla J-M, Kieć-Kononowicz K, Pagès J-M and AlibertS (2016) Efflux Pump Blockers in Gram-Negative Bacteria: The New Generation of Hydantoin Based-Modulators to Improve Antibiotic Activity.

Front. Microbiol. 7:622. doi: 10.3389/fmicb.2016.00622

\section{Efflux Pump Blockers in Gram-Negative Bacteria: The New Generation of Hydantoin Based-Modulators to Improve Antibiotic Activity}

\section{Ewa Otrębska-Machaj1,2, Jacqueline Chevalier², Jadwiga Handzlik¹, Ewa Szymańska1, Jakub Schabikowski', Gérard Boyer ${ }^{2}$, Jean-Michel Bolla², Katarzyna Kieć-Kononowicz ${ }^{1}$, Jean-Marie Pagès ${ }^{2 *}$ and Sandrine Alibert ${ }^{2}$}

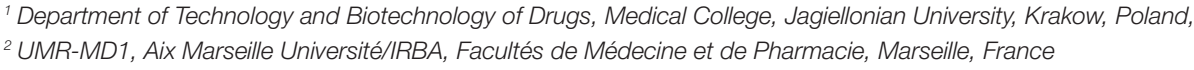

Multidrug resistant (MDR) bacteria are an increasing health problem with the shortage of new active antibiotic agents. Among effective mechanisms that contribute to the spread of MDR Gram-negative bacteria are drug efflux pumps that expel clinically important antibiotic classes out of the cell. Drug pumps are attractive targets to restore the susceptibility toward the expelled antibiotics by impairing their efflux activity. Arylhydantoin derivatives were investigated for their potentiation of activities of selected antibiotics described as efflux substrates in Enterobacter aerogenes expressing or not AcrAB pump. Several compounds increased the bacterial susceptibility toward nalidixic acid, chloramphenicol and sparfloxacin and were further pharmacomodulated to obtain a better activity against the AcrAB producing bacteria.

Keywords: antibiotics, AcrAB pump, efflux pump blocker, hydantoin, multidrug resistance

\section{INTRODUCTION}

Multidrug resistance (MDR) described in Gram-negative bacteria is continuously emerging as a prominent worldwide health concern (Chopra et al., 2008; Laxminarayan et al., 2008; Gandhi et al., 2010). One of the most contributing mechanisms is the overexpression of efflux pumps that are involved in bacterial survival, colonization and virulence (Delmar et al., 2014; Blair et al., 2015; Li et al., 2015; Venter et al., 2015; Davin-Regli et al., 2016). Several efflux pump superfamilies, e.g., major-facilitator (MF), multi-drug and toxic efflux (MATE), ATP-binding cassette (ABC), small multidrug resistance (SMR), resistance-nodulation-division (RND) transporters have been classified $^{1,2}$ and extensively described in well-documented reviews: they differ by their functional structure and organization, their subcellular location inside the bacterium, their energy source (e.g., membrane potential for RND or ATP for ABC) and the involvement of a coupled antiport during the antibiotic expulsion (e.g., proton for AcrAB pump; Delmar et al., 2014; Blair et al., 2015; Li et al., 2015). The overexpression of Gram-negative efflux pumps, especially those belonging to the

\footnotetext{
${ }^{1}$ http://www.membranetransport.org/

${ }^{2} \mathrm{http}: / / \mathrm{www} . t \mathrm{cdb} .0 \mathrm{rg} /$
} 
RND family, is now well-described in resistant isolates (Nikaido and Pagès, 2012; Li et al., 2015). This contributes to the acquisition of additional mechanisms of resistance including the mutation in antibiotic targets (e.g., mutation in gyrase/topoisomerase for quinolone) or the production of enzymes that degrade antibiotics (e.g., $\beta$-lactamases) and this can be associated or not with the alteration of the outer membrane permeability (Davin-Regli et al., 2008; Pagès et al., 2008). Regarding resistant clinical isolates of Gram-negative bacteria, the archetype of the drug active transporter system is the AcrAB-TolC/MexAB-OprM efflux pumps (Nikaido and Pagès, 2012; Li et al., 2015). The structures of components of efflux systems belonging to RND group, have been solved by $\mathrm{X}$-ray crystallography and models of the pump assembly have been obtained (Yao et al., 2010; Du et al., 2014). The structure and function of the RND efflux pumps must be molecularly deciphered thus allowing the rational design and the synthesis of new compounds to combat MDR. The broad selectivity of efflux pumps makes difficult the identification of precise pharmacophoric groups at the drug surface. However, efflux pumps are attractive target by blocking this efflux mechanism in order to restore the intracellular concentration of antibacterial agents (Bolla et al., 2011; Ruggerone et al., 2013; Dreier and Ruggerone, 2015; Opperman and Nguyen, 2015). Recent computer docking analyses have produced some information about the involvement of certain amino acid residues, but clearly more chemical and biological information are needed to improve models (Schulz et al., 2010; Fischer et al., 2014). This is a 'key' point not only regarding the mode of action and dynamics of the process but also regarding the clinical impact of the design of new antibacterial agents: this last aspect is illustrated by the $\beta$-lactamases inhibitors currently used today (Bolla et al., 2011; Chen et al., 2013; Pucci and Bush, 2013).

To enhance the activity of old antibiotics by targeting resistance mechanisms in clinical resistant isolates, they can be combined with adjuvant molecules such as chemosensitizers (e.g., membrane permeabilizer or efflux inhibitor; Jones, 2010; Bolla et al., 2011). In addition, these types of transporter inhibitors may impair the activity of efflux pumps and thus reduce bacterial colonization and virulence (Venter et al., 2015; Davin-Regli et al., 2016). It must be noted that the efficacy of the inhibitors depends on their affinity for transporter binding sites (compared to the antibiotic) and their internal concentration close to the efflux pump. Consequently, due to these parameters associated with penetration rate and affinity for pump sites, some discrepancies can be observed in the level of the internally accumulated antibiotics depending on the bacterial backgrounds (Kašćáková et al., 2012; Cinquin et al., 2015).

Recently, two generations of hydantoin derivatives have been identified as AcrAB-TolC inhibitors with the Enterobacter aerogenes CM 64 strain that overproduces AcrAB (Handzlik et al., 2011). Compounds showing chemosensitizing effect on nalidixic acid activity were the starting point for new pharmacomodulations carried out in this study to obtain a new generation of products with an improved activity.
Moreover, tests were extended to other chemically unrelated antibiotics, chloramphenicol and sparfloxacin, for which the antibacterial activity decreased together with the emergence of multidrug-resistant strains. Several chemical derivatives were synthesized to define pharmacophoric groups important for restoring the activity of antibiotics in $\mathrm{Acr} A \mathrm{~B}$ active bacteria.

\section{MATERIALS AND METHODS}

\section{Bacterial Strains}

All generations of hydantoin derivatives were tested against two strains of E. aerogenes: the reference strain, ATCC 13048 (basal efflux producer) and its derivative strain, CM 64, a chloramphenicol-selected resistant strain that over-produces the AcrAB-TolC EPs (Ghisalberti et al., 2005). In addition, two isogenic strains EA289 (a clinical MDR strain that overproduced the AcrAB pump) and its EA294 derivative (an $\operatorname{acr} A B$ knockout strain) were used (Pradel and Pagès, 2002). These two strains contain additional mechanisms of antibiotic resistance such as Blactamases, targets mutations, etc. (Malléa et al., 1998; Pradel and Pagès, 2002; Kaščáková et al., 2012).

\section{Chemicals}

Hydantoin derivatives used in the pharmacological assays were obtained by chemical synthesis. The new generations IIIA and B were obtained using 3-4-step synthesis (Handzlik et al., 2014; Matys et al., 2015; Figure 1 and Supplementary data). Purity and identity of new compounds were confirmed using spectral analysis (H-NMR, IR), elemental analysis and melting point measurements. Phenylalanine-Arginine $\beta$-naphthylamide $(\mathrm{PA} \beta \mathrm{N}$, dihydrochloride, Sigma) previously described as efflux pump inhibitor was used as reference (Bolla et al., 2011; Misra et al., 2015).

\section{Compound Susceptibility Assays}

Susceptibilities of ATCC 13048, CM 64, EA294, and EA289 were determined by using the twofold standard microbroth dilution method (microplates and automatic analyses Tecan ${ }^{\circledR} ; \mathrm{CLSI}^{3}$ ). Approximately, $10^{5} \mathrm{CFU}$ (colony forming unit) were inoculated in $200 \mu \mathrm{l}$ of Mueller-Hinton II broth (MH II broth cation adjusted, Becton, Dickinson \& Company) containing twofold serial dilutions of the targeted molecule. Experiments were performed in triplicate for each compound and each antibiotic. Results were estimated visually after $18 \mathrm{~h}$ incubation at $37^{\circ} \mathrm{C}$ (Philippe et al., 2015).

\section{Antibiotic Susceptibility Potentiating Assays}

To assay the possible chemosensitizing activity of compounds, serial dilutions of antibiotics, nalidixic acid (NAL, Sigma), chloramphenicol (CHL, Sigma), doxycycline (DOX, hyclate, Sigma), erythromycin (ERY, lactobionate, AMDIPHARM) and

\footnotetext{
${ }^{3}$ http://clsi.org/
} 


\section{Generation IIIA}

\begin{tabular}{ccc}
\hline Compound \\
\hline 32
\end{tabular}<smiles>CCC1NC(=O)N(NN)C1=O</smiles>

Generation IIIB

\begin{tabular}{cc}
\hline Compound & $\mathrm{R}^{1}$ \\
\hline $\mathbf{3 3}$ & $\mathrm{H}$ \\
$\mathbf{3 4}$ & $-\mathrm{CH}_{3}$ \\
$\mathbf{3 5}$ & $-\mathrm{CH}_{2} \mathrm{COOC}_{2} \mathrm{H}_{5}$ \\
& \\
$\mathbf{3 6}$ & \\
\hline
\end{tabular}<smiles>CCC1(c2ccc(F)cc2)NC(=O)N(CC(O)CN2CCN(P)CC2)C1=O</smiles>

FIGURE 1 | Compounds of generation IIIA and generation IIIB of hydantoins.

sparfloxacin (SPX, Sigma), were incubated in the absence or in the presence of compounds. The antibiotics, NAL, CHL, and SPX are substrates of the AcrAB-TolC efflux pump as demonstrated by the increased MIC values obtained in CM 64 strain overexpressing the AcrAB-TolC pump (Table 1)

TABLE 1 | Susceptibility (MIC) of the Enterobacter aerogenes reference strain ATCC 13048, the derivative strain CM 64 overexpressing AcrAB-ToIC pump, Ea289 overproducing the AcrAB pump and its derivative Ea294 (an acrAB knockout strain) to the different compounds belonging to various generations of hydantoins and to nalidixic acid (NAL), chloramphenicol (CHL), sparfloxacin (SPX), doxycycline (DOX), and erythromycin (ERY).

\begin{tabular}{lllll}
\hline Compound & $\begin{array}{l}\text { MIC [mM] } \\
\text { ATCC 13048 }\end{array}$ & $\begin{array}{l}\text { MIC [mM] } \\
\text { CM 64 }\end{array}$ & $\begin{array}{l}\text { MIC [mM] } \\
\text { Ea294 }\end{array}$ & $\begin{array}{l}\text { MIC [mM] } \\
\text { Ea289 }\end{array}$ \\
\hline 29-36 & $>2$ & $>2$ & $>1$ & $>1$ \\
NAL & 0.034 & $0.55(16)^{*}$ & 4.4 & $>17.6$ \\
CHL & 0.012 & $0.79(66)$ & 0.2 & 3.2 \\
SPX & 0.00015 & $0.0025(17)$ & $>2$ & $>2$ \\
DOX & 0.002 & $0.07(35)$ & 0.002 & 0.07 \\
ERY & 0.35 & $0.7(2)$ & 0.087 & 0.35 \\
PAßN & 5 & $5(1)$ & 0.0625 & 3.5 \\
\hline
\end{tabular}

*MIC ratio: efflux overproducer/basal producer strains. compared to the reference one ATCC 13048. Thus we are able to hypothesize that an efflux blocker may reduce the antibiotic MIC in efflux producer strain. Generations IIIA and IIIB of hydantoin derivatives were tested at a concentration of $0.5 \mathrm{mM}$ according to the intrinsic antibacterial activity of each compound (corresponding to the value of $\mathrm{MIC} / 4$ ). To facilitate the comparison of activity and the performance of a rational SAR analysis, they were additionally tested at the concentration corresponding to that of the best first generation of chemosensitizers $(0.0625 \mathrm{mM}$; Handzlik et al., 2011). PABN, the reference inhibitor for AcrAB pump, was used at $0.050 \mathrm{mM}$. Control experiments were carried out without compounds. Experiments were performed in triplicate for each antibiotic, each strain and each condition (without and with compound). The results were assessed after $18 \mathrm{~h}$ at $37^{\circ} \mathrm{C}$ and were presented by using the activity gain parameter A, the ratio of the MIC of the antibiotic (determined in the absence of compound) to its MIC in the presence of the compound.

\section{Determination of the FIC Index}

To determine the fractional inhibitory concentration index (FICi), a two dimensional checkerboard with twofold dilutions of each compound was set up for the study (Berenbaum, 1978; 
TABLE 2 | Effect of the hydantoin derivatives on the susceptibility level of E. aerogenes ATCC 13048 and CM 64 strains to nalidixic acid (NAL), chloramphenicol (CHL), and sparfloxacin (SPX).

\begin{tabular}{|c|c|c|c|c|c|c|c|}
\hline \multirow[t]{2}{*}{ Compound } & \multirow[t]{2}{*}{ Concentration [mM] } & \multicolumn{2}{|c|}{$A_{\text {NAL }}$} & \multicolumn{2}{|c|}{$\mathbf{A}_{\mathrm{CHL}}$} & \multicolumn{2}{|c|}{$A_{\text {SPX }}$} \\
\hline & & ATCC 13048 & СM 64 & ATCC 13048 & CM 64 & ATCC 13048 & CM 64 \\
\hline 30 & " & 2 & 2 & 2 & 1 & 1 & 1 \\
\hline 31 & $"$ & 2 & 2 & 2 & 1 & 1 & 2 \\
\hline 32 & $"$ & 2 & 4 & 1 & 1 & 1 & 2 \\
\hline 35 & $"$ & 1 & 1 & 0.5 & 1 & 0.5 & 0.5 \\
\hline 36 & $"$ & 1 & 1 & 1 & 1 & 0.5 & 0.5 \\
\hline 29 & 0.5 & 8 & 4 & 2 & 4 & 4 & 2 \\
\hline 30 & $"$ & 8 & 4 & 4 & 4 & 8 & 4 \\
\hline 31 & $"$ & 16 & 4 & 4 & 4 & 4 & 4 \\
\hline 35 & $"$ & 2 & 1 & 2 & 1 & 2 & 1 \\
\hline 36 & “ & 2 & 1 & 2 & 1 & 2 & 1 \\
\hline PABN & 0.05 & 64 & 128 & 2 & 64 & 8 & 32 \\
\hline
\end{tabular}

"A" corresponds to the antibacterial activity gain obtained in the presence of the respective compound and evaluated for each antibiotic ( $A=M I C$ without compound/MIC with compound).

Allam et al., 2014). For the first clear well in each row of the microplate containing an antimicrobial agent, the FIC was calculated as follows: FIC of compound A (FIC A) = MIC of compound $\mathrm{A}$ in combination with $\mathrm{B} / \mathrm{MIC}$ of compound $\mathrm{A}$ alone, and FIC of compound $\mathrm{B}$ (FIC B) = MIC of compound $\mathrm{B}$ in combination with A/MIC of compound $\mathrm{B}$ alone. The FICi was calculated as the sum of the FIC of each compound. The nature of the interaction was classified as follows: synergy FICi $\leq 0.5$; additivity $0.5<\mathrm{FICi} \leq 1$; indifference $1<\mathrm{FICi} \leq 2$; and antagonism FICi $>2$ (European Committee for Antimicrobial Susceptibility Testing (EUCAST) of the European Society of Clinical Microbiology and Infectious Diseases (ESCMID), 2000). For each combination, an isobologram, which graphically illustrates the interaction effect, was constructed: FIC A was showed in the abscissa and FIC B in the ordinate, the profile of the corresponding curve reflects the nature of the interaction.

\section{RESULTS}

\section{Antibacterial Activity}

It is important that putative inhibitors that could be used as "adjuvant molecule" for antibiotics, do not display a high intrinsic antibacterial activity (Davin-Regli et al., 2008; Bolla et al., 2011). The determination of the antibacterial activity for each compound was performed and presented in Table 1. Many of the compounds did not inhibit the growth of bacteria even at the highest tested concentration as the previous generations of hydantoin derivatives (Handzlik et al., 2011). The majority of compounds exhibited a MIC > $2 \mathrm{mM}$ in ATCC13048 and in CM64 strains and MIC $\geq 1 \mathrm{mM}$ in Ea289 and Ea294.

\section{Influence on Antibiotic Susceptibility in ATCC 13048 and CM 64 Strains Effect on Nalidixic Acid Susceptibility}

Table 2 presents the chemosensitizing effect of compounds on ATCC 13048 and CM 64 susceptibility to nalidixic acid (NAL). The gain on antibiotic activity (A) was calculated for each compound and each antibiotic. Regarding the CM 64 strain, a moderate or a weak effect was observed on the MIC when compounds were used at $0.0625 \mathrm{mM}$ compared to PA $\beta \mathrm{N}$. Compounds of generations IIIA (29-32) and IIIB (33-36) were used at a concentration of $0.0625 \mathrm{mM}$ and $0.5 \mathrm{mM}$ due to their higher MIC. A higher concentration of compounds of the generation IIIB did not improve the antibiotic activity whereas we observed a significant decrease of the antibiotic MIC, from 4- to 32-fold in CM 64 (see $A_{\text {NAL }}$ in Table 2), in the case of compounds of generation IIIA (29-32). These compounds decreased the MIC of NAL from 8- to 16-fold comparing to the PA $\beta N$ effect (64fold) in the reference ATCC 13048 strain (see $A_{N A L}$ in Table 2). In the case of $\mathrm{PA} \beta \mathrm{N}$ and derivatives 32 the difference in activity in both tested strains was not significant (only a twofold stronger activity in the strain overexpressing the AcrAB pump than in the reference one) whereas compounds 29-31 showed a better action on antibiotic activity in the reference strain. It must be noted that the compound $\mathbf{3 2}$ used at increased concentration exhibits an activity profile similar to PAßN, which has been shown to be an efficient efflux inhibitor at low concentrations (Nikaido and Pagès, 2012; Misra et al., 2015). 
TABLE 3 | Effect of the compounds of generation IIIA (29-32) depends on AcrAB context.

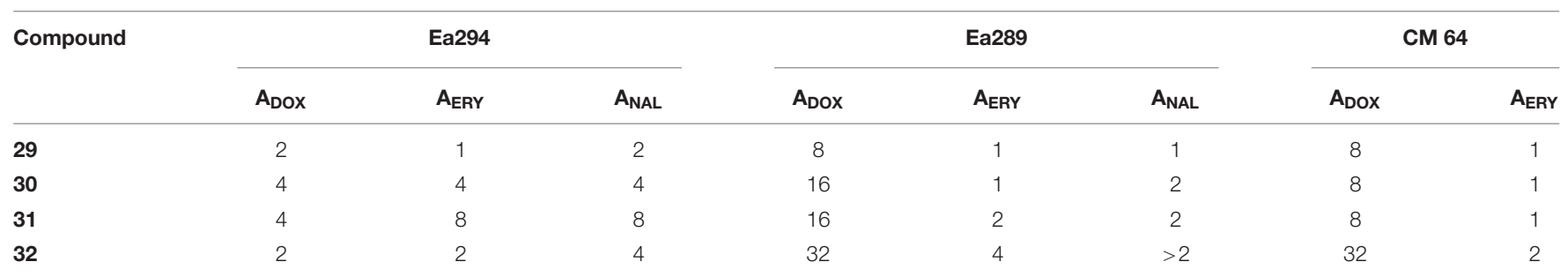

"A" corresponds to the antibacterial activity gain obtained in the presence of the respective compound and evaluated for each antibiotic $(A=$ MIC without compound/MIC with compound). DOX, doxycycline; ERY, erythromycin; NAL, nalidixic acid.

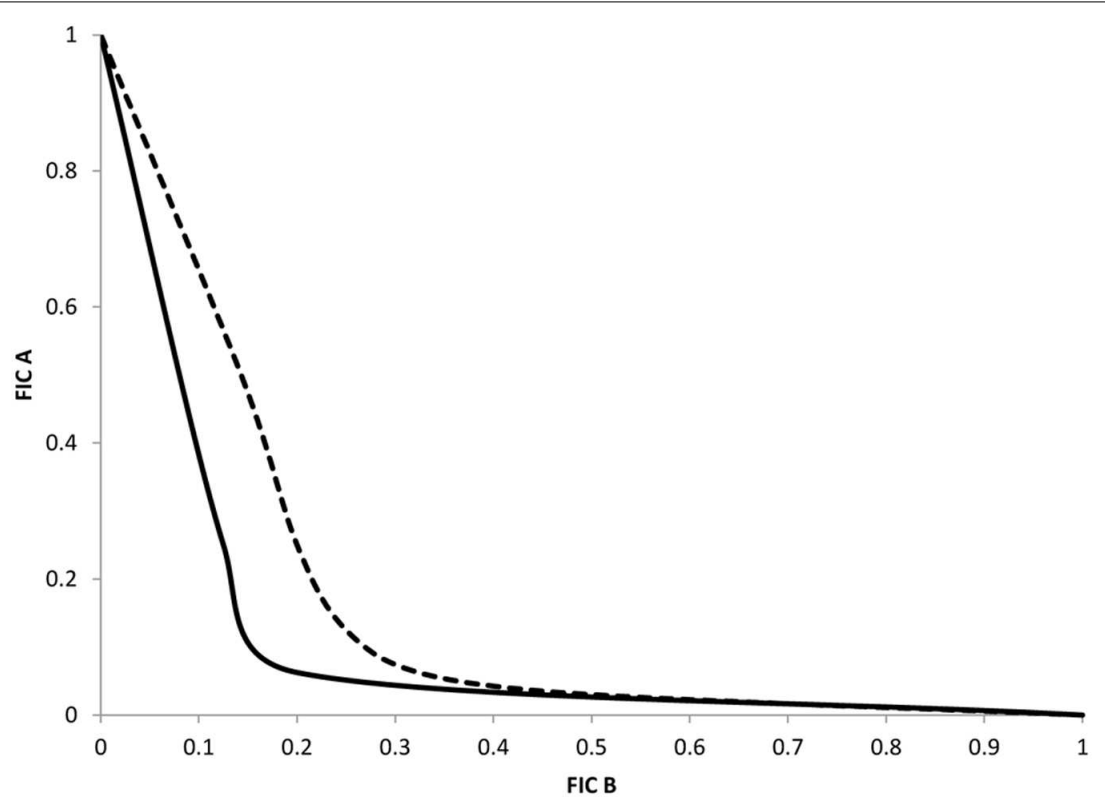

FIGURE 2 | Representative isobologram of interactions between compound 32 and nalidixic acid or chloramphenicol. Nalidixic acid, solid line; chloramphenicol, dotted line. The axis numbers correspond to normalized FICs obtained with Enterobacter aerogenes CM64 strain.

\section{Effect on Chloramphenicol Susceptibility}

The results are presented in Table 2 . In the case of the CM 64 strain, compounds tested at the concentration of $0.0625 \mathrm{mM}$ showed a weak chemosensitizing effect on $\mathrm{CHL}$ antibacterial activity compared to PA $\beta N$. Compounds of generation IIIA (29-32) examined at a concentration of $0.5 \mathrm{mM}$ decreased the chloramphenicol MIC from 2- to 4-fold in the reference strain and from 4- to 32-fold in the strain overexpressing efflux pump (see $\mathrm{A}_{\mathrm{CHL}}$ in Table 2). We observed a noticeable increase in CHL susceptibility in the AcrAB overproducer CM 64, a 32-fold gain in the susceptibility with the compound 32 compared to compounds 29-31. In this assay, the chemosensitizing effect of compound 32 can be compared to $\mathrm{PA} \beta \mathrm{N}$ which was much more active in the strain overexpressing the AcrAB pump than in the parental strain ATCC 13048.

\section{Effect on Sparfloxacin Susceptibility}

Table 2 presents the effects of hydantoins on sparfloxacin (SPX) susceptibility. In CM 64, the chemosensitizing effect of low concentrated hydantoins on SPX was as weak as we observed in the case of CHL. Compounds of generation IIIA (29-32) tested at the highest concentration $0.5 \mathrm{mM}$ caused a $4-8$-fold decrease in the MIC in the ATCC 13048 strain and a 2-8-fold decrease in the MIC in CM 64 one. Comparing the results obtained for the active compounds (29-32) to the results obtained for PA $\beta N$ (see ASPX in Table 2) we observed that the action of hydantoins with SPX was less efficient than $\operatorname{PA} \beta \mathrm{N}$, which was more active in the CM64 strain overexpressing the AcrAB pump than in the reference strain ATCC13048. This may suggest a different conformational site for the two molecules, either for recognition or for binding step, in the AcrB monomer (Delmar et al., 2014; Du et al., 2014; Yamaguchi et al., 2015), inside the pump or a different mode of action on the resistance mechanism.

\section{Effect of Compounds 29-32 on the Resistance Level in MDR E. aerogenes Strains}

In order to evaluate the chemosensitizing effect of the compounds 29-32 (the most effective molecules) on the MDR 
background, the Ea289 and Ea294 strains were assayed. It is important to mention that these strains contain various resistance mechanisms (Malléa et al., 1998; Pradel and Pagès, 2002) such as target mutations (e.g., mutations in QRDR region of gyrase that increase quinolone resistance) and expression of modifying enzymes (e.g., ß-lactamases that contribute to $ß$-lactam resistance). Table 3 presents the activity gain parameter A tested with doxycycline (DOX), erythromycin (ERY), and nalidixic acid (NAL) in parental and $A c r A B$-derivative strain context. It is interesting to note that 32 exhibited significant restoring antibiotic activity with DOX in Ea289 and very weak action in Ea294 that is devoid of the AcrAB efflux pump components. In contrast, a weak chemosensitizing activity was observed with ERY, this effect could be caused by the presence of additional resistance mechanisms for the macrolide antibiotic class as previously reported (Chollet et al., 2004). Regarding the effect on NAL and sparfloxacin susceptibility, the mutations in the quinolone target (DNA gyrase) previously reported in Ea289, can explain the weak effect observed toward these strains (Pradel and Pagès, 2002; Kaščáková et al., 2012).

\section{Determination of the FIC Index for Compound 32}

To precise the type of interaction (synergistic, additive, or indifferent) between compound 32 and selected antibiotics, we carried out analysis based on the FICi as previously described. Combinations of compound $\mathbf{3 2}$ with NAL and CHL respectively were performed in CM 64 strain. This strain overexpresses the AcrAB pump and does not contain target mutation that can impair the effect of compounds on the restoration of antibiotic activity. The nature of the association was determined from the FICi average obtained from each combination and the representation was performed for each combination (Figure 2). In two cases, the isobologram curve obtained was concave indicating a synergy association between antibiotic and compound 32. The synergistic association corresponds to an average of calculated FICi of about 0.32 for NAL and 0.44 for CHL respectively. These curves fitted well with the Table 2 data.

\section{DISCUSSION}

The aim of this study was to identify compounds that are able to modulate the AcrAB pump activity and restore antibiotic activity on efflux producing strains.

The various molecules combined with NAL and CHL increased the susceptibility of the CM 64 strain and of the reference strain ATCC 13048 to antibiotics. On the one hand, we found compounds that showed stronger activity in the ATCC 13048 strain than in the CM 64 strain (29-31 tested with NAL, 29, 30 tested with SPX). These findings could indicate in this case that tested compounds are not highly selective toward the AcrAB-TolC pump involved in the efflux of antibiotics. This suggests that they act not only on the basal AcrAB expressing strain but also that they may recognize other bacterial targets. In contrast, $P A \beta N$ combined with CHL and SPX as well as the 3-aminobutyl-5$\beta$-naphthylhydantoin 32 tested with CHL exhibited a difference between the AcrAB overproducer strain and the parental one. This time these results suggest a significant selectivity of chemosensitizers for the AcrAB pump expressed in bacterial strains. Taking into account the influence of concentration of 3-aminoalkyl-5-naphthylhydantoins (generation IIIA) on the antibiotic activity, we observed that the higher is the concentration, the stronger is the chemosensitizing effect, not only on the CM 64 strain but also on ATCC 13048. These outcomes could suggest additional mechanisms of action besides the effect on the AcrAB-TolC efflux pump.

The comparison of the results in Ea289 and Ea294 (Table 3) indicates the correlation of the restoring activity of antibiotics with the presence of the AcrAB efflux pump. They also illustrate the capability of compound $\mathbf{3 2}$ to increase the antibacterial effect of DOX in these strains. The low effect of 32 on the ERY susceptibility when assayed in Ea289 and Ea294 can be due to additional pump, other than AcrB, active in these clinical derivative strains capable to expel this class of drugs (Chollet et al., 2004).

The different structural features of tested compounds allow showing how the nature and the position of their diverse molecular fragments modulate on the one hand their selectivity regarding AcrAB pump and on the other hand their chemosensitizing activity. The tested compounds exhibiting the common hydantoin scaffold were modified using two of diverse substituents at position 5 as well as amine substituents and a selection of linkers to bind an amine to the hydantoin core. The location of an amine-alkyl fragment at position N1 or N3 to an aryl fragment at position 5 was modified for comparing with the activity of the first generations of hydantoin synthesized (Handzlik et al., 2011). In the case of 32, the hydrophilic primary amine fragment is focused by terminate location at the end of longer 3-hydantoin substitution, opposite to the 5 - $\beta$-nahthyl one.

The most active compounds (29-32) share the same pharmacophore profile than $\mathrm{PA} \beta \mathrm{N}$ suggesting a similar physicochemical outline for an identical target. The amphiphilic nature of the Generation IIIA of optimized derivatives seems to be crucial to inhibit antibiotic resistance mediated by efflux pump and open new ways for generating original active compounds able to inhibit pump activity. With the recent published data regarding piperazine arylideneimidazolone derivatives as potential efflux inhibitors in Escherichia coli cells (Bohnert et al., 2016), the compound 32 characterized in this study will be used for pharmacophoric modulations in order to develop more potent inhibitors.

\section{AUTHOR CONTRIBUTIONS}

JH, KK-K, J-MP, and SA designed research; EO-M, JC, ES, and SA performed research; JS, GB, and J-MB contributed new 
reagents or analytic tools; EO-M, JH, KK-K, J-MP, and SA analyzed data; JH, KK-K, J-MP, and SA wrote the paper.

\section{FUNDING}

This work was supported by French Embassy in Poland, 757/N-POLONIUM/2010/0, by grant ANR-11-BS07-019-01 "IBEF" Agence Nationale de la Recherche (ANR, France) and partly supported by Polish statutory project K/ZDS/005593 and Aix-Marseille University and IRBA.

\section{REFERENCES}

Allam, A., Maigre, L., Alimi, M., Alves de Sousa, R., Hessani, A., Galardon, E., et al. (2014). New peptides with metal binding abilities and their use as drug carriers. Bioconjug. Chem. 25, 1811-1819. doi: 10.1021/bc500317u

Berenbaum, M. C. (1978). A method for testing for synergy with any number of agents. J. Infect. Dis. 137, 122-130. doi: 10.1093/infdis/137.2.122

Blair, J. M., Webber, M. A., Baylay, A. J., Ogbolu, D. O., and Piddock, L. J. (2015). Molecular mechanisms of antibiotic resistance. Nat. Rev. Microbiol. 13, 42-51. doi: $10.1038 /$ nrmicro3380

Bohnert, J. A., Schuster, S., Kern, W. V., Karcz, T., Olejarz, A., Kaczor, A., et al. (2016). Novel piperazine arylideneimidazolones inhibit the AcrAB-TolC pump in Escherichia coli and simultaneously act as fluorescent membrane probes in a combined real-time influx and efflux assay. Antimicrob. Agents Chemother. 60, 1974-1983. doi: 10.1128/AAC.01995-15

Bolla, J. M., Alibert-Franco, S., Handzlik, J., Chevalier, J., Mahamoud, A., Boyer, G., et al. (2011). Strategies for bypassing the membrane barrier in multidrug resistant Gram-negative bacteria. FEBS Lett. 585, 1682-1690. doi: 10.1016/j.febslet.2011.04.054

Chen, J., Shang, X., Hu, F., Lao, X., Gao, X., Zheng, H., et al. (2013). $\beta$-Lactamase inhibitors: an update. Mini Rev. Med. Chem. 13, 1846-1861. doi: 10.2174/13895575113139990074

Chollet, R., Chevalier, J., Bryskier, A., and Pagès, J. M. (2004). The AcrAB-TolC pump is involved in macrolide resistance but not in telithromycin efflux in Enterobacter aerogenes and Escherichia coli. Antimicrob. Agents Chemother. 48, 3621-3624. doi: 10.1128/AAC.48.9.3621-3624.2004

Chopra, I., Schofield, C., Everett, M., O’Neill, A., Miller, K., Wilcox, M., et al. (2008). Treatment of health- care-associated infections caused by Gramnegative bacteria: a consensus statement. Lancet Infect. Dis. 8, 133-139. doi: 10.1016/S1473-3099(08)70018-5

Cinquin, B., Maigre, L., Pinet, E., Chevalier, J., Stavenger, R. A., Mills, S., et al. (2015). Microspectrometric insights on the uptake of antibiotics at the single bacterial cell level. Sci Rep. 5, 17968. doi: 10.1038/srep17968

Davin-Regli, A., Bolla, J. M., James, C. E., Lavigne, J. P., Chevalier, J., Garnotel, E., et al. (2008). Membrane permeability and regulation of drug influx and efflux' in enterobacterial pathogens. Curr. Drug Targets. 9, 750-759. doi: $10.2174 / 138945008785747824$

Davin-Regli, A., Masi, M., Bialek, S., Nicolas-Chanoine, M. H., and Pagès, J.M. (2016). "Antimicrobial resistance and drug efflux pumps in Enterobacter and Klebsiella," in Efflux-Mediated Drug Resistance in Bacteria: Mechanisms, Regulation and Clinical Implications, eds X.-Z. Li, C. A. Elkins, and H. I. Zgurskaya (Berlin: Springer).

Delmar, J. A., Su, C. C., and Yu, E. W. (2014). Bacterial multidrug efflux transporters. Annu. Rev. Biophys. 43, 93-117. doi: 10.1146/annurev-biophys051013-022855

Dreier, J., and Ruggerone, P. (2015). Interaction of antibacterial compounds with RND efflux pumps in Pseudomonas aeruginosa. Front Microbiol. 6:660. doi: 10.3389/fmicb.2015.00660

Du, D., Wang, Z., James, N. R., Voss, J. E., Klimont, E., Ohene-Agyei, T., et al. (2014). Structure of the AcrAB-TolC multidrug efflux pump. Nature 509, 512-515. doi: 10.1038/nature13205

European Committee for Antimicrobial Susceptibility Testing (EUCAST) of the European Society of Clinical Microbiology and Infectious Diseases (ESCMID)

\section{ACKNOWLEDGMENT}

We appreciate greatly fruitful discussions and advices from Dr. Anne Davin-Regli.

\section{SUPPLEMENTARY MATERIAL}

The Supplementary Material for this article can be found online at: http://journal.frontiersin.org/article/10.3389/fmicb. 2016.00622

(2000). Terminology relating to methods for the determination of susceptibility of bacteria to antimicrobial agents. Clin. Microbiol. Infect. 6, 503-508. doi: 10.1046/j.1469-0691.2000.00149.x

Fischer, N., Raunest, M., Schmidt, T. H., Koch, D. C., and Kandt, C. (2014). Efflux pump-mediated antibiotics resistance: insights from computational structural biology. Interdiscip. Sci. 6, 1-12.

Gandhi, T. N., DePestel, D. D., Collins, C. D., Nagel, J. J., and Washer, L. L. (2010). Managing antimicrobial resistance in intensive care units. Crit. Care Med. 38, 315-323. doi: 10.1097/CCM.0b013e3181e6a2a4

Ghisalberti, D., Masi, M., Pagès, J. M., and Chevalier, J. (2005). Chloramphenicol and expression of multidrug efflux pump in Enterobacter aerogenes. Biochem. Biophys. Res. Commun. 328, 1113-1118. doi: 10.1016/j.bbrc.2005.01.069

Handzlik, J., Bojarski, A. J., Satała, G., Kubacka, M., Sadek, B., Ashoor, A., et al. (2014). SAR-Studies on the importance of aromatic ring topologies in search for selective 5-HT7 receptor ligands among phenylpiperazine hydantoin derivatives. Eur. J. Med. Chem. 78, 324-339. doi: 10.1016/j.ejmech.2014. 01.065

Handzlik, J., Szymańska, E., Chevalier, J., Otrebska, E., Kiec-Kononowicz, K., Pagès, J. M., et al. (2011). Amine-alkyl derivatives of hydantoin: new tool to combat resistant bacteria. Eur. J. Med. Chem. 46, 5807-5816. doi: 10.1016/j.ejmech.2011.09.032

Jones, D. (2010). News and analysis: the antibacterial lead discovery challenge. Nat. Rev. Drug Discov. 9, 751-752. doi: 10.1038/nrd3289

Kaščáková, S., Maigre, L., Chevalier, J., Réfrégiers, M., and Pagès, J. M. (2012). Antibiotic transport in resistant bacteria: synchrotron UV fluorescence microscopy to determine antibiotic accumulation with single cell resolution. PLoS ONE 7:e38624. doi: 10.1371/journal.pone.0038624

Laxminarayan, R., Duse, A., Wattal, C., Zaidi, A. K. M., Wertheim, H. F. L., Sumpradit, N., et al. (2008). Antibiotic resistance - the need for global solution. Lancet Infect. Dis. 13, 1057-1098. doi: 10.1016/S1473-3099(13) 70318-9

Li, X. Z., Plésiat, P., and Nikaido, H. (2015). The challenge of efflux-mediated antibiotic resistance in Gram-negative bacteria. Clin. Microbiol. Rev. 28, 337418. doi: 10.1128/CMR.00117-14

Malléa, M., Chevalier, J., Bornet, C., Eyraud, A., Pagès, J.-M., and DavinRegli, A. (1998). Porin alteration and active efflux: two in vivo drug resistance strategies used by Enterobacter aerogenes. Microbiology 144, 3003-3009. doi: 10.1099/00221287-144-11-3003

Matys, A., Podlewska, S., Witek, K., Witek, J., Bojarski, A. J., Schabikowski, J., et al. (2015). Imidazolidine-4-one derivatives in the search for novel chemosensitizers of Staphylococcus aureus MRSA: synthesis, biological evaluation and molecular modeling studies. Eur. J. Med. Chem. 101, 313-325. doi: 10.1016/j.ejmech.2015.06.013

Misra, R., Morrison, K. D., Cho, H. J., and Khuu, T. (2015). Importance of real-time assays to distinguish multidrug efflux pump-inhibiting and outer membranedestabilizing activities in Escherichia coli. J. Bacteriol. 197, 2479-2488. doi: 10.1128/JB.02456-14

Nikaido, H., and Pagès, J. M. (2012). Broad-specificity efflux pumps and their role in multidrug resistance of Gram-negative bacteria. FEMS Microbiol. Rev. 36, 340-363. doi: 10.1111/j.1574-6976.2011.00290.x

Opperman, T. J., and Nguyen, S. T. (2015). Recent advances toward a molecular mechanism of efflux pump inhibition. Front Microbiol. 6:421. doi: $10.3389 /$ fmicb. 2015.00421 
Pagès, J. M., James, C. E., and Winterhalter, M. (2008). The porin and the permeating antibiotic: a selective diffusion barrier in Gram-negative bacteria. Nat. Rev. Microbiol. 6, 893-903. doi: 10.1038/nrmicro1994

Philippe, N., Maigre, L., Santini, S., Pinet, E., Claverie, J. M., Davin-Regli, A., et al. (2015). In vivo evolution of bacterial resistance in two cases of Enterobacter aerogenes infections during treatment with imipenem. PLoS One. 10:e138828. doi: 10.1371 /journal.pone. 0138828

Pradel, E., and Pagès, J. M. (2002). The AcrAB-TolC efflux pump contributes to multidrug resistance in the nosocomial pathogen Enterobacter aerogenes. Antimicrob. Agents Chemother. 46, 2640-2643. doi: 10.1128/AAC.46.8.26402643.2002

Pucci, M. J., and Bush, K. (2013). Investigational antimicrobial agents of 2013. Clin. Microbiol. Rev. 26, 792-821. doi: 10.1128/CMR.00033-13

Ruggerone, P., Murakami, S., Pos, K. M., and Vargiu, A. V. (2013). RND efflux pumps: structural information translated into function and inhibition mechanisms. Curr. Top. Med. Chem. 13, 3079-3100. doi: 10.2174/15680266113136660220

Schulz, R., Vargiu, A. V., Collu, F., Kleinekathoefer, U., and Ruggerone, P. (2010). Functional rotation of the transporter AcrB: insights into drug extrusion from simulations. PLoS Comput. Biol. 6:e1000806. doi: 10.1371/journal.pcbi.10 00806
Venter, H., Mowla, R., Ohene-Agyei, T., and Ma, S. (2015). RND-type drug efflux pumps from Gram-negative bacteria: molecular mechanism and inhibition. Front Microbiol. 6:377. doi: 10.3389/fmicb.2015.00377

Yamaguchi, A., Nakashima, R., and Sakurai, K. (2015). Structural basis of RND-type multidrug exporters. Front Microbiol. 6:327. doi: 10.3389/fmicb.2015.00327

Yao, X. Q., Kenzaki, H., Murakami, S., and Takada, S. (2010). Drug export and allosteric coupling in a multidrug transporter revealed by molecular simulations. Nat. Commun. 1, 1-8. doi: 10.1038/ncomms1116

Conflict of Interest Statement: The authors declare that the research was conducted in the absence of any commercial or financial relationships that could be construed as a potential conflict of interest.

Copyright (c) 2016 Otrębska-Machaj, Chevalier, Handzlik, Szymańska, Schabikowski, Boyer, Bolla, Kieć-Kononowicz, Pagès and Alibert. This is an open-access article distributed under the terms of the Creative Commons Attribution License (CC BY). The use, distribution or reproduction in other forums is permitted, provided the original author(s) or licensor are credited and that the original publication in this journal is cited, in accordance with accepted academic practice. No use, distribution or reproduction is permitted which does not comply with these terms. 


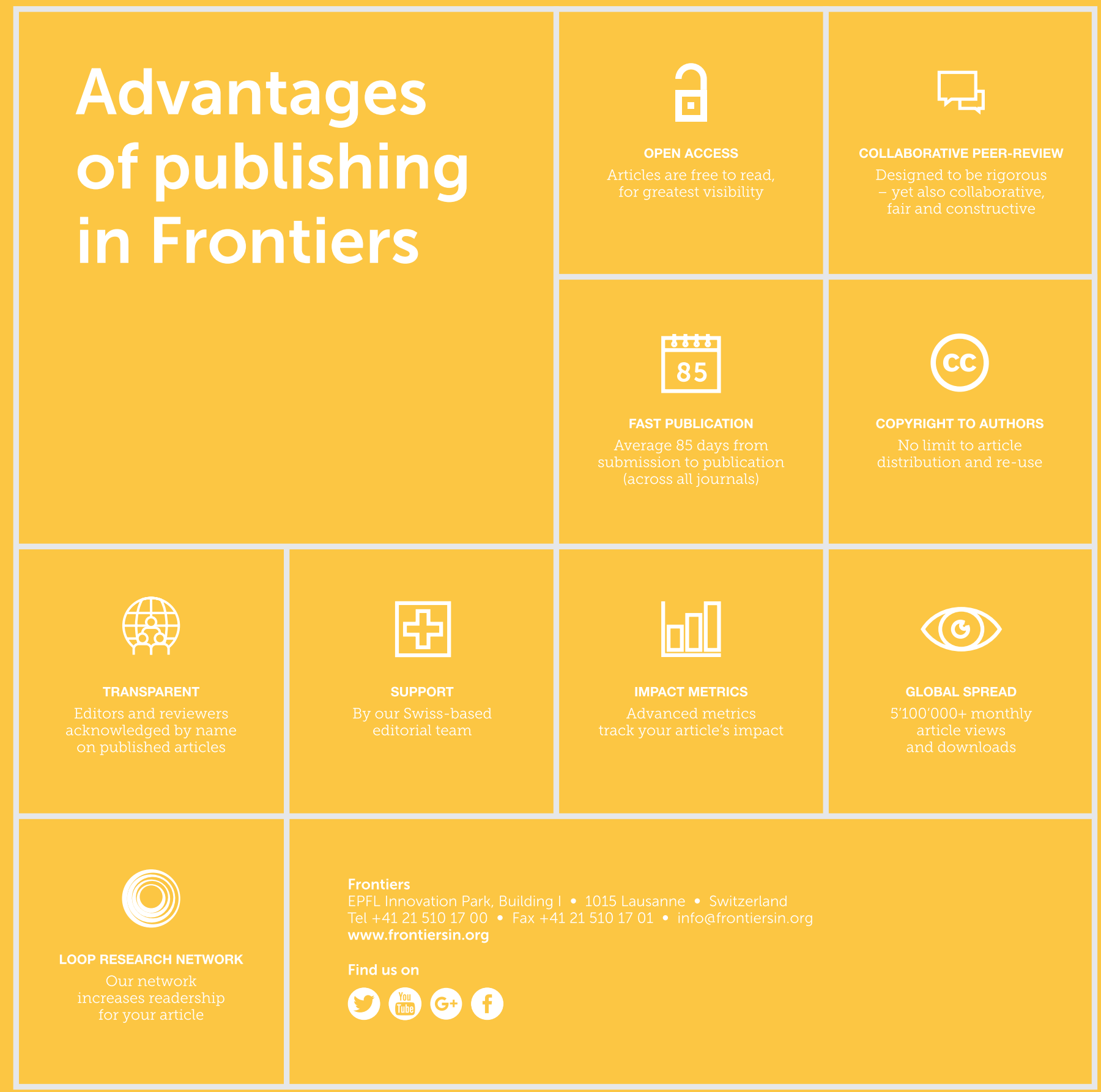

